\title{
Structure-Property Correlations in Luminescent Anthracene Derivatives
}

\author{
Dissertation \\ zur Erlangung des mathematisch-naturwissenschaftlichen Doktorgrades \\ „Doctor rerum naturalium“ \\ der Georg-August-Universität Göttingen \\ im Promotionsprogramm Chemie \\ der Georg-August University School of Science (GAUSS)
}

vorgelegt von

Timo Schillmöller

aus Vechta

Göttingen

2021 



\section{Betreuungsausschuss}

Prof. Dr. Dietmar Stalke

Institut für Anorganische Chemie, Georg-August-Universität Göttingen

Prof. Dr. Sven Schneider

Institut für Anorganische Chemie, Georg-August-Universität Göttingen

\section{Mitglieder der Prüfungskommission}

Referent:

Prof. Dr. Dietmar Stalke

Institut für Anorganische Chemie, Georg-August-Universität Göttingen

Korreferent:

Prof. Dr. Sven Schneider

Institut für Anorganische Chemie, Georg-August-Universität Göttingen

\section{Weitere Mitglieder der Prüfungskommission:}

Prof. Dr. Manuel Alcarazo Institut für Organische und Biomolekulare Chemie, Georg-AugustUniversität Göttingen

Prof. Dr. Konrad Koszinowski Institut für Organische und Biomolekulare Chemie, Georg-AugustUniversität Göttingen

Prof. Dr. Ricardo Mata

Institut für Physikalische Chemie, Georg-August-Universität Göttingen

Jun.-Prof. Dr. Anna Krawczuk Institut für Anorganische Chemie, Georg-August-Universität Göttingen

Tag der mündlichen Prüfung: 06.07.2021 



\section{Publications}

Parts of this thesis have been published separately:

- Timo Schillmöller, Paul Niklas Ruth, Regine Herbst-Irmer, Dietmar Stalke, "Three colour solid-state luminescence from positional isomers of facilely modified thiophosphoranyl anthracenes", Chem. Commun. 2020, 56, 7479-7482.

- Timo Schillmöller, Paul Niklas Ruth, Regine Herbst-Irmer, Dietmar Stalke, "Analysis of Solid-State Luminescence Emission Amplification at Substituted Anthracenes by Host-Guest Complex Formation", Chem. Eur. J. 2020, 26, 17390-17398.

invited cover profile + front cover. DOI: 10.1002/chem.202004509

- Timo Schillmöller, Regine Herbst-Irmer, Dietmar Stalke, "Insights into Excimer Formation Factors from Detailed Structural and Photophysical Studies in the Solid-State", Adv. Opt. Mater. 2021, 9, 2001814. 



\section{Table of Contents}

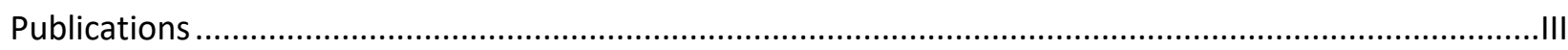

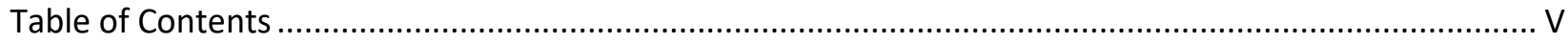

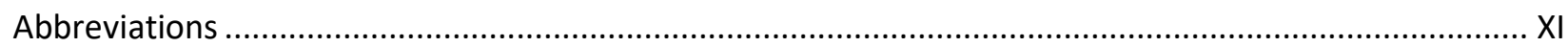

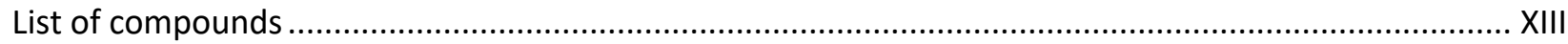

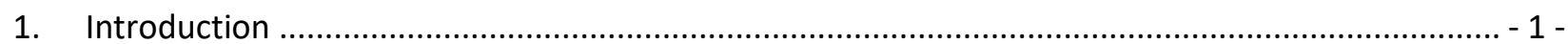

1.1 A brief historical overview on luminescence in aggregated states..................................... - 3 -

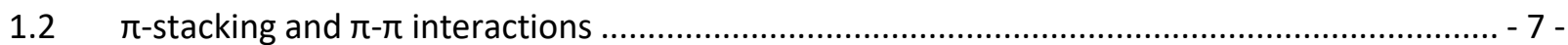

1.3 Photophysical processes in polyaromatic hydrocarbons .................................................. - $11-$

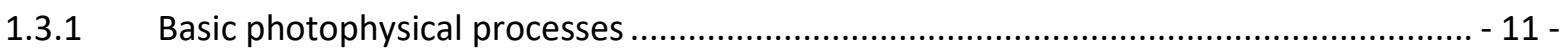

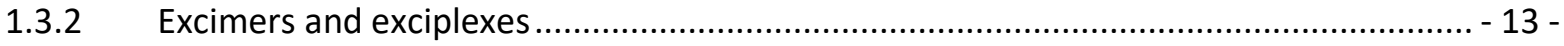

1.4 Aggregation-Induced Emission................................................................................ -24 -

1.5 Previous work regarding solid-state luminescence in the Stalke group ............................. - 27 -

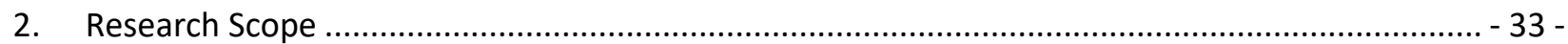

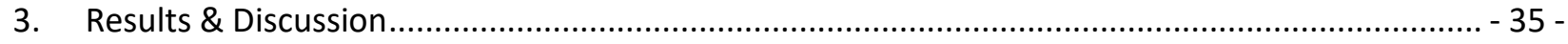

3.1 Structural and photophysical properties of 9,10-functionalized anthracene derivatives ...... - 36 -

3.1.1 Diphenylphosphanyl anthracenes ........................................................................ - 36 -

3.1.2 Investigation of the structural and photophysical influences of different substituents and

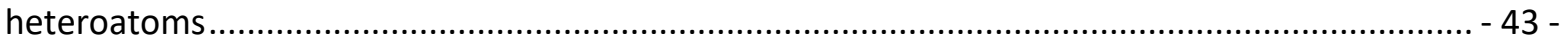

3.1.3 Influence of intra- and intermolecular structural parameters on the solid-state luminescence of thiophosphoryl anthracenes............................................................................. - 64 -

3.1.4 Solid-state emission enhancement by host-guest-complex formation ......................... - 72 -

3.2 Structural and photophysical properties of regioisomers of phosphanyl- and thiophosphoryl

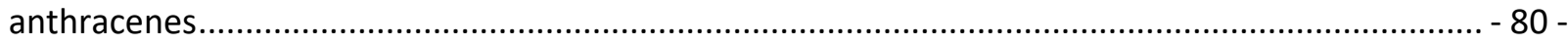

3.2.1 Evaluation of solid-state excimer formation factors based on anthracene dimers........ - 81 -

3.2.2 Examination of the influence of the substituents position in phosphanyl anthracene



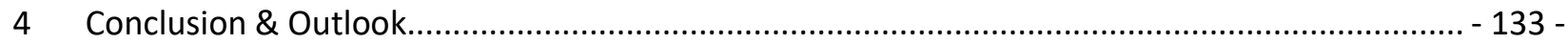

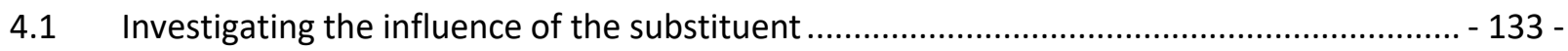

4.2 Structure-property correlations in 9,10-substituted thiophosphoryl anthracenes .............. - 135 - 
4.2.1 Controlling intermolecular interactions in thiophosphoryl anthracenes. $-135-$

4.2.2 Solid-state emission amplification through co-crystallization of small aromatic molecules- 136 -

4.3 Investigation of the phosphanyl- and thiophosphoryl anthracene regioisomers................. 136 -

4.3.1 Insights into solid-state excimer formation factors ................................................ 137 -

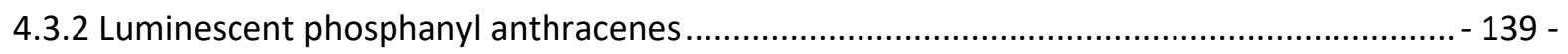

4.3.3 Conformation induced emission enhancement ….......................................................... 140 -

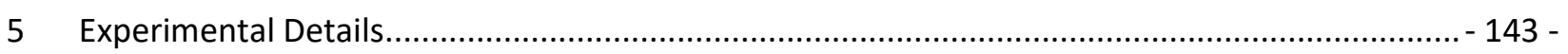

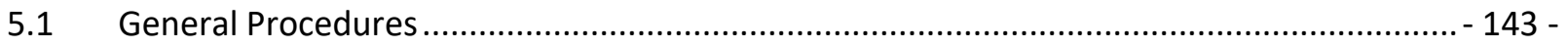

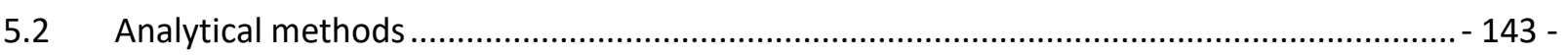

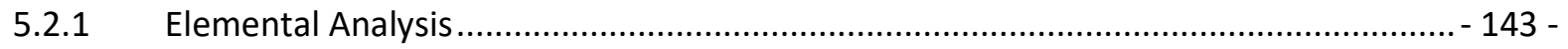

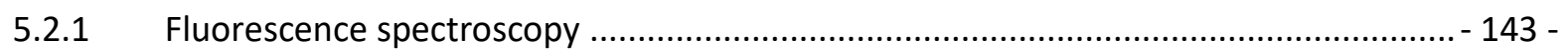

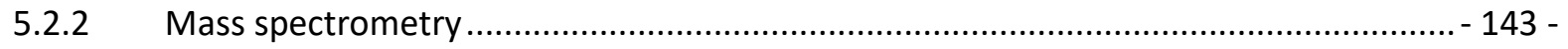

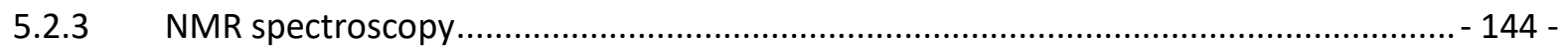

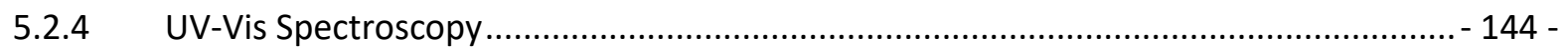

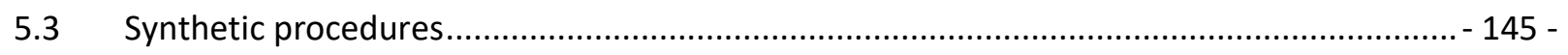

5.3.1 Synthesis of 9-Bromo-10-methylanthracene ......................................................... 145 -

5.3.2 Synthesis of 9-Bromo-10-ethylanthracene.............................................................. 145 -

5.3.3 Synthesis of 9-Bromo-10-trimethylsilylanthracene.................................................. - 146 -

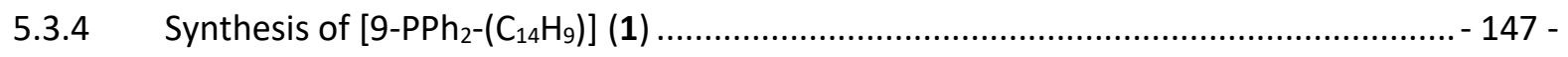

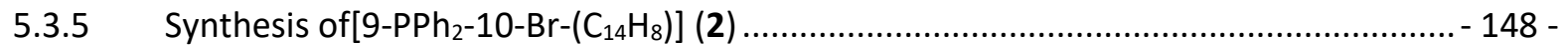

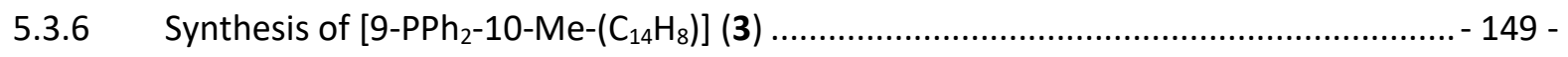

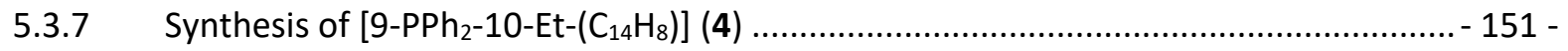

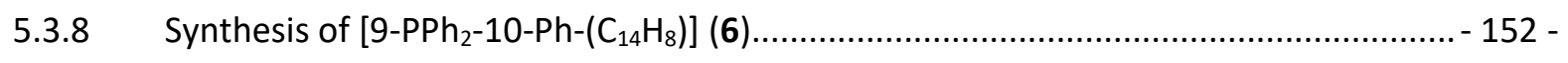

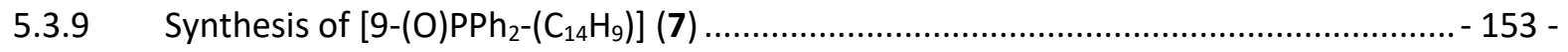

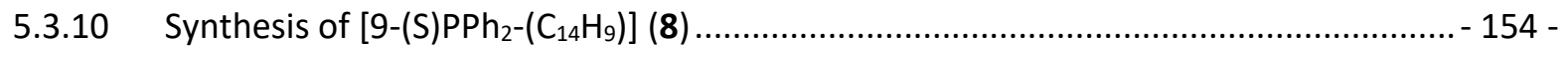

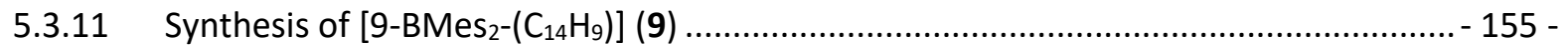

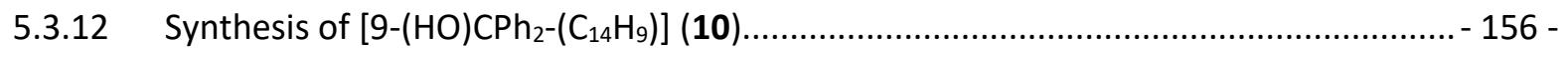

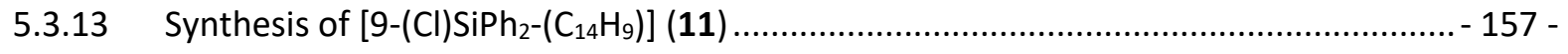

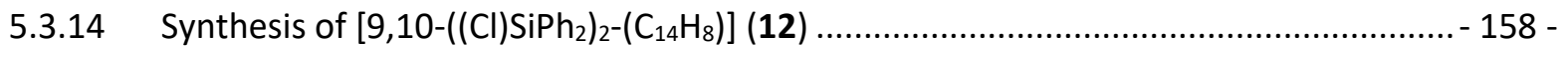

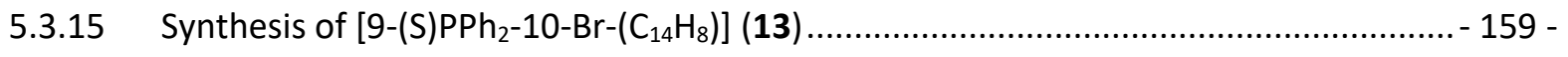




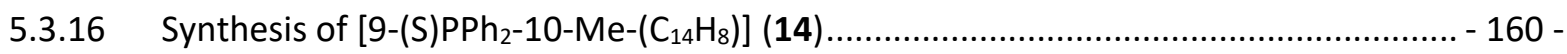

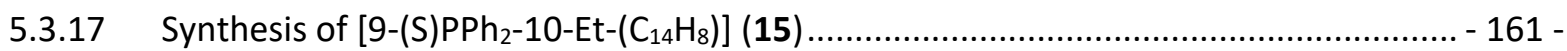

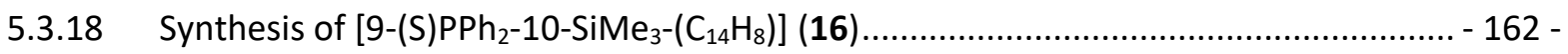

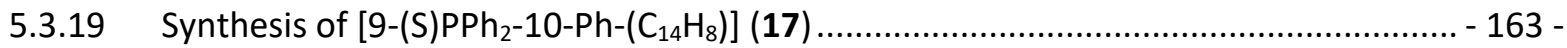

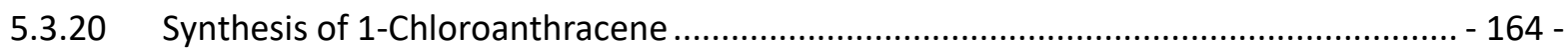

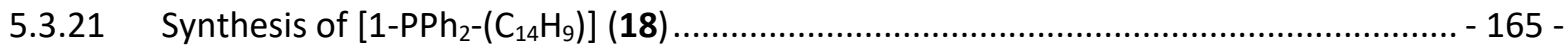

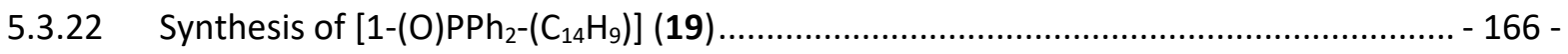

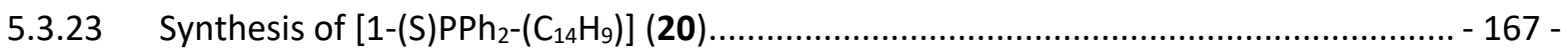

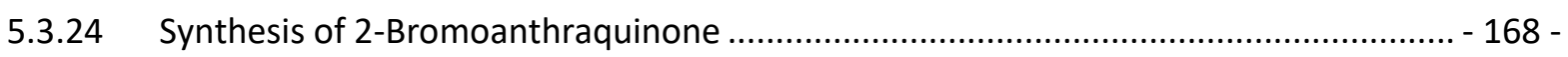

5.3.25 Synthesis of 2-Bromoanthracene ........................................................................... - 169 -

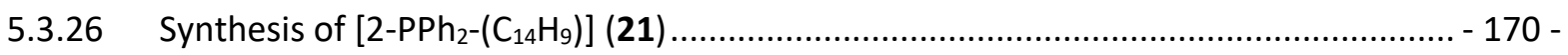

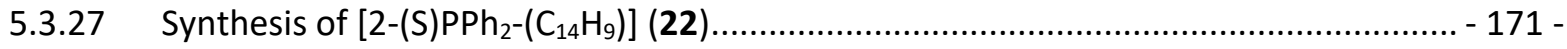

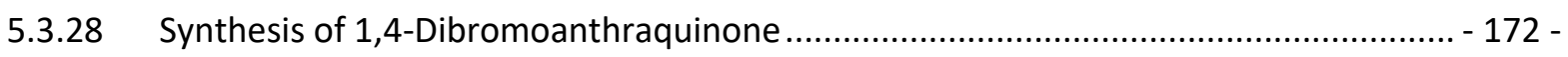

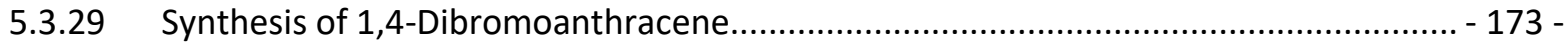

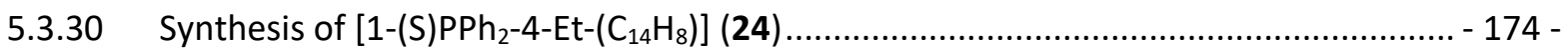

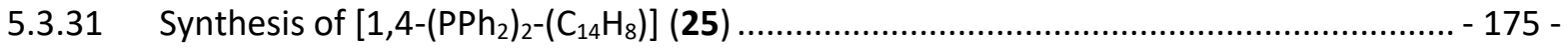

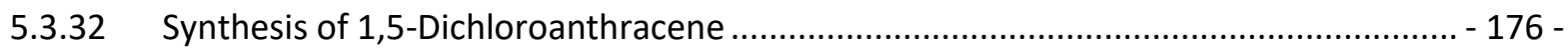

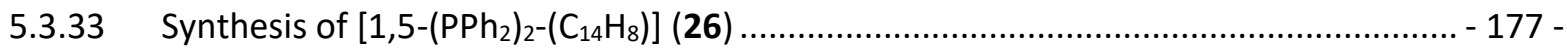

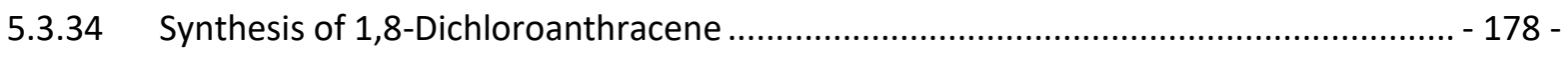

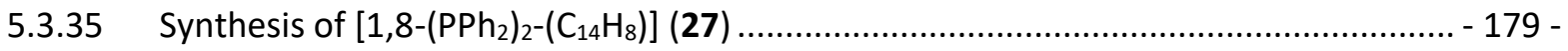

5.3.36 Synthesis of 2,6-Dibromoanthraquinone ................................................................. -180 -

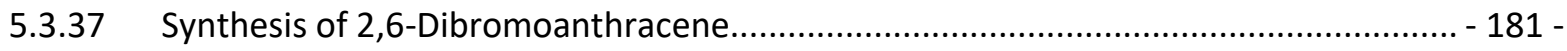

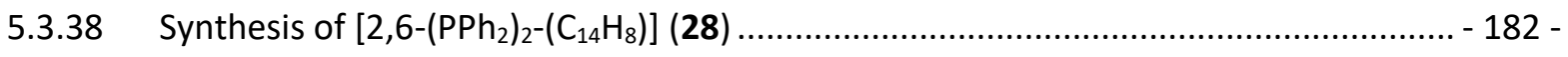

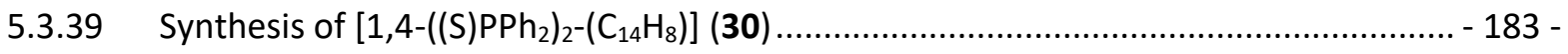

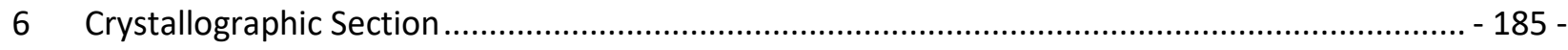

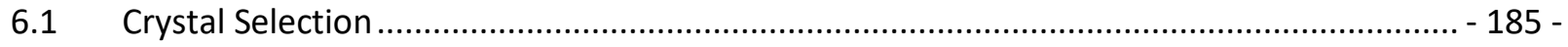

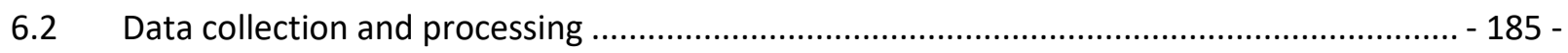

6.3 Structure solution and refinement............................................................................ $185-$

6.4 Structures determined during this work ................................................................... - 186 -

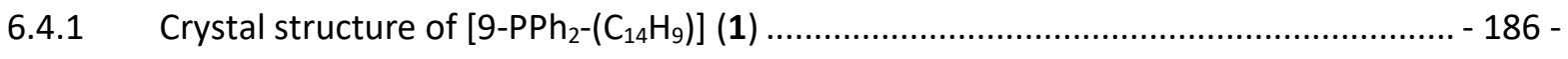




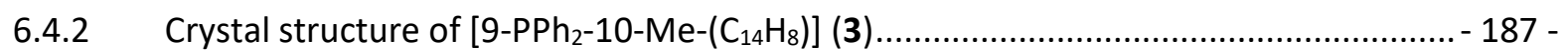

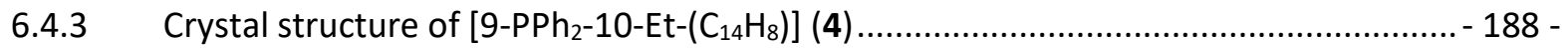

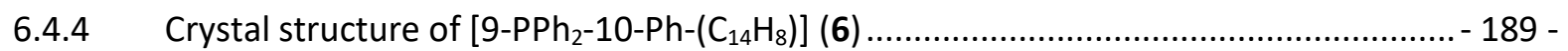

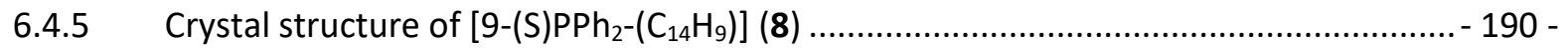

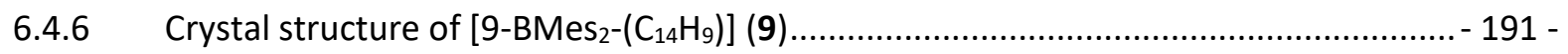



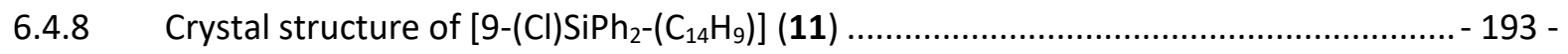

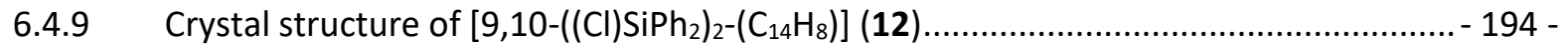

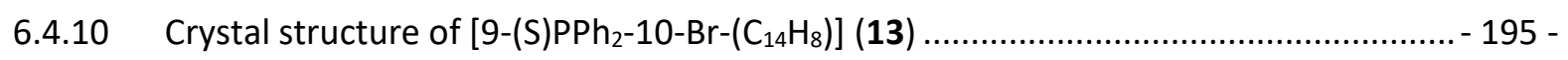

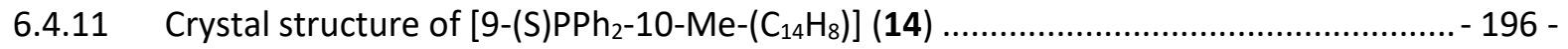

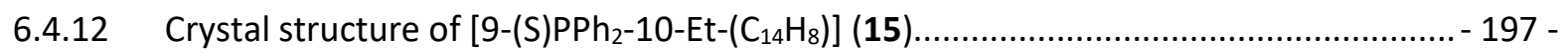

6.4.13 Crystal structure of $\mathrm{C}_{6} \mathrm{H}_{6} @\left[9-(\mathrm{S}) \mathrm{PPh}_{2}-10-\mathrm{Et}-\left(\mathrm{C}_{14} \mathrm{H}_{8}\right)\right]$ (15a) ....................................... 198 -

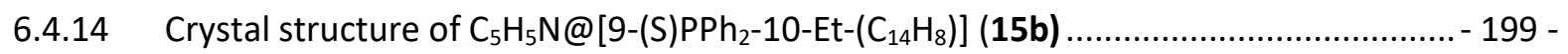

6.4.15 Crystal structure of $\mathrm{C}_{7} \mathrm{H}_{8} @\left[9-(\mathrm{S}) \mathrm{PPh}_{2}-10-\mathrm{Et}-\left(\mathrm{C}_{14} \mathrm{H}_{8}\right)\right]$ (15c) ....................................... 200 -

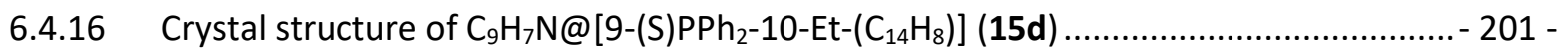

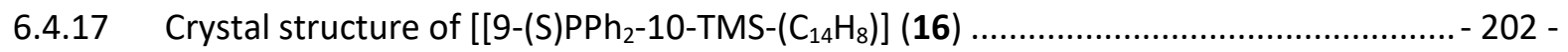

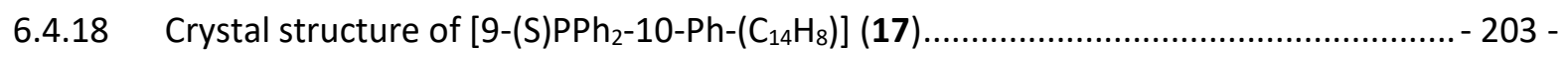

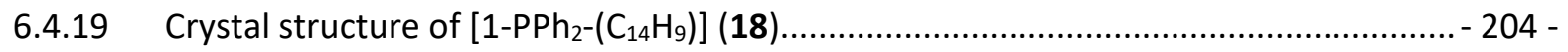

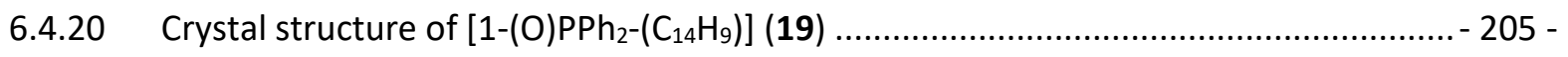

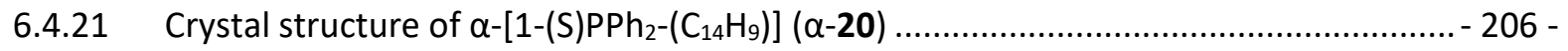

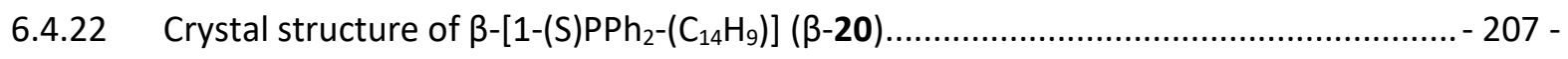

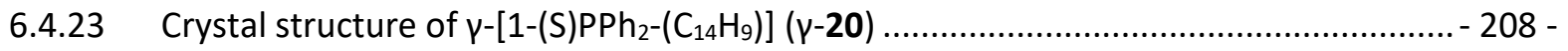

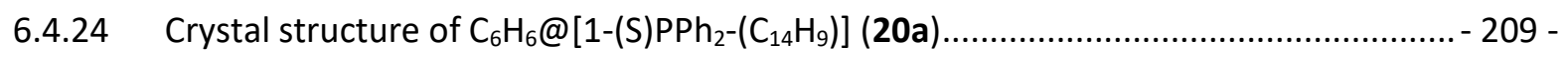

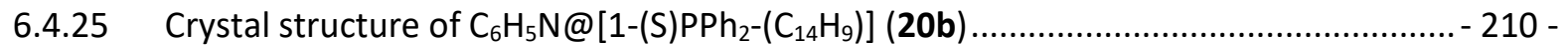

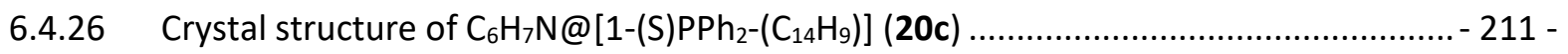

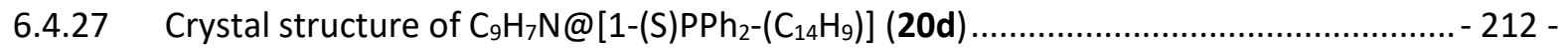

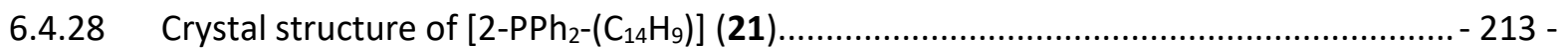

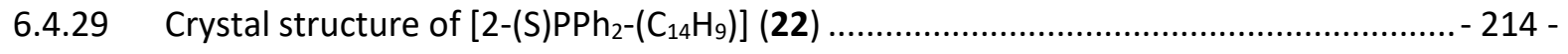

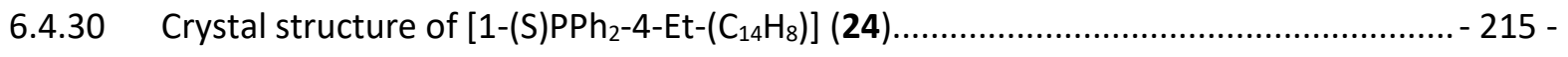

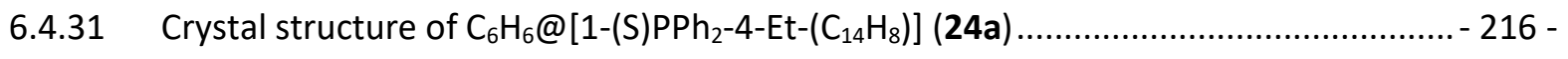




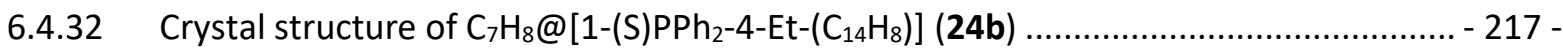

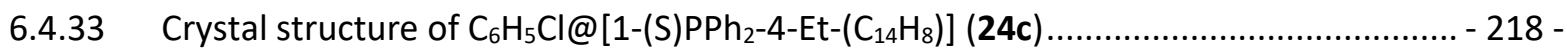



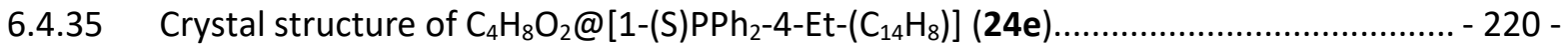

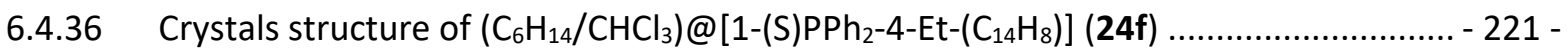

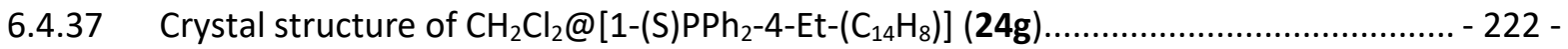

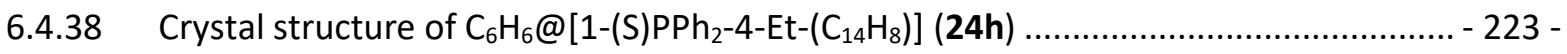

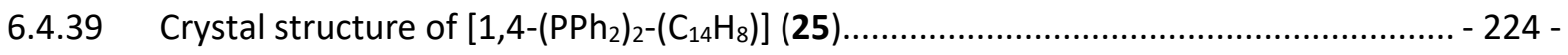

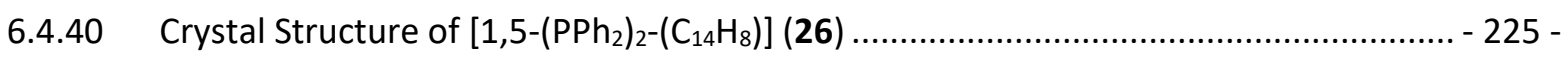

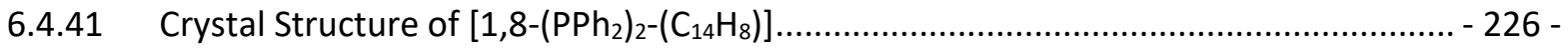

6.4.42 Crystal Structure of 2,6-Dibromo-9,10-dihydroanthracene..................................... - 227 -

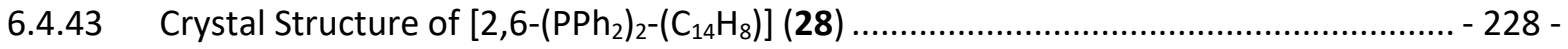

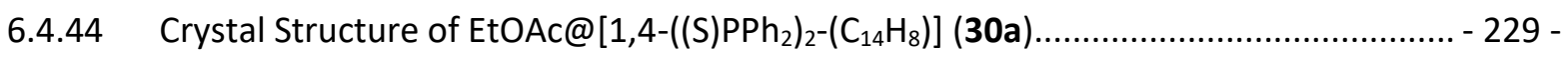

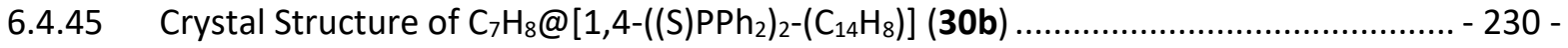

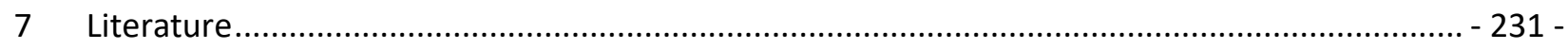

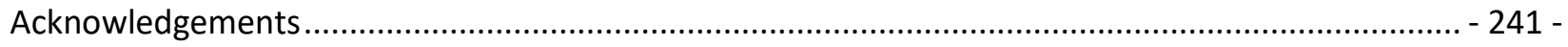





\begin{tabular}{|c|c|}
\hline$\AA$ & Ångstrom \\
\hline AIE & Aggregation-induced emission \\
\hline ACQ & Aggregation-caused quenching \\
\hline a.u. & arbitrary units \\
\hline $\operatorname{Ar}$ & aryl; aromatic substituent \\
\hline $\mathrm{Bu}$ & butyl \\
\hline CCDC & Cambridge Crystallographic Database \\
\hline $\mathrm{Cl}$ & conical intersection \\
\hline CPS & counts per second \\
\hline CSD & Cambridge Structural Database \\
\hline CT & charge transfer \\
\hline$d$ & days \\
\hline DCM & dichloromethane \\
\hline DFT & density functional theory \\
\hline DMF & dimethylformamide \\
\hline EI & electron ionization \\
\hline Eq. & equivalent(s) \\
\hline ESI & electrospray ionization \\
\hline Et & ethyl \\
\hline $\mathrm{Et}_{2} \mathrm{O}$ & diethylether \\
\hline et al. & et alii, and others \\
\hline $\mathrm{EtOH}$ & ethanol \\
\hline excimer & excited dimer \\
\hline exciplex & excited complex \\
\hline h & hour(s) \\
\hline HPS & hexaphenylsilole \\
\hline HOMO & highest occupied molecular orbital \\
\hline i & ipso \\
\hline IC & internal conversion \\
\hline ICT & intramolecular charge transfer \\
\hline iPrOH & isopropyl alcohol / 2-propanol \\
\hline ISC & intersystem crossing \\
\hline LIFDI & liquid injection field desorption ionization \\
\hline LED & light emitting diode \\
\hline LP & lone pair \\
\hline LR & Lawessons's reagent \\
\hline $\begin{array}{l}\text { LUMO } \\
\mathrm{m}\end{array}$ & $\begin{array}{l}\text { lowest unoccupied molecular orbital } \\
\text { meta }\end{array}$ \\
\hline Me & methyl \\
\hline $\mathrm{MECl}$ & minimum energy conical intersection \\
\hline Mes & mesityl (2,4,6-trimethylphenyl) \\
\hline $\mathrm{MHz}$ & megahertz \\
\hline Min & minute(s) \\
\hline MS & mass spectrometry \\
\hline $\mathrm{m} / \mathrm{z}$ & mass/charge \\
\hline$n$ BuLi & $n$-butyllithium \\
\hline
\end{tabular}




$\begin{array}{ll}\text { NBS } & \text { N-bromosuccinimide } \\ \text { NMR } & \text { nuclear magnet resonance } \\ \text { o } & \text { ortho } \\ \text { OLED } & \text { organic light-emitting diode } \\ \text { p } & \text { para } \\ \text { PET } & \text { photoinduced electron transfer } \\ \text { Ph } & \text { phenyl } \\ \text { ppm } & \text { parts per million } \\ \text { RACI } & \text { restricted access to a conical intersection } \\ \text { RIM } & \text { restriction of intramolecular motion } \\ \text { RIR } & \text { restriction of intramolecular rotation } \\ \text { RIV } & \text { restriction of intramolecular vibration } \\ \text { rISC } & \text { reverse intersystem crossing } \\ \text { rt } & \text { room temperature } \\ \text { S, sec } & \text { second } \\ \text { SOC } & \text { spin-orbit-coupling } \\ \text { TADF } & \text { thermally activated delayed fluorescence } \\ \text { TDDFT } & \text { time-dependent density functional theory } \\ \text { THF } & \text { tetrahydrofuran } \\ \text { TPE } & \text { tetraphenylethylene } \\ \text { UV } & \text { ultraviolet } \\ \text { UV/Vis } & \text { ultraviolet/ visible } \\ \text { X-Ray } & \text { X-ray radiation } \\ \text { XRD } & \text { X-ray Diffraction Analysis } \\ & \end{array}$




\section{List of compounds}<smiles>C1=C[C@H]2O[C@H]1O2</smiles>

1<smiles>c1ccc(Pc2ccccc2)cc1</smiles><smiles>Cc1c2ccccc2c([Si](C)(C)C)c2ccccc12</smiles>

5<smiles></smiles>

9<smiles>[O-][PH]([S-])(c1ccccc1)c1c2ccccc2c(Br)c2ccccc12</smiles>

13<smiles>Brc1c2ccccc2c(P(c2ccccc2)c2ccccc2)c2ccccc12</smiles>

2<smiles>c1ccc(Pc2ccccc2)cc1</smiles><smiles>Cc1c2ccccc2c(-c2ccccc2)c2ccccc12</smiles>

6<smiles>Cc1c2ccccc2c(P(c2ccccc2)c2ccccc2)c2ccccc12</smiles>

3<smiles>[O-][PH]([O-])(c1ccccc1)c1c2ccccc2cc2ccccc12</smiles>

7<smiles>Cl[Si](c1ccccc1)(c1ccccc1)c1c2ccccc2cc2ccccc12</smiles>

11<smiles>CCc1c2ccccc2c([PH]([O-])([O-])c2ccccc2)c2ccccc12</smiles>

15<smiles>CCc1c2ccccc2c(P(c2ccccc2)c2ccccc2)c2ccccc12</smiles>

4<smiles>[O-][P+]([O-])(c1ccccc1)c1c2ccccc2cc2ccccc12</smiles>

8<smiles>Cl[Si](Cl)(c1ccccc1)c1ccccc1</smiles><smiles>[Si]c1c2ccccc2c([Si](Cl)(c2ccccc2)c2ccccc2)c2ccccc12</smiles>

12

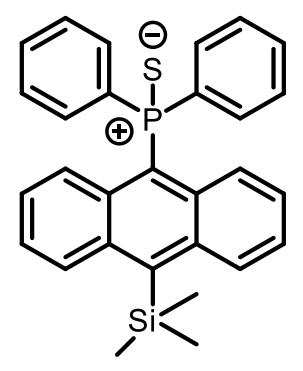

16 
<smiles>C1#[Ge]O[Ge]1</smiles>

17

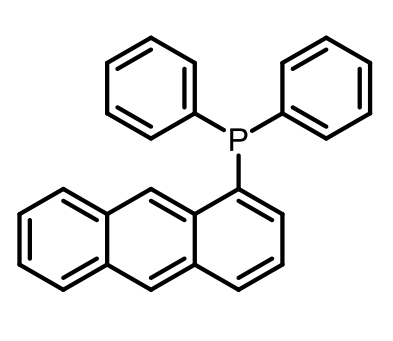

18

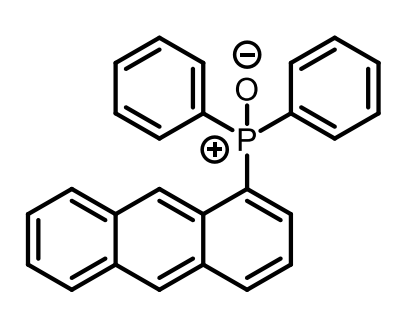

19

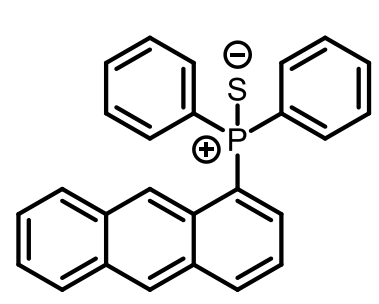

20<smiles>c1ccc(P(c2ccccc2)c2ccc3cc4ccccc4cc3c2)cc1</smiles>

21

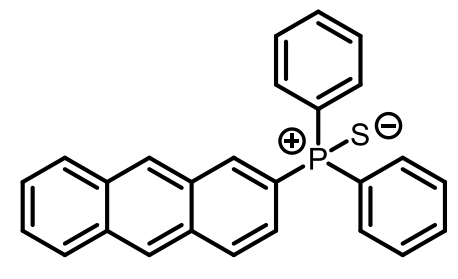

22
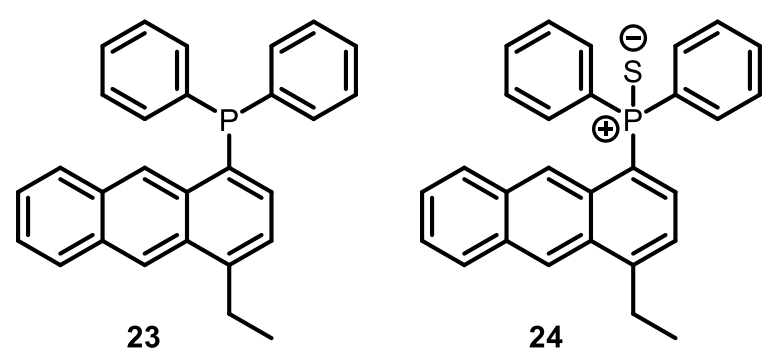<smiles>Pc1cccc(-c2ccc3cc4ccccc4cc3c2-c2ccccc2)c1</smiles>

25<smiles>c1ccc(-c2c3ccccc3c(-c3ccccc3)c3ccccc23)cc1</smiles>

29<smiles></smiles>

26<smiles></smiles>

27<smiles>c1ccc(Pc2ccc3cc4cc(-c5ccccc5)ccc4cc3c2)cc1</smiles>

28

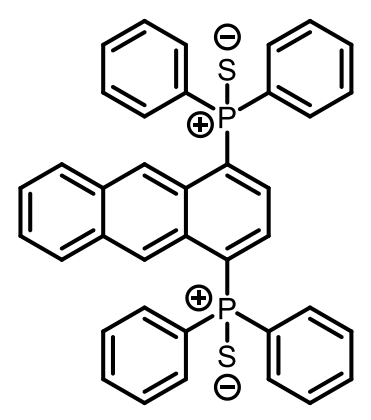

30<smiles>C[Pb]([O-])(c1ccccc1)c1ccccc1</smiles><smiles>c1ccc2cc3ccccc3cc2c1</smiles><smiles>C[Pb]([O-])(c1ccccc1)c1ccccc1</smiles>

31 


\section{Introduction}

Luminescent molecules and materials have been established in a variety of applications and processes in science over the last centuries. ${ }^{[1]}$ Especially in the life sciences, fluorescence plays an important role as a non-destructive analytical tool for labeling, imaging and sensing and allows the study and observation of fundamental biochemical processes. ${ }^{[2]}$ Nowadays, fluorescence spectroscopy and microscopy are probably among the most used analytical tools in the life sciences. ${ }^{[1]}$ The high sensitivity and selectivity of fluorescence probes gives several advantages over comparable techniques. Recent developments allow the observation and investigation of biochemical processes up to the level of single molecules. ${ }^{[2,3]}$ With the discovery and development of fluorescent proteins like the green fluorescent protein (GFP), live-cell imaging became a standardized tool for the observation of biochemical processes since the $1990 \mathrm{~s} .{ }^{[4]}$ The 2008 Nobel prize in chemistry awarded to Shimomura, Chalfie and Tsien underlines the importance of this research area. ${ }^{[5]}$ In the past decades also the progress in fluorescence microscopy was enormous. ${ }^{[6]}$ Several super-resolution imaging techniques like stimulated emission depletion microscopy (STED) ${ }^{[7]}$ and photoactivated localization microscopy (PALM) $)^{[8]}$ allow the circumvention of the diffraction resolution limit and the study of biochemical processes on the nanometer scale. Betzig, Hell and Moerner were awarded with the Nobel prize in chemistry 2014 for their work in this field. ${ }^{\left[{ }^{[9]}\right.}$ The highly sensitive and selective response of fluorescence probes to changes in their environment makes them ideal candidates for sensing and monitoring conformational changes and interactions of biomolecules. ${ }^{[1,2]}$ For these medical and biochemical applications, the methods are usually applied in a fluid medium. ${ }^{[2]}$

Fluorescence applications are not limited only to the life sciences and fluid solutions, but interest in luminescent solid-state materials has been increasing over the last decades. ${ }^{[10]}$ Optoelectronic devices, including organic-light-emitting diodes (OLEDs) ${ }^{[11]}$, organic photovoltaic cells (OPVs) ${ }^{[12]}$, organic fieldeffective transistors (OFETs) ${ }^{[13]}$, lasers ${ }^{[14]}$ and also sensors ${ }^{[15]}$ require solid materials with suitable photophysical properties. Especially, in LEDs and OLEDs, a wide range of solid-state emitting compounds comes into use as these devices bear several advantages over commonly used light sources: low energy use, long lifetimes and great stability. ${ }^{[16,17]}$ Further benefits are the tunability of the emitted spectrum through combination of multiple diodes. ${ }^{[17]}$ OLEDs are built from several thin layers with different functions, whereas the emitting process occurs through electroluminescence from the emitting layer. ${ }^{[17]}$ Recombination of induced holes and electrons in the emitting layer leads to the formation of excitons, which can be described as localized hole-electron pairs. ${ }^{[17]}$ The transition to the electronic ground state can then occur via the emission of a photon. For efficient applications, the control over the photophysical properties of the emitting materials is essential. ${ }^{[18]}$ Several factors, for example emission wavelength, lifetime and quantum efficiency determine the properties of such devices. Therefore, optimization and

tunability of the emitting materials is important for future applications. ${ }^{[19]}$ Especially, the realization of efficient and stable blue- and white light emitting materials is still challenging and in the focus of current research. ${ }^{[20,21]}$ The overall efficiencies of these devices are mainly determined by the efficiency of the emitting layer. 
In general, light-emitting materials are built up from polyaromatic hydrocarbons. The emission of these compounds is usually in the visible region of the electromagnetic spectrum. Several approaches have been developed to improve the photophysical properties and increase in the emission efficiency, especially in OLED applications.

The first developed OLEDs suffered from low efficiencies as the emitting layer were based on purely fluorescent compounds like tris(8-hydroxyquinolinato)aluminum (Alq3 (I), Scheme 1). Besides its emission properties, other factors like stability and conductivity, make Alq3 (I) a suitable candidate. From spin statistics it can be derived that of $25 \%$ of the generated excitons adopt a singlet state and $75 \%$ a triplet state. ${ }^{[22-24]}$ As fluorescence occurs only from the singlet state, $75 \%$ of the excitons could not be used and the theoretical efficiency was limited to a maximum $25 \% .{ }^{\left[{ }^{[20]}\right.}$ Therefore, research in the past decades focused on overcoming this statistical limit through the design of emitting molecules with defined photophysical processes that enable the use of excitons with triplet character and enhancing the efficiency of the devices. ${ }^{[25,26]}$ First approaches were based on phosphorescent emitters, which can use theoretically $100 \%$ of the excitons. ${ }^{[27]}$ Usually, a large spin-orbit-coupling (SOC) is required, to enable a radiative transition from the excited triplet state to the singlet ground state of the molecule. ${ }^{[28]}$ Therefore, organometallic compounds with heavier transition metal centers like iridium or platinum are processed in these devices as they exhibit strong spin-orbit-coupling (Scheme 1). ${ }^{[29]}$ Tuning of the emission properties can be achieved through variation of the ligands around the metal centers. ${ }^{[30]}$ While the higher possible efficiencies are beneficial for applications, the high costs and low environmental compatibility of the heavy transition metals are the main disadvantageous.

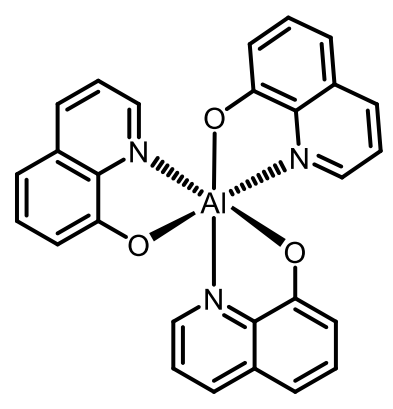

Alq3, (l)

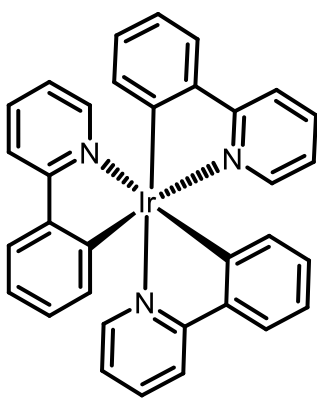

$\operatorname{Ir}(\text { ppy })_{3},(\mathrm{II})$

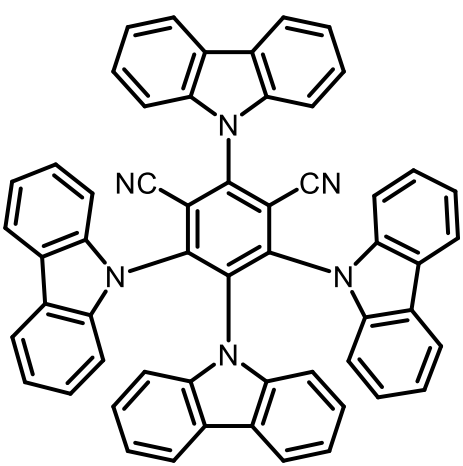

4CzIPN, (III)

Scheme 1. Typical examples of emitter materials used in OLEDs. $1^{\text {st }}$ generation OLEDs are based on fluorescent materials like $\mathrm{Alq}_{3}$ (I) (left). Incorporation of heavier transition metals like iridium and platinum allows the use of triplet excitons through phosphorescence for more efficient devices (middle). Latest research focused on organic molecules like 4CzIPN (III), whose high efficiencies are due to thermally activated delayed fluorescence (TADF) (right).

A different approach for obtaining more efficient emitting materials is based on thermally activated delayed fluorescence (TADF). ${ }^{[31]}$ In contrast to phosphorescence the emission occurs not from the triplet state, but from the singlet state. A reverse intersystem crossing ( $\mathrm{rlSC}$ ) from the triplet to the excited singlet state allows the use of the triplet excitons and enables higher efficiencies even if the emission occurs from the singlet state. ${ }^{[32]}$ For an efficient $\mathrm{rISC}$, a small energetic gap between the singlet and triplet state $\left(\Delta \mathrm{E}\left(\mathrm{S}_{1}\right.\right.$ - 2 - 
$\left.T_{1}\right)$ ) is required that facilitates the rISC from the lower energy triplet state through thermal activation. ${ }^{[32]}$ One class of TADF-molecules is based on $\mathrm{Cu}(\mathrm{I})$ emitters, which reveal a weak overlap of the HOMO and LUMO and therefore a small $\Delta \mathrm{E}\left(\mathrm{S}_{1}-\mathrm{T}_{1}\right) \cdot{ }^{[33]}$ Furthermore, copper complexes are cost-effective in comparison to iridium- and platinum-based complexes. Tuneability of the photophysical properties is given through different coordination geometries and the applied ligands. ${ }^{[33]}$

A second class of TADF emitters is based on purely organic compounds and first examples like 4CZIPN (III) were reported in 2012 (Scheme 1). ${ }^{[34]}$ Thanks to the absence of metal atoms, these derivatives have in general a higher environmental comparability and lower costs. Usually, donor-acceptor systems with a twisted structure are used for organic TADF emitters, as they generate a small overlap between the ground-state HOMO and the excited state LUMO, which induces small singlet-triplet energy gaps. ${ }^{[35]}$ Excited complexes (exciplexes) can also be utilized for achieving delayed fluorescence. Separation of the electron-donating and electron-accepting moieties on two different molecules can result in smaller energy gaps compared to intramolecular donor-acceptor molecules. ${ }^{[31]}$

As shown by the example of OLEDs, the increasing number of applications of luminescent materials require molecules with defined and optimized photophysical properties. For the discussed reasons, organic compounds get in the focus of research as an alternative to metal-based materials. ${ }^{[26]}$ Since these compounds are processed in condensed phase, an understanding of the properties upon aggregation and in the solid-state is essential. Especially in polyaromatic hydrocarbons, the intermolecular interactions can drastically influence the luminescence properties. ${ }^{[36,37]}$ For a long time, these interactions were mostly claimed to be detrimental for possible applications and strong intermolecular interactions were usually avoided. ${ }^{[38,39]}$ In recent years, several working groups investigated the impact of these interactions and evaluated concepts to make use of the resulting photophysical properties. ${ }^{[36,37,40,41]} \mathrm{A}$ short overview of the basic concepts and photophysical properties of polyaromatic hydrocarbons will be given in chapter 1.2. Beforehand, a short historical overview over the research regarding luminescence in aggregates will be given in the next paragraphs.

\subsection{A brief historical overview on luminescence in aggregated states}

A comprehensive historical overview regarding the nowadays widely used term 'Aggregation-inducedemission' (AIE) has been recently given by Würthner (2020). ${ }^{[42]}$ He focused especially on studies, which were published before the term AIE was coined by Tang in 2001.

Early investigations regarding photophysical processes upon aggregation were already made in the late 19th century. Schmidt examined a wide range of compounds and reported on molecules, which reveal fluorescence only in certain solvents as well as on salts, which emit in the solid state but not in solution. ${ }^{[43]}$ Around 50 years later, studies on organic dyes were intensified and the first concepts on fluorescence quenching and enhancement upon aggregation were postulated. Oster and Nishijima introduced the internal rotation of phenyl groups as the main fluorescence quenching process in Auramine $\mathrm{O}$ (IV), a diarylmethane dye (Scheme 2). ${ }^{[4]}$ With increasing viscosity of the solvent, higher fluorescence quantum yields of Auramine $\mathrm{O}$ glycerol solutions were obtained and attributed to the restricted rotation of the 
phenyl groups in viscous solutions. ${ }^{[44]}$ In the 1980 s, Shultz and Fox synthesized tetraphenylethylenes (TPE, V) and investigated the effect of the phenyl ring torsion on the photophysical properties (Scheme 2). Restriction of the torsion through covalent linkage of the geminal phenyl rings led to drastic changes in the emission characteristics and the ring torsion was assigned to be a main deactivation pathway of the excited state. ${ }^{[45]}$ In the following years further reports on molecules that are non-fluorescent in diluted solutions but become emissive in more viscous solvents or in the solid-state, were published. These observations were ascribed to the restriction of torsions in rigid environments and a resulting inhibition of non-radiative decay pathways. ${ }^{[46]}$

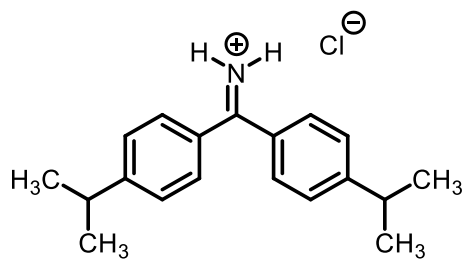

Auramine 0

IV, 1956

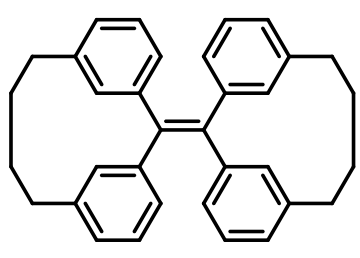

V, 1989

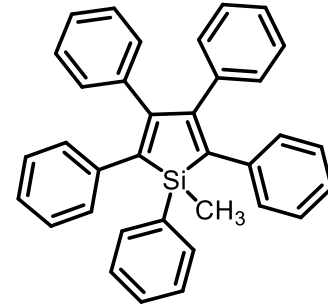

VI, 2001

Scheme 2. Early examples of molecules that change emission properties upon aggregation. The restriction of intramolecular rotation was introduced for the first time during the investigation of the photophysical properties of Auramine O (IV) in 1956. In 1989 the intramolecular rotation of tetraphenylethylene derivatives (V) was examined and became more popular since 2001 through the work of Tang and co-workers. Together with penta- and hexaphenylsiloles (VI), TPE-derivatives became prominent representatives of the AIE-phenomenon.

In 2001, Tang and co-workers observed a similar phenomenon during the investigation of the 1-methyl1,2,3,4,5-pentaphenylsilole (VI, Scheme 2), which revealed increasing fluorescence quantum yields in THF/water mixtures with increasing water fractions. ${ }^{[4]}$ They concluded that the silole forms nanoaggregates in solutions with higher water content. These nanoaggregates are highly luminescent in contrast to the isolated molecules in diluted solution. This behavior was named "Aggregation-inducedemission" (AIE) and is used nowadays in a general way for compounds that are non- or only weakly luminescent in solution but emit upon aggregation. Since 2001, Tang and others investigated a wide range of AIE compounds and introduced them in different applications like biomedical imaging ${ }^{[8,49]}$ and OLEDs. ${ }^{[50]}$ Furthermore, mechanistic studies on the underlying processes of the fluorescence enhancement of hexaphenylsiloles (HPS) upon aggregation were performed. Similar behavior was found for several derivatives of HPS and TPE. ${ }^{[51-53]}$ Diluted solutions of HPS in common organic solvents reveal only weak or no emission. The intensities can be increased whether (i) a poor solvent (water) is added, or (ii) the viscosity is increased, or (iii) temperature is decreased. ${ }^{[51-53]}$ Since further changes in the emission spectra (shift of the emission wavelength or the spectral shape) are negligible, a mechanism based on the planarization of the twisted phenyl groups with a following increased conjugation, was excluded. Instead, the restriction of intramolecular motion (RIM) upon aggregation was ascribed to be responsible for the fluorescence enhancement, which has been already postulated in the earlier works (vide supra). In diluted solutions, the free torsion of the phenyl rings offers an easily accessible non-radiative decay pathway. The rigidification of the environment of the fluorophores hampers the access to the deactivation pathway and 
promotes the radiative decay. This results in higher fluorescence quantum yields in the aggregated state (Scheme 3). ${ }^{[51-53]}$

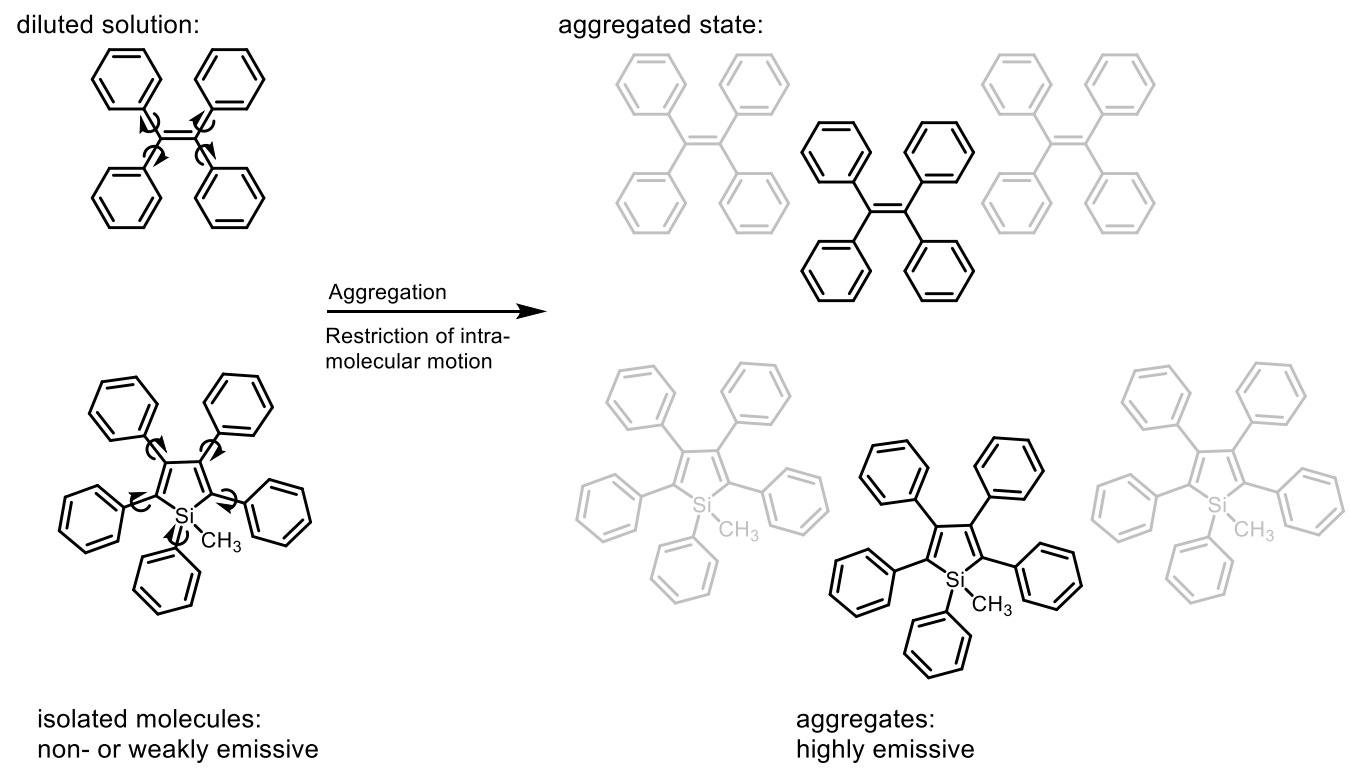

Scheme 3. Illustration of the proposed mechanism of typical AIE-active molecules. In diluted solution the rotation of the phenyl group leads to a fluorescence quenching. Upon aggregation the intramolecular motion is restricted, and an emission enhancement observed.

This mechanism was suitable for molecules with discrete molecular rotors such as the described hexaphenylsiloles. Nevertheless, typical AIE-behavior was observed for molecules without any rotatory elements. Therefore, the concept was extended to vibrational modes as non-radiative deactivation pathways and the restriction of intramolecular vibration (RIV) ascribed to an emission enhancement upon aggregation. ${ }^{[54]}$ The mechanism of the restriction of intramolecular motion (RIM) was transferred to further molecule classes. Also TPE derivatives were reinvestigated and became popular AIE-luminogens. ${ }^{\text {[55- }}$ ${ }^{57]}$ Further insights into the AIE concept are given in chapter 1.4 .

The hitherto described concepts for molecules exhibiting AIE-behavior are mainly based on intramolecular rigidifications upon aggregation. Intermolecular interactions do not seem to affect the photophysical properties of the typical AlE-luminogens, as the basic parameters such as spectral shape, emission wavelength and fluorescence lifetime are comparable in diluted solution and aggregates. The emission in the solid-state of most AIE-luminogens therefore occurs from the monomeric state, similar as in diluted solution. However, the influence of non-covalent interactions on the absorption and emission properties have already sparked interest of scientists in the beginning of the last century. ${ }^{[42]}$ In general, strong $\pi-\pi$ or $\mathrm{C}-\mathrm{H} \cdots \pi$ interactions are considered to be responsible for fluorescence quenching until today. ${ }^{[53,55,57]}$ Therefore, many common dyes, which are highly luminescent in solution, undergo a complete vanishing of the fluorescence in solid phases, which is named as "Aggregation-caused quenching" (ACQ). Early studies on the effect of aggregation were performed in the 1930 s by Jelley and Scheibe. They investigated independently the photophysical behavior of aggregates of pseudoisocyanine chloride (VII) in aqueous solution (Scheme 4) ${ }^{[58-61]}$ Both observed a bathochromic shift of the absorption maximum with increasing 
dye concentration. Along with the new sharp absorption band, a strong fluorescence with a small Stokes shift was observed. As the changes in the spectra were found to be reversible upon heating and cooling, they were attributed to the supramolecular aggregation of the dye molecules at higher concentration. ${ }^{[58-}$ 61]
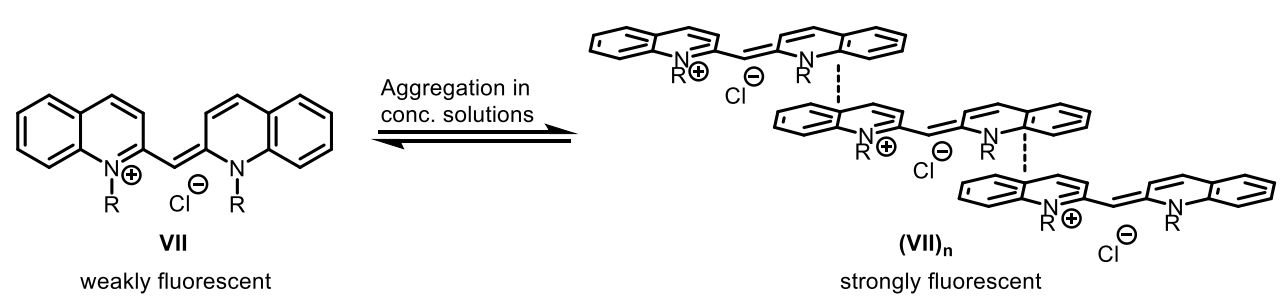

Scheme 4. Jelley and Scheibe investigated the photophysical properties of a pseudoisocyanine derivative (VII) upon aggregation in concentrated aqueous solution and observed an emission enhancement upon aggregation.

Since that discovery by Jelley and Scheibe, a plethora of molecules with similar behavior were reported. ${ }^{[62]}$ In general, dye aggregates that show a bathochromic shift of the absorption compared to the monomeric state and fluorescence with a small Stokes shift are called J-aggregates (named after Jelley). Molecules, which undergo a hypsochromic shift of the absorption band upon aggregation are named $\mathrm{H}$-aggregates. Together with the blue shift of the absorption band, a fluorescence quenching is usually observed in $\mathrm{H}$ aggregates. ${ }^{[63]}$ The changes of the absorption properties were attributed to different molecular arrangements of the aggregated dyes. It was explained with the exciton theory developed by Kasha. ${ }^{\text {[64] }}$ According to this theory, the excited state of the aggregates (for reasons of simplicity a dimer-aggregate will be used in the following) is split into two energy levels due to the coupling of the two transition dipole moments (Figure 1). Depending on the orientation of the transition dipole moments and therefore on the arrangement of the molecules, different electronic transitions are allowed. The two simplest cases are shown in Figure 1, where the transition dipole moments of a dye aggregate are oriented in a parallel fashion (sandwich-type; $\mathrm{H}$-aggregate) and in a linear fashion (J-aggregate). Only the in-phase oscillation of the transition dipole moments may result in an allowed transition. Therefore, only one transition is allowed for each the $\mathrm{H}$ - and J-aggregates. In case of the $\mathrm{H}$-aggregate, a hypsochromic shift is observed for the absorption compared to the monomer, while, for the J-aggregate, a bathochromic shift of the allowed transition is the consequence. Often, an internal conversion into the lowest excitonic state occurs right after excitation. Since the transition from the lowest excitonic state to the ground state in $\mathrm{H}$-aggregates is forbidden, no or only weak fluorescence of the $\mathrm{H}$-aggregates can usually be observed. In contrast, the allowed transition from the lowest excitonic state into the ground state of the J-aggregate often results in a bright fluorescence with a small Stokes-shift. ${ }^{[62,64]}$ 


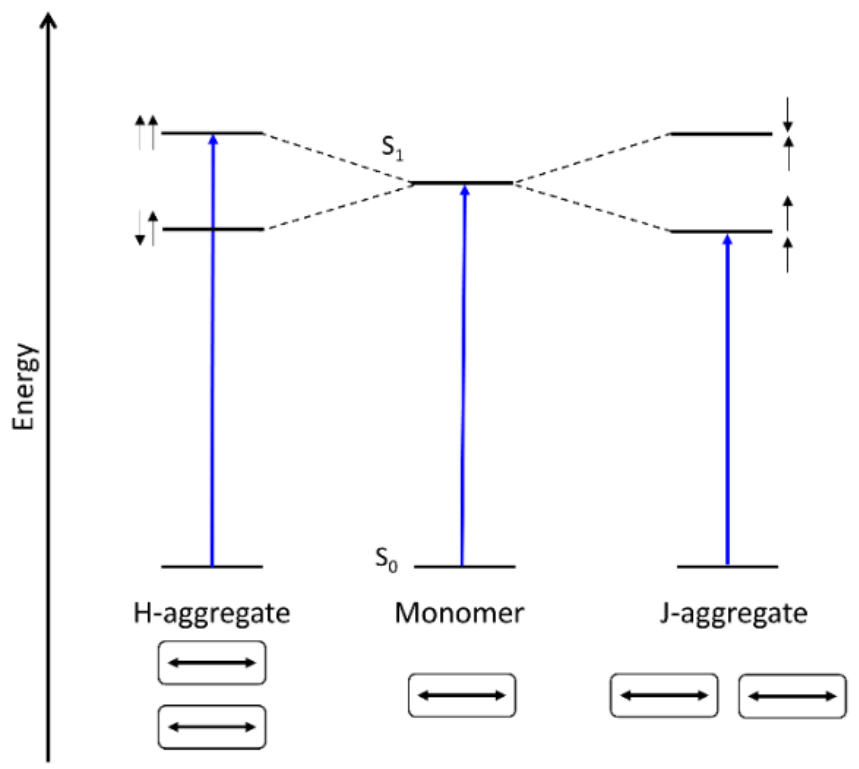

Figure 1. Schematic illustration of the exciton coupling and the resulting hypsochromic and bathochromic shifts of the allowed transitions according to Kasha. ${ }^{[64]}$ The orientations of the transition dipole moments in the $\mathrm{H}$ - and J-aggregates are shown as double arrows.

The described dipole coupling interactions are generally considered as long-range electronic interactions. Also, short-range interactions with an orbital overlap of the involved molecules influence the photophysical properties. Förster and Kasper discovered an unexpected broad emission band at higher wavelengths for concentrated pyrene solutions. ${ }^{[65]}$ They ascribed this broad emission band to the formation of an excited dimer (excimer) between two pyrene molecules. The ability to form excimers has been confirmed in the following years for further polyaromatic hydrocarbons. ${ }^{[66,67]}$ Details on the excimer formation process will be given in chapter 1.3.2.

Strong intermolecular interactions are still avoided in most approaches for obtaining efficient luminescent materials. However, several examples have been reported, in which defined interactions are beneficial for tuning and optimizing the photophysical properties. ${ }^{[36,63,68]}$ Therefore, elucidation of the correlation between intermolecular interactions and the photophysical properties in the solid-state is an important research area. Additionally, controlling the emission properties via non-covalent interactions is still highly challenging.

\section{$1.2 \pi$-stacking and $\pi-\pi$ interactions}

Due to their extended $\pi$-systems and planar structures, most polyaromatic hydrocarbons are ascribed to undergo weak interactions involving the $\pi$-electrons of the aromatic moieties. These interactions influence the resulting packing motif in the solid-state and vice versa. Therefore, $\pi$ - $\pi$-interactions play an important role in supramolecular chemistry, biochemistry and materials science, even if they are rather weak compared to covalent bonds. ${ }^{[40,69]}$ Also, these interactions can affect the excited state and the resulting photophysical properties of common aromatic hydrocarbons, as briefly discussed above. The nature of interactions involving $\pi$-electrons is still under discussion and the basic concepts will be shortly introduced in the following chapter. ${ }^{[70,71]}$ 
The benzene dimer has often been used as a model system for the investigation of interactions involving $\pi$-electrons of aromatic molecules. The two molecules can adopt three basic orientations (Scheme 5). When the two $\pi$-systems are oriented in a cofacial manner with an ideal overlap of the aromatic planes, a so called 'sandwich-dimer' is obtained. A shift of one benzene molecule along the molecular plane results in a parallel displaced or offset face-to-face stacking. In the third motif, the aromatic planes adopt an orthogonal (or T-shape) orientation, with edge-to-face interactions. Different models try to explain these stacking motifs and the influence of substituents with the help of non-covalent interactions involving the $\pi$-electrons of the aromatic systems. ${ }^{[71]}$

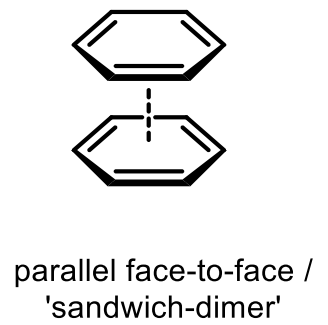

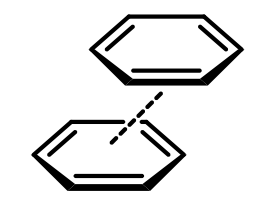

offset face-to-face / parallel displaced

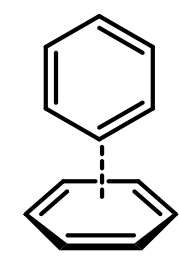

edge-to-face /

T-shape

Scheme 5. Three basic orientations of the benzene dimer in the context of $\pi$-stacking interactions.

A widely used model for the description of $\pi$-interactions in aromatic molecules was developed by Hunter and Sanders in 1990. ${ }^{[72]}$ The model is mainly based on quadrupole moments induced by the $\pi$-electron density in aromatic systems. For unsubstituted aromatic hydrocarbons, a partial negative charge is found above and below the aromatic plane, while a partial positive charge is found in the periphery (Scheme 6). ${ }^{[72]}$ The electrostatic repulsion and attraction of the partial charges is therefore responsible for the observed stacking geometries. For the benzene dimer, an ideal face-to-face stacking ('sandwich-dimer') is therefore unfavorable due to the repulsive interaction of the partial charges. Instead, a parallel displaced (offset face-to-face) or an edge-to-face (T-shape) orientation is preferred. The repulsive interactions are minimized, and the attractive dispersive interactions are more pronounced. Calculations in the gas-phase further support the model, as the two structures with attractive quadrupole interactions (parallel displaced and T-shape) are about $2 \mathrm{kcal} / \mathrm{mol}$ more stable compared to the sandwich-dimer. ${ }^{[73]}$ 

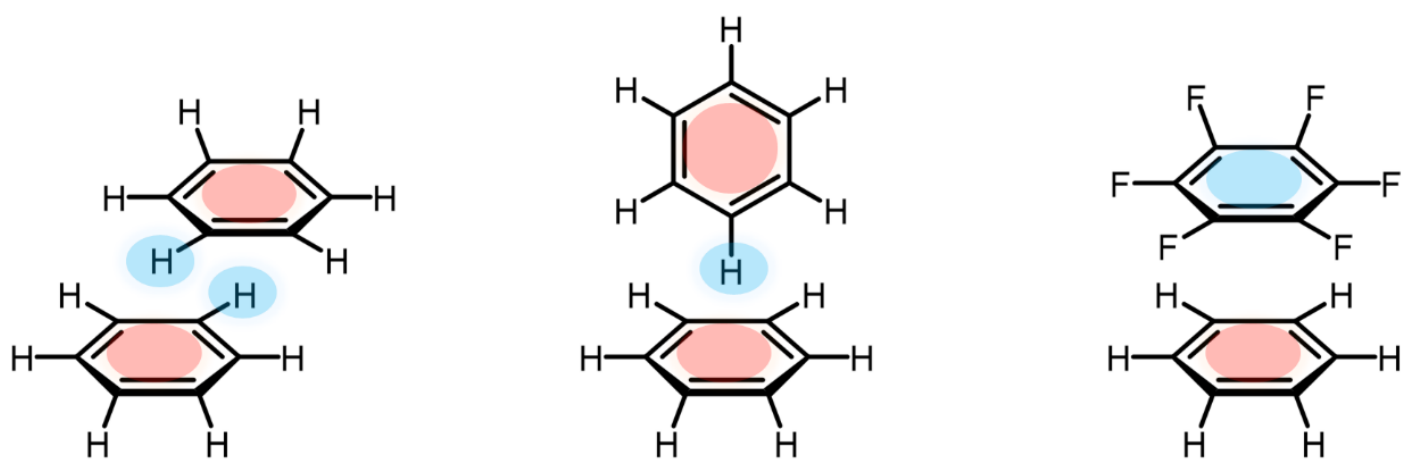

Scheme 6. Schematic representation of the partial charges induced by the quadrupole moments in benzene and hexafluorobenzene and the resulting preferred stacking geometries. Partial negative charges are depicted in red and partial positive charges in blue.

Effects of substitution can also be explained by the Hunter-Sanders model. Introduction of electronwithdrawing groups should lead to a reversal of the polarization in the aromatic system (e.g. in $\mathrm{C}_{6} \mathrm{~F}_{6}$ ). The substituents polarize the $\pi$-electron density away from the aromatic core (towards the substituents). A partial positive charge is then located above and below the aromatic plane and a negative partial charge in the periphery (Scheme 6, right). Interaction of an electron-poor aromatic ring with an electron-rich aromatic ring should therefore result in a more cofacial stacking geometry of the aromatic systems. It has to be noted that this assumption was disproved later as the $\mathrm{C}_{6} \mathrm{H}_{6}-\mathrm{C}_{6} \mathrm{~F}_{6}$ dimer also exhibits a parallel displaced geometry as the most stable form according to computational studies. ${ }^{[74]}$ For electron-donating substituents, the opposite behavior was predicted. The increased negative partial charge above and below the aromatic center should lead to a T-shape interaction with unsubstituted benzene. ${ }^{[72]}$ This proposed model is supported by experimental NMR-studies on 1,8-aryl-substituted naphthalenes by Cozzi and Siegel et al. ${ }^{[75]}$

The interaction of electron rich and electron poor aromatic molecules is also referred as an aromatic donor-acceptor interaction. ${ }^{[71]}$ As the influence of $\pi$-orbital mixing of the two aromatic molecules to the ground-state energy is still unclear, the donor-acceptor term is probably more appropriate. ${ }^{[76]}$ In the excited-state, interaction of the $\pi$-orbitals is often more pronounced resulting in charge-transfer complexes or excimers and exciplexes and will be discussed in chapter 1.3.2.

Even if the Hunter-Sanders model was widely accepted, the nature of $\pi$-stacking interactions was still questioned and widely discussed in the following years. Wheeler and Houk investigated the influence of substituents on the stacking geometry of the benzene dimer. ${ }^{[7]}$ They postulated that the polarization of the $\pi$-density through substituents was less pronounced than predicted by Hunter and Sanders. ${ }^{[77]}$ Instead, direct through-space interactions between the local dipoles of the substituent and the neighboring aromatic ring might be responsible for the enhanced stacking interactions. Furthermore, dispersive interactions between the substituent and the aromatic ring lead to a stabilization of the displaced stacking geometry between the substituted and unsubstituted aromatic system. ${ }^{[77]}$ 
Further computational studies addressed the question whether aromaticity is required for stacking interactions or if related non-aromatic molecules can undergo similar interactions. Grimme reported the interaction energies of linear (poly)acenes and their saturated homologs. ${ }^{[78]}$ For the benzene and naphthalene dimer, the differences in interaction energies between the aromatic and non-aromatic compounds are very small. From this result, it can be assumed that $\pi$-stacking interactions of small aromatic rings may have been overestimated. Especially in biochemistry, these interactions play an important role in protein folding and stacking of nucleobases in DNA for example. ${ }^{[79]}$ Only for larger systems, a stabilizing interaction for the aromatic dimer with a face-to-face orientation has been confirmed. ${ }^{[78]}$ The energies of the T-shape oriented dimers are similar for the saturated and unsaturated systems. ${ }^{[78]}$ Grimme concluded that the binding motif (edge-to-face vs. face-to-face) is more important than the presence of $\pi$-electrons and the term $\pi-\pi$ stacking should be used "as a geometrical descriptor" ${ }^{\prime[78]} \mathrm{A}$ beneficial $\pi$-stacking interaction is therefore only significant for larger $\pi$-systems with 10 or more carbon atoms in a face-to-face interaction.

Bloom and Wheeler investigated the role of delocalized $\pi$-electrons in stacking interactions by calculating the interaction energies between benzene and 2-methylnaphthalene (VIII) or the non-aromatic isomer 2methylene-2,3-dihydronaphthalene (IX). ${ }^{[80]}$ Furthermore, a dimer of homodesmotic dissected benzene and benzene was examined. In all cases, the interaction energy with the non-aromatic isomer was higher in the perfect parallel and parallel displaced conformation. From these results, it was postulated that localized $\pi$-electrons are more favorable in stacking interactions in comparison to their aromatic counterparts and $\pi-\pi$ interactions are not an exclusive feature of aromatic molecules. ${ }^{[80]}$

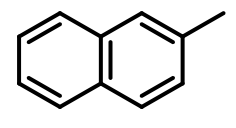

VIII



IX

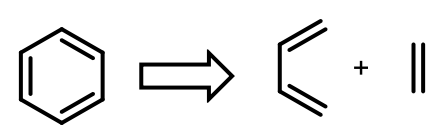

Scheme 7. The role of aromaticity in $\pi$-stacking interactions was investigated by Bloom and Wheeler through calculation of the interaction energies between benzene and 2-methylnaphthalene (VIII) and the non-aromatic 2-methylene-2,3dihydronaphthalene (IX). Furthermore, the interactions in the dimer of benzene and homodesmotic dissected benzene were investigated (right).

In general, $\pi-\pi$ interactions can be described as a combination of electrostatic-, dispersive-, attractive and repulsive orbital interactions. The contribution of each component determines the resulting overall interaction energy and therefore the stacking geometry. The molecular packing is also a main topic of this thesis. The terms $\pi$-stacking and $\pi-\pi$ interactions will therefore be used mostly in a geometrical sense to emphasize the face-to-face orientation of the aromatic moieties.

A special type of interaction involving the $\pi$-system is the $\mathrm{C}-\mathrm{H} \cdots \pi$ bond, which is often described as a weak hydrogen bond with a $\mathrm{C}-\mathrm{H}$ group as hydrogen donor and a $\pi$-system as acceptor. ${ }^{[81]}$ The T-shape or edge-to-face orientation of two aromatic molecules (Scheme 5 ) is often referred as an aromatic $\mathrm{C}-\mathrm{H} \cdots \pi$ hydrogen bond. Computational studies have shown that the energy in nonpolar $\mathrm{C}-\mathrm{H} \cdots \pi$ bond is rather weak with around $1.5 \mathrm{kcal} / \mathrm{mol}$ and the attractive interaction is mainly ascribed to dispersion forces. ${ }^{[82,83]}$ $-10-$ 
More acidic $\mathrm{C}-\mathrm{H}$ groups can undergo stronger interactions. $\mathrm{C}-\mathrm{H} \cdots \pi$ interactions involving aromatic or $\mathrm{Sp}^{2}-\mathrm{C}-\mathrm{H}$ groups are also slightly stronger compared to aliphatic groups. ${ }^{[81,82]}$ Even if the $\mathrm{C}-\mathrm{H} \cdots \pi$ interactions can be considered rather weak, they can play an important role in determining packing motifs in the solid-state. In biological systems, the weak interactions can control the stability of 3D structures of different macromolecules and take part in several recognition processes. ${ }^{[81]}$

Besides the discussed interactions, anions and cations can also interact with a $\pi$-system. These interactions will not be considered in this work but have been discussed in several review articles. ${ }^{[84]}$

Even if the nature of $\pi$-interactions is controversial, these types of interactions and, more generally noncovalent interactions, have been used widely for tuning the properties of optoelectronic materials such as organic semiconductors ${ }^{[85]}$, solar cells ${ }^{[86]}$, sensors ${ }^{[87]}$, and OLEDs ${ }^{[40,88]}$. The resulting stacking geometries in the solid-state can drastically influence the obtained molecular properties and differ strongly from the isolated compound. ${ }^{[36,89]}$ Therefore, the design of molecules with particular interactions is an emerging research field in materials science. As the differences in energy of different stacking geometries is often very small (vide supra), controlling the resulting interactions and packing motifs is still challenging. Nevertheless, the approach of tuning materials properties through intermolecular interactions is promising, as the interactions are reversible and nondestructive. Determination of the solid-state structure with X-Ray diffraction analysis allows a direct insight into the occurring interactions, which then can be correlated to the observed physical properties.

The photophysical properties will be in the focus of this work and their dependency on intermolecular interactions will be examined. Therefore, the fundamental photophysical processes in polyaromatic hydrocarbons will now be presented.

\subsection{Photophysical processes in polyaromatic hydrocarbons}

\subsubsection{Basic photophysical processes}

The basic principles of fluorescence in polyaromatic hydrocarbons are well known and have been investigated since the $19^{\text {th }}$ century. ${ }^{[1]}$ The fundamental processes will be described shortly with the aid of the Jablonski diagram (Figure 2). Upon irradiation with light of suitable energy, a transition of an electron from the electronic ground state $S_{0}$ to an excited state $\left(S_{n}, n=1,2,3 \ldots\right)$ can occur. ${ }^{[28]}$ Excitations into higher excited states $(n>1)$ usually relax radiationless via internal conversion (IC) to the first excited state $S_{1}$ as energy gaps between the higher excited states are usually small. IC occurs on the picosecond timescale and is therefore fast compared to competitive transitions. ${ }^{[1,28]}$ The deactivation of the $S_{1}$ state can proceed via several pathways. A radiative decay (fluorescence) is observed for the transition from the $S_{1}$ state into the ground state $\left(S_{0}\right)$. As fluorescence occurs between two singlet states, the transition is spin-allowed. Therefore, the lifetime of the $S_{1}$ state for common organic chromophores does usually not exceed a few nanoseconds. ${ }^{[1]}$ Radiationless decay from the $\mathrm{S}_{1}$-state can proceed via an intersystem crossing (ISC) to a lower lying triplet state $\left(T_{1}\right)$. For the anthracene fluorophore the ISC to the $T_{1}$ state is the main deactivation pathway with a quantum yield of around $0.7 .{ }^{[90]}$ Reverse intersystem crossing (rISC) into a high vibronic singlet-state and subsequent internal conversion can bring the system radiationless back to the ground 
state $\left(\mathrm{S}_{0}\right)$. Under certain conditions, a rISC can occur from the triplet state back to the excited singlet state. This process is fundamental for obtaining high-efficiency OLEDs based on TADF (vide supra). ${ }^{\left[{ }^{[6]}\right.}$ The decay of the triplet state to the ground state can also result in release of radiation (phosphorescence). As the multiplicity changes in the $T_{1} \rightarrow S_{0}$ transition, it is spin-forbidden and therefore clearly slower than the $\mathrm{S}_{1} \rightarrow \mathrm{S}_{0}$ transition. Phosphorescence lifetimes are therefore in the millisecond range and can reach values up to seconds. ${ }^{[1]}$

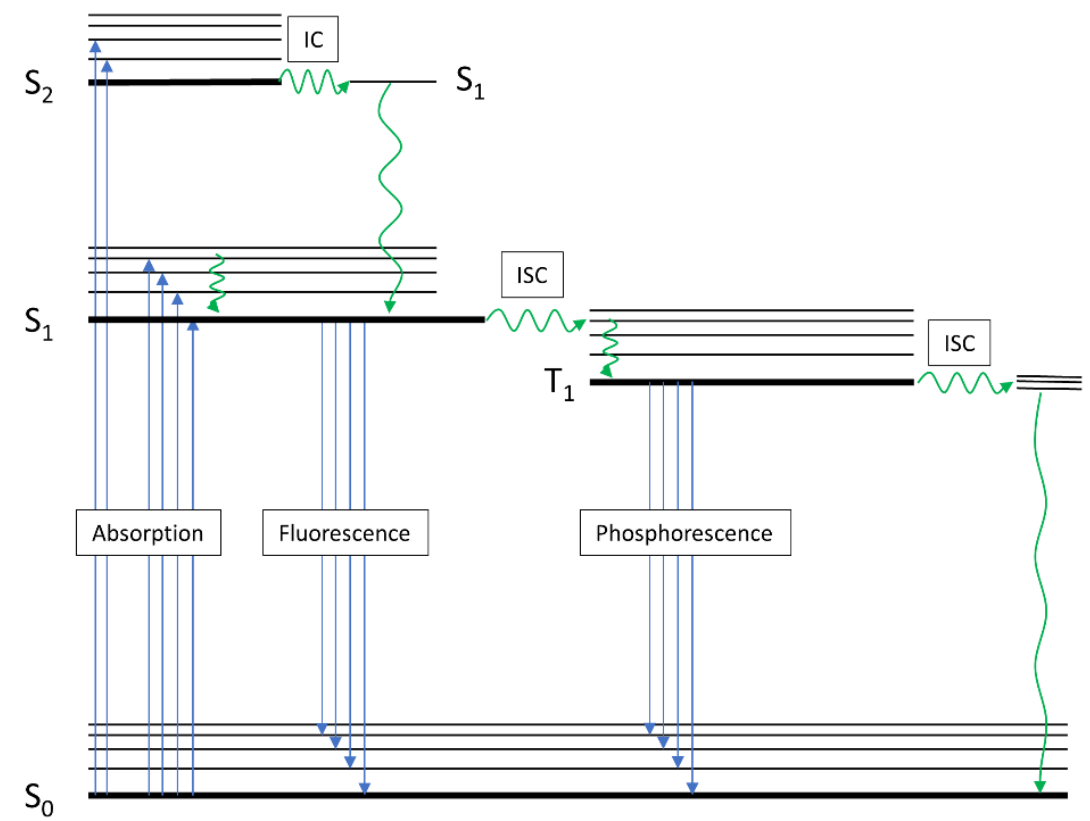

Figure 2. Jablonski-Diagram for representation of the main photophysical processes in polyaromatic hydrocarbons. Blue arrows indicate radiative transitions while green arrows represent non-radiative transitions.

Transitions between the lowest vibrational levels of different electronic states are called 0-0 transitions. According to the Franck-Condon-principle, transitions occur not only between these vibrational ground states. ${ }^{[28]}$ While at room temperature most of the molecules are in the lowest vibrational state, the excitation can result into a transition into a higher vibrational level of the first excited state. These vibronic transitions can be observed in the absorption spectra of common aromatic hydrocarbons, resulting in the structured characteristic absorption bands. Vibrational relaxation is usually fast with $10^{-12} \mathrm{~s}$ to $10^{-10} \mathrm{~s}$ and the radiative decay from the $S_{1}$ state then occurs from the lowest vibrational level of the excited state. ${ }^{[28]}$ Nevertheless, emission spectra often reveal a vibronic structure, which results from decays into the higher vibronic levels of the electronic ground state. Therefore, the absorption spectrum represents the vibronic levels of the first excited state and the emission spectrum the vibronic levels of the ground state. ${ }^{[28]}$

Besides the presented basic transitions, further processes such as fluorescence from higher excited-states or dual-emission from two different states are also known but are beyond the scope of this work. Furthermore, deactivation of excited molecules via photochemical reactions is also possible and widely investigated. ${ }^{[22,23]}$ The $[4+4]$ photocycloaddition of anthracene was an early example of photoreactions and 
led to further investigations. Nowadays, photochemistry is well established and used in several synthetic applications. ${ }^{[22,23]}$

The photophysical properties of simple and isolated fluorophores can be described thanks to the discussed fundamental processes. For many applications, molecules with special features are needed and the underlying processes become more complex. Especially, intermolecular interactions and aggregation can influence the excited states, as briefly discussed before. Formation of excited dimers and complexes can result in drastic changes of the emission properties and the related theory will now be discussed briefly in the following section.

\subsubsection{Excimers and exciplexes}

Excimers and exciplexes can be formed through short-range interactions between several atoms or molecules. This association leads to changes in the electronic structure of the resulting complex. Not only aromatic hydrocarbons, which will be in the focus of this work, but also metals, halogens and noble gases can form excimers and have been used in different technologies.

\subsubsection{Theory of excimer formation}

Identification of the first excimer dates back to 1927, when the emission bands in the spectrum of the mercury dimer were investigated. ${ }^{[91]}$ Later, the formation of associated dimers of noble gases has been postulated, when an intense continuum in the ultraviolet emission spectrum of high-pressure helium was observed. ${ }^{\left[{ }^{[2]}\right.}$ Similar findings were reported in the following years for other noble gases. ${ }^{[93]}$

A comparable phenomenon in solutions of a polyaromatic hydrocarbon was recognized by Förster and Kasper in 1954. ${ }^{[65]}$ The fluorescence spectrum of a pyrene solution was found to be dependent on the pyrene concentration. ${ }^{[65]}$ As no corresponding change in the absorption spectrum could be observed an association of an excited molecule with an unexcited molecule was assumed to be responsible for the spectral changes. The emerging fluorescence band in the concentrated solution was ascribed to these associated dimers. ${ }^{[65]}$ To distinguish this process from the excitation of a 'usual' dimer, Stevens and Hutton introduced the term excimer (=excited dimer) in 1960. ${ }^{[94]}$ The formation process of excimers in solution was further studied by Birks and Christophorou ${ }^{[66]}$ While at first the excimer emission was described to be an exclusive feature of pyrene, later studies revealed that a wide range of hydrocarbons are able to undergo excimer emission in concentrated solutions. ${ }^{[66,67]}$

A basic description of the excimer formation and underlying photophysical processes was given by Birks. According to his model, excimers form through collisional interaction between an excited molecule $\left({ }^{1} \mathrm{M}^{*}\right)$ and a molecule of the same type in the ground state $\left({ }^{1} \mathrm{M}\right)(1)$. In the resulting excimer ${ }^{1}(\mathrm{MM})^{*}$, the excitation energy is considered to be delocalized over the two molecules. ${ }^{[28,67]}$

$$
{ }^{1} \mathrm{M}^{*}+{ }^{1} \mathrm{M} \rightleftarrows{ }^{1}(\mathrm{MM})^{*}
$$

When an excited molecule $\left({ }^{1} \mathrm{M}^{*}\right)$ interacts with a molecule of different type in the ground state $\left({ }^{1} \mathrm{~N}\right)$, an excited complex $\left({ }^{1}(\mathrm{MN})^{*}\right)$ (exciplex) is formed (2). Depending on the interacting molecules, different types of exciplexes can be formed and the polyaromatic hydrocarbon can act as the donor or acceptor molecule. 
An exciplex requires a definite stochiometric composition to distinguish it from an excited molecule interacting with an undefined number of solvent molecules. ${ }^{[28,67]}$

$$
{ }^{1} \mathrm{M}^{*}+{ }^{1} \mathrm{~N} \rightleftarrows{ }^{1}(\mathrm{MN})^{*}
$$

Excimers and exciplexes are often identified through pronounced changes in their fluorescence spectra. As these processes in solution are diffusion controlled, the excimer formation and the corresponding spectral changes are dependent on the fluorophores concentration, viscosity of solvent and temperature. ${ }^{[95]}$ Since the corresponding absorption spectra are not affected by these parameters, the molecules are dissociated or only weakly bonded in the ground state and the excimer is formed only after excitation. The resulting spectral changes can be illustrated by the potential energy diagram of the ground state $\left({ }^{1} \mathrm{M}+{ }^{1} \mathrm{M}\right)$ and the first excited singlet state $\left({ }^{1} \mathrm{M}+{ }^{1} \mathrm{M} *\right)$ of two identical molecules (Figure 3). ${ }^{[28,67]}$ The molecules are considered to be parallelly oriented in a cofacial conformation with partially or complete overlap of the aromatic planes. The separation of the molecules is given by the interplanar distance $r$. When both molecules are largely separated, and no intermolecular interactions occur $(r=\infty)$ monomer fluorescence with the energy $M_{0}$ from a single excited molecule $\left({ }^{1} M^{*}\right)$ can be observed. This is usually the case in diluted solutions and goes along with the typical vibronic structure in the emission spectrum. Association of two molecules through decrease of the distance $r$ below $\sim 3.6 \AA$ results in a repulsive potential $R(r)$ in the ground state between ${ }^{1} \mathrm{M}$ and ${ }^{1} \mathrm{M}$. Also, a repulsive interaction $\left(R^{\prime}(r)\right)$ between ${ }^{1} \mathrm{M} *$ and ${ }^{1} \mathrm{M}$ can be observed in the exited state, which is nearly identical to the potential in the ground state as nuclear and electronic coordinates change only slightly. Moreover, an attractive potential exists in the excited state, which is the excimer interaction potential $V^{\prime}(r)$. The sum of the repulsive and attractive potential is the resulting excimer energy $D^{\prime}(r)$ (3). A stable excimer only forms if the excimer energy $D^{\prime}(r)$ is below the energy of $M_{0}$. The minimum excimer energy is found at the distance $r_{\mathrm{m}}$. The energy difference between $M_{0}$ and $D_{m}^{\prime}$ is the excimer binding energy $B$ relative to the dissociation into the initial states ${ }^{1} \mathrm{M}^{*}$ and ${ }^{1} \mathrm{M}(4) .{ }^{[28,67]}$ The excimer binding energy $B$ for the pyrene excimer was estimated to be around $39 \mathrm{~kJ} / \mathrm{mol}$ at a distance $r=3.4 \AA{ }^{[96]}$

$$
\begin{gathered}
D^{\prime}(r)=V^{\prime}(r)+R^{\prime}(r) \\
B=M_{0}-D_{\mathrm{m}}^{\prime}
\end{gathered}
$$

Excimer fluorescence occurs from the minimum-energy geometry of the excited state with the emission energy $D_{m}$. The emission can be observed for example at concentrated solutions of polyaromatic hydrocarbons, where the probability of two fluorophores in proximity rises. It is not unusual that both excimer and monomer fluorescence happen simultaneously in solution and the intensity ratio is often concentration dependent. ${ }^{[65]}$ 


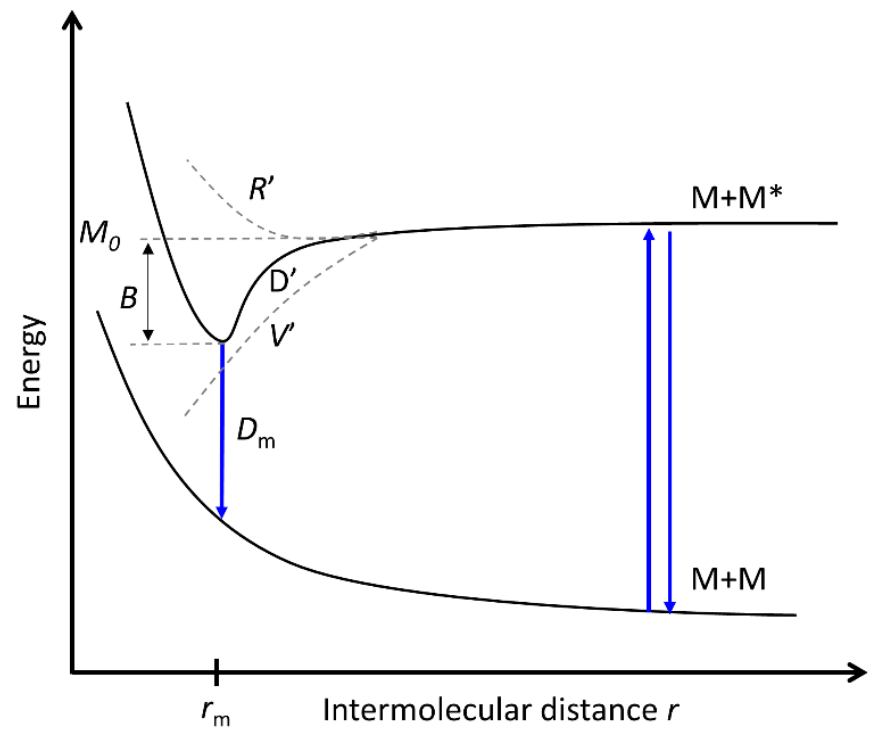

Figure 3. Schematic energy diagram for excimer formation. The excimer is formed through collision of two identical molecules (M), one in the ground state and one in the excited state.

The spectral changes of the excimer fluorescence can be explained by the potential energy diagram (Figure 3). The excimer binding energy $B$ results in a bathochromic shift and a larger Stokes shift of the excimer fluorescence compared to the emission from the monomer. The broad structureless emission, which is characteristic for excimer emission, is the consequence of the dissociative ground state at the excimer geometry. The radiative decay from the symmetrical excimer state to the ground-state is symmetry forbidden and usually results in low quantum yields and increased lifetimes. ${ }^{[67]}$ The occurrence and observability of excimer fluorescence is assigned to vibronic coupling to higher excited states or slight geometric deviations. ${ }^{[28]}$ Formation of excimers therefore contributes to the common observed concentration quenching of polyaromatic hydrocarbons and was usually avoided.

For many hydrocarbons, the formation of excimers in solution was confirmed relatively quickly after the first description of the pyrene excimer. ${ }^{[66]}$ The observation of excimer fluorescence of pure anthracene in solution was not that straightforward. In 1962, Parker and Hatchard investigated the fluorescence of anthracene in ethanol solutions, but the expected excimer fluorescence could not be confirmed. ${ }^{[97]}$ Four years later, Moore reported on the excimer emission of anthracene from a glycerin solution and observed the excimer fluorescence at $520 \mathrm{~nm} \cdot{ }^{[98]}$ About the same time, Barnes and Birks studied the fluorescence of 9-methyl and 9,10-dimethyl anthracene and confirmed the excimer formation in several solvents. ${ }^{\text {[99] }}$

A competitive process to excimer fluorescence is the formation of a stable photodimer, which complicates the observation of excimer emission (Scheme 8). For anthracene, the formation of a photodimer is more likely in comparison to other hydrocarbons like benzene, naphthalene, pyrene and benzanthracene. ${ }^{[100]}$ The first excited state $\left(S_{1}\right)$ of anthracene and higher acenes is polarized along the short molecular axis resulting in relatively high electron density at the 9- and 10-position after photoexcitation. ${ }^{[28]}$ The interaction between an excited monomer and a monomer in the ground state is strong enough for 
formation of covalent bonds between the $\mathrm{C}$ atoms in 9- and 10-position, respectively. The reaction results in a stable photodimer and goes along with the loss of aromaticity in the central anthracene rings. Similar behavior is found for the higher acenes like tetracene or pentacene, whose first excited states are also polarized along the short molecular axis. ${ }^{[28]}$ In contrast, the formation of photodimers is not favored over the excimer emission for other aromatics like naphthalene, pyrene and benzanthracene. The $\mathrm{S}_{1}$ state of these compounds is polarized along the long molecular axis, which results in insufficient interaction between two monomers. The formation of a photodimer is thus unlikely and fluorescence from the excimer occurs. Ferguson and Mau studied the photophysical processes of anthracene dimers and assumed that the excimer is an intermediate state in the formation of the photodimer. ${ }^{[100]}$ The competing processes (excimer fluorescence and photodimerization) are actually temperature-dependent. The efficiency of excimer fluorescence was found to be very low at ambient temperatures and increased at low temperatures. The opposite behavior was found for the formation of the photodimer. ${ }^{[100]}$
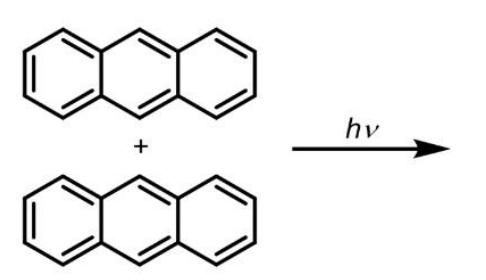
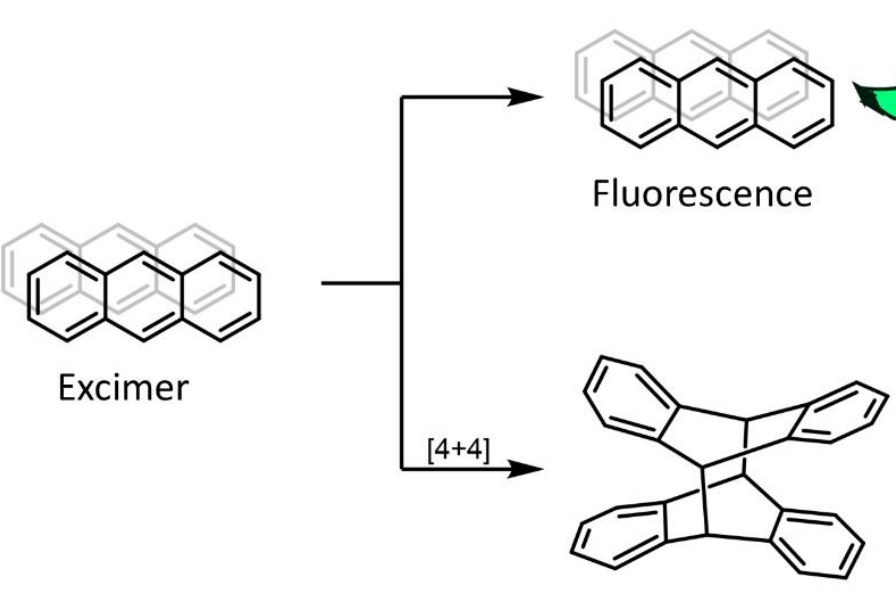

Photodimer

Scheme 8. Possible deactivation pathways of the anthracene excimer through excimer fluorescence (top) or photodimerization via a [4+4] cycloaddition (bottom).

Introduction of appropriate substituents can change the excited state properties of polyaromatic hydrocarbons in terms of excimer and photodimer formation. ${ }^{[28]}$ Both processes require spatial proximity of two acenes in a parallel orientation. Therefore, the introduction of substituents influences the mandatory approach of two monomers. For anthracene, the effect of the steric size has been investigated with different substituents in the 9- and 10-position. ${ }^{[99]}$ Introduction of two phenyl groups, which adopt a twisted conformation towards the anthracene, prevents the formation of excimers as well as photodimers. Therefore, 9,10-diphenylanthracene shows less concentration quenching and an overall high fluorescence quantum yield in solution. ${ }^{[99]}$ Hence, substitution of fluorophores with bulky substituents has become a suitable strategy for avoiding excimer formation and achieving efficient fluorescence emission in solution. For 9,10-dimethylanthracene, Barnes and Birks could observe an excimer fluorescence but no formation of photodimers. ${ }^{[99]}$ The mono substituted 9-methylanthracene forms fluorescent excimers and stable photodimers, although the excimer fluorescence is favored. As no steric hindrance is present in pure anthracene, the formation of the photodimer is preferred over excimer fluorescence. ${ }^{[99]}$

- 16 - 
The basic photophysical processes of aromatic excimers have been investigated shortly after their discovery. The nature of the electronic structure of the excimer was subject of several studies. ${ }^{[67]}$ Even today, the electronic state of the excimer is still under discussion and especially the benzene excimer is part of numerous experimental and theoretical approaches. ${ }^{[101,102,103]}$ It is assumed that the most stable excimer structure is the perfect cofacial dimer that forms after excitation from the ground-state structure. A discussed, in the ground-state a parallelly displaced structure is usually preferred. Upon excitation, the displacements in all three directions are reduced to obtain the cofacial alignment. The ideal, parallel orientation allows an interaction of the orbitals, resulting in a delocalization of the excitation energy over the two molecules. The stabilization of the excimer is usually ascribed to a combination of two types of interactions, which are an exciton resonance (5) and a charge resonance (6). ${ }^{[103,104]}$ The contribution of each energy to the excimer binding in different aromatic hydrocarbons is still investigated.

$$
\begin{gathered}
\mathrm{M}^{*}+\mathrm{M} \rightleftarrows \mathrm{M}^{*} \mathrm{M} \leftrightarrow \mathrm{MM}^{*} \\
\mathrm{M}^{*}+\mathrm{M} \rightleftarrows \mathrm{M}^{-} \mathrm{M}^{+} \leftrightarrow \mathrm{M}^{+} \mathrm{M}^{-}
\end{gathered}
$$

For the benzene dimer, the rearrangement from the minimum energy ground-state structure, which adopts a T-shape geometry, to the sandwich-type structure of the excimer is also subject of theoretical studies. ${ }^{[102,105]}$ Besides the investigation of aromatic excimers in solution and their theoretical study in the gas phase, the formation of excimers in the solid-state was examined shortly after the first discovery of the pyrene excimer.

\subsubsection{Excimers in the solid-state}

One of the first investigations of solid-state excimers concentrated on the photophysical properties of crystalline pyrene, the prototype for excimer emission in solution. ${ }^{[106]}$ Fluorescence of pyrene crystals was found to be similar to the excimer fluorescence of concentrated solutions. As the absorption spectra of the crystals are also comparable to the absorption spectra in solution, the occurrence of excimer fluorescence in the pyrene crystals was postulated. ${ }^{[106]}$ Like observed before, the excimer forms only after excitation and is dissociated in the ground state. After investigations on the emission properties of further polyaromatic hydrocarbons, a correlation between the occurrence of excimer fluorescence and the crystal structure was postulated. ${ }^{[107]}$ Excimer fluorescence was only observed for crystals, in which the aromatic compounds adopted a pairwise parallel structure (in pyrene or perylene for example). For other acenes like naphthalene, anthracene or phenanthrene, the nearly perpendicular arrangement of neighboring molecules resulted in a herringbone packing. The fluorescence spectra of these crystals resemble the structured emission observed for diluted solutions. ${ }^{[107]}$ The emission is assigned to the molecular emission from single excited molecules. As discussed before, substitution of the acenes can change the excimer formation ability. For example, both 9-cyanoanthracene and 1-chloranthracene crystallize in two different polymorphic forms. One resembles the typical herringbone structure of pure anthracene and in the other form pairs of molecules with parallelly oriented aromatic planes are found. Mixtures of both forms reveal structured monomer emission as well as excimer emission at higher wavelengths. This observation further 
supports the postulated hypothesis that the dimer geometry with parallelly oriented aromatic planes is beneficial or even essential for excimer formation. ${ }^{[28]}$

Excimer emission could also be observed from pure anthracene or similar compounds with a herringbone type packing by application of high external pressure. ${ }^{[108]}$ The external pressure induces defects within the crystal structure that adopt a pairwise parallel orientation and are therefore capable of excimer emission. The same method can be used for the observation of monomer fluorescence from pyrene crystals, as the resulting defects show no overlap and emission occurs from the molecular state. ${ }^{[109]}$

\subsubsection{Role of excimers in optoelectronic applications}

After the discovery and investigation of fundamental processes of the excimer formation, several applications were developed to make use of the properties of excimers. The first applications were not based on the excimer formation of polyaromatic hydrocarbons but rather of noble-gases and halides. In the 1970s, excimer and exciplex lasers ${ }^{1}$ based on the UV excimer emission of noble-gas dimers (e.g. $\mathrm{Xe}_{2}$ ) or noble-gas halides (e.g. XeBr) were developed. ${ }^{[110]}$ The first commercially available excimer laser was built in Göttingen in 1977 by Basting and Steyer. Since then, excimer lasers have been used for example in fabrication of microelectronics or medical applications. ${ }^{[111]}$

First applications based on excimers of polyaromatic hydrocarbons were introduced in the 1990s. Excimers were used for the investigation of supramolecular bio assemblies and as sensors. ${ }^{[112]}$ Most research in this field is still based on the pyrene excimer due to its high tendency to form excimers. ${ }^{[113]}$ Rational labeling of nucleic acids or proteins with pyrene and its derivatives allows monitoring of conformational changes. Excimer fluorescence is only observed when the corresponding fluorophores are in close proximity to each other. Therefore, the intensity of excimer emission gives information about intra- and intermolecular interactions, such as protein oligomerization, aggregation or protein folding and unfolding. ${ }^{[114]}$

Besides their usage in biochemical diagnostics, organic excimers are used in electronic applications. ${ }^{[15]}$ While for diagnostics and sensing the fluorophore is usually in a fluid environment, application in organic electronics requires an excimer formation in the solid-state. For many years, excimer formation was ascribed to be responsible for exciton trapping and fluorescence quenching. Corresponding compounds were therefore not suitable for applications in organic electronics. ${ }^{[115]}$ Further investigation of the photophysical properties and rational design of the molecules helped to overcome these drawbacks and established excimers in various organic electronic devices. ${ }^{[115]}$ Besides the noble gases, lasers based on organic excimers have also been introduced in the literature. Wei et al. developed a wavelength switchable laser using of the monomer and excimer emission of 4-(Dicyanomethylene)-2-methyl-6-(4dimethylaminostyryl)-4H-pyran. ${ }^{[116]}$ By controlling the doping concentration of the dye, the emission wavelength could be modulated between monomer and excimer emission. The approach by Wei et al. showed that the excimer formation is not always unfavorable in organic electronics: beneficial excimer

\footnotetext{
${ }^{1}$ In laser technology the term 'excimer-laser' is used in general even if two different type of molecules are used and the term 'exciplex' would be more suitable. - 18 -
} 
properties can be used in suitable devices. This strategy is therefore a promising contribution to the development of organic solid-state lasers. ${ }^{[116]}$

In the research field of organic photovoltaics, perylene bisimides (PBI) have been used as an alternative acceptor material for the common fullerene-based acceptors. PBIs have several advantages like higher stability, lower costs and are easier to modify chemically. ${ }^{[115]}$ Nevertheless, they often suffer from lower efficiencies, which are ascribed to excimer formation due the large $\pi$-system. The co-facial stacking favors the formation of excimers, which can act as exciton traps and reduce the efficiency. Therefore, the PBI moiety is often modified to induce a twisted structure, which prevents excimer formation, but comes with several drawbacks regarding charge-transport and electron mobility. ${ }^{[117]}$ Wasielewski and co-workers investigated the influence of the stacking geometry in perylene bisimides on the performance of organic photovoltaic cells. Through different substituents, the packing of the PBIs could be slightly modified. A slipped-stacking motif reduced the excimer formation rate under conservation of the beneficial $\pi$-stacking geometry for charge transport. The authors concluded that the control of the packing in PBIs through subtle substituents is a promising strategy for obtaining efficient alternatives for fullerene-based acceptors. ${ }^{[117,118]}$

Excimer emitting materials are probably mostly suitable towards applications for OLEDs. The possibility of tuning and controlling emission wavelengths is fundamental in the development of effective light emitting devices (vide supra). Especially, white OLEDs (WOLEDs) can benefit from the excimer emission characteristics. WOLEDs are usually prepared through a combination of separate blue, green and red emitters. This induces several drawbacks regarding stability and color purity. ${ }^{[119]}$ A possible alternative is the preparation of white light emitting materials based on a single emitting structure. However, the design of molecules, whose emission covers the whole visible spectral range is still challenging. ${ }^{[21]}$ The broad excimer emission can be beneficial for addressing a wide range of the emission spectrum. A combination of monomer and excimer emission from the same compound is therefore a suitable strategy for generating white light emission.

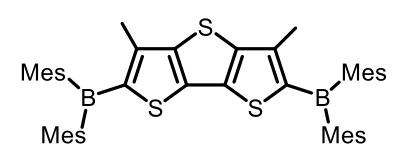

$X, 2005$

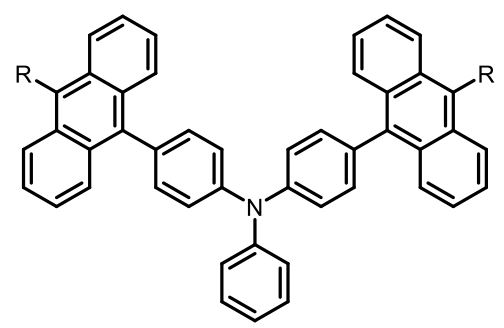

$X I, 2006$

$\mathrm{R}=$ Pyrenyl

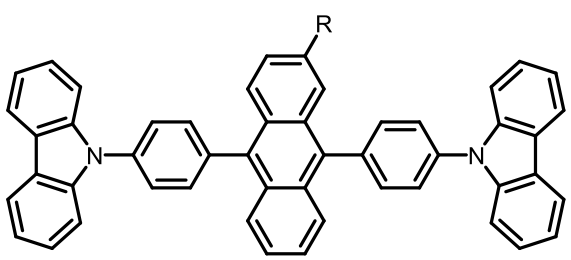

XII, 2016

$\mathrm{R}=\mathrm{H}, \mathrm{tBu}$

Scheme 9. Examples of excimer based single molecular white light emitters.

An early example of this approach, reported by Mazzeo et al. in 2005, used a terthiophene derivative (X) as the only luminescent material for obtaining a white light emitting device (Scheme 9). ${ }^{[120]}$ The white luminescence was attributed to a combination of blue-green monomer emission and red excimer emission. The peripheral dimesitylboryl substituents are essential for the observed cross conjugation motif 
of the terthiophene unit, which allows the excimer formation. Comparable derivatives without cross conjugation showed no excimer emission and were therefore not suitable for application in white light emitting materials. ${ }^{[120]} \mathrm{A}$ further example of a white light emitting organic compound was reported by Tao et al. in 2008. ${ }^{[121]}$ Two phenyl rings of triphenylamine were substituted with pyrenylanthracene in the para position (XI). Besides the blue monomer fluorescence, a broad, red excimer emission was observed in thin films. A light emitting device prepared from this structure emits white light with good performances at the time. ${ }^{[121]}$ Due to its blue monomer emission, anthracene is a suitable fluorophore for obtaining singlemolecular white light emission as the formed excimers emit in longer wavelength regions of the spectrum. The group of Chou investigated two slightly different anthracene derivatives in terms of white light generation. ${ }^{[122]}$ The compound without the tert-butyl group in 2-position of the anthracene (XII) revealed nearly white luminescence, which was attributed to the monomer and excimer emissions. Substitution of the hydrogen in 2-position with a tert-butyl group induces a different packing motif where no excimer formation is possible. A blue fluorescence without excimer contribution is the result. ${ }^{[122]}$ This example further demonstrates how small structural changes can influence the occurring intermolecular interactions and the resulting photophysical properties.

The herein described compounds emit from the singlet excited state, which is connected to lower efficiencies due to the statistical limit of generated singlet excitons. Therefore, excimers have also been used for obtaining white-light emission in the advanced techniques like TADF, which allow higher quantum yields of the devices.

Wang et al. investigated the dependence of thermally activated delayed fluorescence on the supramolecular structure of different xanthen-9-one based derivatives (XIII, XIV, Scheme 10). ${ }^{[123]}$ The donor-acceptor motif with a twisted structure is generally suitable for a small energy gap between the singlet and triplet excited state, due to a spatial separation of the HOMO and LUMO. Further, it could be shown that defined $\pi$-stacking interactions can increase the lifetime of singlet and triplet excited states, which is also advantageous for the TADF process. ${ }^{[123]}$ For the diphenylamino substituted derivative (XIV), three polymorphs with different intermolecular interactions could be isolated. ${ }^{[124]}$ One crystal, which revealed no $\pi-\pi$ interactions, showed blue fluorescence, which was attributed to the locally excited monomer state. The two other polymorphs underwent a decent bathochromic shift resulting in a green and yellow emission. This bathochromic shift was assigned to an excimer emission, which could be supported by the present $\pi-\pi$ interactions in the crystal structure. A combination of all three polymorphs resulted in a white light emission. Furthermore, only the two polymorphs with $\pi$-stacking interactions revealed TADF behavior, which was assigned to a more rigid structure, which prohibits non-radiative decay from the triplet state and promotes the reverse intersystem crossing. ${ }^{[24]}$ 


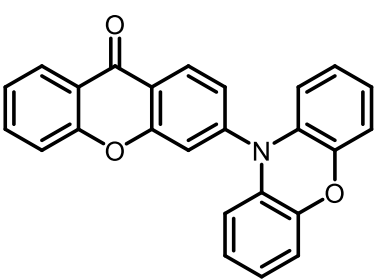

XIII

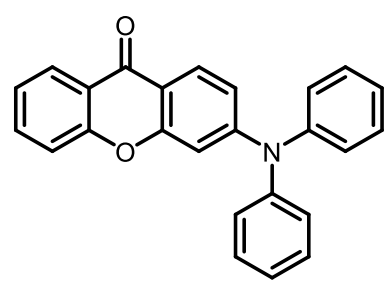

XIV

Scheme 10. Two xanthen-9-one based fluorophores with TADF characteristics dependent on the intermolecular interactions. For the diphenylamine substituted derivative $\mathbf{X I V}$, three polymorphs with different emission wavelengths were identified and a combination of them resulted in white-light emission.

Besides the discussed excimer formation in organic molecules, transition metal complexes have also been investigated regarding their ability for excimer formation and application in white light emitting devices. ${ }^{[21]}$ Due to their large spin-orbit coupling, complexes of heavier transition metals like iridium and platinum are suitable for achieving high efficiency devices as the emission occurs from the triplet state. Furthermore, the square planar geometry of Pt-based complexes is beneficial for excimer formation as both Pt centers as well as ligands can participate in the required inter- or also intramolecular interactions. ${ }^{[125]}$ Also, Iridium complexes have been reported to exhibit excimer emission. For example Aoki et al. investigated two related quinoline based iridium complexes $(\mathbf{X V}) \cdot{ }^{[126]}$ Structural and photophysical investigations revealed that the white light emission is composed of a blue and a longer-wavelength orange emission. The lower energy emission was assigned to the emission of the excimer formed between pyridyl and phenyl moieties of the ligands (Scheme 11). ${ }^{[126]}$

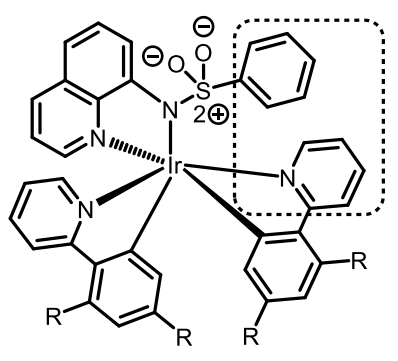

XV, 2016

$\mathrm{R}=\mathrm{H}, \mathrm{F}$

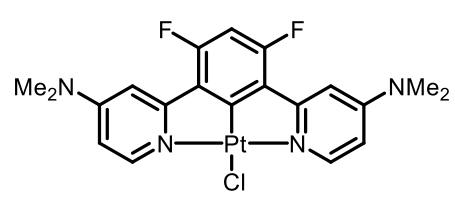

XVI, 2012

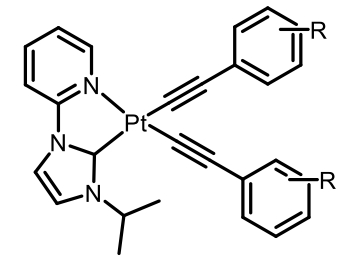

XVII, 2016

Scheme 11. Examples of transition-metal complexes for white-light emission in OLEDs due to intra- (XV) or intermolecular excimer formation (XVI and $\mathbf{X V I I ) . ~}$

The platinum-based complexes reveal similar behavior, although the excimer is usually formed by intermolecular interactions due to the relatively planar geometry of $\mathrm{Pt}(\mathrm{II})$ complexes. Murphy et al. synthesized and investigated a cyclometallated tridentate platinum complex (XVI) with white light emission. ${ }^{[127]}$ Compared, to a similar bidentate system, complex $\mathbf{X V I}$ revealed significantly higher quantum yields. The more efficient emission was ascribed to a higher rigidity, which suppresses the deactivation via $d$ - $d$ transitions. ${ }^{[127]} A$ similar approach was applied by the group of Venkatesan. ${ }^{[128]}$ The pyridine NHC ligand was used in a platinum complex (XVII), as it was expected to separate the non-radiative $d$-d states effectively from the radiative states. The alkyne ligands are able to form an intermolecular excimer. The 
ratio of monomer and excimer emission could be controlled by different concentrations of the complex in the PMMA film, which also allows tuning of the emission wavelength. By this approach, efficient white light phosphorescence with high quantum yields could be obtained. ${ }^{[128]}$ Until now, square-planar Pt(II) complexes capable of excimer formation belong to the best candidates for obtaining white light emission with high quantum yields. ${ }^{[21]}$

The discussed examples show that the unique properties of excimers can be beneficial for different applications in organic electronics, especially for organic light emitting devices. The intermolecular interactions affect the performance of these devices in several ways. Therefore, understanding of the photophysical-structural correlations is essential.

\subsubsection{Current research related to Anthracene excimers}

As excimer emission has found application in several areas of organic electronics, the factors determining the excimer formation in the solid-state are in the focus of current research. The understanding of the interplay between intermolecular interactions and luminescence properties seems to be essential for optimizing future applications. Recent progress concerning excimer emission of the anthracene fluorophore, which will also be used during this work, will be evaluated shortly.

Several groups reported on the structure-property relationship between the emission properties and the intermolecular interactions of the anthracene fluorophore. The applied strategy usually consists in introducing small variations at the substituent of the anthracene, which should induce a change in the crystal packing. Then, evaluation of the solid-state structures and photophysical properties can provide information about the correlation between the structure and the emission properties.

An early approach was reported in 2006 by Zhang et al., who investigated the structural and optical properties of 9-anthrylpyrazole (XVIII). ${ }^{[129]}$ Through variation of the crystallization conditions, five polymorphs with partially different molecular assemblies could be obtained. The $\mathrm{N}-\mathrm{H} \cdots \mathrm{N}$ hydrogen bonds between the substituents of neighboring molecules were attributed to control the crystal packing and the formation of different oligomers. The changed arrangements led to variation in the interactions between the anthracene fluorophores. As the polymorphs furthermore revealed altered emission properties, it was concluded that the optical properties are strongly dependent on the occurring $\pi-\pi$ interactions and on the stacking motifs between the fluorophores. ${ }^{[129]}$ 


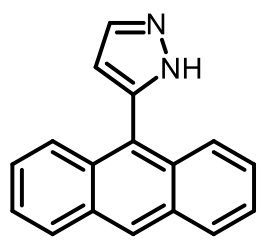

XVIII

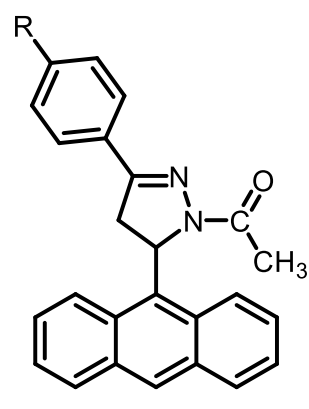

XIX

Scheme 12. Two pyrazole substituted anthracene derivatives, which revealed solid-state emission depending on the occurring intermolecular interactions.

Dong et al. reported in 2012 on the solid-state fluorescence of three co-crystals of a dihydropyrazole substituted anthracene derivative (XIX). ${ }^{[130]}$ The emission properties are controlled by the arrangement of the anthracene fluorophores and the resulting weak interactions. Two crystals showed a bathochromic shift in their emission wavelength up to $500 \mathrm{~nm}$, which was attributed to the $\pi-\pi$ interactions between the anthracene moieties. The estimated quantum yields are higher in the crystalline state than in solution. Additionally, the two crystals with moderate $\pi-\pi$ interactions revealed higher emission efficiencies compared to the third derivative without face-to-face interactions. The authors attributed this unexpected behavior to the immobilization of the fluorophores through several weak intermolecular interactions like $\mathrm{C}-\mathrm{H} \cdots \pi$ and $\pi-\pi$ interactions. Also, co-crystallization with a suitable guest led to the formation of stronger $\mathrm{C}-\mathrm{H} \cdots \mathrm{O}$ and $\mathrm{C}-\mathrm{H} \cdots \mathrm{N}$ hydrogen bonds and a further increase of the emission intensity. ${ }^{[130]}$

The group of Kohmoto designed a variety of anthracene derivatives with different hydrogen bond donors and acceptors as substituents to induce a pairwise packing of the anthracene derivatives through hydrogen bonding of the substituents. ${ }^{[131]}$ Emission of these derivatives in solution was quenched, but moderate quantum yields were measured in the solid-state. The origin of the fluorescence was ascribed to a socalled 'dimer emission' from the anthracene excimer. The separation of the anthracene pairs from each other resulted in a pure dimer emission and an increased quantum yield compared to emission in solution. ${ }^{[131]}$

Separation of anthracene dimers in the solid-state from each other was also in the focus of works by other groups. With suitable substituents, a columnar stacking of the fluorophores was avoided, and the anthracene dimers could be isolated from neighboring pairs. ${ }^{[132-135]}$ The face-to-face interactions between the fluorophores are the dominant interaction resulting in an excimer formation. It was postulated that the absence of further interactions involving the fluorophore is beneficial to achieve higher emission efficiencies. ${ }^{[132-135]}$

From recent examples, it can be concluded that substitution of the anthracene fluorophore on one side with a bulky substituent can induce the dimeric stacking motif. A twisted conformation of the substituent is beneficial for prohibiting a columnar stacking and for obtaining excimer emission. Nevertheless, the examples have also shown that only small modifications at the substituent can result in completely 
different packing motifs without $\pi-\pi$ interaction and therefore wihtou excimer formation. The prediction of the resulting intermolecular interactions and the occurring packing motif is still challenging, which makes the design of compounds with defined intermolecular interactions still an ambitious endeavor.

The excimer formation of the above discussed examples is induced mainly by the functionalization of the anthracene fluorophore and the resulting face-to-face interactions in the solid-state. A different approach is based on supramolecular assemblies and allows better control of the formed aggregates. Stoddart and co-workers synthesized an anthracene based cyclophane. ${ }^{[136]}$ Through guest inclusion of pure anthracene into the cyclophane or the formation of the corresponding homo-catenane, the study of anthracene excimers in diluted solution was possible. ${ }^{[136]}$ With the intention of studying the dynamics of excimer formation of unsubstituted anthracene in solution, Das et al. recently prepared a host-guest complex of anthracene and octa acid. ${ }^{[137]}$ The formed capsule contained two anthracenes in a face-to-face manner. The confined space prohibits a photodimerization of the anthracene, which is usually favored. Instead, the unusual excimer emission of pure anthracene in solution could be studied in detail and novel insights into the excimer formation were obtained. ${ }^{[137]}$

The herein described examples focused on the anthracene fluorophore, as it is also used during this work. For other polyaromatic hydrocarbons, similar trends can be found. Especially, the already wellinvestigated pyrene is still subject of current research regarding the control and tunability of intermolecular interactions towards photophysical properties. ${ }^{[138]}$

\subsection{Aggregation-Induced Emission}

As already introduced in the historical overview (chapter 1.1), the increased interest in the AlEphenomenon since the early 2000s led to an enormous and still increasing number of publications regarding this topic (Figure 4). The basic principles and underlying processes will be shortly evaluated as they give valuable information for general photophysical processes in the solid-state. A detailed overview over the studies will not be given during, but comprehensive summaries regarding the AIE-phenomenon can be found in several review articles. ${ }^{[55,57,139]}$

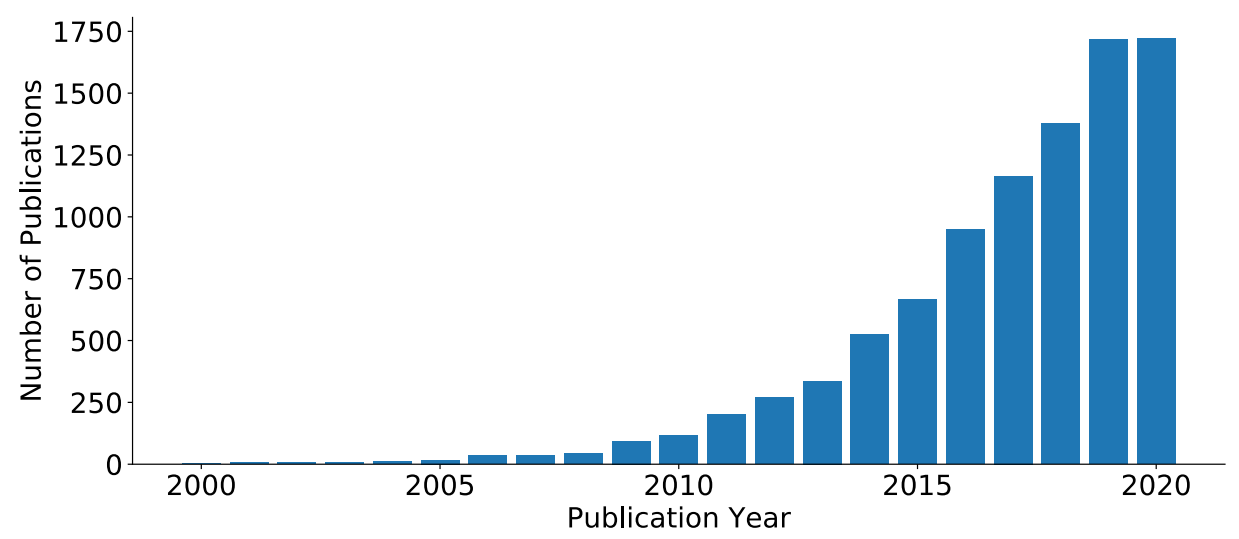

Figure 4. Number of publications per year correlated with the term 'Aggregation-induced-emission'. Data retrieved from SciFinder in December 2020. 
The term 'Aggregation-induced-emission' (AIE) was coined for molecules and materials, which are non- or only weakly fluorescent in diluted solution and become emissive upon aggregation. ${ }^{[4]}$ This behavior is unusual as common organic fluorophores are usually luminescent in solution and non-emissive in the solidstate or higher concentrated solutions due to concentration-quenching. ${ }^{[140]}$ As pointed out before, the underlying mechanism was ascribed to the restriction of intramolecular motion (RIM) upon aggregation (chapter 1.1). Common structural features of AIE-luminogens are therefore rotatable bonds and isomerizable double bonds. Typical representatives are tetraphenylethylene (TPE) ${ }^{[48,141]}$ and penta- or hexaphenylsilole (HPS ${ }^{[47,142]}$ and a plethora of examples containing these structural motifs can be found in the literature. Investigations on the underlying processes suggested that an energy transfer of the excitation energy into rotational modes of the molecule result in a non-radiative deactivation in solution. ${ }^{[51,143]}$ As the rotations are restricted upon aggregation, the deactivation via radiative pathways is possible. In the following years, the concept was expanded to intramolecular vibrations as deactivation pathways and generalized as a restriction of intramolecular motion (RIM). ${ }^{[54]}$

During the last years, molecules and materials without typical AIE structural features were reported, which also undergo an emission enhancement upon aggregation. Especially, the behavior of compounds with comparable structures and different luminescent properties could not be explained solely by the RIMmodel. Therefore, an updated and more general approach for the AIE phenomenon was evaluated based on the restricted access to a conical intersection $(\mathrm{Cl}) \cdot{ }^{[144,145]} \mathrm{A} \mathrm{Cl}$ can be described as the region where two or more potential energy surfaces (PES) are degenerate. ${ }^{[146]}$ For photochemical and -physical processes, especially the intersection of the ground-and first-excited state is important and enables the deactivation of the excited state via internal conversion. The $\mathrm{Cl}$ of a molecule can be accessible if it is lower in energy than the absorption energy, even if the $\mathrm{Cl}$ often adopt largely distorted structures. The access to the $\mathrm{Cl}$ via rotations or vibrations of AIE-luminogens results in an efficient fluorescence quenching in solution via internal conversion (Figure 5). When these molecules are in the aggregated state, the energy of the $\mathrm{Cl}$ rises and cannot be reached by the excited molecules as large structural distortions are usually not possible in the solid-state. Consequently, the non-radiative pathways are blocked, and the fluorescence in the aggregated state is enhanced. This general concept has been named "restricted access to a conical intersection" (RACl) and gives a more general explanation of the AIE-phenomenon. 
(a)

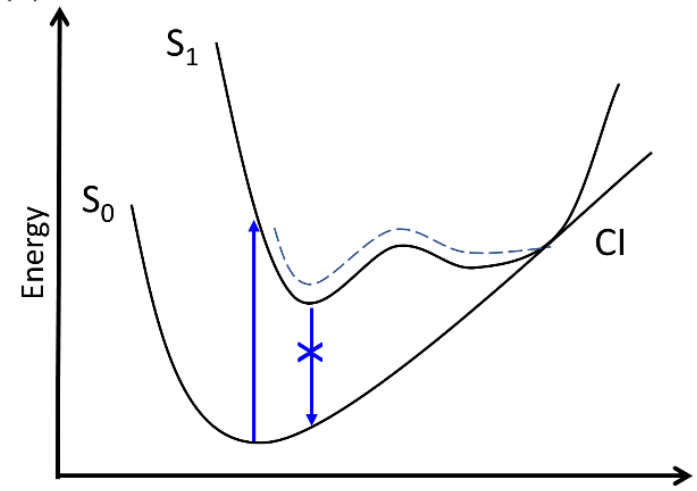

Reaction coordinate (b)

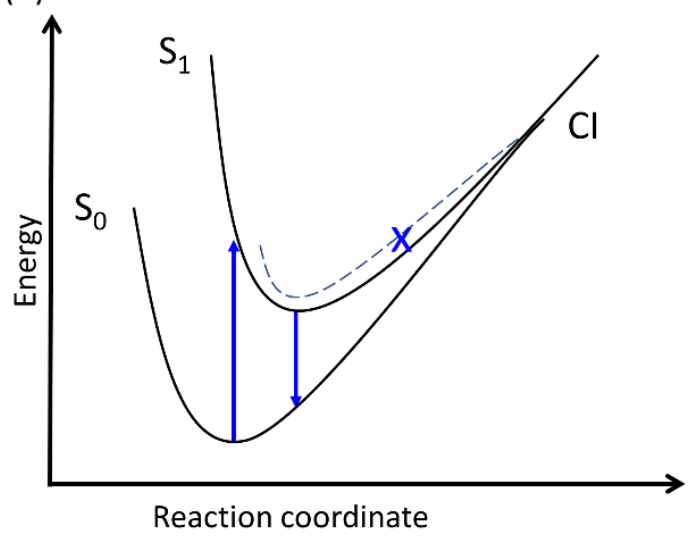

Figure 5. Schematic representation of the restricted access to a conical intersection (RACl) model. (a) In diluted solution a lowlying $\mathrm{Cl}$ is accessible, and deactivation of the excited state occurs via non-radiative internal conversion. (b) In the solid-state the access to the $\mathrm{Cl}$ is hindered as large-amplitude modes are not likely to occur. The result is a radiative decay via fluorescence.

The quantum chemical investigation of possible Cls for typical AIE-molecules also gave further insight into the deactivation pathways. For tetraphenylethylene (TPE) and its derivatives, the double bond rotation was widely accepted to be responsible for fluorescence quenching in solution. More recent studies revealed that the radiationless decay to the ground-state of the major part of the molecules occurs at a $\mathrm{Cl}$ via a photocyclization through formation of a $\mathrm{C}-\mathrm{C}$ bond between neighboring phenyl rings. ${ }^{[147]}$ The photocyclization requires of course a spatial proximity of the carbon atoms, which can be reached through rotation of the phenyl rings. In the aggregated state, the cyclization is blocked, and the typical AIE behavior is observed. The mechanism was confirmed experimentally by ultrafast transient absorption spectroscopy. ${ }^{[147]}$

Furthermore, the photophysical properties of structurally simple bis $(N, N$-dialkylamino $)$ anthracenes (BDAA) were also described by a restricted access to the conical intersection. ${ }^{[148-150]}$ As typical structural features for AIE are absent, the emission properties could not be explained by the RIM-model (Figure 6a). Additionally, the regioisomers of the bis $(N, N$-dialkylamino $)$ anthracenes revealed different photophysical behavior as only the para-substituted isomers 1,4-BPA and 9,10-BPA revealed aggregation-inducedemission, while for the other isomers a quenching upon aggregation was observed. A detailed computational investigation revealed that the $\mathrm{S}_{1} / \mathrm{S}_{0} \mathrm{Cl}$ is located at lower energy than the excited FranckCondon state. The anthracene plane of the structure at the $\mathrm{Cl}$ is largely distorted with an out-of-plane folding of the aromatic moiety (Figure 6b). ${ }^{[148]}$ The authors concluded that the $\mathrm{Cl}$ can be accessed in solution, but not in the solid-state as such a strong deformation is clearly restricted. ${ }^{[150]}$ Moreover, the electron donating amino substituents in para-position were attributed to stabilize the distorted structure at the $\mathrm{Cl}$. Therefore, the AIE behavior was only observed for the 1,4- and 9,10-substituted derivatives with the amino groups in para position. For the other isomers, the largely distorted structure of the $\mathrm{Cl}$ was not accessible and high fluorescence quantum yields in solution could be obtained $\left(\Phi_{\mathrm{F}}=0.80-0.88\right) .{ }^{[148-150]}$ This strategy was also transferred to other polyaromatic hydrocarbons and further AIE-active molecules were obtained. ${ }^{[150]}$ 
(a)

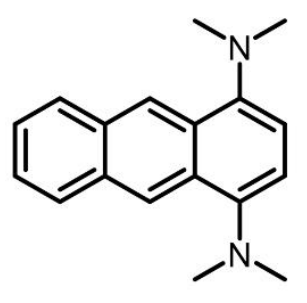

1,4-BDAA

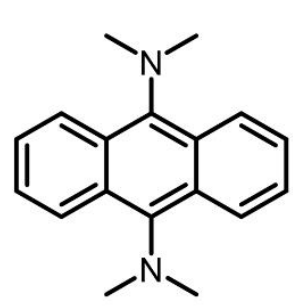

9,10-BDAA

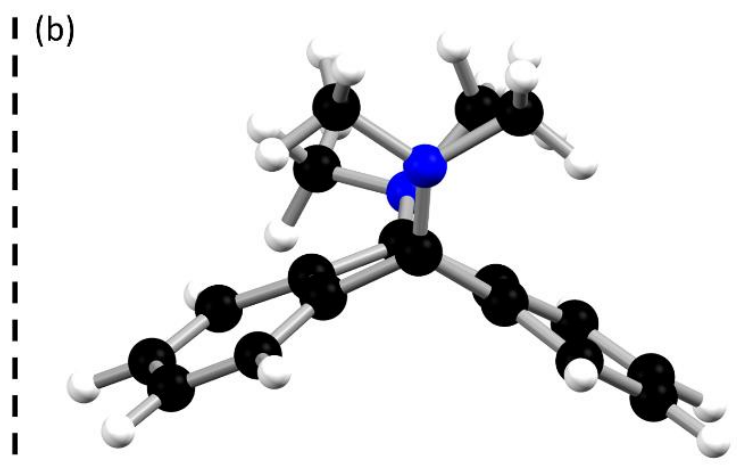

Figure 6. (a) Two regioisomers of bis( $N, N$-dialkylamino)anthracenes, which exhibit AIE-behavior. (b) Largely distorted structure of a 9,10-BDAA at the minimum energy conical intersection. Coordinates taken from reference. ${ }^{[150]}$

At first, the research regarding AIE focused on a few structural features, which were considered to be responsible for the unusual emission behavior. Nowadays, a wide range of different compounds with a variety of structural motifs is known. Their investigation gave insights into the general photophysical processes, which are important for understanding the emission properties of molecules in the solid-state. In general, the control of radiative and non-radiative deactivation pathways is essential for optimizing solid-state luminescent materials. Especially, the influence of intermolecular interactions on these pathways is often complex and less investigated.

\subsection{Previous work regarding solid-state luminescence in the Stalke group}

In the following chapter, an overview of previous studies on solid-state luminescence in the Stalke group will be given. The research began in 2003 when Fei et al. described a phenomenon, which was still not completely understood at the beginning of this work. ${ }^{[151]}$ The disubstituted 9,10bis(diphenylthiophosphoryl) anthracene $\left[9,10-\left((\mathrm{S}) \mathrm{PPh}_{2}\right)_{2}-\left(\mathrm{C}_{14} \mathrm{H}_{8}\right)\right]$ revealed remarkable solid-state luminescence, when co-crystallized with toluene, whereas the emission in diluted solution was nearly quenched. At that time, reports about strongly fluorescent solids were quite rare as aggregation was generally attributed for fluorescence quenching and concepts such as AIE were just recently developed (2001). ${ }^{[47]}$ Further research emphasized the unique properties of the thiophosphoryl anthracene. ${ }^{[152,153]}$ The corresponding oxophosphoryl anthracene $\left[9,10-\left((\mathrm{O}) \mathrm{PPh}_{2}\right)_{2}-\left(\mathrm{C}_{14} \mathrm{H}_{8}\right)\right]$ exhibited a contrary behavior with intense blue emission in solution and only weak emission as single crystals or powder, which is typical for many common organic chromophores (ACQ) ${ }^{[151]}$ When examining the photophysical properties of the sulfur-oxidized derivative, a direct correlation between co-crystallized toluene and the ability for solidstate emission was assumed. Removing the co-crystallized toluene under reduced pressure led to a diminishing fluorescence, which could be restored by subsequent exposure of the dried crystals to toluene. Other aromatic solvents, for instance benzene, were not able to recover the fluorescence, indicating unique structural features of the toluene co-crystals. From these findings, the origin of the pronounced emission in the solid-state was attributed to an exciplex ${ }^{2}$ formation between the anthracene core and the

\footnotetext{
${ }^{2}$ In the original publication the term excimer was used, which is usually applied to an excited dimer of two molecules of the same compound. Excited complexes between two different molecules are usually noted as exciplexes, which will be used in the following for this system.
} 
co-crystallized toluene. The broadened, structureless emission and the redshift compared to pure anthracene gave further evidence for the exciplex emission.
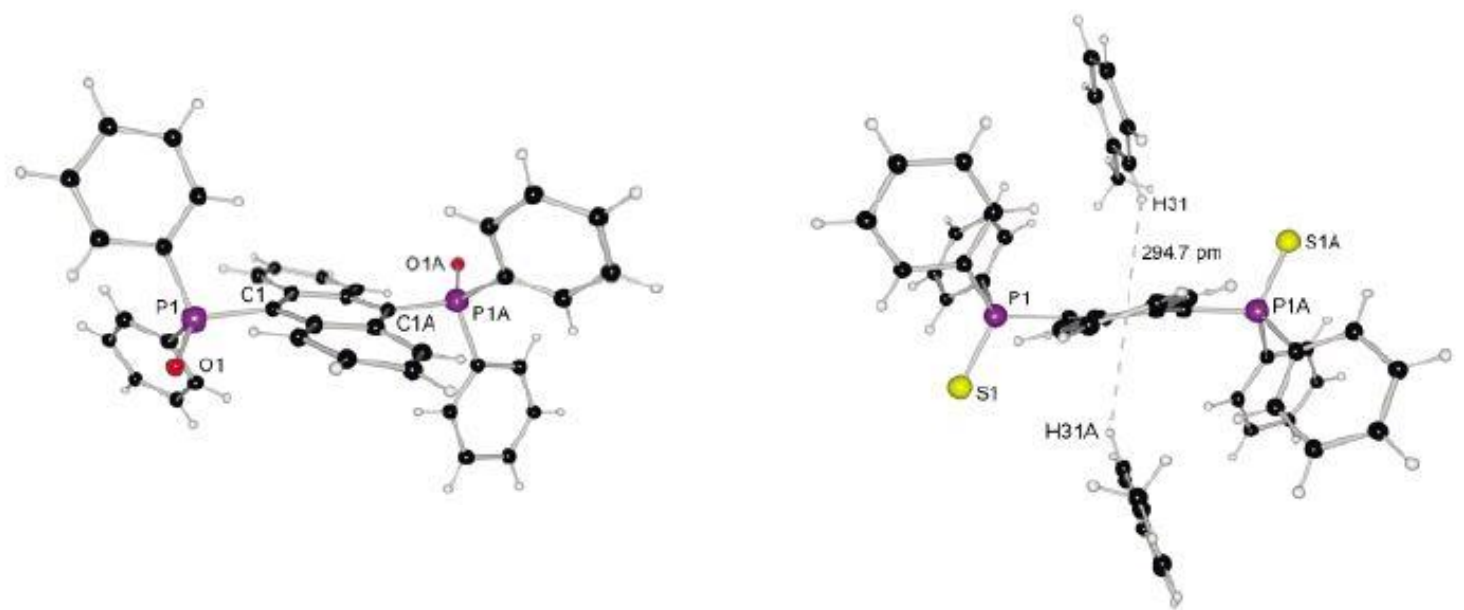

Figure 7. Solid-state structures of $\left[9,10-\left((\mathrm{O}) \mathrm{PPh}_{2}\right)_{2}-\left(\mathrm{C}_{14} \mathrm{H}_{8}\right)\right]$ (left) and $\left[9,10-\left((\mathrm{S}) \mathrm{PPh}_{2}\right)_{2}-\left(\mathrm{C}_{14} \mathrm{H}_{8}\right)\right]$ with co-crystallized toluene (right) revealing different orientations of the $(E) \mathrm{PPh}_{2}(\mathrm{E}=\mathrm{O}, \mathrm{S})$ substituents regarding the anthracene plane and the T-shape orientation of the co-crystallized toluene towards the anthracene. Adapted with permission from reference. ${ }^{[151]}$

The solid-state structure supported these conclusions, revealing a transoid orientation of the (S)PPh substituents, with one sulfur atom above and the other one below the anthracene plane and therefore a nearly orthogonal position of the sulfur towards the anthracene ( $\mathrm{S}-\mathrm{P}-\mathrm{C} 1-\mathrm{C} 2$ torsion angle $\left.=84.7^{\circ}\right)$. This shape forms a cradle for an edge-to-face stacking between toluene and the anthracene moiety (Figure 7). Furthermore, the toluene was fixed in this position by weak $\mathrm{C}-\mathrm{H} \cdots \pi$ interactions of one ortho hydrogen and the anthracene core. The oxygen-homolog revealed a different conformation, with the oxygen atoms almost in-plane $\left(\mathrm{O}-\mathrm{P}-\mathrm{C} 1-\mathrm{C} 2\right.$ torsion angle $\left.=23.6^{\circ}\right)$ and the phenyl-rings therefore oriented one above and one below the anthracene moiety. This alignment seemed not to be feasible for an intercalation of toluene and therefore no exciplex emission was observed in the solid state (Figure 7). The selenium-homolog $[9,10-$ $\left.\left((\mathrm{Se}) \mathrm{PPh}_{2}\right)_{2}-\left(\mathrm{C}_{14} \mathrm{H}_{8}\right)\right]$ also crystallizes with one toluene molecule in the asymmetric unit, but with a slightly different orientation. Nevertheless, for the latter compound no fluorescence could be observed in solution or in the solid-state.

Right after the work of Fei et al., Schwab continued the examination of luminescent host-guest complexes of $\left[9,10-\left((\mathrm{S}) \mathrm{PPh}_{2}\right)_{2}-\left(\mathrm{C}_{14} \mathrm{H}_{8}\right)\right]$. During his diploma and Ph.D. thesis, he was able to identify several new cocrystals with a wide range of aromatic solvents. ${ }^{[154,155]}$ Contrary to the findings of Fei, Schwab discovered strongly emissive compounds with different guests other than toluene, disproving the assumed selectivity towards toluene. The fluorescence spectra as well as the crystal structures of these compounds revealed only minor differences leading to the assumption that the solid-state emission is nearly independent of the intercalated guest. Additionally, some weakly fluorescent host-guest systems of $\left[9,10-\left((S) P P h_{2}\right)_{2}-\right.$ $\left(\mathrm{C}_{14} \mathrm{H}_{8}\right)$ ] were obtained, which adopt a different conformation regarding the orientation of the substituents. In contrast to the previous described transoid orientation, the (S) $\mathrm{PPh}_{2}$ groups adopt a cisoid arrangement with all phenyl groups at one side and both sulfur atoms at the other side referred to the -28 - 
anthracene plane (Figure 8). As the sulfur atoms are still almost orthogonal to the aromatic core the steric strain of the four phenyl groups leads to a deformation and distortion from planarity of the anthracene. For these cisoid oriented structures no preferred alignment for the aromatic guests relative to the anthracene was found.

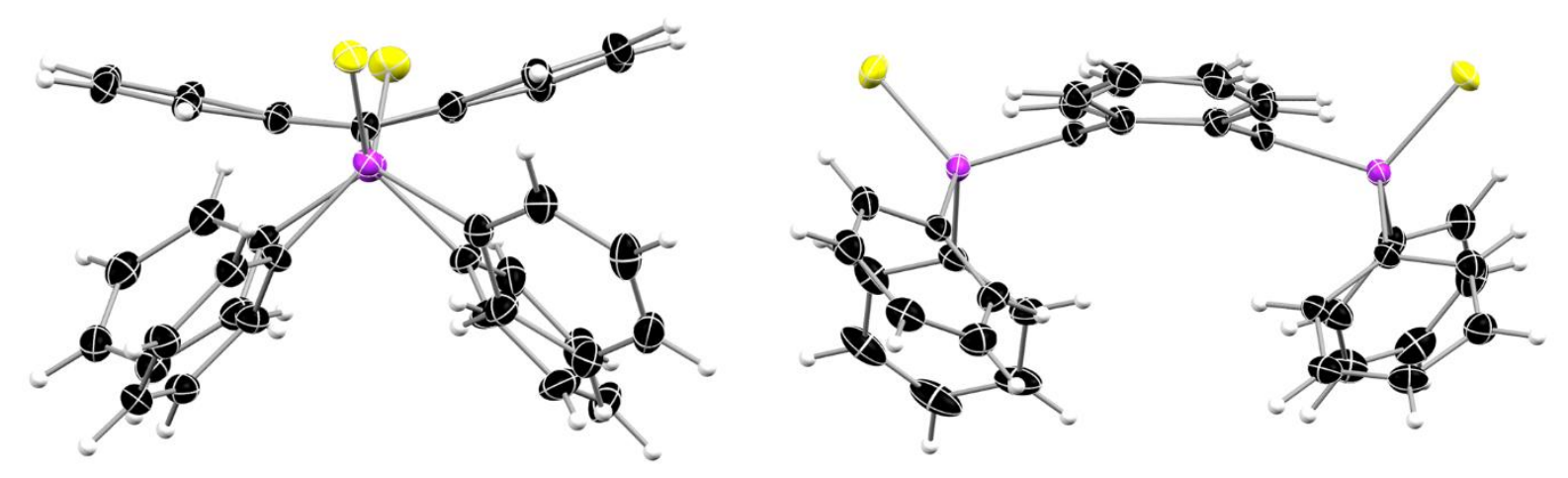

Figure 8. Cisoid conformation of the (S)PPh -substituents in $\left[9,10-(\mathrm{S}) \mathrm{PPh}_{2}-\left(\mathrm{C}_{14} \mathrm{H}_{8}\right)\right]$. The changed orientation of the substituents induces a strong deformation of the anthracene plane. View along the short (left) and along the long anthracene axes (right). The co-crystallized solvent is omitted for clarity.

Until this point, the investigation of the luminescence properties was carried out at ambient temperature. When Schwab performed experiments at low temperature, he made some remarkable observations. Not only the weak emissive co-crystals in the cisoid conformation, but also the vacuum dried toluene cocrystals exhibited strong fluorescence at temperatures below $-135^{\circ} \mathrm{C}$. A phase transition for the cisoid cocrystals could be excluded experimentally by X-Ray diffraction as the orientation of the $(S) \mathrm{PPh}_{2}$ substituents remained the same at different temperatures. These findings led to the conclusion that the origin of the solid-state fluorescence is largely independent of the kind of intercalated aromatic guests and also questioned the exciplex hypothesis. The necessary condition for ambient temperature emission seemed to be the transoid conformation of the (S)PPh $\mathrm{Ph}_{2}$ groups, which could be induced and stabilized by co-crystallization of suitable aromatic guests. Quenching of the emission at ambient temperature of cisoid compounds and of vacuum dried transoid orientated co-crystals was explained by a temperature dependent process, for example inter system crossing into a non-radiative triplet state. ${ }^{[155]}$

Besides varying the co-crystallized solvent, the substituents at the phosphorous center have been modified as well. Cyclohexyl-, o-tolyl- and isopropylphosphoryl anthracenes were studied, but for all these compounds no solid-state fluorescence at ambient temperature has been reported. ${ }^{[154,155]}$ It has to be noted that up to here the emission properties for most of the compounds were investigated by naked-eye observations after irradiation with an UV-lamp and no detailed spectroscopic analyses were performed. Only for several host-guest complexes of $\left[9,10-\left((\mathrm{S}) \mathrm{PPh}_{2}\right)_{2}\right]$ the solid-state emission spectra were presented. ${ }^{[154,155]}$ Nevertheless, these results underline the unique properties of the (S)PPh${ }_{2}$-substituent in terms of luminescence in crystalline solids.

After Schwab finished his work, Finkelmeier continued the research and quantified structural features of host-guest systems of $\left[9,10-\left((\mathrm{S}) \mathrm{PPh}_{2}\right)_{2}-\left(\mathrm{C}_{14} \mathrm{H}_{8}\right)\right]$ as well as their emission properties in more detail. ${ }^{[153]}$ All 
compounds, strongly or weakly fluorescent, revealed a maximum emission wavelength in the narrow range of $\lambda_{\mathrm{em}}=515-530 \mathrm{~nm}$ in the solid-state. Noteworthy, for the first time the guest-free structure of

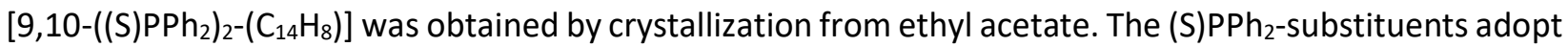
a cisoid conformation which goes along with a rather weak emission. As already noted by Schwab, all cisoid conformers showed a strong deformation and folding of the anthracene moiety while transoid structures seemed to be nearly planar but exhibited a twist of the anthracene. Considering a possible influence on the luminescence properties Finkelmeier developed a protocol for quantification of these structural features (Figure 9). In addition, $\mathrm{C}-\mathrm{H} \cdots \pi$ interactions from guest molecules or phenyl groups to the anthracene core were quantified in case of distance and angle, but an overall influence on the luminescence characteristics could not be stated. In conclusion Finkelmeier attributed the fluorescence quenching to the strong folding of the anthracene, but he also noted that folding goes along with a cisoid structure and therefore the main reasons for fluorescence quenching or enhancement remained unclear.
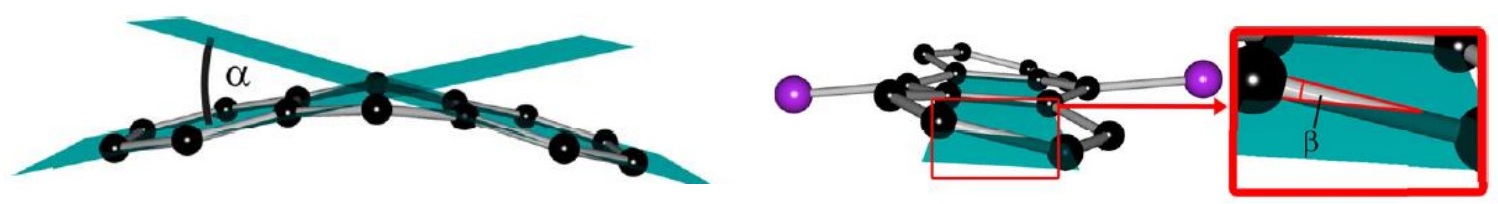

Figure 9. Quantification protocol of the deformation of the anthracene moiety regarding the folding angle $\alpha$ (left) and the twist angle $\beta$ (right) by Finkelmeier. ${ }^{[153]}$

The latest investigations regarding this topic were performed by Krause, who revised the previous obtained data in a more theoretical approach and applied further complementary methods as powder diffraction, neutron diffraction, solid-state NMR-spectroscopy and computational chemistry. ${ }^{[156]} \mathrm{A}$ closer look at the space groups of the emissive co-crystals and experiments with interchanging of guest molecules resulted in new polymorphs and new insights in the formation of the co-crystals. Krause readopted the original experiment by Fei et al. and removed the co-crystallized toluene under reduced pressure from the $\left[9,10-\left((\mathrm{S}) \mathrm{PPh}_{2}\right)_{2}-\left(\mathrm{C}_{14} \mathrm{H}_{8}\right)\right]$ co-crystals in the space group $P 2_{1} / \mathrm{n}$, until the emission was quenched. Afterwards, he exposed the vacuum dried crystals to benzene vapor. As already stated by Fei no subsequent restoring of the fluorescence was observed. Astonishingly after roughly 60 minutes small fluorescent crystals were found in the flask and determined to adopt the space-group $P \overline{1}$. Unfortunately, it could not be monitored if a rearrangement in the original crystals or a new crystallization took place. Nevertheless it seemed, that benzene co-crystals cannot adopt a stable arrangement in the $P 2_{1} / \mathrm{n}$ space group, which led Fei et al. to the conclusion that benzene is not able to restore the fluorescence of dried $\left[9,10-\left((S) P P h_{2}\right)_{2}-\left(C_{14} H_{8}\right)\right]$. The analog experiment, when dried co-crystals of the benzene polymorph in the space group $P \overline{1}$ were exposed to toluene, a new polymorph containing toluene as intercalated guest in the space group $P \overline{1}$ was identified. Again, the substituents adopt a transoid conformation and a green fluorescence of the crystals was observed. Krause concluded that the dried co-crystals somehow act as a matrix that can predefine the structure and space group of the emerging co-crystals.

As Finkelmeier emphasizes $\mathrm{C}-\mathrm{H} \cdots \pi$ interactions as a predominant factor that influences the solid-state fluorescence, Krause focused on the examination of intermolecular interactions. Solid-state NMR experiments and charge density investigations gave rise to a detailed picture of the packing and - 30 - 
corresponding energies. Surprisingly, rather than being dominated by the host-guest interactions, interaction energies are mainly influenced by the outer hydrogen atoms of the anthracene. Nevertheless, no straightforward correlation between the interactions in the solid-state and the photophysical properties of different polymorphs and co-crystals could be found. Krause concluded that a general rigidity of the co-crystals in a transoid conformation may be responsible for the intense solid-state emission. ${ }^{[156]}$ Parallel to this thesis, Bukala continued the work on host-guest systems of $\left[9,10-\left((\mathrm{S}) \mathrm{PPh}_{2}\right)_{2}-\left(\mathrm{C}_{14} \mathrm{H}_{8}\right)\right]$ with a more detailed investigation of the photophysical properties. Until now, no further insights were obtained. Besides the host-guest systems of the disubstituted 9,10-((S)PPh $\left.)_{2}-\left(\mathrm{C}_{14} \mathrm{H}_{8}\right)\right]$, a few further derivatives were assessed in terms of solid-state luminescence in the group of Stalke. Stern started the investigation of the relationship between structure and solid-state fluorescence of asymmetric 9,10-substituted anthracene derivatives. $^{[152,157]} \mathrm{He}$ was able to synthesize and characterize several new compounds of the type [9(E) $\left.\mathrm{PPh}_{2}-10-\mathrm{R}-\left(\mathrm{C}_{14} \mathrm{H}_{8}\right)\right]\left(\mathrm{E}=\mathrm{O}, \mathrm{S}, \mathrm{Se} ; \mathrm{R}=\mathrm{H}, \mathrm{CH}_{3}\right)$. For both sulfur oxidized compounds, a blue-green fluorescence of the crystalline powder could be observed after irradiation with UV-light. Crystals obtained by recrystallization from toluene revealed no co-crystallized solvent present in the structure, but moderate $\pi-\pi$ interactions of two anthracene moieties could be observed. These $\pi-$ stacking motif was assumed to influence the photophysical properties and be responsible for the blue-green emission in the solid-state.

As stated above, the previous studies on solid-state fluorescence were dominated by the host-guest systems of $\left[9,10-\left((\mathrm{S}) \mathrm{PPh}_{2}\right)_{2}-\left(\mathrm{C}_{14} \mathrm{H}_{8}\right)\right]$ and the interactions between aromatic guests and the fluorophore. Only little effort was made in the synthesis and investigation of comparable derivatives, which could give further insights into the underlying processes. Stern introduced the asymmetric derivatives of the type [9(E) $\left.\mathrm{PPh}_{2}-\left(\mathrm{C}_{14} \mathrm{H}_{9}\right)\right]$, which also revealed emission in the solid-state even without co-crystallized solvent. As the structure revealed $\pi-\pi$ interactions between the anthracene chromophores as well as solid-state luminescence, this compound class was identified as a suitable system for further studies regarding the correlation of intermolecular interactions and photophysical properties. 



\section{Research Scope}

The increased demand in daily life for optoelectronic devices has generated considerable research interest in luminescent materials. For several reasons, small and robust metal-free compounds bear numerous benefits over commonly used late transition-metal complexes as emitting materials. Their performance in potential applications is strongly dependent on the photophysical properties in the solid-state. Despite all efforts, the influence of intermolecular interactions on the emission properties, has not been fully understood so far. Due to this fact, the work presented in here contributes to the general understanding of the correlation between intermolecular interactions and solid-state luminescence. The anthracene fluorophore, which is readily accessible, and several functionalized derivatives will be in the focus of this work. One important factor that influences the solid-state emission of polyaromatic hydrocarbons, is the excimer formation. While excimers in solution are already widely investigated, their role in solid-state photophysical processes is still debated.

This thesis deals with the influence of intermolecular interactions on the photophysical properties, with the main focus on the excimer formation of the anthracene fluorophore. Several points will be addressed during this work:

- The influence of different heteroatoms directly bonded, or in periphery of the anthracene fluorophore, will be examined. The phosphanyl- and phosphoryl anthracenes that have already been briefly investigated, showed interesting structural and photophysical properties that were attributed to the substituent and the involved heteroatoms. The emission efficiencies of $[9,10-$ $\left.\left((E) P h_{2}\right)_{2}-\left(C_{14} \mathrm{H}_{8}\right)\right](E=L P, O, S)$ were found to be fundamentally different. To investigate whether electronic or structural changes are responsible for this behavior, further anthracene derivatives with comparable substitution motifs should be prepared and investigated. Variation of the heteroatoms within the substituent should provide further insights, how the structural and optical properties of the anthracene fluorophore are affected. Possible quenching pathways connected to the presence of particular heteroatoms will be explored. The combined structural and photophysical analysis, should identify further potential candidates for solid-state excimer formation.

- The already briefly investigated thiophosphoryl anthracene [9-(S)PPh $\left.{ }_{2}-\left(\mathrm{C}_{14} \mathrm{H}_{9}\right)\right]$ showed several structural features that were attributed to influence the emission properties. Especially the occurring $\pi-\pi$-interactions hinted for a possible excimer formation. This assumption will be clarified by additional spectroscopic measurements. Furthermore, it will be investigated if substituents in the 10-position of the anthracenes are able to change and control the intermolecular interactions in the solid-state. Through synthesis of several, slightly modified thiophosphoryl anthracenes, a correlation of these interactions and the optical properties, will be examined. 
- The previously reported intense solid-state fluorescence of $\left[9,10-\left((\mathrm{S}) \mathrm{PPh}_{2}\right)_{2}-\left(\mathrm{C}_{14} \mathrm{H}_{8}\right)\right]$ was strongly related to the co-crystallized solvent. The conformation of the two thiophosphoryl groups and the structural distortion of the anthracene plane are determined by the co-crystallized solvent. As outlined, both factors are considered to influence the emission efficiency in the solid-state. Furthermore, weak non-covalent interactions between the co-crystallized guest and the anthracene fluorophore, were attributed to promote the emission in the solid-state. Therefore, the ability for co-crystallization of the herein prepared thiophosphoryl anthracenes will be examined. If suitable co-crystals are available, the proposed hypothesis will be tested. Comparison of the photophysical properties of the host-guest systems with the pure host compounds, will provide information about co-crystallization as a strategy for tuning of the emission properties.

- Anthracene excimer formation has so far mainly been reported for derivatives functionalized in the 9- and 10-position of the fluorophore. Anthracenes substituted at the outer benzene perimeters are barely studied in terms of their optical properties. Moving the substituents to the 1- and 2-position should increase the available area for stacking interactions. The synthetic route towards these substitution motifs will be explored. Investigation of the resulting solid-state structures will show if these assumptions are true. Together with the 9,10-substituted derivatives a wide range of different intermolecular interactions involving the anthracene fluorophore, is expected. Novel insights about the structure-property correlation and especially about the mechanism of excimer formation in the solid-state, are awaited. 


\section{Results \& Discussion}

This thesis focuses on the synthesis of novel luminescent compounds based on the anthracene fluorophore and the investigation regarding the correlation of photophysical and structural properties. The main emphasis was set on emission characteristics in the solid-state, as direct insights into the structure and possible intermolecular interactions via X-ray crystallography are possible. Previous works in the Stalke group have demonstrated that diphenyl(thiophosphoryl) anthracenes are able to emit green light upon excitation in the solid-state (chapter 1.5). Hitherto, most effort has been spent on the hostguest systems as they show remarkable luminescent features upon co-crystallization with various aromatic guest molecules (Figure 10). Asymmetric thiophosphoryl derivatives have been investigated only initially ${ }^{[152]}$ and only vague information is available about their emission properties. ${ }^{[152]}$
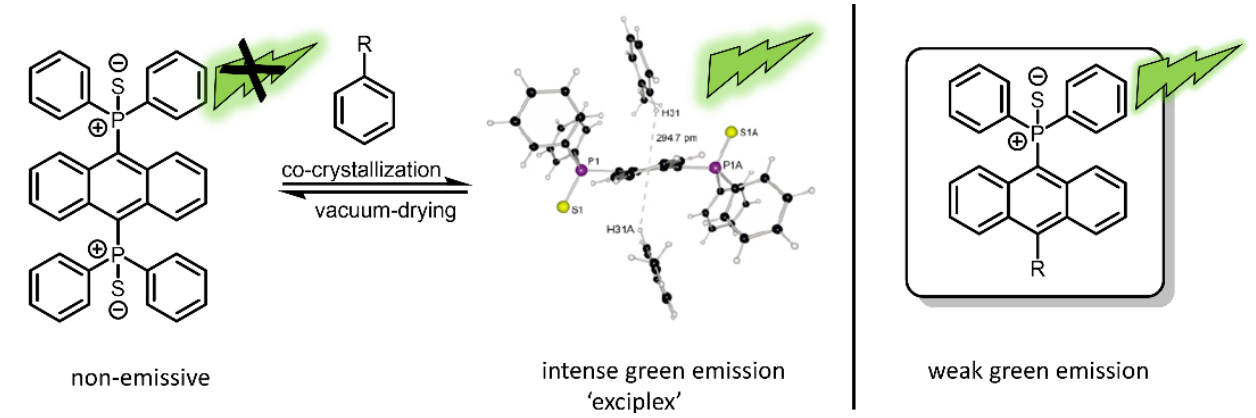

Figure 10. Previous studied luminescent host-guest complexes of $\left[9,10-\left((\mathrm{S}) \mathrm{PPh}_{2}\right)_{2}\left(\mathrm{C}_{14} \mathrm{H}_{8}\right)\right]$ and aromatic guest molecules (left) and asymmetric derivatives of [9-((S)PPh$\left.)-10-R-\left(C_{14} \mathrm{H}_{8}\right)\right]$ (right), which will be in the focus of this work.

In the first part of this work, the group of asymmetric substituted derivatives [9-((S)PPh$\left.)-10-R-\left(C_{14} \mathrm{H}_{8}\right)\right]$ will be introduced and investigated. The thiophosphoryl substituent serves as a bulky group to prevent strong intermolecular interactions in form of columnar stacking of the aromatic planes which are ascribed to fluorescence quenching. ${ }^{[38,55,57]}$ When only one side of the anthracene is occupied by the bulky substituent interactions between the fluorophores $\pi$-systems in form of $\mathrm{C}-\mathrm{H} \cdots \pi$ and/or $\pi-\pi$ interactions are still possible. By varying the second substituent in the 10-position, the possibility of altering these interactions should be investigated and, if as a result, the packing motif can be controlled by the substituent in 10position. The ability of excimer or exciplex formation of this substance class and their structure-property relationship will be evaluated with a detailed investigation of the photophysical and structural properties. Furthermore, any influence of the thiophosphoryl substituent beyond the steric shielding will be investigated. Recent works have revealed drastic changes in the photophysical properties caused by simple replacement of heteroatoms in the fluorophore's substituents. ${ }^{[158,159,160]}$ Therefore, the P-S moiety will be replaced by comparable heteroatoms and the effect on the emission properties will be examined. 


\subsection{Structural and photophysical properties of 9,10-functionalized anthracene}

derivatives

Parts of this chapter have been published in: T. Schillmöller, P. N. Ruth, R. Herbst-Irmer, D. Stalke, "Analysis of solid-state luminescence emission amplification at substituted anthracenes by host-guest complex formation", Chem. Eur. J. 2020, 26, 1739017398.

For the introduction of the phosphanyl group, the established route via lithiation of the respective bromoanthracene and subsequent lithium-halogen exchange with chlorodiphenylphosphine was used. ${ }^{[151,152,155,161]}$ For an asymmetric substitution pattern, the substituent in 10-position is introduced first, followed by the introduction of the diphenylphosphine in 9-position. First attempts by Stern who tried to functionalize the thiophosphoryl anthracenes were not successful. ${ }^{[157]}$ Therefore, the use of already asymmetric substituted bromoanthracenes was found to be more suitable, at least for substituents insensitive towards organolithium reagents such as simple alkyl groups.

\subsubsection{Diphenylphosphanyl anthracenes}

\subsubsection{Synthesis}

The reactivity of the anthracene fluorophore will be discussed briefly as several derivatives were synthesized during this thesis via different reaction types. In later chapters, we will see that when other positions than the 9 and 10 positions are the targets for substitution, an understanding of reactivity is essential. Substitution of the hydrogen atoms in 9,10 position is the most common way of functionalization of anthracene. Therefore, a plethora of compounds bearing this substitution pattern are known and used widely in different applications such as optoelectronic devices, sensing and imaging. ${ }^{[162]}$ Suitable starting materials for further functionalization are halogenated anthracenes, which are commercially available or can be prepared straightforwardly from anthracene. ${ }^{[163]}$ In comparison to benzene, anthracene and other polyaromatic hydrocarbons have a greater tendency to undergo addition reactions rather than direct substitution. ${ }^{[164]}$ Addition products of halogenation reactions can form the substitution product by subsequent elimination of hydrogen halides. Electrophilic addition of equimolar amounts of bromine occurs selectively in the 9- and 10-position resulting in 9,10-dibromo-9,10-dihydroanthracene (Scheme 13). The selectivity originates from the more stable $\sigma$-complex formed upon addition in 9,10-position. The aromaticity in the terminal rings is sustained and compared to the addition products in 1,4- or 2,3-position the 9,10-system is stabilized by approximately $9 \mathrm{kcal} / \mathrm{mol} .{ }^{[165]}$ The intermediate 9,10 -dibromo-9,10dihydroanthracene is unstable at ambient temperature and undergoes rapid elimination of $\mathrm{HBr}$ resulting in the monobrominated 9-bromoanthracene, which is still reactive towards addition of halogens. If an excess of bromine is present another molecule adds in the 9,10-position and subsequent elimination of $\mathrm{HBr}$ provides the 9,10-dibromoanthracene. ${ }^{[163,166]}$ 


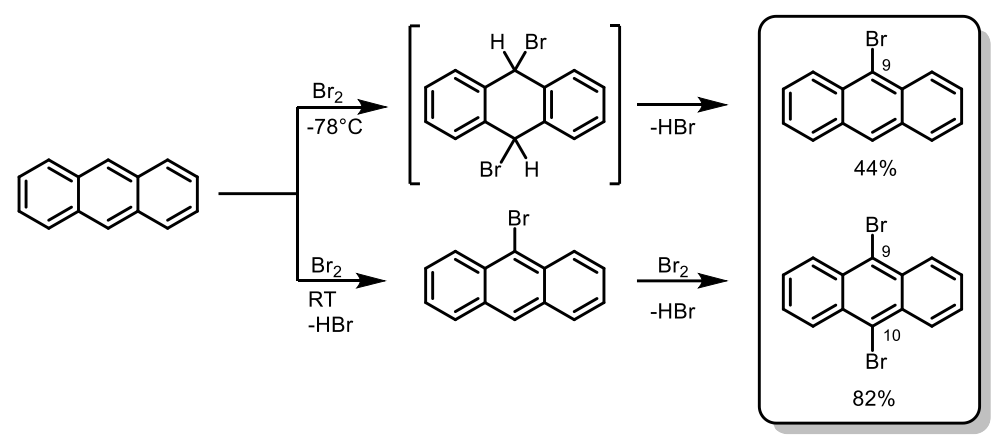

Scheme 13. Synthesis of 9-bromoanthracene and 9,10-dibromoanthracene from anthracene via the addition-elimination mechanism.

As a result of the high reactivity towards the addition of halogens, the preparation of mono brominated anthracene is more challenging. The formed 9-bromoanthracene reacts immediately with bromine towards the disubstituted derivative even if only one equivalent bromine is used. Preventing elimination of $\mathrm{HBr}$ at low temperatures $\left(-78^{\circ} \mathrm{C}\right)$ until the complete anthracene is consumed can afford the mono brominated anthracene in good yields and purity. ${ }^{[152]}$ Also the use of brominating systems such as NBS ${ }^{[167]}$, $\mathrm{CuBr}_{2}{ }^{[168]}$ or more complex agents ${ }^{[169]}$ can afford 9-bromoanthracene. Within this work, 9bromoanthracene was prepared using NBS in moderate yield (44\%) and the 9,10-dibromoanthracene was synthesized using $\mathrm{Br}_{2}$ in multi-gram scale and good yields (82\%).

Further functionalization of the 9,10-dibromoanthracene can afford asymmetric bromoanthracenes (Scheme 14). Stern already investigated the synthesis of the 10-methyl substituted derivative. ${ }^{[152,157]}$ This approach will be adopted for the introduction of further substituents in the 10-position. The best results were obtained by Stern, when the methyl group was introduced prior to the phosphanyl group as the other way round suffered from side reactions and low yields. Therefore, 9,10-dibromoanthracene was reacted with one equivalent $n \mathrm{BuLi}$ at $-78^{\circ} \mathrm{C}$ in THF for a selective exchange of one bromine. Addition of the corresponding electrophile (methyl iodide, ethyl iodide or chlorotrimethylsilane) and stirring overnight afforded the desired products after recrystallization or sublimation up to quantitative yields. Attempts towards 9-bromo-10-isopropylanthracene were not successful via the outlined route. Only 9bromoanthracene could be isolated, which indicates a deprotonation/elimination reaction rather than a lithium/halogen exchange. Different conceivable pathways like palladium-catalyzed cross-coupling or the use of Grignard reagents with anthrones should be suitable for the preparation but were not mandatory for the studies in this work. ${ }^{[170]}$ 

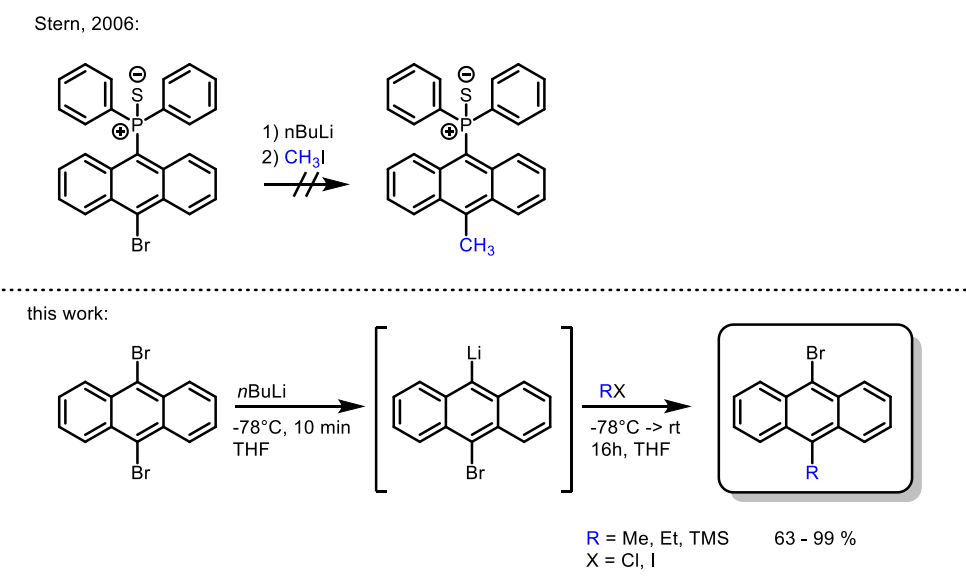

Scheme 14. Synthesis of asymmetric substituted bromoanthracenes via selective mono-lithiation and salt-elimination with corresponding alkyl halides.

The substitution of the second bromine was performed following the established route via lithiation and subsequent addition of chlorodiphenylphosphine (Scheme 15). ${ }^{[151,152,155,161]}$ The already introduced alkyl substituents did not seem to affect the second lithiation. In previous works, different reaction conditions have been reported for this conversion. ${ }^{[152,155]}$ In this work. $\mathrm{Et}_{2} \mathrm{O}$ or THF were used as solvents and the preparations were done at low temperature $\left(-78^{\circ} \mathrm{C}\right)$. After the reaction mixture was warmed to ambient temperature, the precipitated product could be filtered off. Formed $\mathrm{LiCl}$ was removed by dissolving the solid in DCM or toluene with subsequent filtration. The crude products were further purified by washing with hexane and recrystallization from DCM or toluene, affording the target compounds in good yields and purity.

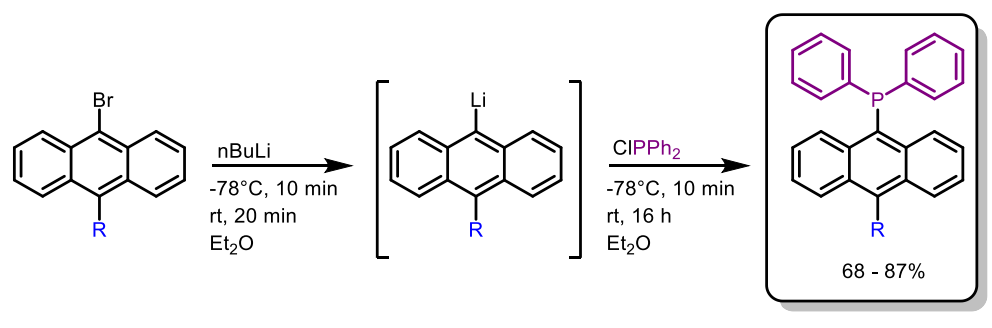

$R=H(1), B r(2), M e(3), \operatorname{Et~(4),~TMS~(5),~Ph~(6)~}$

Scheme 15. Synthesis of 9-diphenylphosphanylanthracenes with various substituents in 10-position. ${ }^{3}$

As other common tertiary aromatic phosphines, the obtained phosphanylanthracenes are stable against air and moisture as solids. No decomposition or oxidation products could be identified after storage of the solids in air for several days. In diluted solution of non-degassed solvents, the P(III) center slowly oxidizes to $P(v)$, which can be monitored by ${ }^{31} \mathrm{P}-\mathrm{NMR}$ spectroscopy and leads to drastic changes of the emission properties (see 3.1.1.3). The ${ }^{31} \mathrm{P}-\mathrm{NMR}$ resonances of the obtained phosphines 1-6 are in a narrow range from -23 to $-25 \mathrm{ppm}$ and therefore reveal a small upfield shift compared to triphenylphosphine ( $-6 \mathrm{ppm})$ but are still in a typical range for tertiary aromatic phosphines. ${ }^{[171]}$

\footnotetext{
${ }^{3}$ The starting material 9-bromo-10-phenylanthracene was not synthesized in the course of this work but purchased commercially and used as obtained. 


\subsubsection{Structural Properties}

Solid-state structures of the phosphines [9-PPh $-10-\mathrm{R}-\left(\mathrm{C}_{14} \mathrm{H}_{8}\right)$ ] with $\mathrm{R}=\mathrm{H}(\mathbf{1}), \mathrm{Me}(\mathbf{3}), \mathrm{Et}(\mathbf{4})$ and $\mathrm{Ph}(\mathbf{6})$ were determined via X-Ray diffraction analysis to get a brief glance about the structural properties and for later comparison with their sulfur oxidized homologs. $\mathbf{1}, \mathbf{3}$ and $\mathbf{4}$ crystallize in the triclinic space group $P \overline{1}$, while 6 adopts a monoclinic crystal system in the space group C2/c. The asymmetric units of $\mathbf{1}$ and $\mathbf{3}$ consist of two slightly different molecules, while in $\mathbf{4}$ only one molecule is present. $\mathbf{6}$ co-crystallizes with one toluene molecule in the asymmetric unit. The anthracene moieties are nearly planar with folding angles $\alpha$ ranging from $2.51^{\circ}$ to $7.50^{\circ}$. Folding angles $\alpha$ were determined as the intersection angle of two planes through the outer carbon atoms ( $\mathrm{C} 1-\mathrm{C} 4$ and $\mathrm{C} 5-\mathrm{C} 8$ ) according to the procedure introduced by Finkelmeier. ${ }^{[153]}$ The phenyl groups are oriented one above and one below the anthracene plane, resulting in a pyramidal molecular shape regarding the phosphorous atom (Figure 11). The phosphorous does not lie exact in the anthracene plane but reveals a small deflection out of the plane (1: $0.17 \AA$, 3: $0.35 \AA$, 4: $0.30 \AA, 6: 0.14 \AA$ ).
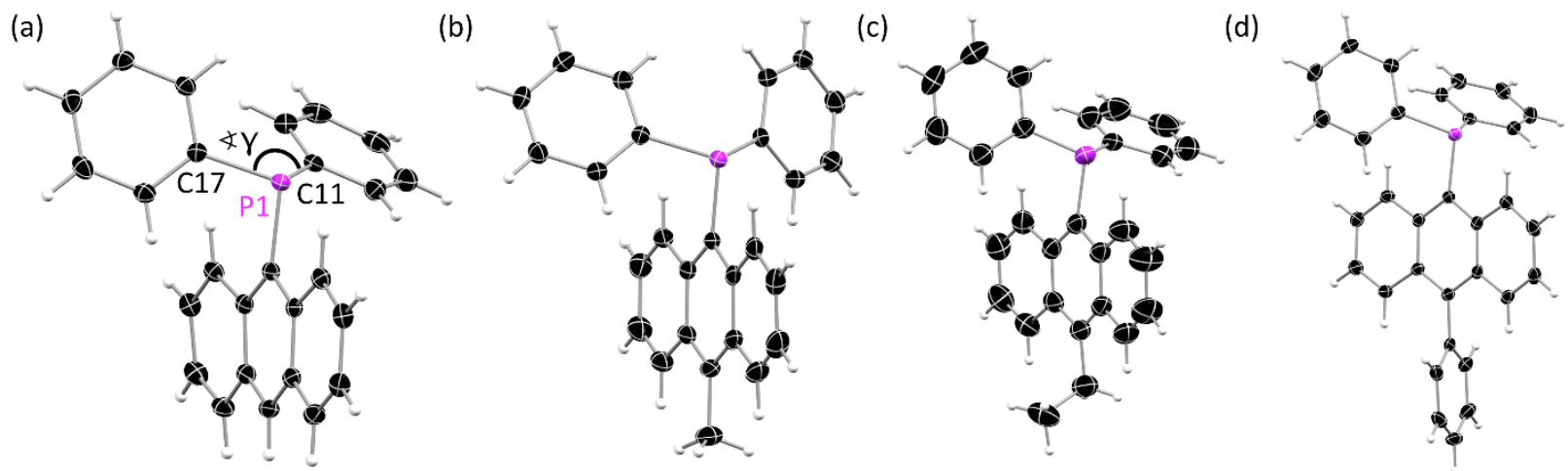

Figure 11. Solid-state structures of the diphenylphosphanyl anthracenes [9-PPh$-10-R-\left(C_{14} H_{8}\right)$ ] with (a) $R=H(\mathbf{1}),(b) R=M e(3)$, (c) $\mathrm{R}=\mathrm{Et}(\mathbf{4})$ and (d) $\mathrm{R}=\mathrm{Ph}(\mathbf{6})$. For $\mathbf{1}$ and $\mathbf{3}$ only one molecule of the asymmetric unit is shown. Co-crystallized toluene in the asymmetric unit of 6 is omitted for clarity.

Bond lengths and -angles of the anthracene core are similar to the unsubstituted anthracene and the $\mathrm{P}-\mathrm{C}$ bond lengths of around $1.83 \AA\left(\mathrm{P}-\mathrm{C}_{\mathrm{Ph}}\right)$ and $1.85 \AA$ ( $\left.\mathrm{P}-\mathrm{C} 9\right)$ are in accordance with typical $\mathrm{P}-\mathrm{C}$ single bonds in tertiary phosphines. ${ }^{[172]}$ The $C-P$ bond towards the anthracene is slightly elongated in all structures compared to the bond towards the phenyl groups, probably due to the greater steric demand of the anthracenyl group compared to the phenyl moieties. The C11-P-C17 angle $\gamma$ adopts values between $105.6^{\circ}$ and $107.3^{\circ}$ and is therefore slightly decreased compared to the ideal tetrahedral angle of $109.5^{\circ}$ indicating for a greater steric demand of the lone pair.

The crystal packing of the phosphines is mainly organized by weak $\mathrm{C}-\mathrm{H} \cdots \pi$ interactions between the different aromatic moieties. For the three compounds with the smaller substituents in 10-position $(\mathrm{R}=\mathrm{H}$ (1), Me (3), Et (4)) only weak face-to-face interactions of two neighboring anthracene $\pi$-systems could be identified. In the solid-state structure of $\mathbf{1}$ without a substituent in the 10-position, edge-to-face interactions of adjacent anthracenes are present, and the overall structure resembles a herringbone type stacking relating to the anthracene moieties (Figure 9a). When a substituent is introduced in the 10postion edge-to-face interactions between the anthracenes are prohibited. The crystal packing of $\mathbf{3}$ and $\mathbf{4}$ resembles a slipped stacking where all anthracene planes are parallelly oriented (Figure 12). As expected, 
the phenyl substituent in the 10-position of 6 adopts a twisted conformation towards the anthracene plane with an intersecting angle of the two aromatic planes of $66.78(6)^{\circ}$. This conformation leads to a shielding of both anthracene sides and makes face-to-face interactions and other strong interactions towards the anthracene $\pi$-system impossible.

(a)
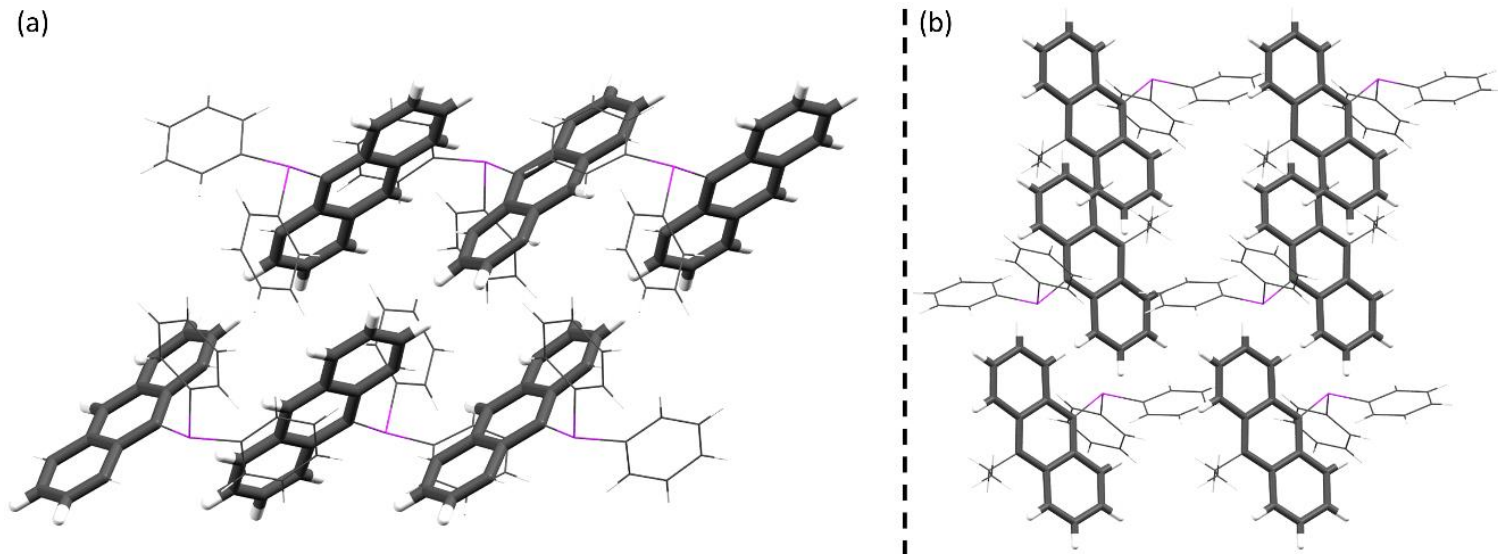

Figure 12. (a) Excerpt of the crystal packing of [9-PPh$\left.{ }_{2}-\left(\mathrm{C}_{14} \mathrm{H}_{9}\right)\right](\mathbf{1})$ and $(\mathrm{b})$ of $\left[9-\mathrm{PPh}_{2}-10-\mathrm{Et}-\left(\mathrm{C}_{14} \mathrm{H}_{8}\right)\right](\mathbf{4})$ with all anthracenes parallelly oriented. The anthracene moieties are highlighted.

At this point, the role of the substituents can already be demonstrated. The bulky phosphanyl group occupies one side of the anthracene and prevents columnar stacking. When the second side is unsubstituted, edge-to-face interactions of two anthracenes are possible and a herringbone-like packing is observed. If the $\mathrm{H}$-atom in the 10-position is replaced by a bulkier group, the crystal packing changes, and edge-to-face interactions vanish. Increasing the size of the substituent again changes the possible intermolecular interactions. The twisted phenyl group of 6 also blocks both sites of the anthracene and prevents any stronger interactions between two anthracene chromophores. The investigation of the structural properties of the phosphanyl anthracenes could already show how sensitive the intermolecular interactions and the resulting crystal packings are to small changes in the chemical structure. The variation of the substituent in the 10-position therefore seems to be a suitable strategy to achieve different packing motifs with diverse intermolecular interactions.

\subsubsection{Photophysical Properties}

The photophysical properties of the phosphanylanthracenes will be discussed briefly to investigate the influence of the phosphanyl group. The absorption spectra resemble the absorption of parent unsubstituted anthracene and reveal transitions into the two lowest excited states, which are assigned to the $\pi-\pi^{*}$ transition (Figure 13). The higher energy absorption is located between 250 and $260 \mathrm{~nm}$ and assigned to the $L_{b}$ state with a transition dipole moment along the long molecular axes. The lower energy absorptions between 320 and $430 \mathrm{~nm}$ represent the transition into the $\mathrm{L}_{a}$ state and give rise to the yellow color of the compounds. The corresponding transition dipole moment is oriented along the short molecular axes of the anthracene. The vibronic fine structure is broadened indicating a less rigid scaffold of the molecules. The phosphanyl substituent induces a small red shift of the absorption compared to 
unsubstituted anthracene. Disubstituted derivatives experience a stronger bathochromic shift, which is slightly stronger for the phenyl substituted anthracene 6 .
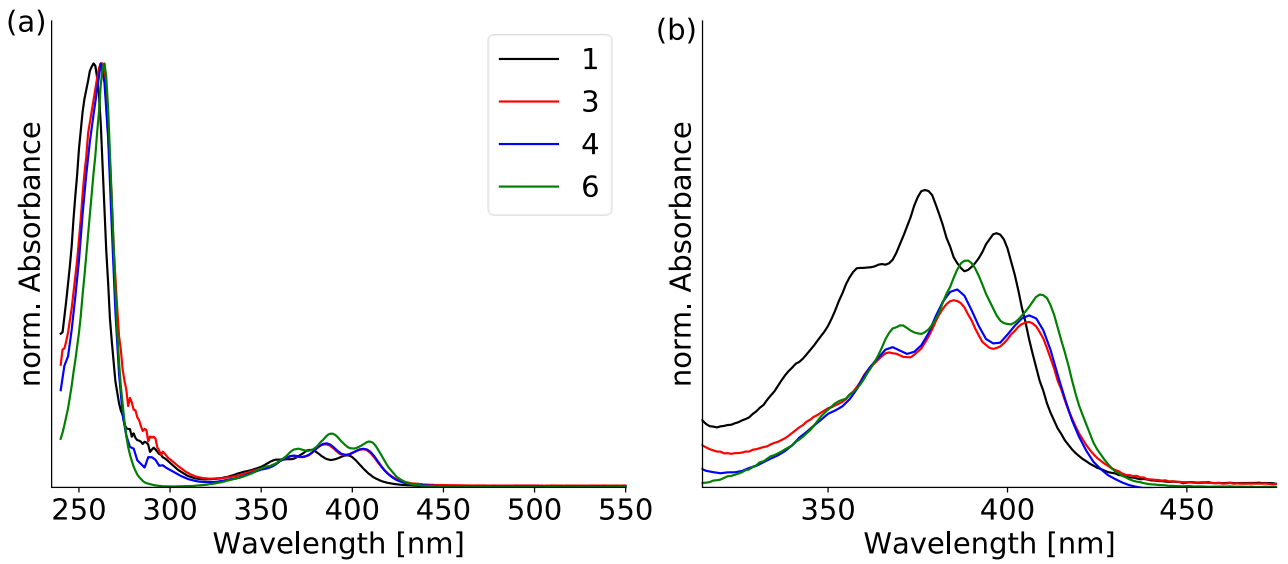

Figure 13. (a) Normalized absorption spectra of phosphanyl anthracenes 1, 3, 4 and $\mathbf{6}$ in diluted THF solution (10-5 M). (b) Excerpt of the lower energy absorption around $340-420 \mathrm{~nm}$.

At higher wavelengths, no absorption bands could be observed indicating the absence of CT-like transitions. For the corresponding 9-diphenyalminoanthracene, a $\mathrm{S}_{0} \rightarrow{ }^{1} \mathrm{CT}$ transition from the amine to the anthracene could be identified as a broadened band at higher wavelengths, which strongly affects its photophysical properties. ${ }^{[173]}$ As for the phosphanyl anthracenes a CT is absent, the absorption spectra are mainly determined by the anthracene fluorophore. Fluorescence spectra of the phosphanyl anthracenes could not be obtained as emission is almost completely quenched both in solution and solid-state. The quenching can be ascribed to a photoinduced electron transfer (PET) from the phosphorous lone pair towards the anthracene and has been reported for several phosphine substituted fluorophores before. ${ }^{[174,175]}$ After excitation, the vacancy in the ground state orbital is filled by an electron from the phosphorous lone pair, resulting in a radiationless decay of the excited state via internal conversion. $A$ more detailed description of the PET is given in chapter 3.1.2.3.

The PET process has been studied widely for amines and usually a fluorophore-spacer-donor geometry suits best for an efficient quenching. ${ }^{[176,177]}$ Amine derivatives without spacer are typically not affected from quenching as the molecules are not flexible enough for the required orbital overlap of the nitrogen lone pair and the fluorophore. For example, 9-diphenylaminoanthracene exhibits efficient emission from the CT-state resulting in high quantum yields in solution $\left(\Phi_{\mathrm{F}}=0.92\right) \cdot{ }^{[173]}$ Nevertheless, the phosphine group in [9-PPh $-\left(\mathrm{C}_{14} \mathrm{H}_{9}\right)$ ] seems to have suitable energy level and geometry even without a spacer for efficient emission quenching. Compared to the amino derivative, the $C_{9}-P$ bond in 1 is elongated $(1.851(2) \AA$ (C-P) vs. $1.442 \AA(\mathrm{C}-\mathrm{N})$ ) and a pyramidal instead of a trigonal geometry is observed. Furthermore, the lone pair at the phosphorous should be more diffuse and of more s-character compared to the nitrogen lone pair. Therefore, the overlap with the anthracene orbitals is possible without any spacer and a fluorescence quenching occurs. When the lone pair at the phosphorous is part of a bond, for example in case of oxidation to $\mathrm{P}(\mathrm{V})$ or through coordination to a transition metal, the compound becomes emissive, which further supports the PET-quenching-mechanism (see chapter 3.1.2). The time dependent emission spectra 
of 1 in non-degassed THF solutions demonstrate this behavior (Figure 14). In the beginning, the fluorescence is nearly quenched, but intensity increases continuously upon slow oxidation of the $\mathrm{P}$ (III) center. The emission rises until a maximum is reached after roughly $16 \mathrm{~h}$. The maximum emission wavelength at around $445 \mathrm{~nm}$ is in accordance with the emission of the oxidation product [9-(O)PPh2$\left(\mathrm{C}_{14} \mathrm{H}_{9}\right)$ ] (chapter 3.1.2.3). The oxidation can additionally be monitored via ${ }^{31} \mathrm{P}-\mathrm{NMR}$ spectroscopy in nondegassed THF- $d_{8}$. The initial resonance of 1 at $-25 \mathrm{ppm}$ slowly decreases and a new peak at $+27 \mathrm{ppm}$ emerges after several hours, which can be ascribed to the oxidation product [9-(O)PPh $\left.-\left(\mathrm{C}_{14} \mathrm{H}_{9}\right)\right]$. After roughly $72 \mathrm{~h}$, the initial resonance of [9- $\left.\mathrm{PPh}_{2}-\left(\mathrm{C}_{14} \mathrm{H}_{9}\right)\right]$ has nearly vanished and only the signal of the oxidized compound is present. The discrepancy of the time scale can be ascribed to a higher concentration in the NMR sample compared to the diluted solution used for the fluorescence measurements. Furthermore, this experiment underlines the stability of the phosphines as oxidation occurs only slowly. The oxidation in the presence of peroxides occurs much faster and therefore, phosphine substituted hydrocarbons have been used as fluorescent chemosensors for peroxides. ${ }^{[174]}$ Coordination of the phosphine to various transition metals can also turn on the fluorescence as demonstrated for several anthracenyl phosphines. ${ }^{[178]}$
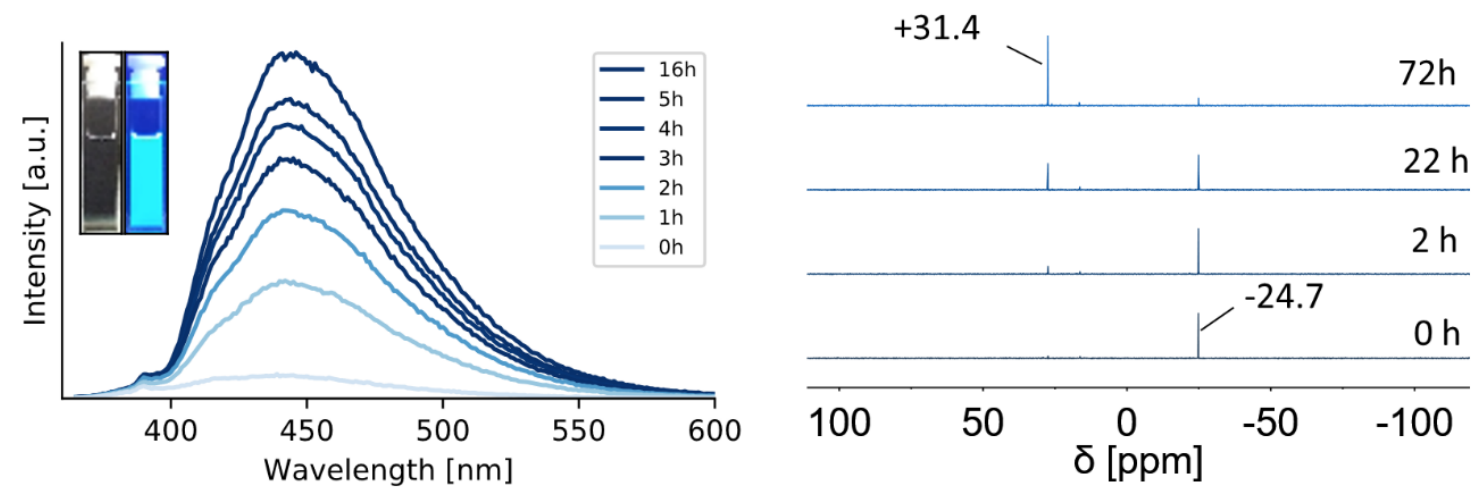

Figure 14. Emission spectra of $\left[\left(9-\mathrm{PPh}_{2}-\left(\mathrm{C}_{14} \mathrm{H}_{9}\right)\right](1)\right.$ in a non-degassed THF solution $\left(10^{-5} \mathrm{M}, \lambda_{\mathrm{ex}}=350 \mathrm{~nm}\right)$ recorded over $16 \mathrm{~h}$. The inset picture shows the solution after $16 \mathrm{~h}$ under daylight (left) and irradiated with UV-light (right). ${ }^{31 \mathrm{P}-N M R}$ spectra of 1 in nondegassed THF-d8 showing the slow oxidation of 1 to strongly emissive [9-(O)PPh $\left.-\left(\mathrm{C}_{14} \mathrm{H}_{9}\right)\right]$ (right).

Even if the hitherto studied phosphanyl anthracenes are widely non-luminescent, their investigation of the solid-state structures and photophysical properties gave valuable insights for the examination of the following compounds. The initially introduced idea that small variations of the steric demand of the substituent in 10-position can change the packing motifs could be confirmed for the phosphanyl anthracenes. Therefore, it can be assumed that this approach has a great chance to be also successful for further derivatives. Furthermore, the influence of the phosphanyl substituent was briefly investigated, indicating that small variations in the nature of the substituent can drastically change the emission characteristics. Even if the intermolecular interactions are in the focus of this work, the electronic influence of the substituent(s) should not be underestimated. Therefore, several anthracene derivatives with different substituents and involved heteroatoms will be examined briefly in the following chapter. 


\subsubsection{Investigation of the structural and photophysical influences of different substituents and heteroatoms}

The $\mathrm{P}$ (III) center of the phosphanyl anthracenes can be easily oxidized with oxygen or sulfur to the corresponding oxo- or thiophosphoryl anthracenes. As described in the previous chapter, the oxidation to $\mathrm{P}(\mathrm{v})$ changes the luminescence properties drastically. Especially, the influence on the disubstituted system $\left[9,10-\left((E) P h_{2}\right)_{2}-\left(C_{14} H_{8}\right)\right]$ has been already investigated in detail in previous works (see chapter 1.5).

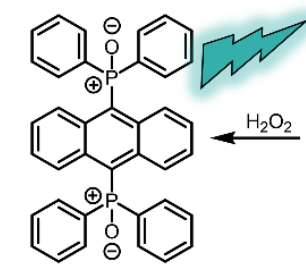

intense blue-green emission in solution

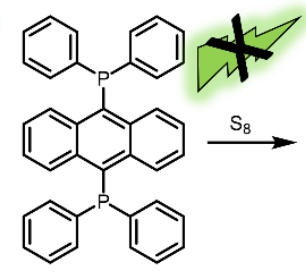

no emission

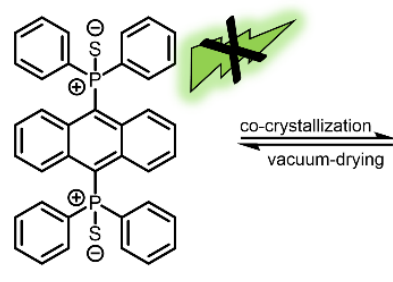

weak emission

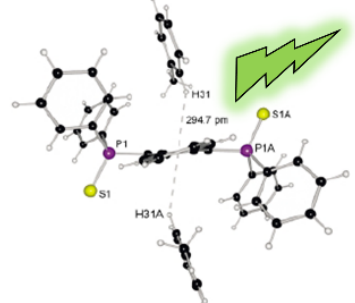

intense green exciplex emission in the solid-state

Figure 15. Illustration of changes in luminescence properties upon oxidation of $\left[9,10-\left(\mathrm{PPh}_{2}\right)_{2}\left(\mathrm{C}_{14} \mathrm{H}_{8}\right)\right]$ with oxygen and sulfur.

Generally speaking, the oxidation of the phosphine with oxygen led to an intense blue-green emission in solution with excellent quantum efficiencies $\left(\Phi_{F}=75.3-95 \%^{[179,180]}\right.$ ) (Figure 15). Despite this, the emission in the solid-state is nearly quenched. The opposite behavior is observed when sulfur is used as oxidant. The luminescence of the obtained product in solution is very weak but can be restored upon cocrystallization with small aromatic molecules in the solid-state. ${ }^{[151]}$ These findings again demonstrate the challenge in rational design of luminescent solid materials, as the emission properties from diluted solution to the condensed phase can change drastically. Small structural modifications can be accompanied by an inversion of its luminescence properties. Synthesis and characterization of the asymmetric substituted phosphoryl anthracenes should clarify if the observed luminescence features are a general attribute of the phosphoryl substituent or must be ascribed to the special structural situation in the disubstituted system. Furthermore, the phosphoryl group will be replaced with different comparable heteroatoms to investigate their influence on the structural properties and on the luminescence properties. Therefore, a few related derivatives with comparable substituents at the anthracene will be examined regarding their structural and photophysical properties.

\subsubsection{Synthesis}

The oxidation of the phosphines is performed analog to literature procedures with urea hydrogenperoxide as the oxygen source or elemental sulfur as the sulfur source (Scheme 16). Urea hydrogen peroxide was used in DCM and [9-(O) $\left.\mathrm{PPh}_{2}-\left(\mathrm{C}_{14} \mathrm{H}_{9}\right)\right](7)$ was obtained in good yields after stirring the mixture at ambient temperature overnight and following aqueous work-up. The sulfurization was performed in toluene at temperatures around $80{ }^{\circ} \mathrm{C}$ for $6-16 \mathrm{~h}$. Following filtration and recrystallization from common organic solvents afforded the thiophosphoryl anthracene [9-(S)PPh $\left.-\left(\mathrm{C}_{14} \mathrm{H}_{9}\right)\right](\mathbf{8})$ as yellow crystals. Crystallization of the thiophosphoryl anthracenes was much easier compared to their oxygen-homologs. The latter are very soluble in nearly all common organic solvents, while the S-derivatives are only moderate soluble at 
ambient temperature and can therefore be easily crystallized from hot saturated solutions and subsequent slow cooling. The obtained crystals showed a green solid-state fluorescence, which can be already observed by naked-eye, after irradiation with UV-light.
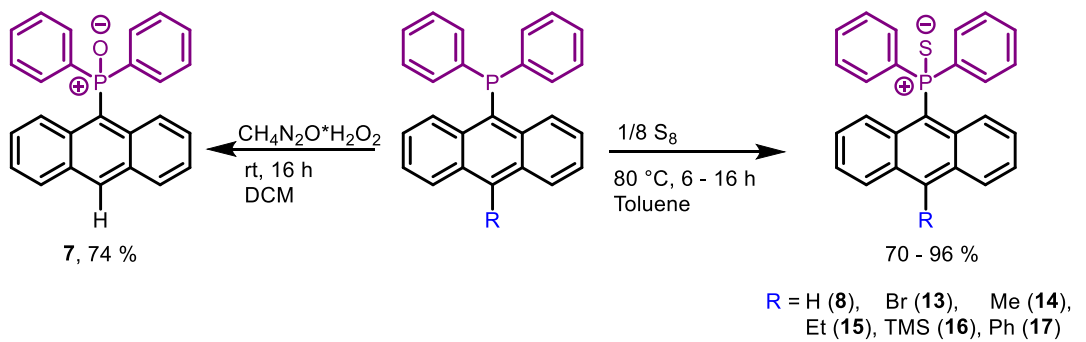

Scheme 16. Synthesis of oxo- and thiophosphoryl anthracenes from the corresponding phosphanyl derivatives through oxidation with hydrogen peroxide - urea or elemental sulfur.

The ${ }^{31} \mathrm{P}-\mathrm{NMR}$ resonance of the oxophosphoryl anthracene 7 lies at $+31.4 \mathrm{ppm}$ and is comparable to triphenylphosphine oxide $\left(\delta\left({ }^{31} \mathrm{P}\right)=+29 \mathrm{ppm} \text { in } \mathrm{CDCl}_{3}\right)^{[181]}$ and in accordance with other aromatic phosphoryl derivatives. ${ }^{[182]}$ The thiophosphoryl anthracenes undergo a slightly larger downfield shift to around +34 ppm and deviate sparsely when compared to similar (diphenyl)thiophosphoryl substituted hydrocarbons, which chemical shifts are typically in a narrow region around $+42 \mathrm{ppm} .{ }^{[183,184]}$

For examination of the influence of the phosphanyl and phosphoryl group on the structural and luminescence properties, comparable anthracene derivatives were synthesized. The phosphanyl substituent with a lone pair on the P(III) center can be classified as an electron donating substituent. The interaction of the lone pair with the anthracene $\pi$-system resulted in a fluorescence quenching both in solution and in the solid-state, which is typical for phosphine substituted polyaromatic hydrocarbons. As a counterpart, the dimesitylenboryl anthracene 9 was prepared (Scheme 17). Through the vacant p-orbital at the boron center, a boryl substituent can be considered as an electron withdrawing group. Through the absence of the lone pair, the dominant non-radiative decay pathway via the PET should be closed. For synthetic reasons, the more stable dimesitylenboryl anthracene was prepared as the boron center is protected by the bulky mesityl groups from any nucleophilic attacks. Furthermore, the starting material dimesitylboron fluoride is commercially available in contrast to the diphenylboron halides. The influence of the mesityl groups at the boron center on the photophysical should be negligible. In comparison to the diphenylphosphanyl anthracene the change of the heteroatom substituted at the anthracene should overlay the small influence of different aryl substituents at the heteroatom.

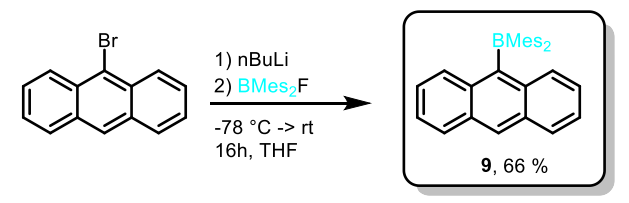

Scheme 17. Synthesis of 9-(dimesitylboryl) anthracene (9) according to reported procedures. ${ }^{[185]}$

The dimesitylboryl anthracene was synthesized analog to the diphenylphosphanyl anthracenes via lithiation of 9-bromoanthracene at $-78^{\circ} \mathrm{C}$ in THF and quenching with dimesitiylboron fluoride (Scheme 17). 
The synthesis and crystal structure of [9-BMes $\left.\left.2-\mathrm{C}_{14} \mathrm{H}_{9}\right)\right]$ (9) have already been published earlier, but the photophysical properties have not been reported. ${ }^{[185,186]}$ The compound was obtained in high purity and good yields after recrystallization from toluene or ethyl acetate. For verification of the successful synthesis and for better comparability of the data, the crystal structure was determined again in the scope of this work and the obtained dataset was used for evaluation of the structural parameters.

The boryl anthracene 9 serves as the counterpart for the phosphanyl anthracenes as the electronic properties of these derivatives should completely differ. For the comparison with the oxo and thiophosphoryl anthracenes, related derivatives with similar electronic and structural properties should be prepared. To ensure a good comparability, the Pauling electronegativity and covalent radii of the involved elements must be considered as they are the dominant criteria determining the structure and electronic properties. For obtaining a tetrahedral geometry similar as expected for the phosphoryl substituent, a carbon or silicon center is obviously suitable. Furthermore, chlorine should serve as an appropriate candidate for replacing the sulfur as especially the covalent radii are very similar (Table 1). Based on these considerations and the synthetic availability, the two 9-substituted anthracene derivatives $\left[9-(\mathrm{HO}) \mathrm{CPh}_{2}-\left(\mathrm{C}_{14} \mathrm{H}_{9}\right)\right](\mathbf{1 0})$ and $\left[9-(\mathrm{Cl}) \mathrm{SiPh}_{2}-\left(\mathrm{C}_{14} \mathrm{H}_{9}\right)\right](\mathbf{1 1})$ were prepared and investigated.

Table 1. Excerpt of the periodic table with Pauling electronegativity and covalent radii in parentheses of the corresponding elements. Elements shaded in grey were not considered during this work.

\begin{tabular}{lllll}
\hline Group 13 & Group 14 & Group 15 & Group 16 & Group 17 \\
\hline $\mathrm{B}$ & $\mathrm{C}$ & $\mathrm{N}$ & $\mathrm{O}$ & $\mathrm{F}$ \\
$1.88(0.84)$ & $2.45\left(0.76, \mathrm{sp}^{3}\right) ;$ & $2.93(0.71)$ & $3.61(0.66)$ & $4.14 /(0.57)$ \\
& $\left(0.73, \mathrm{sp}^{2}\right)$ & & & $\mathrm{Cl}$ \\
$\mathrm{Al}$ & $\mathrm{Si}$ & $\mathrm{P}$ & $\mathrm{S}$ & $3.05 /(1.02)$ \\
\hline $1.62(1.21)$ & $2.12(1.11)$ & $2.46(1.07)$ & $2.64(1.05)$ & \\
\hline
\end{tabular}

Both compounds were prepared from 9-bromoanthracene according to the well-established route via lithiation and quenching with a suitable electrophile. For the synthesis of $\mathbf{1 0}$, benzophenone was used as the electrophile. After aqueous work up and subsequent recrystallization, compound $\mathbf{1 0}$ was obtained in good yields (Scheme 18). The preparation of $\mathbf{1 0}$ and the stability and reactivity of its radicals have been reported recently in 2019 by Tomohiko et al., but the photophysical properties were not investigated and will therefore be evaluated during this work. ${ }^{[187]}$ From the previous works on the disubstituted $[9,10$ $\left.\left((E) P h_{2}\right)_{2}-\left(\mathrm{C}_{14} \mathrm{H}_{8}\right)\right]$ system drastic changes in the emission properties are known when oxygen was replaced by sulfur. Therefore, the corresponding thiol of $\mathbf{1 0}$ could also give information on the influence of heteroatoms on the photophysical properties. Unfortunately, the conversion of $\left[9-(\mathrm{HO}) \mathrm{CPh}_{2}-\left(\mathrm{C}_{14} \mathrm{H}_{9}\right)\right]$ into the corresponding thiol by treatment with Lawesson's reagent (LR) in refluxing toluene was not successful. The formation of the thiol could be confirmed by mass spectrometry but isolation in sufficient purity for the investigation of the photophysical properties failed.

[9-(Cl) $\left.\mathrm{SiPh}_{2}-\left(\mathrm{C}_{14} \mathrm{H}_{9}\right)\right]$ (11) was also synthesized from 9-bromoanthracene and dichlorodiphenylsilane and obtained in moderate yields (Scheme 18). Considering the size and electronegativity of the involved 
elements, the properties should be comparable to the thiophosphoryl anthracene and a similar solid-state structure is expected.

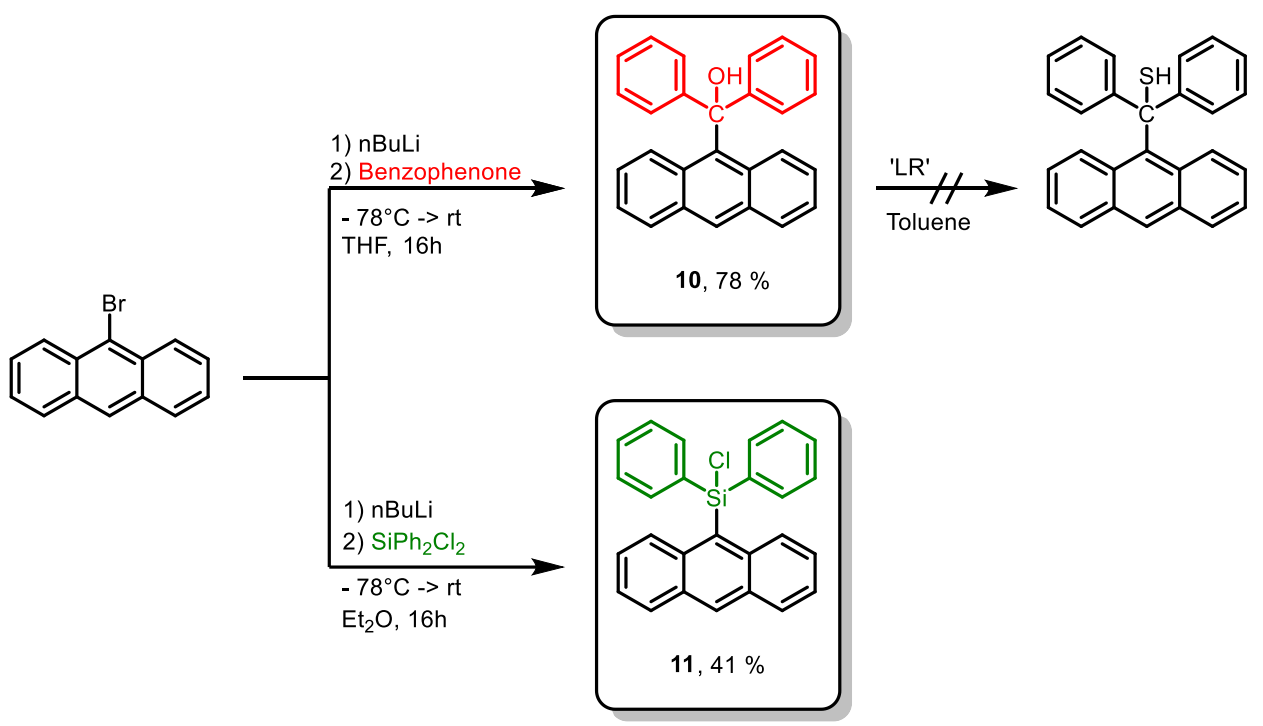

Scheme 18. Synthesis of the anthracene derivatives $\left[9-(\mathrm{HO}) \mathrm{CPh}_{2^{-}}\left(\mathrm{C}_{14} \mathrm{H}_{9}\right)\right](\mathbf{1 0})$ and $\left[9-\left((\mathrm{Cl}) \mathrm{SiPh}_{2^{-}}\left(\mathrm{C}_{14} \mathrm{H}_{9}\right)\right](\mathbf{1 1})\right.$ from 9bromoanthracene via the established route.

As the synthesis of $\mathbf{1 1}$ was straightforward, the disubstituted $\left[9,10-\left((\mathrm{Cl})\left(\mathrm{SiPh}_{2}\right)_{2}-\left(\mathrm{C}_{14} \mathrm{H}_{8}\right)\right]\right.$ (12) was also prepared even if it does not perfectly fit in the scope of this work as the two bulky silyl groups will prevent the anthracene chromophore from any strong intermolecular interactions. Nevertheless, it is interesting to see if the chlorosilyl anthracene shows a similar behavior as the $\left[9,10-\left((\mathrm{S})\left(\mathrm{PPh}_{2}\right)_{2}-\left(\mathrm{C}_{14} \mathrm{H}_{8}\right)\right]\right.$, if it is able to crystallize in different conformations, and if a co-crystallization with aromatic solvents can enhance the fluorescence efficiency in the solid-state. The synthesis was performed analog to 11 starting from 9,10dibromoanthracene using two equivalents of butyllithium and the dichlorodiphenylsilane (Scheme 19). The target compound was obtained as a pale-yellow solid in clearly higher yields compared to the monosubstituted derivative 11.

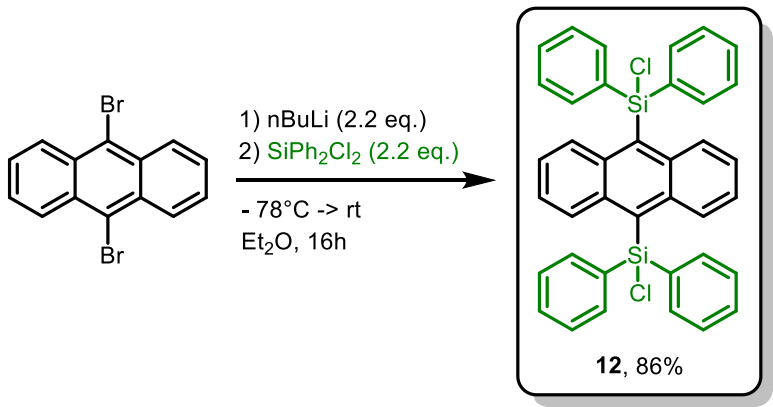

Scheme 19. Synthesis of $\left[9,10-\left((\mathrm{Cl}) \mathrm{SiPh}_{2}\right)_{2}-\left(\mathrm{C}_{14} \mathrm{H}_{8}\right)\right](\mathbf{1 2})$ from 9,10-dibromoanthracene.

\subsubsection{Structural Properties}

The solid-state structures of single-side substituted anthracenes $\mathbf{8}$ - $\mathbf{1 1}$ were determined via X-Ray diffraction analysis. For the oxophosphoryl anthracene 7, the prior obtained data by Stern ${ }^{[157]}$ and 
Katagiri $^{[188]}$ et al. will be used for comparison. As already noted, the crystal structure of 9 was also published before this work. ${ }^{[186]}$ The reported structure was obtained at $173 \mathrm{~K}$ and $\mathrm{x}$-ray diffraction experiments during this work were performed at $100 \mathrm{~K}$. For consistency, the obtained data during this work will be used for the following discussion.

(a)

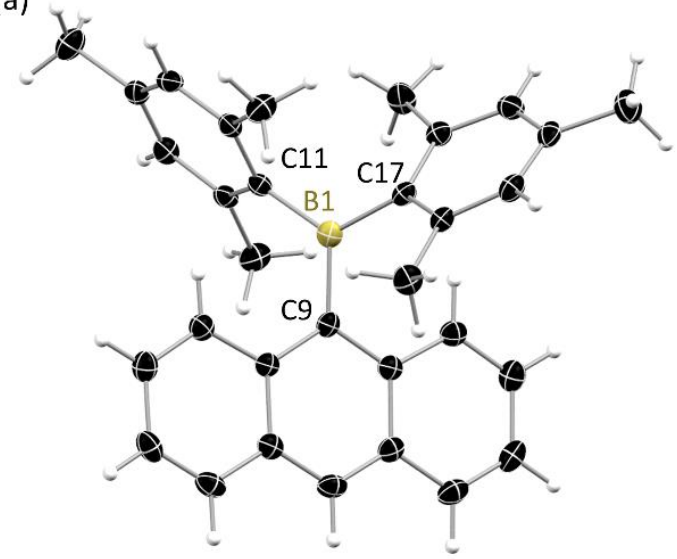

(b)



Figure 16. (a) Front view and (b) side view of the solid-state structure of [9-BMes ${ }_{2}-\left(\mathrm{C}_{14} \mathrm{H}_{9}\right)$ ] (9). Only one molecule of the asymmetric unit is shown.

The boryl anthracene [9-BMes $\left.{ }_{2}-\left(\mathrm{C}_{14} \mathrm{H}_{9}\right)\right](9)$ crystallizes in the monoclinic space group $P 2_{1} / \mathrm{n}$ with two independent molecules in the asymmetric unit. As expected, the boryl center adopts a trigonal planar geometry. The $\mathrm{C}-\mathrm{B}-\mathrm{C}$ angles range from $115.13(19)^{\circ}$ to $122.5(2)^{\circ}$. The $\mathrm{C}-\mathrm{B}$ bonds are as expected shortened to around 1.582(3) A compared to the C9-P bonds for the discussed phosphines with lengths of around $1.85 \AA$. The mesityl groups are also located one above and one below the anthracene plane but adopt a propeller like arrangement due to the increased steric demand (Figure 16). The crystal packing is organized by weak $\mathrm{C}-\mathrm{H} \cdots \pi$ interactions, but no characteristic packing regarding the anthracene moieties is found.

The oxophosphoryl anthracene [9-(O) PPh $\left.{ }_{2}-\left(\mathrm{C}_{14} \mathrm{H}_{9}\right)\right](7)$ crystallizes in the monoclinic space group $P 2_{1} / \mathrm{n}$ with four slightly different molecules in the asymmetric unit. In general, the molecular structure of the parent phosphine [9- $\left.\mathrm{PPh}_{2}-\left(\mathrm{C}_{14} \mathrm{H}_{9}\right)\right]$ is resembled with a few minor changes. The phenyl groups are still located one above and one below the anthracene plane. However, with a torsion angle O-P-C9-C9A of nearly $27^{\circ}$ the $\mathrm{P}-\mathrm{O}$ fragment is not completely in plane of the anthracene core like observed for the lone pair of 1. Moreover, the anthracene scaffold is not planar anymore and a small butterfly-like bending can be observed and quantified by the folding angle $\alpha$ to an average of $7.83^{\circ}$. The C9-P bond length is slightly shortened to around $1.83 \AA$ compared to $1.85 \AA$ found for the phosphines. Further bond lengths and angles are as expected, and a selection can be found in Table 2. 
Table 2. Basic structural parameters of the monosubstituted anthracene derivatives [9-(E)YPh$\left.-\left(\mathrm{C}_{14} \mathrm{H}_{9}\right)\right]$.

\begin{tabular}{llllll} 
& $\mathbf{7}(\mathrm{Y}=\mathrm{P}, \mathrm{E}=\mathrm{O})^{[\mathrm{a}]}$ & $\mathbf{8}(\mathrm{Y}=\mathrm{P}, \mathrm{E}=\mathrm{S})$ & $\mathbf{9}(\mathrm{Y}=\mathrm{B})$ & $\mathbf{1 0}(\mathrm{Y}=\mathrm{C}, \mathrm{E}=\mathrm{OH})$ & $\mathbf{1 1}(\mathrm{Y}=\mathrm{Si}, \mathrm{E}=\mathrm{Cl})$ \\
\hline $\mathrm{C} 9-\mathrm{Y}^{[\mathrm{b}]} / \AA$ & 1.827 & $1.8327(13)$ & $1.582(3)$ & $1.5576(17)$ & $1.891(3)$ \\
$\mathrm{Y}-\mathrm{E}^{[\mathrm{b}]} / \AA$ & 1.483 & $1.9553(8)$ & - & $1.4568(15)$ & $2.0840(9)$ \\
$\mathrm{Y}(\mathrm{C} 11-\mathrm{Y}-\mathrm{C} 17)^{[\mathrm{b}]} /{ }^{\circ}$ & 107.7 & $101.30(6)$ & $121.3(2)$ & $112.15(10)$ & $108.95(11)$ \\
$\omega(\mathrm{Y}-\mathrm{E}-\mathrm{C} 9-\mathrm{C} 9 \mathrm{a})^{[\mathrm{b}]} /{ }^{\circ}$ & 26.97 & $85.38(10)$ & - & $38.44(14)$ & $53.06(18)$ \\
$\alpha /{ }^{\circ}$ & 7.83 & $14.27(13)$ & $6.06(11)$ & $11.31(14)$ & $6.78(13)$ \\
\hline
\end{tabular}

[a] published data from CCDC used ${ }^{[188]}[\mathrm{b}]$ if more than molecule is present in the asymmetric unit the average value was taken as differences between the independent molecules are marginal.

When the phosphine is oxidized with sulfur to the thiophosphoryl anthracene, the structural changes are more pronounced. [9-(S) $\mathrm{PPh}_{2}-\left(\mathrm{C}_{14} \mathrm{H}_{9}\right)$ ] (8) crystallizes in the triclinic space group $P \overline{1}$ with two independent molecules in the asymmetric unit. The phenyl groups are now located on the same anthracene side and a nearly orthogonal orientation of the P-S moiety towards the anthracene plane is observed ( $\omega$ (S-P-C9$\left.\mathrm{C} 9 \mathrm{~A})=85.38(10)^{\circ}\right)$ and the sulfur atom occupies the other anthracene side (Figure 17). This reorientation of the substituent is ascribed to the larger and more diffuse character of the sulfur lone pairs compared to the oxygen. The increased steric demand can be further confirmed by a widening of the S-P-C9 angle up to $116^{\circ}$, and the smaller C11-P-C17 angle $\left(\gamma=101.30(6)^{\circ}\right)$ goes along with this hypothesis. As well, the changed orientation with the two phenyl groups at one side induces a strong butterfly-like folding of the anthracene moiety. The folding angle $\alpha$ is determined to an average of $14.27(13)^{\circ}$. The P-S bond is elongated in comparison to the $\mathrm{P}-\mathrm{O}$ bond about $0.47 \AA$ and further bond lengths and angles are as expected and comparable to the oxophosphoryl anthracene.

(a)

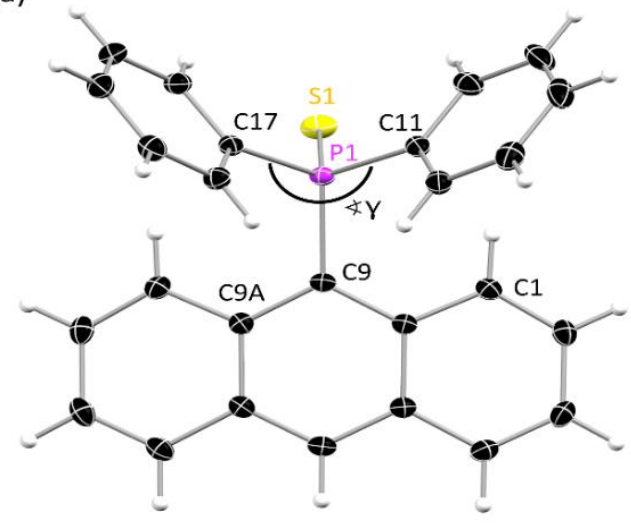

(b)

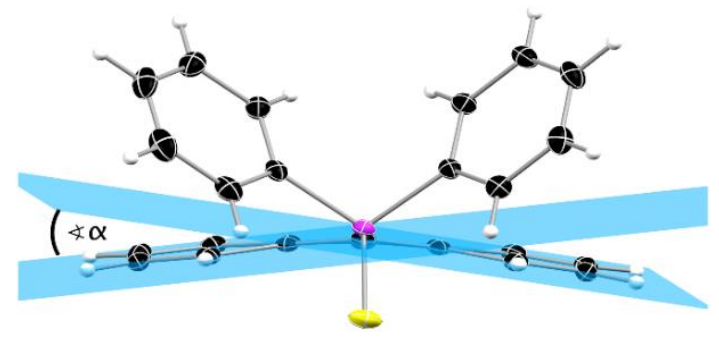

Figure 17. (a) Front view of the molecular structure of [9-(S)PPh $\left.-\left(\mathrm{C}_{14} \mathrm{H}_{9}\right)\right](\mathbf{8})$ and (b) side view of 8 revealing the changed orientation of the $\mathrm{SPPh}_{2}$-substituent inducing a strong butterfly-like distortion of the anthracene plane.

Replacing the phosphoryl moiety leads to only minor structural changes. The solid-state structures of [9$\left.(\mathrm{HO}) \mathrm{CPh}_{2}-\left(\mathrm{C}_{14} \mathrm{H}_{9}\right)\right](\mathbf{1 0})$ and $\left[9-(\mathrm{Cl}) \mathrm{SiPh}_{2}-\left(\mathrm{C}_{14} \mathrm{H}_{9}\right)\right]$ (11) are overall comparable to the oxophosphoryl anthracene 7. 10 crystallizes in the monoclinic space group $P 2_{1} / \mathrm{n}$ with one molecule in the asymmetric unit. The orientation of the substituent is similar as seen for [9-(O) $\left.\mathrm{PPh}_{2}-\left(\mathrm{C}_{14} \mathrm{H}_{9}\right)\right]$ with one phenyl group above and one below the anthracene plane (Figure 18). The O-C23-C9-C9A torsion angle $\omega$ is slightly larger with $38.44(14)^{\circ}$ and the bending of the anthracene plane is also slightly increased $\left(\alpha=11.31(14)^{\circ}\right)$. As excepted the C9-C23 bond is significant shorter than the C9-P bond, but the C23-O and P-O bond -48 - 
lengths are in good accordance. Unfortunately, $\mathbf{1 0}$ could not be converted into to the corresponding thiol in satisfying purity and no single-crystals suitable for structure determination were obtained. It would be interesting to see if the substitution of the oxygen with sulfur is followed by similar drastic changes in the structural and photophysical properties as seen for the phosphoryl derivatives. Nevertheless, from the structural point of view, the exchange of the $\mathrm{P}-\mathrm{O}$ against a $\mathrm{C}-\mathrm{OH}$ group leads to only small changes and the anthracenyl methanol is a suitable candidate for comparison with the oxophosphoryl anthracene concerning the photophysical properties.

The fourth investigated system is the chlorosilyl anthracene 11, which crystallizes in the triclinic space group $P \overline{1}$ with two molecules in the asymmetric unit. As the $\mathrm{Si}-\mathrm{Cl}$ and $\mathrm{P}-\mathrm{S}$ moieties are comparable regarding the size of the atoms, similar solid-state structures are anticipated, but this expectation could not be confirmed completely. The orientation of the $\mathrm{ClSiPh}_{2}$ group is changed and more similar to the $\mathrm{OPPh}_{2}$ and $\mathrm{HOCPh}_{2}$ groups with one phenyl group above and one below the anthracene plane (Figure 18). Indeed, the $\mathrm{Cl}-\mathrm{Si}-\mathrm{C9}-\mathrm{C9A}$ torsion angle is widened to $\omega=53.06(18)^{\circ}$ but still not in range of the almost orthogonal orientation found for the P-S group $(\omega=85.38(10))$. However, the relevant bond lengths C9$\mathrm{Si}$ and $\mathrm{Si}-\mathrm{Cl}$ are in good accordance with the corresponding $\mathrm{C}-\mathrm{P}$ and $\mathrm{P}-\mathrm{S}$ lengths found in $\mathbf{8}$. Therefore, the steric demand of the $\mathrm{Cl}-\mathrm{Si}$ substituent seems not to be large enough to induce the reorientation of the substituent with both phenyl groups at the same side of the anthracene. This is further confirmed by a larger C11-Si-C17 angle $\left(\gamma=108.95(11)^{\circ}\right)$ and the clearly reduced folding angle $\left(\alpha=6.78(13)^{\circ}\right)$.

(a)



(b)

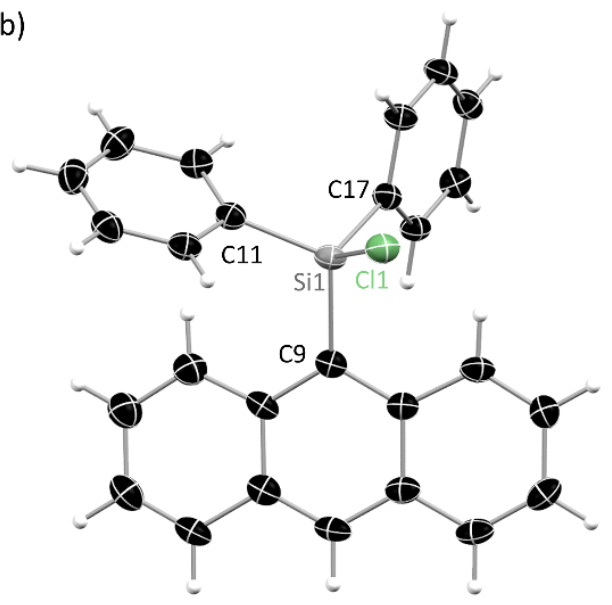

Figure 18. Solid-state structures of (a) [9-(HO) $\left.\mathrm{CPh}_{2}-\left(\mathrm{C}_{14} \mathrm{H}_{9}\right)\right](\mathbf{1 0})$ and $(\mathrm{b})\left[9-(\mathrm{Cl}) \mathrm{SiPh}_{2}-\left(\mathrm{C}_{14} \mathrm{H}_{9}\right)\right](\mathbf{1 1})$.

The investigated structures with slightly modified substituents revealed as expected several similarities among each other. Especially the structures of 7, $\mathbf{1 0}$ and $\mathbf{1 1}$ are in a good accordance considering the general structural motif and the basic parameters. In contrast, the thiophosphoryl anthracene showed strong variations in the structure. The examination of the different substituents revealed that these variations are attributed to the slightly increased steric demand of the sulfur atom in comparison to the oxygen or chlorine. Probably, due to its slightly larger van der Waals radius and the more diffuse character, an increased space is required resulting in a new orientation of the diphenyl substituent and a strong distortion of the anthracene plane. 
Before the intermolecular interactions and the crystal packing will be investigated, a brief look into the crystal structure of the disubstituted $\left[9,10-\left((\mathrm{Cl}) \mathrm{SiPh}_{2}\right)_{2}-\left(\mathrm{C}_{14} \mathrm{H}_{8}\right)\right](\mathbf{1 2})$ will be given. As initially mentioned, the chlorosilyl anthracene could serve as a model compound for the disubstituted $\left[9,10-\left((\mathrm{S}) \mathrm{PPh}_{2}\right)_{2}-\left(\mathrm{C}_{14} \mathrm{H}_{8}\right)\right]$ with its remarkable luminescence features. Crystallization of $\left[9,10-\left((\mathrm{Cl}) \mathrm{SiPh}_{2}\right)_{2}-\left(\mathrm{C}_{14} \mathrm{H}_{8}\right)\right]$ from toluene afforded crystals suitable for structure determination. The compound crystallizes in the monoclinic space group $P 2_{1} / c$ with half a molecule in the asymmetric unit and without co-crystallized solvent. The orientation of the substituents is similar as seen in the monosubstituted derivative 11 with $\mathrm{Cl}-\mathrm{Si}-\mathrm{C} 9-\mathrm{C} 9 \mathrm{~A}$ torsion angles of $52.05(18)^{\circ}$. Through the changed orientation of the substituents, the chlorosilyl anthracene is probably not able to form co-crystals with aromatic molecules. No cradle is formed alike as in the packing of the thiophosphoryl anthracene, which was assigned to a preferred orientation for cocrystallization of aromatic guest molecules in a T-shaped fashion. The arrangement of the chlorosilyl groups can be considered as a transoid orientation with the chlorine atoms on different sites of the anthracene plane (Figure 19a). The transoid orientation is assumed to be mandatory for the occurrence of intense solid-state luminescence in the thiophosphoryl anthracene and was only observed in combination with a co-crystallized aromatic solvent.

(a)

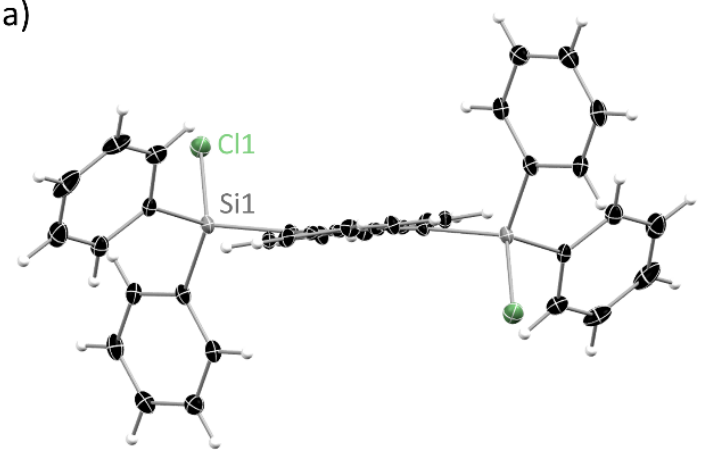

(b)

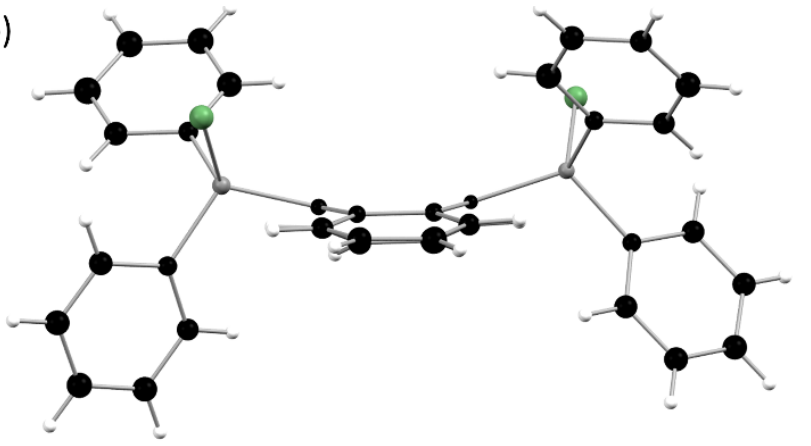

Figure 19. (a) Solid-state structure of $\left[9,10-\left((\mathrm{Cl}) \mathrm{SiPh}_{2}\right)_{2}-\left(\mathrm{C}_{14} \mathrm{H}_{8}\right)\right](12)$ with a transoid orientation of the $\mathrm{Cl}-\mathrm{Si}$ moiety. (b) Polymorph of 12 with a cisoid confirmation and a strong deformation of the anthracene plane. The cisoid polymorph could not be crystallized in sufficient quality and no complete data set was obtained.

During the crystal selection process, a few crystals with a different shape and appearance were observed. Unfortunately, the crystals were not suitable for obtaining a complete data set of sufficient quality. However, from the collected data, the structure could be solved to get a brief glance of the crystallized compound. In contrast to the structure described above a different orientation of the chlorosilyl substituents was found. Both chlorine atoms are now located on the same anthracene side and the conformation can be referred as cisoid (Figure 19b). Furthermore, the changed orientation induces a strong deformation of the aromatic plane with a pronounced folding of the anthracene as also observed for cisoid-[9,10-((S)PPh $\left.)_{2}-\left(\mathrm{C}_{14} \mathrm{H}_{8}\right)\right]$.

In contrast to the thiophosphoryl derivative, the bis(diphenylchlorosilyl) anthracene seems able to crystallize in two polymorphic forms, even without the presence of the lattice solvent. From several crystallization approaches, it could be derived that the transoid form appeared to be the more stable polymorph as the crystallization of the cisoid form could not be reproduced. If a suitable method and 
condition for reliable crystallization of the cisoid polymorph could be found, this would allow a direct insight into a possible dependence of the cisoid/transoid conformation on the emission properties. For the thiophosphoryl anthracene $\left[9,10-\left((\mathrm{S}) \mathrm{PPh}_{2}-\left(\mathrm{C}_{14} \mathrm{H}_{8}\right)\right]\right.$, the cisoid conformation was attributed to be responsible for the fluorescence quenching. Until now, this hypothesis could not be confirmed completely as the transoid conformation could only be obtained upon co-crystallization with aromatic solvents. Therefore, it could not be clarified finally if the transoid conformation, the co-crystallization of the solvent or a combination of both effects induces the enhanced emission in the solid-state.

Investigation of the structural properties of the mono-substituted derivatives revealed some slight changes regarding the orientation of the substituent and the deformation of the anthracene scaffold. Whether these intramolecular structural variations or electronic effects of the heteroatoms in general, influence the intermolecular interactions will be evaluated in the following. Especially, direct $\pi-\pi$ interactions between the anthracenes are of interest, as they can potentially influence the solid-state emission and are essential for excimer formation. For a major part of the compounds presented up to here, no $\pi-\pi$ interactions are observed. Regarding the anthracene moieties, no specific packing motifs are found in the solid-state structures. Most non-covalent interactions are identified as weak $\mathrm{C}-\mathrm{H} \cdots \pi$ interactions between the phenyl groups and the anthracene scaffold. Examples of the crystal packing motifs for [9-( $\left.\mathrm{HO}) \mathrm{CPh}_{2}-\left(\mathrm{C}_{14} \mathrm{H}_{9}\right)\right](10)$ and $\left[(\mathrm{Cl}) \mathrm{SiPh}_{2}-\left(\mathrm{C}_{14} \mathrm{H}_{9}\right)\right](\mathbf{1 1})$ are given in Figure 20 and clearly reveal the absence of any strong $\pi-\pi$ interactions, as no face-to-face orientations of neighboring anthracenes are present.

(a)

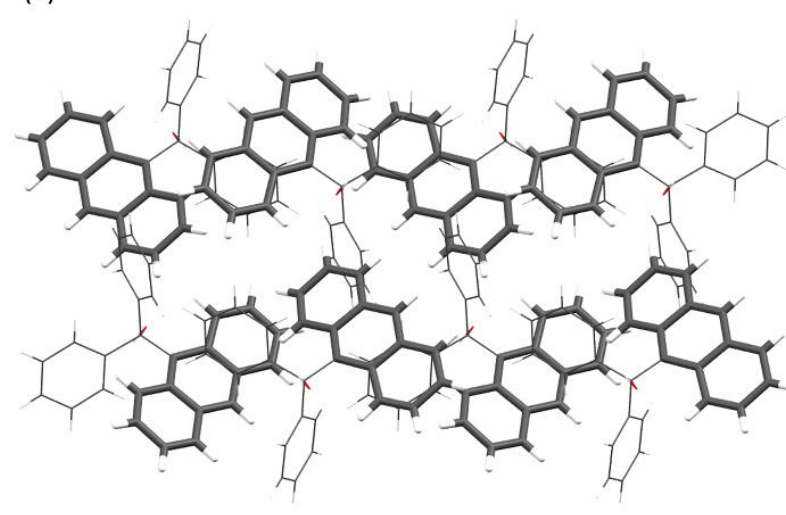

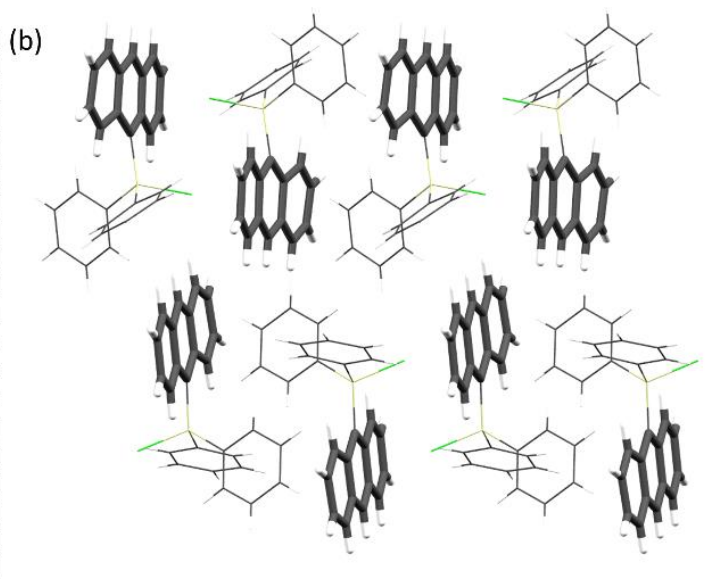

Figure 20. Excerpt of the crystal packing motif as found in (a) $\left[9-(\mathrm{HO}) \mathrm{CPh}_{2}-\left(\mathrm{C}_{14} \mathrm{H}_{9}\right)\right](\mathbf{1 0})$ and (b) $\left[9-(\mathrm{Cl}) \mathrm{SiPh}_{2}-\left(\mathrm{C}_{14} \mathrm{H}_{9}\right)\right](\mathbf{1 1})$. Anthracene moieties are highlighted in dark grey.

In order to evaluate the strength of the interactions between the anthracene fluorophores, the shortest distances $d_{c c}$ between the centroids of the central anthracene rings of neighboring molecules were estimated. The values are given in Table 3 and indicate that the interaction between the anthracene moieties in 7, 9 and $\mathbf{1 0}$ are rather weak as the shortest observed distances are in a range of $7.41 \AA$ to $8.27 \AA$ and comparable to the investigated phosphine derivatives in the previous chapter. The chlorosilyl anthracene 11 reveals a slightly shorter distance $(6.15 \AA)$ between neighboring anthracenes, but the crystal packing diagram clearly shows an offset of the nearest anthracenes and no overlapping area of the 
anthracene planes. Therefore, the occurring interactions can be considered as $\mathrm{C}-\mathrm{H} \cdots \pi$ type rather than $\pi-\pi$ interactions.

Table 3. Shortest distances between the centroids of the central anthracene ring of neighboring anthracene moieties.

\begin{tabular}{ll}
\hline & $\mathrm{d}_{\mathrm{cc}} / \AA$ \\
\hline$\left[9-\mathrm{PPh}_{2}-\left(\mathrm{C}_{14} \mathrm{H}_{8}\right)\right](\mathbf{1})$ & 7.00 \\
{$\left[9-(\mathrm{O}) \mathrm{PPh}_{2}-\left(\mathrm{C}_{14} \mathrm{H}_{9}\right)\right](\mathbf{7})$} & 8.27 \\
{$\left[9-(\mathrm{S}) \mathrm{PPh}_{2}-\left(\mathrm{C}_{14} \mathrm{H}_{9}\right)\right](\mathbf{8})$} & 5.01 \\
{$\left[9-\mathrm{BMes}_{2}-\left(\mathrm{C}_{14} \mathrm{H}_{9}\right)\right](\mathbf{9})$} & 8.11 \\
{$\left[9-\left(\mathrm{HO}_{2} \mathrm{CPh}_{2}-\left(\mathrm{C}_{14} \mathrm{H}_{9}\right)\right](\mathbf{1 0})\right.$} & 7.41 \\
{$\left[9-(\mathrm{Cl}) \mathrm{SiPh}_{2}-\left(\mathrm{C}_{14} \mathrm{H}_{9}\right)\right](\mathbf{1 1})$} & 6.15 \\
\hline
\end{tabular}

For the thiophosphoryl anthracene $\mathbf{8}$ a different behavior is found. Strong interactions between the anthracene units are present resulting in an overall dimeric herringbone packing motif and closest distances between the centroids of neighboring anthracenes is reduced to around $5.01 \AA$ (Figure 21). Edge-to-face stacking with $\mathrm{H} 4, \mathrm{H} 5$ and $\mathrm{H} 9$ pointing on the aromatic plane of a neighboring anthracene as well as direct face-to-face interactions between the aromatic planes

of neighboring anthracenes are observed. The $\pi-\pi$ distance is estimated $3.242 \AA$, which is slightly shorter than typical for $\pi-\pi$ interactions between polyaromatic hydrocarbons and attributed to the bent structure of the anthracene plane. ${ }^{[71]}$ As desired, the bulky thiophosphoryl substituent induces an antiparallel orientation in the dimer and prevents a columnar stacking of the anthracene moieties. Instead, dimeric pairs are formed with only two molecules interacting via direct face-to-face interactions. Other neighboring molecules show edge-to-face interactions resulting in the herringbone-type packing motif. It is notable that the two anthracenes of the dimer are bent towards each other. Therefore, the question arises if the strong folding of the anthracene is induced by the $\pi-\pi$ interactions or by the steric demand of the substituent as claimed earlier. For verification, a structure optimization in the gas phase was performed by Ruth and revealed also a folding angle of $\alpha=11.4^{\circ}$ and nearly the same orientation of the substituent $\left(\omega(\mathrm{S}-\mathrm{P}-\mathrm{C} 9-\mathrm{C} \mathrm{A})=80.14^{\circ}\right) \cdot{ }^{[189]}$ As a consequence, these features were attributed to be inherent to the structure and no to the crystal packing effect. Nevertheless, it cannot be excluded that at least the orientation of the substituent is beneficial for the formation of $\pi-\pi$ interactions. Besides the $\pi-\pi$ distance, the interactions in the anthracene dimer can be quantified by further structural parameters. The overlapping area of the two anthracene is assigned to be representative for the strength of the interactions. In collaboration with Ruth, a procedure was developed to calculate the overlapping area of two anthracenes. Therefore, a mean plane is fitted through the carbon atoms of one anthracene. The carbon atoms of both anthracenes are projected onto this plane and two polygons are obtained. From the two polygons, an intersection polygon is calculated, and the overlapping area is calculated as the ratio of the intersection polygon and the area of the polygon from one anthracene. Additionally, this procedure provides the offsets of the dimer along the short $\left(d_{x}\right)$ and the long anthracene axis $\left(d_{y}\right)$, which reveal small changes in the dimeric motif in more detail. For [9-(S)PPh$\left.-\left(\mathrm{C}_{14} \mathrm{H}_{9}\right)\right]$ an overlap ratio of $21.1 \%$ of the anthracene planes and offsets of $d_{x}=1.344 \AA$ and $d_{y}=3.227 \AA$ are obtained. 

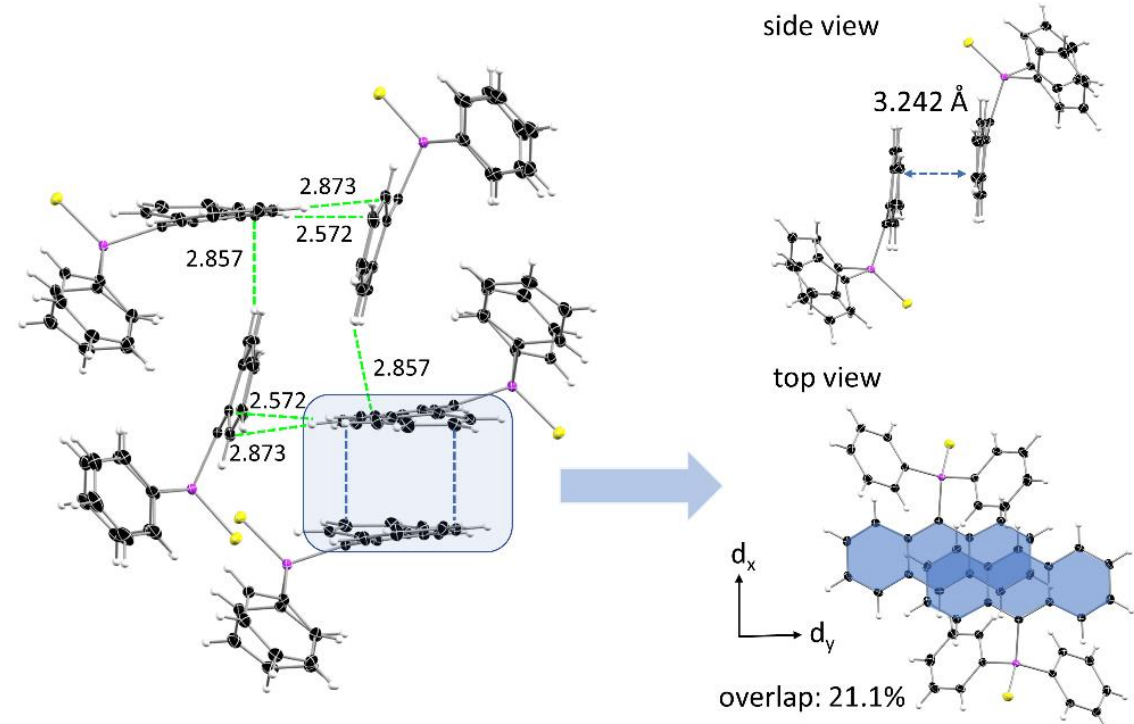

Figure 21. Dimeric herringbone packing as found in the solid-state structure of [9-(S)PPh$\left.-\left(\mathrm{C}_{14} \mathrm{H}_{9}\right)\right](8)$ with resulting edge-to-face (green) and face-to-face (blue) interactions between the anthracene moieties. On the right the dominant dimeric motif is shown in more detail.

In contrast to the related derivatives, the thiophosphoryl anthracene is the only compound which reveals direct $\pi-\pi$ interactions between the anthracene scaffolds. Therefore, 8 and similar thiophosphoryl anthracenes should be suitable candidates for an investigation of the correlation between the intermolecular interactions and the solid-state luminescence. By varying of the substituent in 10-position, it should be possible to tailor the intermolecular interactions and the resulting packing motif. This approach is presented in chapter 3.1.3.

\subsubsection{Photophysical Properties}

\section{In solution}

The photophysical properties of the phosphoryl anthracenes and their related derivatives in diluted solutions will be investigated as follows. Diluted solutions of fluorophores, with suitable concentrations, can serve as an idealized system of the monomeric form of the fluorophore without any strong interactions. The information obtained from these experiments is crucial for comparison to the condensed phase, where intermolecular interactions can influence the absorption and emission processes.

The absorption spectra of 7-11 in diluted THF solution are very similar regarding the shape and the observed transitions, except for the boryl anthracene 9 (Figure 22). All compounds reveal a sharp high energy band absorption between $254 \mathrm{~nm}$ and $260 \mathrm{~nm}$, which was already observed for the diphenyphosphine anthracenes. The $\mathrm{S}_{0} \rightarrow \mathrm{S}_{2}$ absorption is nearly unaffected by the substituent in 9-position as the transition is mainly localized on the anthracene moiety and oriented along the long molecular anthracene axes. Substituents in 1,4,9 or 10-position should therefore not affect the high energy transition, which has already been demonstrated for other anthracene derivatives by experimental and computational studies. ${ }^{[190]}$ 

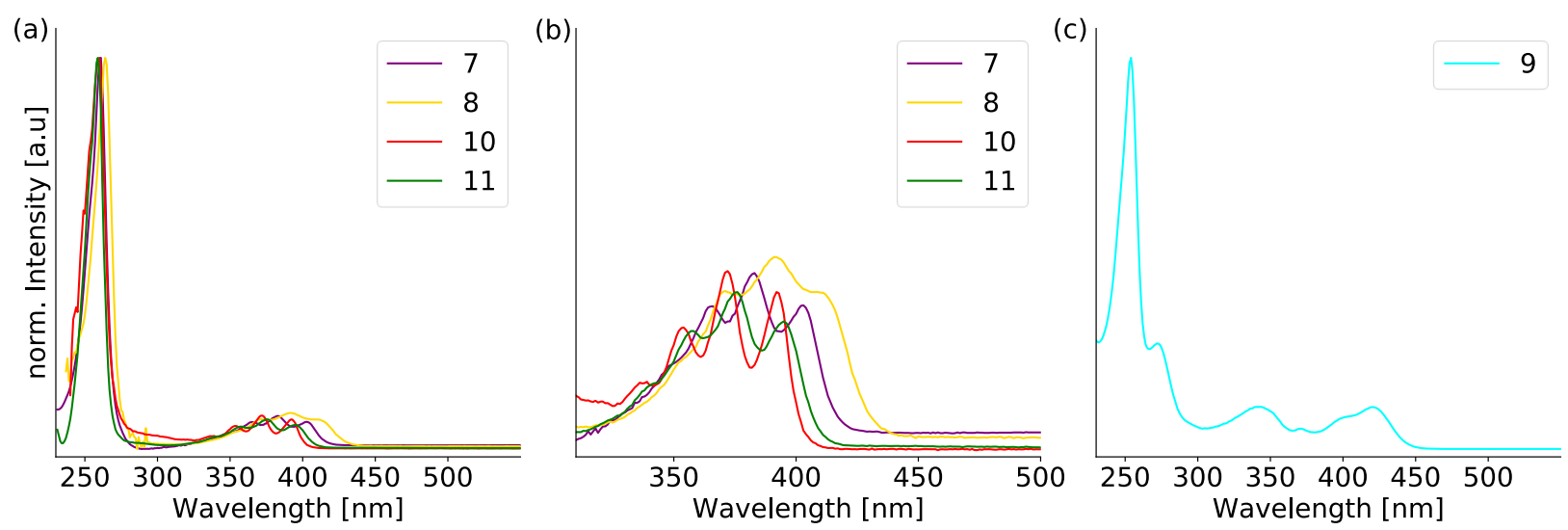

Figure 22. (a) UV-Vis absorption spectra of the anthracene derivatives [9-(O) $\left.\mathrm{PPh}_{2}-\left(\mathrm{C}_{14} \mathrm{H}_{9}\right)\right](7)$, [9-(S)PPh$\left.)^{-}\left(\mathrm{C}_{14} \mathrm{H}_{9}\right)\right](8)$, [9-( $\left.\mathrm{HO}\right) \mathrm{CPh}_{2^{-}}$ $\left.\left(\mathrm{C}_{14} \mathrm{H}_{9}\right)\right](\mathbf{1 0})$ and $\left[9-(\mathrm{Cl}) \mathrm{SiPh}_{2}-\left(\mathrm{C}_{14} \mathrm{H}_{9}\right)\right](\mathbf{1 1})$ in diluted THF solution $\left(10^{-5} \mathrm{M}\right)$; (b) more detailed view of the transition around $370 \mathrm{~nm}$ and (c) UV-Vis absorption spectra of $\left[9-\mathrm{BMes}_{2}-\left(\mathrm{C}_{14} \mathrm{H}_{9}\right)\right](9)$.

The more important transition is the $S_{0} \rightarrow S_{1}$ absorption as fluorescence occurs in general from the $S_{1}$ state. The transition dipole moment is polarized along the short anthracene axis and can therefore be affected by the different substituents in 9-position. As typical for anthracene derivatives and as already observed for the phosphanyl anthracenes, a structured absorption band in the region from $350 \mathrm{~nm}$ to $430 \mathrm{~nm}$ is obtained for the herein investigated derivatives. The vibronic structure is less pronounced for the thiophosphoryl anthracene 8 , which also reveals a slightly stronger bathochromic shift with a 0-0 transition at $411 \mathrm{~nm}$ (Table 4). In general, the different substituents seem to affect the absorption properties only moderately. For $\mathbf{7}, \mathbf{8}, \mathbf{1 0}$ and $\mathbf{1 1}$, the absorption properties are therefore mainly determined by the anthracene scaffold and assigned to typical $\pi-\pi^{*}$ transitions. Contribution from charge-transfer processes involving the substituents could not be identified.

A more complex spectrum is found for [9-BMes $\left.{ }_{2}-\left(\mathrm{C}_{14} \mathrm{H}_{9}\right)\right](9)$, which reveals further absorptions than the just evaluated transitions (Figure 22c). Close to the $S_{0} \rightarrow S_{2}$ transition, a second band at $272 \mathrm{~nm}$ is observed. A further absorption band is located at $343 \mathrm{~nm}$. At longer wavelengths up to $420 \mathrm{~nm}$, another band arises with slight vibronic splitting. As the absorption spectrum clearly differs from the previous described derivatives it is likely that the boryl substituent has a larger impact on the absorption properties. The spacing of the vibronic bands of the lower energy absorption at $420 \mathrm{~nm}$ is similar as seen in the spectra before. Therefore, the transition is assigned to the red-shifted $\pi-\pi^{*}$ transition of the anthracene moiety. In 2019, Gabbaï et al. reported the synthesis and properties of the 1-(dimesitylboryl)anthracene, which should have comparable electronic transitions as 9. ${ }^{[191]}$ Indeed, the absorption spectrum reveals two low energy bands centered at $328 \mathrm{~nm}$ and $407 \mathrm{~nm}$, with similar shape as observed for the 9-substituted derivative (9). The lower energy band was assigned to the $\pi-\pi^{*}$ transition, which was confirmed by TD-DFT calculations. Furthermore, the bathochromic shift of around $30 \mathrm{~nm}$ compared to the $0-0$ transition of anthracene was assigned to a conjugation of the vacant $p$-orbital located at the boron with the $\pi$ * orbitals of the anthracene moiety resulting in a slightly lower energy. As the absorption centered at $328 \mathrm{~nm}$ vanishes upon binding of small anions $\left(\mathrm{F}^{-}, \mathrm{CN}^{-}\right)$at the boron, the origin of the transition was assigned to the boryl substituent and mainly attributed to a charge transfer from the mesityl groups towards the boron 
center. Even if no studies on the anion binding capabilities of 9 were performed during this thesis, one can probably assign the absorption band at $343 \mathrm{~nm}$ also predominantly to the mesitylboryl substituent. Through absence of the transition around $272 \mathrm{~nm}$ in the other derivatives, it is also assigned to the substituent, but the nature of the transition could not be identified.

The evaluation of the UV-Vis spectra of 7-11 revealed that, except for $\mathbf{9}$, the absorption properties are only minorly affected by the substituent in 9-position. Besides a slight bathochromic shift and a partially less pronounced vibronic structure the absorption spectra are comparable to parent anthracene and thus the transitions are assigned mainly to be located on the anthracene moiety.

For the emission properties of 7-11 in diluted solution a similar behavior is expected and the emission process should mainly be determined by the anthracene fluorophore. At first glance, the emission spectra differ stronger compared to the absorption spectra considering shape and emission wavelength for the different substituted derivatives.
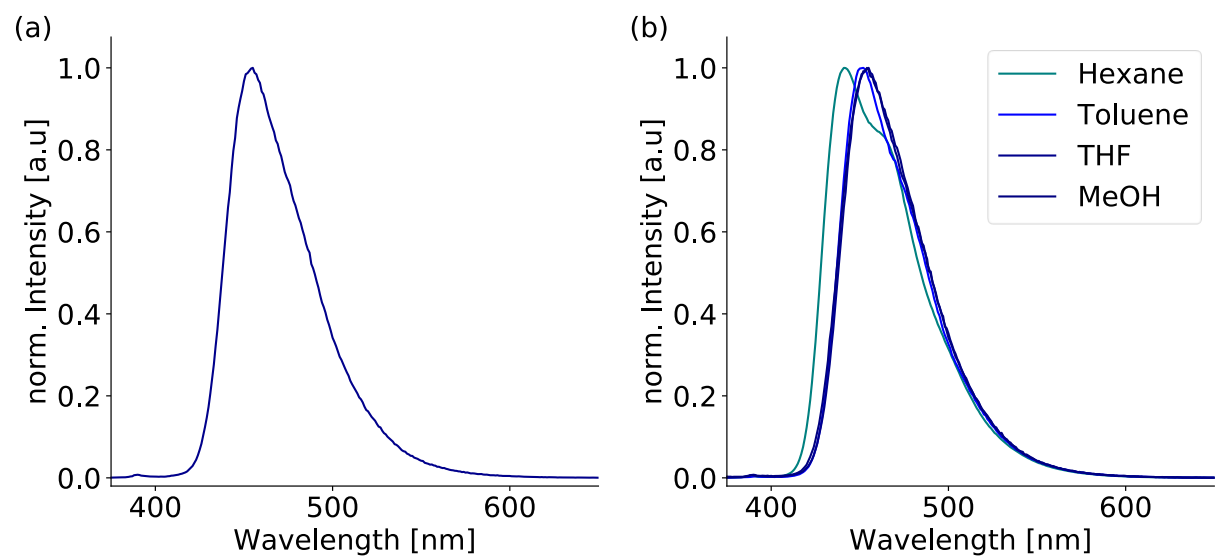

Figure 23. (a) Emission spectrum of [9-BMes $\left.{ }_{2}-\left(\mathrm{C}_{14} \mathrm{H}_{9}\right)\right](9)$ in diluted THF solution $\left(10^{-5} \mathrm{M}, \lambda_{\mathrm{ex}}=350 \mathrm{~nm}\right)$ and (b) emission spectra in solvents with different polarity.

The emission of the anthracenyl phosphines $\mathbf{1}$ - $\mathbf{6}$ was nearly completely quenched, which was ascribed to the PET of the phosphorous lone pair. Substitution of the phosphine group with the boryl group should therefore lead to an enhanced emission as the absence of the lone pair prohibits a PET. Indeed, a strong blue-green emission for [9-BMes ${ }_{2}-\left(\mathrm{C}_{14} \mathrm{H}_{9}\right)$ ] (9) is observed in diluted THF solution. The spectrum reveals no vibronic structure and the emission peaks at $455 \mathrm{~nm}$. The quantum yield reaches values up to $50.7 \%$ and is therefore significantly increased compared to the anthracenyl phosphines and also to unsubstituted anthracene $\left(\phi_{\mathrm{F}}=0.36\right.$ in $\left.\mathrm{C}_{6} \mathrm{H}_{12}\right) .{ }^{[192]}$ As the determined fluorescence lifetimes are in a few nanoseconds and no longer-wavelength emission bands are present, the emission is assigned to pure fluorescence from the $\mathrm{S}_{1}$ state. To investigate a possible intramolecular charge transfer (ICT), emission spectra of 9 in solvents with different polarity were performed, but the emission wavelength is nearly independent of the solvent polarity (Figure 23). The bathochromic shift of the emission wavelength from hexane to the more polar toluene is only $13 \mathrm{~nm}$ and changes therefore only little. The spectral shape is slightly changed as the small shoulder found in the spectrum in hexane is absent in toluene. For more polar solvents like THF and methanol, no further shift could be observed. Nevertheless, emission processes involving a charge transfer 
from the anthracene to the electron deficient boryl moiety, which could be assumed from the absorption spectra, could not be identified as the solvatochromic behavior should be more pronounced. Introduction of an electron donating substituent like an amine or phosphine in the 10-position of the anthracene would lead to a donor- $\pi$-acceptor motif. This motif should be capable to undergo an ICT from the donor to the acceptor resulting in a more pronounced solvatochromic behaviour. Such a behavior was reported for a trianthrylborane substituted with diphenyl amine in the para position of the anthracene. This compound emits over a range of nearly $100 \mathrm{~nm}$ in solvents with different polarity. ${ }^{[193]}$

The previously observed slow oxidation of [9- $\left.\mathrm{PPh}_{2}-\left(\mathrm{C}_{14} \mathrm{H}_{9}\right)\right](\mathbf{1})$ already suggested an intense blue emission for the oxophosphoryl 7 in solution, which can now be confirmed by the targeted synthesis and investigation of [9-(O) $\left.\mathrm{PPh}_{2}-\left(\mathrm{C}_{14} \mathrm{H}_{9}\right)\right]$. The emission wavelength at $443 \mathrm{~nm}$ is identical to the observed emission for the oxidation product of $\left[9-\mathrm{PPh}_{2}-\left(\mathrm{C}_{14} \mathrm{H}_{9}\right)\right]$ in aerated THF solution. The emission spectrum is broadened and the typical vibronic structure for anthracene derivatives is completely absent (Figure 24). The quantum yield in solution reaches $48.6 \%$ and a bright emission is observed compared to the nearly completely quenched fluorescence of the parent phosphine (Table 4). The quenching of the emission of 1 was addressed to a PET from the phosphorous lone pair and therefore, oxidation of the $\mathrm{P}(\mathrm{III})$ center prevents the PET and turns on the emission. This effect has already been applied to several phosphines in sensing application of peroxides. ${ }^{[174,175,194]}$ The well investigated disubstituted $\left[9,10-\left((\mathrm{O}) \mathrm{PPh}_{2}\right)-\left(\mathrm{C}_{14} \mathrm{H}_{8}\right)\right]$ revealed a stronger bathochromic shift of the emission wavelength up to $470 \mathrm{~nm}$ and an even higher quantum yield of $75-95 \%{ }^{[179,180]}$ These properties make the phosphoryl anthracene a suitable triplet acceptor in photon upconversion systems via triplet-triplet annihilation. ${ }^{[179,180]}$ The mono substituted derivative 7 seems also to be a suitable candidate in upconversion processes as the emission wavelength is even more in the blue region, which is usually a strongly desired criterium. The quantum yield of nearly $50 \%$ should also be high enough for efficient upconversion via triplet-triplet annihilation.
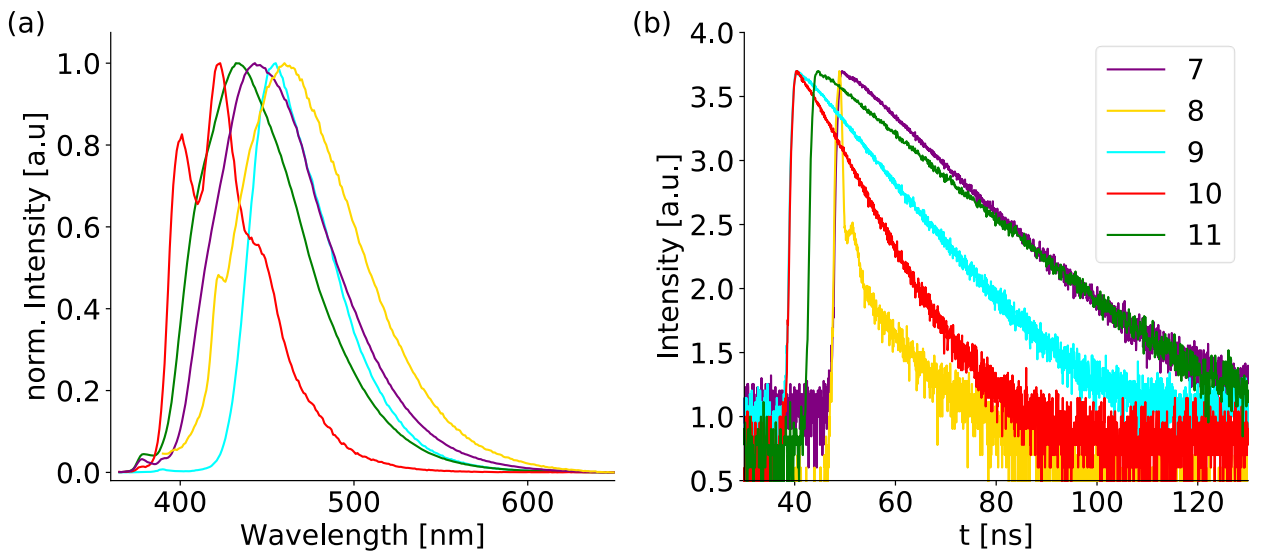

Figure 24. (a) Fluorescence emission spectra of [9-(O)PPh $\left.-\left(\mathrm{C}_{14} \mathrm{H}_{9}\right)\right](7)$, [9-(S)PPh$\left.-\left(\mathrm{C}_{14} \mathrm{H}_{9}\right)\right](8)$, [9--BMes $\left.2^{-}\left(\mathrm{C}_{14} \mathrm{H}_{9}\right)\right](9)$, [9-( $\left.\mathrm{HO}\right) \mathrm{CPh}_{2-}$ $\left.\left(\mathrm{C}_{14} \mathrm{H}_{9}\right)\right](10)$ and $\left[9-(\mathrm{Cl}) \mathrm{SiPh}_{2}-\left(\mathrm{C}_{14} \mathrm{H}_{9}\right)\right](\mathbf{1 1})$ in diluted THF solution $\left(10^{-5} \mathrm{M}, \lambda_{\mathrm{ex}}=350 \mathrm{~nm}\right)$ and $(\mathrm{b})$ fluorescence decay plots of the corresponding solutions. Color code is the same in both figures.

Single atom replacement of the oxygen with sulfur changes the photophysical properties again. As already reported in the previous works for $\left[9-(\mathrm{S}) \mathrm{PPh}_{2}-\left(\mathrm{C}_{14} \mathrm{H}_{9}\right)\right](8)$ and for the disubstituted $\left[9,10-\left((\mathrm{S}) \mathrm{PPh}_{2}\right)_{2}-\left(\mathrm{C}_{14} \mathrm{H}_{8}\right)\right]$ 
the emission in solution is nearly completely quenched. The emission is further red-shifted to around $462 \mathrm{~nm}$ and a structureless emission is obtained. The fluorescence quenching is again ascribed to a PET. The sulfur lone pairs are probably suitable in energy to undergo an electron transfer to the anthracene core, resulting in a rapid quenching after excitation. The thiophosphoryl anthracene reveals a comparable geometry to the prototypes of PET systems, which consist of a fluorophore, spacer and an amine with a lone pair for coordination. ${ }^{[177,195]}$ For $\mathbf{8}$, the P-atom can serve as the spacer and the amine is replaced by the sulfur atom. Upon excitation of the anthracene, an electron transfer from the sulfur to the anthracene HOMO can occur, which prohibits a radiative decay and results in a fluorescence quenching (Figure 25). The oxygen derivative [9-(O) $\left.\mathrm{PPh}_{2}-\left(\mathrm{C}_{14} \mathrm{H}_{9}\right)\right]$ is probably not suitable in energy to undergo a PET and therefore no fluorescence quenching occurs. The drastic change of the emission properties upon single-atom replacement (O vs. S) has been observed for several comparable compounds like thiocarbonyls or thioamides and has already found application in biological imaging. ${ }^{[159,196]}$ The observed fluorescencequenching of the sulfur-homologs was also attributed to the different ability of oxygen and sulfur to undergo a photoinduced-electron transfer.

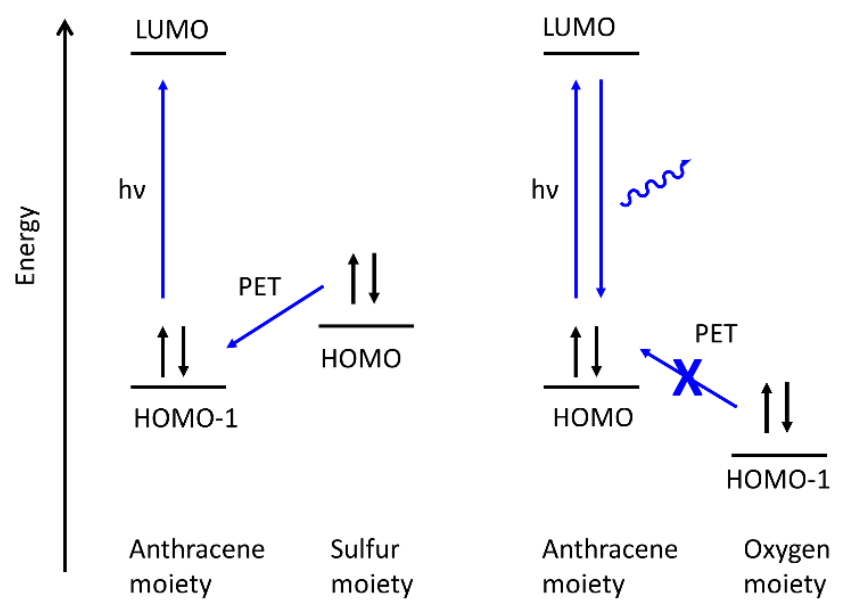

Figure 25. Schematic representation of the photoinduced electron transfer (PET), which is supposed to be responsible for the fluorescence quenching of the thiophosphoryl anthracenes and the strong differences in the emission efficiencies of oxo- and thiophosphoryl anthracenes.

The emission spectrum of $\left[9-(\mathrm{HO}) \mathrm{CPh}_{2}-\left(\mathrm{C}_{14} \mathrm{H}_{9}\right)\right](\mathbf{1 0})$ in THF revealed the most similarities to the pure anthracene fluorophore (Figure 24). A clear vibronic structure is present, and the bathochromic shift is less pronounced compared to the derivatives with a heteroatom directly bond to the anthracene fluorophore. The quantum yield is increased to nearly $60 \%$ and no PET quenching occurs. Even, if the thiol 10 could not be synthesized, a fluorescence quenching through a PET can be expected for this compound, as the sulfur moiety should be more appropriate in energy for an efficient quenching as outlined above.

The chlorosilyl anthracene $\left[9-(\mathrm{Cl}) \mathrm{SiPh}_{2}-\left(\mathrm{C}_{14} \mathrm{H}_{9}\right)\right](\mathbf{1 1})$ reveals also only a slight bathochromic shift in the emission spectrum in solution with a maximum emission wavelength at $432 \mathrm{~nm}$. The vibronic structure is completely absent and a structureless emission is observed. The quantum yield increases near unity to $96.8 \%$ resulting in a bright, blue emission. The diphenylchlorosilyl group seems to be beneficial for 
increasing the radiative decay and suppress non-radiative pathways. A PET from the chlorosilyl moiety is therefore also unlikely. Silyl substituted anthracene derivatives have been reported earlier as highly luminescent chromophores. ${ }^{[197,198]}$ Perturbation of the electronic structure of the anthracene $\pi$-system through $\sigma-\pi$ and $\sigma^{*}-\pi^{*}$ conjugation results in a slight decrease of the HOMO-LUMO gap and a small bathochromic shift. The high quantum yields are attributed to a less effective ISC to the triple state, which is usually the dominating deactivation pathway in anthracene, and a rapid fluorescence decay. Recently, the excellent photophysical properties of silyl anthracenes were used for photon upconversion from nearinfrared to violet light via triplet-triplet annihilation. ${ }^{[199]}$ A 9,10-bis(diisopropylsilyl)anthracene, which also exhibits quantum yields near unity, was used as the triplet acceptor and subsequent emission of violet light.

\begin{tabular}{l} 
Table 4. Photophysical properties of the 9-substituted anthracenyl derivatives 7-11 in diluted \\
THF solution (10 \\
\hline
\end{tabular}

The investigation of differently substituted anthracene derivatives revealed that the photophysical properties are slightly affected by the nature of the substituent in 9-position. The absorption spectra are still dominated by the anthracene fluorophore and the different substituents induce a slight bathochromic shift. Only the dimesitylboryl group with its electron deficient character showed a more complex absorption spectrum with additional transitions, which possess probably charge transfer character. For the emission spectra, more changes are observed for the different derivatives. Introduction of a substituent with a heteroatom bound in 9-position resulted in a structureless emission in solution. Only for the diphenylmethanol group, the typical vibronic structure of anthracene derivatives was observed. Furthermore, the substituents are responsible for bathochromic shifts of varying strength. Again, the emission wavelength of the $\left[9-(\mathrm{HO}) \mathrm{CPh}_{2}-\left(\mathrm{C}_{14} \mathrm{H}_{9}\right)\right]$ derivative without a heteroatom directly bound to the anthracene shows the greatest accordance with the parent anthracene, while the heteroatom substituted derivatives reveal stronger red-shifts. Overall, the maximum emission wavelengths range from around $420 \mathrm{~nm}$ to $460 \mathrm{~nm}$. Fluorescence lifetimes are in the range of a few nanoseconds and indicate the absence of any charge transfer or contribution of a triplet state in the emission processes. The biggest differences in the photophysical properties are found in the quantum yields, which vary from almost 0 (8) to nearly $100 \%$ (11). The effective quenching of the fluorescence of [9-(S)PPh $\left.{ }_{2}-\left(\mathrm{C}_{14} \mathrm{H}_{9}\right)\right]$ was attributed to a PET from the sulfur moiety to the anthracene fluorophore. For oxygen or chlorine, no PET could be identified, resulting in a bright blue emission for these compounds.

The greatest influence of the different substituents at the anthracene on the emission properties, was found in the quantum yields. The spectral shape, lifetimes and emission wavelengths are only moderately affected by the different heteroatoms. The photophysical properties in solution represent the monomeric -58 - 
state without intermolecular interactions. Nevertheless, these slightly changes must be taken into account when considering the impact of the intermolecular interaction on the solid-state luminescence properties.

The change of the emission properties when transitioning from solution to the solid state can often be monitored by increasing the aggregation of the fluorophore in diluted solution. The degree of aggregation can be altered by the addition of increasing amounts of water to the diluted solution at a constant fluorophore concentration. As common organic fluorophores are nearly insoluble in water, the aggregation increases with larger water fraction. Typical AIE luminogens, whose fluorescence quenching is addressed to the intramolecular motion of the substituents, show a strong emission enhancement upon increasing water content due to a restriction of the rotation in highly aggregated solutions. For the oxophosphoryl anthracene [9-(O) $\left.\mathrm{PPh}_{2}-\left(\mathrm{C}_{14} \mathrm{H}_{9}\right)\right](7)$, the opposite behavior is observed. With increasing water fraction, the emission intensity clearly decreases indicating a fluorescence quenching upon aggregation (ACQ) (Figure 26). For the sulfur homolog, an increase in the emission intensity is observed up to a water fraction of $70 \%$. Beyond $70 \%$, the intensity drops to the initial level. As already mentioned, it is not assumed that the fluorescence quenching of $\left[9-(\mathrm{S}) \mathrm{PPh}_{2}-\left(\mathrm{C}_{14} \mathrm{H}_{9}\right)\right](8)$ is caused by an intramolecular motion of the substituent, but rather through a PET. When the rotation of the diphenylgroups would be responsible for the fluorescence quenching, it should have been observed in all previously investigated derivatives. Instead, for comparable derivatives, a clear increased quantum yield compared to anthracene was determined. Nevertheless, a certain degree of flexibility in the molecule is also needed for a PET to guarantee a suitable overlap of the involved orbitals. The slightly increase of emission intensity of $\mathbf{8}$ up to $70 \%$ water content can probably be ascribed to a less efficient PET.
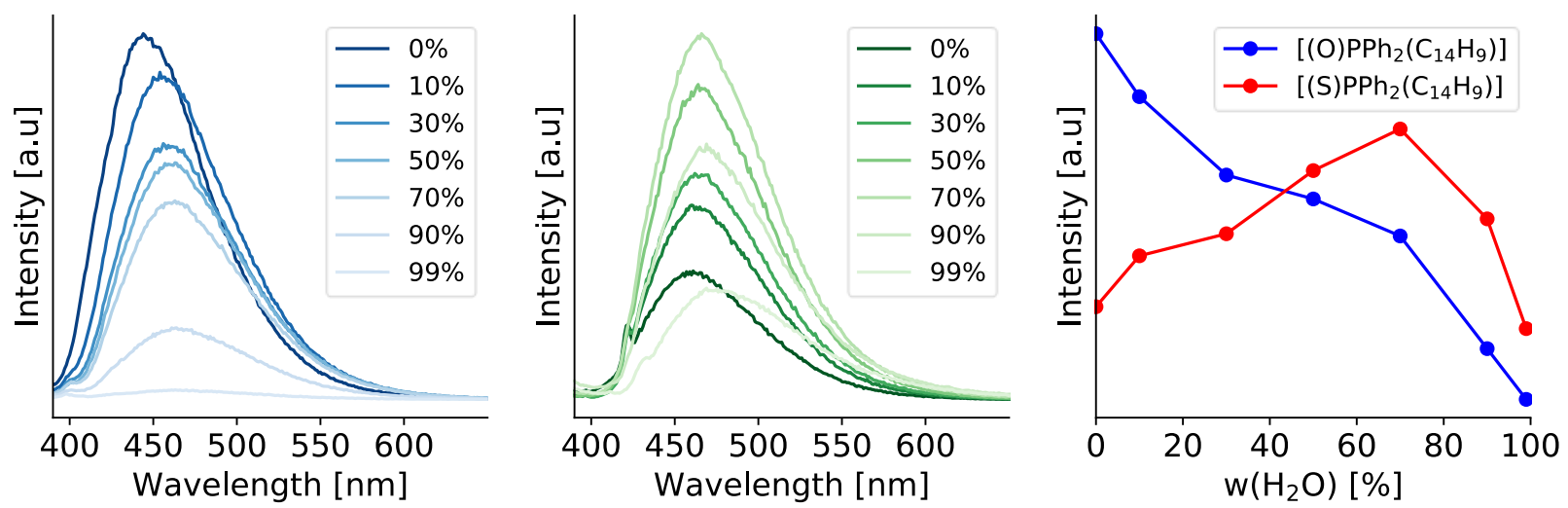

Figure 26. Emission spectra of (a) [9-(O)PPh$\left.-\left(\mathrm{C}_{14} \mathrm{H}_{9}\right)\right](7)$ and (b) [9-(S)PPh $\left.\mathrm{PP}_{2}\left(\mathrm{C}_{14} \mathrm{H}_{9}\right)\right]$ (8) in diluted THF solution with increasing water fraction. Concentration was held constant at $10^{-5} \mathrm{M}$. (c) Plot of the emission intensities against the water fraction.

\section{In the solid-state}

The investigation of the photophysical properties in diluted solution of anthracene derivatives with various diphenyl substituents revealed that the absorption and emission processes are mainly centered on the anthracene moieties. Changes in the emission wavelength are only little and the greatest differences are found in the different quantum yields. Therefore, interactions between the anthracene $\pi$-systems should be able to influence the photophysical properties in the solid-state. The analysis of the solid-state 
structures revealed that only the thiophosphoryl anthracene 8 shows direct $\pi-\pi$ interactions between two anthracenes. A possible influence of these interactions on the photophysical properties in the solid state will be discussed in the next paragraphs.

The investigation of five anthracene derivatives 7-11 with different substituents in 9-position revealed solid-state fluorescence in the range of $430-498 \mathrm{~nm}$. Additionally, for all compounds, a slight bathochromic shift compared to the emission in solution is observed, which is typical for organic chromophores (Figure 27).

(a)

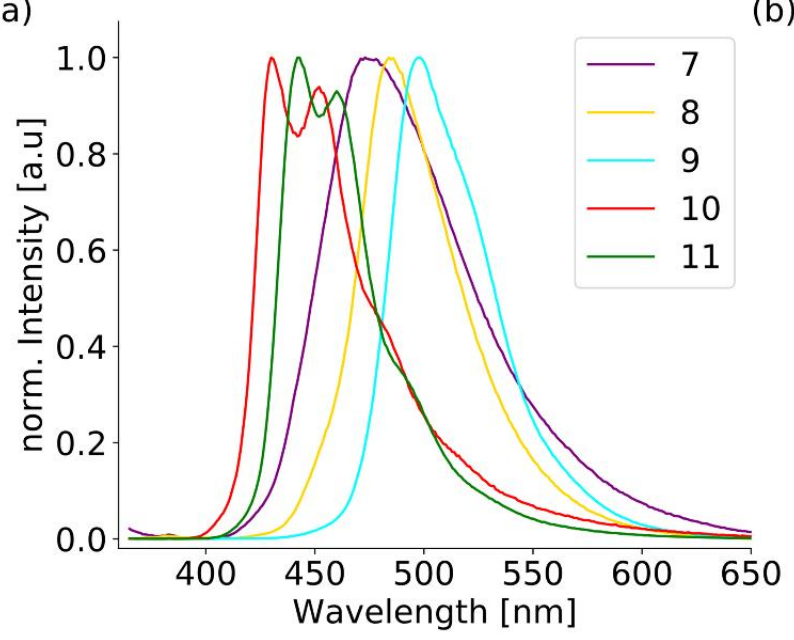

(b)
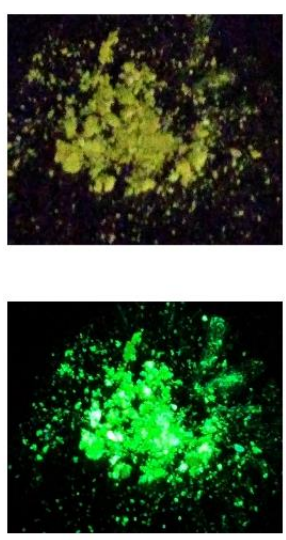

$\left[9-\mathrm{BMes}_{2}-\left(\mathrm{C}_{14} \mathrm{H}_{9}\right)\right](9) \quad\left[9-(\mathrm{Cl}) \mathrm{SiPh}_{2}-\left(\mathrm{C}_{14} \mathrm{H}_{9}\right)\right](\mathbf{1 1})$
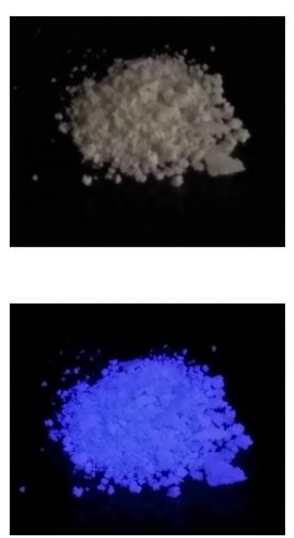

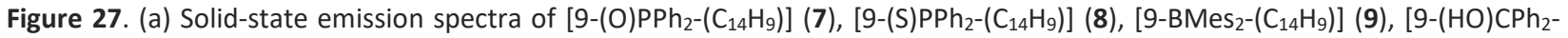
$\left.\left(\mathrm{C}_{14} \mathrm{H}_{9}\right)\right](\mathbf{1 0})$ and $\left[9-(\mathrm{Cl}) \mathrm{SiPh}_{2}-\left(\mathrm{C}_{14} \mathrm{H}_{9}\right)\right](\mathbf{1 1})$ and $(\mathrm{b})$ photographs of bulk samples of $\mathbf{9}$ and $\mathbf{1 1}$ under daylight (top) and under UV irradiation (bottom).

Changes in the emission behavior upon aggregation were initially explored for [9-(O) $\left.\mathrm{PPh}_{2}-\left(\mathrm{C}_{14} \mathrm{H}_{9}\right)\right](7)$ and [9-(S)PPh $\left.-\left(\mathrm{C}_{14} \mathrm{H}_{9}\right)\right](8)$ by increasing the degree of aggregation in diluted THF solutions through addition of different amounts of water. Both compounds revealed a slightly red shift of the emission wavelength at higher water fractions and therefore at higher degree of aggregation. For 7, a clear decrease of the emission intensity at higher water fractions was also observed. Both findings are in accordance with the results of the measurements of the solid sample of 7 . The emission wavelength is about $30 \mathrm{~nm}$ longer and the maximum is located at around $474 \mathrm{~nm}$. The high quantum yield in solution is clearly reduced and 7 undergoes typical aggregation-caused quenching (ACQ), which has also been noted for the disubstituted $\left[9,10-\left((\mathrm{O}) \mathrm{PPh}_{2}\right)_{2}-\left(\mathrm{C}_{14} \mathrm{H}_{8}\right)\right]$ in previous studies. ${ }^{[151]}$ Contrary to the findings of Fei et al., the solid-state emission of the sulfur derivative [9-(S) $\left.\mathrm{PPh}_{2}-\left(\mathrm{C}_{14} \mathrm{H}_{9}\right)\right](8)$ did not increase drastically compared the oxygen homolog. With quantum yields of $4.0 \%$, the intensity is increased compared to the almost completely quenched emission in solution, but an intense emission as found for the host-guest systems of the disubstituted anthracene with quantum yields up to $60 \%$ could not be confirmed. ${ }^{[200]}$ The analysis of the solid-state structure of $\mathbf{8}$ revealed several non-covalent interactions between the anthracene moieties. Besides the $\pi-\pi$ interactions between two anthracene moieties, several $\mathrm{C}-\mathrm{H} \cdots \pi$ interactions in an edgeto-face manner were observed. These various interactions are predestined for opening a plethora of non- 
radiative decay pathways, which could explain the lower quantum yields. Through introduction of the second thiophosphoryl group strong interaction between the anthracene moieties in the disubstituted $\left[9,10-(\mathrm{S}) \mathrm{PPh}_{2}-\left(\mathrm{C}_{14} \mathrm{H}_{8}\right)\right]$ are prohibited, which could be an explanation for the exceptional higher quantum yields of the latter in comparison to $\mathbf{8}$. Furthermore, the bathochromic shift in $\mathbf{8}$ is less pronounced resulting in a blue-green emission at $484 \mathrm{~nm}$ instead of a pure green emission $(520 \mathrm{~nm})$ seen for the disubstituted compound. The tiny red shift of about $22 \mathrm{~nm}$, as well as the still short lifetime of a few nanoseconds, indicate a good comparability to the emission in solution.

Overall, the changes in the photophysical properties observed for $\mathbf{8}$ are very small from solution to the solid-state. A clear influence of the occurring $\pi-\pi$ interactions between the anthracene moieties on the luminescence properties could therefore not be identified. An excimer formation in the solid-state, which should be possible considering the structural motif, is therefore unlikely. Typical spectral features for excimer emission are either also present in solution (broad, structureless emission band and red shift) or not identified (elongated lifetime).

The estimated overlap of the anthracene planes in the dimer was about $21.1 \%$ and is therefore rather small compared to previous reported anthracene derivatives, which were able to undergo an excimer formation in the solid-state. ${ }^{[131,133-135]}$ Increasing the overlap could therefore be a suitable strategy to induce an excimer formation in the solid-state and will be presented in a following chapter (3.1.3).

\begin{tabular}{|c|c|c|c|}
\hline & $\lambda_{\text {em }} / \mathrm{nm}$ & $\Phi_{\mathrm{F}} / \%$ & $\tau / n s$ \\
\hline$\left[9-(\mathrm{O}) \mathrm{PPh}_{2}-\left(\mathrm{C}_{14} \mathrm{H}_{9}\right)\right](7)$ & 474 & 3.9 & 7.0 \\
\hline [9-(S)PPh2-( $\left.\left.\mathrm{C}_{14} \mathrm{H}_{9}\right)\right](8)$ & 484 & 4.0 & $1.4 / 3.2$ \\
\hline [9-BMes2-( $\left.\left.\mathrm{C}_{14} \mathrm{H}_{9}\right)\right](9)$ & 498 & 48.5 & 7.7 \\
\hline$\left[9-(\mathrm{HO}) \mathrm{CPh}_{2}-\left(\mathrm{C}_{14} \mathrm{H}_{9}\right)\right](10)$ & 430 / 452 / 484 & 5.1 & 1.5 \\
\hline$\left[9-(\mathrm{Cl}) \mathrm{SiPh}_{2}-\left(\mathrm{C}_{14} \mathrm{H}_{9}\right)\right](\mathbf{1 1})$ & $442 / 460$ & 30.5 & 6.6 \\
\hline
\end{tabular}

For [9-( $\left.\mathrm{HO}) \mathrm{CPh}_{2}-\left(\mathrm{C}_{14} \mathrm{H}_{9}\right)\right]$ also an ACQ-behavior like [9-(O)PPh $\left.-\left(\mathrm{C}_{14} \mathrm{H}_{9}\right)\right]$ could be identified from the investigation of the emission properties in the solid-state. The intense blue emission in solution is nearly quenched upon aggregation and the quantum yield reduces to $5.1 \%$. The typical vibronic structure of the anthracene chromophore is still present in the emission spectrum and a bathochromic shift of around $30 \mathrm{~nm}$ is observed. As in solution compound $\mathbf{1 0}$ resembles best the emission behavior of the parent anthracene chromophore regarding the spectral shape and emission wavelength. The introduction of heteroatoms as substituents apparently leads to a stronger perturbation of the anthracene $\pi$-system.

The strongest emission in the solid-state is observed for [9-BMes $2_{2}-\left(\mathrm{C}_{14} \mathrm{H}_{9}\right)$ ] (9). The quantum yield of $48.5 \%$ is only slightly reduced compared to the emission in solution (50.7\%). The bathochromic shift of $43 \mathrm{~nm}$ is more pronounced resulting in a bright green emission. Due to their electronic properties, triarylboranes like $\left[9-\right.$ BMes $\left._{2}-\left(\mathrm{C}_{14} \mathrm{H}_{9}\right)\right]$ have been used in OLED-devices already since the late $1990 \mathrm{~s}^{[201,202]}$ The vacant $\mathrm{p}_{\pi^{-}}$ orbital at the boron center enables conjugation with adjacent aryl moieties and allows efficient electron transport. Furthermore, triarylboranes are used as emitting materials due to their efficient emission in the 
solid-state. ${ }^{[201]}$ Their electron accepting character allows tuning of their emission properties such as emission wavelength. Introduction of an electron donating group in conjugation to the boryl group leads to an intramolecular charge transfer. Through variation of the donor groups, the strength of the charge transfer, and therefore the emission wavelength, becomes tunable. ${ }^{[203]}$ This substitution motif should be also achievable for the boryl anthracene 9 through introduction of an electron donating group in the 10position of the anthracene. As these effects are clearly intramolecular, further investigations, would be beyond the scope of this thesis focusing on non-covalent intermolecular interactions. Therefore, no attempts for synthesizing a boryl anthracene with intramolecular charge transfer properties have been made.

The changes of the emission properties between solution and the solid-state are also minor for the chlorosilyl anthracene 11. A slight vibronic structure is found in the emission spectrum in the solid-state with maximum emission wavelengths of 442 and $460 \mathrm{~nm}$, which means a bathochromic shift of around 20 $-30 \mathrm{~nm}$. The remarkable high quantum yield observed in solution is decreased to $30.5 \%$, but a bright blue emission of crystals and powder of $\left[9-(\mathrm{Cl}) \mathrm{SiPh}_{2}-\left(\mathrm{C}_{14} \mathrm{H}_{9}\right)\right]$ can be clearly observed by naked eye after UVirradiation. Especially, the intense blue fluorescence makes the chlorosilyl anthracenes interesting candidates for light-emitting materials in OLEDs. Blue emitting materials, which meets the requirements for OLED-applications, are still rare. ${ }^{[204]}$ Some related silyl anthracenes have already been successful used in such devices. ${ }^{[198,205]}$

\subsubsection{Conclusion}

Analysis of several anthracene derivatives revealed the influence of heteroatoms substituted at the anthracene on the luminescence properties. It could be shown that the emission wavelength varies in a range of around $70 \mathrm{~nm}$. A heteroatom bound to the $\mathrm{C} 9$ atom of the anthracene induces a stronger bathochromic shift compared to the carbon analog. Differences in the emission properties between solution and the solid-state are only rare. Emission wavelengths are only slightly affected upon aggregation as only a moderate bathochromic shift can be observed. Lifetimes also remain nearly unaffected in the range of a few nanoseconds indicating that the emission occurs still from the $\mathrm{S}_{1}$ state without contribution of any charge transfer processes. Stronger distinctions are monitored in the efficiency of the emission. For [9-(O) $\left.\mathrm{PPh}_{2}-\left(\mathrm{C}_{14} \mathrm{H}_{9}\right)\right]$ and [9-( $\left.\left.\mathrm{HO}\right) \mathrm{CPh}_{2}-\left(\mathrm{C}_{14} \mathrm{H}_{9}\right)\right]$, the bright emission in solution is nearly quenched in solution and only low quantum yields are obtained. For these two derivatives, typical ACQ behavior is observed. A decrease in the emission intensity is also found for the chlorosilyl anthracene. The remarkably high quantum yield in solution is reduced to a third to around $30 \%$. For [9-BMes $-\left(\mathrm{C}_{14} \mathrm{H}_{9}\right)$ ], the quantum yield remains nearly constant in solution and in the solid-state. One possible explanation can be the bulkier mesityl groups at the boron center compared to the phenyl groups in the other derivatives. The increased steric demand reduces the intermolecular interactions between the chromophores, which are ascribed to fluorescence quenching. An overall rigid structure is also beneficial for obtaining high quantum yields as intramolecular motion is also referred for fluorescence quenching.

From the investigated compounds, it can be derived that it is in general hard to predict the emission properties and especially the quantum yields of solid-state emitters. A direct transfer from the results $-62-$ 
determined in solution to the solid-state is clearly not possible. Due to the plethora of possible interactions in the solid-state, a variety of non-radiative deactivation pathways must be considered. An influence of the intermolecular interactions on the emission wavelength and the lifetime could not be determined. Even the thiophosphoryl anthracene, which bears the greatest potential for excimer formation through the dimeric structural motif, showed only a slight bathochromic shift of the emission wavelength and an unchanged lifetime. Therefore, no excimer formation could be confirmed. The comparable small overlap of the anthracene planes with around $21 \%$ could be an explanation for the insufficient excimer formation. Therefore, in the following chapter the substituents in the 10 position of [9-(S)PPh$\left.)_{2}\left(\mathrm{C}_{14} \mathrm{H}_{9}\right)\right]$ will be varied in order to tune the electrostatic potential of the anthracene and realize an increased overlapping area of the anthracene dimer, which should increase the possibility for excimer formation. 


\subsubsection{Influence of intra- and intermolecular structural parameters on the solid-state luminescence of thiophosphoryl anthracenes}

The investigation of differently substituted anthracenes revealed some structural and photophysical changes depending on the substituent. Variation of only one or two heteroatoms led to changes in the solid-state structure and luminescence properties. As evaluated, the photophysical changes can be ascribed mainly to the different electronic properties of the substituents. $\pi-\pi$ interactions in a face-to-face manner between the anthracene chromophores were only observed for the thiophosphoryl anthracene, but a strong influence on the emission properties or an excimer formation could not be confirmed.

In the following chapter, further thiophosphoryl derivatives will be prepared in order to vary the occurring $\pi-\pi$ interactions and to investigate the influence on the solid-state luminescence in more detail. Through variation of the substituents size in the 10-position (further denoted ' $R$ '), the resulting packing motif and intermolecular interactions should be changed (Scheme 20). While all other parameters stay unchanged, the derivatives will be comparable among each other regarding their electronic properties. Changes in the solid-state photophysical properties can therefore be assigned to the intermolecular interactions. Therefore, the 9,10-substituted thiophosphoryl anthracenes seem to be a suitable system to investigate the structure-property relationship.

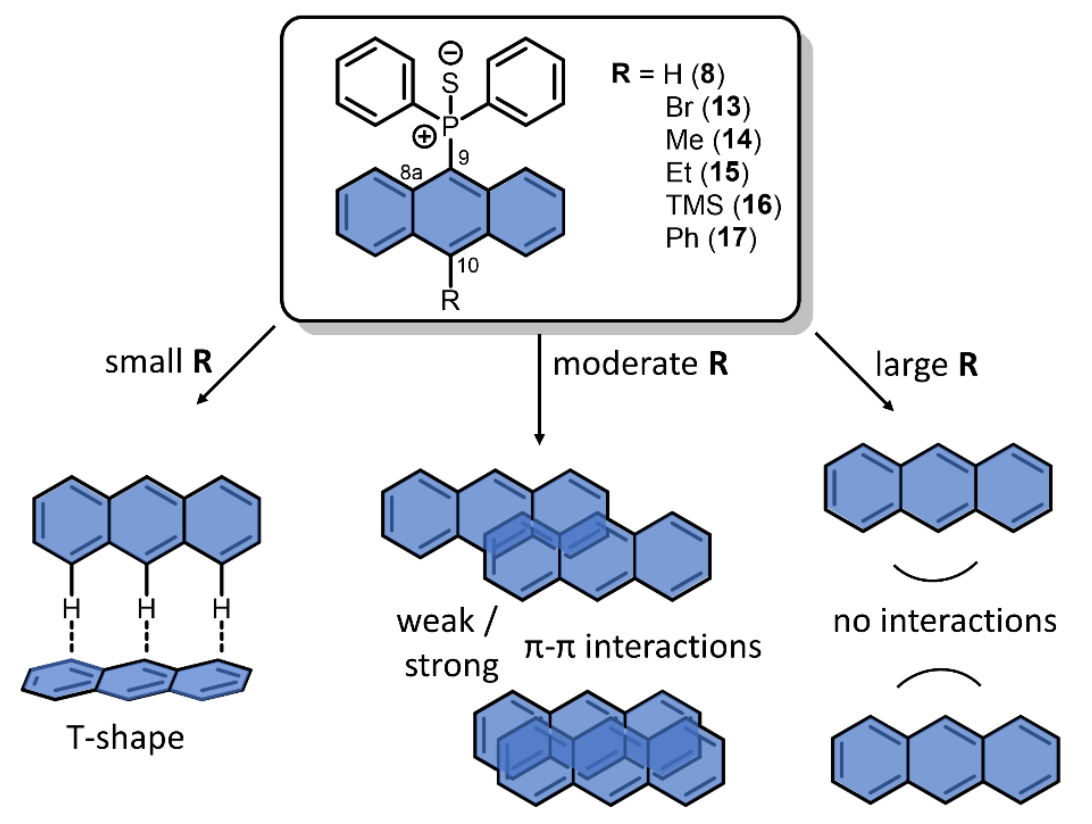

Scheme 20. Applied strategy for the investigation of the influence of the intermolecular interactions on the solid-state luminescence properties. Through variation of the substituent in 10-position of the thiophosphoryl anthracene different packing motifs and intermolecular interactions should be obtained.

\subsubsection{Structural Properties}

The synthesis of the compounds was described before (chapter 3.1.1.1), and single crystals were obtained by recrystallization from common organic solvents like toluene, EtOAc or DCM. Except for 16, all compounds crystallize without lattice solvent. Two derivatives (15 and $\mathbf{1 6}$ ) adopt the triclinic space group 
$P \overline{1}$ as seen earlier for [9-(S)PPh $\left.{ }_{2}-\left(\mathrm{C}_{14} \mathrm{H}_{9}\right)\right](8)$. For two compounds, a monoclinic crystal system in the space group $P 2_{1} / c$ was found (13 and 14 ) and 17 reveals an orthorhombic crystal lattice in the noncentrosymmetric space group $P 2{ }_{1} 2_{1} 2_{1}$. The derivatives with the bromine (13) and methyl-group (14) in the 10-position can be considered as isomorphous as cell parameters differ only slightly (see crystallographic section for details). For the derivatives with the smaller substituents (13-15), two slightly different molecules were found in the asymmetric unit, while for the other compounds only one molecule is present. As already discussed, the sulfur-oxidation leads to a new orientation of the (S) $\mathrm{PPh}_{2}$-substituent compared to the parent phosphines. A nearly orthogonal orientation of the P-S moiety towards the anthracene is also observed for the herein described derivatives. The S-P-C9-C8A torsion angles $\omega$ (see Scheme 20 for numbering) are in a range from $80.84^{\circ}$ to $89.91^{\circ}$ (Table 6) and the phenyl groups are both located above the anthracene, while the sulfur lies below the anthracene plane. Furthermore, the angle $\gamma$ between the phenyl groups (C11-P-C17) decreases and results in a distorted tetrahedral geometry at the phosphorous. The main structural differences besides the substituent in 10-position are found in the folding angle $\alpha$ of the anthracene core, which ranges from $10.7^{\circ}$ to $20.08^{\circ}$. Bond lengths and angles are only slightly affected by the second substituent. As expected, for all derivatives a shortening of the three $\mathrm{C}_{\text {aryl }}-\mathrm{P}$ bonds to around $1.82-1.84 \AA$ compared to the parent phosphines (ca. $1.85 \AA$ ) is observed, which is to some extent more pronounced for the $\mathrm{P}-\mathrm{C} 9$ bond.

Table 6. Structural parameters of thiophosphoryl anthracenes [9-(S)PPh $-10-R-\left(C_{14} H_{8}\right)$ ] with different substituents $R$ in $10-$ position.

\begin{tabular}{lllllll}
\hline $\mathrm{R}=$ & $\mathrm{H}(\mathbf{8})$ & $\mathrm{Br}(\mathbf{1 3})$ & $\mathrm{Me} \mathrm{(14)}$ & $\mathrm{Et} \mathrm{(15)}$ & $\mathrm{TMS}(\mathbf{1 6})$ & $\mathrm{Ph}(\mathbf{1 7})$ \\
\hline space group & $P \overline{1}$ & $P 2_{1} / \mathrm{c}$ & $P 2_{1} / \mathrm{c}$ & $P \overline{1}$ & $P \overline{1}$ & $P 2_{1} 2_{1} 2_{1}$ \\
$\gamma(\mathrm{C} 11-\mathrm{P}-\mathrm{C} 17)^{[\mathrm{a}]} /^{\circ}$ & $101.30(6)$ & $99.10(10)$ & $98.92(6)$ & $98.89(8)$ & $100.64(7)$ & $98.24(13)$ \\
$\omega(\mathrm{S}-\mathrm{P}-\mathrm{C} 9-\mathrm{C} 8 \mathrm{~A})^{[\mathrm{a}]} /^{\circ}$ & $85.38(10)$ & $86.12(18)$ & $85.63(11)$ & $88.45(15)$ & $81.89(12)$ & $86.1(2)$ \\
$\alpha^{[\mathrm{a}]} /^{\circ}$ & $14.27(12)$ & 17.60 & $17.15(10)$ & $15.22(17)$ & $20.08(11)$ & $10.7(2)$ \\
overlap $/ \%^{\mathrm{d}_{\pi-\pi} / \AA}$ & 21.1 & 30.0 & 33.3 & 42.8 & 40.9 & 0 \\
\hline
\end{tabular}

[a] If more than one molecule is present in the asymmetric unit the average value was taken.

Evaluation of the intermolecular interactions and the crystal packing validates the applied strategy as the thiophosphoryl anthracenes adopt different packing motifs. The herringbone motif with strong edge-toface interactions and a T-shape orientation of the anthracenes was only found for the mono substituted derivative 8. As desired, substitution of the hydrogen in 10-position by a larger group prohibited those interactions. The disubstituted compounds with a moderate steric bulk on the 10-position $(\mathbf{1 3}, \mathbf{1 4}, \mathbf{1 5})$ revealed a dimeric motif in their solid-state structure. Two molecules are antiparallelly oriented with an offset face-to-face stacking in a typical distance for $\pi-\pi$ interactions around $3.3-3.4 \AA$ (Figure 28 ). For 13 and 14 , the overlap ratio was estimated to $30-33 \%$ and is significantly increased compared to 8 (21.1\%). A further increase is observed for the bulkier ethyl- and trimethylsilyl substituents with an anthracene overlap of nearly $43 \%$. 
top view

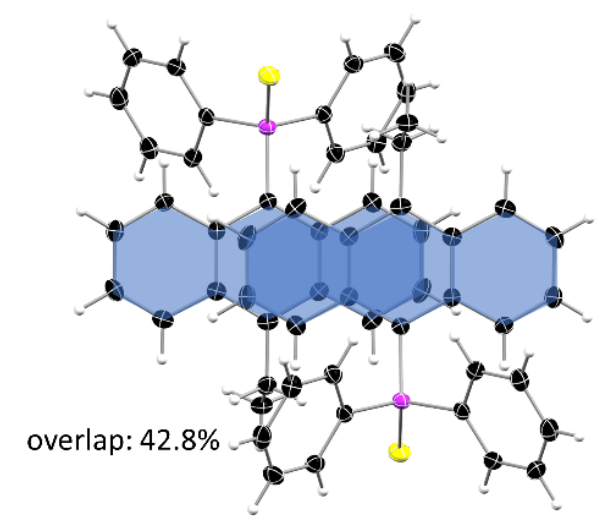

side view

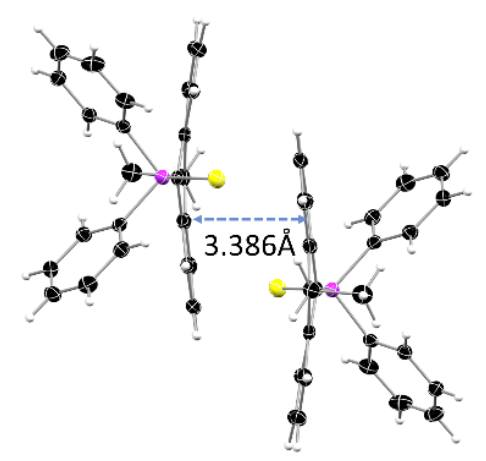

Figure 28. Offset $\pi$-stacking with face-to-face interactions of two adjacent anthracenes in $\left[9-(S) P P h_{2}-10-E t-\left(C_{14} H_{8}\right)\right](15)$. The overlap of the aromatic planes is shown in dark blue and calculated to $42.8 \%$ with a mean distance of $3.386 \AA$ of the anthracenes.

As both structures can be considered isomorphous, the crystal packing for $\mathbf{1 3}$ and $\mathbf{1 4}$ is nearly identical. The anthracene dimers adopt two different orientations regarding the anthracene planes (Figure 29a). For 15, a similar packing motif is observed, but with an apparently less dense packing as the anthracene dimers are more separated from each other (Figure 29b). The $\pi-\pi$ interactions are the dominant intermolecular interactions found for the anthracene moieties. Between the dimers, several $\mathrm{C}-\mathrm{H} \cdots \pi$ interactions in a range of $2.7 \AA$ to $2.9 \AA$ organizing the overall packing can be found.

(a)

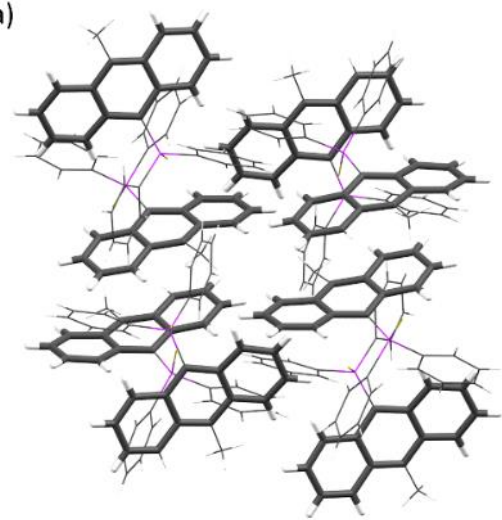

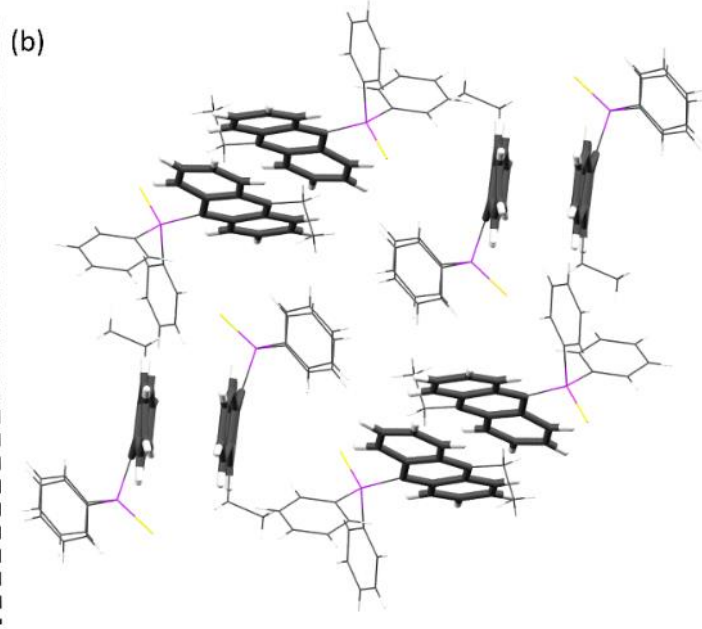

Figure 29. (a) Crystal packing in the solid-state structures of 13 and (b) 15 with the dominant dimeric motif. The anthracene moieties are highlighted in dark-grey. Disorder is omitted for clarity.

In 15, the ethyl group is oriented on the same side as the phenyl groups of the thiophosphoryl substituent, which allows the occurrence of face-to-face interactions between the anthracene $\pi$-systems. A sterically more demanding substituent, which would block both anthracene sites should prevent close contacts of the anthracene planes and reduce the interchromophoric interactions. Surprisingly, the 10-trimethylsilyl substituted derivative $\mathbf{1 6}$ still reveals the dimeric motif with strong face-to-face interactions, even when the corresponding site is occupied by one methyl group. As a consequence, the folding angle $\alpha$ is increased - 66 - 
to the maximum observed value of over $20^{\circ}$ and the Phosphorous and Silicon atoms are moved significantly out of the plane spanned by the central anthracene ring (P: $0.398 \AA$, Si: $0.617 \AA$ ) (Figure 31). Furthermore, the crystal packing of $\mathbf{1 6}$ changes and all anthracene planes are nearly parallelly oriented resulting in a layered structure (Figure 30). The closest distance between two chromophores (except for the face-to-face interactions) is found between two layers to be $7.196 \AA$. Strong interchromophoric interactions, except from $\pi-\pi$ interactions, are therefore negligible. As the structure could not be obtained without co-crystallized DCM, it cannot be excluded that the co-crystallization induces the different packing motif in comparison to 15.
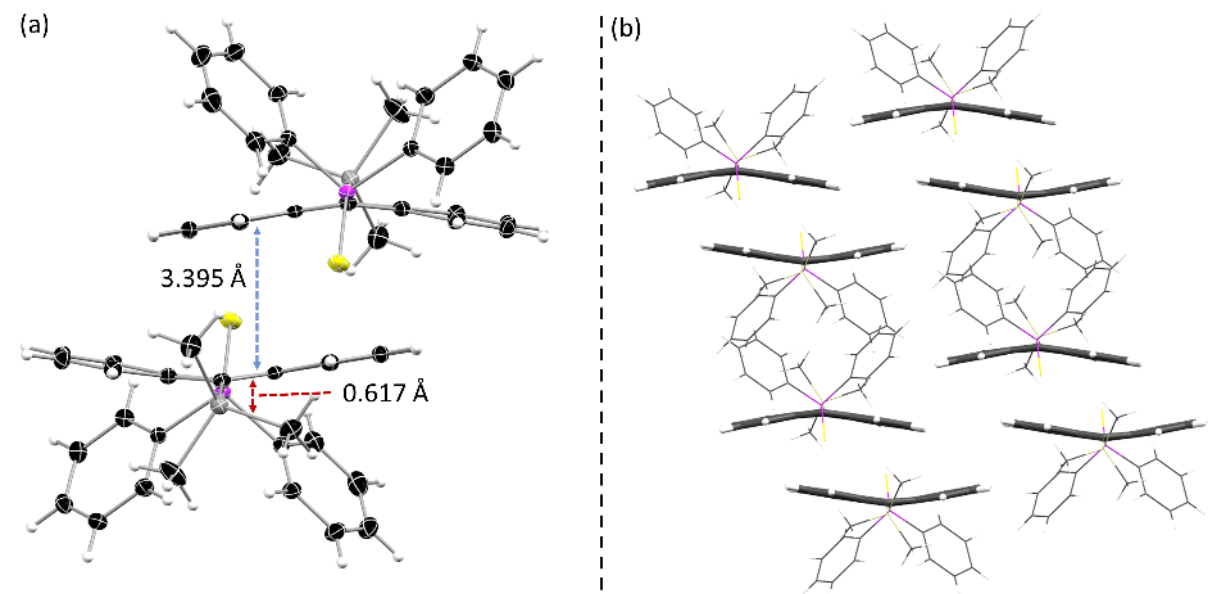

Figure 30. (a) Dimeric motif of [9-(S)PPh $\left.-10-T M S-\left(\mathrm{C}_{14} \mathrm{H}_{8}\right)\right]$ (16) with a strongly bent anthracene plane and $\mathrm{P}$ and $\mathrm{Si}$ atoms pushed out of the anthracene plane. (b) Layered stacking motif, with all anthracenes almost parallelly oriented. Co-crystallized solvent is omitted for clarity.

To obtain the desired packing motif without face-to-face interactions, a phenyl group was introduced in 10-position. Like in the well-known 9-phenylanthracene and 9,10-diphenylanthracene, the phenyl group in $\mathbf{1 7}$ reveals a twisted orientation towards the anthracene scaffold, with an intersecting angle of the aromatic planes of $76.38^{\circ}$ (Figure 31). ${ }^{[206]}$ Therefore, both sites of the anthracene are shielded by the phenyl group and, as desired, no face-to-face interactions in the solid-state structure are found. $\mathrm{C}-\mathrm{H} \cdots \pi$ interactions can be considered as the dominating intermolecular interactions.

(a)
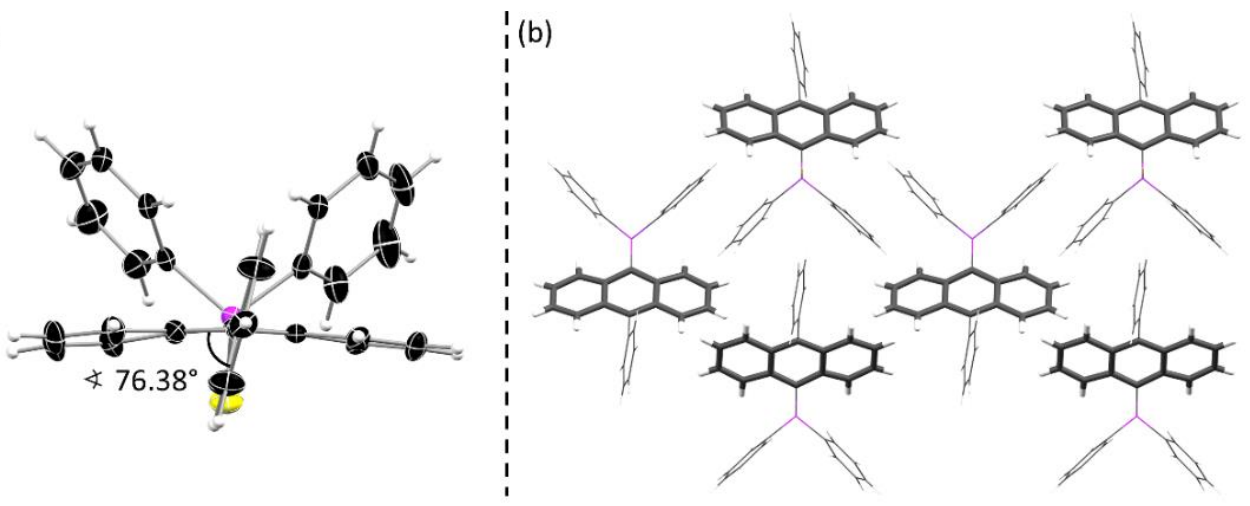

Figure 31. (a) Molecular structure of [9-(S)PPh$\left.-10-\mathrm{Ph}-\left(\mathrm{C}_{14} \mathrm{H}_{8}\right)\right](\mathbf{1 7})$ with the twisted orientation of the phenyl substituent and the anthracene plane. (b) Crystal packing of 17 without any $\pi-\pi$ interactions between the anthracene moieties. 
All six investigated thiophosphoryl anthracenes reveal several similarities in their molecular structure. Sulfur oxidation induces a new orientation of the diphenylphosphoryl substituent with both phenyl groups located at the same anthracene site. The increased steric demand leads to a butterfly bent structure of the anthracene, which was quantified by the folding angle $\alpha$. By variation of the substituent in the 10position, the intramolecular structural features are only slightly changed. The more pronounced differences are found in the different packing motifs. The dimeric motif with strong face-to-face interactions of overlapping anthracenes is dominant in the solid-state of the thiophosphoryl anthracenes. The overlap ratio increases from $21 \%$ to $43 \%$, resulting in $\pi-\pi$ interactions of different strength. Introduction of the bulkier phenyl group with a twisted conformation suppresses the formation of the dimeric motif, and the crystal structure reveals no $\pi-\pi$ interactions between the anthracene chromophores.

From the structural point of view, the thiophosphoryl anthracenes fulfill the requirements for an excimer formation in the solid-state. During this thesis, the group of Yang reported several anthracene derivatives with similar structural motifs, which revealed an efficient excimer emission in the solid-state (Figure 32). ${ }^{[133-135]}$ The reported $\pi-\pi$ distances are comparable and the estimated overlaps are in the range of $26-$ $56 \%$. Due to smaller or more flexible substituents, no deformation of the anthracene core is observed for the reported structures. All compounds reveal a significant bathochromic shift of the emission wavelength in the solid-state compared to derivatives with less or no overlap of the anthracene planes. Furthermore, the emission properties in solution and in the solid-state differ strongly, indicating a sizable influence of the intermolecular interactions. Together with further photophysical properties, such as an increased lifetime and a broadened spectrum, an excimer formation was assumed for these derivatives in the solidstate.
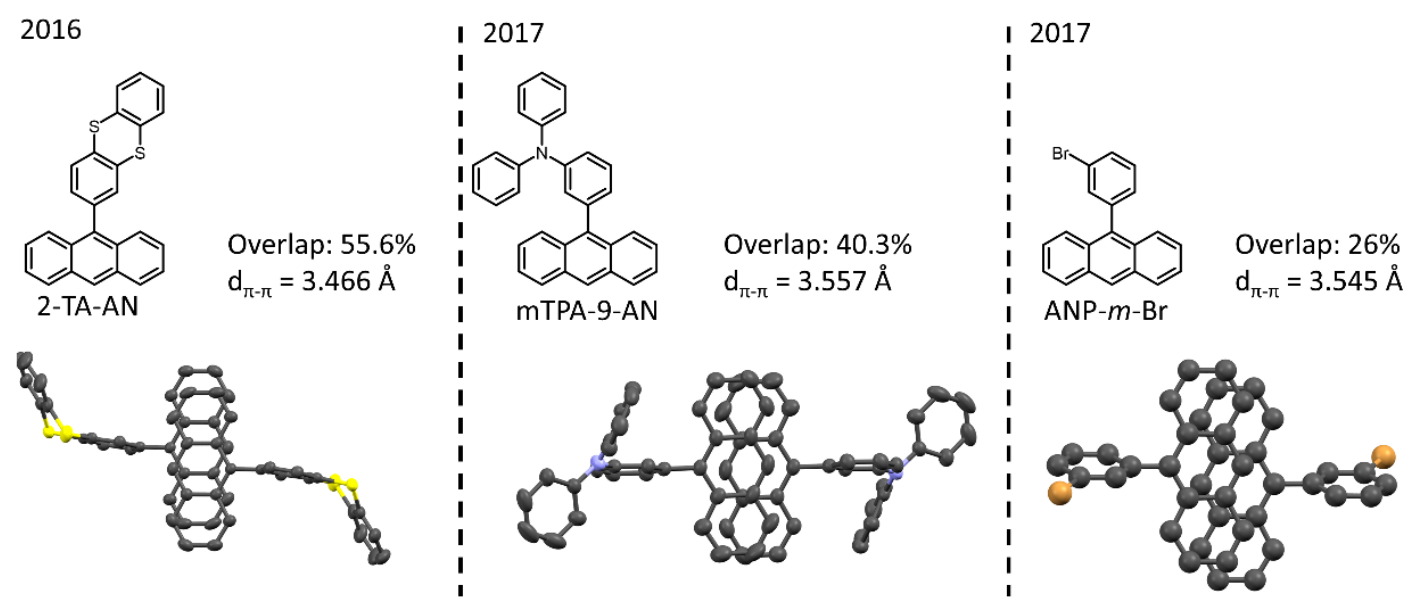

Figure 32. Three recently reported compounds by Yang et al., which reveal an excimer emission in the solid-state due to strong $\pi-\pi$ interactions of the anthracene dimers. Coordinates taken from reference. ${ }^{[133-135]}$

Compared with the reported systems, the synthesized and investigated thiophosphoryl anthracenes are therefore suitable candidates for excimer formation. As the intermolecular interactions and especially the degree of $\pi-\pi$ overlap could be modified through small structural variations, an appropriate system for 
investigation of the structure-property relationship is obtained. The following evaluation of the photophysical properties will determine if an excimer formation can be confirmed and if a dependence of the solid-state luminescence properties on the non-covalent interactions is existing.

\subsubsection{Photophysical Properties}

\section{In solution}

At first, the photophysical properties in diluted solution are examined as they can bear valuable information and represent the monomeric species without any interactions between the chromophores. The emission properties in the solid-state are investigated afterwards to get insight into the photophysical processes upon aggregation and how the intermolecular interactions influence the emission characteristics.

Absorption spectra of the thiophosphoryl anthracenes are similar to the parent phosphines and show a structured emission band around $400 \mathrm{~nm}$, which is assigned to the $S_{0} \rightarrow S_{1}$ transition mainly located on the anthracene moiety and of $\pi-\pi^{*}$ character typical for polyaromatic hydrocarbons (Figure 33). The absorption of the disubstituted derivatives undergoes a small bathochromic shift up to $10 \mathrm{~nm}$ compared to 8, but are still all in a narrow range. Therefore, introduction of the second substituent decreases the HOMO-LUMO gap slightly, independently of the substituent type. As expected, the different substituents in the 10-position reveal no strong influence on the ground-state electronic structure of the molecule.
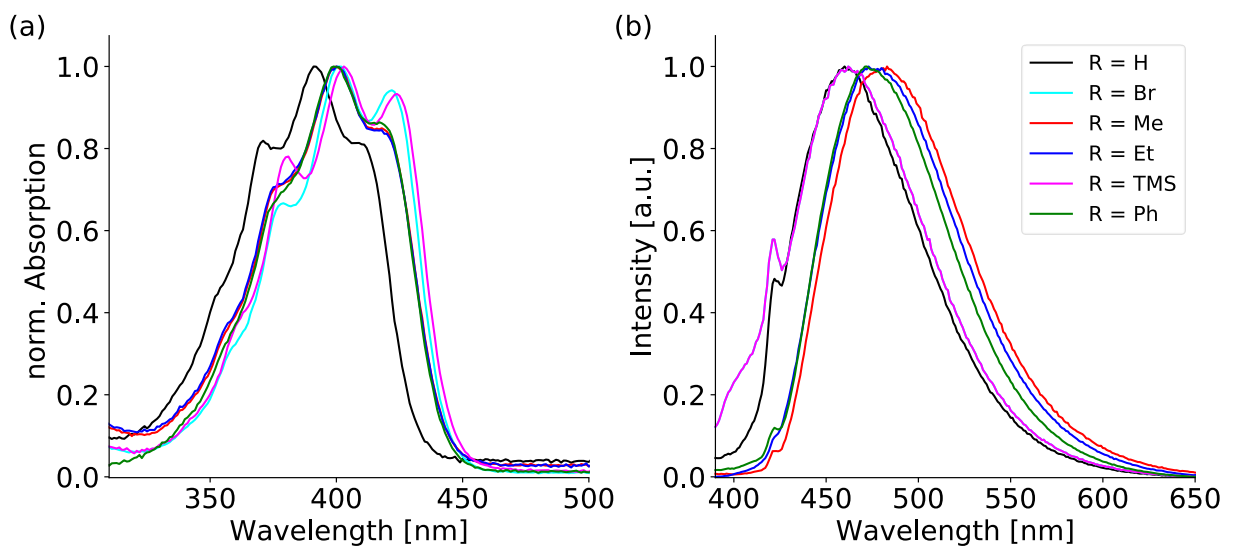

Figure 33. (a) Normalized UV-Vis spectra of [9-(S)PPh $\left.-10-\mathrm{R}-\left(\mathrm{C}_{14} \mathrm{H}_{8}\right)\right]$ in THF $\left(10^{-5} \mathrm{M}\right)$. (b) Normalized emission spectra of [9(S)PPh $\left.-10-R-\left(C_{14} \mathrm{H}_{8}\right)\right]$ in diluted THF solution $\left(\left(10^{-5} \mathrm{M}, \lambda_{\mathrm{ex}}=375 \mathrm{~nm}\right)\right.$. The small peaks at $415 \mathrm{~nm}$ are assigned to scattering of the solvent as it shifts with the excitation wavelength. Color code is the same in both spectra.

All thiophosphoryl anthracenes show only very weak blue-green emission in diluted THF solution (Figure 33 and Table 7). The spectral shape is comparable to mono-substituted 8 and a broad emission band without any vibronic structure is obtained. The small shoulder observed at $415 \mathrm{~nm}$ results from Raman scattering of the solvent as it shifts with the excitation wavelength and is only observable due to the low fluorescence intensity. The emission wavelengths of maximum intensity for $\mathbf{1 3 - 1 7}$ are in a range from 462 to $480 \mathrm{~nm}$, which is a rather strong bathochromic shift for the anthracene chromophore in diluted solution. Comparable derivatives like the compounds reported by Yang et al. reveal maximum emission 
wavelengths at 406/424 nm (2-TA-AN) or 398/418/438 nm (ANP-m-Br) and also show a typical vibronic structure in the emission band.

As already seen for the absorption spectra, the addition of a second substituent in the 10-position has only a minor effect on the emission properties. Even if the emission wavelengths are further red shifted, the involvement of a triplet state or a charge transfer process seems to be unlikely, as that would probably result in an increased fluorescence lifetime.

Table 7. Photophysical Properties of [9-(S)PPh $\left.-10-\mathrm{R}-\left(\mathrm{C}_{14} \mathrm{H}_{8}\right)\right]$ derivatives in diluted THF solutions $\left(10^{-5} \mathrm{M}\right)$. The samples were excited at $\lambda_{\mathrm{ex}}=350$ or $375 \mathrm{~nm}$.

\begin{tabular}{llllll}
\hline & $\boldsymbol{\lambda}_{\text {abs }}\left(\mathbf{S}_{\mathbf{0}}-\mathbf{S}_{\mathbf{1}}\right) / \mathbf{n m}$ & $\boldsymbol{\lambda}_{\text {em }} / \mathbf{n m}$ & $\boldsymbol{\Phi}_{\mathrm{F}} / \%$ & $\boldsymbol{\tau} / \mathbf{n s}$ & FWHM / nm \\
\hline $\mathbf{8}(\mathrm{R}=\mathrm{H})$ & $352 / 371 / 392 / 411$ & 462 & $<1$ & 9.3 & 80 \\
$\mathbf{1 3}(\mathrm{R}=\mathrm{Br})$ & $358 / 378 / 401 / 422$ & 462 & $<1$ & 5.0 & 90 \\
$\mathbf{1 4}(\mathrm{R}=\mathrm{Me})$ & $357 / 376 / 400 / 420$ & 480 & 6.7 & 1.4 & 87 \\
$\mathbf{1 5}(\mathrm{R}=\mathrm{Et})$ & $358 / / 375 / 400 / 421$ & 477 & 7.6 & 1.8 & 87 \\
$\mathbf{1 6}\left(\mathrm{R}=\mathrm{SiMe} \mathrm{S}_{3}\right)$ & $359 / 380 / 403 / 424$ & 462 & $<1$ & 11.7 & 84 \\
$\mathbf{1 7}(\mathrm{R}=\mathrm{Ph})$ & $355 / 373 / 400 / 421$ & 472 & 2.4 & 0.5 & 81 \\
\hline
\end{tabular}

\section{In the solid state}

The fluorescence of the thiophosphoryl derivatives in solution is nearly completely quenched, which was ascribed to the PET from the sulfur moiety. Previous works reported a green emission for the derivatives 8 and $\mathbf{1 4}$ in the solid-state, which was attributed to the strong intermolecular interactions. ${ }^{[152,157]}$ The luminescence properties have only been studied by naked-eye after irradiation with a UV-lamp. Therefore, these two compounds among with the further synthesized derivatives will be investigated regarding their emission wavelengths, quantum yields and fluorescence lifetimes to get more detailed insights about the underlying photophysical processes.

The emission spectra in the solid-state show a broad emission band without any vibrational structure as already seen for the emission in diluted solution. The emission is further red shifted and emission wavelengths with maximum intensity are in a narrow range of $484-506 \mathrm{~nm}$. The small bathochromic shift upon aggregation is typical for organic solid-state emitters. Quantum yields are only partially increased compared to the weak emission in solution. A bright, green emission can clearly be observed by nakedeye after UV-irradiation as reported earlier (Figure 34b), but an extraordinary efficient emission could not be confirmed by the estimation of the quantum yields. The highest quantum yield is found for the phenylsubstituted derivative $\mathbf{1 7}$ with $\mathbf{2 3 . 3} \%$, which is a good value for a comparatively structurally simple compound. 

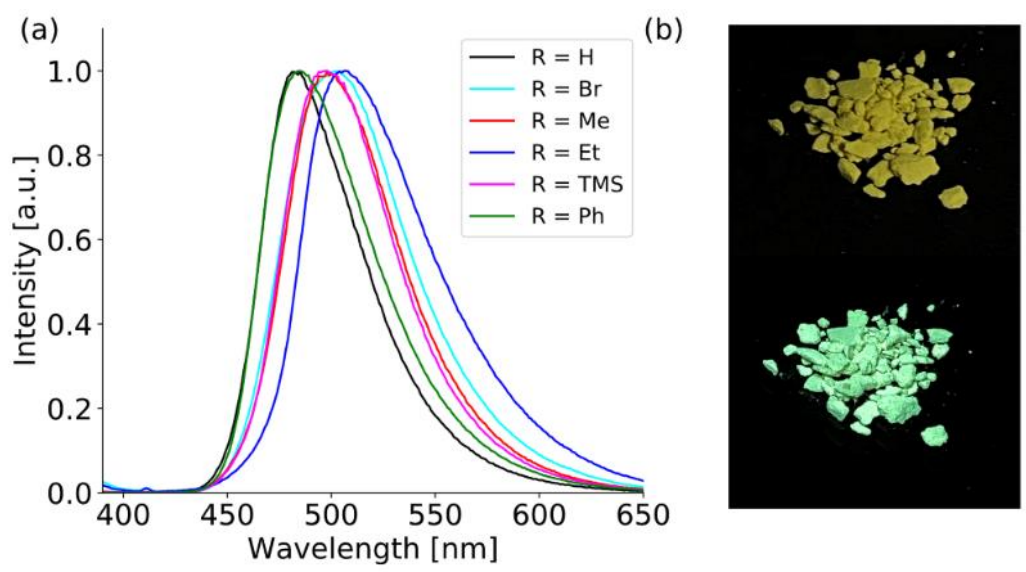

Figure 34. (a) Solid-state emission spectra of thiophosphoryl anthracenes [9-(S)PPh$\left.-10-R-\left(C_{14} \mathrm{H}_{8}\right)\right](8,13-17)\left(\lambda_{\mathrm{ex}}=375 \mathrm{~nm}\right)$. $(\mathrm{b})$ Photographs of powder of [9-(S)PPh $\left.-10-\mathrm{Ph}-\left(\mathrm{C}_{14} \mathrm{H}_{8}\right)\right]$ (17) under daylight (top) and UV-light (bottom).

As evaluated in the structural analysis, the formation of excimers should be possible from the structural point of view for 13-16, which show a dimeric motif with a decent overlap of adjacent anthracenes. The broad, structureless emission in the solid-state, together with the bathochromic shift to around $500 \mathrm{~nm}$, are further indications for an excimer emission process. The anthracene derivatives with excimer emission reported by Yang revealed intense emission at wavelengths around $526 \mathrm{~nm}$ (2-TA-AN), $507 \mathrm{~nm}$ (mTPA-9$\mathrm{AN}$ ) and $526 \mathrm{~nm}$ (ANP- $\mathrm{m}$-Br), which are only slightly further red-shifted compared to the thiophosphoryl anthracenes. ${ }^{[133-135]}$ Lifetimes for these excimers range from 95 ns to 186 ns and are therefore clearly longer than the obtained excited state lifetimes for 13-16. Furthermore, the spectral shape, emission wavelengths and lifetimes of the reported excimers differ significantly from their monomeric species in solution or in a comparable monomeric form in the solid-state. For the thiophosphoryl anthracenes, the changes upon aggregation are rather small. The most characteristic changes, which hint for an excimer emission like spectral broadening, increased lifetimes, and a strong bathochromic shift, could not be affirmed. Moreover, the photophysical properties of the phenyl substituted derivative, which revealed no $\pi-\pi$ interactions, are comparable to 13-16. Again, to confirm an excimer emission, more drastic changes of the emission properties would have been expected.

Table 8. Photophysical data of the thiophosphoryl anthracenes $\mathbf{8}$, and $\mathbf{1 3}$ - $\mathbf{1 7}$ in the solid-state.

\begin{tabular}{lllll}
\hline & $\boldsymbol{\lambda}_{\text {em }} / \mathbf{n m}$ & $\boldsymbol{\Phi}_{\mathrm{F}} / \boldsymbol{\%}$ & $\boldsymbol{\tau} / \mathbf{n s}$ & FWHM / nm \\
\hline $\mathbf{8}(\mathrm{R}=\mathrm{H})$ & 484 & 4.0 & 2.1 & 56 \\
$\mathbf{1 3}(\mathrm{R}=\mathrm{Br})$ & 501 & $<1$ & 1.2 & 70 \\
$\mathbf{1 4}(\mathrm{R}=\mathrm{Me})$ & 495 & 4.7 & 2.4 & 63 \\
$\mathbf{1 5}(\mathrm{R}=\mathrm{Et})$ & 506 & 5.2 & 4.9 & 71 \\
$\mathbf{1 6}(\mathrm{R}=\mathrm{SiMe})$ & 498 & 4.6 & 3.3 & 62 \\
$\mathbf{1 7}(\mathrm{R}=\mathrm{Ph})$ & 485 & 23.3 & 6.4 & 61 \\
\hline
\end{tabular}

\subsubsection{Conclusion}

The investigation of the structural and photophysical properties of the thiophosphoryl anthracenes leads to the conclusion that the blue-green emission in the solid-state is most likely not attributed to an excimer 
emission. Even if the structural requirements with a dimeric structure and a decent overlap of the anthracene planes were given, the analysis of the emission properties could not confirm the excimer formation. The occurring $\pi-\pi$ interactions between the anthracene moieties observed in the structure for 13-16 may lead to a small decrease of the HOMO-LUMO gap, as the obtained emission wavelengths are slightly longer compared to the derivatives $\mathbf{8}$ and $\mathbf{1 7}$ with weak or no anthracene overlap. As the relatively large bathochromic shift for an anthracene-based fluorophore and the structureless emission is also present in solution, these effects are referred to be an intrinsic feature of the thiophosphoryl substituent.

The structural analysis of the thiophosphoryl anthracenes revealed a strong folding of the anthracene core due to the changed orientation of the substituent. Finkelmeier claimed this strong deformation to be influencial on the photophysical properties of $\left[9,10-\left((\mathrm{S}) \mathrm{PPh}_{2}\right)_{2}-\left(\mathrm{C}_{14} \mathrm{H}_{8}\right)\right]$, which revealed an even stronger distortion of the anthracene plane. Recent studies also investigated the influence of structural distortion of conjugated organic molecules. ${ }^{[207]}$ The distortion of the aromatic planes resulted in a perturbation of the overlap of the $\pi$-orbitals, which results in a decreased HOMO-LUMO gap and a bathochromic shift in the absorption spectra. Therefore, the distortion of the anthracene can also be responsible for the bathochromic shift observed in the absorption and emission spectra. For a conclusive confirmation of this hypothesis computational studies or the comparison with a similar non-distorted derivative could be helpful.

\subsubsection{Solid-state emission enhancement by host-guest-complex formation}

\subsubsection{Structural Properties}

During the investigation of the thiophosphoryl anthracenes the ability of co-crystal formation of [9(S)PPh 2 -10-Et- $\left.\left(\mathrm{C}_{14} \mathrm{H}_{8}\right)\right]$ (15) was observed. As summarized in Chapter 1.5, Fei et al. reported on the importance of the co-crystallized toluene for the intense solid-state luminescence of $\left[9,10-\left((\mathrm{S}) \mathrm{PPh}_{2}\right)_{2}\right.$ $\left(\mathrm{C}_{14} \mathrm{H}_{8}\right)$ ]. In following works, further co-crystallized aromatic guests were investigated and ascribed to have a major impact on the emission properties. ${ }^{[151,153,156]}$ In the original publication, the bright emission was ascribed to a T-shaped exciplex between the anthracene and co-crystallized toluene.

The herein described derivative [9-(S)PPh$\left.-10-E t-\left(\mathrm{C}_{14} \mathrm{H}_{8}\right)\right](15)$ also forms co-crystals with small aromatic molecules, which will be described shortly regarding their structural and photophysical properties in the solid-state. Besides the solvent free structure, which was obtained by recrystallization from ethyl acetate or cyclohexane, four co-crystals with co-crystallized benzene (15a), pyridine (15b), toluene (15c) and quinoline (15d), were obtained through crystallization from the corresponding solvent. For the three smaller aromatic guests, nearly identical structural motifs are found. The solvent molecules are located above one outer anthracene ring in a T-shaped manner (Figure 35) and the host/guest ratio is found to be 1:1 in all structures. The aromatic planes of the anthracene and the guest enclose angles from $61^{\circ}$ to $66^{\circ}$. Several $\mathrm{C}-\mathrm{H} \cdots \pi$ interactions in the range of 2.7 to $3.0 \AA$ between the guest and each the anthracene and the phenyl groups fix the position of the guest (Figure 36 ). 

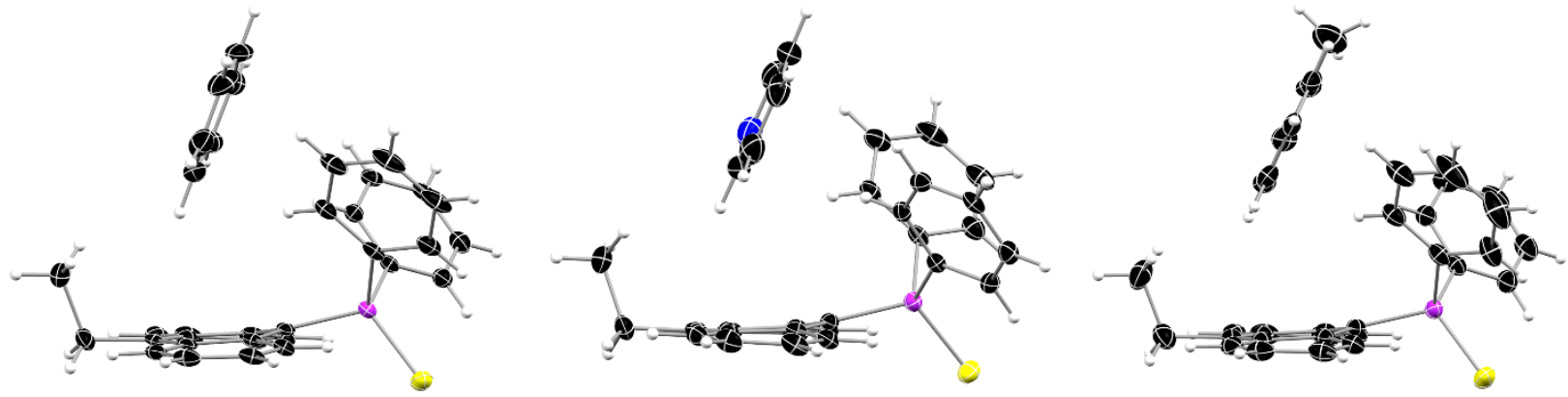

Figure 35. Host-guest complexes of [9-(S)PPh $\left.-10-\mathrm{Et}-\left(\mathrm{C}_{14} \mathrm{H}_{8}\right)\right](15)$ with benzene 15a (left), pyridine $15 \mathrm{~b}$ (middle) and toluene $15 \mathrm{c}$ (right) with a T-shape orientation of the co-crystallized solvent regarding the anthracene plane. Disorder is omitted for clarity.

The three co-crystals resemble the structural motif of the disubstituted $\left[9,10-\left((\mathrm{S}) \mathrm{PPh}_{2}\right)_{2}-\left(\mathrm{C}_{14} \mathrm{H}_{8}\right)\right]$ reported by Fei et al., except that the host/guest ratio was found to be 1:2 in the original system with one toluene located above and one below the anthracene plane. ${ }^{[151]}$ When 15 was crystallized from the bicyclic quinoline, a different structural motif was obtained. The quinoline co-crystallizes in a 1:1 ratio but adopts a different position regarding the anthracene plane compared to $\mathbf{1 5 a - 1 5 c}$. It is now placed on the site with the sulfur atom and not located inside the formed cradle of the ethyl- and phenyl groups (Figure 36). Furthermore, the quinoline is shifted to a more parallel orientation and the enclosed angle of the aromatic planes decreases to $34.46^{\circ}$.

(a)

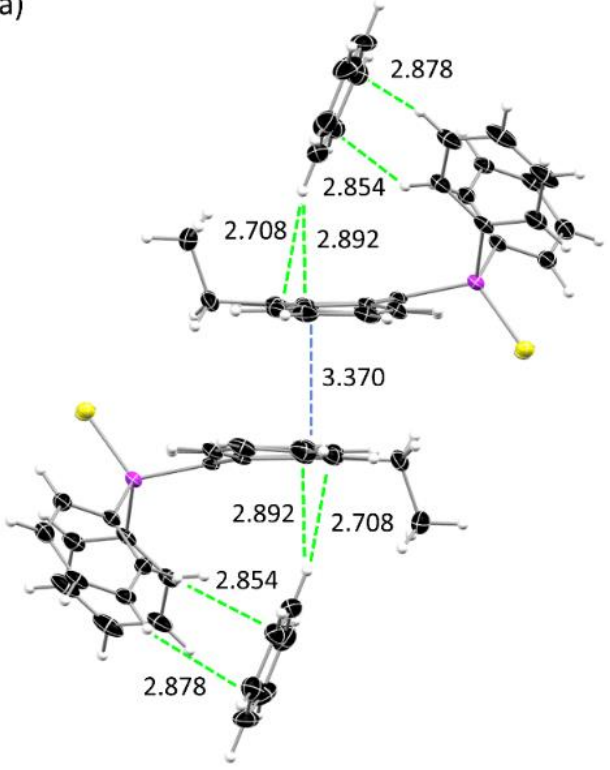

(b)

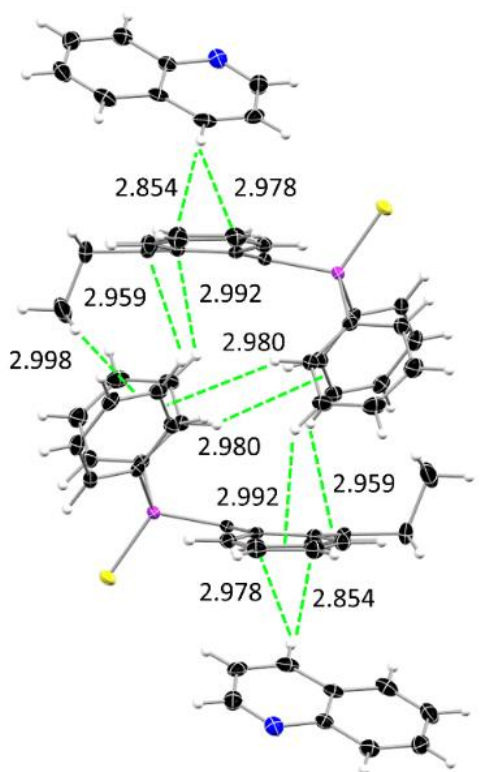

Figure 36. (a) Crystal packing of 15a revealing the dimeric motif with face-to-face interactions (blue) of two adjacent anthracenes and multiple $\mathrm{C}-\mathrm{H} \cdots \pi$ interactions (green) between the host and the co-crystallized benzene. (b) The structure of $15 \mathrm{~d}$ shows a different orientation of the guest and no $\pi-\pi$ interactions anymore.

As expected, bond lengths and angles are not noteworthy affected by the co-crystallization of the solvent. Changes of the structure can be observed in the folding angle $\alpha$ of the anthracene and the S-P-C9-C8A torsion angle $\omega$ (Table 9). The weak interactions of the co-crystallized solvent molecule towards the anthracene in 15a - 15c induce a decrease of the folding angle of around $6-8^{\circ}$ and therefore a more planar 
structure compared to the solvent-free structure is obtained. Interactions of the phenyl groups of the diphenylphosphoryl substituent with the guest leading to a small reorientation of the whole substituent, which can be quantified by slightly smaller S-P-C9-C8A torsion angles. For $\mathbf{1 5 d}$, with the quinoline located on the other anthracene side, only minor changes are observed. The weak host-guest interactions slightly increase the folding angle as the outer anthracene rings bend towards the quinoline. Furthermore, the decrease of the S-P-C9-C8A torsion angle is less pronounced than in 15a-15c.

Table 9: Structural parameters of the co-crystals of [9-(S)PPh $\left.-10-\mathrm{Et}-\left(\mathrm{C}_{14} \mathrm{H}_{8}\right)\right]$.

\begin{tabular}{llllll}
\hline & $\mathbf{1 5}$ & $\mathbf{1 5 a}$ & $\mathbf{1 5 b}$ & $\mathbf{1 5 c}$ & $\mathbf{1 5 d}$ \\
\hline Space group $^{[\mathrm{a}}(\mathrm{C} 11-\mathrm{P}-\mathrm{C} 17) /^{\circ}$ & $P \overline{1}$ & $P \overline{1}$ & $P \overline{1}$ & $P \overline{1}$ & $P 21 / \mathrm{c}$ \\
$\boldsymbol{\omega}^{[\mathrm{a}]}(\mathrm{S}-\mathrm{P}-\mathrm{C}-\mathrm{C}) /^{\circ}$ & $98.89(7)$ & $99.99(8)$ & $100.39(9)$ & $100.37(6)$ & $98.47(5)$ \\
$\alpha^{[\mathrm{a]}} /{ }^{\circ}$ & $88.76(15)$ & $79.43(13)$ & $78.54(15)$ & $80.06(10)$ & $84.19(9)$ \\
Overlap $/ \%$ & $15.22(17)$ & $9.42(11)$ & $7.78(11)$ & $8.05(8)$ & $16.60(9)$ \\
$\mathrm{d}_{\pi-\pi} / \AA$ & 42.8 & 22.2 & 22.9 & 16.9 & 0 \\
\hline
\end{tabular}

[a] If more than one molecule is present in the asymmetric unit the average value was taken.

Besides the discussed intramolecular changes upon co-crystallization, variations in the crystal packing are observed. The dimeric motif of $\mathbf{1 5}$ with an antiparallel overlap of the anthracene planes and distinct $\pi-\pi$ interactions could also be found in the co-crystals 15a-15c (Figure 36). While the $\pi-\pi$ distances between the anthracenes change only slightly, the overlap ratio is clearly reduced to values between $16.9 \%$ and $\mathbf{2 2 . 9} \%$, which is around half as much as found in the structure of $\mathbf{1 5}$ without co-crystallized solvent. The orientation of the anthracene scaffold in the solid-state structure also changes. In the unit cell of $\mathbf{1 5}$ four molecules are present, two of which are arranged in a parallel manner. For 15a-15c, only two molecules of the thiophosphoryl anthracene are found and two molecules of the co-crystallized solvent. All anthracene planes are oriented parallelly to each other, and the anthracene moieties adopt a sheet structure (Figure 37). Again, 15d differs from the three other co-crystals in the crystal packing. The dimeric motif with face-to-face interactions between two adjacent anthracenes has vanished. Instead, interactions of the phosphoryl groups among each other and towards the anthracene core via multiple $\mathrm{C}-\mathrm{H} \cdots \pi$ interactions exist. As already noted, the interactions between the quinoline and the aromatic anthracene plane seem to be less pronounced compared to the T-shape orientation in 15a-15c. 

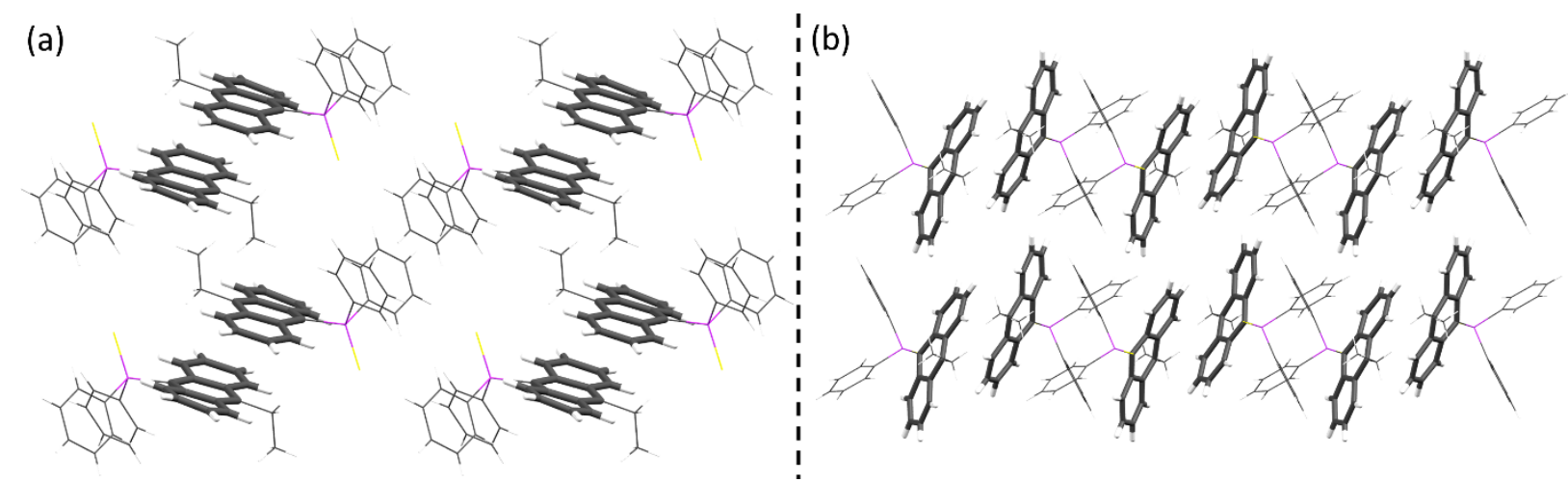

Figure 37. (a) Crystal packing of the thiophosphoryl anthracene moieties in the co-crystal of 15a and (b) 15d. Anthracene scaffolds are highlighted and co-crystallized solvent is omitted for clarity.

As discussed, the structural motifs of the obtained co-crystals are in good agreement with the published system by Fei et al. regarding the position and interactions of the guest towards the anthracene. Therefore, the photophysical analysis of the co-crystals of $\mathbf{1 5}$ could lead to new insights into the role of the guest molecule also for the disubstituted $\left[9,10-\left((\mathrm{S}) \mathrm{PPh}_{2}\right)_{2}\left(\mathrm{C}_{14} \mathrm{H}_{8}\right)\right]$ system. A formation of a T-shaped exciplex, as postulated by Fei et al., should be possible considering the structural motif. Furthermore, the dissimilar packing of $\mathbf{1 5 d}$ allows direct comparison with a different structural motif of the same compound.

Besides varying the substituent in 10-position as described in the previous chapter co-crystallization was recognized as a suitable method for obtaining different structural motifs through the variations of noncovalent interactions, too. Crystallization from different solvents is used to check the ability of co-crystal formation. It is a fast and straightforward method in comparison to the synthesis of novel derivatives. Therefore, co-crystallization of various solvents is a suitable strategy for the thiophosphoryl anthracenes for changing the crystal packing and the occurring intermolecular interactions. The influence on the photophysical properties will be discussed in the following part.

\subsubsection{Photophysical Properties}

To gain insights into the role of the co-crystallized solvent in the thiophosphoryl anthracenes, the emission wavelengths, quantum yields, and lifetimes of the four obtained co-crystals were investigated. The cocrystals were freshly prepared by recrystallization and their successful formation was confirmed via XRD analysis. To obtain a homogenous sample, the co-crystals were slightly grounded and then used for the measurements.

Emission wavelengths are slightly blue-shifted in all co-crystals compared to the solvent-free structure $\mathbf{1 5}$ (Figure 38). The largest hypsochromic shift was observed for the pyridine containing derivative (15b) with an emission maximum at $480 \mathrm{~nm}$. As already discussed, both the intramolecular changes (namely the folding of the anthracene plane) and the intermolecular interactions in form of $\pi$-stacking interactions are considered to induce a bathochromic shift of the emission. The structural analysis revealed that both parameters have decreased for the co-crystals 15a-15c. The folding angle of the anthracene is reduced from $15.62(12)^{\circ}$ in 15 to values between $7.78(11)^{\circ}$ and $9.42(11)^{\circ}(\mathbf{1 5 a}-\mathbf{1 5 c})$. The $\pi-\pi$ interactions between two adjacent anthracenes have decreased, as the overlap ratio of the planes is lowered, and the observed 
offset is enlarged. From these two facts can result the observed blue shift of the emission wavelength upon co-crystallization. For $15 \mathrm{~d}$, the folding angle has increased by $1.5^{\circ}$ and the $\pi-\pi$ interactions have vanished as the packing motif changed. These findings further suggest that both parameters influence the photophysical properties. If the $\pi-\pi$ interactions would be the only factor determining the emission wavelength, a stronger hypsochromic shift, below the $480 \mathrm{~nm}$ observed for 15b, would have been expected as $\pi$-interactions are completely absent. On the other hand, if the distortion of the anthracene plane has the major influence, a stronger bathochromic shift would be expected as the folding angle is slightly increased. Consequently, it is likely that both aspects have to be considered and influence the emission wavelength. A quantification of these factors within the obtained structures is hardly possible and would require a larger data set.

(a)

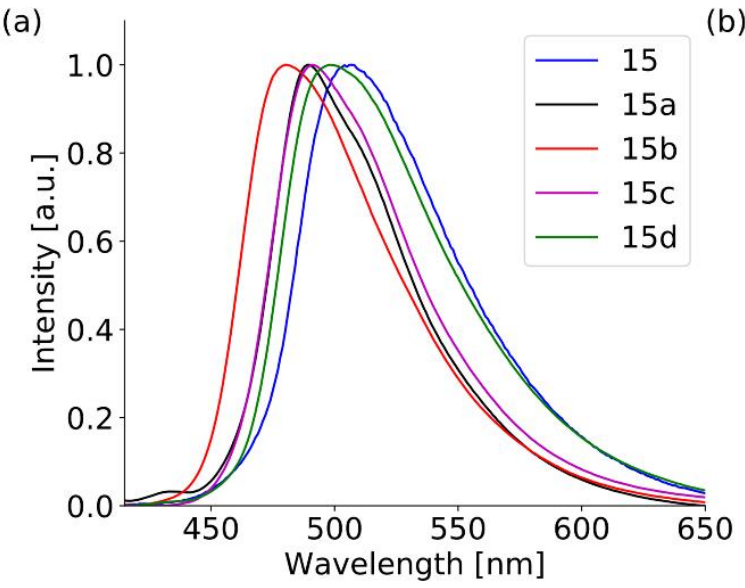

(b)

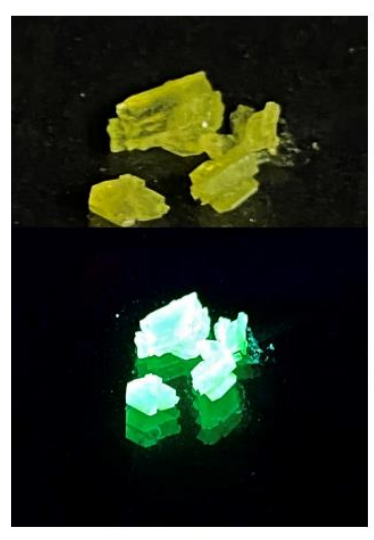

Figure 38. (a) Normalized solid-state emission spectra of 15 and its co-crystals with benzene (15a), pyridine (15b), toluene (15c) and quinoline (15d). (b) Crystals of 15c under daylight (top) and after irradiation with UV-light (bottom).

Considered as whole, the differences in the emission wavelengths are moderate with a maximum variation of $26 \mathrm{~nm}$. The decrease of the emission wavelength upon co-crystallization suggests that the formation of an exciplex upon excitation is not likely. This hypothesis is further supported by the fact that the obtained lifetimes are still in the range of a few nanoseconds and did not increase much (Table 10).

Table 10. Overview of the photophysical data in the solid-state of the co-crystals of [9-(S)PPh$\left.-10-E t-\left(\mathrm{C}_{14} \mathrm{H}_{8}\right)\right]$.

\begin{tabular}{llllll}
\hline & $\boldsymbol{\lambda}_{\max } / \mathbf{n m}$ & $\boldsymbol{\Phi}_{\mathrm{F}}^{\text {[a] }}$ & $\boldsymbol{\tau} / \mathbf{n s}$ & $\mathbf{k}_{\mathbf{r}} / \boldsymbol{\mu s}^{-\mathbf{1}}$ & $\mathbf{k n r}_{\mathbf{n r}} / \boldsymbol{\mu s}^{\mathbf{- 1}}$ \\
\hline $\mathbf{1 5}$ & 506 & 5.2 & 4.9 & 10.6 & 192.7 \\
$\mathbf{1 5 a}$ & 489 & 14.6 & 5.8 & 25.1 & 147.0 \\
$\mathbf{1 5 b}$ & 480 & 22.2 & 5.6 & 39.6 & 138.9 \\
$\mathbf{1 5 c}$ & 494 & 18.2 & 4.9 & 36.5 & 163.9 \\
$\mathbf{1 5 d}$ & 498 & 25.4 & 8.4 & 30.2 & 88.8 \\
\hline
\end{tabular}

[a] as $\Phi_{\mathrm{F}}$ decreases during the measurements the initial value is given (see text for details).

Even if an exciplex mechanism could not be confirmed, a significant increase of the quantum yields of the host-guest complexes could be observed. The quantum yields of the co-crystals are about three to five times higher than for the solvent free compound $\mathbf{1 5}$ and reach values up $25 \%$. Again, several factors must 
be examined to determine which can influence the emission efficiency. Considering that the wavelengths and lifetimes are only slightly affected upon co-crystallization, a strong electronic interaction between the guests and the anthracene system was excluded. Therefore, the induced changes in the crystal packing upon co-crystallization should be more important. As already discussed, the overlap and the occurring $\pi-$ $\pi$ interaction decrease slightly, which results in less interchromophoric interactions. Furthermore, the change of the crystal packing to a sheet structure with all anthracenes oriented parallelly reduces the interactions between single molecules of [9-(S)PPh$\left.-10-E t-\left(\mathrm{C}_{14} \mathrm{H}_{8}\right)\right]$. Accordingly, non-radiative pathways via direct chromophore interactions are less available and the probability for a radiative decay increases.

Additionally, the formation of several $\mathrm{C}-\mathrm{H} \cdots \pi$ interactions upon co-crystallization probably leads to an enhanced rigidity of the anthracene scaffold and of the phenyl rings of the substituent. This rigidity of the structure can suppress or weaken intramolecular vibrations, which are important non-radiative decay pathways in solid-state emitters. In 15a-15c, the T-shape orientation of the co-crystallized solvent and the resulting interactions lead to the higher rigidity, while in $\mathbf{1 5 d}$, the structural motif changes and weak interactions between the phenyl groups of two molecules of $\mathbf{1 5}$ reduce intramolecular motion. The restriction of intramolecular motion (RIM) is in general a promising strategy for increasing the quantum efficiencies in the solid-state and has been applied successfully in typical AIE-luminogens (see chapter 1.4). Calculation of the radiative $\left(k_{r}\right)$ and non-radiative rate-constants $\left(k_{n r}\right)$ from the experimentally obtained quantum yields and lifetimes can further support this hypothesis. The radiative rate constant has clearly increased upon co-crystallization, while $\mathrm{k}_{\mathrm{nr}}$ is reduced (Table 10). Consequently, higher quantum yields are obtained through the formation of co-crystals.

During the reproduction of the measurements of the co-crystals 15a-15d, a slow decrease of the quantum yields over time was observed (Figure 39). When the sample was exposed to eight subsequent measurements, which corresponds to a time scale of around $3 \mathrm{~h}$, a continuous decrease of the obtained quantum yield was measured. The diminution is ascribed to the loss of co-crystallized solvent upon evaporation, and to a slightly increased intramolecular motion in the solid-state. Samples without cocrystallized solvent revealed no decrease of the quantum yield, which should exclude a general photobleaching of the compounds. Nevertheless, the irradiation with UV light during the measurements could facilitate the release of the solvent. 




Figure 39. Slow decrease of the quantum yield of co-crystals $15 \mathrm{a}-\mathbf{1 5 d}$ in subsequent measurements.

The four obtained co-crystals $\mathbf{1 5 a - 1 5 d}$ revealed similar behavior as the already thoroughly investigated cocrystals of the disubstituted $\left[9,10-\left((\mathrm{S}) \mathrm{PPh}_{2}\right)_{2}-\left(\mathrm{C}_{14} \mathrm{H}_{8}\right)\right]$. The solid-state emission was significantly enhanced upon co-crystallization of the aromatic solvent. The photophysical properties argue against an emission from an exciplex state, as postulated for the original system. The increased quantum yields can rather be explained by the RIM-model due to weak $\mathrm{C}-\mathrm{H} \cdots \pi$ interactions between the solvent and the anthracene scaffold (15a-15c) or between the phenyl groups of two thiophosphoryl anthracenes (15d). The change of the crystal packing motif can also influence the efficiency of radiation as co-crystallization leads to a new orientation of the anthracene moieties and therefore to less interchromophoric interactions.

If the type of the co-crystallized solvent has any strong effect on the photophysical properties, could not be evaluated as the data set with four co-crystals is not sufficient. Furthermore, obtaining accurate data is challenging due to the continuous release of the solvent upon sample preparation and the measurement process. Detailed studies on the role of the co-crystallized solvent in the $\left[9,10-\left((\mathrm{S}) \mathrm{PPh}_{2}\right)_{2}\left(\mathrm{C}_{14} \mathrm{H}_{8}\right)\right]$ system, which is more suitable as it co-crystallizes with a lot more solvents, are in progress parallelly to this thesis by Bukala. Up to now, it could not be confirmed that the nature of the co-crystallized solvent has a strong impact on the obtained quantum yields.

\subsubsection{Conclusion}

The investigation of the thiophosphoryl anthracenes lead to valuable insights in the structure-property relationship regarding their photophysical properties. The applied strategy of varying the substituent in the 10-position to obtain different crystal packing motifs and intermolecular interactions was successful. The desired dimeric motif with $\pi-\pi$ interactions between the anthracene moieties was obtained for five compounds with different groups in 10-position. Furthermore, the strength of the interactions between the dimers could be varied through different overlap ratios of the anthracene planes. The obtained structural motif with a decent $\pi$-overlap seemed to fulfill all requirements for an excimer formation in the solid-state. Nevertheless, the analysis of the photophysical properties could not confirm this hypothesis, 
as emission properties of the monomeric and dimeric form are comparable, and as no drastic changes in the photophysical properties, besides a small bathochromic shift, were identified. Instead, the investigation revealed that the emission mostly originates from the monomeric state. The unusual bathochromic shift up to an emission of $500 \mathrm{~nm}$ was therefore attributed to an intramolecular feature of the thiophosphoryl anthracenes. The strong distortion of the anthracene core induced by the steric demand of the substituent could be a factor for the shift of the emission wavelength.

Furthermore, four co-crystals of [9-(S)PPh$\left.-10-E t-\left(\mathrm{C}_{14} \mathrm{H}_{8}\right)\right]$ with a similar behavior as the disubstituted complex $\left[9,10-\left((\mathrm{S}) \mathrm{PPh}_{2}\right)_{2}-\left(\mathrm{C}_{14} \mathrm{H}_{8}\right)\right]$ were investigated. For all co-crystals, the quantum yield increased upon co-crystallization of the aromatic solvent. An exciplex mechanism, which was postulated by Fei et al., could not be confirmed by the photophysical data. Instead, the emission enhancement was ascribed to the restriction of intramolecular motion due to weak $\mathrm{C}-\mathrm{H} \cdots \pi$ interactions between the co-crystallized solvent and the anthracene scaffold. In general, the co-crystallization of different solvents seems to be an effective way for changing the crystal packing and the occurring intermolecular interactions between the fluorophores.

In the next chapter, two positional isomers of $\left[9-(\mathrm{S}) \mathrm{PPh}_{2}-\left(\mathrm{C}_{14} \mathrm{H}_{9}\right)\right](8)$ are presented. Due to a shift of the substituent to the outer anthracene ring, the aromatic plane is less shielded, and a larger area for $\pi$ stacking interactions is available. This could be more suitable for an excimer formation in the solid-state. Furthermore, the induced steric strain by the substituent in the 1- and 2-positions of the anthracene should be reduced as it can probably adopt different conformations compared to [9-(S)PPh$\left.-\left(\mathrm{C}_{14} \mathrm{H}_{9}\right)\right]$. Because of the larger flexibility, a less distorted structure and a more planar anthracene core is expected. Therefore, these two isomers are promising candidates for further insights on the influence of intra- and intermolecular features on the solid-state luminescence. 


\subsection{Structural and photophysical properties of regioisomers of phosphanyl- and thiophosphoryl anthracenes}

Parts of this chapter (especially section 3.2.1) have been published in:

T. Schillmöller, P. N. Ruth, R. Herbst-Irmer, D. Stalke, "Three colour solid-state luminescence from positional isomers of facilely modified thiophosphoranyl anthracenes", Chem. Commun. 2020, 56, 7479-7482.

T. Schillmöller, R. Herbst-Irmer, D. Stalke, "Insights into excimer formation factors from detailed structural and photophysical studies in the solid-state", Adv. Opt. Mater. 2021, 9, 2001814.

The evaluation of the structural and photophysical properties of the anthracene derivatives substituted with the diphenylthiophosphoryl substituent in 9-position revealed only slightly dependency of the emission properties on the solid-state structure. All derivatives revealed only very weak green-blue emission in solution. In comparison to the diluted solution, the emission wavelength was red shifted in the solid-state, resulting in a green emission around $480-510 \mathrm{~nm}$. Furthermore, the spectral shape was very similar for all compounds both in solution and in the solid-state. As even drastic changes in the intermolecular interactions involving the anthracene fluorophore led to only minor influences on the emission properties, it was concluded that the photophysical properties are mainly determined by intramolecular structural and electronic effects induced by the substituents. Excimer emission, which was observed for comparable compounds and attributed to a pairwise stacking of the anthracenes, could not be confirmed, but a small influence of the $\pi-\pi$ interaction on the emission wavelength is likely. The evaluation of the host-guest complexes of $\left[9-(\mathrm{S}) \mathrm{PPh}_{2}-10-\mathrm{Et}-\left(\mathrm{C}_{14} \mathrm{H}_{8}\right)\right](\mathbf{1 5})$ revealed that the packing motifs and resulting interactions between the anthracenes can be changed upon co-crystallization of suitable small aromatic molecules. Furthermore, an enhancement of the solid-state emission could be observed and was ascribed to a more rigid structure and a restriction of internal motion due to weak interactions between the solvent and the thiophosphoryl anthracene. This result already demonstrated nicely that small changes in the solid-state structure can affect the luminescence properties.
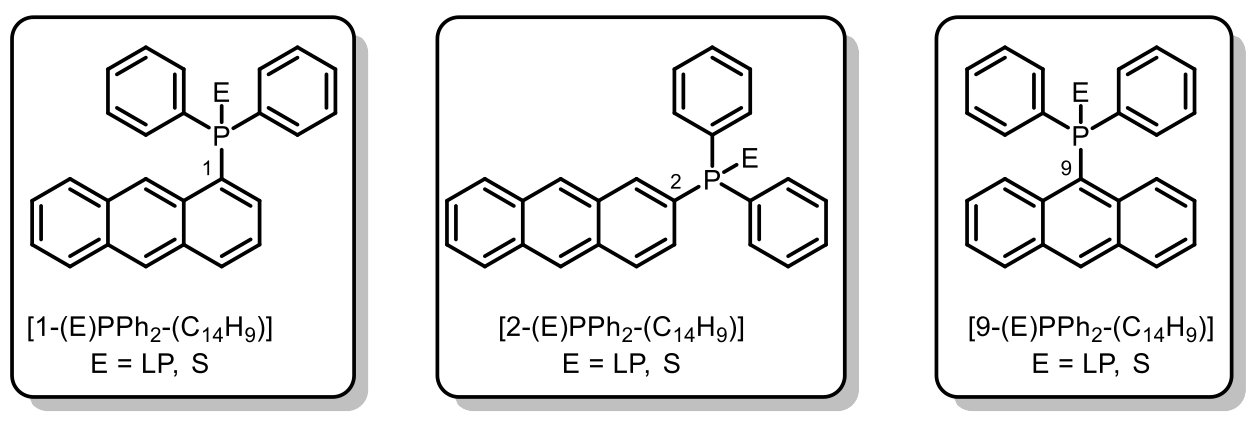

Scheme 21. Three possible positional isomers of $\left[(E) \mathrm{PPh}_{2}-\left(\mathrm{C}_{14} \mathrm{H}_{9}\right)\right](E=L P, S)$ with the substituent in the 1-, 2- and 9-position of the anthracene core.

For further studies, the positional isomers of [9-(S)PPh$\left.-\left(\mathrm{C}_{14} \mathrm{H}_{9}\right)\right](8)$, namely [1-( $\left.\mathrm{S}\right) \mathrm{PPh}_{2}-\left(\mathrm{C}_{14} \mathrm{H}_{9}\right)$ ] and [2(S) $\mathrm{PPh}_{2}-\left(\mathrm{C}_{14} \mathrm{H}_{9}\right)$ ], will be investigated (Scheme 21). Moving the thiophosphoryl group to the outer anthracene ring should lead to a decrease of the steric strain, which is cleary present in the derivatives substituted in 9-position. At the outer ring, the substituent should be more flexible and consequently a 
more planar anthracene core can be expected, which would allow an examination of the role of the anthracene distortion. Additionally, when the substituent is located at the outer ring, less space of the anthracene core is shielded. Thus, it should be available for stronger intermolecular interactions with a larger overlapping area. At the same time, a less protected anthracene plane could lead to a columnar stacking motif, which is usually avoided since it quenches fluorescence. Studies regarding the photophysical properties of anthracene derivatives substituted in 1- or 2-position are rare and therefore valuable insights are expected.

\subsubsection{Evaluation of solid-state excimer formation factors based on anthracene dimers}

As outlined before, (chapter 3.1.1.1) anthracene tends to form 9,10-addition products upon reaction with halogens, which can be transferred into the substitution products upon subsequent elimination of the corresponding hydrogen halides. Therefore, a direct synthesis of 1- and 2-haloanthracene from anthracene via halogenation is not possible and a different reaction pathway has to be adopted.

\subsubsection{Synthesis and structural properties of the diphenylphosphanyl anthracene regioisomers}

Hitherto, the introduction of the diphenylphosphanyl group was done via lithiation of the corresponding bromoanthracene and subsequent quenching with chlorodiphenylphosphine. Therefore, 1bromoanthracene and 2-bromoanthracene appeared to be suitable precursors for the synthesis of the positional isomers of diphenylphosphanyl anthracene.
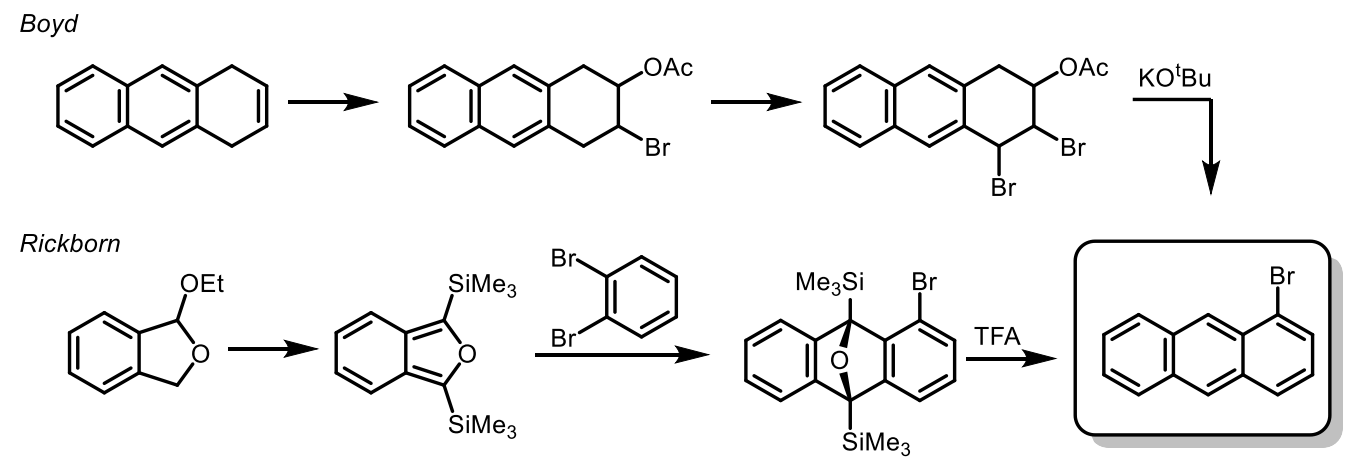

Sandmeyer

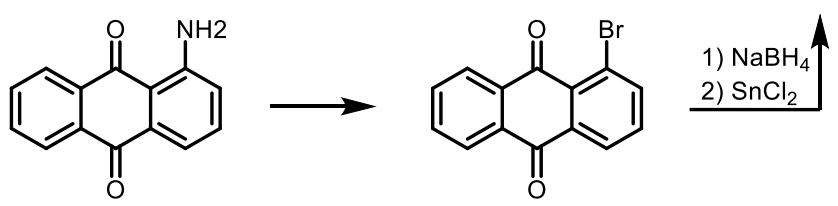

Scheme 22. Different strategies towards the synthesis of 1-bromoanthracene. Starting from 1,4-dihydroanthracene via halogenation and dehydrohalogenation (top). ${ }^{[208]}[4+2]$ Cycloaddition of isobenzofurane with the aryne formed from 1,2dibromobenzene and conversions into the corresponding anthrone and anthracene (middle). ${ }^{[209]}$ Sandmeyer-like reaction followed by reduction of the anthraquinone (bottom). ${ }^{[210]}$

The preparation of 1-bromoanthracene is not trivial and three different methods have been published (Scheme 22). ${ }^{[208-210]}$ Boyd et al. obtained 1-bromoanthracene, when investigating the synthesis of anthracene-1,2-oxide. ${ }^{[208]}$ The intermediate product 2-acetoxy-3,4-dibromo-1,2,3,4-tetrahydroanthracene was synthesized from 1,4-dihydroanthracene. Dehydrobromination in the presence of an alkoxide base resulted in the formation of 1-bromoanthracene instead of the desired anthracene-1,2-oxide with an 
overall yield of $18 \%$. A different method was described by Rickborn et al. in 1985. ${ }^{[209]}$ 1-bromoanthracene was prepared via [4+2] cycloaddition of 1,3-bis(trimethylsilyl)isobenzofurane with an aryne generated in situ from 1,2-dibromobenzene. Treatment of the cycloadduct with trifluoroacetic acid provided the corresponding anthrone (4-Bromo-10H-anthracen-9-one), which was converted into 1-bromoanthracene via reduction with $\mathrm{LiAlH}_{4}$. Starting from the cycloaddition, the product was obtained after three steps with an overall yield of around $43 \%$. The third method is based on a Sandmeyer-like reaction where 1aminoanthraquinone reacts to 1-bromoanthraquinone. Reduction of the anthraquinone with $\mathrm{NaBH}_{4}$ and $\mathrm{SnCl}_{2}$ gave 1-bromoanthracene in an overall yield of $27 \%$. A slightly higher yield (33\%) was obtained with the analog reaction towards 1,4-dibromoanthracene followed by selective substitution of one bromine via lithiation and quenching with aqueous solution.

All three reported methods towards 1-bromoanthracene require several steps of synthesis and purification and furthermore suffer from modest overall yields. Therefore, a different synthetic pathway towards [1-PPh $\left.-\left(\mathrm{C}_{14} \mathrm{H}_{9}\right)\right]$ was considered, starting from 1-chloroanthracene instead of the bromoanthracene.

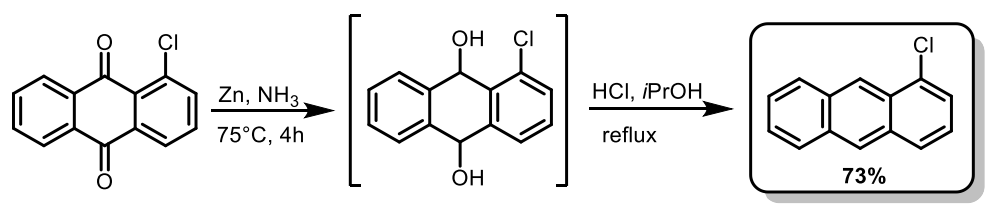

Scheme 23. Reduction of 1-chloroanthraquinone with zinc in aqueous $\mathrm{NH}_{3}$ solution and following dehydration to 1chloroanthracene.

1-Chloroanthracene is prepared from commercially available 1-chloroanthraquinone adopting a procedure for the synthesis of 1,5-dichloroanthracene (Scheme 23). ${ }^{[211]}$ The anthraquinone is reduced with zinc-dust in aqueous ammonia to the 1-chloro-9,10-dihydroanthracene-9,10-diol. Without isolation, the intermediate is dehydrated with hydrochloric acid in refluxing isopropanol. Upon cooling, 1chloroanthracene coagulates as a fluffy, yellow solid in good yields. Further functionalization to more complex monosubstituted derivatives has been barely reported. The few known examples propose nickel or palladium-catalyzed cross-couplings to introduce carbon or nitrogen based substituents. ${ }^{[212]}$ Anthracene derivatives with phosphorous based substituents solely in 1-position are rarely known.

As aryl chlorides, in comparison to aryl bromides, are less reactive in lithium-halogen exchange, a different synthetic route for the introduction of the phosphine was considered. Zorn, Schindlbauer and Hagen described a method for the synthesis of tertiary aromatic and aliphatic phosphines via reaction of aryl/alkyl halides with alkali metal phosphides (Scheme 24). ${ }^{[213]}$ Their first studies were performed with benzyl and naphthyl halides (iodide and bromide), which could be converted to the corresponding phosphines upon reaction with lithium or potassium phosphides in yields up to $86 \%$. Later, an optimized synthesis for aromatic phosphines was established by the same group. Instead of aryl halides, the sulfonic acid of the aromatic compounds was used for the reaction with the alkali metal phosphides. Besides phenyl- and naphthyl derivatives, tertiary anthracenyl phosphines were also synthesized. 


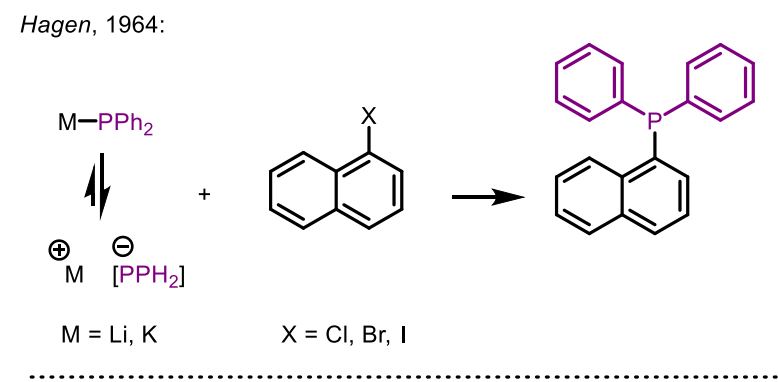

Krüger, 1990:

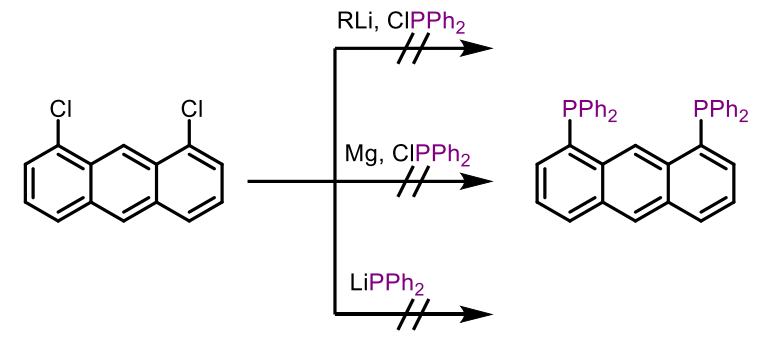

Scheme 24. Synthesis of aromatic tertiary phosphines via alkali metal phosphides (top). Reported synthetic attempts towards 1,8-bis(diphenylphosphanyl) anthracene (bottom). ${ }^{[214]}$

The preparation of the disubstituted 1,8-bis(diphenylphosphanyl) anthracene has been investigated more widely as the compound serves as a ligand for transition metal complexes. However, the reactivity of the mono- and disubstituted derivative should be comparable. In the group of Krüger, the disubstituted anthracene was synthesized by the reaction of anthracene disulfonate with potassium diphenylphosphide under harsh conditions $\left(180^{\circ} \mathrm{C}, 20 \mathrm{~h}\right) \cdot{ }^{[215]}$ Lithiation of 1,8-dichloroanthracene as well as the transfer into a Grignard compound were not successful before (Scheme 24). Also, nucleophilic substitution of the chlorides with lithium diphenylphosphide gave only poor yields. An optimized synthesis was reported seven years later, when the target compound was obtained in higher yields and under milder conditions from 1,8-fluoroanthracene via substitution with potassium diphenylphosphide. ${ }^{[214]}$

The method of choice in this work, for the synthesis of [1-PPh $\left.-\left(\mathrm{C}_{14} \mathrm{H}_{9}\right)\right](\mathbf{1 8})$, is also a nucleophilic substitution with an alkali metal phosphide. In contrast, to the reported poor yields by Krüger et al. for the 1,8-substituted derivative, the introduction of only one diphenylphosphine group was successful via this route. A solution of 1-chloroanthracene in THF was treated with one equivalent of a lithium diphenylphosphide solution in THF (Scheme 25). The target compound was obtained by precipitation through addition of water and subsequent workup.

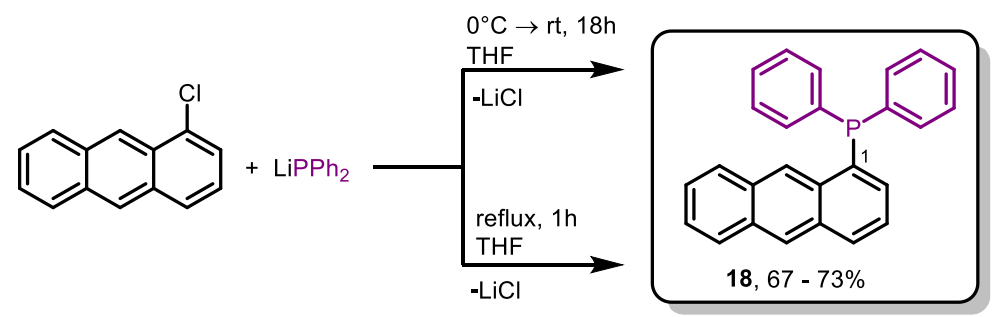

Scheme 25.Synthesis of 1-diphenylphosphanyl anthracene [1-PPh $-\left(\mathrm{C}_{14} \mathrm{H}_{9}\right)$ ] (18) via nucleophilic substitution of 1chloroanthracene with lithiumdiphenyl phosphide. 
The reaction at ambient temperature for $5 \mathrm{~h}$ gave a moderate yield of the product. Higher yields up to $73 \%$ were obtained when the reaction time is extended to $18 \mathrm{~h}$. Refluxing the mixture for $1-3 \mathrm{~h}$ also gave the compound in similar yield and purity. The ${ }^{1} \mathrm{H}-\mathrm{NMR}$ and ${ }^{31} \mathrm{P}-\mathrm{NMR}$ spectra of the crude product revealed only minor side products, which could be removed through recrystallization from DCM or toluene. The ${ }^{31} \mathrm{P}$ NMR signal was slightly downfield shifted to $-14.1 \mathrm{ppm}$ when compared to the previous described compound with the substituent in 9-position of the anthracene. The synthesis and isolation of $\left[1-\mathrm{PPh}_{2}\right.$ $\left(\mathrm{C}_{14} \mathrm{H}_{9}\right)$ ] (18) was straightforward, and it could not be evaluated, why the similar reported synthesis by Krüger et al. was not successful.

Crystallization of $\mathbf{1 8}$ from toluene afforded crystals suitable for solid-state structure determination via XRD analysis. The compound crystallizes in the triclinic space group $P \overline{1}$ with one molecule in the asymmetric unit. Bond lengths and angles of the anthracene and the phenyl rings are as expected and comparable to the prior discussed phosphanyl anthracenes. The C1-P bond is slightly shortened with $1.843 \AA$ compared to the C9-P bond in the regioisomer [9-PPh $\left.-\left(\mathrm{C}_{14} \mathrm{H}_{9}\right)\right](1)(1.851 \AA)$. As expected, the diphenylphosphine group adopts a different orientation regarding the anthracene plane. The lone pair is not located inside the aromatic plane anymore but adopts a more orthogonal position with a torsion angle of the lone pair towards the anthracene plane of roughly $60^{\circ}$ (Figure 40). The phenyl groups reorientate away from the anthracene to minimize the steric strain. As for the previously described phosphines a nearly planar anthracene core was obtained $\left(\alpha=3.52^{\circ}\right)$ and no face-to-face interactions between the anthracenes could be observed. The crystal packing resembles a sheet structure regarding the anthracene, with all anthracene scaffolds oriented in parallel.
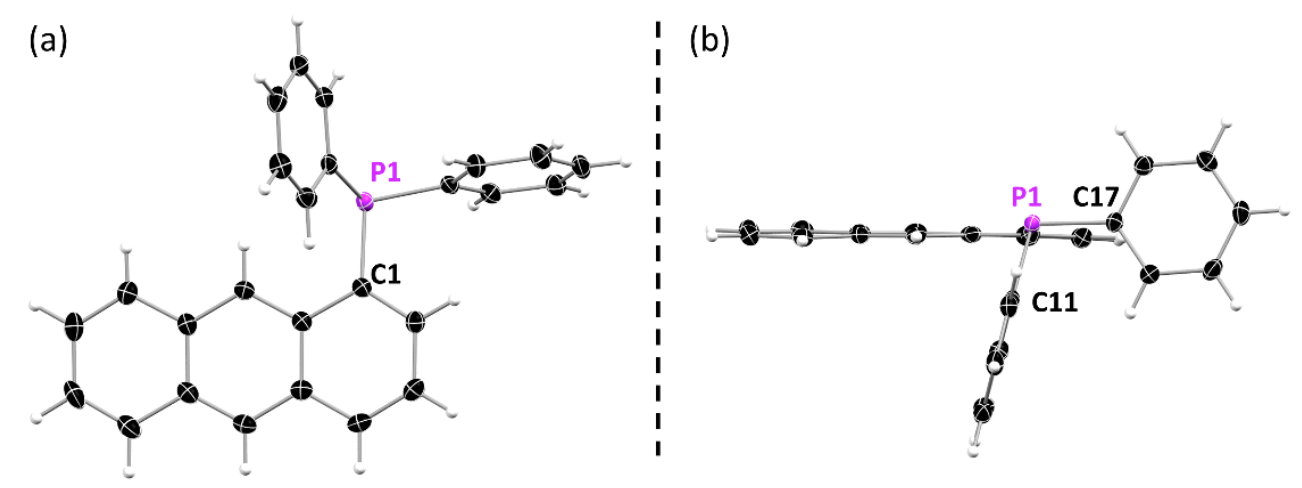

Figure 40.(a) Front view of the solid-state structure of [1-PPh $\left.-\left(\mathrm{C}_{14} \mathrm{H}_{9}\right)\right]$ (18). (b) Side view of 18 revealing the planar anthracene core and changed orientation of the substituent compared to the 9-substitued isomer.

To obtain the third positional isomer, the anthracene core needs to be substituted in the 2-position. Therefore, 2-bromoanthracene seems suitable as a precursor for the introduction of the phosphanyl group. Substitution of the bromide is accessible via lithium-halogen exchange and subsequent salt elimination as described earlier. Synthesis of 2-bromoanthracene was reported earlier in several works and seems to be more promising in comparison to the synthesis of 1-bromoanthracene. ${ }^{[216]}$ The reported procedures were also adopted during this work. 2-Aminoanthraquinone was converted into the diazonium salt with tert-butyl nitrite in acetonitrile at $0{ }^{\circ} \mathrm{C}$ (Scheme 26). In the following Sandmeyer-like reaction a 
stochiometric amount of $\mathrm{CuBr}_{2}$ was added and the 2-bromoanthraquinone obtained after work-up in moderate yields. Reduction of the anthraquinone with zinc powder adopting the procedure for 1chloroanthraquinone failed and led to the formation of unsubstituted anthracene upon debromination. A sequence of stepwise reduction and dehydration was more convenient. In the first step 2bromoanthraquinone is reduced to the dihydroxyanthracene with $\mathrm{NaBH}_{4}$. Dehydration in conc. $\mathrm{HCl}$ yielded the 2-bromoanthrone. Another cycle of reduction and dehydration afforded the 2-bromoanthracene in only modest yields of $24 \%$.

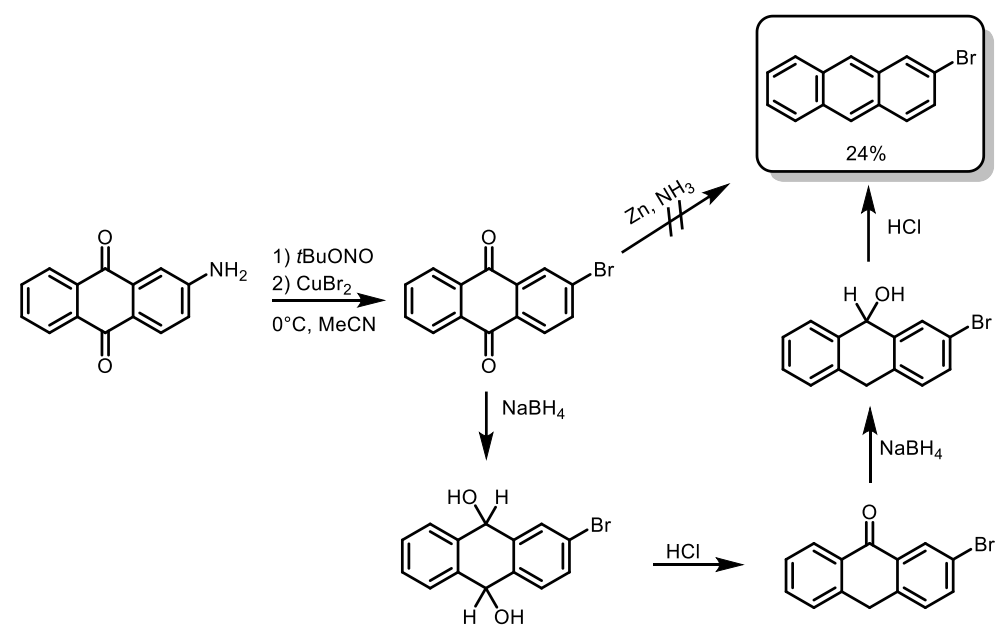

Scheme 26. Synthesis of 2-bromoanthracene via conversion of 2-aminoanthraquinone to 2-bromoanthracene in a Sandmeyerlike reaction and following stepwise reduction.

Lithiation of 2-bromoanthracene and following reaction with chlorodiphenylphosphine afforded the isomer [2- $\left.\mathrm{PPh}_{2}-\left(\mathrm{C}_{14} \mathrm{H}_{9}\right)\right]$ (21) in moderate yields (Scheme 27). Purification of the crude product was achieved by recrystallization from toluene. The ${ }^{31} \mathrm{P}-\mathrm{NMR}$ resonance of the phosphine is located at $-4.4 \mathrm{ppm}$ and therefore further downfield shifted compared to the two previously investigated isomers.

Reaction of 2-bromoanthracene with alkali metal phosphides $\mathrm{MPPh}_{2}(\mathrm{M}=\mathrm{Li}, \mathrm{K})$ was not straightforward as observed for 1-chloroanthracene before (Scheme 27). After aqueous workup the ${ }^{31} \mathrm{P}-\mathrm{NMR}$ spectrum revealed besides the resonance of the desired product, a second signal of a side-product around -14 ppm, which could hint for an isomerization to the 1-substituted phosphine. The isolation and identification of the product mixture was not successful and not further investigated as the reaction via $n$ BuLi already afforded the desired compound in sufficient yield and purity.

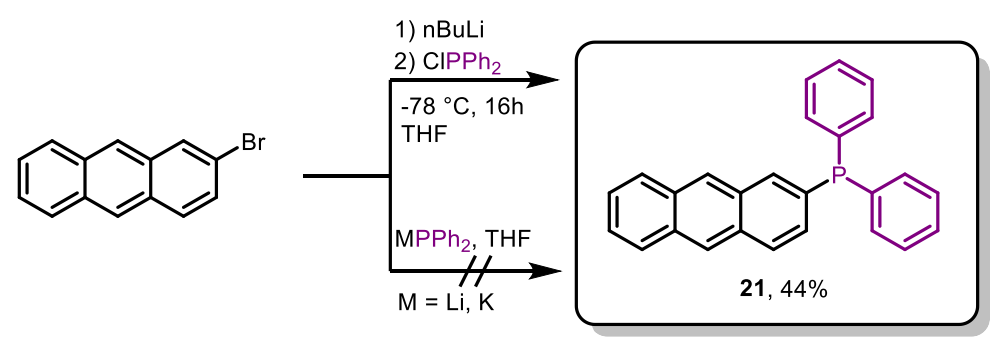

Scheme 27. Two investigated synthetic pathways towards [2-PPh $\left.-\left(\mathrm{C}_{14} \mathrm{H}_{9}\right)\right](21)$ starting from 2-bromoanthracene. 
Obtaining suitable crystals for structure determination was surprisingly challenging, as recrystallization from common organic solvents led to only microcrystalline solids. Only slow diffusion of $n$-hexane into a concentrated solution of $\mathbf{2 1}$ in benzene afforded crystals of sufficient quality. [2-PPh $-\left(\mathrm{C}_{14} \mathrm{H}_{9}\right)$ ] crystallizes in a monoclinic crystal system in the space group $\mathrm{C2} / \mathrm{c}$ with one molecule in the asymmetric unit. The $\mathrm{C2}-$ $P$ bond is again slightly shortened ( $1.838 \AA$ ) compared to the other two positional isomers. As anticipated, the phosphanyl substituent rotated away from the anthracene, and each phenyl group is located above and below the anthracene plane, respectively (Figure 41). The conformation of the substituent assumes that the lone pair is not located completely inside the anthracene plane as seen for the 9-substituted derivative, but rather adopts a slight torsion with the anthracene of roughly $30^{\circ}$. The different distances of C11 and C17 towards the anthracene plane (C11: $0.88 \AA$, C17: $1.81 \AA$ ) support this assumption. For [9$\mathrm{PPh}_{2}-\left(\mathrm{C}_{14} \mathrm{H}_{9}\right)$ ], the corresponding distances are in a narrow range of $1.35-1.53 \AA$, and the tetrahedral geometry around the phosphorous predicts that the lone pair is located nearly inside the anthracene plane.
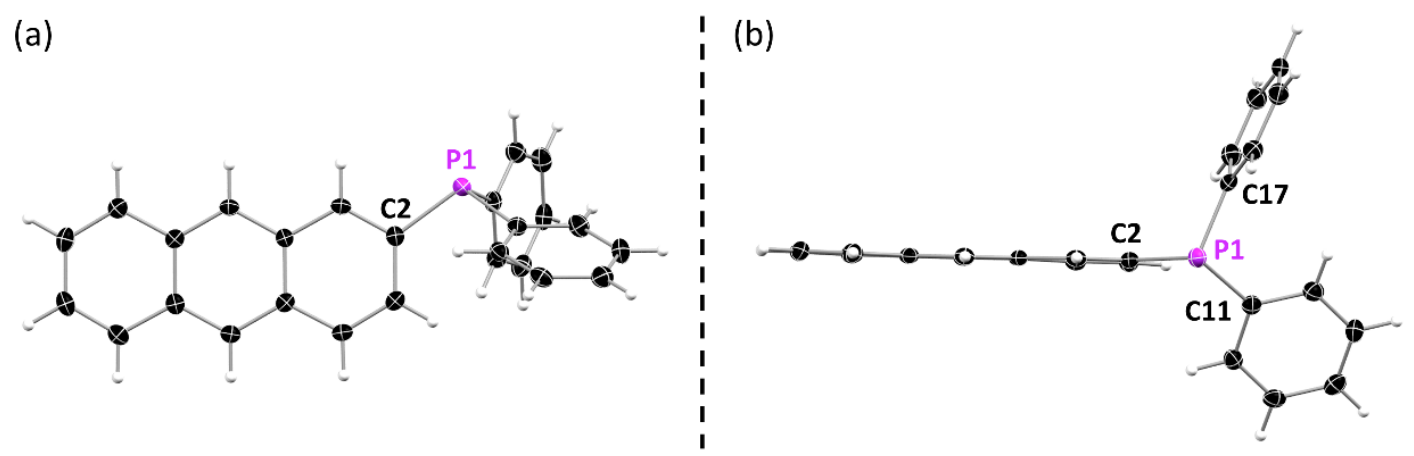

Figure 41. (a) Front view of the solid-state structure of [2-PPh $\left.-\left(\mathrm{C}_{14} \mathrm{H}_{9}\right)\right]$ (21). (b) The side view reveals the orientation of the substituent and indicates that the lone pair is located almost inside the anthracene plane but adopts a slightly torsion.

Considering only the anthracene moieties, the herringbone-type packing of pure anthracene is resembled in the packing of 21. Weak interactions between two anthracene moieties in a T-shape manner are present. Furthermore, several weak $\mathrm{C}-\mathrm{H} \cdots \pi$ interactions involving neighboring anthracenes and between anthracenes and phenyl groups can be observed. Direct face-to-face interactions of the anthracenes $\pi$ systems are not present, which is in accordance with the previously examined phosphines.

\subsubsection{Synthesis of oxo- and thiophosphoryl anthracene regioisomers}

Synthesis of the oxo- and thiophosphoryl anthracenes is again straightforward and performed via the established routes. [1- $\mathrm{PPh}_{2}-\left(\mathrm{C}_{14} \mathrm{H}_{9}\right]$ ] (18) was oxidized by urea hydrogen peroxide to give the oxophosphoryl anthracene [1-(O)PPh $\left.-\left(\mathrm{C}_{14} \mathrm{H}_{9}\right)\right]$ (19) or with elemental sulfur to give the thiophosphoryl anthracene [1-(S)PPh $-\left(\mathrm{C}_{14} \mathrm{H}_{9}\right)$ ] (20) (Scheme 28). For [2- $\mathrm{PPh}_{2}-\left(\mathrm{C}_{14} \mathrm{H}_{9}\right)$ ] (21), only the sulfur oxidized derivative 22 was prepared. All compounds were analyzed via multinuclear NMR-spectroscopy, mass spectrometry, elemental analysis and XRD solid state analysis. As already discussed briefly the ${ }^{31} \mathrm{P}-\mathrm{NMR}$ chemical shifts of $\mathbf{2 0}$ and $\mathbf{2 2}$ are in the expected region around $+42 \mathrm{ppm}$ and therefore slightly downfield shifted compared to the 9-substituted thiophosphoryl anthracene 8 with a ${ }^{31} \mathrm{P}-\mathrm{NMR}$ resonance around 
+34 ppm. The solid-state structures reveal more differences and interesting properties and will be discussed in the following paragraphs in more detail.

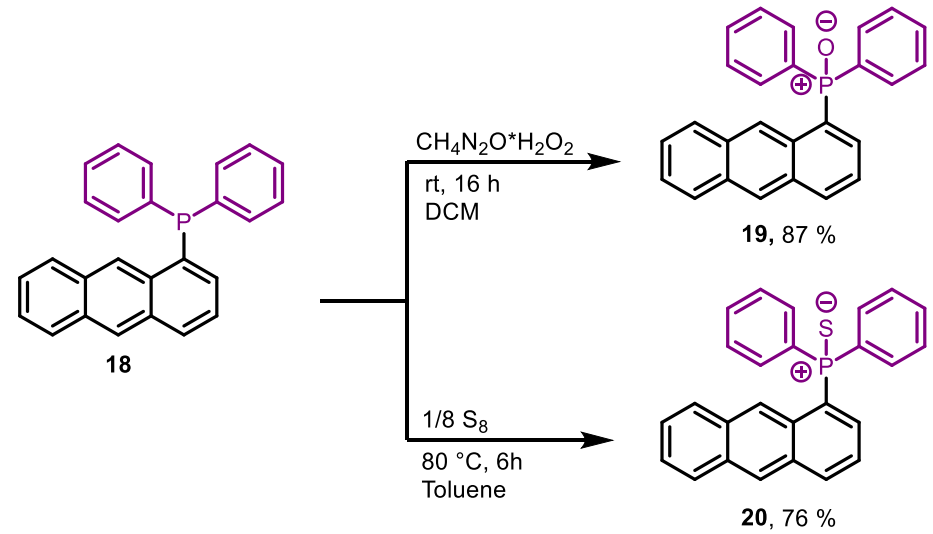

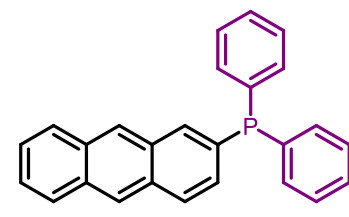

21

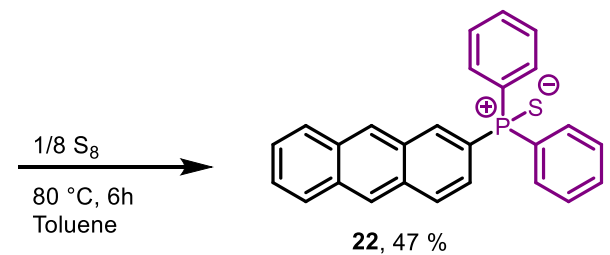

Scheme 28. Synthesis of oxo- and thiophosphoryl anthracene regioisomers [1-(O)PPh $\left.-\left(\mathrm{C}_{14} \mathrm{H}_{9}\right)\right](19)$, [1-(S)PPh $\left.-\left(\mathrm{C}_{14} \mathrm{H}_{9}\right)\right](20)$ and [2-(S) $\left.\mathrm{PPh}_{2}-\left(\mathrm{C}_{14} \mathrm{H}_{9}\right)\right](22)$ with substituent in the 1- or 2-position of the anthracene.

\subsubsection{Structural Properties of [1-(O)PPh $\left.-\left(\mathrm{C}_{14} \mathrm{H}_{9}\right)\right]$}

The oxophosphoryl anthracene $\left[1-(\mathrm{O}) \mathrm{PPh}_{2}-\left(\mathrm{C}_{14} \mathrm{H}_{9}\right)\right](19)$ crystallizes in the triclinic space group $P \overline{1}$ with one molecule in the asymmetric unit. Compared to the parent phosphine, the structural properties change only slightly and the orientation of the substituent in each structure is very similar. In contrast to the isomer [9-(O)PPh $\left.-\left(\mathrm{C}_{14} \mathrm{H}_{9}\right)\right](7)$, the $\mathrm{O}-\mathrm{P}-\mathrm{C}-\mathrm{C}$ torsion angle $\omega$ is widened by around $20^{\circ}\left(19: \omega=47.96^{\circ}, 7\right.$ : $\left.\omega=26.97^{\circ}\right)$ and the folding angle $\alpha$ decreased by $6^{\circ}$ to a nearly planar anthracene core $\left(\alpha=1.8^{\circ}\right)$.

In contrast to the 9-substituted isomer 7, the crystal packing of [1-(O) $\left.\mathrm{PPh}_{2}-\left(\mathrm{C}_{14} \mathrm{H}_{9}\right)\right]$ (19) reveals face-toface interactions between the anthracene moieties with an overlapping area of $21 \%$ in a distance of $3.406 \AA$ A. These interactions construct a columnar packing motif (Figure 42). Columnar stacking is usually avoided in luminescent solid-state materials as it is often responsible for emission quenching, and a separation of face-to-face dimers from each other is preferable. The solid-state structure of $\mathbf{7}$ already reveals the opportunities and challenges of the 1- and 2-substituted anthracene derivatives: due to a less shielded anthracene, a larger overlap and stronger face-to-face interactions are possible, which are desirable in case of excimer formation. At the same time, a columnar stacking motif, which is usually unwanted and addressed to aggregation-caused-quenching (ACQ), is also possible. 



Figure 42. Columnar stacking of [1-(O) $\left.\mathrm{PPh}_{2}-\left(\mathrm{C}_{14} \mathrm{H}_{9}\right)\right](19)$ with all anthracenes parallelly oriented and connected by face-to-face interactions (left). Detailed view on the $\pi-\pi$ interactions and overlapping anthracene area. (right).

\subsubsection{Polymorphism and co-crystal formation of [1-(S)PPh $\left.-\left(\mathrm{C}_{14} \mathrm{H}_{9}\right)\right]$}

During the investigation of the structural properties of the thiophosphoryl anthracene [1-(S)PPh $\left.-\left(\mathrm{C}_{14} \mathrm{H}_{9}\right)\right]$, three structural polymorphs $(\alpha-20, \beta-20, \gamma-20)$ with drastically changes in the crystal packing and intermolecular interactions could be identified.

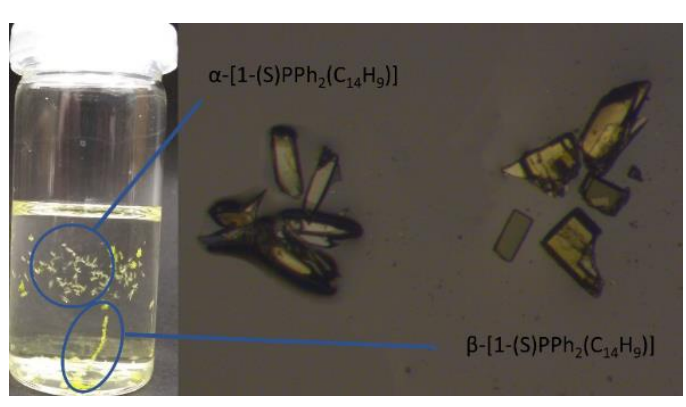

Figure 43. Two polymorphs pf $\left[1-(\mathrm{S}) \mathrm{PPh}_{2}-\left(\mathrm{C}_{14} \mathrm{H}_{9}\right)\right]$ formed during the crystallization process and under the microscope with 10x magnification.
The polymorph $\alpha-20$ was crystallized through slow diffusion of $n$-hexane into a concentrated toluene solution of $\mathbf{2 0}$. After several hours, pale yellow crystals formed at the side of the vial. The XRD solid-state analysis revealed a triclinic crystal system in the space group $P \overline{1}$. The compound crystallizes with one molecule in the asymmetric unit. Crystallization methods and conditions like solvent and temperature were varied and investigated but the polymorph $\alpha-20$ could not be isolated without crystallization of the two other polymorphs. The most reliable method to obtain the $\alpha$ polymorph was the initially described slow diffusion of hexane into a toluene solution of 20 . The polymorphs could be distinguished by color and shape of the crystals under the microscope (Figure 43). Due to the challenging isolation, $\alpha-20$ was ascribed to be only a metastable polymorph.

$\beta-20$ seems to be a more stable polymorph as it could be obtained via various crystallization methods and conditions. For example, recrystallization from a hot saturated solution of $\mathbf{2 0}$ in toluene and slow cooling afforded the $\beta$ polymorph with only minor amounts of the other polymorphs. $\beta-20$ crystallizes in a monoclinic crystal system in the space group $P 2_{1} / c$ with one molecule in the asymmetric unit. 


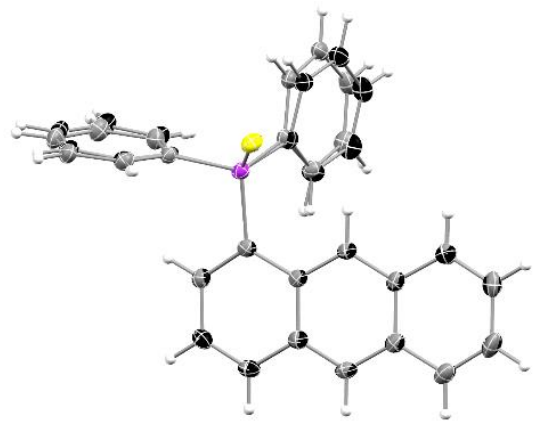

Figure 44. Structural overlay of the starting material [1- $\left.\mathrm{PPh}_{2}-\left(\mathrm{C}_{14} \mathrm{H}_{9}\right)\right]$ (grey) and its sulfur oxidation product [1-(S)PPh $\left.\mathrm{PP}_{2}\left(\mathrm{C}_{14} \mathrm{H}_{9}\right)\right]$ (black) in the $\alpha$-form, showing only minor changes in the orientation of the phenyl substituents.
The third polymorph was identified through recrystallization of $\mathbf{2 0}$ from a DMF solution. In general, solvent polarity can be a factor, which is important for the formation of different polymorphic forms in organic crystals. ${ }^{[217]}$ Therefore, the formation of another polymorph $(\gamma-20)$ can probably be attributed to the high polarity of DMF. $\gamma$-20 also crystallizes in a monoclinic crystal system in the space group $P 2_{1} / c$. Further non-aromatic solvents like EtOH or EtOAc also afforded the $\gamma$-polymorph. It has to be noted that the $\beta$ and $\gamma$-form could not be distinguished under the microscope as color and form of the crystals are nearly identical.

The intramolecular structural differences in the three polymorphs are marginal but are important to understand the crystallization process and the differences in stability of the polymorphs. Therefore, the three polymorphic forms will be discussed briefly and compared with the starting material of the synthesis [1-PPh $\left.{ }_{2}\left(\mathrm{C}_{14} \mathrm{H}_{9}\right)\right]$ (18). Like the parent phosphine, the $\alpha$-form crystallizes in the space group $P \overline{1}$. Furthermore, the cell parameters are also very similar to each other and differ only slightly (Table 11). Another similarity is found in the orientation of the phenyl substituents, which are also nearly identical in both structures (Figure 44).

Table 11. Structural and intermolecular interaction parameters of the three polymorphs of $\left[1-(\mathrm{S}) \mathrm{PPh}_{2}-\left(\mathrm{C}_{14} \mathrm{H}_{9}\right)\right]$ (20) and the starting material [1-PPh $\left.-\left(\mathrm{C}_{14} \mathrm{H}_{9}\right)\right](18)$ for comparison.

\begin{tabular}{lllll} 
& $\mathbf{1 8}$ & $\boldsymbol{\alpha - 2 0}$ & $\boldsymbol{\beta}-\mathbf{2 0}$ & $\boldsymbol{\gamma}-\mathbf{2 0}$ \\
\hline Space group & $P \overline{1}$ & $P \overline{1}$ & $P 21 / \mathrm{c}$ & $P 21 / \mathrm{c}$ \\
Unit cell & $\mathrm{a}=9.492(2) \AA$ & $\mathrm{a}=9.948(2) \AA$ & $\mathrm{a}=13.967(2) \AA$ & $\mathrm{a}=17.662(2) \AA$ \\
& $\mathrm{b}=10.216(2) \AA$ & $\mathrm{b}=10.252(2) \AA$ & $\mathrm{b}=8.892(2) \AA$ & $\mathrm{b}=7.158(2) \AA$ \\
& $\mathrm{c}=10.582(3) \AA$ & $\mathrm{c}=10.815(3) \AA$ & $\mathrm{c}=17.201(3) \AA$ & $\mathrm{c}=17.051(3) \AA$ \\
& $\alpha=86.87(3)^{\circ}$ & $\alpha=83.72(4)^{\circ}$ & $\alpha=90^{\circ}$ & $\alpha=90^{\circ}$ \\
& $\beta=83.46(2)^{\circ}$ & $\beta=80.19(3)^{\circ}$ & $\beta=110.46(4)^{\circ}$ & $\beta=114.25(2)^{\circ}$ \\
$\alpha /{ }^{\circ}$ & $\gamma=67.74(2)^{\circ}$ & $\gamma=64.76(3)^{\circ}$ & $\gamma=90^{\circ}$ & $\gamma=90^{\circ}$ \\
$\omega(\mathrm{S}-\mathrm{P}-\mathrm{C}-\mathrm{C}) /{ }^{\circ}$ & - & 2.09 & 1.47 & 2.23 \\
Overlap & 0 & $55.64(15)$ & $64.12(14)$ & $66.75(13)$ \\
$\mathrm{d}_{\pi-\pi} / \AA$ & -52 & 0 & 42.3 & 45.9 \\
$\mathrm{~d}_{\mathrm{x}} / \AA$ & - & - & 3.325 & 3.346 \\
$\mathrm{~d}_{\mathrm{y}} / \AA$ & - & - & 1.070 & 0.891 \\
$E_{\text {Latt }}[\mathrm{a}] / \mathrm{kJ} \mathrm{mol}^{-1}$ & & -168.1 & 1.057 & 1.342 \\
\hline
\end{tabular}

[a] calculated for a $25 \AA$ cluster around the central molecule and converged within $1 \mathrm{~kJ} \mathrm{~mol}^{-1}$ to a $20 \AA$ cluster.

The analysis of the intermolecular interactions in $\alpha-20$ revealed no face-to-face interactions between the anthracene moieties. All anthracenes are parallelly oriented and $\mathrm{C}-\mathrm{H} \cdots \pi$ interactions between the $\mathrm{H}$ atoms of the phenyl rings towards the anthracene $\pi$-system are the dominant interactions. The crystal packing of $\mathbf{1 8}$ is clearly resembled in $\alpha-\mathbf{2 0}$ (Figure 45 ). From the structural analysis it is assumed that the $\alpha$-form is the product that is initially formed upon oxidation of 18 as it shows only minor structural changes. 
The investigation of the photophysical properties (chapter 3.2.1.6) further supports this assumption and suggests that the formed precipitate of the reaction mixture also mainly consist of the $\alpha$-polymorph.
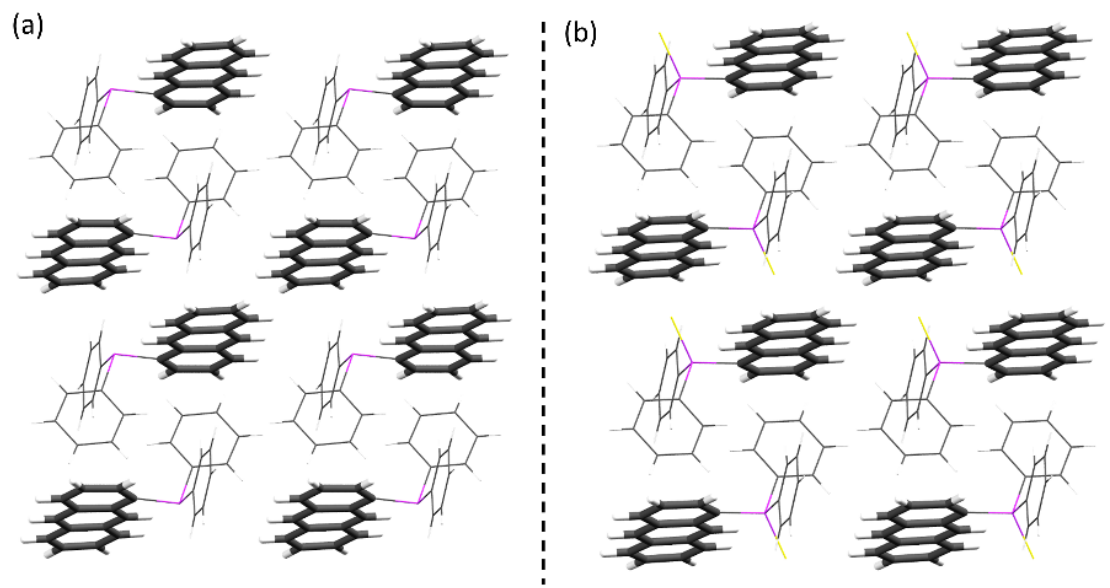

Figure 45. (a) Nearly identical crystal packing of [1- $\left.\mathrm{PPh}_{2}-\left(\mathrm{C}_{14} \mathrm{H}_{9}\right)\right](\mathbf{1 8})$ and (b) the corresponding thiophosphoryl anthracene [1(S)PPh $\left.{ }_{2}-\left(\mathrm{C}_{14} \mathrm{H}_{9}\right)\right](20)$ in the $\alpha$-form. View along the a-axis. The anthracene moieties are all oriented in parallel, but no face-to-face interactions are present.

The $\beta$ - and $\gamma$-form are very similar to each other but reveal some structural changes compared to the $\alpha$ form. Besides the different space group, the S-P-C1-C9A torsion angle is enlarged by about $10^{\circ}$. Also, the phenyl groups of the substituents adopt a slightly different orientation (Figure 46a).
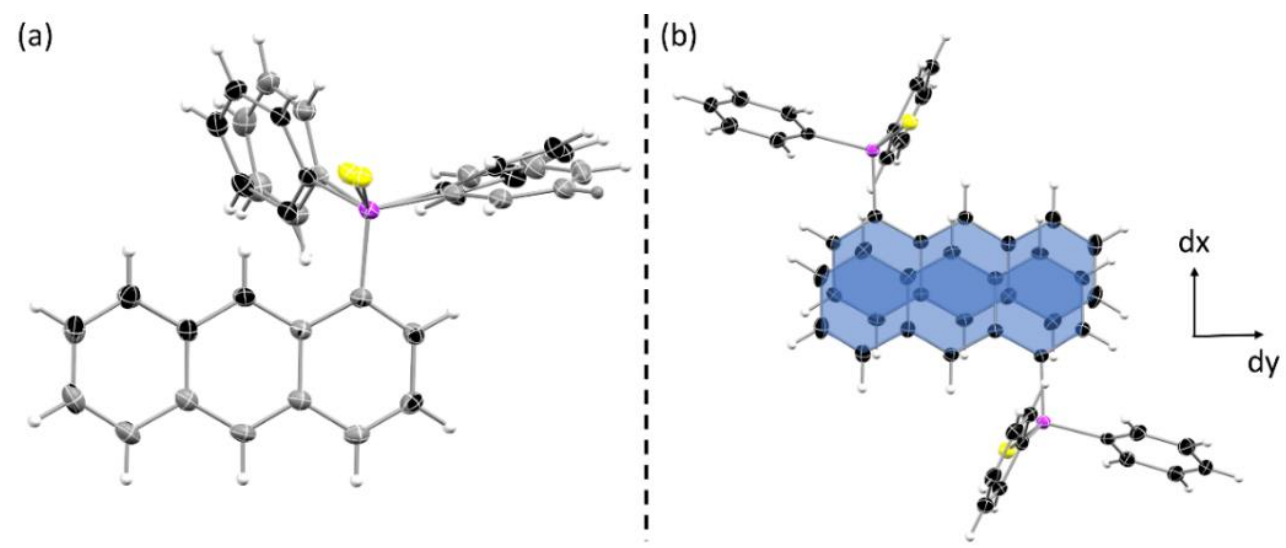

Figure 46. (a) Structural overlay of $\alpha-20$ (grey) and $\beta-20$ (black) with slight changes in the orientation of the phenyl groups. (b) Dimer motif with a large overlap of the anthracene planes as found in $\beta-\mathbf{2 0}$ and $\gamma-\mathbf{2 0 .}$

Most prominent changes are found in the crystal packing. While in the $\alpha$-form no face-to-face interactions were observed, the $\beta$ - and $\gamma$-form reveal strong $\pi-\pi$ interactions between neighboring anthracene moieties. The dimeric motif shows an overlapping area of around $42 \%(\beta-20)$ and $46 \%(\gamma-20)$ in a typical $\pi-\pi$ distances of around $3.3 \AA$. The overall crystal packings of the $\beta$ - and $\gamma$-form are slightly different, but for both the dimeric structure with its strong face-to-face interactions is the dominant motif (Figure 47). As further substituents are absent, edge-to-face interactions of the anthracene moieties are also observed. The columnar stacking of the anthracene moieties, which was found for the oxygen-homolog 19, could not be identified for the sulfur oxidized compounds, and the dimeric pairs can be considered as more isolated. 
Interactions between the dimeric pairs are still found via edge-to-face interactions. Further separation of the dimers from each other can probably be induced by addition of a second substituent in the 4- or 10position, which should inhibit the edge-to-face interactions (similar to the approach discussed in chapter 3.1.3).
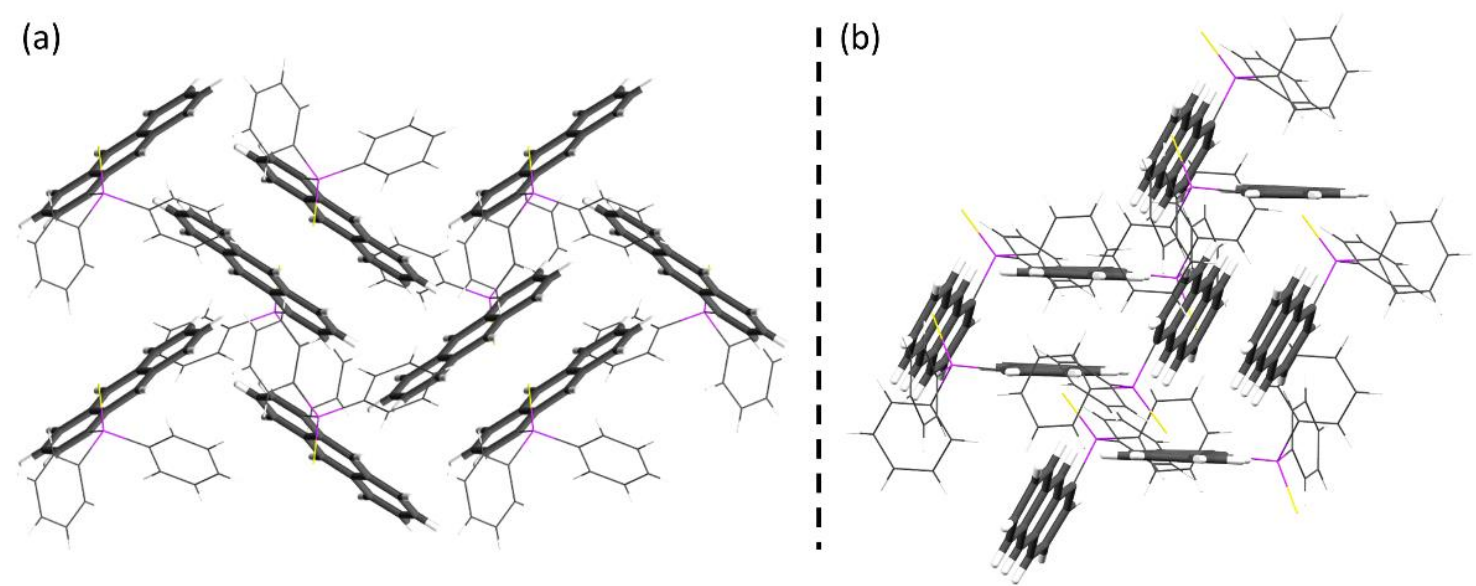

Figure 47. (a) Crystal packing of the $\beta$ - and (b) $\psi$-polymorph of [1-(S)PPh $\left.\mathrm{P}_{2}-\left(\mathrm{C}_{14} \mathrm{H}_{9}\right)\right]$ (20) with the anthracene moieties highlighted in bold. In both structures, strong face-to-face interactions between two anthracene moieties are the dominant interactions.

From the observations during the preparation and crystallization, it is assumed that the $\beta$ - and $\gamma$-form are the thermodynamic stable forms of $\mathbf{2 0}$, as no suitable crystallization method was found for crystallization of the pure $\alpha$-polymorph. As more than one form could often be found in a crystallization batch, the energy differences of the polymorphs are probably rather small and within a few $\mathrm{kJ} / \mathrm{mol}$, which is of course typical for polymorphs. To support the experimental observations, the lattice energies $E_{\text {lat }}$ of the three polymorphic forms were estimated on a basic level using the program package Crystal Explorer. ${ }^{[218,219]}$ Following the reported procedure, the interaction energies for each molecule of a cluster around the central molecule were calculated at the B3LYP/6-31G(d,p) level of theory. ${ }^{[218,219]}$ The lattice energy is obtained from the converged sum of pairwise interaction energies. The procedure was repeated for a larger cluster until $\Delta E_{\text {lat }}$ is converged to smaller than $1 \mathrm{~kJ} / \mathrm{mol}$, which was the case for clusters with a radius of 20 to $25 \AA$. Based on this calculation, the $\gamma$-form is the most stable form with $E_{\text {lat }}=-170.75 \mathrm{~kJ} / \mathrm{mol}$, which goes along with the experimental observations as the polymorph could be crystallized under various conditions. According to the calculated energies, the $\alpha$-polymorph $\left(E_{\text {lat }}=-168.1 \mathrm{~kJ} / \mathrm{mol}\right)$ should be more stable than the $\beta$-polymorph $\left(E_{\text {lat }}=-166.35 \mathrm{~kJ} / \mathrm{mol}\right)$. Thus, the $\beta$-polymorph would be the least stable form, which is surprising as it was the first identified polymorph and could also be crystallized from different solvents and conditions. As expected, the differences in energy are rather small and can explain the challenges in crystallizing pure forms of the polymorphs. However, the calculated differences should not be overinterpreted as they are very small and could be caused by approximations in the calculation.

The observations during the crystallization process and the investigations regarding the stability of the polymorphs go along with Ostwald's step rule. According to this rule it is not the most stable polymorph with the lowest energy that crystallizes first, but the polymorph that is closest in energy to the original 
state. ${ }^{[220]}$ The analysis of the solid-state structures could show the similarities in the structures of the $\alpha$ form and the starting material [1-PPh $\left.-\left(\mathrm{C}_{14} \mathrm{H}_{9}\right)\right](\mathbf{1 8})$. Therefore, the $\alpha$-polymorph is probably closest in energy to the original state and thus forms first. As the photophysical properties of the formed precipitate and the $\alpha$-polymorph are also nearly identical, it is assumed that the precipitate consists mostly of the $\alpha$ form (see chapter 3.2.1.6). When the precipitate is dissolved in hot solutions and crystallized afterwards, the thermodynamically more stable polymorphs are obtained.

During the investigation of the polymorphs and their crystallization conditions, the ability of [1-(S)PPh $2^{-}$ $\left.\left(\mathrm{C}_{14} \mathrm{H}_{9}\right)\right](20)$ for co-crystal formation was discovered. By recrystallisation from the appropriate solvent four co-crystals of 20 with the corresponding solvents benzene (20a), pyridine (20b), aniline (20c) and quinoline (20d) could be obtained. As already observed for [9-(S)PPh$\left.-10-E t-\left(\mathrm{C}_{14} \mathrm{H}_{8}\right)\right](15)$, the unsubstituted aromatic molecules benzene, pyridine and quinoline are suitable for co-crystal formation. Even if co-crystal formation with aniline is possible, further mono-substituted benzene derivatives like toluene, fluorobenzene, chlorobenzene, benzonitrile and anisole were not able to co-crystallize. Non-aromatic molecules like DCM, EtOAc, THF or $\mathrm{MeOH}$ gave also the solvent free polymorphs $(\beta / \gamma-20)$.

Table 12. Structural and intermolecular interaction of the obtained co-crystals of [1-(S)PPh $\left.2-\left(\mathrm{C}_{14} \mathrm{H}_{9}\right)\right](20)$.

\begin{tabular}{lllll}
\hline & $\mathbf{2 0 a}$ & $\mathbf{2 0 b}$ & $\mathbf{2 0 c}$ & $\mathbf{2 0 d}$ \\
\hline $\begin{array}{l}\text { co-crystallized } \\
\text { solvent }\end{array}$ & $\mathrm{C}_{6} \mathrm{H}_{6}$ & $\mathrm{C}_{5} \mathrm{H}_{5} \mathrm{~N}$ & $\mathrm{C}_{6} \mathrm{H}_{7} \mathrm{~N}$ & $\mathrm{C}_{9} \mathrm{H}_{7} \mathrm{~N}$ \\
Space group & $P \overline{1}$ & & & \\
Unit cell & $\mathrm{a}=9.448(2) \AA$ & $\mathrm{A}=9.230(2) \AA$ & $\mathrm{A}$ & $\overline{1}$ \\
& $\mathrm{~b}=10.737(2) \AA$ & $\mathrm{b}=9.706(2) \AA$ & $\mathrm{b}=10.926(2) \AA$ & $\mathrm{a}=10.544(2) \AA$ \\
& $\mathrm{c}=12.653(3) \AA$ & $\mathrm{c}=13.905(3) \AA$ & $\mathrm{d}=11.403(2) \AA$ \\
& $\alpha=78.97(2)^{\circ}$ & $\alpha=78.17(2)^{\circ}$ & $\alpha=79.18(2)^{\circ}$ & $\mathrm{c}=12.930(3) \AA$ \\
& $\beta=89.69(3)^{\circ}$ & $\beta=81.21(3)^{\circ}$ & $\beta=88.58(3)^{\circ}$ & $\beta=102.89(2)^{\circ}$ \\
$\alpha /{ }^{\circ}$ & $\gamma=74.95(2)^{\circ}$ & $\gamma=76.45(2)^{\circ}$ & $\gamma=74.97(2)^{\circ}$ & $\gamma=112.01(3)^{\circ}$ \\
$\omega(\mathrm{S}-\mathrm{P}-\mathrm{C}-\mathrm{C}) /{ }^{\circ}$ & 55.77 & 6.36 & 4.15 & 2.62 \\
Overlap /\% & 25.2 & 56.09 & 56.85 & 56.02 \\
$\mathrm{~d}_{\pi-\pi} / \AA$ & 3.230 & 31.3 & 25.5 & 22.3 \\
$\mathrm{~d}_{\mathrm{x}} / \AA$ & 1.191 & 3.316 & 3.357 & 3.259 \\
$\mathrm{~d}_{\mathrm{y}} / \AA$ & 3.189 & 1.066 & 1.214 & 1.381 \\
\hline
\end{tabular}

All four co-crystals crystallize in the triclinic space group $P \overline{1}$ with one molecule each of 20 and the solvent in the asymmetric unit. The co-crystals formed from benzene (20a) and aniline (20c) have similar cell parameters and are therefore considered as isomorphous. The intramolecular changes of the thiophosphoryl anthracene upon co-crystallization are negligible. The anthracene cores are still nearly planar, and the orientation of the substituent is only slightly affected by co-crystallization. The more pronounced influence of the co-crystallization can be seen in the intermolecular interactions and the crystal packing. Similar to the previously investigated co-crystals of [9-(S)PPh$\left.-10-E t-\left(\mathrm{C}_{14} \mathrm{H}_{8}\right)\right]$, a T-shape orientation of the co-crystallized solvent towards the anthracene plane is observed for 20a-20c (Figure 48). The intersection of the aromatic planes through the anthracene core and of the solvent encloses angles in the range from around $58^{\circ}(20 \mathrm{a}$ and $20 \mathrm{c})$ to $74.36^{\circ}(\mathbf{2 0 b})$. The co-crystallized solvent is involved $-92-$ 
in several weak intermolecular interactions in form of $\mathrm{C}-\mathrm{H} \cdots \pi$ interactions to neighboring anthracene units. For $20 \mathrm{c}$ hydrogen bonds in the form of $\mathrm{N}-\mathrm{H} \cdots \mathrm{N}$ between two aniline molecules and in the form of $\mathrm{N}-\mathrm{H} \cdots \mathrm{S}$ towards the thiophosphoryl substituent could be identified. The co-crystallized quinoline in $20 \mathrm{~d}$ adopts a different orientation, which was also observed for the quinoline co-crystal of [9-(S)PPh $\mathrm{Ph}_{2}-10-\mathrm{Et}-$ $\left.\left(\mathrm{C}_{14} \mathrm{H}_{8}\right)\right]$. The larger size is probably responsible for the different orientation compared to the smaller arenes. Nevertheless, $\mathrm{C}-\mathrm{H} \cdots \pi$ interactions between the quinoline and the anthracene moiety can also be found in the structure of $\mathbf{2 0 d}$.

(a)

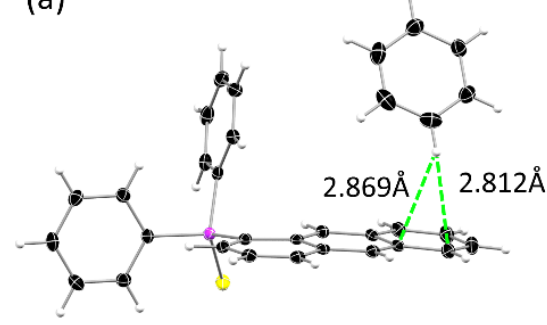

(b)

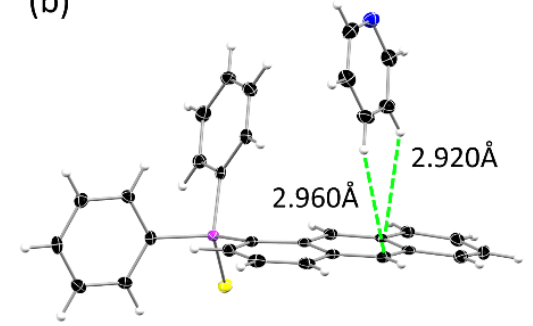

(c)

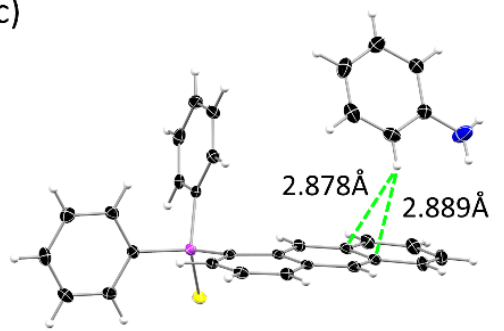

Figure 48. Orientation and observed weak $\mathrm{C}-\mathrm{H} \cdots \pi$ interactions (green) of the co-crystallized solvent towards the anthracene moieties in the co-crystals 20a (a), 20b (b), 20c (c).

Again, the crystal packing changes remarkably upon co-crystallization. The overall structure can be described as a sheet structure with all anthracenes parallelly oriented and resembles the solid-state structure of the $\alpha$-form of $\mathbf{2 0}$ when considering the orientation of the anthracene scaffold. This is further supported by the same space group and similarities in the unit cell dimensions (Table 12). A fundamental difference compared to $\alpha-\mathbf{2 0}$ is the presence of the dimeric motif in all four co-crystals, which was also found for the $\beta$ - and $\gamma$-polymorphs. Again, co-crystallization of the solvent has a drastic influence on the dimer parameters. As already seen for the co-crystals of [9-(S)PPh$\left.-10-E t-\left(\mathrm{C}_{14} \mathrm{H}_{8}\right)\right]$, the overlap of the two anthracene planes is clearly reduced. For 20a-20d, overlap ratios from $22-32 \%$ are obtained. From the offsets of the dimer along the short $\left(d_{x}\right)$ and long $\left(d_{y}\right)$ anthracene axes, the reduced overlap can be ascribed to a shift of the anthracene pair along both molecular axes. The offset $d_{y}$ along the long molecular axis increases by at least $1.5 \AA$, while for the short molecular axis a maximum increase of around $0.5 \AA$ is observed. $d_{x}$ - values are in the range from $1.06 \AA$ to $1.38 \AA$ and are comparable to the offsets found for the $\beta$ and $\gamma-20$ ( 0.89-1.07 $\AA$ ). The increase of the offset $d_{y}$ along the long molecular axes seems therefore to be more pronounced and to have a greater impact on the decreased overlap. The distance of the anthracene planes is still in the typical range for $\pi-\pi$ interactions around $3.3 \AA$.

\subsubsection{Structural properties of [2-(S)PPh $\left.{ }_{2}-\left(\mathrm{C}_{14} \mathrm{H}_{9}\right)\right]$}

For the third and last positional isomer [2-(S)PPh $\left.-\left(\mathrm{C}_{14} \mathrm{H}_{9}\right)\right](22)$, only one polymorph was obtained from different crystallization conditions. [2-(S)PPh $\left.\mathrm{P}_{2}\left(\mathrm{C}_{14} \mathrm{H}_{9}\right)\right]$ crystallizes in the monoclinic space group $P 2_{1} / \mathrm{n}$ with one molecule in the asymmetric unit. As already observed for the parent phosphine 21, the conformation of the substituent is again different compared to the isomer substituted in 1-position. Each phenyl group is now located above and below the anthracene, respectively, while the sulfur atom is inside the plane with an $\mathrm{S}-\mathrm{P}-\mathrm{C} 2-\mathrm{C} 1$ torsion angle close to $0^{\circ}$. The anthracene scaffold is again nearly planar as the 
substituent in the outer position has enough freedom to rotate away from the anthracene plane. Therefore, the anthracene aromatic plane is now nearly de-shielded and not protected by the substituent.
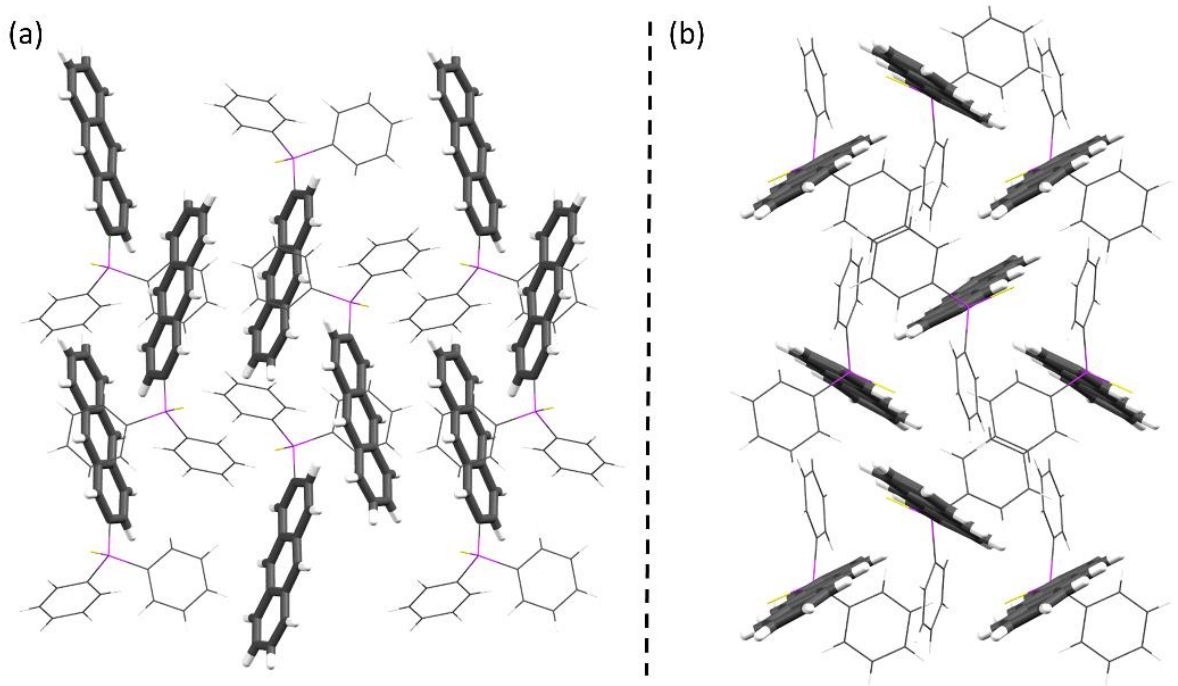

Figure 49. (a) Crystal packing of [2-(S)PPh $\left.-\left(\mathrm{C}_{14} \mathrm{H}_{9}\right)\right](22)$ with view along the crystallographic a-axes and (b) with view along the crystallographic c-axes.

Nevertheless, the crystal structure of $\mathbf{2 2}$ reveals no face-to-face interactions. Moreover, the structure resembles the crystal packing of unsubstituted anthracene with edge-to-face interactions between the anthracene moieties (Figure 49). Further intermolecular interactions are found between the phenyl groups and the anthracene scaffold. The electronic properties on the anthracene core should change only slightly, with the different position of the substituent. However, in contrast to [1-(S)PPh$-\left(C_{14} \mathrm{H}_{9}\right)$ ], no $\pi-\pi$ interactions are found for 22. As discussed in chapter 1.2, the nature of the interactions between $\pi$ systems is still under discussion, and the ground-state interaction energy is usually small. The investigation of the isomers can clearly show that already small changes in the structure can result in different intermolecular interactions. It could not be evaluated why [2-(S)PPh $\left.2_{2}-\left(\mathrm{C}_{14} \mathrm{H}_{9}\right)\right]$ (22) undergoes no $\pi-\pi$ interaction. A possible explanation can be the changed orientation of the substituent, which prohibits interactions between the sulfur and hydrogen atoms from the neighboring anthracene in a face-to-face orientation. These weak interactions are found for the $\beta$ - and $\gamma$-form of $\left[1-(S) P P_{2}-\left(C_{14} H_{9}\right)\right](20)$ in a distance of around $3.1 \AA$ and may contribute to the formation of the observed packing motif. Nevertheless, it could be shown, that only small factors determine the resulting intermolecular interactions between the anthracene $\pi$-systems.

While [1-(S)PPh $\left.-\left(\mathrm{C}_{14} \mathrm{H}_{9}\right)\right]$ (20) showed a great tendency for polymorphism, a number of crystallization approaches under varying conditions and with different methods were performed for [2-(S)PPh $\mathrm{Ph}_{2}\left(\mathrm{C}_{14} \mathrm{H}_{9}\right)$ ] (22), but only one crystalline form could be isolated. However, in the obtained crystal structure a disorder of the molecule over two positions is observed. Interestingly, in the second position, the substituents conformation is changed by around $180^{\circ}$, which can be a hint at a conformational polymorph with otherwise identical cell parameters (Figure 50). 
(a)
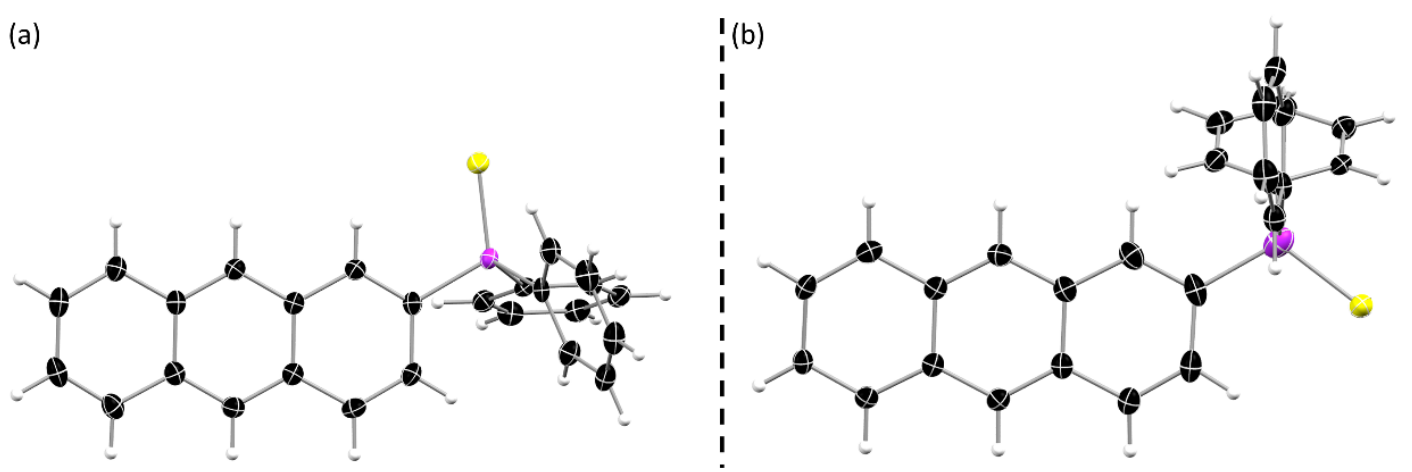

Figure 50. (a) Molecular structure of [2-(S)PPh$\left.-\left(\mathrm{C}_{14} \mathrm{H}_{9}\right)\right](22)$ and (b) a disordered position with a different orientation of the substituent. The occupancy of the minor component shown in (b) refined to 0.0652(15).

The synthesis of the two positional isomers of $\left[(\mathrm{S}) \mathrm{PPh}_{2}-\left(\mathrm{C}_{14} \mathrm{H}_{9}\right)\right]$ and evaluation of the structural properties exhibited great potential for the following analysis of the structure-property relationship regarding the solid-state luminescence. As expected, the isomers with the thiophosphoryl substituent in 1- and 2position reveal a nearly planar anthracene core and can provide insights into the influence of the folding and distortion of the anthracene core on the photophysical properties. In addition, two of the three found polymorphs of [1-(S) $\mathrm{PPh}_{2}-\left(\mathrm{C}_{14} \mathrm{H}_{9}\right)$ ] (20) revealed a fundamental different crystal packing. A direct investigation of the dependency of the photophysical properties on the intermolecular interactions is possible, as the electronic properties of the polymorphs do not change. Together with the co-crystals of 20, a dataset with significant different interchromophoric interactions is obtained. It can provide valuable information for further understanding of the relationship between the non-covalent interactions and luminescence properties.

\subsubsection{Photophysical properties and excimer formation of phosphoryl anthracene regioisomers}

\section{In solution}

At first the photophysical properties in diluted solution will be investigated representing the monomeric species without any intermolecular interactions between the chromophores. The oxophosphoryl anthracene [1-(O) $\mathrm{PPh}_{2}-\left(\mathrm{C}_{14} \mathrm{H}_{9}\right)$ ] (19) reveals similar behavior as described for the isomer in 9-position. The absorption spectrum is also slightly broadened and red shifted compared to unsubstituted anthracene. It reveals the typical vibronic absorption in the region from $320 \mathrm{~nm}$ to $400 \mathrm{~nm}$, which is assigned to the $\pi-\pi^{*}$ absorption of the anthracene $\pi$-system (Figure 51). The emission spectrum is slightly broadened and the vibronic structure less pronounced. An intense blue emission can be observed upon irradiation with UVlight with an emission wavelength of maximum intensity around $420 \mathrm{~nm}$. The quantum yield was determined to $42.8 \%$ and the lifetime with $5.5 \mathrm{~ns}$ is as expected in the typical range for fluorescence emission. As already seen for the 9-substituted derivative, a strong emission enhancement in solution upon oxidation with oxygen can be observed as the quenching process of the PET is suppressed. 


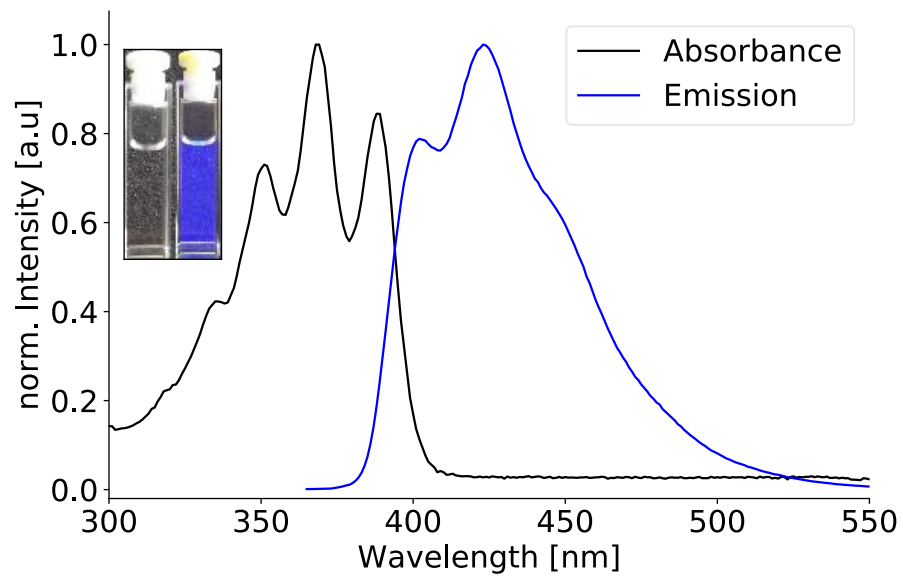

Figure 51. Excerpt of the normalized UV-Vis absorption spectrum (black) and emission spectrum (blue) of [1-(O) $\left.\mathrm{PPh}_{2}-\left(\mathrm{C}_{14} \mathrm{H}_{9}\right)\right](19)$ in diluted THF solution $\left(10^{-5} \mathrm{M}, \lambda_{\mathrm{ex}}=350 \mathrm{~nm}\right)$. The photographs show the solution under day light (left) and under UV-irradiation (right).

The UV-Vis absorption spectra of the three positional isomers of the thiophosphoryl anthracenes [(S)PPh $2^{-}$ $\left(\mathrm{C}_{14} \mathrm{H}_{9}\right)$ ] are very similar. For all three compounds, two major absorptions are visible in the spectrum. The high-energy absorption is located at around $260 \mathrm{~nm}$ and the lower energy absorption at $350-420 \mathrm{~nm}$, which is assigned to the $\pi-\pi^{*}$ transition located mainly on the anthracene core (Figure 52a). The latter reveals a vibronic structure, which is much more pronounced for the 1- and 2- substituted isomers. Besides the broadening of the absorption band of $\mathbf{8}$, a red shifted of around $20 \mathrm{~nm}$ compared to $\mathbf{2 0}$ and $\mathbf{2 2}$ is observed.
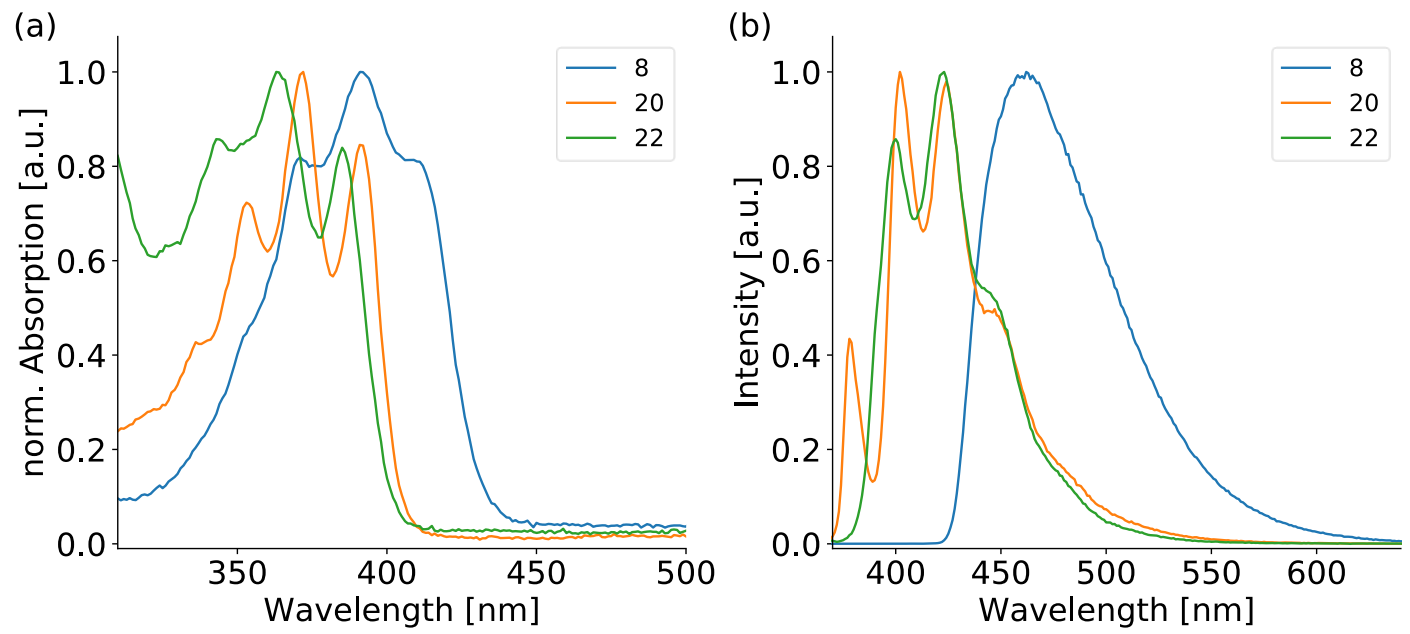

Figure 52. (a) Normalized UV-Vis absorption spectra and (b) normalized emission spectra of the three positional isomers [1$\left.(\mathrm{S}) \mathrm{PPh}_{2}-\left(\mathrm{C}_{14} \mathrm{H}_{9}\right)\right](20)$, [2-(S)PPh$\left.-\left(\mathrm{C}_{14} \mathrm{H}_{9}\right)\right](22)$ and [9-(S)PPh $\left.2^{-}\left(\mathrm{C}_{14} \mathrm{H}_{9}\right)\right](8)$ in diluted THF solution $\left(10^{-5} \mathrm{M}, \lambda_{\text {ex }}=350 \mathrm{~nm}\right)$.

In the emission spectra, the differences within the isomers are more pronounced. Although all emit only weakly in solution, the shape and the emission wavelength alter noteworthy. The 1- and 2-isomer reveal a structured emission band ranging from 400 to $450 \mathrm{~nm}$ and therefore differ in comparison to the broad emission of 8, which also reveals a bathochromic shift of around $20 \mathrm{~nm}$ regarding the emission with highest intensity (Figure 52b). As discussed in the previous chapter, the unusual broadening and comparably large - 96 - 
bathochromic shift was also observed for all thiophosphoryl anthracenes with the substituent in 9position. Along with the bathochromic shift, a strong distortion of the anthracene plane in form of a butterfly-like bending was observed in the solid-state structures. The folding was postulated as a possible reason for the unusual red-shift and spectral broadening. The isomers [1-(S)PPh$\left.-\left(\mathrm{C}_{14} \mathrm{H}_{9}\right)\right](20)$ and [2(S) $\left.\mathrm{PPh}_{2}-\left(\mathrm{C}_{14} \mathrm{H}_{9}\right)\right]$ (22) reveal a nearly planar anthracene core, a less pronounced bathochromic shift and a clear vibronic structure in the emission spectra. These findings support the hypothesis that the strong distortion of the anthracene core has an influence on the photophysical properties however are not conclusive. The low quantum yields are again assigned to the effective quenching due to the sulfur lone pairs via a PET (Table 13).

Table 13. Photophysical properties of the positional isomers [1-(S)PPh$\left.-\left(\mathrm{C}_{14} \mathrm{H}_{9}\right)\right](20)$, [2-(S)PPh $2_{2}^{-}$ $\left.\left(\mathrm{C}_{14} \mathrm{H}_{9}\right)\right](\mathbf{2 2})$ and $\left[9-(\mathrm{S}) \mathrm{PPh}_{2}-\left(\mathrm{C}_{14} \mathrm{H}_{9}\right)\right]$ (8) in diluted THF solution.

\begin{tabular}{lllll}
\hline & $\lambda_{\text {abs }} / \mathrm{nm}$ & $\lambda_{\text {em }} / \mathrm{nm}$ & $\Phi_{\mathrm{F} / \%}$ & $\tau / \mathrm{ns}$ \\
\hline$\left[1-(\mathrm{S}) \mathrm{PPh}_{2}-\left(\mathrm{C}_{14} \mathrm{H}_{9}\right)\right](\mathbf{2 0})$ & $336 / 354 / 371 / 391$ & $378 / 402 / 424 / 450$ & $<1.0$ & $1.1 / 5.3$ \\
{$\left[2-(\mathrm{S}) \mathrm{PPh}_{2}-\left(\mathrm{C}_{14} \mathrm{H}_{9}\right)\right](\mathbf{2 2})$} & $343 / 364 / 385$ & $401 / 423 / 448$ & $<1.0$ & $0.9 / 8.8$ \\
{$\left[9-(\mathrm{S}) \mathrm{PPh}_{2}-\left(\mathrm{C}_{14} \mathrm{H}_{9}\right)\right](8)$} & $352 / 371 / 392 / 411$ & 462 & $<1.0$ & 9.3 \\
\hline
\end{tabular}

Before the photophysical properties in the solid-state are investigated, the emission behavior of THF solutions with different water fractions will be presented. As already discussed in chapter 1.4, this experiment is typical for AlE-active compounds. With increasing water content, the aggregation of the molecules is forced due to the reduced solubility. This experiment resembles the transition between the solution and aggregated state and can therefore give insights into the photophysical properties during the aggregation process.
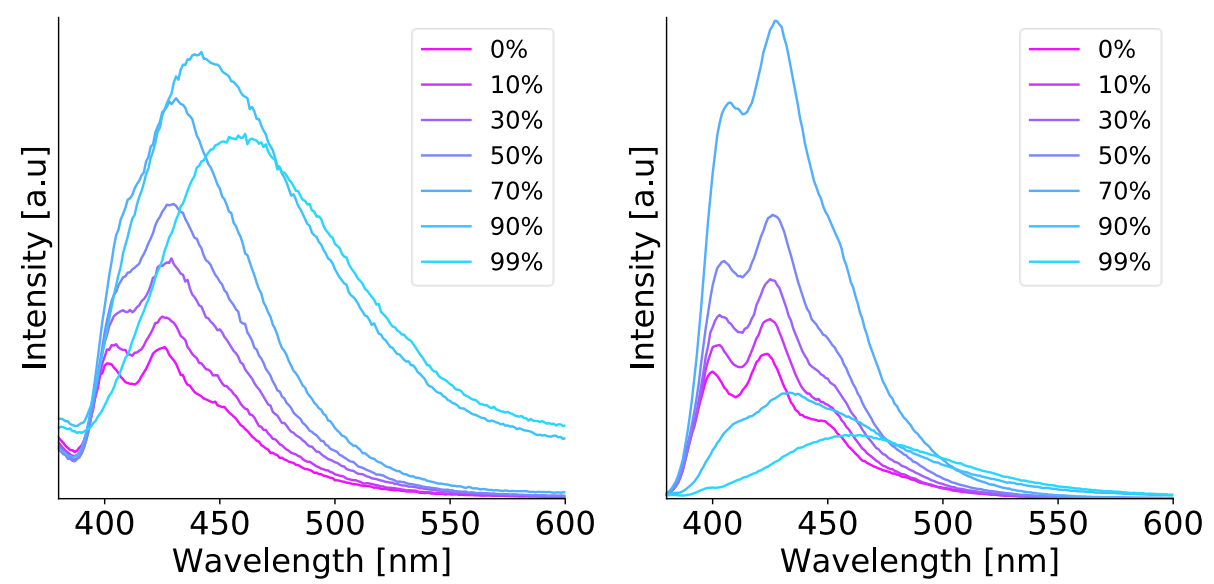

Figure 53. Emission spectra of the two positional isomers [1-(S)PPh $\left.-\left(\mathrm{C}_{14} \mathrm{H}_{9}\right)\right](20)$ (left) and [2-(S)PPh $\left.-\left(\mathrm{C}_{14} \mathrm{H}_{9}\right)\right](22)$ (right) in diluted THF solution with increasing water fractions (in \%). For both compounds the increased water fraction leads to spectral broadening and a slight bathochromic shift.

For the isomers $\mathbf{2 0}$ and $\mathbf{2 2}$, a broadening of the emission spectra with increasing water fraction can be observed (Figure 53). Upon 90\% water content, the vibronic structure is clearly lost, and a slight bathochromic shift of the emission wavelength is observed, which is typical upon aggregation. Furthermore, a slight increase in the emission intensities can be detected up to $70-90 \%$ water fraction, 
which hints for an AIE process. In general, the flexibility of a molecule is decreased upon aggregation, resulting in the restriction of internal motion. Therefore, non-radiative pathways can be blocked upon aggregation resulting in higher emission intensities. For the thiophosphoryl anthracenes, the PET of the sulfur lone pair was ascribed as the main non-radiative pathway. Due to the less flexible conformation upon aggregation, the overlap of the sulfur lone pair with the anthracene $\pi$-system is probably less effective and resultins in slightly increased emission intensities in the aggregated state. Nevertheless, emission intensities are still very low and the increase is only marginal, when compared to typical AIEluminogens. ${ }^{[53,57]}$

\section{In the solid-state}

In the solid-state, the differences in the photophysical properties within the three positional isomers are more pronounced. Besides the small intramolecular structural variations, the occurring intermolecular interactions of the isomers and polymorphs are fundamentally different. Therefore, this system is suitable for investigating the effect of the intermolecular interactions on the photophysical properties. The emission spectrum of [2-(S)PPh $\left.2_{2}\left(\mathrm{C}_{14} \mathrm{H}_{9}\right)\right](22)$ changes only slightly upon aggregation. A vibronic structure is also clearly present in the solid-state, and the emission is still located in the blue region of the spectrum (Figure 54). The quantum yield is increased to $15.7 \%$ compared to the very weak emission in solution and the lifetime is still within a few nanoseconds. Similar emission properties are observed for the $\alpha$-form of 20. The photophysical properties were determined for single-crystals, which were identified as the $\alpha$ polymorph as well as for the precipitate formed during the oxidation of the phosphine. Both emission properties are very comparable and, together with the results from the structural analysis, it was concluded that the precipitate mainly consists of the $\alpha$-form of 20 . The emission spectra in the solid-state are comparable to the spectra in solution regarding the shape and bathochromic shift. Compared to 22, only a slight broadening is observed, but overall, the emission properties of $\alpha-\mathbf{2 0}$ and $\mathbf{2 2}$ are very similar with nearly identical maximum emission wavelengths and a slight vibronic structure. A clear difference is found in the quantum yields, which remains low in the solid-state for $\alpha-20$.

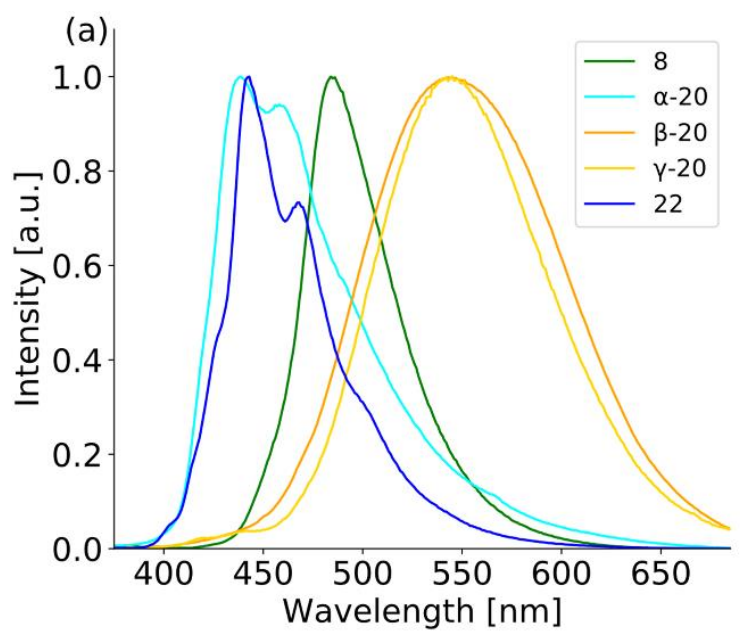

(b)

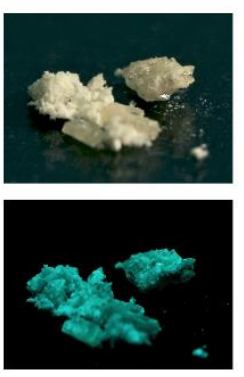

8

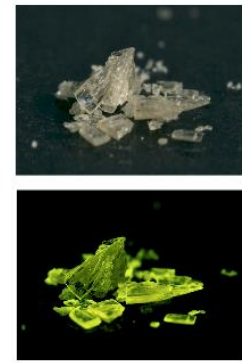

$\beta-20$

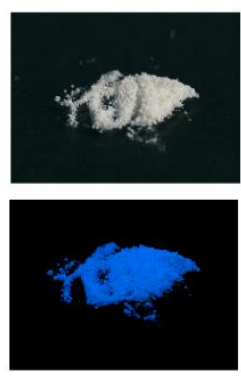

22

Figure 54. (a). Solid-state emission spectra of the positional isomers 8, 20 (and its polymorphs) and 22. (b) Photographs of powder and crystals of the positional isomers $\mathbf{8}$ (left) $\beta-\mathbf{2 0}$ (middle) and $\mathbf{2 2}$ (right) under daylight (top) and UV-light (bottom).

- 98 - 
For the isomer with the substituent in 9-position (8), the vibronic structure is absent in the emission spectrum, which was already the case for the emission in solution (see chapter 3.1.2.3). The emission wavelength is also red shifted by around $20 \mathrm{~nm}$ resulting in a blue-green emission. Most drastic changes are observed for the $\beta$ - and $\gamma$-polymorph of 20 . Both show nearly identical emission characteristics. Emission spectra with a maximum emission at around $545 \mathrm{~nm}$ are obtained from the solid-state samples. Compared to $\mathbf{8}$, the emission band is further broadened and ranges over nearly $200 \mathrm{~nm}$. For a quantification of the broadening, the full width at half maximum (FWHM) was evaluated. The emission spectra of the $\beta$ - and $\psi$-polymorph are clearly broadened, with an increased FWHM from around $75 \mathrm{~nm}$ in the $\alpha$-form to over $100 \mathrm{~nm}$ in the $\beta$ - and $\gamma$-form (Table 14). The two positional isomers 8 and 22 reveal smaller FWHM with around $50 \mathrm{~nm}$. Additionally, an increase in the fluorescence lifetime up to $30 \mathrm{~ns}$ is observed for the $\beta$ - and $\gamma$-polymorphs compared to the typical fluorescence lifetime of 1-10 ns for the other three compounds. The quantum yield increases in comparison to the nearly quenched emission in solution but is still only moderate.

By variation of the substituents' position, a three-color solid-state luminescence was obtained with blue, green, and yellow emission with differences in the emission wavelength of over $100 \mathrm{~nm}$ (Table 14). This is remarkable as the electronic structure of the compounds should be comparable as also seen in the UV-Vis spectra, which revealed only small differences. At least for the three polymorphs of 20, an identical molecular electronic structure can be assumed. The drastic changes in the solid-state photophysical properties are therefore clearly induced by the intermolecular interactions.

Table 14. Overview of the photophysical properties of the three positional isomers [1-(S)PPh $\left.-\left(\mathrm{C}_{14} \mathrm{H}_{9}\right)\right](20)$, $\left[2-(\mathrm{S}) \mathrm{PPh}_{2}-\left(\mathrm{C}_{14} \mathrm{H}_{9}\right)\right](22)$ and the already discussed [9-(S)PPh$\left.-\left(\mathrm{C}_{14} \mathrm{H}_{9}\right)\right](8)$ in the solid-state.

\begin{tabular}{lllll}
\hline & $\lambda_{\max } / \mathbf{n m}$ & $\boldsymbol{\tau} / \mathbf{n s}$ & $\Phi_{\mathrm{F}} / \%$ & FWHM / nm \\
\hline$\alpha-\left[1-(\mathrm{S}) \mathrm{PPh}_{2}-\left(\mathrm{C}_{14} \mathrm{H}_{9}\right)\right](\mathbf{2 0})$ & $437 / 458 / 493$ & 6.6 & $<1$ & 75 \\
$\beta-\left[1-(\mathrm{S}) \mathrm{PPh}_{2}-\left(\mathrm{C}_{14} \mathrm{H}_{9}\right)\right](\mathbf{2 0})$ & 545 & 29.0 & 5.9 & 117 \\
$\gamma$-[1-(S)PPh $\left.2-\left(\mathrm{C}_{14} \mathrm{H}_{9}\right)\right](\mathbf{2 0})$ & 546 & 30.0 & 5.7 & 100 \\
{$\left[2-(\mathrm{S}) \mathrm{PPh}_{2}-\left(\mathrm{C}_{14} \mathrm{H}_{9}\right)\right](\mathbf{2 2})$} & $426 / 441 / 469 / 502$ & $3.2 / 7.8$ & 15.7 & 50 \\
{$\left[9-(\mathrm{S}) \mathrm{PPh}_{2}-\left(\mathrm{C}_{14} \mathrm{H}_{9}\right)\right](8)$} & 484 & $1.4 / 3.2$ & 4.0 & 50 \\
\hline
\end{tabular}

The $\alpha$-polymorph of $\mathbf{2 0}$ and the isomer substituted in 2-position of the anthracene (22) revealed similar emission properties and similar solid-state structures. The photophysical properties change only slightly when going from solution to the solid-state, indicating an emission from the monomer also in the solidstate. The crystal structure confirms this assumption as no noteworthy $\pi-\pi$ interactions are present between the anthracene moieties. The solid-state structure consists mainly of $\mathrm{C}-\mathrm{H} \cdots \pi$ interactions as the dominant interactions between neighboring molecules. The observed edge-to-face and $\mathrm{C}-\mathrm{H} \cdots \pi$ interactions do not seem to have a great influence on the photophysical properties as the emission wavelengths and lifetimes are only slightly different as observed in solution. An excimer formation from this structural motif can therefore be excluded as no face-to-face interactions between the anthracenes $\pi$-system occur. The emission is therefore assigned to occur from the pure locally excited (LE) state. 
The bathochromic shift and the resulting blue-green emission of [9-(S)PPh $\left.-\left(\mathrm{C}_{14} \mathrm{H}_{9}\right)\right]$ (8) was discussed earlier (chapter 3.1.2.3) and ascribed to the bent structure of the anthracene core as well as to the weak $\pi-\pi$ interactions between two neighboring anthracenes. Nevertheless, the formation of an excimer could not be confirmed. This hypothesis is further supported as the FWHM is only slightly increased compared to the $\alpha$-form of $\mathbf{2 0}$ and to $\mathbf{2 2}$. Thus, the emission of all three compound is ascribed to a monomeric state with no or only little contribution from the intermolecular interactions.

However, a clearly different behavior is observed for the two polymorphs $\mathbf{\beta - 2 0}$ and $\boldsymbol{\gamma}-\mathbf{2 0}$. For both forms, a strong bathochromic shift of the emission in the solid-state is observed resulting in an anthraceneuntypical yellow emission. In their solid-state structures, the dimeric motif is present with an overlap ratio of the anthracene chromophore between $42 \%$ and $45 \%$. The structural motif together with the elongated lifetime and the broadened emission spectrum with a FWHM of around $100 \mathrm{~nm}$ and larger, clearly hint for an excimer emission. In good agreement with the theory for excimer formation, two molecules of [1(S) $\left.\mathrm{PPh}_{2}-\left(\mathrm{C}_{14} \mathrm{H}_{9}\right)\right]$, one in the ground state and one in the excited state, form an excimer. The delocalization of the excitation energy over two chromophores results in an increased interaction energy, compared to the two dimers in the ground-state. The emission from the energetic lower excimer state results in the strong bathochromic shift of the emission wavelength. As the ground-state is dissociative or only weakly bonded, the emission is structureless and broadened (see chapter 1.3.2).

Compared to the $\alpha$-form, which shows only very weak emission, the quantum yield for the excimer emission of $\beta-20$ and $\gamma-20$ is slightly increased. Recent studies have reported a more drastic emission enhancement upon excimer formation with quantum yields up to $80 \%{ }^{[68,133-135]}$ This emission enhancement was assigned to a rigidification of the solid-state structure due to the strong intermolecular interactions between the chromophores. Typical non-radiative pathways like vibration are hindered by the rigidification upon excimer formation. For [1-(S) $\left.\mathrm{PPh}_{2}-\left(\mathrm{C}_{14} \mathrm{H}_{9}\right)\right](\mathbf{2 0})$ only a slight increase of the emission intensity could be observed upon excimer formation. The PET from the sulfur lone pairs is probably still active in the solid-state, albeit less efficient as the more rigid structure complicates the required orbital overlap. In consequence, only a slight emission amplification is observed by excimer formation.

For the three polymorphs of [1-(S)PPh$\left.-\left(\mathrm{C}_{14} \mathrm{H}_{9}\right)\right](20)$ a clear dependence of the emission wavelength on the intermolecular interactions could be confirmed. Strong $\pi-\pi$ interaction between the anthracene moieties allow an excimer formation, resulting in drastic changes in the luminescence properties. For a more detailed insight, the co-crystals of $\mathbf{2 0}$ will be investigated. The evaluation of the structural properties has revealed a dimeric motif similar to $\beta$ - and $\gamma$-20. The overlap ratio of the anthracene chromophores in the co-crystals was decreased and therefore a decrease of the intermolecular interactions is assumed. The investigation of the photophysical properties of the co-crystals can therefore address the question whether the co-crystals of $\mathbf{2 0}$ are also able of excimer emission or whether the decreased overlap ratio prohibits the excimer formation. 

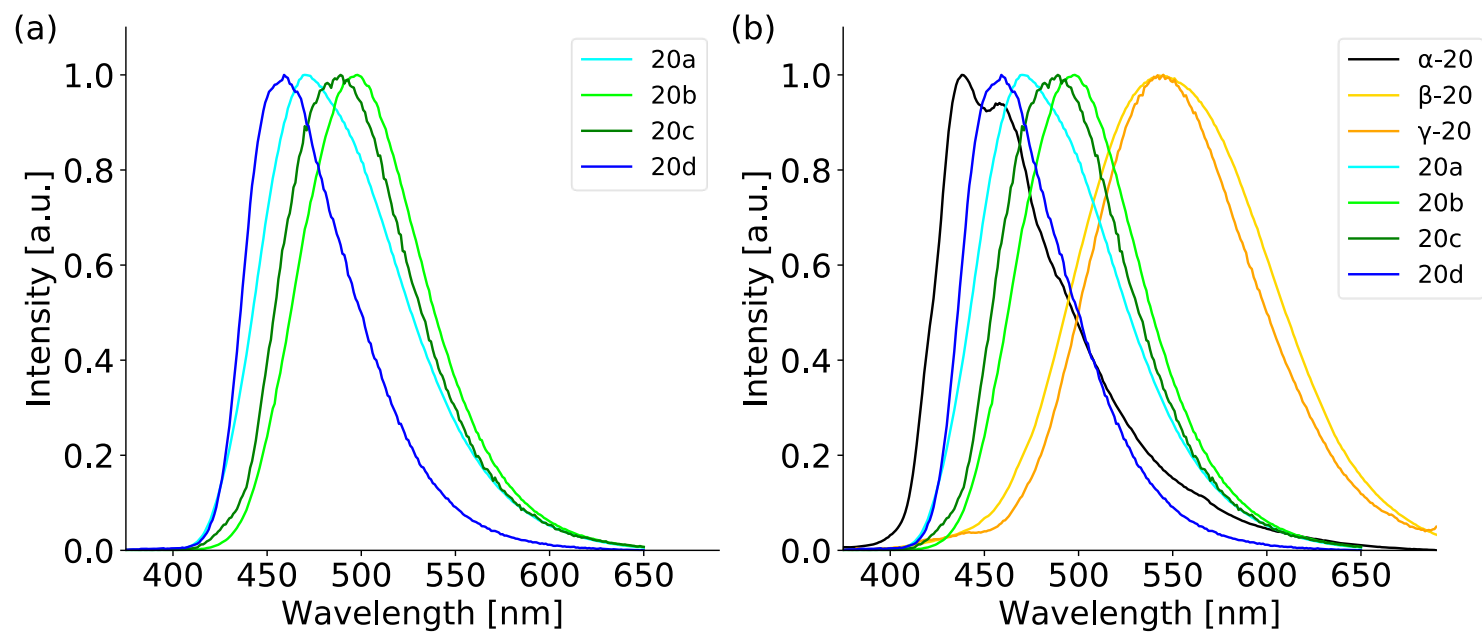

Figure 55. (a) Solid-state emission spectra of co-crystals 20a - 20d and (b) solid-state emission spectra of all polymorphs and cocrystals of [1-(S)PPh$\left.-\left(\mathrm{C}_{14} \mathrm{H}_{9}\right)\right](20)$.

The maximum emission of the co-crystals 20a-20d is observed in the range between 458 and $512 \mathrm{~nm}$, and therefore as expected in between the emission of the polymorphs $\alpha-20$ and $\beta / \gamma-\mathbf{2 0}$ (Figure 55). All four cocrystals reveal also a structureless emission with a broadened emission band. The FWHM are in a range between 64 and $84 \mathrm{~nm}$, and therefore, smaller than the observed broad emission for $\beta / \gamma-20$ (Table 15). It is noteworthy that the emissions for the pyridine and quinoline co-crystals are very weak and the lifetimes are very short, while, for the benzene and aniline derivatives, even higher quantum yields than for the $\beta$ and $\gamma$-polymorph of $\mathbf{2 0}$ are obtained. Also, an increase of the fluorescence lifetime can be observed for 20a and 20c. For the latter co-crystals, an excimer emission can therefore clearly be confirmed. For 20b and $\mathbf{2 0 d}$, the short lifetime speaks against the contribution from an excimer state, while the bathochromic shift and the spectral shape together with the structural properties indicate an excimer formation. The low quantum yields, and short lifetime are therefore probably caused by other non-radiative pathways, which are hard to determine with the obtained data set.

\begin{tabular}{|c|c|c|c|c|c|}
\hline & $\begin{array}{l}\text { co-cryst. } \\
\text { solvent }\end{array}$ & $\lambda_{\max } / \mathrm{nm}$ & $\Phi_{\mathrm{F}} / \%$ & $\tau / \mathrm{ns}$ & FWHM / nm \\
\hline $20 a$ & $\mathrm{C}_{6} \mathrm{H}_{6}$ & 462 & 12.3 & 21.4 & 84 \\
\hline $20 b$ & $\mathrm{C}_{5} \mathrm{H}_{5} \mathrm{~N}$ & 512 & $<1$ & 2.9 & 76 \\
\hline $20 c$ & $\mathrm{C}_{6} \mathrm{H}_{7} \mathrm{~N}$ & 491 & 16.6 & 38.2 & 78 \\
\hline $20 d$ & $\mathrm{C}_{9} \mathrm{H}_{7} \mathrm{~N}$ & 458 & $<1$ & 2.8 & 64 \\
\hline
\end{tabular}

Considering all polymorphs and co-crystals of $\mathbf{2 0}$, an emission range of over $100 \mathrm{~nm}$ is covered (Figure 55). It is remarkable, that for only one structurally simple compound all emissions from blue, green, and yellow color can be observed. The main reason for the shifted emission wavelengths was ascribed to the formation of an excimer. The excimer state can be described as a hybridized local and charger-transfer (HLCT) excited state. With an increasing CT character, the $S_{1}$ state is strongly stabilized resulting in a 
bathochromic shift of the emission wavelength. In recent computational studies, several structural factors have been investigated, which influence the CT contribution to the excimer state of an anthracene modelsystem. ${ }^{[138,221]}$ At first glance, the overlap ratio of the aromatic planes is an important factor. The overlapping area is determined by the offset along the short $\left(d_{x}\right)$ and long $\left(d_{y}\right)$ molecular axes of the two anthracenes. For the perfect cofacial dimer with $100 \%$ overlap, which is usually assumed to be the case in the excimer, both offsets are zero and, only the $\pi-\pi$ distance influences the occurring interactions. In their computational study, Yang et al. have found the minimum energy in the ground state at a $\pi-\pi$ distance of around $3.5 \AA$, which is the typical distance for $\pi-\pi$ interactions in many polyaromatic hydrocarbons. ${ }^{[221]}$ When the distance is decreased, the CT contribution increases and results in a fast stabilization of the $S_{1}$ energy with a minimum at around $3.1 \AA$, while the ground state remains nearly unchanged. A red-shifted emission from the excimer is the consequence. For the hitherto presented co-crystals and polymorphs of 20, the $\pi-\pi$ distances vary from $3.230 \AA$ to $3.357 \AA$, and no direct correlation with the emission wavelength can be found. The $\pi-\pi$ distance of the ground-state seems therefore not to be determining the emission wavelength. The observed $\pi-\pi$ distances in the ground state are probably short enough in all cases to allow the contribution of the CT state to the resulting emission. While evaluating the $\pi-\pi$ distances of earlier reported anthracene excimers, it appears that the distances are in a narrow range of $3.40 \AA$ to $3.58 \AA$, and are not correlated with the emission wavelength. ${ }^{[131,133-135,221]}$ Remarkably, all derivatives of 20 show shorter $\pi-\pi$ distances than for the reported literature-known anthracene excimers. Even if the dataset is small, this could be a hint for the comparatively large bathochromic shift in the solid-state emission.

Considering the overlap, the general trend of an increasing emission wavelength with a larger overlapping area of the anthracenes can clearly be observed (Figure 56a). For the polymorphs and co-crystals of [1(S) $\left.\mathrm{PPh}_{2}-\left(\mathrm{C}_{14} \mathrm{H}_{9}\right)\right]$, the overlap of the anthracene dimers ranges from around $20 \%$ to $45 \%$, and the emission wavelength increases gradually from around $460 \mathrm{~nm}$ to nearly $550 \mathrm{~nm}$. The $\alpha$-polymorph shows no overlap and therefore no excimer character. The emission wavelength of $\alpha-\mathbf{2 0}$ can therefore be considered as the starting point with zero overlap. The emission wavelengths of $\alpha-20$ were determined at 437 and $458 \mathrm{~nm}$. The next longest emission of $462 \mathrm{~nm}$ was observed for $\mathbf{2 0 d}$, which shows an overlap of $22 \%$. Therefore, the increase of the emission wavelength from $0 \%$ to $22 \%$ overlap is rather small, about $25 \mathrm{~nm}$. In comparison, the emission wavelength raises over $80 \mathrm{~nm}$ when the overlap is increased from $22 \%$ (20d) to $46 \%(\gamma-20)$. A clearly linear correlation between the overlap ratio and the emission wavelength over the whole range of the investigated compounds is therefore not likely. Instead, it seems that a threshold exists after which the emission wavelength increases more rapidly. 

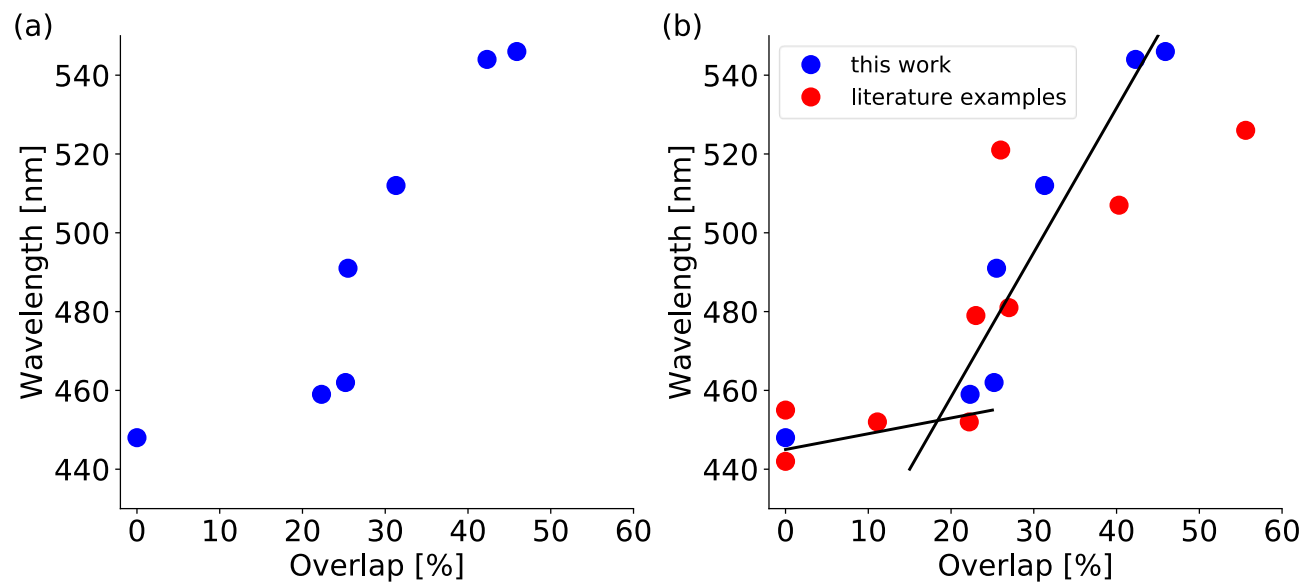

Figure 56. (a) Correlation between the overlap ratio of the anthracene dimers and the emission wavelength in the polymorphs and co-crystals of [1-(S) $\left.\mathrm{PPh}_{2}-\left(\mathrm{C}_{14} \mathrm{H}_{9}\right)\right](20)$ (blue) and (b) in comparison with literature known anthracene excimers ${ }^{4}$ (red). The black lines in the plot are given for clarification and do not represent a fit of the data.

In their computational study, Yang et al. investigated the correlation between the CT contribution to the excited state and the emission wavelength and postulated only a slow increase of the emission wavelength until the $\mathrm{CT}$ contribution to the excited state increased to a value of 0.4 . After this point, the emission wavelength increases more rapidly with increasing CT content. The experimental values obtained in this work can support this hypothesis as the emission wavelength only slightly increases until a critical overlap ratio of around $20 \%$ is reached. For the computationally investigated compound, the CT content of 0.4 correlates with an overlap of $26 \%$, which is in the range of the overlap of $20 \mathrm{~d}$. (22\%).

For a more complete picture, literature known anthracene excimers are also investigated regarding their overlap and emission wavelength (Figure 56b). ${ }^{4}$ The observed trend of the [1-(S)PPh$\left.-\left(\mathrm{C}_{14} \mathrm{H}_{9}\right)\right]$ derivatives stands also when a broader compound range is considered. In the range from $0 \%$ to about $20 \%$ overlap, the emission wavelength is nearly constant and increases only slightly. When the overlap ratio reaches $20 \%$ the emission wavelength increases faster. It has to be noted, that especially at longer wavelengths of over $500 \mathrm{~nm}$ the data points scatter over a broad range of overlaps. Furthermore, the role of the different substituents and their influence on the electronic structure is completely neglected in this picture. The data from the literature was taken from four publications and therefore at least four different substituted anthracene derivatives were examined for the further nine data points. This underlines the benefit of the ability of [1-(S)PPh$\left.-\left(\mathrm{C}_{14} \mathrm{H}_{9}\right)\right]$ to form polymorphs and co-crystal allowing a detailed investigation without changing the fluorophores' electronic structure through different substituents.

A major part of the anthracene excimers reveals an overlap between 22 and $32 \%$. An overlap below $22 \%$ was only observed for one compound (derivatives with no overlap are excluded) and an overlap ratio larger than $32 \%$ was estimated for four compounds, while three are in the region of $40 \%$ and only one above $50 \%$. Nevertheless, a closer look into the parameter, which determine the overlap, can give a more

\footnotetext{
${ }^{4}$ Only anthracene excimers with a given overlap in the publication were investigated. Often no information on the procedure for overlap determination is given. Therefore, deviations in the overlap ratio are possible.
} 
detailed view of the excimer formation process. As pointed out earlier, the overlap ratio of two parallel anthracene scaffolds is determined by the offset along the short $\left(d_{x}\right)$ and long $\left(d_{y}\right)$ molecular anthracene axes. A perfect cofacial dimer with $100 \%$ overlap therefore would show no offset along the axes.

For the polymorphs and co-crystals of $\mathbf{2 0}$, the offsets $d_{x}$ and $d_{y}$ were determined from the crystal structures. As expected, both correlate with the overlap and a decreased offset result in a larger overlap and therefore in an increased emission wavelength. The short molecular axis is around $2.8 \AA$ long and this value represents the maximum offset for obtaining a measurable overlap. For larger offsets, the anthracene scaffolds would not overlap anymore. The observed offsets for the polymorphs and co-crystals of 20 range from around $0.9 \AA$ to nearly $1.4 \AA$ and are nearly uniformly distributed over this range (Figure 57a). The long anthracene axis extends over $7.3 \AA$ and therefore a wider range of observable offsets are possible. For the investigated compounds, offsets from $1.0 \AA$ to $3.2 \AA$ are found, which can be further divided into two subgroups (Figure $57 \mathrm{~b}$ ). The $\beta$ - and $\gamma$-polymorph with the largest overlap reveal the smallest offsets between $1.0 \AA$ and $1.4 \AA$. For the co-crystals $20 \mathrm{a}-\mathbf{2 0 d}, \mathrm{d}_{\mathrm{y}}$ values in the range from $2.7 \AA$ to $3.2 \AA$ are obtained. None of the structures reveal offsets with values in between, and the question arises whether these offsets are in any way disadvantageous. A possible explanation could be the proximity of the ground state to the excimer geometry in the excited state. For the two polymorphs with the short $d_{y}$ offset, the crystal structure reveals that all three six-membered rings of the anthracene are participating in the $\pi-\pi$ interactions between the dimer. For the co-crystals, only two of the rings are involved while the third ring seems to be unaffected by the $\pi$-interactions.
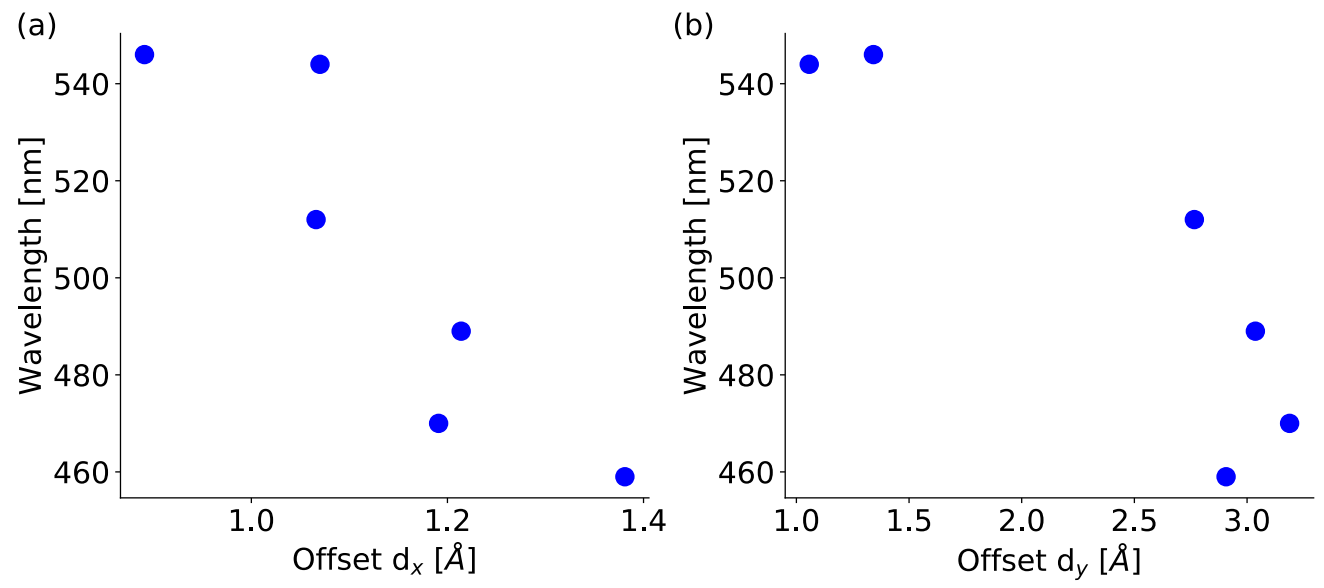

Figure 57. (a) Plot of the offset along the short molecular axis $d_{x}$ and (b) plot of the offset along the long molecular axis $d_{y}$ against the emission wavelength.

As evaluated in the theoretical description of the excimer formation, it is assumed, that the excimer adopts a nearly perfect cofacial geometry (chapter 1.3.2). Therefore, upon excitation, the offsets in all three directions will decrease to form the excimer. The obtained data, with clearly different emission wavelengths, indicate that not always the same excimer species is formed from the different polymorphs and co-crystals. From the ground-state geometry, it rather can be assumed that two different types of excimers are formed. The two polymorphs $\beta$ - and $\gamma$-20 will most likely form an anthracene-type excimer, 
where all three rings of the anthracenes are involved. The contribution of all three rings leads to an enhanced stabilization of the excited state and therefore to the observed large bathochromic shift (Figure 58). The co-crystals 20a-20d with a significant larger $d_{y}$-offset reveal non-ideal preconditions to form an anthracene-like excimer. For an efficient overlap of all rings, a large decrease of the offset $d_{y}$ is required after excitation. Instead, it is supposed that a naphthalene-like excimer geometry with only two rings of each anthracene contributing is favored in the excited state. Therefore, the excimer stabilization energy is less pronounced, and the bathochromic shift is slightly weaker. The observed offsets $d_{y}$ in the structures of $\mathbf{2 0}$ are consequently preferred for the formation either of an anthracene- or a naphthalene-type excimer.

(a)
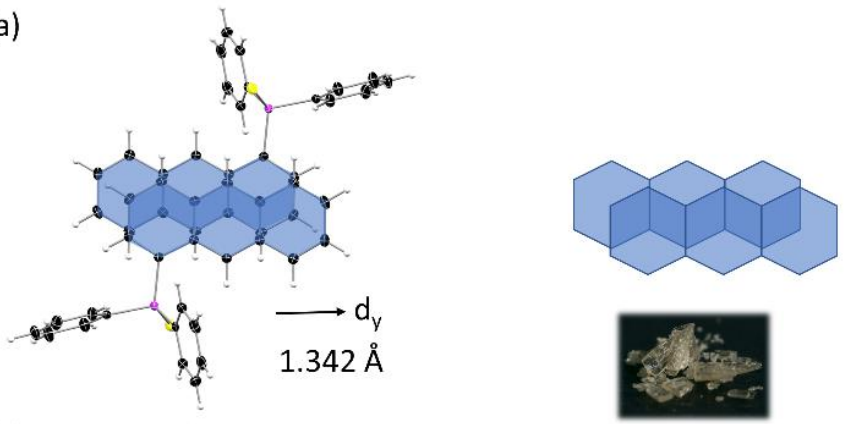

Anthracene-type excimer

(b)
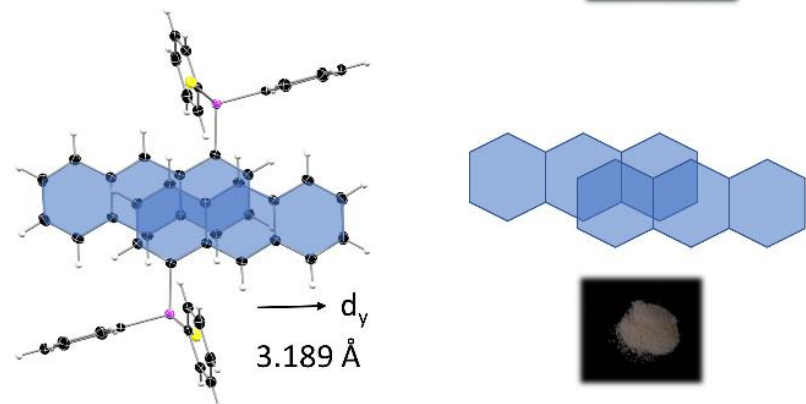
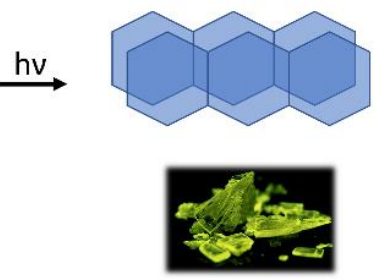

Naphtalene-type excimer
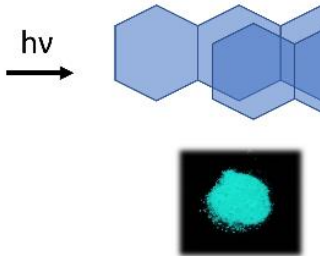

Figure 58. (a) Dimeric structure of $y-20$ with a small $d_{y}$ offset and all three rings of the anthracene interacting resulting in an anthracene-type excimer and (b) dimeric structure as found in 20a with a larger offset $d_{y}$ and only two rings involved in the $\pi$ interactions. After excitation, a naphthalene-type excimer is formed.

The investigation of the polymorphs and co-crystals of $\mathbf{2 0}$ regarding their structural and photophysical properties gave detailed insight in the factors determining the excimer-formation in the solid-state for this compound class. With their structural variety, the clear dependence of the emission wavelength from the overlapping area of the involved anthracenes could be shown. Through the shift of the substituent to the more uncommon 1-position of the anthracene, the overlap ratio was enlarged and an unusual yellow solidstate emission from an anthracene derivative was achieved. As shown in this and previous chapters, cocrystallization with small molecules is a suitable strategy to obtain further derivatives of one compound with varying intermolecular interactions. As the solvent and further non-covalent interactions besides the $\pi-\pi$ interactions seem not to affect the emission wavelengths directly, a wide range of solvents could be used for the investigation of further compounds. 
In general, luminescent co-crystals are also potential candidates for mechanochromic behavior. Several compounds are known, which change their emission behavior upon external stimuli like grinding, heating, fuming or under reduced pressure. ${ }^{[222]}$ For the polymorphs and co-crystals of $\mathbf{2 0}$ similar behavior could not be observed. Emission properties are only marginally affected upon grinding of solid samples. A transformation between the different polymorphs upon heating or grinding could not be observed. Cocrystal formation upon fuming samples of $\alpha-20$ or $\beta-20$ with benzene was also not successful and the emission wavelength did not change after the fuming process. The only response to an external stimulus was observed upon removing the co-crystallized solvent of 20a under reduced pressure at higher temperatures. The release of the solvent results in a yellow emission, which corresponds to the formation of either the $\beta$ - or $\gamma$-form of $\mathbf{2 0}$. The process does not seem to be reversible as fuming of the obtained powder with benzene did not lead to a hypsochromic shift of the emission wavelength. As harsh conditions are required for removal of the solvent, it cannot be excluded that a recrystallization occurs during this process. A pure mechanochromic behavior could therefore not be confirmed.

\subsubsection{Investigation of [1-(S)PPh $\left.-4-E t-\left(\mathrm{C}_{14} \mathrm{H}_{8}\right)\right]$}

\section{Synthesis and Structural Properties}

The investigation of the thiophosphoryl anthracene [1-(S)PPh $\left.-\left(\mathrm{C}_{14} \mathrm{H}_{9}\right)\right](20)$ demonstrated impressively the structure-property relationship regarding the solid-state emission. Two polymorphs of $\mathbf{2 0}$ revealed an unusual large bathochromic shift of the emission wavelength in the solid-state. It was attributed to the excimer formation of the anthracene fluorophore due to a large overlap and due to the resulting strong $\pi$ - $\pi$-interactions. The large structural variety allowed a detailed insight into the factors determining the excimer formation.

In chapter 3.1.3, the 9,10-substituted thiophosphoryl anthracenes were investigated. Even if an excimer emission could not be verified, valuable information regarding the intermolecular interactions could be gained. The introduction of alkyl substituents in the 10-position of the thiophosphoryl anthracene led to an increase of the overlapping anthracene area. For example, the [9-(S)PPh$\left.-10-E t-\left(\mathrm{C}_{14} \mathrm{H}_{8}\right)\right](15)$ derivative revealed a doubled overlap compared to the in 10-position unsubstituted [9-(S)PPh$\left.{ }_{2}-\left(\mathrm{C}_{14} \mathrm{H}_{9}\right)\right]$ (8). Furthermore, the crystal packing changed drastically and allowed co-crystallization of small aromatic molecules. Therefore, alkyl-substitution of the anthracene seems a suitable strategy for varying the electrostatic potential of the anthracene core, which can result in increased $\pi$-stacking interactions.

For this reason, [1-(S)PPh $\left.-\left(\mathrm{C}_{14} \mathrm{H}_{9}\right)\right]$ (20) should be substituted with an ethyl group in the 4-position to obtain a para substitution motif comparable to the 9,10-derivatives. [1-(S)PPh ${ }_{2}-\left(\mathrm{C}_{14} \mathrm{H}_{9}\right)$ ] already revealed a large overlap ratio with up to $45 \%$, without a second substituent present on the anthracene. By introduction of the ethyl group into the 4-position, the overlapping area of the anthracenes may be further increased. The ability of co-crystallization should also be investigated to gather further data points for validation of the previously introduced hypotheses. Moreover, substitution of the 4-position could prohibit edge-to-face interactions, which were observed for $\beta$ - and $\gamma$-20. More isolated dimers were assigned to be responsible for enhanced excimer emission in recent studies. ${ }^{[133-135]}$ 
The synthesis of [1-(S)PPh$\left.-4-E t-\left(\mathrm{C}_{14} \mathrm{H}_{8}\right)\right]$ (24) was similar to the previously described [9-(S)PPh$-10-E t-$ $\left(\mathrm{C}_{14} \mathrm{H}_{8}\right)$ ] and started from 1,4-dibromoanthracene ${ }^{5}$. Introduction of the ethyl group was achieved via selective mono-lithiation with $n \mathrm{BuLi}$ and subsequent addition of ethyl iodide (Scheme 29). The obtained brown-yellow oil was used without further purification. Lithium-halogen exchange of the second bromine and addition of chlorodiphenylphosphine afforded the phosphine, which was oxidized with elemental sulfur according to the already described procedure. The target compound [1-(S)PPh $\left.2-4-E t-\left(\mathrm{C}_{14} \mathrm{H}_{8}\right)\right]$ (24) was obtained as a yellow solid and could be recrystallized from common organic solvents. NMRspectroscopic parameters are as expected with a ${ }^{31} \mathrm{P}-\mathrm{NMR}$ shift of $42.3 \mathrm{ppm}$, which is similar to the observed shift of 20.
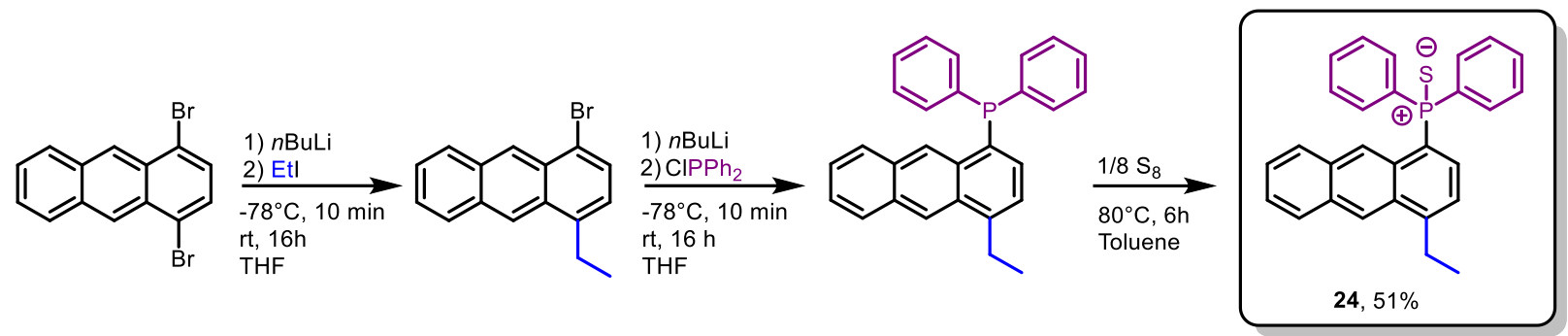

Scheme 29. Synthesis of [1-(S)PPh$\left.-4-E t-\left(C_{14} \mathrm{H}_{8}\right)\right]$ (24) from 1,4-dibromoanthrace via the established route.

Several crystallization approaches in different solvents were performed and, again, a variety of solid-state structures could be obtained. Diffusion of $n$-hexane into a solution of $\mathbf{2 4}$ in toluene afforded the pure structure without any co-crystallized solvent. The compound crystallizes in a monoclinic crystal system in the space group $P 2_{1} / \mathrm{n}$ with one molecule in the asymmetric unit. The general structural motif is comparable to the polymorphs of [1-(S)PPh$\left.-\left(\mathrm{C}_{14} \mathrm{H}_{9}\right)\right](20)$ and only small differences can be determined. The anthracene core is slightly bent and not completely planar (folding angle $\alpha=7.5^{\circ}$ ). Furthermore, the orientation of the thiophosphoryl substituent is slightly changed and the S-P-C1-C9A torsion angle is decreased to $47.79^{\circ}$ (Table 16). The ethyl group in 4-position adopts a more orthogonal orientation considering the anthracene plane with a torsion angle of $72.6(3)^{\circ}$ and is similar to the orientation of the ethyl group in [9-(S)PPh 2 -10-Et- $\left.\left(\mathrm{C}_{14} \mathrm{H}_{8}\right)\right]$.

Crystallization of $\mathbf{2 4}$ from hot saturated solutions of common organic solvents afforded a plethora of cocrystals. Besides small aromatic molecules, which have been favored to co-crystallize with [9-(S) $\mathrm{PPh}_{2}-10-$ Et- $\left.\left(\mathrm{C}_{14} \mathrm{H}_{8}\right)\right]$ (15) and [1-(S)PPh$\left.-\left(\mathrm{C}_{14} \mathrm{H}_{9}\right)\right](20)$, also non-aromatic solvents like cyclohexane, EtOAC or DCM co-crystallize with 24. For [1-(S) $\mathrm{PPh}_{2}-\left(\mathrm{C}_{14} \mathrm{H}_{9}\right)$ ] (20) a plethora of different structures were obtained and only two co-crystals were found to be isomorphous. The ethyl-substituted derivative $\mathbf{2 4}$ reveals less diversity and the obtained co-crystals can be divided into two groups, which members can be considered as isomorphous (Table 16).

\footnotetext{
${ }^{5}$ The preparation of the starting material 1,4-dibromoanthracene will be described in chapter 3.2.2.1.
} 
Table 16. Structural and intermolecular interactions parameters of 24 and its co-crystals 24a-24c.

\begin{tabular}{|c|c|c|c|c|}
\hline & 24 & $24 a$ & $24 b$ & $24 c$ \\
\hline Space group & $P 2_{1} / \mathrm{n}$ & $C 2 / c$ & $C 2 / \mathrm{c}$ & $C 2 / \mathrm{c}$ \\
\hline $\begin{array}{l}\text { Co-crystallized } \\
\text { solvent }\end{array}$ & - & benzene & toluene & chlorobenzene \\
\hline \multirow[t]{4}{*}{ Unit cell } & $a=11.711(2) \AA$ & $a=16.448(2) \AA$ & $a=16.506(2) \AA$ & $a=16.557(2) \AA$ \\
\hline & $b=10.962(2) \AA$ & $b=14.176(2) \AA$ & $b=14.293(2) \AA$ & $b=14.273(2) \AA$ \\
\hline & $c=17.881(3) \AA$ & $c=21.798(3) \AA$ & $c=21.854(3) \AA$ & $c=21.811(3) \AA$ \\
\hline & $\beta=109.63(2)^{\circ}$ & $\beta=109.73(2)^{\circ}$ & $\beta=109.93(2)^{\circ}$ & $\beta=109.69(2)^{\circ}$ \\
\hline$\omega(S-P-C 1-C 9 A) /{ }^{\circ}$ & $47.79(18)$ & $58.09(12)$ & $56.98(13)$ & $57.04(15)$ \\
\hline Overlap / \% & 38.5 & 34.0 & 33.7 & 33.4 \\
\hline $\mathrm{d}_{\pi-\pi} / \AA$ & 3.34 & 3.44 & 3.45 & 3.47 \\
\hline$d_{x} / \AA$ & 1.06 & 0.97 & 0.98 & 0.98 \\
\hline$d_{y} / \AA$ & 1.47 & 2.88 & 2.87 & 2.90 \\
\hline
\end{tabular}

When small aromatic solvents like benzene (24a), toluene (24b) and chlorobenzene (24c) are used for crystallization, the compound tends to crystallize in the monoclinic space group $C 2 / c$ in a 1:1 ratio. The anthracene moieties are almost planar. The orientation of the thiophosphoryl substituent is nearly identical as found in the co-crystals of 20 with S-P-C $-\mathrm{C}$ torsion angles $\omega$ around $57^{\circ}$ and therefore slightly enlarged compared to the solvent-free structure of $\mathbf{2 4}$. The change of the orientation of the ethyl group is more pronounced and the alkyl-substituent lies now inside the anthracene plane. It is turned away from the anthracene core, probably to minimize steric interactions with the hydrogen atom in 9-position (Figure 59).

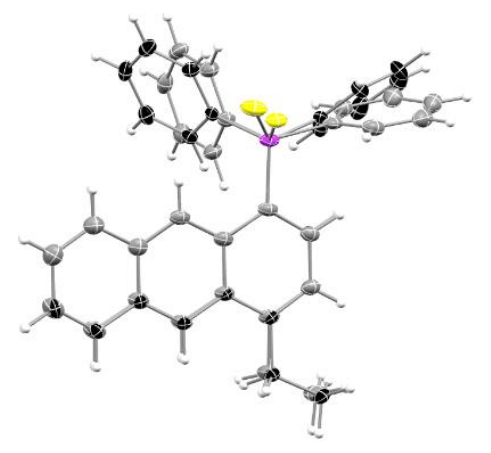

Figure 59. Structural overlay of $\mathbf{2 4}$ crystallized from toluene (grey) and EtOAC (black).
The co-crystallized solvents do not adopt specific positions regarding the anthracene core as seen for the other investigated co-crystals and seem to fill the voids in the crystal structure. These can further explain the wide range of solvents that can co-crystallize with $\mathbf{2 4}$. Nevertheless, a variety of $\mathrm{C}-\mathrm{H} \cdots \pi$ interactions between the arene and the anthracene scaffolds and phenyl groups of neighboring molecules are present, which also influence the interchromophoric interactions between the anthracene moieties.

When cyclohexane (24d) and EtOAc (24e) are used for crystallization, a triclinic crystal system in the space group $P \overline{1}$ with one anthracene moiety and one solvent molecule in the asymmetric unit is preferred (Table 17). An isomorphous structure could also be crystallized through diffusion of $n$-hexane into a solution of $\mathbf{2 4}$ in $\mathrm{CHCl}_{3}$ (24f). The anthracene moiety in these co-crystals is nearly planar and the torsion angle $\omega$ is enlarged up to $70^{\circ}$, which goes along with a slight reorientation of the phenyl groups of the substituent (Figure 59). The position of the ethyl group is nearly identical as found in the co-crystals 24a-24c. It has to be mentioned that benzene was also suitable to afford crystals in the triclinic crystal system with identical cell parameters. The structure could be determined, but no photophysical properties were measured. -108 - 
After storage for a few days, the crystals transitioned to the monoclinic crystal system, which is probably more stable when small arenes are co-crystallized. Further attempts to crystallize the co-crystal in the $P \overline{1}$ space group were not successful. Therefore, only the described structure of $24 a$ will be considered in the upcoming investigations. Co-crystals with the small aliphatic solvent DCM crystallize in a different space group (24g) and a monoclinic crystal system in the space group $P 2_{1} / \mathrm{n}$ is obtained. Probably due to the smaller size of the solvent a 2:1 ratio in the asymmetric unit is adopted and cell parameters differ clearly compared to the structures $\mathbf{2 4 d - 2 4 f . ~ N e v e r t h e l e s s , ~ t h e ~ i n t r a m o l e c u l a r ~ s t r u c t u r a l ~ p a r a m e t e r s ~ c h a n g e ~ o n l y ~}$ slightly, and an overview is given in Table 17.

Table 17. Structural and intermolecular interactions parameters of the co-crystals $\mathbf{2 4 d - 2 4 g}$.

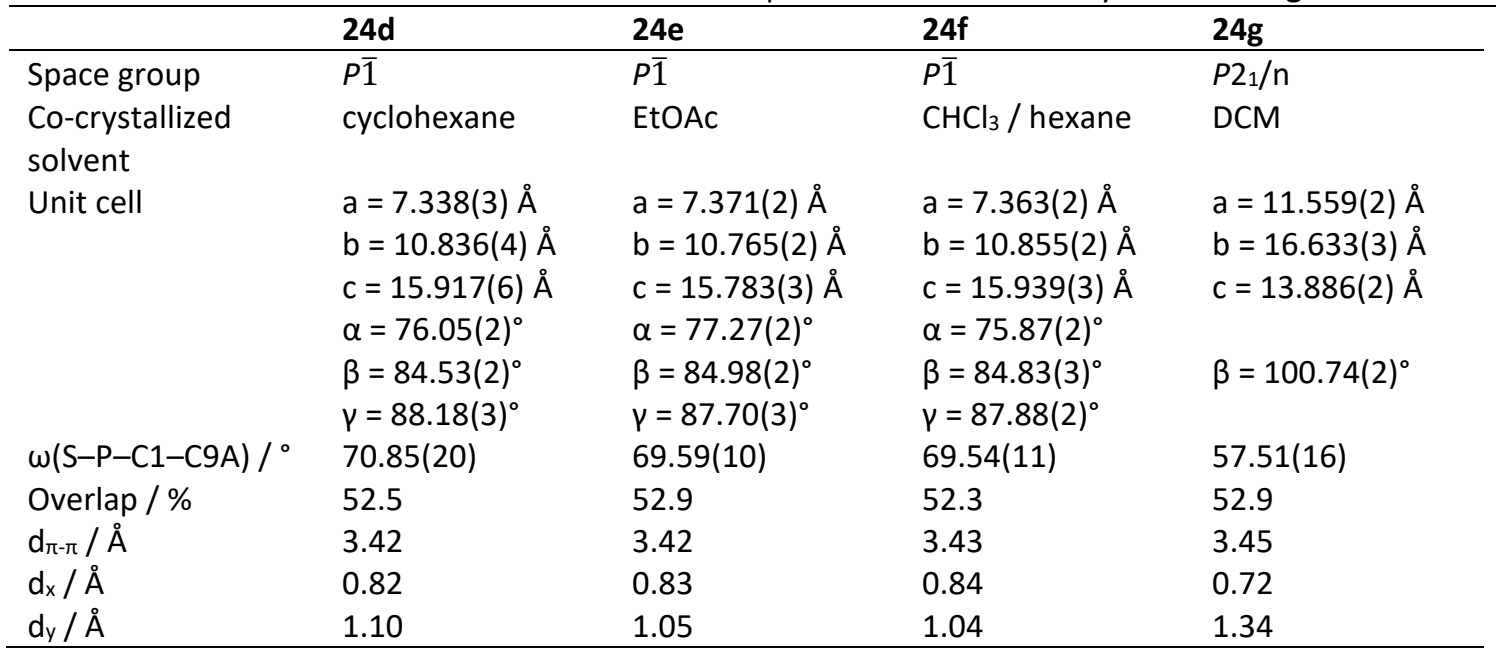

Investigation of the intermolecular interactions revealed small changes in the two groups of co-crystals. The packing motif and occurring interactions are comparable to these of $\mathbf{2 0}$. The dimeric motif with strong $\pi-\pi$ interactions between the anthracene planes is also found in the different forms of $\mathbf{2 4}$. For the pure structure of $\mathbf{2 4}$ without co-crystallized solvent the $\pi-\pi$ distance amounts to $3.34 \AA$ and is similar to the structures of 20. The overlapping area of the anthracene dimer is estimated to $38.5 \%$ and therefore slightly reduced compared to $\beta$ - and $\gamma$-20 (overlap of nearly $46 \%$ ). Increasing the overlap through simple ethyl substitution in para position was therefore not successful.

The $\pi-\pi$ distances for the co-crystals $24 a-24 c$ are slightly elongated to values around $3.45 \AA$. For these structures of the $C 2 / c$ group with aromatic guest molecules, the overlapping area of the anthracene planes is estimated to $33.4-34.0 \%$ and is therefore slightly larger than found for the co-crystals of $\mathbf{2 0}$, which revealed overlaps in the range from $21 \%-32 \%$. As seen for all co-crystals so far, the overlapping area is also decreased in comparison to the pure structure 24 . The offsets, compared to a perfect co-facial dimer, are around $0.98 \AA$ along the short molecular anthracene axis $\left(d_{x}\right)$ and around $2.87 \AA$ along the long axis $\left(d_{y}\right)$ (Table 16).

Surprisingly, co-crystals of the $P \overline{1}$ group, which mainly consist of non-aromatic guests, reveal an increased overlapping area of the anthracene dimer, and differ from the herein discussed co-crystals. The overlap 
ratio was estimated to around 52.3-52.9\%, which is the highest value obtained during this work. Cocrystallization of $\mathbf{2 4}$ with non-aromatic solvents seems to enlarge the interchromophoric interactions. Therefore, offsets are also shortened to around $0.82 \AA\left(d_{x}\right)$ and $1.05 \AA\left(d_{y}\right)$. When comparing the two groups of structures, it is assumed that the occurring $\mathrm{C}-\mathrm{H} \cdots \pi$ interactions between the co-crystallized arenes and the thiophosphoryl anthracene reduce the direct $\pi-\pi$ interactions between the anthracene dimers. When aliphatic guests are in the solid-state structure $(\mathbf{2 4} \mathbf{d}-\mathbf{2 4} \mathbf{g})$, the $\pi-\pi$ interactions are less affected, as they cannot be compensated by $\mathrm{C}-\mathrm{H} \cdots \pi$ contacts between the guest and the thiophosphoryl anthracene.

Even if the guest free structure of $\mathbf{2 4}$ could not confirm the expected overlap enlargement due to ethylsubstation, an overlap increase was achieved for the co-crystals with non-aromatic solvents. The occurring intermolecular interactions of $\mathbf{2 4}$ and its co-crystals are comparable to the investigated [1-( $\mathrm{S} \mathrm{PPh}_{2}-\left(\mathrm{C}_{14} \mathrm{H}_{9}\right)$ ] and should therefore be suitable for excimer formation. As the overlap ratio is further increased, stronger $\pi-\pi$ interactions between the anthracene dimers are expected and could lead to a further bathochromic shift of the emission wavelength. Therefore, the examination of the photophysical properties will be promising, as it may confirm the hypothesis concerning the structure-property relationship and the excimer formation factors.

\section{Photophysical Properties}

The absorption and emission spectra of [1-(S)PPh $\left.2-4-E t-\left(\mathrm{C}_{14} \mathrm{H}_{8}\right)\right](24)$ in diluted solution are comparable to the prior discussed derivatives. A vibronic band from the $\pi-\pi^{*}$ absorption is obtained and located between 340 and $400 \mathrm{~nm}$ (Figure 60a). The UV-Vis spectrum is similar to [1-(S)PPh $\left.-\left(\mathrm{C}_{14} \mathrm{H}_{9}\right)\right]$ and only a minor bathochromic shift can be observed, which is probably induced by the inductive effect of the ethyl group. The emission spectrum reveals a structured emission band in the blue region of the spectrum and is overall comparable to [1-(S)PPh $-\left(\mathrm{C}_{14} \mathrm{H}_{9}\right)$ ] and [2-(S)PPh${ }_{2}-\left(\mathrm{C}_{14} \mathrm{H}_{9}\right)$ ] (Figure 60a). As expected, the photophysical properties in solution are again dominated by the anthracene scaffold and only marginal changes are induced by the ethyl group.

In the solid-state, the emission is affected clearly by the intermolecular interactions between the anthracene chromophores. As the isomorphous co-crystals reveal nearly identical interactions, also similar photophysical properties are expected. Indeed, the emission spectra of the isomorphous compounds are very similar and will be discussed as the whole group. The complete photophysical data can be found in Table 18. 

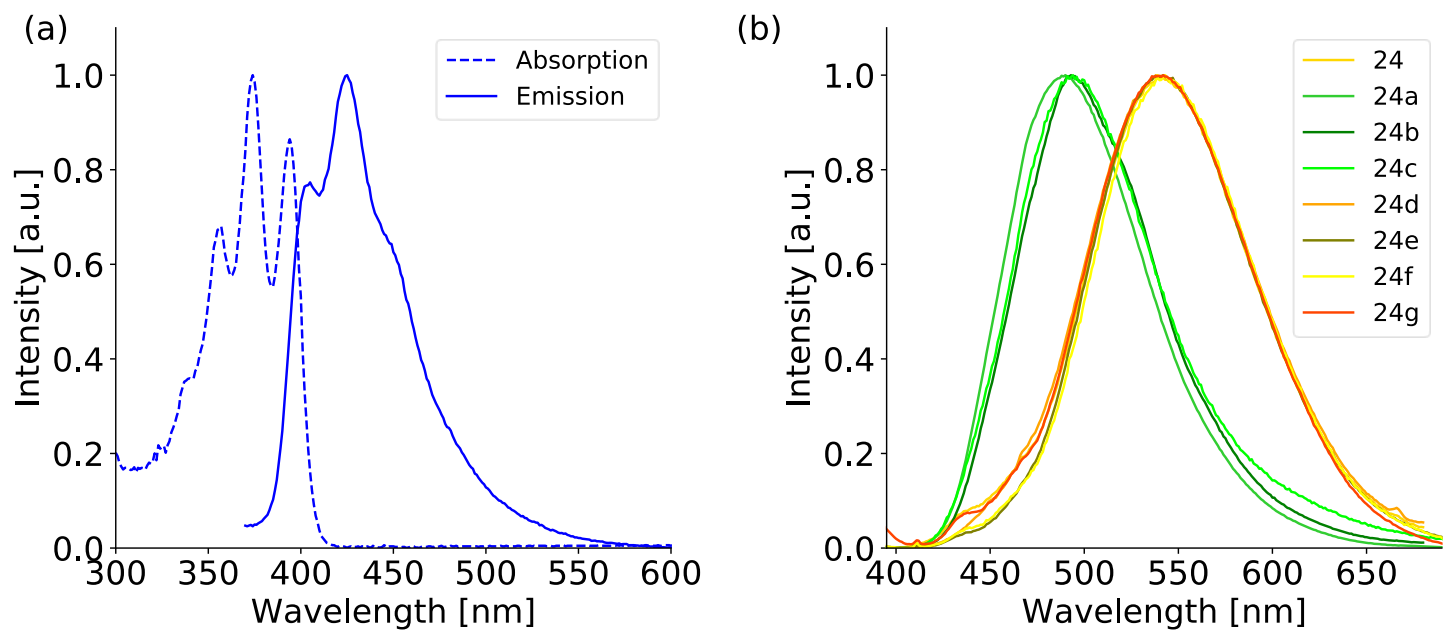

Figure 60. (a) UV-Vis absorption and emission spectra of [1-(S)PPh$\left.-4-E t-\left(\mathrm{C}_{14} \mathrm{H}_{8}\right)\right]$ (24) in diluted THF solution (10-5 $\left.\mathrm{M}\right)$ and $(\mathrm{b})$ emission spectra of $\mathbf{2 4}$ and its co-crystals in the solid-state $\left(\lambda_{\mathrm{ex}}=375 \mathrm{~nm}\right)$.

The pure structure of $\mathbf{2 4}$ without co-crystallized solvent shows a broad emission band with a maximum at $539 \mathrm{~nm}$ (Figure 60b), which is slightly blue-shifted compared to the $\beta$ - and $\gamma$-polymorph of $\mathbf{2 0}$, which goes along with the slightly decreased overlap. The lifetime of $16.4 \mathrm{~ns}$ is also shorter, but still slightly elongated to the lifetime in solution and therefore the emission is assigned to an excimer emission.

The co-crystals 24a-24c with a co-crystallized arene reveal a broad structureless emission, which reaches a maximum at around $490 \mathrm{~nm}$ in the green region of the spectrum. The obtained lifetimes are in a nanoseconds range. Even if the quantum yields are slightly lower the emission properties are related to the co-crystals of [1-(S)PPh $\left.{ }_{2-}\left(\mathrm{C}_{14} \mathrm{H}_{9}\right)\right]$ 20a-22d.

For the co-crystals $\mathbf{2 4 d - 2 4 g}$ co-crystallized with an aliphatic solvent, the emission spectrum is further broadened and red-shifted. The emission intensity reaches its maximum at wavelengths around $540 \mathrm{~nm}$ $542 \mathrm{~nm}$. The broadening is quantified by the FWHM and displays an increase compared to the co-crystals 24a-24c. Together with the elongated lifetimes the expected excimer emission can be confirmed. Even if the overlap of the anthracene $\pi$-system in $\mathbf{2 4 d - 2 4 g}$ is enlarged compared to $\beta$ - and $\gamma$-20 no further increase of the bathochromic shift is observed. The initial approach of further increasing the emission wavelength by an enlarged overlap is therefore not successful.

\begin{tabular}{|c|c|c|c|c|c|}
\hline & $\begin{array}{l}\text { co-cryst. } \\
\text { solvent }\end{array}$ & $\lambda_{\max } / \mathrm{nm}$ & $\Phi_{F} / \%$ & $\tau / n s$ & FWHM / nm \\
\hline 24 & - & 539 & 2.1 & 16.4 & 101 \\
\hline $24 a$ & $\mathrm{C}_{6} \mathrm{H}_{6}$ & 490 & 3.9 & 6.3 & 86 \\
\hline $24 b$ & $\mathrm{C}_{7} \mathrm{H}_{8}$ & 493 & 4.4 & 8.4 & 80 \\
\hline $24 c$ & $\mathrm{C}_{6} \mathrm{H}_{5} \mathrm{Cl}$ & 493 & 5.4 & 4.8 & 92 \\
\hline 24d & $\mathrm{C}_{6} \mathrm{H}_{12}$ & 542 & $<1.0$ & 13.2 & 104 \\
\hline $24 e$ & $\mathrm{C}_{4} \mathrm{H}_{8} \mathrm{O}_{2}$ & 541 & 2.9 & 25.7 & 102 \\
\hline $24 f$ & $\mathrm{CHCl}_{3} / \mathrm{C}_{6} \mathrm{H}_{14}$ & 542 & 2.2 & 21.7 & 104 \\
\hline $24 \mathrm{~g}$ & $\mathrm{CH}_{2} \mathrm{Cl}_{2}$ & 540 & 2.0 & 26.0 & 101 \\
\hline
\end{tabular}


As the emission wavelength does not increase further with an enlarged overlap, the herein obtained data seem not to fit in the plot of the correlation between overlap and emission wavelength at the first glance. Considering the molecular offsets $d_{x}$ and $d_{y}$ of the dimers, no correlation between the offset $d_{x}$ along the short molecular anthracene axis is present as the offsets are distributed over a broad range (Figure 61b). The offsets $d_{y}$ along the long anthracene axis match very well with the previous obtained data. The offsets of the structures with a large overlap $(\mathbf{2 4}, \mathbf{2 4} \mathbf{d}-\mathbf{2 4 g})$ are in a range from $0.72 \AA$ to $1.34 \AA$ and integrate well in the group of offsets obtained for $\mathbf{2 0}$ (Figure 61c). The same is true for the offsets $d_{y}$ of the co-crystals 24a-24c with a reduced overlap. The values are in a narrow range of $2.87 \AA$ to $2.90 \AA$ and therefore comparable to the co-crystals of $\mathbf{2 0 a - 2 0 d}$. These results further support the hypothesis that in the groundstate different geometries of the anthracene dimers are present, which determine the nature of the excimer formed.

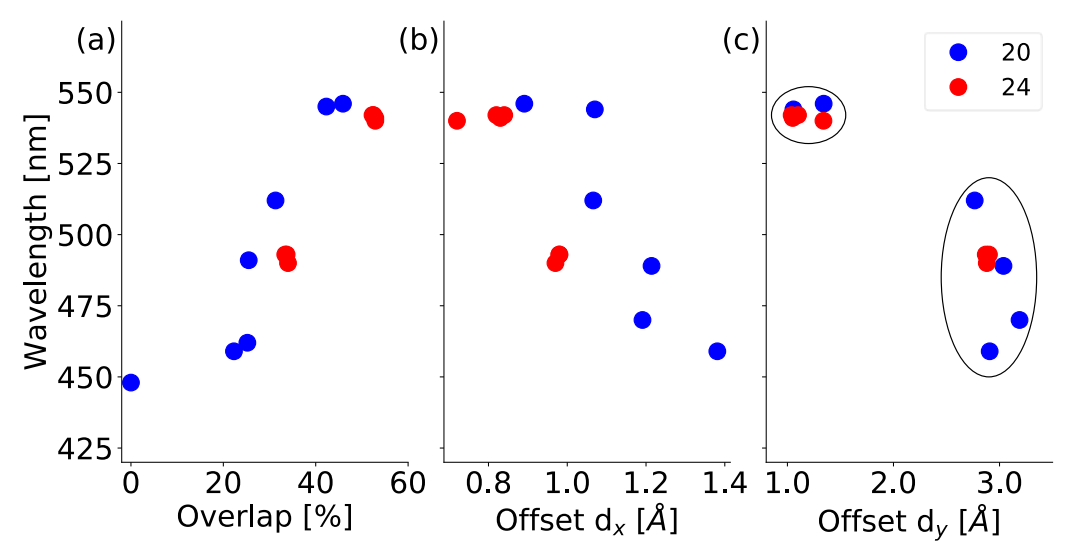

Figure 61. Correlation between (a) anthracene overlap, (b) offset $d_{x}$ along the short anthracene axis and (c) offset $d_{y}$ along the long anthracene axis with the emission wavelength in the solid-state of the polymorphs and co-crystals of $\left[1-(\mathrm{S}) \mathrm{PPh}_{2}-\left(\mathrm{C}_{14} \mathrm{H}_{9}\right)\right](20$, blue) and $\left[1-(\mathrm{S}) \mathrm{PPh}_{2}-4-\mathrm{Et}-\left(\mathrm{C}_{14} \mathrm{H}_{8}\right)\right](\mathbf{2 4}$, red) in the solid-state.

In comparison with the polymorphs and co-crystals of 20, a slightly larger bathochromic shift would have

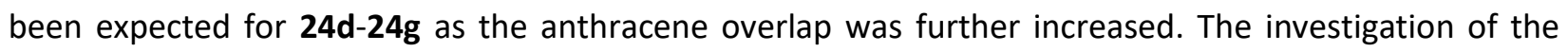
photophysical properties could not confirm this assumption, as the obtained emission wavelengths from 540-542 $\mathrm{nm}$ are in the range of $\beta$ - and $\gamma$-20. The question arises, if further factors influence the excimer formation or if at some point a maximum shift of the emission wavelength is reached. From the obtained results, it is likely that, besides the lower threshold for excimer formation, also an upper limit exists for the increase of the emission wavelength. Considering the values for the overlap ratio and the resulting emission nearly constant values of the emission wavelength are found for an overlap area between $38 \%$ and $53 \%$. At a certain degree of overlap, the emission wavelength probably does not increase further with the increasing overlap. When a suitable ground-state geometry is existent, the molecule is able to form an anthracene-like excimer in the excited state with all three aromatic rings participating. As a result, the $\mathrm{S}_{1}$ state is stabilized due to the excimer binding energy. When the overlap in the ground state is further enlarged beyond a certain value, the stabilization of the $S_{1}$ state does not increase any further, as probably a similar excimer-state is formed. Therefore, comparable emission wavelengths from the same excimer state are obtained even if the overlap is further increased. 
In their computational investigation concerning anthracene excimers, Yang et al. did not comment on an upper limit of the emission wavelength, but their reported data can support the herein postulated hypothesis. ${ }^{[221]}$ As already mentioned, they attributed a higher charge-transfer contribution to the excitedstate to the increased bathochromic shift of the excimer emission. A rapid increase of the emission wavelength was shown for the small range of dimers with a charge transfer content of 0.4 to around 0.45 , which was assigned to anthracene dimers with an overlap in the range of $25 \%$ - $50 \%$. For smaller overlaps, the CT contribution was estimated to 0.4 or lower and only a small increase in the emission wavelength was predicted. This threshold for excimer formation could be confirmed with the herein obtained experimental data. Yang et al. did not explicitly comment on the case when the CT contributions reaches values larger than $0.45 .{ }^{[221]}$ From the calculated data, it can be seen that the CT content increases only slowly for overlaps larger than $50 \%$. Also, for the maximum calculated overlap of nearly $64 \%$ the CT content was found to be around $0.45 .^{[221]}$ These results show that an upper limit probably exists for the increase of the CT content to the excited state. Therefore, it seems likely that the bathochromic shift of the emission wavelength also reaches a maximum at a certain point.

\section{Conclusion}

The synthesis and investigation of the different positional isomers revealed detailed insight into the dependence of the solid-state luminescence properties on the intermolecular interactions. The shift of the thiophosphoryl group from the central anthracene ring (9-position) to the outer anthracene ring (1- and 2-position) could fulfill the expected structural changes. Due to the decreased steric strain in the outer positions, changed orientations of the substituent are obtained. The more flexible position leads to a decrease of the anthracene distortion, and nearly planar aromatic planes could be confirmed by the crystal structure analysis. In contrast to the derivatives with the substituent in 9-position, a structured emission band is found for [1-(S)PPh $\left.-\left(\mathrm{C}_{14} \mathrm{H}_{9}\right)\right]$ and [2-(S)PPh$\left.{ }_{2}-\left(\mathrm{C}_{14} \mathrm{H}_{9}\right)\right]$ in solution. These results give further evidence that the strong distortion of the anthracene core in the 9-substituted derivatives could be responsible for the observed structureless emission both in solution and in the solid-state.

As desired, clear insights into the relationship between the intermolecular interactions and the photophysical properties in the solid-state could be obtained. Already, the two isomers [1-(S)PPh $-\left(\mathrm{C}_{14} \mathrm{H}_{9}\right)$ ] and $\left[2-(\mathrm{S}) \mathrm{PPh}_{2}-\left(\mathrm{C}_{14} \mathrm{H}_{9}\right)\right]$ revealed fundamental differences in their emission properties. The emission spectrum of $\left[2-(\mathrm{S}) \mathrm{PPh}_{2}-\left(\mathrm{C}_{14} \mathrm{H}_{9}\right)\right]$ in the solid-state resembles the emission in diluted solution. As no prominent face-to-face interactions could be identified in the crystal structure, the emission in the solidstate was also assigned to a monomer-like emission. Similar behavior was found for the $\alpha$-polymorph of [1-(S) $\mathrm{PPh}_{2}-\left(\mathrm{C}_{14} \mathrm{H}_{9}\right)$ ], which also revealed no $\pi-\pi$ interactions. In contrast, the $\beta$ - and $\gamma$-polymorph display an unusual yellow emission with emission wavelengths of maximum intensity around $545 \mathrm{~nm}$ and therefore of around $100 \mathrm{~nm}$ longer. A closer look into the solid-state structures exhibits for both forms strong $\pi-\pi$ interactions between two anthracene chromophores with a large overlapping area. Together with the clearly changed spectral shape and increased lifetimes, an excimer formation is proposed for the two polymorphs. Again, co-crystallization with small arenes could alter the resulting intermolecular 
interactions. The overlap of the anthracene planes could be varied over a broad range and a correlation with the emission wavelength could be confirmed.

Together with the co-crystals of [(1-(S)PPh$\left.-4-E t-\left(\mathrm{C}_{14} \mathrm{H}_{8}\right)\right]$ (24), further insights into the excimer formation process were achieved. The threshold for excimer formation postulated by Yang et al. through computational studies, could be confirmed by experimental results. In addition, an upper limit of the bathochromic shift is suggested at which the emission wavelength does not further increase with increasing overlap. The detailed structural investigation revealed two preferred ground-state geometries of the anthracene dimers. For one geometry only two anthracene rings participate in the $\pi-\pi$ interactions, while for the second geometry all three rings contribute to the intermolecular interactions. Accordingly, two different types of excimers are formed upon excitation. The naphthalene-type excimer with two overlapping rings emits in the green region with a moderate bathochromic shift. For the anthracene-type excimer a yellow emission with emission wavelengths up to $545 \mathrm{~nm}$ was identified. These results assume that a further increase of the overlap ratio in the ground-state also leads to the formation of an anthracene-type excimer with a similar stabilization energy of the excited state. 


\subsubsection{Examination of the influence of the substituents position in phosphanyl anthracene regioisomers}

In the previous chapter the synthesis of anthracenes substituted with the diphenylphosphine group in the uncommon 1- or 2-position was introduced. Their sulfur oxidation products revealed interesting luminescence properties with remarkable changes in the emission wavelengths due to excimer formation depending on the occurring intermolecular interactions. During the preparation of the thiophosphoryl anthracenes a solid-state emission of the precursor phosphine [1- $\mathrm{PPh}_{2}-\left(\mathrm{C}_{14} \mathrm{H}_{9}\right)$ ] (18) was observed. In general, phosphines are known to quench fluorescence when substituted to a polyaromatic hydrocarbon. ${ }^{[174,223]}$ This common quenching is referred to a PET from the phosphorous lone pair and was also observed and discussed for the 9,10-substituted diphenylphosphine anthracenes (chapter 3.1.1.3). Therefore, the question arises, if a transfer of the phosphanyl substituent to the outer anthracene ring can restore the fluorescence. This approach will be briefly discussed in the following paragraphs.

Recently, the structural and photophysical properties of a series of $\mathrm{N}, \mathrm{N}$-dialkylaminoanthracenes were investigated. ${ }^{[149,150]}$ With the nitrogen atoms bonded to the anthracene, the compounds are structurally comparable to the phosphanyl anthracenes. The different regioisomers of the dialkylaminoanthracenes revealed drastic changes in their emission properties regarding the emission wavelengths and quantum yields. ${ }^{[149,150]}$ The different conformations of the amino-substituents and the resulting ability of conjugation with the anthracene $\pi$-system were attributed to be responsible for the observed changes. The authors concluded that para substitution of anthracene (and other fluorophores) with electron-donating substituents (like amines) can introduce a strong AlE-behavior, even without the presence of typical rotatable groups or bonds. ${ }^{[149,150]}$

Inspired by these studies and the observation of the solid-state emission of [1-PPh $\left.-\left(\mathrm{C}_{14} \mathrm{H}_{9}\right)\right](\mathbf{1 8})$, further regioisomers of the diphenylphosphanyl anthracene will be synthesized and investigated regarding their emission properties. As the so far investigated phosphanyl anthracenes revealed no prominent intermolecular interactions involving the anthracene, it is assumed that the photophysical properties are dominated by intramolecular factors like the PET from the phosphorous lone pair. The synthesis and investigation of further regioisomers should give additional insights into the underlying processes.

\subsubsection{Synthesis}

According to the described synthesis in the previous chapter dichloroanthraquinones and diaminoanthraquinones are suitable starting materials for the synthesis of the bis(diphenylphosphanyl) anthracene regioisomers.

1,5- and 1,8-dichloroanthracene were prepared according to literature procedures via reduction of the corresponding anthraquinones with zinc in aqueous ammonia solution and obtained in moderate yields (Scheme 30). ${ }^{[211,224]}$ The introduction of the diphenylphosphine group followed the described procedure for [1-PPh $\left.-\left(\mathrm{C}_{14} \mathrm{H}_{9}\right)\right](\mathbf{1 8})$ via nucleophilic substitution with lithiumdiphenylphosphide in refluxing THF. After aqueous work-up the target compounds were obtained in good yields and purity. ${ }^{31} \mathrm{P}-\mathrm{NMR}$ spectra reveal similar chemical shifts as observed for the mono-substituted derivative [1-PPh $\left.-\left(\mathrm{C}_{14} \mathrm{H}_{9}\right)\right]$. The ${ }^{1} \mathrm{H}-\mathrm{NMR}$ 
spectrum of $\left[1,8-\left(\mathrm{PPh}_{2}\right)_{2}-\left(\mathrm{C}_{14} \mathrm{H}_{8}\right)\right]$ (27) reveals a further downfield shift of the H9-atom to $9.82 \mathrm{ppm}$. The coupling to the phosphorous atoms results in a triplet splitting of the resonance $(J=5.3 \mathrm{~Hz})$, which was already reported by Krüger et al. ${ }^{[215]}$ The synthesis of the 1,8-bis(diphenylphosphanyl) anthracene (27) has been reported earlier via different routes as it was used as ligand in a few transition metal complexes. ${ }^{[214,215]}$ The solid-state structure was also determined, but photophysical properties besides the absorption spectrum were not investigated.

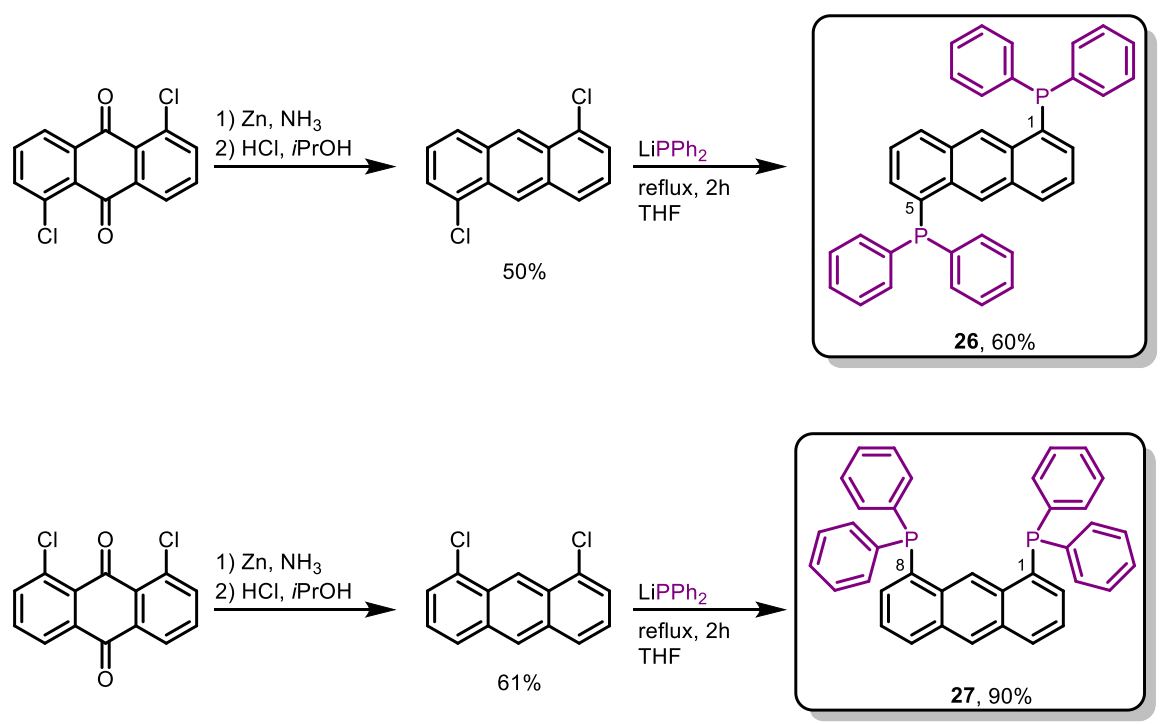

Scheme 30. Synthesis of the two regioisomers [1,5- $\left.\left(\mathrm{PPh}_{2}\right)_{2}-\left(\mathrm{C}_{14} \mathrm{H}_{8}\right)\right](26)$ and [1,8- $\left.\left(\mathrm{PPh}_{2}\right)_{2}-\left(\mathrm{C}_{14} \mathrm{H}_{8}\right)\right](27)$ from the corresponding dichloroanthraquinones via reduction to the dichloroanthracenes and subsequent nucleophilic substitution with lithiumdiphenylphosphide.

The commercially availability of 1,4- and 2,6-chloroanthraquinones is rare. Therefore, the corresponding aminoanthraquinones were used as starting materials for the preparation of 1,4- and 2,6- substituted anthracene derivatives. The transformation to the bromoanthraquinones was straightforward with the Sandmeyer-like procedure as already described for 2-bromoanthraquinone (chapter 3.2.1.1). The reduction of the anthraquinones was more challenging and different procedures were evaluated to obtain the desired 1,4- and 2,6-dibromoanthracenes.

As reduction of the chloroanthraquinones with zinc in aqueous $\mathrm{NH}_{3}$ was straightforward, this method was consequently tested for the bromoanthraquinones. Indeed, the reduction of the anthraquinone was successful but also a debromination occurred and only unsubstituted anthracene could be isolated.

In chapter 3.2.1.1 different literature-known procedures for the reduction of 1-bromoanthraquinone were introduced. Two of them were successfully applied for the preparation of the disubstituted 1,4dibromoanthracene. Stepwise reduction and dehydration of 1,4-dibromoanthraquinone with $\mathrm{NaBH}_{4}$ and $\mathrm{HCl}$ afforded 1,4-dibromoanthracene in $43 \%$ yield. When the reduction was performed with $\mathrm{NaBH}_{4}$ and $\mathrm{SnCl}_{2}$ a slightly higher yield of $65 \%$ was obtained. Therefore, this route was chosen for the preparation of 1,4-dibromoanthracene throughout this work (Scheme 31). Introduction of the diphenylphosphine group was performed via the established way of lithiation and quenching with chlorodiphenylphosphine. 
[1,4-( $\left.\left(\mathrm{PPh}_{2}\right)_{2-}\left(\mathrm{C}_{14} \mathrm{H}_{8}\right)\right](25)$ was obtained in moderate yields of $67 \%$ as a yellow solid, which could be crystallized from toluene. The ${ }^{31} \mathrm{P}-\mathrm{NMR}$ resonance is found at $-13.3 \mathrm{ppm}$ similar to the other isomers with the phosphine group in 1-, 5- or 8-position.

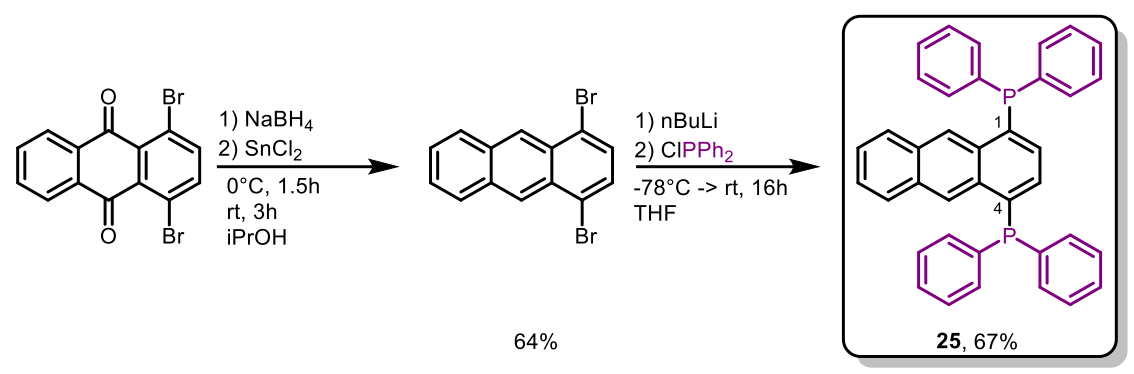

Scheme 31. Reduction of 1,4-dibromoanthraquinone to the corresponding dibromoanthracene and following synthesis of [1,4$\left.\left(\mathrm{PPh}_{2}\right)_{2}-\left(\mathrm{C}_{14} \mathrm{H}_{8}\right)\right](25)$ via the established route.

The reduction of the 2,6-dibromoanthraquinone was more challenging. The previous used methods were not successful and did not afford the 2,6-dibromoanthracene in acceptable yields for further synthetic steps. Another reported procedure for the reduction of anthraquinones makes use of hydroiodic acid and hypophosphorous acid as reducing agents. When this procedure was adopted for the reduction of 2,6dibromoanthraquinone a yellow solid was isolated. The ${ }^{1} \mathrm{H}-\mathrm{NMR}$ spectrum revealed a high purity of the compound, but the resonances could not be assigned adequately to the target compound as apparently ten instead of the expected eight protons were found. Mass spectrometry also could not confirm the successful synthesis of 2,6-dibromoanthracene as a signal at slightly higher $\mathrm{m} / \mathrm{z}$ ratio were found, which goes along with the two additional protons observed in the ${ }^{1} \mathrm{H}-\mathrm{NMR}$ spectrum. Crystallization and X-Ray structure determination could confirm the results of the NMR- and mass spectrometric analysis. The obtained reaction product was identified as 2,6-dibromo-9,10-dihydroanthracene (Figure 62). Obviously, the reaction did not stop at the anthracene level as reported by previous studies and resulted in further reduction to the dihydroanthracene. This observation could be reproduced in several reaction batches.

(a)

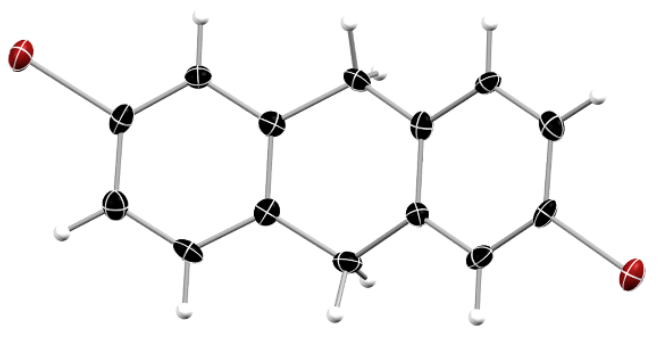

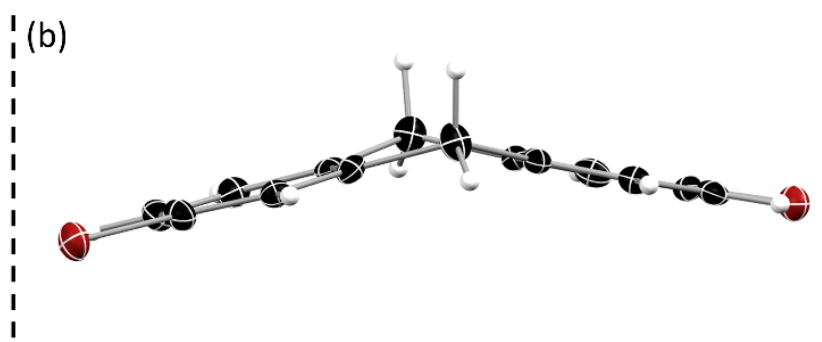

Figure 62. (a) Front view and (b) side view of the solid-state structure of the isolated reaction product 2,6-dibromo-9,10dihydroanthracene obtained through the reduction of 2,6-dibromoanthraquinone.

The desired 2,6-dibromoanthracene could be obtained easily from the dihydroanthracene by oxidation with chloranil. In the last step the diphenylphosphine groups were introduced via the established route and [2,6-( $\left.\left(\mathrm{PPh}_{2}\right)_{2}-\left(\mathrm{C}_{14} \mathrm{H}_{8}\right)\right]$ (28) was isolated as a yellow powder in moderate yields (Scheme 32). Crystallization was performed via slow diffusion of $n$-hexane into a solution of $\mathbf{2 8}$ in benzene. Compared 
to the isomers with the substituent in 1-,4-,5- and 8-position, the ${ }^{31} \mathrm{P}-\mathrm{NMR}$-resonance is shifted slightly downfield to around $-5 \mathrm{ppm}$. The chemical shift is in good accordance with the monosubstituted [2-PPh $2^{-}$ $\left.\left(\mathrm{C}_{14} \mathrm{H}_{9}\right)\right](21)$.
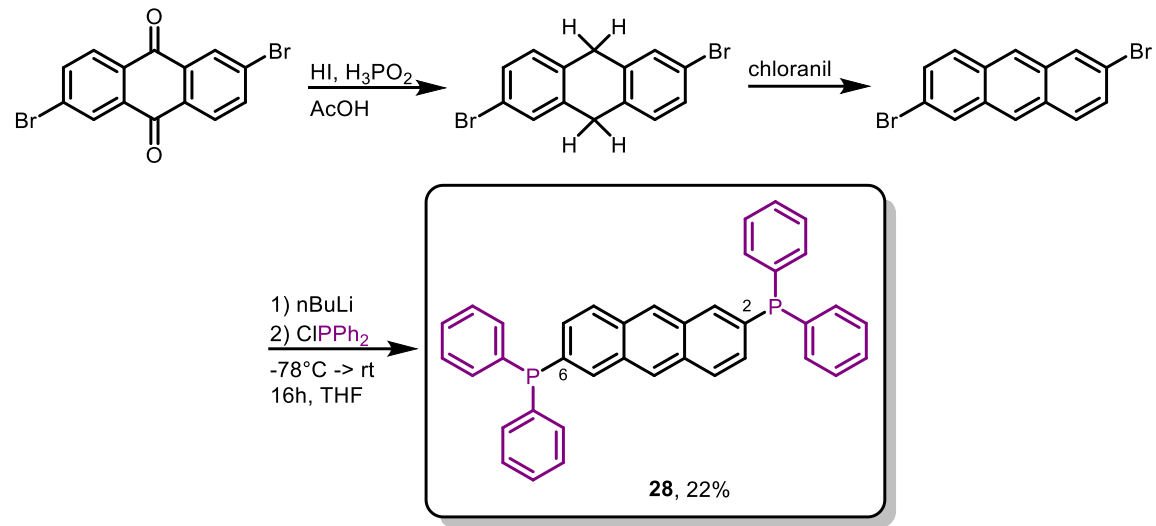

Scheme 32. Synthesis of 2,6-dibromoanthracene and following preparation of [2,6-( $\left.\left(\mathrm{PPh}_{2}\right)_{2}-\left(\mathrm{C}_{14} \mathrm{H}_{8}\right)\right](\mathbf{2 8})$.

\subsubsection{Structural Properties}

The solid-state structures of the synthesized bis(diphenylphosphanyl) anthracenes are in general comparable among each other and with the already discussed mono-substituted isomers [1- $\left.\mathrm{PPh}_{2}-\left(\mathrm{C}_{14} \mathrm{H}_{9}\right)\right]$ (18) and [2- $\left.\mathrm{PPh}_{2}-\left(\mathrm{C}_{14} \mathrm{H}_{9}\right)\right](21)$. The solid-state structures will be discussed briefly and a possible influence of the changed orientation on the photophysical properties will be examined.

[1,4- $\left.\left(\mathrm{PPh}_{2}\right)_{2}-\left(\mathrm{C}_{14} \mathrm{H}_{8}\right)\right](25)$ crystallizes in the triclinic space group $P \overline{1}$ with one molecule in the asymmetric unit. The anthracene is as expected almost planar and the orientation of the phosphanyl group similar as found in [1- $\left.\mathrm{PPh}_{2}-\left(\mathrm{C}_{14} \mathrm{H}_{9}\right)\right](\mathbf{1 8})$. The phosphorous lone pair lies not inside the anthracene plane as seen for the 9,10-substituted derivatives but rotates slightly to a more perpendicular orientation regarding the anthracene scaffold. Furthermore, the two phosphanyl groups are oriented in the same direction with both lone pairs above the anthracene plane. In the well-investigated $\left[9,10-\left(\mathrm{PPh}_{2}\right)_{2}-\left(\mathrm{C}_{14} \mathrm{H}_{8}\right)\right]$ the lone pairs at the phosphorous atoms point in different directions and the phenyl groups are located one above and one below the anthracene plane (Figure 63). ${ }^{[151]}$ 
(a)
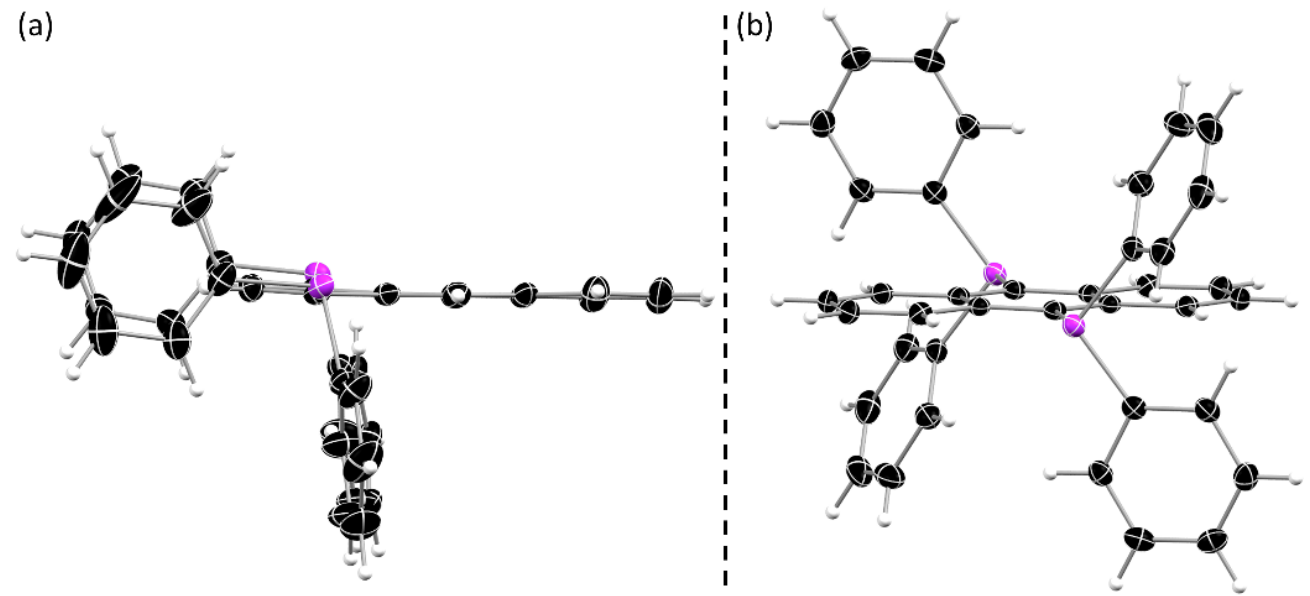

Figure 63. Different orientations of the dipenylphosphine group in (a) $\left[1,4-\left(\mathrm{PPh}_{2}\right)_{2}-\left(\mathrm{C}_{14} \mathrm{H}_{8}\right)\right](25)$ with a cisoid conformation with both phosphorous lone pairs pointing in the same direction and $(\mathrm{b})$ in $\left[9,10-\left(\mathrm{PPh}_{2}\right)_{2}-\left(\mathrm{C}_{14} \mathrm{H}_{8}\right)\right]$ with a transoid conformation and the lone pairs located in the anthracene plane. Crystal structure data for $\left[9,10-\left(\mathrm{PPh}_{2}\right)_{2}-\left(\mathrm{C}_{14} \mathrm{H}_{8}\right)\right]$ was taken from reference. ${ }^{[151]}$

[1,5- $\left.\left(\mathrm{PPh}_{2}\right)_{2}-\left(\mathrm{C}_{14} \mathrm{H}_{9}\right)\right](26)$ crystallizes in the triclinic space group $P \overline{1}$ with only half a molecule in the asymmetric unit. As the second half is generated by an inversion center the molecule adopts a transoid orientation with the phosphorous lone pairs on different sites of the anthracene plane (Figure 64a). The orientation of the phosphanyl group is like in [1- $\left.\mathrm{PPh}_{2}-\left(\mathrm{C}_{14} \mathrm{H}_{9}\right)\right]$ and $\left[1,4-\left(\mathrm{PPh}_{2}\right)_{2}-\left(\mathrm{C}_{14} \mathrm{H}_{8}\right)\right]$ with the lone pairs enclosing an angle of around $45^{\circ}$ regarding the anthracene plane.

The last synthesized isomer with the phosphanyl group in the closely related 1-, 4-, 5-, or 8-position, [1,8$\left.\left(\mathrm{PPh}_{2}\right)_{2}-\left(\mathrm{C}_{14} \mathrm{H}_{8}\right)\right](27)$ adopts a monoclinic crystal system in the space group $P 2_{1} / n$ with one molecule of 27 and one co-crystallized DCM molecule in the asymmetric unit. A cisoid conformation is obtained with both lone pairs on the same site of the anthracene and again not inside the plane (Figure 64b). Minor changes in the orientation of the phenyl groups of each substituent are observed, but the differences are only marginal. The distance between the phosphorous atoms is around 5.0 $\AA$. Deprotonation of C9 leads to a pincer-type ligand, which was used for preparation of its $\mathrm{Ni}, \mathrm{Pd}$, and $\mathrm{Pt}$ complexes as reported by Krüger et al. ${ }^{[215]}$ 
(a)

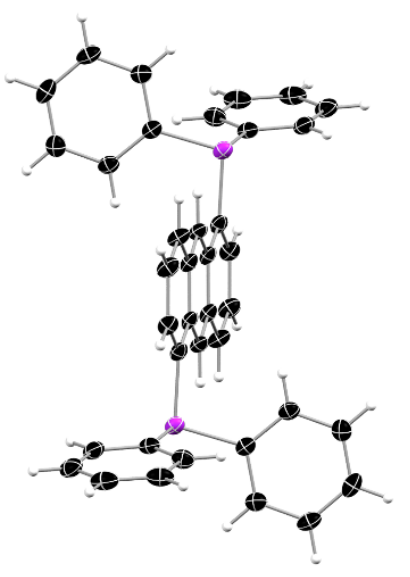

i (b)

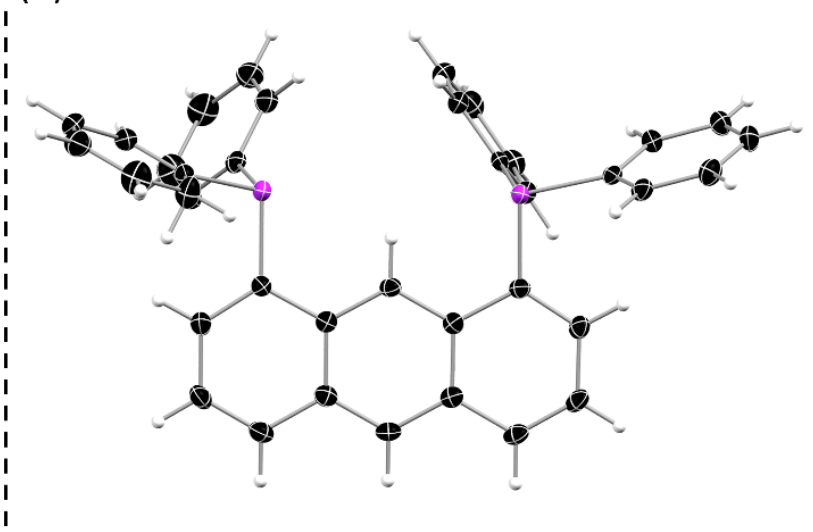

Figure 64. Solid-state structures of (a) $\left[1,5-\left(\mathrm{PPh}_{2}\right)_{2}-\left(\mathrm{C}_{14} \mathrm{H}_{8}\right)\right](26)$ and $(\mathrm{b})\left[1,8-\left(\mathrm{PPh}_{2}\right)_{2}-\left(\mathrm{C}_{14} \mathrm{H}_{8}\right)\right](27)$. Co-crystallized solvent is omitted for clarity.

For the fourth positional isomer [2,6-( $\left.\left.\mathrm{PPh}_{2}\right)_{2}-\left(\mathrm{C}_{14} \mathrm{H}_{8}\right)\right]$ (28) the phosphanyl substituent was introduced in the 2- and 6-position of the anthracene. The obtained compound crystallizes in the triclinic space group $P \overline{1}$ with half a molecule of $\mathbf{2 8}$ and half a molecule of co-crystallized benzene in the asymmetric unit. As the second half is again generated by the inversion center a transoid conformation regarding the substituents is found. Compared to the monosubstituted derivative [2-PPh $\left.-\left(\mathrm{C}_{14} \mathrm{H}_{9}\right)\right](\mathbf{2 1})$ a change in the orientation of the phosphine groups is observed (Figure 65). For the mono-substituted $\mathbf{2 1}$ the phosphorous lone pair is located more inside the anthracene plane and the phenyl groups are located one above and one below the anthracene moiety, while for the di-substituted $\mathbf{2 8}$ a shift into the more orthogonal direction regarding the anthracene scaffold is recognized, similar to the derivatives 25-27.

(a)

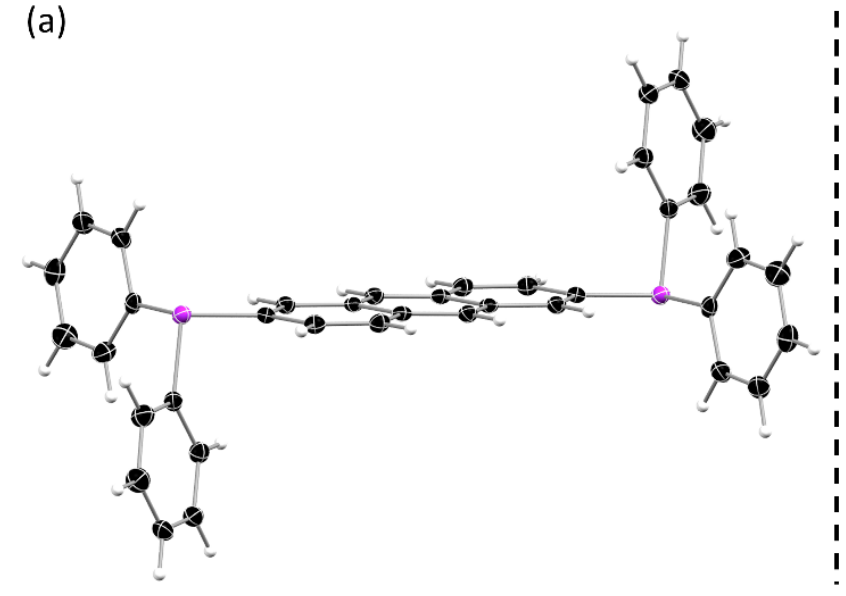

I (b)

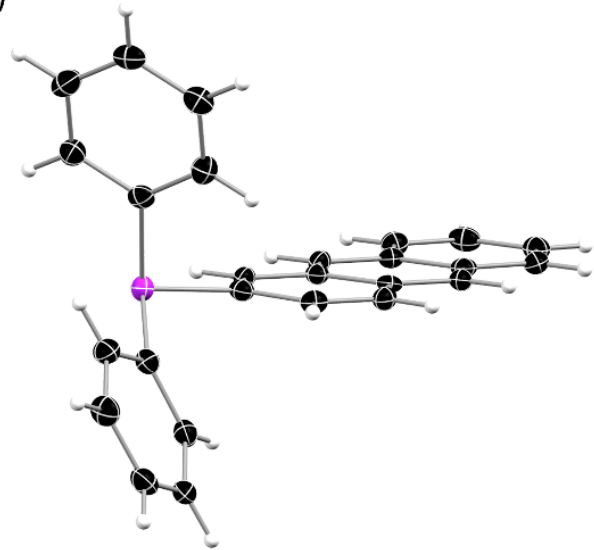

Figure 65. Solid-state structures of (a) [2,6-( $\left.\left.\mathrm{PPh}_{2}\right)_{2}-\left(\mathrm{C}_{14} \mathrm{H}_{8}\right)\right](28)$ and (b) [2- $\left.\mathrm{PPh}_{2}-\left(\mathrm{C}_{14} \mathrm{H}_{9}\right)\right](21)$ revealing a different orientation of the substituent and position of the phosphorous lone pair. Co-crystallized solvent is omitted for clarity.

Bond lengths and angles of the positional isomers are as expected and differ only slightly. As already discussed earlier, a marginal elongation of the $C_{\text {Anth }}-P$ bond is observed for the 9,10-substituted phosphines in comparison to the other compounds, probably induced by the larger steric strain present in the 9,10-position. 
Like in the previously investigated phosphanyl anthracenes, no strong intermolecular interactions between the anthracene moieties are found for most of the bis(diphenylphosphanyl) anthracenes. Only for [1,8-( $\left.\left(\mathrm{PPh}_{2}\right)_{2}-\left(\mathrm{C}_{14} \mathrm{H}_{8}\right)\right]$ (27) $\pi-\pi$ interactions between two anthracenes could be identified. As the overlapping area with around $15 \%$ is very small and only the outer anthracene ring is involved, a strong influence and especially an excimer formation is unlikely. For the other derivatives the two bulky substituents prohibit close contacts between the anthracenes $\pi$-systems and shield the anthracene plane. The overall crystal packing resembles a sheet structure with all anthracene moieties parallelly oriented (Figure 66).

(a)

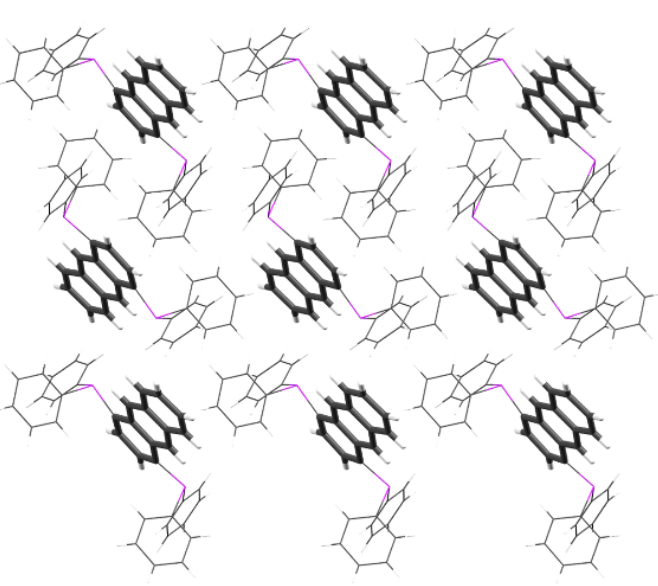

i (b)

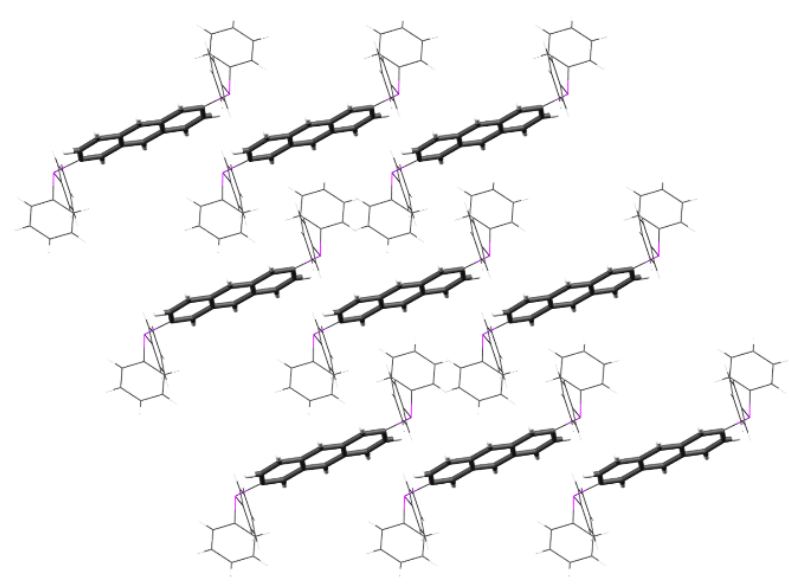

Figure 66. Crystal packing of (a) [1,4-( $\left.\left.\mathrm{PPh}_{2}\right)_{2}-\left(\mathrm{C}_{14} \mathrm{H}_{8}\right)\right](25)$ and (b) [2,6-( $\left.\left(\mathrm{PPh}_{2}\right)_{2}-\left(\mathrm{C}_{14} \mathrm{H}_{8}\right)\right]$ (28) with all anthracene scaffolds parallelly oriented. No face-to-face interactions between the anthracenes are observed. Anthracene moieties are highlighted, and cocrystallized solvent is omitted for clarity.

Overall, the most prominent differences in the solid-state structures of the positional isomers of [PPh $2^{-}$ $\left.\left(\mathrm{C}_{14} \mathrm{H}_{9}\right)\right]$ and $\left[\left(\mathrm{PPh}_{2}\right)_{2}-\left(\mathrm{C}_{14} \mathrm{H}_{8}\right)\right]$ are found in the orientation of the substituents and consequently of the phosphorous lone pair. For the 9,10-derivatives the lone pair is positioned inside the anthracene plane as one phenyl group is located above and one below the anthracene scaffold. The other isomers (except [2$\left.\left(\mathrm{PPh}_{2}-\left(\mathrm{C}_{14} \mathrm{H}_{9}\right)\right]\right)$ revealed a changed orientation with the lone pairs clearly outside the aromatic plane of the anthracene adopting a more orthogonal orientation. This small structural rearrangement can probably influence the observed conjugation in the different phosphanyl anthracenes. The orientation towards a more orthogonal position should be beneficial for a conjugation of the lone pair with the anthracene $\pi$ system. Examination of the photophysical properties can help to investigate this effect and a possible influence on the emission properties.

\subsubsection{Photophysical Properties}

While the absorption properties of the previously investigated 9,10-phosphanyl anthracenes are comparable to the parent anthracene, the emission properties differ strongly. Fluorescence could not be observed neither in solution nor in the solid-state (chapter 3.1.1.3). The quenching of the fluorescence of aromatic phosphines is in general ascribed to the PET from the phosphorous lone pair. Fluorescence in solution can be restored upon oxidation with hydrogen peroxide or coordination of the lone pair to a metal center. ${ }^{[174,223]}$ In both cases the lone pair is not accessible for the PET and a blue emission is observed in 
solution. These properties make phosphines suitable candidates for sensing of reactive oxygen species (ROS) or transition metals, which was shown in several studies. ${ }^{[223,225]}$ Furthermore, aromatic phosphines have been used as ligands in $\mathrm{Cu}(\mathrm{I})$ complexes, which exhibit TADF characteristics. ${ }^{[26]}$ In general, the PET has been used in a wide range of sensing applications in solution. Up to now, investigations regarding a PET of aromatic phosphines in the solid-state and the influence on the emission intensity are barely reported. ${ }^{[227]}$

The absorption spectra of the phosphanyl anthracene regioisomers are overall very similar and comparable to the earlier investigated phosphanyl anthracenes. Two main absorption bands are present, while the higher energy band is located around $280 \mathrm{~nm}$ (Figure 67). For [2,6- $\left.\left(\mathrm{PPh}_{2}\right)_{2}-\left(\mathrm{C}_{14} \mathrm{H}_{8}\right)\right](28)$ a splitting of the signal into two peaks is observed.

The lower energy absorption band lies in the region from 325 to $420 \mathrm{~nm}$ and reveals the typical vibronic structure of the anthracene fluorophore. The isomers with the substituent in 1-, 4-, 5- or 8-position undergo a slightly stronger bathochromic shift compared to [2-PPh $\left.-\left(\mathrm{C}_{14} \mathrm{H}_{9}\right)\right](21)$ and [2,6- $\left.\left(\mathrm{PPh}_{2}\right)_{2}-\left(\mathrm{C}_{14} \mathrm{H}_{8}\right)\right]$ (28). As already mentioned, the lower energy absorption is polarized along the short molecular anthracene axis. Therefore, substituents introduced along the short axis have a stronger influence on the lower energy-absorption. A similar behavior is found for the higher energy absorption, which is polarized along the long anthracene axis. Substituents in the 2- and 6-position of the anthracene have a stronger stabilization effect on the high-energy absorption and result in slightly stronger bathochromic shift of the high-energy absorption.
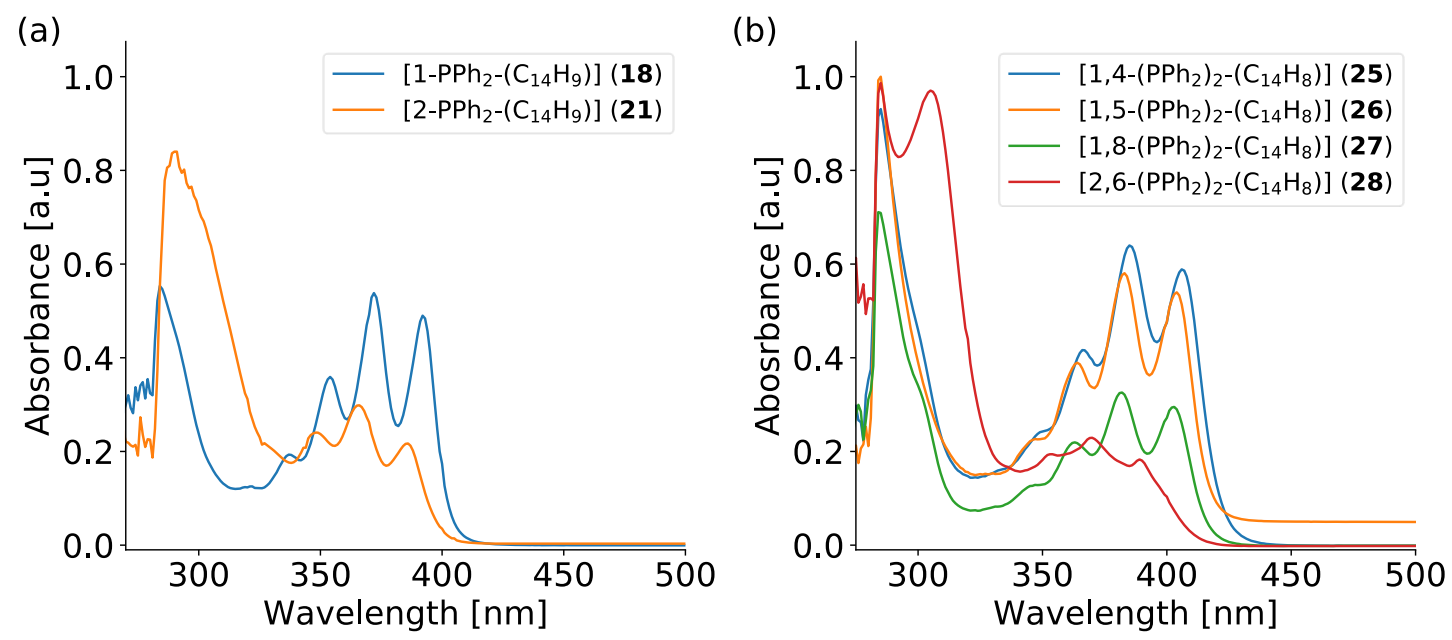

Figure 67. (a) UV-Vis absorption spectra in toluene of the positional isomers [1-PPh $\left.-\left(\mathrm{C}_{14} \mathrm{H}_{9}\right)\right](\mathbf{1 8})$ and $\left[2-\mathrm{PPh}_{2}-\left(\mathrm{C}_{14} \mathrm{H}_{9}\right)\right](\mathbf{2 1})$ and (b) of the disubstituted isomers [1,4- $\left.\left(\mathrm{PPh}_{2}\right)_{2}-\left(\mathrm{C}_{14} \mathrm{H}_{8}\right)\right](25)$, [1,5- $\left.\left(\mathrm{PPh}_{2}\right)_{2}-\left(\mathrm{C}_{14} \mathrm{H}_{8}\right)\right](26)$, [1,8- $\left.\left(\mathrm{PPh}_{2}\right)_{2}-\left(\mathrm{C}_{14} \mathrm{H}_{8}\right)\right](27)$ and $\left[2,6-\left(\mathrm{PPh}_{2}\right)_{2}-\right.$ $\left.\left(\mathrm{C}_{14} \mathrm{H}_{8}\right)\right](28)$.

The emission in solution of all phosphanyl anthracenes is nearly completely quenched. Only after storage of diluted solutions for several hours an emission band arises in the blue region of the spectrum, which is assigned to the oxidation product and was also observed and discussed for [9-PPh $-\left(\mathrm{C}_{14} \mathrm{H}_{9}\right)$ ] (1) (chapter 3.1.1.3). The absence of any luminescence in solution is again ascribed to an efficient quenching from the PET of the phosphorous lone pair. As widely investigated, oxidation of the phosphorous deactivates the - 122 - 
PET and turns on the emission. As mentioned, coordination of the phosphorous lone pair to a metal center will probably have a similar effect but was not investigated during this work.

As photophysical properties in solution are as expected, it is more astonishing, that the situation changes in the solid-state. During the synthesis and workup of [1-PPh $\left.-\left(\mathrm{C}_{14} \mathrm{H}_{9}\right)\right](\mathbf{1 8})$ a weak, greenish emission of the obtained powder was observed under UV-light. Therefore, the emission behavior of all synthesized phosphanyl anthracenes in the solid-state was investigated.

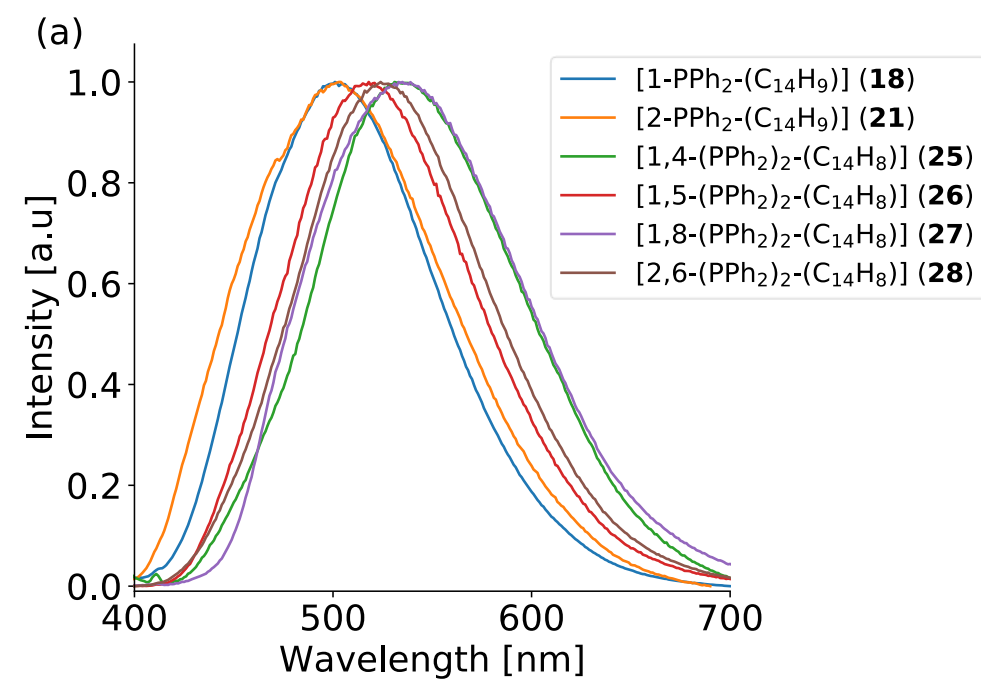

Figure 68. Solid-state emission spectra of the different regioisomers revealed a broad, structureless emission of all isomers with slightly different maximum emission wavelengths.

The solid-state emission spectra of all isomers are overall very comparable. For all fluorescent derivatives a broad, structureless emission band is obtained, which undergoes a bathochromic shift compared to unsubstituted anthracene (Figure 68). The emission wavelengths with maximum intensity are located around $500 \mathrm{~nm}$ for the mono-substituted isomers [1-PPh $\left.-\left(\mathrm{C}_{14} \mathrm{H}_{9}\right)\right]$ (18) and [2-PPh $\left.-\left(\mathrm{C}_{14} \mathrm{H}_{9}\right)\right]$ (21). Introduction of the second diphenylphosphine group results in a further bathochromic shift and the emission of 25-28 peaks in the range from 518 to $533 \mathrm{~nm}$. The obtained lifetimes are within a few nanoseconds and typical for fluorescence emission from the singlet state (Table 19). Even if the obtained quantum yields are only low to moderate, a clear emission from the phosphanyl anthracenes is observed in the solid-state. In contrast, the 9- and 9,10-substituted phosphanyl anthracenes revealed no fluorescence at all, which is the common case for aromatic phosphines.

Table 19. Overview of the solid-state photophysical properties of the investigated positional isomers of $\left[\mathrm{PPh}_{2}-\left(\mathrm{C}_{14} \mathrm{H}_{9}\right)\right]$ and $\left.\left[\mathrm{PPh}_{2}\right)_{2}-\left(\mathrm{C}_{14} \mathrm{H}_{8}\right)\right]$.

\begin{tabular}{llll}
\hline & $\boldsymbol{\lambda}_{\max } / \mathbf{n m}$ & $\boldsymbol{\Phi}_{\mathrm{F}} / \%$ & $\boldsymbol{\tau} / \mathbf{n s}$ \\
\hline$\left[1-\mathrm{PPh}_{2}-\left(\mathrm{C}_{14} \mathrm{H}_{9}\right)\right](18)$ & 501 & 11.1 & 2.3 \\
{$\left[2-\mathrm{PPh}_{2}-\left(\mathrm{C}_{14} \mathrm{H}_{9}\right)\right](\mathbf{2 1})$} & 503 & $<1$ & 2.6 \\
{$\left[1,4-\left(\mathrm{PPh}_{2}\right)_{2}-\left(\mathrm{C}_{14} \mathrm{H}_{8}\right)\right](25)$} & 531 & 4.3 & 1.9 \\
{$\left[1,5-\left(\mathrm{PPh}_{2}\right)_{2}-\left(\mathrm{C}_{14} \mathrm{H}_{8}\right)\right](26)$} & 518 & 8.7 & 3.4 \\
{$\left[1,8-\left(\mathrm{PPh}_{2}\right)_{2}-\left(\mathrm{C}_{14} \mathrm{H}_{8}\right)\right](27)$} & 533 & 4.9 & 2.9 \\
{$\left[2,6-\left(\mathrm{PPh}_{2}\right)_{2}-\left(\mathrm{C}_{14} \mathrm{H}_{8}\right)\right](28)$} & 525 & 7.5 & 4.3 \\
\hline
\end{tabular}


The discussed solid-state structures revealed no face-to-face interactions between the anthracenes $\pi$ systems. Therefore, a strong influence of the intermolecular interactions on the photophysical properties is not likely and the emission is probably determined by intramolecular effects. The obtained lifetimes support this assumption. The shape of the spectra and location of the emission maxima is comparable to the reported emission spectra of the $N, N$-dialkylamino anthracenes. ${ }^{[149,150]}$ For the aminoanthracenes a twisted-intra-molecular charge transfer (TICT) mechanism was postulated by Sasaki et al. to determine the photophysical properties. ${ }^{[149,150]}$ In the ground-state the nitrogen atoms adopt a pyramidal structure with the lone pair located inside the anthracene plane. As no conjugation with the anthracene $\pi$-system is possible the absorption was mainly assigned to a $\pi-\pi^{*}$ transition located on the anthracene. After excitation a relaxation occurs, which involves a reorientation of the amine groups. TD-DFT calculations identified two minima in the excited state. One minimum structure revealed a changed orientation of the lone pair through rotation of around $30^{\circ}$. The authors assumed that for this structure a conjugation with the anthracene $\pi$-system is possible. For both minima the $S_{1}$ state was found to be a charge-transfer dominant state, which is responsible for the red-shift of the emission. In contrast to the phosphines, the aminoanthracenes also emit in solution. A PET, which results in fluorescence-quenching, could not be observed. Amines usually require a fluorophore-spacer-amine structure to undergo an efficient PET. ${ }^{[195]}$ Without a spacer, the orbital overlap is not suitable for the PET and the intense emission is maintained. ${ }^{[195]}$

Similar to the described aminoanthracenes, it is assumed that a CT-state also contributes to the excited state of the investigated phosphanyl anthracenes. The two isomers [9- $\left.\mathrm{PPh}_{2}-\left(\mathrm{C}_{14} \mathrm{H}_{9}\right)\right]$ and $\left[9,10-\left(\mathrm{PPh}_{2}\right)_{2^{-}}\right.$ $\left(\mathrm{C}_{14} \mathrm{H}_{8}\right)$ ] with the lone pair oriented inside the anthracene plane revealed no emission. The position of the lone pair allows no conjugation with the anthracene $\pi$-system. When the diphenylphosphine group is shifted to the outer anthracene rings, a different orientation is adopted. The lone pair(s) of the phosphorous are now rotated by about $30^{\circ}$ and not located inside the anthracene plane anymore. Possibly, the changed orientation allows a conjugation of the lone pair with the anthracene $\pi$-system. A broad, structureless emission in the blue-green region can be observed. Moreover, it is assumed, that the changed orientation of the phosphorous lone pair is less suitable for an efficient PET. Through the reduced flexibility in the solid-state the non-radiative rate is decreased, and the fluorescence is restored. As the emission in solution is nearly completely quenched, a comparison of the radiative and non-radiative rates in solution and in the solid-state is not meaningful. Therefore, the given explanation about the origin of the observed solid-state emission of the diphenylphosphanyl anthracenes is only vague. For a better understanding further investigation like computational studies are necessary. For example, TD-DFT calculations could bring more insights into the nature of the excited state and the occurring electronic transitions related to the emission.

\subsubsection{Conclusion}

The synthesis and investigation of the diphenylphosphanyl anthracenes, revealed an unusual solid-state fluorescence, as phosphines are known to suffer from fluorescence quenching. The position and orientation of the substituent and especially the phosphorous lone pair seem to influence the excited state and radiative and non-radiative rates. The influence of a PET on the emission properties in the solid-state 
is rarely investigated up to now. This concept can be a promising strategy for developing new AlE-based molecules. A potential compound must consist of a fluorophore and a substituent with a lone pair suitable in energy and geometry for a PET. The high flexibility of the molecule in diluted solution allows the required orbital overlap for a PET. A high non-radiative rate is the consequence, and an efficient fluorescence quenching is observed. Upon aggregation, the flexibility of the molecules decreases, which prohibits the required orbital overlap for the PET. Consequently, the fluorescence quenching in the solid-state should be less pronounced and an emission enhancement upon aggregation is observed. A rational design and investigation of this type of compounds can potentially lead to a novel design-principle for AlE-based materials. 


\subsubsection{A short comment on the solid-state fluorescence of $\left[9,10-\left((S) P P h_{2}\right)_{2}-\left(C_{14} H_{8}\right)\right]$ and its host-guest}

complexes

The discovery of the bright solid-state fluorescence of co-crystals of the disubstituted thiophosphoryl anthracene $\left[9,10-\left((\mathrm{S}) \mathrm{PPh}_{2}\right)_{2}-\left(\mathrm{C}_{14} \mathrm{H}_{8}\right)\right]$ marks the starting point of the research on luminescent solid materials in the working group of Stalke (chapter 1.5). Since then, much effort has been given in understanding this unusual phenomenon. During this work several related compounds were synthesized and investigated resulting in a better understanding of the influence of the intra- and intermolecular structural features on the photophysical properties. Therefore, the results obtained during this thesis will be set in relation to the earlier studies on the $\left[9,10-\left((\mathrm{S}) \mathrm{PPh}_{2}\right)_{2}-\left(\mathrm{C}_{14} \mathrm{H}_{8}\right)\right]$ host-guest systems.

As the synthesis of $\left[1,4-\left(\mathrm{PPh}_{2}\right)-\left(\mathrm{C}_{14} \mathrm{H}_{8}\right)\right](\mathbf{2 5})$ was introduced in the previous chapter, it was obvious to synthesize the corresponding thiophosphoryl anthracene $\left[1,4-\left((\mathrm{S}) \mathrm{PPh}_{2}\right)_{2}-\left(\mathrm{C}_{14} \mathrm{H}_{8}\right)\right]$ (30). With two thiophosphoryl groups in para position, the structural properties should be comparable to $\left[9,10-\left((\mathrm{S}) \mathrm{PPh}_{2}\right)_{2^{-}}\right.$ $\left.\left(\mathrm{C}_{14} \mathrm{H}_{8}\right)\right](31)$. Crystallization of [1,4-((S)PPh $\left.)_{2}-\left(\mathrm{C}_{14} \mathrm{H}_{8}\right)\right]$ from EtOAc (30a) and toluene (30b) confirmed the ability of co-crystal formation and two different co-crystals were obtained. Both co-crystals adopt a monoclinic crystal system in the space group $P 2_{1} / c$ but with different cell parameters and are therefore not considered as isomorphous. For both co-crystals one molecule of $\mathbf{3 0}$ and one solvent molecule is present in the asymmetric unit. The anthracene scaffolds are nearly planar as the largest deformation is found for $30 \mathrm{~b}$ with a folding angle of $6.6^{\circ}$ (Figure 69c). The folding is far less pronounced compared to the strongly bent structure found for $\left[9,10-\left((\mathrm{S}) \mathrm{PPh}_{2}\right)_{2}-\left(\mathrm{C}_{14} \mathrm{H}_{8}\right)\right](31 \mathrm{a})$ in the cisoid conformation with folding angles up to $30^{\circ}$ (Figure 69a). For $\mathbf{3 0}$ only a transoid conformation was found with the sulfur atoms on different sites of the anthracene scaffold (Figure 69c). The shift of the substituents to the outer anthracene ring reduces the steric strain within the molecule as not all phenyl groups are located on the same anthracene site as in 31a. The changed substituent orientation in $\mathbf{3 0}$ leads also to a decrease of the S-PC1-C9A torsion angles to values between $52.95^{\circ}$ and $68.59^{\circ}$ in comparison to the nearly orthogonal orientation found in $\left[9,10-\left((\mathrm{S}) \mathrm{PPh}_{2}\right)_{2}-\left(\mathrm{C}_{14} \mathrm{H}_{8}\right)\right](31)$.

(a)

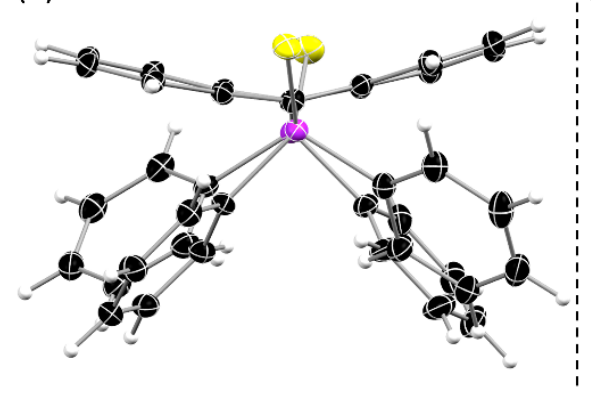

i(b)



i (c)

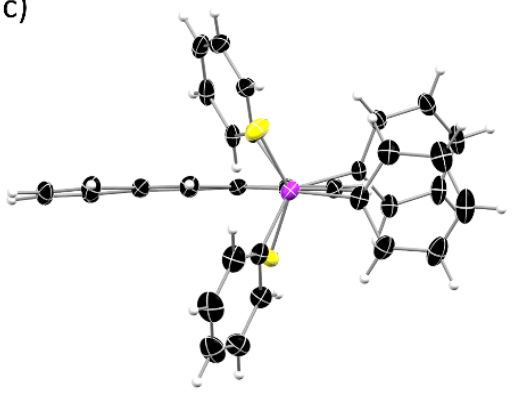

Figure 69. (a) Cisoid conformation of the (S)PPh 2 groups in $\left[9,10-\left((\mathrm{S}) \mathrm{PPh}_{2}\right)_{2}-\left(\mathrm{C}_{14} \mathrm{H}_{8}\right)\right](31 \mathrm{a})$ and the resulting strong bent structure of the anthracene scaffold. (b) Transoid conformer of $\left[9,10-\left((\mathrm{S}) \mathrm{PPh}_{2}\right)_{2}-\left(\mathrm{C}_{14} \mathrm{H}_{8}\right)\right](31 \mathrm{~b})$ with a nearly planar, but twisted anthracene plane and (c) transoid conformation of $\left[1,4-\left((\mathrm{S}) \mathrm{PPh}_{2}\right)_{2}-\left(\mathrm{C}_{14} \mathrm{H}_{8}\right)\right](30)$ with different orientation of the substituents and only little deformation of the anthracene plane. Co-crystallized solvents in (b) and (c) are omitted for clarity.

The EtOAc molecule in the co-crystal 30a has no special position regarding the anthracene scaffold and reveals only weak interactions towards the phenyl groups of the phosphoryl group. Similar behavior was 
observed for the co-crystals of $\left[9,10-\left((\mathrm{S}) \mathrm{PPh}_{2}\right)_{2}-\left(\mathrm{C}_{14} \mathrm{H}_{8}\right)\right](31 \mathrm{a})$ in the cisoid conformation. Often a disorder of the solvent molecule goes along with this structural motif, which could also be confirmed for the EtOAc molecule in 30a. The solvent in the co-crystals in a transoid orientation, adopts a more prominent position. In the work by Fei et al. the overall structure was described as a T-shaped orientation. Several $\mathrm{C}-\mathrm{H} \cdots \pi$ interactions between the crystallized solvent and the anthracene moiety were attributed to keep the solvent inside the formed cradle (Figure 70b). This orientation and the occurring interactions probably induce the transoid orientation of the thiophosphoryl groups. The steric strain within the molecule is still present but the transoid orientation allows no folding of the anthracene. Instead, a twisting of the anthracene plane was identified and quantified by Finkelmeier. ${ }^{[153]}$ In the solid-state structure of [1,4$\left.\left((\mathrm{S}) \mathrm{PPh}_{2}\right)_{2}-\left(\mathrm{C}_{14} \mathrm{H}_{8}\right)\right](\mathbf{3 0 b})$ the toluene is located above the anthracene in a similar T-shape orientation as in $\left[9,10-\left((\mathrm{S}) \mathrm{PPh}_{2}\right)_{2}-\left(\mathrm{C}_{14} \mathrm{H}_{8}\right)\right](\mathbf{3 1 b})$. Due to the changed position of the substituents the solvent is now placed above the outer anthracene ring and the $\mathrm{CH}_{3}$-group is pointing away from the anthracene plane. The intersection angle of the aromatic planes of toluene and anthracene is about $63.5^{\circ}$ and only slightly smaller compared to the $70.9^{\circ}$ found in $\left[9,10-\left((\mathrm{S}) \mathrm{PPh}_{2}\right)_{2}-\left(\mathrm{C}_{14} \mathrm{H}_{8}\right)\right]$. In $30 \mathrm{~b}$ the toluene is disordered over a second position generated by a rotation about the $\mathrm{C}_{6}$-perimeter midpoint of about $180^{\circ}$, while no disorder is observed in the co-crystals of the 9,10-derivative adopting a transoid orientation. ${ }^{6}$ Considering the intermolecular interactions in both regioisomers several $\mathrm{C}-\mathrm{H} \cdots \pi$ interactions between the co-crystallized solvent and the thiophosphoryl anthracene are present. The shortest distances between the corresponding hydrogens and $\mathrm{C}-\mathrm{C}$ bond are shown in Figure 70. As these values are in a similar range it cannot be estimated, if a difference in the strength of the non-covalent interactions in both co-crystals exist. The higher degree of disorder of the toluene in $\left[1,4-\left((\mathrm{S}) \mathrm{PPh}_{2}\right)_{2}-\left(\mathrm{C}_{14} \mathrm{H}_{8}\right)\right]$ hints for a less fixed position of the co-crystallized solvent but is not a final proof.

\footnotetext{
${ }^{6}$ In recent investigations by $P$. N. Ruth a disorder of the toluene molecule in $\left[9,10-\left((\mathrm{S}) \mathrm{PPh}_{2}\right)_{2}-\left(\mathrm{C}_{14} \mathrm{H}_{8}\right)\right]$ was identified. The occupation of the second position is dependent of the temperature and increases at higher temperatures and is only small at $100 \mathrm{~K}$.
} 

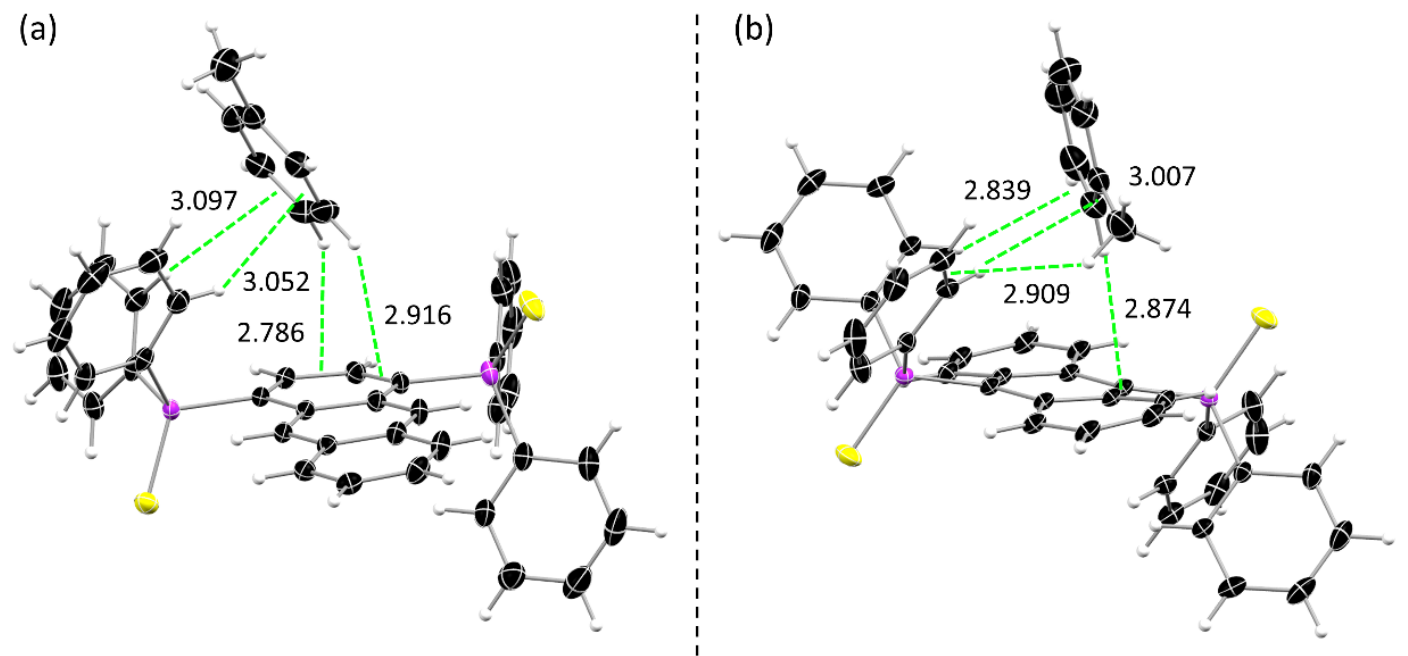

Figure 70. Shortest $\mathrm{C}-\mathrm{H} \cdots \pi$ interactions in $\AA$ between the co-crystallized toluene and the thiophosphoryl anthracene as observed in (a) [1,4-((S)PPh $\left.)_{2}-\left(\mathrm{C}_{14} \mathrm{H}_{8}\right)\right](\mathbf{3 0 b})$ and (b) [9,10-((S)PPh$\left.)_{2}-\left(\mathrm{C}_{14} \mathrm{H}_{8}\right)\right](\mathbf{3 1 b})$. Disorder of the toluene in (a) is omitted for clarity.

From the structural point of view the novel synthesized isomer $\left[1,4-\left((\mathrm{S}) \mathrm{PPh}_{2}\right)_{2}-\left(\mathrm{C}_{14} \mathrm{H}_{8}\right)\right](30)$ reveals several similarities to the well-investigated co-crystals of $\left[9,10-\left((\mathrm{S}) \mathrm{PPh}_{2}\right)_{2}-\left(\mathrm{C}_{14} \mathrm{H}_{8}\right)\right](31)$ regarding the orientation of the substituents and the position of the co-crystallized solvent. If these factors are predominant in determining the luminescence properties in the solid-state as postulated earlier, [1,4-((S)PPh $\left.)_{2}-\left(\mathrm{C}_{14} \mathrm{H}_{8}\right)\right]$ should also be able to show a bright fluorescence in the aggregated state.

(a)

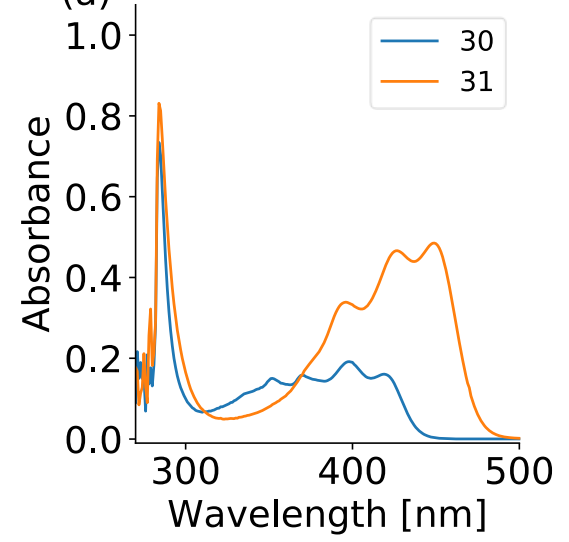

(b)

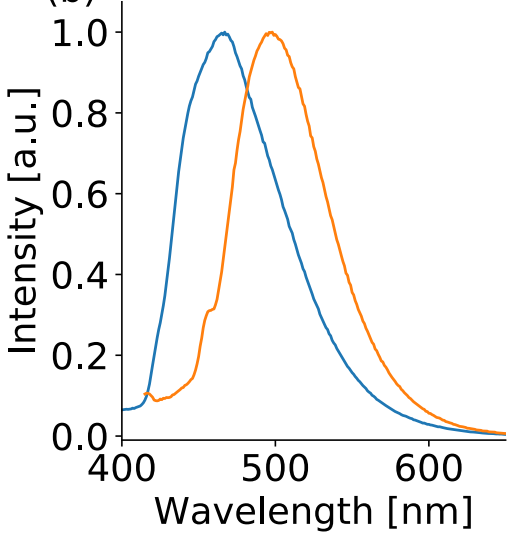

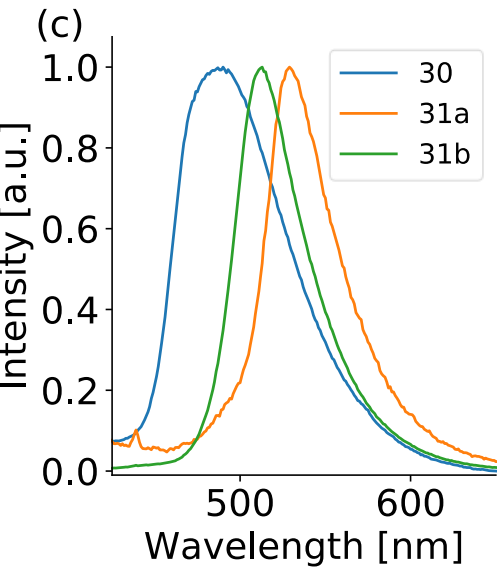

Figure 71. (a) UV-Vis absorption spectra and (b) normalized emission spectra of the two isomers $\left[1,4-\left((\mathrm{S}) \mathrm{PPh}_{2}\right)_{2}-\left(\mathrm{C}_{14} \mathrm{H}_{8}\right)\right](30)$ and $\left[9,10-\left((\mathrm{S}) \mathrm{PPh}_{2}\right)_{2}-\left(\mathrm{C}_{14} \mathrm{H}_{8}\right)\right](31)$ in diluted toluene solution $\left(10^{-5} \mathrm{M}\right)$.

Photophysical properties of both isomers in diluted solution are overall very similar. The UV-Vis spectra show the typical absorptions of anthracene derivatives with one sharp and intense band in the UV-region and a broad absorption of the $\mathrm{S}_{0} \rightarrow \mathrm{S}_{1}$ transition with a vibronic structure at longer wavelengths (Figure 71a). The $\mathrm{S}_{0} \rightarrow \mathrm{S}_{1}$ absorption of $\left[9,10-\left((\mathrm{S}) \mathrm{PPh}_{2}\right)_{2}-\left(\mathrm{C}_{14} \mathrm{H}_{8}\right)\right]$ is slightly stronger and undergoes a bathochromic shift by about $30 \mathrm{~nm}$ compared to $\left[1,4-\left((\mathrm{S}) \mathrm{PPh}_{2}\right)_{2}-\left(\mathrm{C}_{14} \mathrm{H}_{8}\right)\right]$. The emission of $\left[9,10-\left((\mathrm{S}) \mathrm{PPh}_{2}\right)_{2}-\left(\mathrm{C}_{14} \mathrm{H}_{8}\right)\right]$ is also red-shifted by around $30 \mathrm{~nm}$ compared to the 1,4-substituted derivative and peaks in the green region at 
nearly $500 \mathrm{~nm}$ (Figure 71b). For both compounds no vibronic structure is visible in the emission spectra and the fluorescence in solution is nearly completely quenched, as already observed for the other thiophosphoryl anthracenes.

In the solid-state the changes in the emission properties are more pronounced especially in terms of the emission efficiency. While the co-crystals of $\left[9,10-\left((\mathrm{S}) \mathrm{PPh}_{2}\right)_{2}-\left(\mathrm{C}_{14} \mathrm{H}_{8}\right)\right](31)$ in a transoid orientation show a bright green fluorescence with quantum yields up to $60 \%{ }^{[200]}$ the emission of $\left[1,4-\left((\mathrm{S}) \mathrm{PPh}_{2}\right)_{2}-\left(\mathrm{C}_{14} \mathrm{H}_{8}\right)\right]$ is nearly completely quenched and quantum yields around $1 \%$ are obtained. The crystals of $\mathbf{3 1}$ in a cisoid orientation revealed similar low quantum efficiencies. Differences in the emission wavelengths are comparable to the shifts in solution as $\mathbf{3 1}$ shows an emission in the green region at around $515-530 \mathrm{~nm}$, while emission of $\mathbf{3 0}$ peaks at $490 \mathrm{~nm}$ (Figure 71c).

For [1,4-((S)PPh $\left.)_{2}-\left(\mathrm{C}_{14} \mathrm{H}_{8}\right)\right]$ (30) no strong fluorescence could be observed neither in solution nor in the solid-state. The behavior in the aggregated state is therefore comparable to the cisoid co-crystals of $[9,10$ $\left.\left((\mathrm{S}) \mathrm{PPh}_{2}\right)_{2}-\left(\mathrm{C}_{14} \mathrm{H}_{8}\right)\right]$, which also revealed only very weak luminescence. The results from the newly obtained 1,4-substituted derivative, together with the previous studies allow an updated description of the unusual photophysical properties of the co-crystals of $\left[9,10-\left((\mathrm{S}) \mathrm{PPh}_{2}\right)_{2-}\left(\mathrm{C}_{14} \mathrm{H}_{8}\right)\right](31)$.

The still fundamental question is why only the transoid co-crystals of $\left[9,10-\left((\mathrm{S}) \mathrm{PPh}_{2}\right)_{2}-\left(\mathrm{C}_{14} \mathrm{H}_{8}\right)\right](31)$ reveal a bright fluorescence in the solid-state while the emission of cisoid congeners and the structurally related $\mathbf{3 0}$ is nearly completely quenched. A strong electronic interaction between the co-crystallized solvent and the anthracene moiety, like formation of an exciplex as postulated in earlier works, seems to be unlikely for several reasons. An exciplex formation in the solid-state would imply several changes of the photophysical properties like spectral broadening, a strong bathochromic shift, and an increased fluorescence lifetime. The lifetime of the transoid co-crystals is within a few ns and comparable to the lifetimes obtained in solution and for other co-crystals and derivatives. A structureless emission is found for all derivatives in solution and in the solid-state and is not a special feature of the transoid co-crystals. A clear broadening of the spectra could not be observed. Actually, the emission spectrum of $\mathbf{3 0}$ in the solidstate, which is nearly non-fluorescent is slightly broadened compared to the spectrum of the strong fluorescent co-crystals of 31. Furthermore, no shift of the emission wavelength upon removing the cocrystallized toluene of $\mathbf{3 1}$ occurred and only the decrease in the emission efficiency is observed. The discussed findings indicate that the emission of $\left[9,10-\left((\mathrm{S}) \mathrm{PPh}_{2}\right)_{2}-\left(\mathrm{C}_{14} \mathrm{H}_{8}\right)\right]$ (31) originates from the monomeric state rather than an exciplex, which was also confirmed for other 9,10 substituted thiophosphoryl anthracenes as shown in chapter 3.1.3. Therefore, the availability of non-radiative pathways seems to be determined by the molecular conformation and/or the interaction of the thiophosphoryl anthracene with the co-crystallized solvent.

A photoinduced-electron transfer (PET) from the sulfur lone pairs to the anthracene moiety was postulated as one predominant non-radiative decay pathway for thiophosphoryl anthracenes in solution. The emission efficiency in the solid-state is probably also affected by the PET. As the quantum yields of the most herein investigated thiophosphoryl anthracenes are higher in the solid-state than in solution the PET is probably less effective in the solid-state. Previous studies regarding the PET have shown that an efficient 
PET requires a decent structural flexibility for a suitable orbital overlap. ${ }^{[177,195]}$ The structural flexibility is in general decreased in the solid-state compared to the solution-state. So, a less efficient PET can explain the general trend that emission of the thiophosphoryl anthracenes is brighter in the solid-state than in solution. The drastic differences found in the emission efficiencies of the cisoid and transoid co-crystals of 31 can probably not be attributed only to a less efficient PET. Instead, it seems likely that another nonradiative pathway is blocked upon co-crystallization in a transoid orientation.

As discussed in chapter 1.4, the AIE phenomenon can be described by the restricted access to a conical intersection (RACl) model in a more general way. The fluorescence quenching in solution is ascribed to internal conversion through a low-lying $\mathrm{S}_{1}-\mathrm{S}_{0}$ conical intersection $(\mathrm{Cl})$. The structural flexibility in solution allows an access to the $\mathrm{Cl}$ through vibrational motion, which results in high non-radiative rates and consequently in an emission quenching. The accessibility of the $\mathrm{Cl}$ through large amplitude modes is less probable in the aggregated state as the structure is less flexible. Therefore, the non-radiative rates in the solid-state are decreased, the emission is more efficient, and a typical AIE-behavior can be observed.

The structures of several polyaromatic hydrocarbons including anthracene at their minimum energy conical intersections (MECls) have been calculated and reported in the literature. ${ }^{[228]}$ Furthermore, the structure at the $\mathrm{MECl}$ of the in-detail investigated AIE-luminogen 9,10-bis( $N, N$-dimethylamino) anthracene has been calculated by Sasaki et al. ${ }^{[148-150]}$ For the latter as well as for pure anthracene a highly distorted structure at the $\mathrm{MECl}$ was obtained (Figure 72 ). The anthracene moiety of 9,10-bis $(\mathrm{N}, \mathrm{N}$-dimethylamino) anthracene reveals a strong folding with two upraised substituents. Sasaki et al. concluded that the access to this highly distorted structure is restricted in the solid-state and only possible in solution. The AIEbehavior of these structurally simple compounds was therefore ascribed to the bending mode of the anthracene. ${ }^{[150,173]}$

(a)

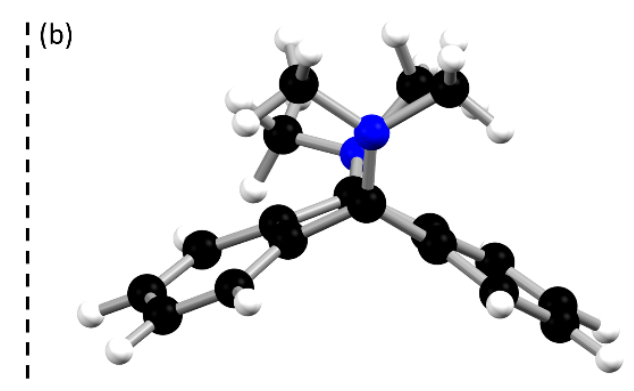

Figure 72. Calculated structures at the minimum energy conical intersections for (a) anthracene and (b) 9,10-bis(N,Ndimethylamino) anthracene. Coordinates taken from references. ${ }^{[150,228]}$

It is not unlikely that the structure at the $\mathrm{MECl}$ for the thiophosphoryl anthracenes is similar to the structures of unsubstituted anthracene and the bisdialkylamino anthracenes. With this assumption, the fundamental differences in the luminescence behavior of the cisoid and transoid co-crystals of $\mathbf{3 1}$ can be explained. Comparing the solid-state structure of the cisoid co-crystals with the described structure at the MECls several similarities are found. The anthracene plane in $\mathbf{3 1}$ reveals a strong distortion in terms of a bent structure (vide supra). Furthermore, the phosphorous atoms are upraised like the nitrogen atoms in the bisdialkylamino anthracene. The ground-state solid-state structure of the cisoid conformers is - 130 - 
therefore already close to the proposed structure at the $\mathrm{MECl}$. It is likely that the $\mathrm{S}_{1}-\mathrm{S}_{0} \mathrm{Cl}$ can be reached easily even in the solid-state and radiationless internal conversion can occur after excitation. The consequence is a high non-radiative rate and nearly completely quenched emission (Figure 73).

A contrary situation is found for the transoid co-crystals of 31. Apart from the described twist the anthracene is less distorted, and no bending of the aromatic moiety is observed. The two bulky thiophosphoryl substituents induce an overall rigid structure. Moreover, the weak $\mathrm{C}-\mathrm{H} \cdots \pi$ interactions between the co-crystallized aromatic solvent and the thiophosphoryl anthracene lead to a further rigidification. All in all, the anthracene moieties in the transoid conformation seem to be less flexible and more rooted in their ground-state geometry. These structural features indicate that a large amplitude mode towards a bent structure is not likely for the transoid co-crystals of $\mathbf{3 1}$. Therefore, the $\mathrm{S}_{1}-\mathrm{S}_{0}$ conical intersection cannot be reached in the solid-state and the non-radiative decay rate is small. As a result, the deactivation occurs via the radiative pathway and an intense solid-state fluorescence with large quantum yields is observed (Figure 73). Removal of the co-crystallized solvent upon drying decreases the rigidity and enhances the structural flexibility as the weak host-guest interactions are vanished. The $\mathrm{S}_{1}-\mathrm{S}_{0} \mathrm{Cl}$ is accessible again and the fluorescence quantum yield decreases. This interpretation goes along with the increased emission efficiencies of the co-crystals of [9-(S)PPh $\left.-10-E t-\left(\mathrm{C}_{14} \mathrm{H}_{8}\right)\right](\mathbf{1 5})$ as described in chapter 3.1.4. Upon co-crystallization the distortion of the anthracene moiety was reduced and weak intermolecular interactions between the co-crystallized solvent and the anthracene moiety are present, which resulted in higher quantum yields.

(a)

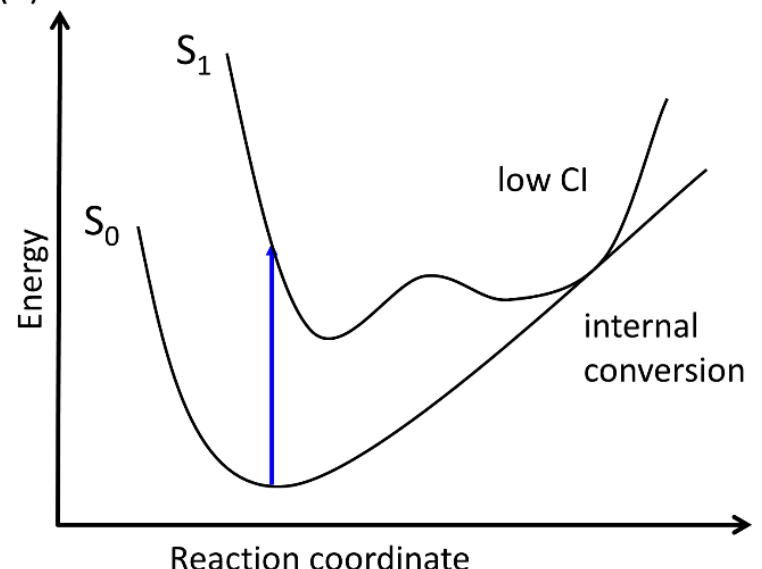

(b)

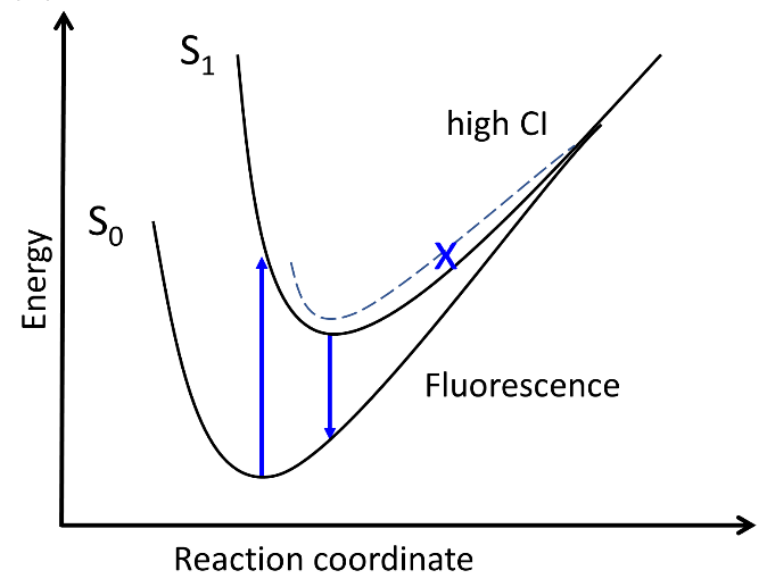

Figure 73. Schematic representation of the photophysical processes in the different co-crystals of $\left[9,10-(S) P P h_{2}-\left(C_{14} \mathrm{H}_{8}\right)\right](31)$. In the cisoid co-crystals of the low lying $\mathrm{S}_{1}-\mathrm{S}_{0}$ conical intersection $(\mathrm{Cl})$ is accessible in the solid-state and deactivation occurs via internal conversion. In the transoid co-crystals the energy of the $\mathrm{Cl}$ is increased and not accessible and an intense fluorescence can be observed in the solid-state.

The co-crystals of [1,4-(S)PPh $\left.-\left(\mathrm{C}_{14} \mathrm{H}_{8}\right)\right](30)$ revealed similar emission properties as the cisoid co-crystals of $\mathbf{3 1}$ with a nearly quenched emission in the solid-state. From the low quantum yields, it can be assumed that the $\mathrm{Cl}$ is also accessible for $\mathbf{3 0}$ in the solid-state. Due to the shift of the substituents to the outer anthracene ring, the steric strain within the molecule is clearly reduced as the orientation of phenyl rings 
is more flexible. The obtained solid-state structure is therefore less rigid, and the $\mathrm{S}_{1}-\mathrm{S}_{0} \mathrm{Cl}$ is available, and deactivation of the excited state can occur via internal conversion.

The updated interpretation of the unusual luminescence properties of $\left[9,10-(S) P P h_{2}-\left(C_{14} \mathrm{H}_{8}\right)\right]$ (31) attributes the observed behavior to both intramolecular and intermolecular effects. A strong electron coupling between host and guest molecules like an exciplex formation was excluded, but the weak interactions between the co-crystallized solvents are probably essential for the enhanced rigidity of the structure. Furthermore, the co-crystallized aromatic solvent seems to be necessary to induce the transoid orientation, as this orientation could not be obtained without co-crystallized solvent until now. The cisoid co-crystals adopt a geometry which is already close to the proposed structure at the $\mathrm{MECl}$, which is therefore easily accessible also in the solid-state.

It has to be noted again, that several factors influence the radiative and non-radiative rates in solution and in the solid-state. The photophysical processes of $\left[9,10-(\mathrm{S}) \mathrm{PPh}_{2}-\left(\mathrm{C}_{14} \mathrm{H}_{8}\right)\right](31)$ could therefore be even more complex. Probably, detailed quantum chemical calculations are necessary for obtaining a complete picture of the involved radiative and non-radiative deactivation pathways. 


\section{Conclusion \& Outlook}

This thesis was devoted to investigate the influence of intermolecular interactions on the solid-state luminescence of the anthracene fluorophore. Especially, the structural requirements for solid-state excimer formation and its impact on the emission properties were studied.

\subsection{Investigating the influence of the substituent}

To investigate the influence of different substituents, several anthracene derivatives, substituted in the 9position, were synthesized, and examined regarding their structural and photophysical properties (Figure 74). Besides the already known phosphanyl- and phosphoryl anthracenes ([9- $\left.\mathrm{PPh}_{2}-\left(\mathrm{C}_{14} \mathrm{H}_{9}\right)\right]$ (1), [9-(O) $\mathrm{PPh}_{2^{-}}$ $\left.\left(\mathrm{C}_{14} \mathrm{H}_{9}\right)\right](7)$ and [9-(S)PPh$\left.\left.\left.-\left(\mathrm{C}_{14} \mathrm{H}_{9}\right)\right]\right)(8)\right)^{[152,155]}$, three further compounds were prepared and studied. [9BMes $\left._{2}-\left(\mathrm{C}_{14} \mathrm{H}_{9}\right)\right](\mathbf{9})$, [9-( $\left.\left.\mathrm{HO}\right) \mathrm{CPh}_{2}-\left(\mathrm{C}_{14} \mathrm{H}_{9}\right)\right](\mathbf{1 0})$ and $\left[9-(\mathrm{Cl}) \mathrm{SiPh}_{2}-\left(\mathrm{C}_{14} \mathrm{H}_{9}\right)\right](\mathbf{1 1})$ showed comparable substitution motifs with different heteroatoms bonded to the anthracene. The structural and photophysical changes, connected to the different substituents and heteroatoms, were examined. The absorption and emission properties of the monomeric forms, which exist in diluted solutions, are dominated by the anthracene fluorophore. The two anthracene-typical absorptions were also found for the substituted anthracenes. Only for [9-BMes $\left.{ }_{2}-\left(\mathrm{C}_{14} \mathrm{H}_{9}\right)\right](9)$, additional absorption bands were present in the absorption spectrum and assigned to a CT from the anthracene to the electron deficient boron center. The influence of the other substituents is expressed mainly by a bathochromic shift and a slightly broadened vibronic structure of the lower energy absorption. These observations indicated a similar electronic ground state of all anthracene derivatives, with exception of [9-BMes $\left.2-\left(\mathrm{C}_{14} \mathrm{H}_{9}\right)\right](\mathbf{9})$.

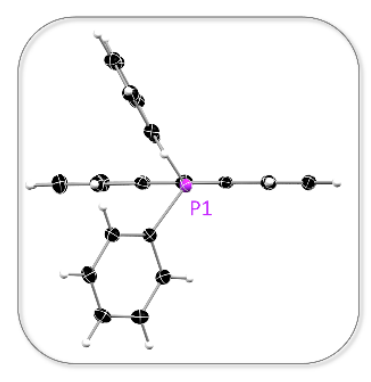

$\left[9-\mathrm{PPh}_{2}-\left(\mathrm{C}_{14} \mathrm{H}_{9}\right)\right](1)$

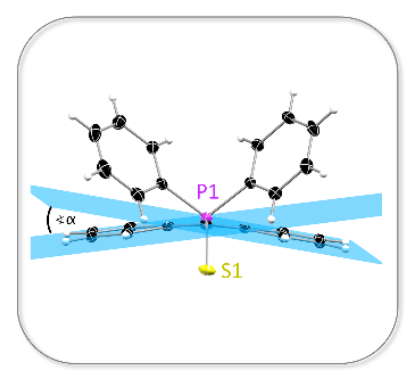

$\left[9-(\mathrm{S}) \mathrm{PPh}_{2}-\left(\mathrm{C}_{14} \mathrm{H}_{9}\right)\right](8)$

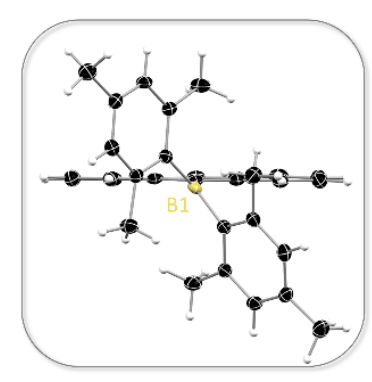

$\left[9-\mathrm{BMes}_{2}-\left(\mathrm{C}_{14} \mathrm{H}_{9}\right)\right](9)$

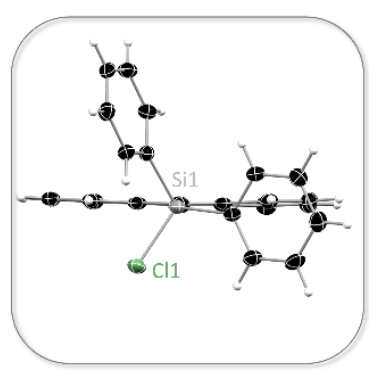

$\left[9-(\mathrm{Cl}) \mathrm{SiPh}_{2}-\left(\mathrm{C}_{14} \mathrm{H}_{9}\right)\right](11)$

Figure 74. Excerpt of the investigated anthracene derivatives with different substituents in the 9-position. Small structural variations, like a different heteroatom bonded to the anthracene, resulted in different orientations of the substituent. The increased steric demand of the substituents can induce a distortion of the anthracene plane, which was quantified by the folding angle $\alpha$ (as shown for 8 ).

The emission spectra in diluted solution revealed only minor differences, which were attributed to the slightly different electronic properties of the substituents. Fluorescence lifetimes in the nanosecond range, as well as only small bathochromic shifts, indicated the absence of CT or excimer emission in solution. As expected, the emission in solution occurred from the monomeric species. The anthracene typical vibronic structure of the emission band was only found for [9-( $\left.\mathrm{HO}) \mathrm{CPh}_{2}-\left(\mathrm{C}_{14} \mathrm{H}_{9}\right)\right](\mathbf{1 0})$. The substitution of the carbon atom bonded to $\mathrm{C} 9$, with a heteroatom, resulted in a loss of the vibronic structure. Strongest differences within the investigated derivatives, were found in their emission efficiencies. The determined quantum 
yields in diluted solution varied over the whole possible range, from almost zero ([9-PPh $\left.-\left(\mathrm{C}_{14} \mathrm{H}_{9}\right)\right](\mathbf{1})$ and $\left.\left[9-(\mathrm{S}) \mathrm{PPh}_{2}-\left(\mathrm{C}_{14} \mathrm{H}_{9}\right)\right](\mathbf{8})\right)$ to nearly unity $\left(\phi_{\mathrm{F}}=96.8 \%\right.$ for [9- $\left.(\mathrm{Cl}) \mathrm{SiPh}_{2}-\left(\mathrm{C}_{14} \mathrm{H}_{9}\right)\right]$ (11)). The observed fluorescence quenching of $\mathbf{1}$ and $\mathbf{8}$ was attributed to a PET from the lone-pair towards the anthracene fluorophore. This quenching mechanism has already been reported earlier for other aromatic phosphines. ${ }^{[175,223]}$ The lone pairs of the other derivatives are probably not suitable in energy to undergo a PET. Instead, perturbation of the anthracenes $\pi$-system by the heteroatoms led to an emission enhancement and increased quantum yields compared to that of anthracene.

The main differences in the solid-state structures were determined in the orientation of the substituent and the distortion of the anthracene plane. The butterfly-like bending of the anthracene core was attributed to the steric demand of the substituent and quantified by the folding angle $\alpha$ (Figure 74). The torsion of the substituent influenced the distortion, and the largest folding angle was found for $\left[9-\left(\mathrm{S}^{2} \mathrm{PPh}_{2}-\right.\right.$ $\left(\mathrm{C}_{14} \mathrm{H}_{9}\right)$ ] (8) with $\alpha=14.27^{\circ}$. The P-S moiety adopted a nearly orthogonal orientation towards the anthracene plane and forces both phenyl groups onto the opposite anthracene side. The outer anthracene rings bended away to decrease the steric strain. A possible impact of the distortion onto the photophysical properties (bathochromic shift or spectral broadening), as postulated by earlier studies ${ }^{[153,184]}$, is still under discussion.

Investigation of the crystal packing modes gave insights into the occurring intermolecular interactions. From the investigated derivatives with different substituents, only the thiophosphoryl anthracene [9(S) $\left.\mathrm{PPh}_{2}-\left(\mathrm{C}_{14} \mathrm{H}_{9}\right)\right](8)$ showed $\pi-\pi$ interactions in a face-to-face manner. These interactions between the two chromophores are ascribed to influence the photophysical properties and are necessary for an excimer formation in the solid-state. The overlapping area of the two anthracenes was estimated to $21.1 \%$ and is therefore rather small. Accordingly, the examination of the photophysical properties could not confirm an excimer formation in the solid-state. Only a small bathochromic shift and a slightly increased quantum yield, compared to the solution-state, were estimated. Also, fluorescence lifetimes were still in the nanosecond range. The other derivatives showed even a decrease of the quantum yields compared to the solution-state. This ACQ-behavior is typical for many polyaromatic hydrocarbons. According to the analysis of the structural and photophysical data, the emission in the solid-state was ascribed also to a monomeric state. A strong influence of the intermolecular interactions, and the targeted excimer formation, could not be confirmed. From the structural point of view, the used substituents fulfilled their purpose and prevented the anthracene moieties from detrimental, columnar stacking. Therefore, a solid-state fluorescence could be observed for all derivatives, except for [9-PPh $\left.-\left(\mathrm{C}_{14} \mathrm{H}_{9}\right)\right](\mathbf{1})$.

The quantum yields of the investigated derivatives seemed to be highly dependent on the used substituent. Therefore, a combination of two different substituents could lead to interesting photophysical properties. A combination of an electron-donating (e.g., the phosphanyl group) and an electron deficient (e.g., a boryl group) substituent, may induce an intramolecular charge transfer. Further variations of the substituents could then allow a control of the derived optical properties. A similar approach to control the optical properties through a combination of different main group elements has been recently investigated for different phenyleneethynylenes. ${ }^{[160]}$

- 134 - 


\subsection{Structure-property correlations in 9,10-substituted thiophosphoryl anthracenes}

Thanks to the presence of the $\pi-\pi$ interactions in [9-(S)PPh $\left.-\left(\mathrm{C}_{14} \mathrm{H}_{9}\right)\right](8)$, the thiophosphoryl anthracenes were investigated in more detail. Even if no excimer formation could be confirmed for $\mathbf{8}$, the observed structural properties showed great potential for further investigations. Therefore, more thiophosphoryl anthracenes were prepared and investigated.

\subsubsection{Controlling intermolecular interactions in thiophosphoryl anthracenes}

By substituting the hydrogen atom in the 10-position of [9-(S) $\left.\mathrm{PPh}_{2}-\left(\mathrm{C}_{14} \mathrm{H}_{9}\right)\right](8)$, the packing motif and the occurring intermolecular interactions could be varied (Figure 75). Introduction of small alkyl groups preserved the desired dimeric motif and could increase the anthracenes overlapping. The inductive effect of the alkyl group was assigned to change the electrostatic potential of the anthracene and therefore, allow a larger overlap. The overlap ratio could be increased from $21.1 \%(8)$ to $42.8 \%$ for [9-(S)PPh $\mathrm{Ph}_{2}-10$-Et$\left.\left(\mathrm{C}_{14} \mathrm{H}_{8}\right)\right](15)$. The doubled overlapping area should also result in stronger $\pi-\pi$ interactions and therefore, increase the chance for excimer formation. Introduction of a phenyl group in the 10-position resulted in complete absence of $\pi-\pi$ interactions in the solid-state structure of [9-(S)PPh$\left.-10-P h-\left(C_{14} \mathrm{H}_{8}\right)\right](17)$. Both sides of the anthracene aromatic plane are now shielded by the substituents and therefore not accessible for $\pi$-stacking interactions. The approach of controlling the intermolecular interactions and the packing motif via small structural modifications, was therefore successful.

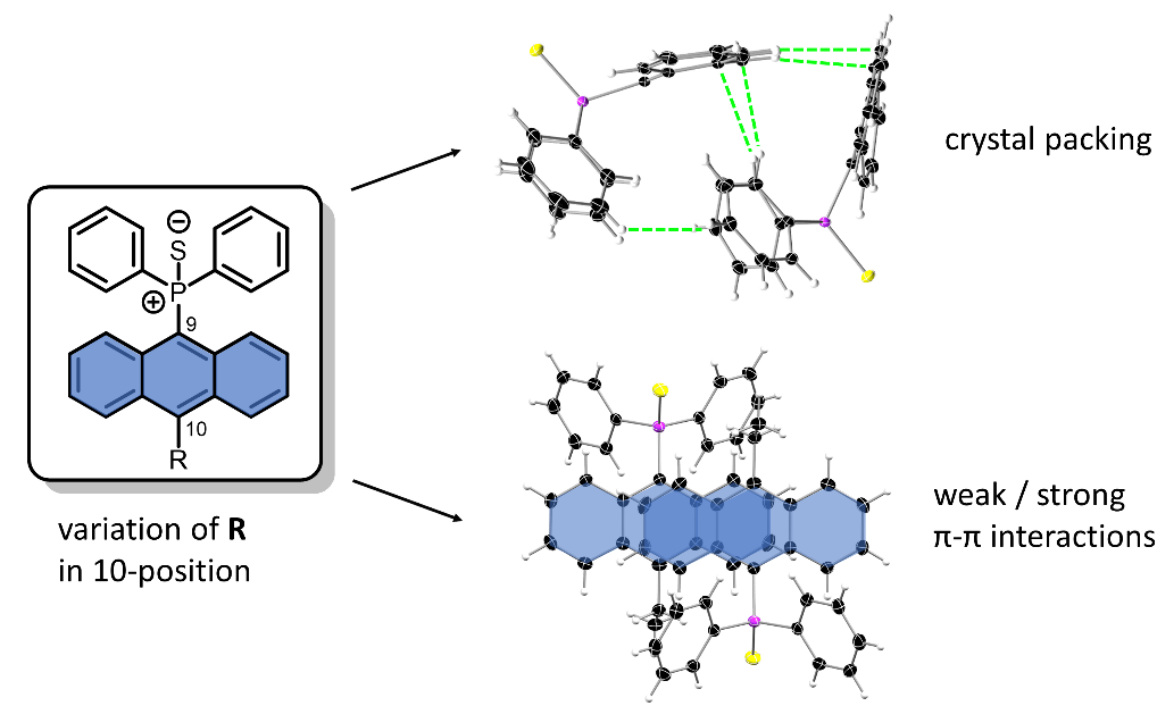

Figure 75. Through variation of the substituent $\mathbf{R}$ in the 10-position, the crystal packing, and the intermolecular interactions in the thiophosphoryl anthracenes could be varied. The investigation of their solid-state structures revealed the desired face-toface dimeric motif for most derivatives. The occurring interactions were quantified via the overlap ratio, $\pi-\pi$ distance and the offset along the anthracene axes.

All thiophosphoryl anthracenes revealed a blue-green luminescence in the solid-state with maximum emission wavelengths up to $506 \mathrm{~nm}$. Even if these wavelengths are in a typical range of anthracene excimer emission ${ }^{[131,134,135]}$, the further obtained photophysical data could not support the excimer formation. The emission properties of the investigated thiophosphoryl anthracenes are very comparable among each other, even if the occurring intermolecular interactions differed strongly. Only a small 
bathochromic shift could be determined for the derivatives that undergo stronger $\pi$-stacking interactions (13-16). Overall, the emission in the solid-state was also ascribed to occur from the monomeric species. The determined quantum yields were generally higher in the solid-state than in solution. Therefore, nonradiative deactivation (for example PET) seemed to be less efficient in the solid-state and the thiophosphoryl anthracenes revealed typical AIE-behavior.

\subsubsection{Solid-state emission amplification through co-crystallization of small aromatic molecules}

During the investigation of its crystallization conditions, the ability of $\left[9-(\mathrm{S}) \mathrm{PPh}_{2}-10-\mathrm{Et}-\left(\mathrm{C}_{14} \mathrm{H}_{8}\right)\right](15)$ to cocrystallize with small aromatic molecules, was discovered. The guest molecules crystallized in a T-shape orientation regarding the anthracene plane, similar as described earlier by Fei et al for $\left[9,10-\left((\mathrm{S}) \mathrm{PPh}_{2}\right)_{2^{-}}\right.$ $\left(\mathrm{C}_{14} \mathrm{H}_{8}\right)$ ] (Figure 76). ${ }^{[151]}$ Upon co-crystallization, the packing motifs and the resulting interchromophoric interactions changed. Three co-crystals revealed a decrease of the dimer overlap ratio (15a-15c), while for $\mathbf{1 5 d}$ the dimeric motif was completely absent. Therefore, co-crystallization of small guest molecules was proposed as another suitable strategy for varying the intermolecular interactions, without changing the electronic structure. A T-shape exciplex formation, as postulated by Fei et al., could not be confirmed for the herein investigated host-guest systems. Instead, the co-crystallization led to an unexpected amplification of the emission intensity. As further photophysical characteristics changed only slightly, the emission enhancement was attributed to the increased structural rigidity through weak $\mathrm{C}-\mathrm{H} \cdots \pi$ interactions between the anthracene and the co-crystallized arenes. Calculated radiative and nonradiative rates supported this assumption. The restriction of intramolecular motion (RIM) is a general strategy for achieving efficient solid-state emission and was found as the underlying working principle of typical AIE-luminogens. ${ }^{[54]}$

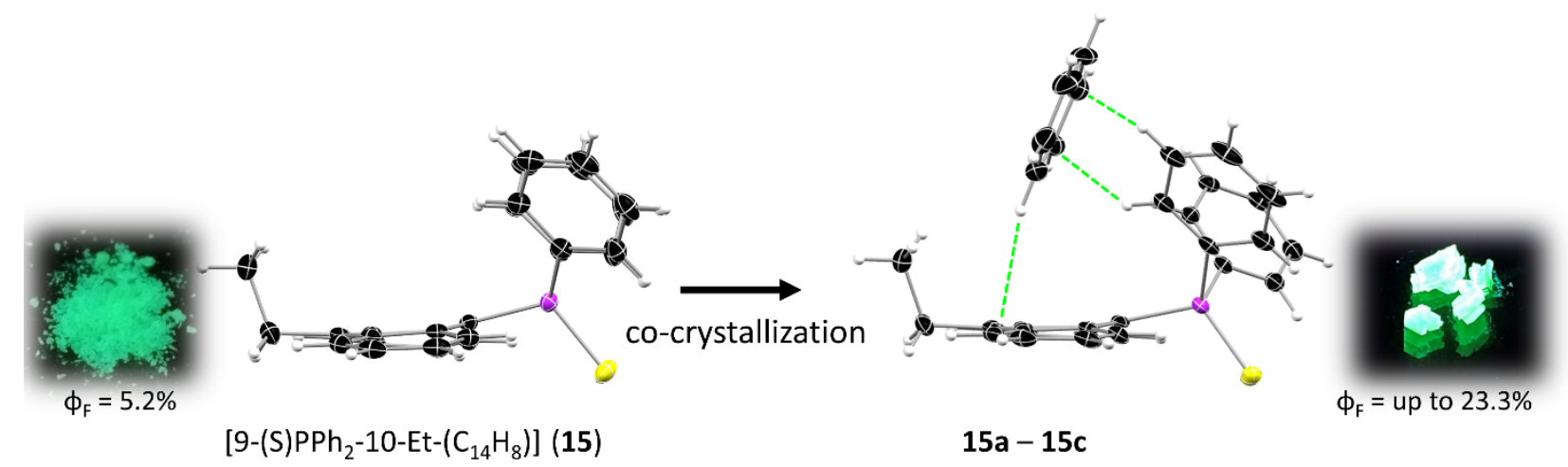

Figure 76. Co-crystallization of [9-(S)PPh $\left.-10-E t-\left(\mathrm{C}_{14} \mathrm{H}_{8}\right)\right](15)$ with small aromatic molecules, led to an amplification of the solidstate emission intensity. The emission enhancement was attributed to a rigidification of the solid-state structure through weak $\mathrm{C}-\mathrm{H} \cdots \pi$ interactions between the thiophosphoryl anthracene and the co-crystallized guest.

\subsection{Investigation of the phosphanyl- and thiophosphoryl anthracene regioisomers}

In order to increase the structural flexibility and maintain the benefits from the thiophosphoryl group, the two positional isomers [1-(S)PPh$\left.-\left(\mathrm{C}_{14} \mathrm{H}_{9}\right)\right]$ (20) and [2-(S)PPh${ }_{2}-\left(\mathrm{C}_{14} \mathrm{H}_{9}\right)$ ] (22) were synthesized and investigated. It was expected that the shift of the substituent to the outer anthracene ring, would decrease the steric strain and increase the structural flexibility. Accordingly, the anthracene $\pi$-system would be less 
shielded by the substituent and a larger area for $\pi$ - $\pi$-interactions would be available, increasing the chance of excimer formation in the solid-state.

\subsubsection{Insights into solid-state excimer formation factors}

The synthesis of the novel isomers with the uncommon substitution motif was performed, starting from the corresponding chloro- and bromoanthraquinones. Analysis of the solid-state structures of [1-(S)PPh $2^{-}$ $\left.\left(\mathrm{C}_{14} \mathrm{H}_{9}\right)\right](20)$ and [2-(S)PPh$\left.-\left(\mathrm{C}_{14} \mathrm{H}_{9}\right)\right](22)$ revealed different orientations of the substituent and therefore, the increased flexibility (Figure 77). Furthermore, the reduced steric strain brought back the planarity of the anthracene scaffold $\left(\alpha<3.6^{\circ}\right)$. Probably induced by the increased flexibility, three different polymorphs of [1-(S)PPh$\left.{ }_{2}-\left(\mathrm{C}_{14} \mathrm{H}_{9}\right)\right]$ (20) were identified via X-ray crystallography. Their intramolecular structural parameters varied only slightly, whereas the intermolecular interactions were fundamentally different. As desired, for two polymorphs ( $\beta-20$ and $\gamma-20)$ a dimeric motif with a large overlapping area of the anthracene moieties were found (Figure 77$)$. The third polymorph $(\alpha-20)$ revealed no face-to-face interactions and adopted a herringbone type packing. A similar motif was also found for the third regioisomer [2-(S)PPh $\left.-\left(\mathrm{C}_{14} \mathrm{H}_{9}\right)\right]$ (22). The crystal packing is similar to unsubstituted anthracene and no $\pi-\pi$ interactions were found. The positional isomers and especially the polymorphs of [1-(S)PPh $\left.{ }_{2}\left(\mathrm{C}_{14} \mathrm{H}_{9}\right)\right](20)$ were therefore ideal candidates for the intended investigations of the relationship between the intermolecular interactions and the solid-state luminescence.

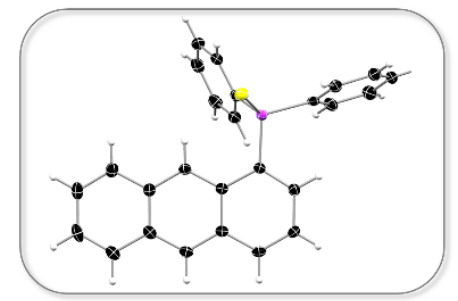

$\left[1-(\mathrm{S}) \mathrm{PPh}_{2}-\left(\mathrm{C}_{14} \mathrm{H}_{9}\right)\right](20)$

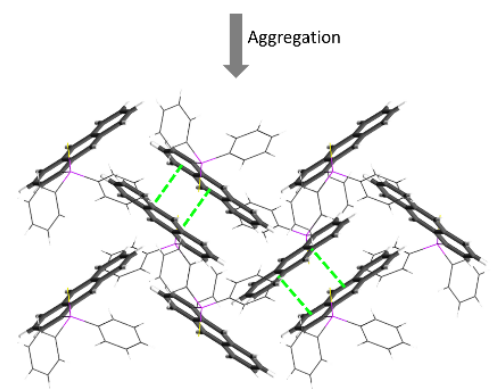

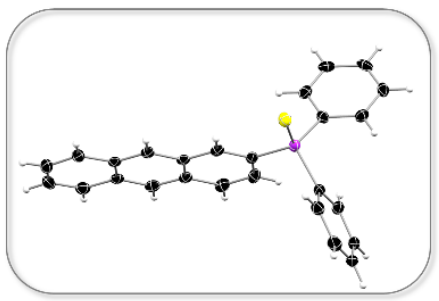

$\left[2-(\mathrm{S}) \mathrm{PPh}_{2}-\left(\mathrm{C}_{14} \mathrm{H}_{9}\right)\right](\mathbf{2 2})$

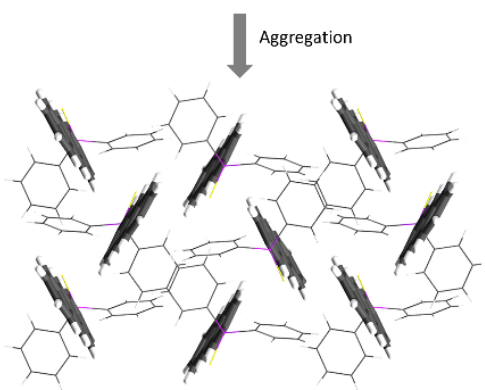

Figure 77. The two regioisomers [1-(S)PPh $\left.-\left(\mathrm{C}_{14} \mathrm{H}_{9}\right)\right](20)$ (left) and [2-( $\left.\left.\mathrm{S}\right) \mathrm{PPh}_{2}-\left(\mathrm{C}_{14} \mathrm{H}_{9}\right)\right]$ (22) (right) showed fundamentally different packing motifs and intermolecular interactions. The two polymorphs of [1-(S)PPh $\left.-\left(\mathrm{C}_{14} \mathrm{H}_{9}\right)\right], \beta-20$ and $\gamma-20$, revealed strong $\pi-\pi$ interactions (green) between the anthracene moieties, which are essential for an excimer formation.

The photophysical properties of [1-(S)PPh $\left.-\left(\mathrm{C}_{14} \mathrm{H}_{9}\right)\right](20)$ and [2-(S)PPh $\left.-\left(\mathrm{C}_{14} \mathrm{H}_{9}\right)\right](22)$ in diluted solution are closely related to unsubstituted anthracene in terms of the vibronic structure and emission wavelength. The low quantum yields were again attributed to the PET from the sulfur lone pairs. The restorage of the 
vibronic structure in the emission spectra supports the initial hypothesis that the deformation of the anthracene plane can contribute to the loss of the vibronic structure.

The emission properties of the regioisomers changed drastically in the solid-state and are fundamentally different. [2-(S)PPh $-\left(\mathrm{C}_{14} \mathrm{H}_{9}\right)$ ] (22) as well as the $\alpha$-form of [1-(S)PPh ${ }_{2}-\left(\mathrm{C}_{14} \mathrm{H}_{9}\right)$ ] (20) revealed a blue emission that differs only slightly from the emission in solution. The solid-state structures revealed no $\pi-\pi$ interaction, and the emission was consequently assigned to their monomeric forms. The $\beta$ - and $\gamma$ polymorphs of [1-(S) $\mathrm{PPh}_{2}-\left(\mathrm{C}_{14} \mathrm{H}_{9}\right)$ ] (20) exhibited an anthracene-unusual yellow emission. Furthermore, the vibronic structure was completely lost, and a large broadening of the emission spectra could be observed. Together with the increased lifetimes, a solid-state excimer formation could be confirmed.

Through introduction of a second substituent in the 4-position, another comparable derivative was obtained. Furthermore, co-crystallization with solvent molecules afforded further compounds with different intermolecular interactions. Overall, a plethora of compounds with overlap ratios varying from 0 - 53\% were examined and allowed a detailed study of the correlation between the $\pi-\pi$ interactions and the solid-state emission. A clear trend in the increase of the emission wavelength with larger overlap ratio could be determined. The red shift of the emission wavelength was attributed to the excimer formation and the resulting stabilization of the excited state.

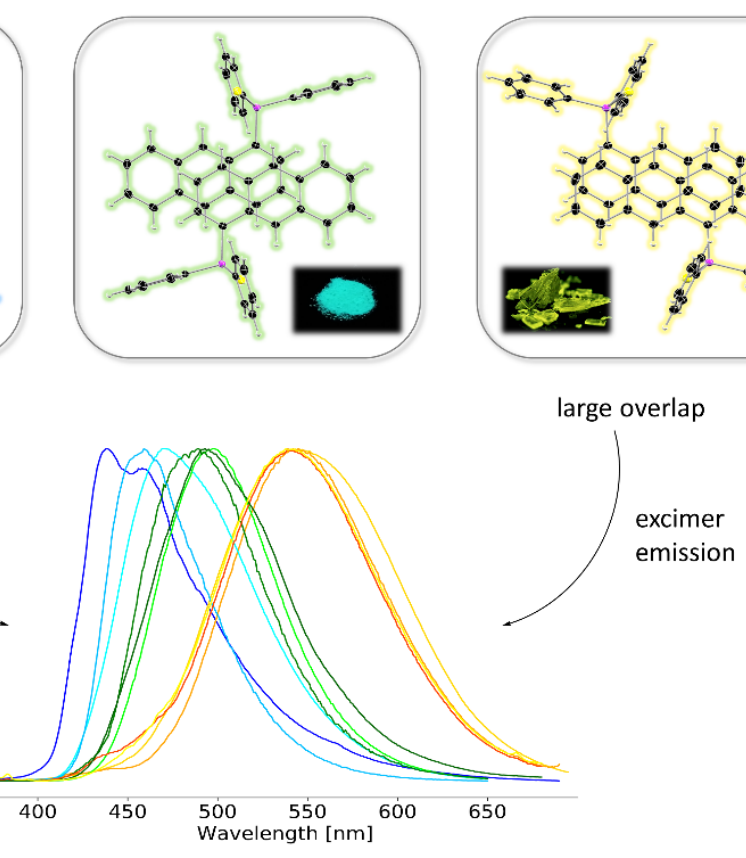

Figure 78. Several polymorphs and co-crystals of the thiophosphoryl anthracenes [1-(S)PPh $\left.\mathrm{Ph}_{2}\left(\mathrm{C}_{14} \mathrm{H}_{9}\right)\right](20)$ and $\left[1-(\mathrm{S}) \mathrm{PPh}_{2}-4-\mathrm{Et}-\right.$ $\left.\left(\mathrm{C}_{14} \mathrm{H}_{8}\right)\right](24)$, with different emission properties were obtained. A solid-state excimer formation could be confirmed, and the overlap ratio of the anthracene dimers determined the emission wavelengths.

Thanks to the variety of obtained structures with different pronounced $\pi-\pi$ interactions between the anthracene moieties, further insights into the excimer formation factors in the solid-state were possible. A linear correlation of the emission wavelength with the overlap ratio was found only for a small area of overlap ratios (from around $20-45 \%$ ). It was assumed that a threshold for excimer formation exists. 
Below $20 \%$ overlap, the increase of the emission wavelength with increasing overlap is only marginal. Beyond the threshold, the emission wavelength increased more rapid. Furthermore, an upper limit for the shift of the emission wavelength was found at around 45\% overlap. Even if the overlapping area was further increased, no additional shift of the emission wavelength could be determined. This indicated for a maximum stabilization of the excited state at a certain overlap ratio in the ground-state. The analysis of the solid-state structures revealed two preferred geometries of the anthracene dimers. It was postulated that the geometry in the ground-state determines the type of excimer formed in the excited state. For a naphthalene-type excimer, two of the three anthracene rings are participating in the $\pi-\pi$ interactions in the ground-state. The corresponding overlap ratio was determined between $22.3 \%$ and $34.0 \%$. The observed excimer emission is located in the green region and reaches values up to $512 \mathrm{~nm}$. When all three anthracene rings are overlapping in the ground-state, an anthracene-type excimer is formed upon excitation. The excimer stabilization energy is even larger, and a further bathochromic shift of the emission wavelength was observed.

The investigation of the positional isomers of the thiophosphoryl anthracene revealed a strong dependency of the solid-state emission from the intermolecular interaction. The obtained results gave valuable insights into the understanding of the solid-state luminescence and especially the excimer formation. The variation of the emission wavelength of only one compound, over a broad range of the visible spectrum, brings several advantages for possible applications. White light emitting organic diodes (WOLEDs) consist usually of three components that emit in a different color each. For reasons of preparation, stability, and color-purity, it can be beneficial, if two or more of the components are derived from the same compound. Varying the emission wavelength by control of the intermolecular interactions, could be a suitable strategy for this approach. The combination of monomer and excimer fluorescence is a promising approach for single-molecular white light emission and is already subject of current research. ${ }^{[21,122]}$ Moreover, the herein investigated compounds [1-(S) $\left.\mathrm{PPh}_{2}-\left(\mathrm{C}_{14} \mathrm{H}_{9}\right)\right](20)$ and [1-(S)PPh$-4-E t-$ $\left.\left(\mathrm{C}_{14} \mathrm{H}_{8}\right)\right]$ (24) bear potential for mechanochromic applications. Those materials often show a strong correlation between the intermolecular interactions and the emission properties. External stimuli (fuming with appropriate solvent; grinding and annealing) could force a change in the occurring interactions, resulting in an altered emission wavelength. Furthermore, a piezochromic behavior can be expected from the thiophosphoryl anthracenes. The application of external pressure probably influences the structure of the anthracene dimers. A compression and an increase of the overlap ratio is expected that could go along with a shift of the emission wavelength. In combination with high-pressure XRD-analysis, the structural changes can be monitored and may give further insights into the excimer formation process.

\subsubsection{Luminescent phosphanyl anthracenes}

During the synthesis and investigation of the phosphoryl anthracene regioisomers, an unusual solid-state luminescence of the precursors [1-PPh $\left.-\left(\mathrm{C}_{14} \mathrm{H}_{9}\right)\right](\mathbf{1 8})$ and [2- $\left.\mathrm{PPh}_{2}-\left(\mathrm{C}_{14} \mathrm{H}_{9}\right)\right](\mathbf{2 1})$ was observed. The emission of aromatic phosphines is usually efficiently quenched due to the PET from the phosphorous lone pair. Such a behavior was also observed for the herein 9,10 substituted phosphanyl anthracenes. While the emission in solution of $\mathbf{1 8}$ and $\mathbf{2 1}$ was still nearly quenched, the observed emission in the solid-state was 
therefore unexpected. For further investigations, the regioisomers of the disubstituted $\left[\left(\mathrm{PPh}_{2}\right)_{2}-\left(\mathrm{C}_{14} \mathrm{H}_{8}\right)\right]$ (25-28) were synthesized. All showed a similar solid-state emission with moderate quantum yields, while the emission in solution remained quenched. The crystal packing of the phosphines revealed no strong intermolecular interactions. The emission enhancement in the solid-state was therefore attributed to an intramolecular effect. In comparison to the non-emissive 9,10-disubstituted derivatives, the positional isomers revealed a changed orientation of the phosphanyl group. The phosphorous lone pair is turned towards a more orthogonal orientation regarding the anthracene. In contrast, in the 9,10-substituted derivatives the lone pair was located inside the anthracene plane. The changed orientation was attributed to be responsible for the emission enhancement in the solid-state. The main non-radiative deactivation pathway, the PET from the phosphorous lone pair, is probably less effective in the changed orientation. In solution, the molecules are flexible enough to reach the required geometry for a PET. Upon aggregation the flexibility is decreased, and an efficient PET is prohibited. Consequently, a blue-green emission could be observed in the solid-state. A transfer of this concept to other fluorophores or substituents that can undergo a PET, can proof this concept. In general, AIE-materials based on a PET are only barely investigated yet but bear potential as a new subclass of AIE-luminogens. Rational design of fluorophores could probably further optimize the suppression of the PET upon aggregation and emphasize the emission enhancement in the solid-state.

\subsubsection{Conformation induced emission enhancement}

In the last part of this work, the results of prior studies on the unusual luminescence of the $\left[9,10-\left((\mathrm{S}) \mathrm{PPh}_{2}\right)_{2^{-}}\right.$ $\left(\mathrm{C}_{14} \mathrm{H}_{8}\right)$ ] co-crystals were set into relation with the results obtained during this work. The intense, green emission of the co-crystals showed similar characteristics as the herein investigated 9,10-disubstituted thiophosphoryl anthracenes (13-17). Therefore, an exciplex emission as initially postulated by Fei et al. was excluded. The fluorescence was also ascribed to occur from the thiophosphoryl anthracene monomer. Intramolecular effects induced by the two thiophosphoryl groups were supposed to be responsible for the large bathochromic shift. The drastic differences in the quantum yields of the two types of co-crystals resulted from the cisoid and transoid conformation of the substituents. The high quantum yields of the cocrystals with a transoid conformation were ascribed to the structural rigidity, induced by the two thiophosphoryl groups. The large steric strain within the molecule led to a twist of the anthracene moiety, as already described by Finkelmeier. ${ }^{[153]}$ In contrast, the co-crystals that adopted a cisoid conformation, with a large folding of the anthracene, revealed only weak emission (Figure 79). 


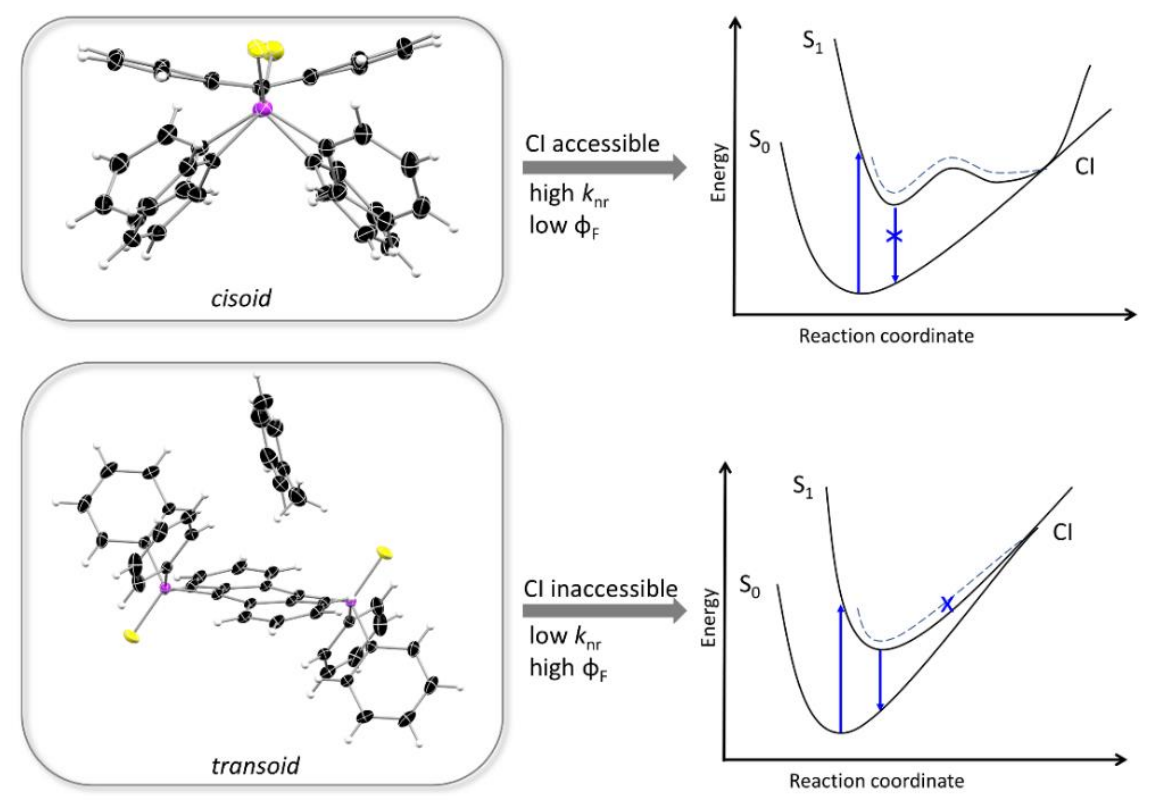

Figure 79. The already known $\left[9,10-\left((\mathrm{S}) \mathrm{PPh}_{2}\right)_{2}-\left(\mathrm{C}_{14} \mathrm{H}_{8}\right)\right]$ and its co-crystals were reinvestigated during this work. The unusual photophysical behavior was attributed to the two different conformations of the co-crystals. Due to the strong deformation of the anthracene plane in the cisoid conformation, the $\mathrm{S}_{0}-\mathrm{S}_{1}$ conical intersection is accessible upon excitation, and a fluorescence quenching is observed. In the transoid conformation, the thiophosphoryl groups and the co-crystallized solvent prevent a folding of the anthracene scaffold. The $\mathrm{Cl}$ cannot be reached, and the non-radiative decay is blocked. Thus, an intense fluorescence is observed.

Generally speaking, a conical intersection of the first excited state and the ground state of a molecule, results in a non-radiative deactivation and a fluorescence quenching. Therefore, knowledge about the energies and structures at the conical intersection, is helpful in understanding photophysical processes. Computational investigations by several groups have identified the structures of anthracene and other fluorophores at the minimum energy conical intersection. ${ }^{[145,148,228]}$ Based on these results, a general concept concerning fluorescence quenching in solution and in the solid-state was postulated. ${ }^{[148]}$

For anthracene, the structure at the conical intersection revealed a strong distortion through a bending of the two outer rings. ${ }^{[228]} \mathrm{A}$ similar distortion is found in the cisoid co-crystals of $\left[9,10-(\mathrm{S}) \mathrm{PPh}_{2}-\left(\mathrm{C}_{14} \mathrm{H}_{8}\right)\right]$ (Figure 79). Thus, the ground-state structure is already similar to the expected geometry at the conical intersection. Upon excitation only little structural changes are necessary, to reach the conical intersection. In contrast, the co-crystals in a transoid orientation are not able to undergo such a large-amplitude motion to reach the $\mathrm{Cl}$. The substituents in the transoid conformation prohibit a folding of the anthracene scaffold. The conical intersection and therefore the main non-radiative pathway cannot be reached. The deactivation occurs radiative via fluorescence. An intense green emission with high quantum yields can be observed. The $\mathrm{C}-\mathrm{H} \cdots \pi$ interactions between the co-crystallized solvents and the thiophosphoryl anthracene further increase the rigidity of the whole system.

The described concept was introduced in the literature as "restricted access to a conical intersection" $(\mathrm{RACl}){ }^{[145]} \mathrm{A}$ few molecules have been reported, whose properties could be explained by this concept. ${ }^{[148]}$ A direct observation of one compound in two conformations with such drastic structural and 
photophysical differences (like the co-crystals of $\left.\left[9,10-\left((\mathrm{S}) \mathrm{PPh}_{2}\right)_{2}-\left(\mathrm{C}_{14} \mathrm{H}_{8}\right)\right]\right)$, has not been reported up to now. For a complete verification of the proposed process, computational studies, and a calculation of the structure at the conical intersections of $\left[9,10-\left((\mathrm{S}) \mathrm{PPh}_{2}\right)_{2}-\left(\mathrm{C}_{14} \mathrm{H}_{8}\right)\right]$ are required. The already long-known compound can then still be helpful in the understanding of the photophysical processes in the solid-state.

A further increase of the molecule's rigidity can be a possibility for achieving even higher quantum yields. A conceivable modification would be the replacement of the phenyl groups of the diphenylphosphine substituents by bulkier mesityl groups, for example. Another approach could be the use of a phosphole that would result in a more rigid structure. If these compounds crystallize also in a transoid-orientation, even higher quantum yields could be expected. Furthermore, a transfer of this concept to other compounds is desirable. The already briefly investigated $\left[9,10-\left((\mathrm{Cl}) \mathrm{SiPh}_{2}\right)_{2}-\left(\mathrm{C}_{14} \mathrm{H}_{8}\right)\right](\mathbf{1 2})$ is also able to crystallize in two different conformations, even without the co-crystallization of the solvent. An isolation and detailed investigation of the two conformers could give further insights into the underlying photophysical processes of the conformation dependent luminescence. 


\section{Experimental Details}

\subsection{General Procedures}

Air- and moisture sensitive compounds were handled and stored using modified Schlenk techniques in a dry nitrogen or argon atmosphere or in an argon glovebox LABmaster SP by MBRAUN. Solvents for air and moisture sensitive reactions were dried by distillation from sodium ( $n$-hexane, toluene), potassium (THF), sodium-potassium alloy ( $\mathrm{Et}_{2} \mathrm{O}, n$-pentane) or phosphorous pentoxide (DCM) and degassed using standard laboratory techniques. Commercially available chlorodiphenylphosphine was purified by vacuum distillation, elemental sulfur was purified by sublimation. $n$ Butyllithium was filtered through Celite ${ }^{\odot}$ to remove formed lithium hydroxide and the concentration was determined by titration. All other commercially available compounds were used as received and without further purification. If not otherwise noted, all starting materials and reactants were synthesized according to the given procedures.

\subsection{Analytical methods}

\subsubsection{Elemental Analysis}

Elemental analyses ( $\mathrm{C}, \mathrm{H}, \mathrm{N}, \mathrm{S})$ were performed by the Analytisches Labor of the Institute for Inorganic Chemistry at the Georg-August-Universität, Göttingen.

\subsubsection{Fluorescence spectroscopy}

Fluorescence measurements were performed on a Horiba Fluoromax 4 spectrofluorometer with a $150 \mathrm{~W}$ xenon, continuous output, lamp. Slit widths of excitation and emission monochromators were adjusted through the software. Usually, small slit widths in the range of 1-5 nm were selected. Emission spectra in solution were measured in diluted solution $\left(10^{-3}-10^{-6} \mathrm{~mol} \mathrm{~L}^{-1}\right)$ to avoid self-quenching and inner filter effects. Quartz glass cuvettes and analytical grade solvents were used. Solid samples were measured with the solid-sample holder at an angle of rotation of $30^{\circ}$ or $60^{\circ}$. Absolute quantum yields were determined with the Quanta- $\varphi$ integrating sphere with an internal diameter of $15 \mathrm{~cm}$. Solid samples were measured in spectralon sample holders. Fluorescence lifetimes were measured with the TCSPC setup with a pulsed laser diode $(375 \mathrm{~nm})$ as excitation source. The obtained decays were usually fitted as one or twoexponential decays to obtain the lifetime(s) of the compounds. Short lifetimes in the ps range were usually attributed to scattering effects and neglected.

\subsubsection{Mass spectrometry}

Mass spectrometric analyses were performed by the Zentrale Analytik of the Institute for Organic and Biomolecular Chemistry at the Georg-August-Universität, Göttingen. El-MS spectra were recorded with a Finnigan MAT 95, $70 \mathrm{eV}$. ESI-MS spectra were recorded with a Bruker Daltonics microTOF or maXis ESIQTOF-MS. Signals are given as a mass to charge ratio $\mathrm{m} / \mathrm{z}$ of the fragment ions, based on the molecular mass of the isotopes with the highest natural abundancies (e.g., ${ }^{1} \mathrm{H},{ }^{13} \mathrm{C},{ }^{14} \mathrm{~N},{ }^{16} \mathrm{O},{ }^{28} \mathrm{Si},{ }^{31} \mathrm{P},{ }^{32} \mathrm{~S},{ }^{35} \mathrm{Cl} /{ }^{37} \mathrm{Cl}$. $\left.{ }^{79} \mathrm{Br} /{ }^{81} \mathrm{Br}\right)$. 


\subsubsection{NMR spectroscopy}

NMR spectra were recorded on either a Bruker Avance III 300 or a Bruker Avance III 400 NMR spectrometer. All spectra were referenced to residual solvent signals of the deuterated solvent. ${ }^{[229]}{ }^{31} \mathrm{P}$ chemical shifts are reported relative to the external standard phosphoric acid $(\delta=0.0 \mathrm{ppm})$. Coupling constants $J$ are reported in $\mathrm{Hz}$ and the following abbreviations are used indicating the multiplicity: $\mathrm{s}$ =singlet, $\mathrm{d}=$ doublet, $\mathrm{t}=$ triplet, $\mathrm{q}=$ quartet, $\mathrm{m}=$ multiplet, $\mathrm{br}=$ broad, and their combinations. Assignment of the signals was done, if possible, via $2 \mathrm{D}$-correlation spectra $\left({ }^{1} \mathrm{H}-{ }^{1} \mathrm{H} \operatorname{COSY},{ }^{1} \mathrm{H}-{ }^{13} \mathrm{C}-\mathrm{HSQC},{ }^{1} \mathrm{H}-{ }^{13} \mathrm{C} \mathrm{HMBC}\right) .{ }^{[230]}$

\subsubsection{UV-Vis Spectroscopy}

UV-Vis Absorption spectra were obtained on an Agilent Cary spectrometer using the Scan software. Standard quartz glass cuvettes and analytical grade solvent were used. Background measurements of the solvent were performed in identical cuvettes. Concentration of the used solutions were usually in the range of $10^{-4}-10^{-6} \mathrm{M}$. 


\subsection{Synthetic procedures}

\subsubsection{Synthesis of 9-Bromo-10-methylanthracene}

9,10-Dibromoanthracene (6.08 g, $18.1 \mathrm{mmol}, 1.0$ eq.) was dissolved in THF (100 mL) and cooled to $-78{ }^{\circ} \mathrm{C}$. $n$-Butyllithium in hexane $(2.4 \mathrm{M}, 8.0 \mathrm{~mL}, 19.0 \mathrm{mmol}, 1.1 \mathrm{eq}$.) was added dropwise over $20 \mathrm{~min}$. After the addition was completed the mixture was stirred for $10 \mathrm{~min}$ and then lodomethane $(5.1 \mathrm{~g}, 2.24 \mathrm{~mL}$, $36.0 \mathrm{mmol}, 2.0$ eq.) was added slowly. The reaction was warmed to room temperature and stirred for $16 \mathrm{~h}$. $\mathrm{NH}_{4} \mathrm{Cl}$-solution $\left(2 \times 100 \mathrm{~mL}\right.$ ) was added, and the mixture extracted with $\mathrm{DCM}$. Drying over $\mathrm{MgSO}_{4}$ and removal of the solvent under reduced pressure gave the desired compound as yellow powder. Recrystallization from ethylacetate/toluene (95:5) afforded the pure compound as yellow needles.

Yield:

Chemical formula:

Molecular weight:

${ }^{1} \mathrm{H}-\mathrm{NMR}$

$\left(\mathrm{CDCl}_{3}, 300 \mathrm{MHz}\right)$

${ }^{13} \mathrm{C}\left\{{ }^{1} \mathrm{H}\right\}-N M R$

$\left(\mathrm{CDCl}_{3}, 75 \mathrm{MHz}\right)$

\section{$3.1 \mathrm{~g}(11.4 \mathrm{mmol}, 63 \%)$}

$\mathrm{C}_{15} \mathrm{H}_{11} \mathrm{Br}$

$271.16 \mathrm{~g} / \mathrm{mol}$

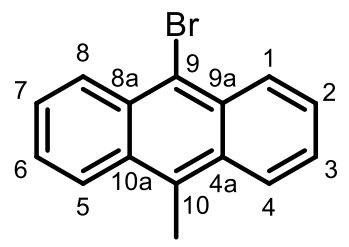

$\delta[p p m]=8.61-8.57\left(\mathrm{~m}, 2 \mathrm{H}, \mathrm{H}_{1,8}\right), 8.29-8.26\left(\mathrm{~m}, 2 \mathrm{H}, \mathrm{H}_{3,5}\right), 7.62-7.50$ (m, $4 \mathrm{H}, \mathrm{H}_{2,3,6,7)}, 3.05$ (s, $3 \mathrm{H}, \mathrm{CH}_{3}$ ).

$$
\begin{aligned}
& \delta[p p m]=131.0\left(\mathrm{~s}, 1 \mathrm{C}, \mathrm{C}_{10}\right), 131.0\left(\mathrm{~s}, 1 \mathrm{C}, \mathrm{C}_{8 \mathrm{a}, 9 \mathrm{a}}\right), 130.3\left(\mathrm{~s}, 2 \mathrm{C}, \mathrm{C}_{4 \mathrm{a}, 10 \mathrm{a}}\right), 128.4 \\
& \left(\mathrm{~s}, 2 \mathrm{C}, \mathrm{C}_{1,8}\right), 126.8\left(\mathrm{~s}, 2 \mathrm{C}, \mathrm{C}_{2,7}\right), 125.6\left(\mathrm{~s}, 2 \mathrm{C}, \mathrm{C}_{3,6}\right), 125.1\left(\mathrm{~s}, 2 \mathrm{C}, \mathrm{C}_{4,5}\right), 121.6(\mathrm{~s} \text {, } \\
& \left.\left.1 \mathrm{C}, \mathrm{C}_{9}\right), 14.5 \text { (s, 1C, } \mathrm{CH}_{3}\right) \text {. }
\end{aligned}
$$

\section{EI-MS}

$m / z(\%):$

$270.0(100)[\mathrm{M}]^{+}, 191.1(75)[\mathrm{M}-\mathrm{Br}]^{+}$.

\section{HR-MS (EI+)}

$\mathrm{m} / \mathrm{z}$ (calculated): $\quad 270.0051(270.0044)$.

\subsubsection{Synthesis of 9-Bromo-10-ethylanthracene}

9,10-dibromoanthracene $(1.14 \mathrm{~g}, 3.4 \mathrm{mmol}, 1.0$ eq.) was suspended in THF $(15 \mathrm{~mL})$ and cooled to $-78{ }^{\circ} \mathrm{C}$. ${ }^{n}$ Butyllithium in hexane $(4.05 \mathrm{M}, 0.24 \mathrm{~g}, 3.8 \mathrm{mmol}, 1.1 \mathrm{eq})$ was added over the curse of $30 \mathrm{~min}$ and the dark-yellow solution was stirred for $10 \mathrm{~min}$ before ethyl iodide $(1.12 \mathrm{~g}, 0.58 \mathrm{~mL}, 7.2 \mathrm{mmol}, 2.1 \mathrm{eq}$.) was added. The solution was warmed to ambient temperature overnight. $\mathrm{NH}_{4} \mathrm{Cl}$-solution $(20 \mathrm{~mL})$ was added, and the mixture extracted with hexane $(2 \times 20 \mathrm{~mL})$. The solvent of the combined organic phases was removed under reduced pressure. The pure material was obtained as a yellow powder. 
Yield:

0.96 g (3.4 mmol, $99 \%)$

Chemical formula: $\quad \mathrm{C}_{16} \mathrm{H}_{13} \mathrm{Br}$

Molecular weight: $\quad 285.18 \mathrm{~g} / \mathrm{mol}$

${ }^{1} \mathrm{H}-\mathrm{NMR}$

$\left(\mathrm{CDCl}_{3}, 300 \mathrm{MHz}\right)$

$\delta[\mathrm{ppm}]=8.62\left(\mathrm{dd},{ }^{3} J_{\mathrm{HH}}=8.3 \mathrm{~Hz},{ }^{4} J_{\mathrm{HH}}=1.8 \mathrm{~Hz}, 2 \mathrm{H}, \mathrm{H}_{1,8}\right), 8.30(\mathrm{dd}$, $\left.{ }^{3} J_{H H}=8.0 \mathrm{~Hz},{ }^{4} J_{H H}=1.4 \mathrm{~Hz}, 2 \mathrm{H}, \mathrm{H}_{4,5}\right), 7.52-7.66\left(\mathrm{~m}, 4 \mathrm{H}, \mathrm{H}_{2,3,6,7}\right)$, ), 3.64 (q, $\left.{ }^{3} J_{\mathrm{HH}}=7.6 \mathrm{~Hz}, 2 \mathrm{H}, \mathrm{CH}_{2}\right), 1.47\left(\mathrm{t},{ }^{3} J_{\mathrm{HH}}=7.6 \mathrm{~Hz}, 3 \mathrm{H}, \mathrm{CH}_{3}\right)$

\section{${ }^{13} \mathrm{C}\left\{{ }^{1} \mathrm{H}\right\}-\mathrm{NMR}$}

$\left(\mathrm{CDCl}_{3}, 75 \mathrm{MHz}\right)$

$\delta[p p m]=137.3\left(\mathrm{~s}, 1 \mathrm{C}, \mathrm{C}_{10}\right), 130.5\left(\mathrm{~s}, 2 \mathrm{C}, \mathrm{C}_{8 \mathrm{a}, 9 \mathrm{a}}\right), 130.0$ (s, 2C, $\left.\mathrm{C}_{4 \mathrm{a}, 10 \mathrm{a}}\right), 128.7$

(s, 2C, $\left.C_{1,8}\right), 126.7\left(\mathrm{~s}, 2 \mathrm{C}, \mathrm{C}_{3,6}\right), 125.6\left(\mathrm{~s}, 2 \mathrm{C}, \mathrm{C}_{2,7}\right), 124.7$ (s, 2C, $\left.\mathrm{C}_{4,5}\right), 121.6$ (s, $\left.1 \mathrm{C}, \mathrm{C}_{9}\right), 21.4\left(\mathrm{~s}, 1 \mathrm{C}, \mathrm{CH}_{2}\right), 15.4\left(\mathrm{~s}, 1 \mathrm{C}, \mathrm{CH}_{3}\right)$.

\section{EI-MS}

$\mathrm{m} / \mathrm{z}(\%):$

$189.1(100)\left[\mathrm{C}_{15} \mathrm{H}_{9}\right]^{+}, 269.0(100)\left[\mathrm{M}-\mathrm{CH}_{3}\right]^{+}, 284.0(46)[\mathrm{M}]^{+}$.

\section{HR-MS (EI+)}

$\mathrm{m} / \mathrm{z}$ (calculated): $\quad 284.0199(284.0201)$.



\subsubsection{Synthesis of 9-Bromo-10-trimethylsilylanthracene}

9,10-dibromoanhtracene $(2.12 \mathrm{~g}, 6.3 \mathrm{mmol}, 1.0$ eq.) was suspended in THF (15 mL) and cooled to $-78{ }^{\circ} \mathrm{C}$. "Butyllithium in hexane $(4.0 \mathrm{M}, 1.7 \mathrm{~mL}, 1.05$ eq.) was added over the course of $15 \mathrm{~min}$ and the darkyellow solution was stirred for $20 \mathrm{~min}$. Trimethylsilylchloride (1.03 g, $1.2 \mathrm{~mL}, 9.5 \mathrm{mmol}, 1.5 \mathrm{eq}$.) was added within $20 \mathrm{~min}$ and the mixture was stirred for another $30 \mathrm{~min}$ at $-78{ }^{\circ} \mathrm{C}$. Afterwards it was allowed to warm to ambient temperature and water $(20 \mathrm{~mL})$ was added. The mixture was extracted with hexane $(2 \times 20 \mathrm{~mL})$. The combined organic phases were dried over $\mathrm{MgSO}_{4}$, filtrated and the solvent removed under reduced pressure, which afforded the target compound as a yellow solid.

\section{Yield: $\quad 1.75 \mathrm{~g}(6.2 \mathrm{mmol}, 98 \%)$}

Chemical formula: $\quad \mathrm{C}_{17} \mathrm{H}_{17} \mathrm{SiBr}$ 
Molecular weight: $\quad 329.31 \mathrm{~g} / \mathrm{mol}$

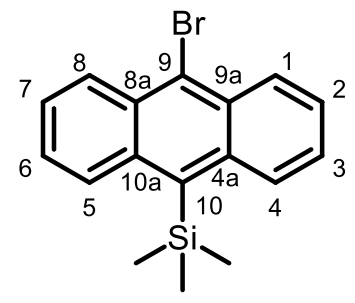

${ }^{1}$ H-NMR

$\left(\mathrm{CDCl}_{3}, 300 \mathrm{MHz}\right) \quad \delta[\mathrm{ppm}]=8.63\left(\mathrm{ddd}, J_{\mathrm{HH}}=8.8,1.5,0.7 \mathrm{~Hz}, 2 \mathrm{H}, \mathrm{H}_{1,8}\right), 8.44$ (ddd, $J_{\mathrm{HH}}=8.8$, $\left.1.3,0.7 \mathrm{~Hz}, 2 \mathrm{H}, \mathrm{H}_{4,5}\right), 7.60-7.46\left(\mathrm{~m}, 4 \mathrm{H}, \mathrm{H}_{2,3,6,7}\right), 0.71\left(\mathrm{~s}, 9 \mathrm{H}, \mathrm{CH}_{3}\right)$.

${ }^{13} \mathrm{C}\left\{{ }^{1} \mathrm{H}\right\}-N M R$

$\left(\mathrm{CDCl}_{3}, 75 \mathrm{MHz}\right) \quad \delta[\mathrm{ppm}]=137.8\left(\mathrm{~s}, 2 \mathrm{C}, \mathrm{C}_{4 \mathrm{a}, 10 \mathrm{a}}\right), 137.3\left(\mathrm{~s}, 1 \mathrm{C}, \mathrm{C}_{10}\right), 130.3\left(\mathrm{~s}, 2 \mathrm{C}, \mathrm{C}_{8 \mathrm{a}, 9 \mathrm{a}}\right), 129.2$ $\left(\mathrm{s}, 2 \mathrm{C}, \mathrm{C}_{4,5}\right), 129.0\left(\mathrm{~s}, 2 \mathrm{C}, \mathrm{C}_{1,8}\right), 126.7$ (s, 1C, $\left.\mathrm{C}_{9}\right), 126.5$ (s, 2C, $\left.\mathrm{C}_{2,7}\right), 124.9$ (s, $\left.2 \mathrm{C}, \mathrm{C}_{3,6}\right), 4.7\left(\mathrm{~s}, 3 \mathrm{C}, \mathrm{CH}_{3}\right)$.

\section{EI-MS}

$\mathrm{m} / \mathrm{z}(\%): \quad 328.1(89)[\mathrm{M}]^{+}, 313.0(79)\left[\mathrm{M}-\mathrm{CH}_{3}\right]^{+}, 234.1(100)\left[\mathrm{M}-\mathrm{CH}_{3} \mathrm{Br}\right]^{+}$.

HR-MS (EI $)$

$\mathrm{m} / \mathrm{z}$ (calculated): $\quad 328.0293(328.0282)$.

\subsubsection{Synthesis of $\left[9-\mathrm{PPh}_{2}-\left(\mathrm{C}_{14} \mathrm{H}_{9}\right)\right](1)$}

9-Bromoanthracene (1.00 g, $3.89 \mathrm{mmol}, 1.0$ eq.) was suspended in $\mathrm{Et}_{2} \mathrm{O}(15 \mathrm{~mL})$ and cooled to $-78^{\circ} \mathrm{C} . n$ Butyllithium in hexane $(4.05 \mathrm{M}, 0.26 \mathrm{~g}, 1.0 \mathrm{~mL}, 4.08 \mathrm{mmol}, 1.05 \mathrm{eq}$.) was added slowly and the reaction stirred for $10 \mathrm{~min}$. Afterwards the mixture was warned to ambient temperature for about $10 \mathrm{~min}$ until a clear yellow solution formed. Cooling again to $-78{ }^{\circ} \mathrm{C}$ was followed by the addition of Chlorodiphenylphosphine $(0.88 \mathrm{~g}, 0.73 \mathrm{~mL}, 4.08 \mathrm{mmol}, 1.05$ eq.). The mixture warmed to ambient temperature and stirred for $2 \mathrm{~h}$. The precipitate was filtered off and washed with $\mathrm{Et}_{2} \mathrm{O}(2 \times 5 \mathrm{~mL})$. The crude product was dissolved in DCM $(20 \mathrm{~mL})$ and Lithium chloride removed by filtration. Removing the solvent under reduced pressure yields the desired product as a yellow powder. Recrystallization from DCM lead to suitable crystals for X-Ray structure determination.

Yield:

$1.03 \mathrm{~g}(2.83 \mathrm{mmol}, 72.7 \%)$

Chemical formula: $\quad \mathrm{C}_{26} \mathrm{H}_{19} \mathrm{P}$ 
Molecular weight: $\quad 362.41 \mathrm{~g} / \mathrm{mol}$

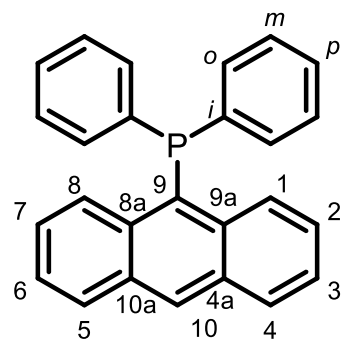

${ }^{1}$ H-NMR

$\left(\mathrm{CDCl}_{3}, 300 \mathrm{MHz}\right)$

$\delta[\mathrm{ppm}]=8.79\left(\mathrm{dd},{ }^{3} J_{\mathrm{HH}}=8.8 \mathrm{~Hz},{ }^{4} J_{\mathrm{PH}}=4.6 \mathrm{~Hz}, 2 \mathrm{H}, \mathrm{H}_{1,8}\right), 8.67\left(\mathrm{~s}, 1 \mathrm{H}, \mathrm{H}_{10}\right)$, $8.07\left(\mathrm{dd},{ }^{3} \mathrm{~J}_{\mathrm{HH}}=8.4 \mathrm{~Hz}, 2 \mathrm{H}, \mathrm{H}_{4,5}\right), 7.49-7.41\left(\mathrm{~m}, 6 \mathrm{H}, o-\mathrm{Ph}, \mathrm{H}_{3,6}\right), 7.37-$ $7.32\left(\mathrm{~m}, 2 \mathrm{H}, \mathrm{H}_{2,7}\right), 7.30-7.26(\mathrm{~m}, 6 \mathrm{H}, m-\mathrm{Ph}, p-\mathrm{Ph})$.

${ }^{13} \mathrm{C}\left\{{ }^{1} \mathrm{H}\right\}-N M R$

$\left(\mathrm{CDCl}_{3}, 75 \mathrm{MHz}\right)$

$\delta[p p m]=137.0\left(\mathrm{~d},{ }^{3} \mathrm{~J}_{\mathrm{CP}}=6.0 \mathrm{~Hz}, 2 \mathrm{C}, \mathrm{C}_{4 \mathrm{a}}, 10 \mathrm{a}\right), 136.8\left(\mathrm{~d},{ }^{2} \mathrm{~J}_{\mathrm{CP}}=6.8 \mathrm{~Hz}, 2 \mathrm{C}\right.$, $\mathrm{C}_{8 \mathrm{a}, 9 \mathrm{a}}$ ), $132.1\left(\mathrm{~s}, 1 \mathrm{C}, \mathrm{C}_{10}\right), 132.0\left(\mathrm{~d},{ }^{1} \mathrm{~J}_{\mathrm{CP}}=4.5 \mathrm{~Hz}, 2 \mathrm{C}, i-\mathrm{Ph}\right), 131.7$ (d, ${ }^{2} \mathrm{~J}_{\mathrm{CP}}=$ $18.3 \mathrm{~Hz}, 4 \mathrm{C}, o-\mathrm{Ph}), 129.6\left(\mathrm{~s}, 1 \mathrm{C}, \mathrm{C}_{9}\right), 129.4\left(\mathrm{~s}, 2 \mathrm{C}, \mathrm{C}_{4,5}\right), 128.7$ (d, ${ }^{3} \mathrm{JPP}_{\mathrm{CP}}=$ $\left.24.8 \mathrm{~Hz}, 2 \mathrm{C}, \mathrm{C}_{1,8}\right), 128.5$ (d, ${ }^{3} \mathrm{JP}_{\mathrm{CP}}=5.7 \mathrm{~Hz}, 4 \mathrm{C}, m-\mathrm{Ph}$ ), 127.7 (s, 2C, $p-\mathrm{Ph}$ ), 126.1 (s, 2C, $\left.C_{2,7}\right), 125.2$ (s, 2C, $C_{3,6}$ ).

${ }^{31} \mathrm{P}\left\{{ }^{1} \mathrm{H}\right\}-N M R$

$\left(\mathrm{CDCl}_{3}, 121 \mathrm{MHz}\right) \quad \delta[\mathrm{ppm}]=-24.69$.

EI-MS

$\mathrm{m} / \mathrm{z}(\%)$ :

$362.1(100)[\mathrm{M}]^{+}, 283.1(54)[\mathrm{M}-(\mathrm{Ph}+2 \mathrm{H})]^{+}, 254.1(57)\left[\mathrm{M}-\left(\mathrm{Ph}+\mathrm{C}_{2} \mathrm{H}_{7}\right)\right]$.

HR-MS (EI+)

m/z (calculated): $\quad 363.1291(363.1297)$.

Elemental analysis

in \% (calculated): $\quad$ C: 85.01 (86.17), H: 5.35 (5.28).

\subsubsection{Synthesis of $\left[9-\mathrm{PPh}_{2}-10-\mathrm{Br}-\left(\mathrm{C}_{14} \mathrm{H}_{8}\right)\right](2)$}

9,10-dibromoanthracene (1.30 g, $1.74 \mathrm{~mL}, 3.7 \mathrm{mmol}, 1.0$ eq.) was suspended in $\mathrm{Et}_{2} \mathrm{O}(20 \mathrm{~mL})$ and cooled to $-15^{\circ} \mathrm{C}$. ${ }^{n}$ Butyllithium ( $2.36 \mathrm{M}, 1.74 \mathrm{~mL}, 4.1 \mathrm{mmol}, 1.1$ eq.) was added slowly and the mixture was stirred for $20 \mathrm{~min}$. Afterwards chlorodiphenylphosphine $(0.83 \mathrm{~g}, 0.67 \mathrm{~mL}, 3.7 \mathrm{mmol}, 1.0$ eq.) was added. The solution stirred for $2 \mathrm{~h}$ at ambient temperature. The solvent was removed under reduced pressure and the residue dissolved in DCM $(20 \mathrm{~mL})$ and filtrated. Removal of the solvent afforded a yellow powder, which could be recrystallized from Toluene. 
Yield:

Chemical formula: $\quad \mathrm{C}_{26} \mathrm{H}_{18} \mathrm{PBr}$

Molecular weight:

${ }^{1} \mathrm{H}-\mathrm{NMR}$

$\left(\mathrm{CDCl}_{3}, 300 \mathrm{MHz}\right)$ $\mathrm{Ph})$.

\section{${ }^{13} \mathrm{C}\left\{{ }^{1} \mathrm{H}\right\}-\mathrm{NMR}$}

$\left(\mathrm{CDCl}_{3}, 75 \mathrm{MHz}\right)$

$440.31 \mathrm{~g} / \mathrm{mol}$

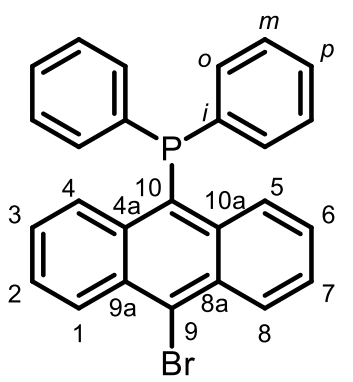

$\delta[p p m]=8.87-8.82\left(m, 2 \mathrm{H}, \mathrm{H}_{1,8}\right), 8.69-8.66\left(\mathrm{~m}, 2 \mathrm{H}, \mathrm{H}_{4,5}\right), 7.59-7.53$ $\left(\mathrm{m}, 2 \mathrm{H}, \mathrm{H}_{3,6}\right) 7.43-7.32\left(\mathrm{~m}, 6 \mathrm{H}, \mathrm{H}_{2,7}, o-\mathrm{Ph}\right), 7.27-7.24(\mathrm{~m}, 6 \mathrm{H}, m-\mathrm{Ph}, p-$
$\delta[\mathrm{ppm}]=137.1\left(\mathrm{~d},{ }^{1} J_{\mathrm{C}, \mathrm{P}}=12.9 \mathrm{~Hz}, 1 \mathrm{C}, \mathrm{C}_{10}\right), 136.2\left(\mathrm{~d},{ }^{1} \mathrm{~J}_{\mathrm{C}, \mathrm{P}}=12.9 \mathrm{~Hz}, 2 \mathrm{C}, i-\right.$ $\mathrm{Ph}), 131.4\left(\mathrm{~d},{ }^{2} \mathrm{~J}_{\mathrm{C}, \mathrm{P}}=18.3 \mathrm{~Hz}, 4 \mathrm{C}, \mathrm{o}-\mathrm{Ph}\right), 130.9$ (d, ${ }^{3} \mathrm{~J}_{\mathrm{C}, \mathrm{P}}=4.5 \mathrm{~Hz}, 2 \mathrm{C}, \mathrm{C}_{8 \mathrm{a}, 9 \mathrm{a}}$ ), $129.6\left(\mathrm{~d},{ }^{2} \mathrm{~J}_{\mathrm{C}, \mathrm{P}}=21.8 \mathrm{~Hz}, 2 \mathrm{C}, \mathrm{C}_{4 a}, \mathrm{C}_{10 \mathrm{a}}\right), 129.1\left(\mathrm{~s}, 2 \mathrm{C}, \mathrm{C}_{1,8}\right), 128.8\left(\mathrm{~s}, 2 \mathrm{C}, \mathrm{C}_{4,5}\right)$, $128.5\left(\mathrm{~d},{ }^{3} \mathrm{~J}_{\mathrm{C}, \mathrm{P}}=5.5 \mathrm{~Hz}, 4 \mathrm{C}, m-\mathrm{Ph}\right), 127.7\left(\mathrm{~s}, 1 \mathrm{C}, \mathrm{C}_{9}\right), 126.9$ (d, ${ }^{4} \mathrm{~J}_{\mathrm{C}, \mathrm{P}}=1.6 \mathrm{~Hz}$, $\left.2 \mathrm{C}, \mathrm{C}_{3,6}\right), 126.1\left(\mathrm{~s}, 2 \mathrm{C}, \mathrm{C}_{2,7}\right)$.

\section{EI-MS}

${ }^{31} \mathrm{P}\left\{{ }^{1} \mathrm{H}\right\}-\mathrm{NMR}$

$\left(\mathrm{CDCl}_{3}, 121 \mathrm{MHz}\right) \quad \delta[\mathrm{ppm}]=-23.22$.

$\mathrm{m} / \mathrm{z}(\%): \quad \quad 440.0(100)[\mathrm{M}]^{+}, 283.0(40)\left[\mathrm{C}_{20} \mathrm{H}_{13} \mathrm{P}\right]^{+}$.

\section{HR-MS $\left(\mathrm{EI}^{+}\right)$}

$\mathrm{m} / \mathrm{z}$ (calculated): $\quad 441.0406(441.0402)$.

\section{Elemental analysis}

in \% (calculated): $\quad$ C: 70.10 (70.76), $\mathrm{H}: 4.13(4.11)$.

\subsubsection{Synthesis of [9-PPh $\left.-10-\mathrm{Me}-\left(\mathrm{C}_{14} \mathrm{H}_{8}\right)\right]$ (3)}

9-Bromo-10-methylanthracene $\left(0.57 \mathrm{~g}, 2.1 \mathrm{mmol}, 1.0\right.$ eq.) was suspended in $\mathrm{Et}_{2} \mathrm{O}(12 \mathrm{~mL})$ and cooled to $60{ }^{\circ} \mathrm{C}$. $n$-Butyllithium in hexane $(4.25 \mathrm{M}, 0.15 \mathrm{~g}, 2.3 \mathrm{mmol}, 1.1 \mathrm{eq}$.) was added slowly and the reaction stirred for $10 \mathrm{~min}$. Afterwards the mixture was warmed to ambient temperature until a clear yellow solution formed. Cooling again to $-60{ }^{\circ} \mathrm{C}$ was followed by the addition of Chlorodiphenylphosphine $(0.51 \mathrm{~g}, 0.42 \mathrm{~mL}, 2.3 \mathrm{mmol}, 1.1 \mathrm{eq}$.). The mixture warmed to ambient temperature and stirred for $16 \mathrm{~h}$. The formed precipitate was filtered off and washed with $\mathrm{Et}_{2} \mathrm{O}(3 \times 5 \mathrm{~mL})$. The crude product was dissolved in DCM $(20 \mathrm{~mL})$ and Lithium chloride removed by filtration. Evaporating of the solvent under reduced 
pressure yields the desired product as a yellow powder. Recrystallization from DCM leads to suitable crystals for X-Ray structure determination.

Yield:

Chemical formula: $\quad \mathrm{C}_{27} \mathrm{H}_{21} \mathrm{P}$

Molecular weight: $\quad 376.44 \mathrm{~g} / \mathrm{mol}$

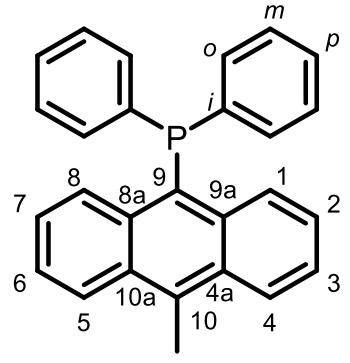

${ }^{1} \mathrm{H}-\mathrm{NMR}$

$\left(\mathrm{CD}_{2} \mathrm{Cl}_{2}, 300 \mathrm{MHz}\right): \quad \delta[\mathrm{ppm}]=8.83\left(\mathrm{dd},{ }^{3} \mathrm{~J}_{\mathrm{HH}}=8.9 \mathrm{~Hz},{ }^{4} \mathrm{~J}_{\mathrm{HH}}=1.2 \mathrm{~Hz}, 2 \mathrm{H}, \mathrm{H}_{4,5}\right), 8.41$ (ddd, $\left.{ }^{3} J_{\mathrm{HH}}=8.9 \mathrm{~Hz},{ }^{4} J_{\mathrm{PH}}=3.0 \mathrm{~Hz},{ }^{4} \mathrm{~J}_{\mathrm{HH}}=1.2 \mathrm{~Hz}, 2 \mathrm{H}, \mathrm{H}_{1,8}\right), 7.49$ (ddd, , ${ }^{3} J_{\mathrm{HH}}=8.9 \mathrm{~Hz}$, $\left.{ }^{3} J_{\mathrm{HH}}=6.4 \mathrm{~Hz},{ }^{4} J_{\mathrm{HH}}=1.2 \mathrm{~Hz}, 2 \mathrm{H}, \mathrm{H}_{2,7}\right), 7.43-7.37(\mathrm{~m}, 4 \mathrm{H}, o-\mathrm{Ph}$ ), 7.32 (ddd, $\left.{ }^{3} J_{\mathrm{HH}}=8.9 \mathrm{~Hz},{ }^{3} J_{\mathrm{HH}}=6.4 \mathrm{~Hz},{ }^{4} J_{\mathrm{HH}}=1.3 \mathrm{~Hz}, 2 \mathrm{H}, \mathrm{H}_{3,6}\right), 7.27-7.22(\mathrm{~m}, 6 \mathrm{H}, m-$ $\mathrm{Ph}, \mathrm{p}-\mathrm{Ph}), 3.21\left(\mathrm{~s}, 3 \mathrm{H}, \mathrm{CH}_{3}\right)$.

${ }^{13} \mathrm{C}\left\{{ }^{1} \mathrm{H}\right\}-\mathrm{NMR}$

$\left(\mathrm{CD}_{2} \mathrm{Cl}_{2}, 75 \mathrm{MHz}\right): \quad \delta[\mathrm{ppm}]=137.7\left(\mathrm{~d},{ }^{1} \mathrm{~J}_{\mathrm{CP}}=14.7 \mathrm{~Hz}, 2 \mathrm{C}\right.$, ipso- $\left.\mathrm{Ph}\right), 136.8\left(\mathrm{~d},{ }^{2} J_{\mathrm{CP}}=12.7 \mathrm{~Hz}, 2 \mathrm{C}\right.$, $\left.\mathrm{C}_{8 \mathrm{a}, 9 \mathrm{a}}\right), 136.6\left(\mathrm{~d},{ }^{3} \mathrm{JPP}_{\mathrm{CP}}=1.6 \mathrm{~Hz}, 2 \mathrm{C}, \mathrm{C}_{4 \mathrm{a}, 10 \mathrm{a}}\right), 131.8\left(\mathrm{~d},{ }^{2} \mathrm{~J}_{\mathrm{CP}}=18.5 \mathrm{~Hz}, 2 \mathrm{C}, 0-\mathrm{Ph}\right)$, $131.2\left(\mathrm{~s}, 1 \mathrm{C}, \mathrm{C}_{10}\right), 129.7\left(\mathrm{~d},{ }^{4} \mathrm{~J}_{\mathrm{CP}}=25.6 \mathrm{~Hz}, 2 \mathrm{C}, \mathrm{C}_{4,5}\right), 128.9\left(\mathrm{~d},{ }^{3} \mathrm{~J}_{\mathrm{CP}}=5.6 \mathrm{~Hz}\right.$, $4 \mathrm{C}, m-\mathrm{Ph}), 128.0(\mathrm{~s}, 2 \mathrm{C}, p-\mathrm{Ph}), 127,1\left(\mathrm{~d},{ }^{1} \mathrm{~J}_{\mathrm{CP}}=18.6 \mathrm{~Hz}, 1 \mathrm{C}, \mathrm{C}_{9}\right), 126.1(\mathrm{~s}, 2 \mathrm{C}$, $\left.\mathrm{C}_{1,8}\right), 125.6\left(\mathrm{~d},{ }^{4} \mathrm{JP}_{\mathrm{CP}}=1.7 \mathrm{~Hz}, \mathrm{C}_{2,7}\right), 15.3\left(\mathrm{~s}, 1 \mathrm{C}, \mathrm{CH}_{3}\right)$.

\section{${ }^{31} \mathrm{P}\left\{{ }^{1} \mathrm{H}\right\}-N M R$}

$\left(\mathrm{CD}_{2} \mathrm{Cl}_{2}, 121 \mathrm{MHz}\right) \quad \delta[\mathrm{ppm}]=-24.30$.

\section{EI-MS}

$\mathrm{m} / \mathrm{z}(\%):$

$376.1(100)[\mathrm{M}]^{+}, 297.1(21)[\mathrm{M}-\mathrm{Ph}]^{+}, 283.1(21)\left[\mathrm{M}-\mathrm{Ph}-\mathrm{CH}_{3}\right]^{+}$.

\section{HR-MS (EI+)}

m/z (calculated): $\quad 376.1373$ (376.1381).

\section{Elemental analysis}

in \% (calculated):

C: 83.96 (86.15), H: 5.64 (5.62). 


\subsubsection{Synthesis of [9-PPh2-10-Et- $\left.\left(\mathrm{C}_{14} \mathrm{H}_{8}\right)\right](4)$}

10-Ethyl-9-bromoanthracene ( $1.75 \mathrm{~g}, 6.1 \mathrm{mmol}, 1.0$ eq.) was suspended in $\mathrm{Et}_{2} \mathrm{O}(20 \mathrm{~mL})$ and cooled to $78{ }^{\circ} \mathrm{C}$. " Butyllithium in hexane $(4.05 \mathrm{M}, 0.41 \mathrm{~g}, 6.4 \mathrm{mmol}, 1.05$ eq.) was added slowly and the reaction stirred for $10 \mathrm{~min}$ leading to a dark-yellow solution. The mixture was warmed to ambient temperature and stirred for another $5 \mathrm{~min}$. Chlorodiphenylphosphine $(1.42 \mathrm{~g}, 1.2 \mathrm{~mL}, 6.4 \mathrm{mmol}, 1.05 \mathrm{eq}$.) was added at $78{ }^{\circ} \mathrm{C}$ over the course of $10 \mathrm{~min}$. The mixture warmed to ambient temperature and stirred for $16 \mathrm{~h}$ while a yellow precipitate was formed. Solvent was removed under reduced pressure and the yellow solid dissolved again in DCM $(25 \mathrm{~mL})$. The formed $\mathrm{LiCl}$ was filtered off and drying of the filtrate yielded the desired product as a yellow powder. Crystallization from DCM leads to suitable crystals for X-Ray structure determination.

Yield:

Chemical formula: $\quad \mathrm{C}_{28} \mathrm{H}_{23} \mathrm{P}$

Molecular weight: $\quad 390.47 \mathrm{~g} / \mathrm{mol}$

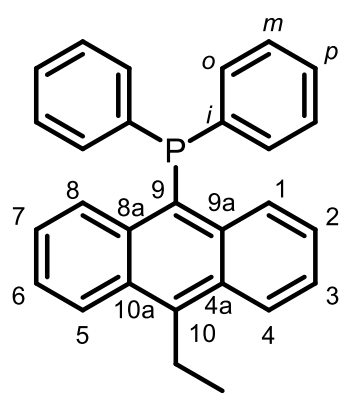

\section{${ }^{1} \mathrm{H}-\mathrm{NMR}$}

$\left(\mathrm{CDCl}_{3}, 300 \mathrm{MHz}\right)$

$\delta[p p m]=8.90\left(\mathrm{dd}, \mathrm{J}_{\mathrm{HH}}=8.8,5.3 \mathrm{~Hz}, 2 \mathrm{H}, \mathrm{H}_{1,8}\right), 8.41\left(\mathrm{dd}, \mathrm{J}_{\mathrm{HH}}=8.8 \mathrm{~Hz}, 1.1 \mathrm{~Hz}\right.$, $\left.2 \mathrm{H}, \mathrm{H}_{4,5}\right), 7.53-7.43\left(\mathrm{~m}, 6 \mathrm{H}, \mathrm{H}_{3,6}, \mathrm{o}-\mathrm{Ph}\right), 7.37-7.25\left(\mathrm{~m}, 8 \mathrm{H}, \mathrm{H}_{2,7}, m-\mathrm{Ph}, p-\right.$ $\mathrm{Ph}), 3.77\left(\mathrm{q}, \mathrm{J}_{\mathrm{HH}}=7.6 \mathrm{~Hz}, 2 \mathrm{H}, \mathrm{CH}_{2}\right), 1.59\left(\mathrm{t}, \mathrm{J}_{\mathrm{HH}}=7.6 \mathrm{~Hz}, 3 \mathrm{H}, \mathrm{CH}_{3}\right)$.

\section{${ }^{13} \mathrm{C}\left\{{ }^{1} \mathrm{H}\right\}-\mathrm{NMR}$}

$\left(\mathrm{CDCl}_{3}, 75 \mathrm{MHz}\right)$

$\delta[\mathrm{ppm}]=141.8\left(\mathrm{~d},{ }^{4} \mathrm{~J}_{\mathrm{CP}}=1.9 \mathrm{~Hz}, 1 \mathrm{C}, \mathrm{C}_{10}\right), 137.0\left(\mathrm{~d},{ }^{1} J_{\mathrm{CP}}=14.3 \mathrm{~Hz}, i-\mathrm{Ph}\right)$, $136.3\left(\mathrm{~d},{ }^{1} J_{\mathrm{CP}}=13.0 \mathrm{~Hz}, \mathrm{C}_{9}\right), 131.5\left(\mathrm{~d},{ }^{2} \mathrm{~J}_{\mathrm{CP}}=18.3 \mathrm{~Hz}, 4 \mathrm{C}, o-\mathrm{Ph}\right), 129.6(\mathrm{~d}$, $\left.{ }^{3} J_{\mathrm{CP}}=6.2 \mathrm{~Hz}, 2 \mathrm{C}, \mathrm{C}_{1,8}\right) 129.3\left(\mathrm{~s}, 2 \mathrm{C}, \mathrm{C}_{4 \mathrm{a}, 10 \mathrm{a}}\right) 128.4\left(\mathrm{~d},{ }^{3} \mathrm{~J}_{\mathrm{CP}}=5.5 \mathrm{~Hz}, 4 \mathrm{C}, m-\mathrm{Ph}\right)$, $127.4\left(\mathrm{~s}, 2 \mathrm{C}, \mathrm{C}_{3,6}\right), 126.7$ (d, J $\left.\mathrm{J}_{\mathrm{CP}}=18.5 \mathrm{~Hz}, \mathrm{C}_{8 \mathrm{a}, 9 \mathrm{a}}\right), 125.3\left(\mathrm{~d},{ }^{4} \mathrm{~J}_{\mathrm{CP}}=1.8 \mathrm{~Hz}, 2 \mathrm{C}\right.$, $\left.\mathrm{C}_{2,7}\right), 125.2\left(\mathrm{~d},{ }^{4} \mathrm{~J}_{\mathrm{CP}}=1.6 \mathrm{~Hz}, 2 \mathrm{C}, \mathrm{p}-\mathrm{Ph}\right), 125.0\left(\mathrm{~s}, 2 \mathrm{C}, \mathrm{C}_{4,5}\right), 21.9\left(\mathrm{~s}, \mathrm{CH}_{2}\right), 15.5$ (s, $\mathrm{CH}_{3}$ ).

\section{${ }^{31} \mathrm{P}\left\{{ }^{1} \mathrm{H}\right\}-N M R$}

$\left(\mathrm{CDCl}_{3}, 121 \mathrm{MHz}\right)$

$\delta[p p m]=-24.13$.

ESI-MS

$\mathrm{m} / \mathrm{z}(\%)$ :

$391.2(100)[\mathrm{M}+\mathrm{H}]^{+}$.

HR-MS (EI+) 
m/z (calculated): $\quad 391.1602(391.1610)$.

Elemental analysis

in \% (calculated): $\quad$ C: $85.63(86.13), H: 5.94(5.94)$.

\subsubsection{Synthesis of [9-PPh $\left.-10-P h-\left(\mathrm{C}_{14} \mathrm{H}_{8}\right)\right](6)$}

Lithiumdiphenylphosphide ( $0.19 \mathrm{~g}, 1.0 \mathrm{mmol}, 1.0$ eq.) was dissolved in THF ( $4 \mathrm{~mL}$ ) and cooled to $0{ }^{\circ} \mathrm{C}$. To the red solution 9-Bromo-10-phenylanthracene $(0.33 \mathrm{~g}, 1.0 \mathrm{mmol}, 1.0 \mathrm{eq})$ in THF $(4 \mathrm{~mL})$ was added dropwise over $10 \mathrm{~min}$ and the mixture was stirred for $10 \mathrm{~min}$. Afterwards it was allowed to warm to ambient temperature and was refluxed for $60 \mathrm{~min}$. After cooling to ambient temperature again $\mathrm{EtOH} / \mathrm{H}_{2} \mathrm{O}$ (5:1) was added until a yellow precipitate formed. The yellow solid was filtered off and washed with water several times. The desired compound was obtained after drying under reduced pressure as a yellow solid and could be further purified by recrystallization from DCM.

Yield:

Chemical formula: $\quad \mathrm{C}_{32} \mathrm{H}_{23} \mathrm{P}$

Molecular weight: $\quad 438.51 \mathrm{~g} / \mathrm{mol}$
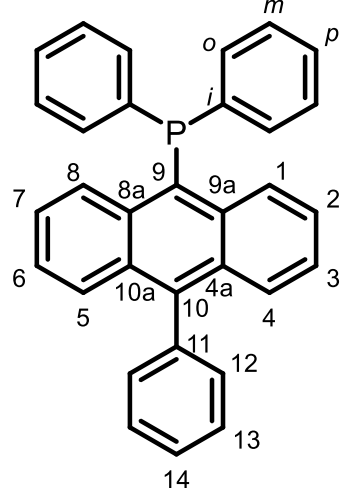

14

\section{${ }^{1} \mathrm{H}-\mathrm{NMR}$}

$\left(\mathrm{C}_{6} \mathrm{D}_{6}, 300 \mathrm{MHz}\right)$

$\delta[p p m]=8.91-8.87\left(m, 2 H, H_{4,5}\right), 7.73-7.71\left(m, 2 H, H_{1,8}\right), 7.66-7.59$ $\left(\mathrm{m}, 3 \mathrm{H}, \mathrm{H}_{12,14,16}\right), 7.52-7.48\left(\mathrm{~m}, 6 \mathrm{H}, \mathrm{H}_{13,15}, \mathrm{o}-\mathrm{Ph}\right), 7.34-7.27(\mathrm{~m}, 10 \mathrm{H}$,

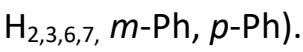

${ }^{13} \mathrm{C}\left\{{ }^{1} \mathrm{H}\right\}-N M R$

$\left(\mathrm{C}_{6} \mathrm{D}_{6}, 75 \mathrm{MHz}\right)$

$\delta[p p m]=141.9\left(\mathrm{~s}, 1 \mathrm{C}, \mathrm{C}_{10}\right), 138.9\left(\mathrm{~s}, 1 \mathrm{C}, \mathrm{C}_{11}\right), 136.8\left(\mathrm{~d},{ }^{1} \mathrm{~J}_{\mathrm{CP}}=14.2 \mathrm{~Hz}, 1 \mathrm{C}\right.$, $\left.C_{9}\right), 136.9\left(\mathrm{~d},{ }^{1} J_{\mathrm{CP}}=13.1 \mathrm{~Hz}, 2 \mathrm{C}, i-\mathrm{Ph}\right), 131.7\left(\mathrm{~d},{ }^{2} J_{\mathrm{CP}}=18.3 \mathrm{~Hz}, 4 \mathrm{C}, o-\mathrm{Ph}\right)$, 131.0 (s, 2C, $C_{4 a, 10 a}$ ), 130.5 (d, ${ }^{2} J_{C P}=4.5 \mathrm{~Hz}, 2 \mathrm{C}, \mathrm{C}_{8 \mathrm{a}, 9 \mathrm{a}}$ ), 128.7 (s, 2C, C4.5), $128.6\left(\mathrm{~d},{ }^{3} \mathrm{~J}_{\mathrm{CP}}=5.3 \mathrm{~Hz}, 4 \mathrm{C}, \mathrm{m}-\mathrm{Ph}\right), 128.2\left(\mathrm{~s}, 2 \mathrm{C}, \mathrm{C}_{13,15}\right.$ ), 127.9 (s, 3C, $\mathrm{C}_{12,14,16}$ ), $127.6\left(\mathrm{~d},{ }^{2} \mathrm{JPP}_{\mathrm{CP}}=12.0 \mathrm{~Hz}, 2 \mathrm{C}, \mathrm{C}_{1,8}\right), 125.5\left(\mathrm{~s}, 2 \mathrm{C}, \mathrm{C}_{2,7}\right), 124.9\left(\mathrm{~s}, 2 \mathrm{C}, \mathrm{C}_{3,6}\right)$.

${ }^{31} \mathrm{P}\left\{{ }^{1} \mathrm{H}\right\}-\mathrm{NMR}$

$\left(\mathrm{CDCl}_{3}, 121 \mathrm{MHz}\right) \quad \delta[\mathrm{ppm}]=-24.11$. 


\section{LIFDI-MS}

$\mathrm{m} / \mathrm{z}(\%):$

$438.1(100)[\mathrm{M}]^{+}$.

HR-MS (ESI $\left.{ }^{+}\right)$

$\mathrm{m} / \mathrm{z}$ (calculated): $\quad 439.1612(439.1610)$.

Elemental analysis

in \% (calculated): $\quad$ C: $86.41(87.65), \mathrm{H}: 5.20(5.20)$.

\subsubsection{Synthesis of [9-(O)PPh2- $\left.\left(\mathrm{C}_{14} \mathrm{H}_{9}\right)\right](7)$}

9-(Diphenylphosphanyl)anthracene (1) $(0.18 \mathrm{~g}, 0.5 \mathrm{mmol}, 1.0$ eq.) was dissolved in DCM (20 mL) and urea hydrogen peroxide $(0.047 \mathrm{~g}, 0.5 \mathrm{mmol}, 1.0 \mathrm{eq})$ was added at $0{ }^{\circ} \mathrm{C}$. Afterwards the mixture was stirred at ambient temperature for $16 \mathrm{~h}$. The reaction was quenched with $\mathrm{H}_{2} \mathrm{O}(10 \mathrm{~mL})$ and washed with $\mathrm{H}_{2} \mathrm{O}(2 \mathrm{x}$ $15 \mathrm{~mL}$ ). The combined organic phase was dried over $\mathrm{MgSO}_{4}$, filtered and the solvent was removed under reduced pressure. The target compound was obtained as a yellow powder.

Yield:

Chemical formula: $\quad \mathrm{C}_{26} \mathrm{H}_{19} \mathrm{PO}$

Molecular weight:

${ }^{1} \mathrm{H}-\mathrm{NMR}$

$\left(\mathrm{CDCl}_{3}, 300 \mathrm{MHz}\right)$

$\delta[\mathrm{ppm}]=8.71\left(\mathrm{~s}, 1 \mathrm{H}, \mathrm{H}_{10}\right), 8.63-8.60\left(\mathrm{~m}, 2 \mathrm{H}, \mathrm{H}_{1,8}\right), 8.05-8.01(\mathrm{~m}, 2 \mathrm{H}$, $\left.\mathrm{H}_{5,4}\right), 7.74-7.67(\mathrm{~m}, 4 \mathrm{H}, o-\mathrm{Ph}), 7.53-7.48(\mathrm{~m}, 2 \mathrm{H}, p-\mathrm{Ph}), 7.44-7.37(\mathrm{~m}$, $\left.6 \mathrm{H}, m-\mathrm{Ph}, \mathrm{H}_{3,6}\right), 7.28-7.22\left(\mathrm{~m}, 2 \mathrm{H}, \mathrm{H}_{2,7}\right)$.

$378.41 \mathrm{~g} / \mathrm{mol}$

${ }^{13} \mathrm{C}\left\{{ }^{1} \mathrm{H}\right\}-N M R$

$\left(\mathrm{CDCl}_{3}, 75 \mathrm{MHz}\right)$

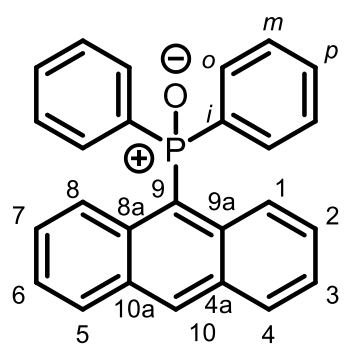


$\left(\mathrm{CDCl}_{3}, 121 \mathrm{MHz}\right) \quad \delta[\mathrm{ppm}]=31.36$.

ESI-MS

$\mathrm{m} / \mathrm{z}(\%): \quad 379.1(100)[\mathrm{M}+\mathrm{H}]^{+}, 401.1(43)[\mathrm{M}+\mathrm{Na}]^{+}$.

HR-MS (ESI $\left.{ }^{+}\right)$

$\mathrm{m} / \mathrm{z}$ (calculated): $\quad 379.1240(379.1246)$.

Elemental analysis

in \% (calculated): $\quad$ C: $82.53(81.03), \mathrm{H}: 5.06(5.12)$.

\subsubsection{Synthesis of $\left[9-(\mathrm{S}) \mathrm{PPh}_{2}-\left(\mathrm{C}_{14} \mathrm{H}_{9}\right)\right](8)$}

9-(Diphenylphosphanyl)anthracene (1) $(0.80 \mathrm{~g}, 2.2 \mathrm{mmol}, 1.0 \mathrm{eq})$ and Sulphur (0.85 g, $3.3 \mathrm{mmol}, 1.5 \mathrm{eq})$ were dissolved in Toluene $(20 \mathrm{~mL})$ and heated up to $80^{\circ} \mathrm{C}$ for $6 \mathrm{~h}$. After cooling to ambient temperature, the mixture was filtrated, and the solvent of the filtrate was removed under reduced pressure. Recrystallization from toluene leads to the pure product as yellow crystals, which were suitable for X-Ray structure determination.

Yield:

$0.84 \mathrm{~g}(2.13 \mathrm{mmol}, 96 \%)$

Chemical formula: $\quad \mathrm{C}_{26} \mathrm{H}_{19} \mathrm{PS}$

Molecular weight: $\quad 394.47 \mathrm{~g} / \mathrm{mol}$

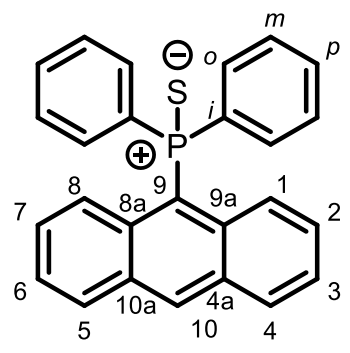

${ }^{1}$ H-NMR

$\left(\mathrm{CDCl}_{3}, 300 \mathrm{MHz}\right): \quad \delta[\mathrm{ppm}]=8.66\left(\mathrm{~s}, 1 \mathrm{H}, \mathrm{H}_{10}\right), 8.05-7.96\left(\mathrm{~m}, 4 \mathrm{H}, \mathrm{H}_{1,8}, \mathrm{H}_{4,5}\right), 7.87-7.75(\mathrm{~m}$, $4 \mathrm{H}, o-\mathrm{Ph}), 7.40-7.25\left(\mathrm{~m}, 8 \mathrm{H}, \mathrm{H}_{2,7}, m-\mathrm{Ph}, p-\mathrm{Ph}\right), 7.10-7.03\left(\mathrm{~m}, 2 \mathrm{H}, \mathrm{H}_{3,6}\right)$.

${ }^{13} \mathrm{C}\left\{{ }^{1} \mathrm{H}\right\}-\mathrm{NMR}$

$\left(\mathrm{CDCl}_{3}, 75 \mathrm{MHz}\right): \quad \delta[\mathrm{ppm}]=137.2\left(\mathrm{~d},{ }^{1} J_{\mathrm{CP}}=82.5 \mathrm{~Hz}, 2 \mathrm{C},{ }_{i p s o}-\mathrm{Ph}\right), 133.8\left(\mathrm{~d},{ }^{4} \mathrm{JP}_{\mathrm{CP}}=3.8 \mathrm{~Hz}, 1 \mathrm{C}\right.$, $\left.\mathrm{C}_{10}\right), 133.4\left(\mathrm{~d},{ }^{2} \mathrm{~J}_{\mathrm{CP}}=8.0 \mathrm{~Hz}, 2 \mathrm{C}, \mathrm{C}_{8 \mathrm{a}, 9 \mathrm{a}}\right), 131.6\left(\mathrm{~d},{ }^{3} \mathrm{~J}_{\mathrm{CP}}=11.2 \mathrm{~Hz}, 2 \mathrm{C}, \mathrm{C}_{4 \mathrm{a}, 10 \mathrm{a}}\right)$, $131.0\left(\mathrm{~d},{ }^{2} \mathrm{~J}_{\mathrm{CP}}=10.5 \mathrm{~Hz}, 4 \mathrm{C}, o-\mathrm{Ph}\right), 130.7\left(\mathrm{~d},{ }^{4} \mathrm{~J}_{\mathrm{CP}}=3.0 \mathrm{~Hz}, 2 \mathrm{C}, p-\mathrm{Ph}\right), 129.2$ $\left(\mathrm{s}, 2 \mathrm{C}, \mathrm{C}_{4,5}\right), 128.6\left(\mathrm{~d},{ }^{3} \mathrm{~J}_{\mathrm{CP}}=12.6 \mathrm{~Hz}, 4 \mathrm{C}, m-\mathrm{Ph}\right), 127.5\left(\mathrm{~d},{ }^{3} \mathrm{~J}_{\mathrm{CP}}=10.0 \mathrm{~Hz}, 2 \mathrm{C}\right.$, $\left.\mathrm{C}_{1,8}\right), 125.8\left(\mathrm{~s}, 2 \mathrm{C}, \mathrm{C}_{3,6}\right), 125.2\left(\mathrm{~s}, 2 \mathrm{C}, \mathrm{C}_{2,7}\right), 122.7\left(\mathrm{~d},{ }^{1} \mathrm{~J}_{\mathrm{CP}}=87.3 \mathrm{~Hz}, 1 \mathrm{C}, \mathrm{C}_{9}\right.$ ).

\section{${ }^{31} \mathrm{P}\left\{{ }^{1} \mathrm{H}\right\}-\mathrm{NMR}$}

$\left(\mathrm{CDCl}_{3}, 121 \mathrm{MHz}\right) \quad \delta[\mathrm{ppm}]=34.2$. 


\section{EI-MS}

$\mathrm{m} / \mathrm{z}(\%):$

$394.1(100)[\mathrm{M}]^{+}, 285.1(39)[\mathrm{M}-(\mathrm{S}) \mathrm{Ph}]^{+}, 183.0(48)\left[\mathrm{PPh}_{2}-2 \mathrm{H}\right]^{+}$.

\section{HR-MS (EI+)}

$\mathrm{m} / \mathrm{z}$ (calculated): $\quad 394.0943$ (394.0945).

\section{Elemental analysis}

in \% (calculated): $\quad$ C: 77.09 (79.17), H: 4.83 (4.86), S: 8.13 (8.63).

\subsubsection{Synthesis of $\left[9-\right.$ BMes$\left._{2}-\left(\mathrm{C}_{14} \mathrm{H}_{9}\right)\right](9)$}

9-Bromoanthracene $\left(0.257 \mathrm{~g}, 1.0 \mathrm{mmol}, 1.0\right.$ eq.) was dissolved in $\mathrm{Et}_{2} \mathrm{O}(20 \mathrm{~mL})$ and cooled to $-20^{\circ} \mathrm{C}$. $n \mathrm{BuLi}$ in hexane ( $0.55 \mathrm{~mL}, 1.2 \mathrm{mmol}, 1.2 \mathrm{eq}$.) was added dropwise over the course of $20 \mathrm{~min}$. The reaction was allowed to warm to ambient temperature until a clear solution formed $(20 \mathrm{~min})$. It was cooled to $-20^{\circ} \mathrm{C}$ again and dimesitylenboron fluoride $\left(0.322 \mathrm{~g}, 1.2 \mathrm{mmol}, 1.2 \mathrm{eq}\right.$.) in $\mathrm{Et}_{2} \mathrm{O}(10 \mathrm{~mL})$ was added. The orange solution was warmed to ambient temperature and stirred for $16 \mathrm{~h}$. The solvent was removed under reduced pressure and a yellow powder obtained, which was recrystallized from DCM.

Yield:

Chemical formula: $\quad \mathrm{C}_{32} \mathrm{H}_{31} \mathrm{~B}$

Molecular weight: $\quad 462.41 \mathrm{~g} / \mathrm{mol}$

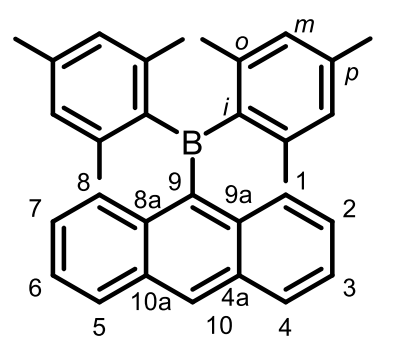

${ }^{1} \mathrm{H}-\mathrm{NMR}$

$\left(\mathrm{CDCl}_{3}, 300 \mathrm{MHz}\right) \quad \delta[\mathrm{ppm}]=8.47\left(\mathrm{~s}, 1 \mathrm{H}, \mathrm{H}_{10}\right), 8.05-7.96\left(\mathrm{~m}, 4 \mathrm{H}, \mathrm{H}_{1,4,5,8}\right), 7.36\left(\mathrm{~m}, 2 \mathrm{H}, \mathrm{H}_{2,7}\right)$, 7.20 (ddd, $\left.J_{\mathrm{HH}}=8.9,6.5,1.4 \mathrm{~Hz}, 2 \mathrm{H}, \mathrm{H}_{3,6}\right), 6.7$ (s (br), 4H, m-Mes), 2.27 (s (br), $12 \mathrm{H}, o-\mathrm{CH}_{3}$ ), 1.54 (s (br), $6 \mathrm{H}, p-\mathrm{CH}_{3}$ ).

\section{${ }^{13} \mathrm{C}\left\{{ }^{1} \mathrm{H}\right\}-\mathrm{NMR}$}

$\left(\mathrm{CDCl}_{3}, 75 \mathrm{MHz}\right)$

$\delta[\mathrm{ppm}]=139.8$ (s, 4C, o-Mes), 134.1 (s, $\left.\mathrm{C}_{\text {Anth }}\right), 131.4$ (s, $\left.\mathrm{C}_{\text {Anth }}\right), 129.8$ (s, 1C, $\mathrm{C}_{10}$ ), 129.2 (s, $\mathrm{C}_{1,8}$ ), 129.0 (s, 4C, m-Mes), 128.5 (s, 2C, C 4,5 ), 125.4 (s, 2C, $\left.\mathrm{C}_{3,6}\right) 125.0\left(\mathrm{~s}, 2 \mathrm{C}, \mathrm{C}_{2,7}\right), 23.5\left(\mathrm{~s}, 2 \mathrm{C}, \mathrm{p}-\mathrm{CH}_{3}\right), 21.4\left(\mathrm{~s}, 4 \mathrm{C}, \mathrm{o}-\mathrm{CH}_{3}\right)$.

\section{EI-MS}

$\mathrm{m} / \mathrm{z}(\%):$

$362.1(100)[\mathrm{M}]^{+}, 283.1(54)[\mathrm{M}-(\mathrm{Ph}+2 \mathrm{H})]^{+}, 254.1(57)\left[\mathrm{M}-\left(\mathrm{Ph}+\mathrm{C}_{2} \mathrm{H}_{7}\right)\right]$. 


\section{HR-MS (EI $\left.{ }^{+}\right)$}

$\mathrm{m} / \mathrm{z}$ (calculated): $\quad 363.1291(363.1297)$.

\subsubsection{Synthesis of [9-( $\left.\mathrm{HO}) \mathrm{CPh}_{2}-\left(\mathrm{C}_{14} \mathrm{H}_{9}\right)\right](10)$}

9-Bromoanthracene $\left(0.54 \mathrm{~g}, 2.1 \mathrm{mmol}, 1.0\right.$ eq.) was dissolved in THF $(8 \mathrm{~mL})$ and cooled to $-78^{\circ} \mathrm{C}$. $\mathrm{nBuLi}$ in hexane (2.2M, $1.0 \mathrm{~mL}, 2.2 \mathrm{mmol}, 1.05$ eq.) was added dropwise. After stirring for $20 \mathrm{~min}$ a solution of benzophenone $(0.36 \mathrm{~g}, 2.1 \mathrm{mmol}, 1.0 \mathrm{eq})$ in THF $(7 \mathrm{~mL})$ was added over the course of $10 \mathrm{~min}$. The mixture was allowed to warm to ambient temperature overnight. The reaction was quenched with water and extracted with $\mathrm{DCM}(2 \times 15 \mathrm{~mL})$. The combined organic phases were dried over $\mathrm{MgSO}_{4}$. After filtration and evaporation of the solvent under reduced pressure the target compound was obtained as a yellow powder and purified by recrystallization from toluene.

Yield:

$0.59 \mathrm{~g}(1.64 \mathrm{mmol}, 78 \%)$.

Chemical formula: $\quad \mathrm{C}_{27} \mathrm{H}_{20} \mathrm{O}$

Molecular weight: $\quad 360.45 \mathrm{~g} / \mathrm{mol}$

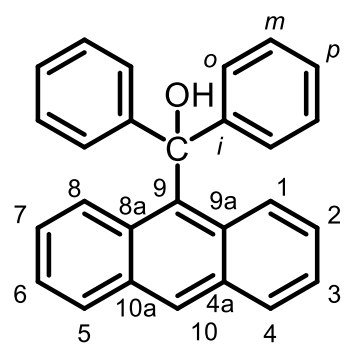

${ }^{1} \mathrm{H}-\mathrm{NMR}$

$\left(\mathrm{CDCl}_{3}, 300 \mathrm{MHz}\right)$

$\delta[\mathrm{ppm}]=8.46\left(\mathrm{~s}, 1 \mathrm{H}, \mathrm{H}_{10}\right), 7.98-7.89\left(\mathrm{~m}, 4 \mathrm{H}, \mathrm{H}_{1,4,5,8}\right), 7.33-7.31(\mathrm{~m}, 12 \mathrm{H}$, $\left.\mathrm{H}_{2,7}, \mathrm{Ph}-\mathrm{H}\right), 7.08-7.03\left(\mathrm{~m}, 2 \mathrm{H}, \mathrm{H}_{3,6}\right), 3.09(\mathrm{~s}, 1 \mathrm{H}, \mathrm{OH})$.

${ }^{13} \mathrm{C}\left\{{ }^{1} \mathrm{H}\right\}-\mathrm{NMR}$

$\left(\mathrm{CDCl}_{3}, 75 \mathrm{MHz}\right)$

$\delta[\mathrm{ppm}]=148.5$ (s, 2C, i-Ph) 139.2 (s, 1C, C9) 132.2 (s, 2C, $\left.\mathrm{C}_{8 \mathrm{a}, 9 \mathrm{a}}\right), 130.9$ (s, 2C, $\mathrm{C}_{4 \mathrm{a}, 10 \mathrm{a}}$ ), 129.4 (s, 1C, $\mathrm{C}_{10}$ ), 128.8 (s, 2C, C 1,8 ), 128.6 (s, 4C, o-Ph), 128.2 (s, 2C, $\left.C_{4,5}\right), 127.9$ (s, 4C, m-Ph), 127.7 (s, 2C, p-Ph), 124.2 (s, 2C, $\left.C_{2,7}\right), 124.0$ $\left(s, 2 C, C_{3,6}\right), 84.2\left(s, 1 C, C_{11}\right)$.

ESI-MS

$m / z(\%):$

$343.2(100)\left[\mathrm{C}_{27} \mathrm{H}_{18}+\mathrm{H}\right]^{+}, 383.1(65)[\mathrm{M}+\mathrm{Na}]^{+}, 743.3(0.85)[2 \mathrm{M}+\mathrm{Na}]^{+}$.

\section{$\mathrm{HR}-\mathrm{MS}\left(\mathrm{ESI}^{+}\right)$}

$\mathrm{m} / \mathrm{z}$ (calculated): $\quad 383.1398(383.1406)$ for $\mathrm{C}_{27} \mathrm{H}_{20} \mathrm{ONa}, 343.1478$ (343.1481) for $\mathrm{C}_{27} \mathrm{H}_{19}$.

\section{Elemental analysis}

in \% (calculated): $\quad$ C: 89.26 (89.97), H: 5.46 (5.59). 


\subsubsection{Synthesis of $\left[9-(\mathrm{Cl}) \mathrm{SiPh}_{2}-\left(\mathrm{C}_{14} \mathrm{H}_{9}\right)\right](11)$}

9-Bromoanthracene $\left(0.76 \mathrm{~g}, 3.0 \mathrm{mmol}, 1.0\right.$ eq.) was dissolved in $\mathrm{Et}_{2} \mathrm{O}(25 \mathrm{~mL})$ and cooled to $-50{ }^{\circ} \mathrm{C} . n \mathrm{BuLi}$ in hexane ( $2.2 \mathrm{M}, 1.5 \mathrm{~mL}, 3.3 \mathrm{mmol}, 1.1$ eq.) was added dropwise and the mixture was stirred for $30 \mathrm{~min}$. Chlorodiphenylsilane $(0.84 \mathrm{~g}, 0.7 \mathrm{~mL}, 3.3 \mathrm{mmol}, 1.1 \mathrm{eq}$.) was added over the course of $15 \mathrm{~min}$ and the yellow suspension was allowed to warm to ambient temperature overnight. The solvent was removed under reduced pressure and the residue dissolved in DCM $(8 \mathrm{~mL})$ and filtrated. The solvent was again concentrated, and a brown oil was obtained. Recrystallization from DCM afforded the target compound as pale-yellow crystals.

Yield:

Chemical formula: $\quad \mathrm{C}_{26} \mathrm{H}_{19} \mathrm{SiCl}$

Molecular weight: $\quad 394.97 \mathrm{~g} / \mathrm{mol}$

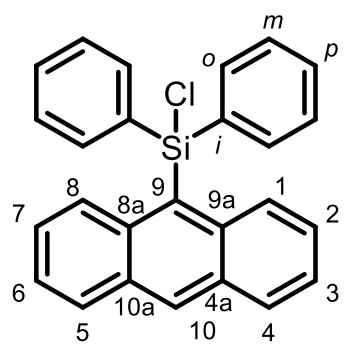

\section{${ }^{1} \mathrm{H}-\mathrm{NMR}$}

$\left(\mathrm{CDCl}_{3}, 300 \mathrm{MHz}\right)$

$\delta[p p m]=8.65\left(\mathrm{~s}, 1 \mathrm{H}, \mathrm{H}_{10}\right), 8.17\left(\mathrm{dd}, J_{\mathrm{HH}}=9.0 \mathrm{~Hz}, 0.9 \mathrm{~Hz}, 2 \mathrm{H}, \mathrm{H}_{1,8}\right), 8.03$ (dd, $\left.J_{\mathrm{HH}}=8.5,1.5 \mathrm{~Hz}, 2 \mathrm{H}, \mathrm{H}_{4,5}\right), 7.70-7.67(\mathrm{~m}, 4 \mathrm{H}, o-\mathrm{Ph}), 7.54-7.50(\mathrm{~m}, 2 \mathrm{H}, p-$ $\mathrm{Ph}), 7.44-7.37\left(\mathrm{~m}, 6 \mathrm{H}, m-\mathrm{Ph}, \mathrm{H}_{3,6}\right), 7.17$ (ddd, $\mathrm{J}_{\mathrm{HH}}=9.0,6.5,1,5 \mathrm{~Hz}, 2 \mathrm{H}$, $\left.\mathrm{H}_{2,7}\right)$.

${ }^{13} \mathrm{C}\left\{{ }^{1} \mathrm{H}\right\}-\mathrm{NMR}$

$\left(\mathrm{CDCl}_{3}, 75 \mathrm{MHz}\right)$

$\delta[p p m]=137.8\left(\mathrm{~s}, 2 \mathrm{C}, \mathrm{C}_{4 \mathrm{a}, 10 \mathrm{a}}\right), 137.7$ (s, 2C, i-Ph), 135.1 (s, 4C, o-Ph), 133.0 (s, 1C, $\left.\mathrm{C}_{10}\right), 131.5$ (s, 2C, C $\left.8 \mathrm{a}, 9 \mathrm{a}\right), 130.7$ (s, 2C, p-Ph), 129.5 (s, 2C, C4,5), 129.4

(s, 2C, $\left.C_{1,8}\right), 128.6(s, 4 C, m-P h), 126.2\left(s, 1 C, C_{9}\right), 125.5$ (s, 2C, $\left.C_{2,7}\right), 125.0$

$\left(\mathrm{s}, 2 \mathrm{C}, \mathrm{C}_{3,6}\right)$.

\section{LIFDI-MS}

$\mathrm{m} / \mathrm{z}(\%):$

$394.1(100)[\mathrm{M}]^{+}$.

\section{HR-MS (EI+)}

m/z (calculated): $\quad 376.1276(376.1278)$ for $\mathrm{C}_{26} \mathrm{H}_{20} \mathrm{SiO}$.

\section{Elemental analysis}

in \% (calculated):

C: 77.70 (79.07), H: 4.87 (4.85). 


\subsubsection{Synthesis of $\left[9,10-\left((\mathrm{Cl}) \mathrm{SiPh}_{2}\right)_{2}-\left(\mathrm{C}_{14} \mathrm{H}_{8}\right)\right](12)$}

9,10-Dibromoanthracene ( $0.67 \mathrm{~g}, 2.0 \mathrm{mmol}, 1.0$ eq.) was suspended in $\mathrm{Et}_{2} \mathrm{O}(15 \mathrm{~mL})$ and cooled to $0{ }^{\circ} \mathrm{C}$. $n$ BuLi in hexane $(2.2 \mathrm{M}, 0.28 \mathrm{~g}, 4.4 \mathrm{mmol}, 2.2$ eq.) was added dropwise over the course of $10 \mathrm{~min}$. Afterwards the mixture was stirred at ambient temperature for $10 \mathrm{~min}$ and Dichlorodiphenylsilane $(1.11 \mathrm{~g}$, $0.92 \mathrm{~mL}, 4.4 \mathrm{mmol}, 2.2 \mathrm{eq}$.) was added. While stirring for $16 \mathrm{~h}$ a yellow precipitate formed which was filtered off and dried. The solid was dissolved in DCM $(10 \mathrm{~mL})$ and again filtered. The target compound was obtained after removing the solvent under reduced pressure as a pale-yellow powder, which could be crystalized from toluene.

Yield:

Chemical formula: $\quad \mathrm{C}_{38} \mathrm{H}_{28} \mathrm{Si}_{2} \mathrm{Cl}_{2}$

Molecular weight: $\quad 611.71 \mathrm{~g} / \mathrm{mol}$

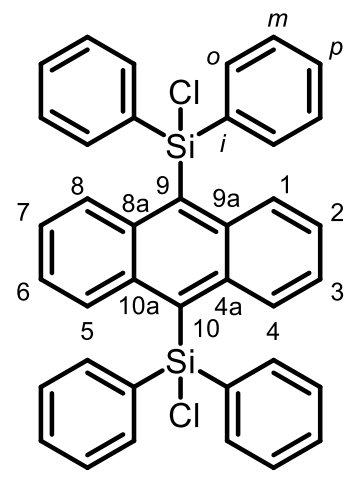

\section{${ }^{1} \mathrm{H}-\mathrm{NMR}$}

$\left(\mathrm{CDCl}_{3}, 300 \mathrm{MHz}\right)$

$\delta[p p m]=8.22\left(\mathrm{~d}, J_{\mathrm{HH}}=6.9 \mathrm{~Hz}, 3.3 \mathrm{~Hz}, 4 \mathrm{H}, \mathrm{H}_{1,4,5,80)}\right) 7.73-7.70(\mathrm{~m}, 8 \mathrm{H}, 0-$ $\mathrm{Ph}), 7.52-7.48(\mathrm{~m}, 4 \mathrm{H}, p-\mathrm{Ph}), 7.44-7.41(\mathrm{~m}, 8 \mathrm{H}, m-\mathrm{Ph}), 7.05$ (dd, $J_{H H}=6.9,3.3 \mathrm{~Hz}, 4 \mathrm{H}, \mathrm{H}_{2,3,6,7}$.

${ }^{13} \mathrm{C}\left\{{ }^{1} \mathrm{H}\right\}-\mathrm{NMR}$

$\left(\mathrm{CDCl}_{3}, 75 \mathrm{MHz}\right)$

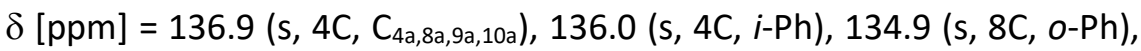
133.0 (s, 2C, $\left.C_{9,10}\right), 130.6$ (s, 4C, p-Ph), 129.8(s,4C, $\left.C_{1,4,5,8}\right), 128.4$ (s, 8C, m$\mathrm{Ph})$, 124. (s, 4C, $\left.\mathrm{C}_{2,3,6,7}\right)$.

\section{LIFDI-MS}

$\mathrm{m} / \mathrm{z}(\%):$

$610.1(100)$

HR-MS (EI+)

m/z (calculated): $\quad 603.2159(603.2170)$ for $\mathrm{C}_{38} \mathrm{H}_{28} \mathrm{Si}_{2} \mathrm{C}_{2} \mathrm{H}_{6} \mathrm{O}_{2}$.

Elemental analysis

in \% (calculated): $\quad$ C: 74.12 (74.61), H: 4.45 (4.61). 


\subsubsection{Synthesis of [9-(S)PPh $\left.-10-B r-\left(\mathrm{C}_{14} \mathrm{H}_{8}\right)\right](13)$}

9-Brom-10-diphenylphosphanylanthracene $(0.46 \mathrm{~g}, 1.05 \mathrm{mmol}, 1.0 \mathrm{eq})$ was dissolved in Toluene and $\mathrm{S}_{8}$ was added. The mixture was heated to $100{ }^{\circ} \mathrm{C}$ for $6 \mathrm{~h}$. After cooling to ambient temperature the solvent was removed under reduce pressure to give a yellow powder. The target compound was achieved by recrystallization from toluene.

Chemical formula: $\quad \mathrm{C}_{26} \mathrm{H}_{18} \mathrm{PSBr}$

Molecular weight:

$473.31 \mathrm{~g} / \mathrm{mol}$
Yield:

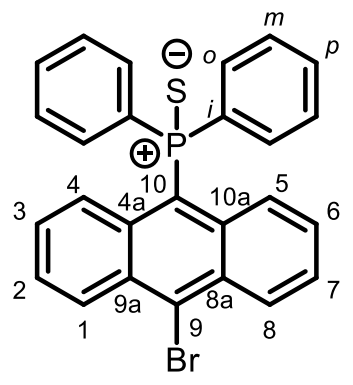

${ }^{1}$ H-NMR

(THF-d8, $300 \mathrm{MHz}$ )

$\delta[p p m]=8.62-8.58\left(m, 2 \mathrm{H}, \mathrm{H}_{1,8}\right), 8.14-8.11\left(\mathrm{~m}, 2 \mathrm{H}, \mathrm{H}_{4,5}\right), 7.83-7.75$ $(\mathrm{m}, 4 \mathrm{H}, o-\mathrm{Ph}), 7.51-7.46\left(\mathrm{~m}, 2 \mathrm{H}, \mathrm{H}_{2,7}\right), 7.36-7.23(\mathrm{~m}, 6 \mathrm{H}, \mathrm{m}-\mathrm{Ph}, \mathrm{p}-$ $\mathrm{Ph}), 7.10-7.04\left(\mathrm{~m}, 2 \mathrm{H}, \mathrm{H}_{3,6}\right)$.

\section{${ }^{13} \mathrm{C}\left\{{ }^{1} \mathrm{H}\right\}-\mathrm{NMR}$}

(THF-d8, $75 \mathrm{MHz}$ )

$\delta[\mathrm{ppm}]=137.7\left(\mathrm{~d},{ }^{1} \mathrm{~J}_{\mathrm{C}, \mathrm{P}}=82.8 \mathrm{~Hz}, 2 \mathrm{C}, \mathrm{i}-\mathrm{Ph}\right), 133.4\left(\mathrm{~d},{ }^{2} \mathrm{~J}_{\mathrm{C}, \mathrm{P}}=8.0 \mathrm{~Hz}, 2 \mathrm{C}\right.$, $\left.\mathrm{C}_{4 \mathrm{a} 10 \mathrm{a}}\right), 130.7$ (d, $\left.{ }^{2} J_{\mathrm{C}, \mathrm{P}}=10.4 \mathrm{~Hz}, 2 \mathrm{C}, \mathrm{o}-\mathrm{Ph}\right), 130.4\left(\mathrm{~s}, \mathrm{C}_{9}\right), 130.3\left(\mathrm{~d},{ }^{4} \mathrm{~J}_{\mathrm{C}, \mathrm{P}}=3.0\right.$ $\mathrm{Hz}, 2 \mathrm{C}, p-\mathrm{Ph}), 129.3\left(\mathrm{~d},{ }^{3} \mathrm{~J}_{\mathrm{C}, \mathrm{P}}=4.6 \mathrm{~Hz}, 2 \mathrm{C}, \mathrm{C}_{8 \mathrm{a}, 9 \mathrm{a}}\right), 128.2\left(\mathrm{~d},{ }^{3} \mathrm{~J}_{\mathrm{C}, \mathrm{P}}=12.6 \mathrm{~Hz}, 2 \mathrm{C}\right.$, $m-\mathrm{Ph}$ ), 127.9 (s, 2C, $\left.\mathrm{C}_{1,8}\right), 127.8\left(\mathrm{~d},{ }^{3} \mathrm{~J}_{\mathrm{C}, \mathrm{P}}=10.3 \mathrm{~Hz}, \mathrm{C}_{4,5}\right), 126.9\left(\mathrm{~s}, 2 \mathrm{C}, \mathrm{C}_{2,7}\right)$, $125.3\left(\mathrm{~d},{ }^{1} \mathrm{~J}_{\mathrm{C}, \mathrm{P}}=89.2 \mathrm{~Hz}, \mathrm{C}_{10}\right), 125.2\left(\mathrm{~s}, 2 \mathrm{C}, \mathrm{C}_{3,6}\right)$.

\section{${ }^{31} \mathrm{P}\left\{{ }^{1} \mathrm{H}\right\}-\mathrm{NMR}$}

$(\mathrm{THF}-\mathrm{d} 8,121 \mathrm{MHz}) \quad \delta[\mathrm{ppm}]=34.63$.

\section{EI-MS}

$\mathrm{m} / \mathrm{z}(\%):$

$473.9(100)[\mathrm{M}]^{+}, 185.0(95)\left[\mathrm{PPh}_{2}\right]^{+}$.

\section{HR-MS (EI+)}

$\mathrm{m} / \mathrm{z}$ (calculated): $\quad 473.0123$ (473.0119).

\section{Elemental analysis}

in \% (calculated):

C: 66.46 (65.97), H: 3.84 (3.83), S: 7.0 (6.77). 


\subsubsection{Synthesis of [9-(S)PPh $\left.-10-\mathrm{Me}-\left(\mathrm{C}_{14} \mathrm{H}_{8}\right)\right](14)$}

10-Methyl-9-(diphenyl)phosphanylanthracene $\left(0.20 \mathrm{~g}, 0.53 \mathrm{mmol}, 1.0 \mathrm{eq}\right.$.) and Sulphur $\mathrm{S}_{8}(0.04 \mathrm{~g}$, $1.25 \mathrm{mmol}, 2.4$ eq.) were dissolved in toluene $(10 \mathrm{~mL})$. The mixture was heated to $80{ }^{\circ} \mathrm{C}$ for $6 \mathrm{~h}$. After cooling to ambient temperature, the reaction was filtered, and the solvent removed under reduced pressure. The yellow powder can be crystallized from toluene, which afforded crystals suitable for X-Ray structure determination.

Yield:

Chemical formula: $\quad \mathrm{C}_{27} \mathrm{H}_{21} \mathrm{PS}$

Molecular weight: $\quad 408.49 \mathrm{~g} / \mathrm{mol}$

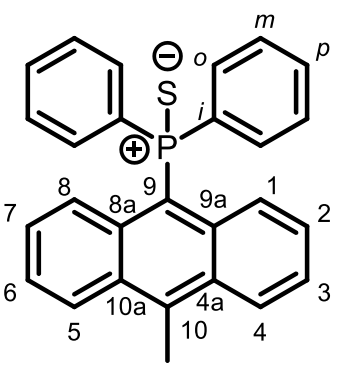

${ }^{1} \mathrm{H}-\mathrm{NMR}$

$\left(\mathrm{CDCl}_{3}, 300 \mathrm{MHz}\right)$

$\delta[p p m]=8.34-8.31\left(\mathrm{~m}, 2 \mathrm{H}, \mathrm{H}_{4,5}\right), 8.10-8.07\left(\mathrm{~m}, 2 \mathrm{H}, \mathrm{H}_{1,8}\right), 7.79-7.72$ (m, $4 \mathrm{H}, o-\mathrm{Ph}), 7.40-7.35\left(\mathrm{~m}, 2 \mathrm{H}, \mathrm{H}_{3,6}\right), 7.36-7.22(\mathrm{~m}, 6 \mathrm{H}, m-\mathrm{Ph}, p-\mathrm{Ph})$, $7.07-7.02\left(\mathrm{~m} \mathrm{2H}, \mathrm{H}_{2,7}\right), 3.20\left(\mathrm{~s}, 3 \mathrm{H}, \mathrm{CH}_{3}\right)$.

${ }^{13} \mathrm{C}\left\{{ }^{1} \mathrm{H}\right\}-\mathrm{NMR}$

$\left(\mathrm{CDCl}_{3}, 75 \mathrm{MHz}\right)$

$\delta[\mathrm{ppm}]=137.8\left(\mathrm{~d},{ }^{4} \mathrm{~J}_{\mathrm{CP}}=4.1 \mathrm{~Hz}, 2 \mathrm{C}, \mathrm{C}_{4 \mathrm{a}, 10 \mathrm{a}}\right), 137.3\left(\mathrm{~d},{ }^{1} J_{\mathrm{C}, \mathrm{P}}=82.8 \mathrm{~Hz}, 2 \mathrm{C}, i-\right.$ Ph), 133.0 (d, J = 7.6 Hz,2C, C $8 \mathrm{a}, 9 \mathrm{a}), 130.8\left(\mathrm{~d},{ }^{2} \mathrm{~J}_{\mathrm{C}, \mathrm{P}}=10.3 \mathrm{~Hz}, 2 \mathrm{C}, o-\mathrm{Ph}\right), 130.4$ $\left(\mathrm{d},{ }^{4} \mathrm{~J}_{\mathrm{C}, \mathrm{P}}=2.9 \mathrm{~Hz}, 2 \mathrm{C}, p-\mathrm{Ph}\right), 130.2\left(\mathrm{~s}, \mathrm{C}_{10}\right), 128.3\left(\mathrm{~d},{ }^{3} \mathrm{~J}_{\mathrm{C}, \mathrm{P}}=12.5 \mathrm{~Hz}, 2 \mathrm{C}, m-\mathrm{Ph}\right)$, $128.0\left(\mathrm{~d},{ }^{3} J_{C, P}=10.7 \mathrm{~Hz}, C_{1,8}\right), 125.0\left(\mathrm{~m}, 6 \mathrm{C}, C_{2,3,4,5,6,7}\right), 120.7\left(\mathrm{~d},{ }^{1} J_{C, P}=\right.$ $\left.92.1 \mathrm{~Hz}, \mathrm{C}_{9}\right), 15.3\left(\mathrm{~s}, \mathrm{CH}_{3}\right)$.

${ }^{31} \mathrm{P}\left\{{ }^{1} \mathrm{H}\right\}-\mathrm{NMR}$

$\left(\mathrm{CDCl}_{3}, 121 \mathrm{MHz}\right) \quad \delta[\mathrm{ppm}]=34.44$.

EI-MS

$m / z(\%):$

$408.1(100)[\mathrm{M}]^{+}, 223.1(65)\left[\mathrm{C}_{15} \mathrm{H}_{12} \mathrm{P}\right]^{+}, 299.1(48)\left[\mathrm{M}-\left(\mathrm{C}_{6} \mathrm{H}_{5} \mathrm{~S}\right)\right]$.

HR-MS (EI+)

$\mathrm{m} / \mathrm{z}$ (calculated): $\quad 408.1110(408.1102)$.

Elemental analysis

in \% (calculated): $\quad$ C: 78.53 (79.39), H: 5:21 (5.18), S: 7.90 (7.58). 


\subsubsection{Synthesis of [9-(S)PPh2-10-Et- $\left.\left(\mathrm{C}_{14} \mathrm{H}_{8}\right)\right](15)$}

10-Ethyl-9-(diphenyl)phosphanylanthracene $(0.50 \mathrm{~g}, 1.3 \mathrm{mmol}, 1.0 \mathrm{eq})$ and $\mathrm{S}_{8}(0.05 \mathrm{~g}, 0.2 \mathrm{mmol}, 1.2$ eq.) were dissolved in Toluene $(10 \mathrm{~mL})$ and heated to $80^{\circ} \mathrm{C}$ for $6 \mathrm{~h}$. After cooling to ambient temperature and filtration the solvent was removed under reduced pressure. The target compound was obtained as a yellow powder and could be further purified by recrystallization from EtOAC or Cyclohexane.

Yield:

Chemical formula:

Molecular weight:

\section{${ }^{1} \mathrm{H}-\mathrm{NMR}$}

(THF-d8, $300 \mathrm{MHz}$ )

$0.43 \mathrm{~g}(1.0 \mathrm{mmol}, 77 \%)$

$$
\mathrm{C}_{28} \mathrm{H}_{23} \mathrm{PS}
$$

$422.53 \mathrm{~g} / \mathrm{mol}$
$\delta$ $\left.{ }^{3} J_{\mathrm{HH}}=8.9 \mathrm{~Hz}, 2 \mathrm{H}, \mathrm{H}_{4,5}\right), 7.75\left(\mathrm{dd},{ }^{3} J_{\mathrm{HH}}=8.0 \mathrm{~Hz},{ }^{4} \mathrm{~J}_{\mathrm{HH}}=1.4 \mathrm{~Hz}, 4 \mathrm{H}, 0-\mathrm{Ph}\right), 7.37$ $\left(\mathrm{dd},{ }^{3} \mathrm{~J}_{\mathrm{HH}}=8.9 \mathrm{~Hz},{ }^{3} \mathrm{~J}_{\mathrm{HH}}=6.5 \mathrm{~Hz}, 2 \mathrm{H}, \mathrm{H}_{2,7}\right), 7.30-7.19(\mathrm{~m}, 6 \mathrm{H}, m-\mathrm{Ph}, p-\mathrm{Ph})$, $6.99\left(\mathrm{dd},{ }^{3} \mathrm{~J}_{\mathrm{HH}}=8.9 \mathrm{~Hz},{ }^{3} J_{\mathrm{HH}}=6.5 \mathrm{~Hz}, 2 \mathrm{H}, \mathrm{H}_{3,6}\right), 3.75\left(\mathrm{q},{ }^{3} \mathrm{~J}_{\mathrm{HH}}=7.5 \mathrm{~Hz}, 2 \mathrm{H}\right.$, $\left.\mathrm{CH}_{2}\right), 1.52\left(\mathrm{t}, 3^{3} \mathrm{HH}_{\mathrm{H}}=7.5 \mathrm{~Hz}, 3 \mathrm{H}, \mathrm{CH}_{3}\right)$.
$\delta[\mathrm{ppm}]=8.38\left(\mathrm{dd},{ }^{3} \mathrm{~J}_{\mathrm{HH}}=8.9 \mathrm{~Hz},{ }^{4} \mathrm{~J}_{\mathrm{PH}}=2.2 \mathrm{~Hz}, 2 \mathrm{H}, \mathrm{H}_{1,8}\right), 8.09(\mathrm{~d}$,

\section{${ }^{13} \mathrm{C}\left\{{ }^{1} \mathrm{H}\right\}-\mathrm{NMR}$}

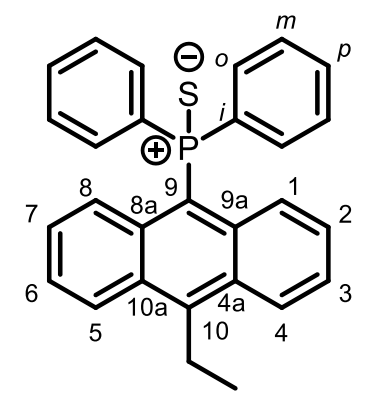
$($ THF- $d 8,75 \mathrm{MHz}) \quad \delta[\mathrm{ppm}]=144.5\left(\mathrm{~d},{ }^{3} \mathrm{~J}_{\mathrm{CP}}=3.9 \mathrm{~Hz}, 2 \mathrm{C}, \mathrm{C}_{4 \mathrm{a}}, 10 \mathrm{a}\right), 139.5\left(\mathrm{~d},{ }^{1} \mathrm{~J}_{\mathrm{CP}}=82.3 \mathrm{~Hz}, 2 \mathrm{C}\right.$, ipso-Ph,), $134.2\left(\mathrm{~d},{ }^{2} \mathrm{~J}_{\mathrm{CP}}=7.7 \mathrm{~Hz}, 2 \mathrm{C}, \mathrm{C}_{8 \mathrm{a}, 9 \mathrm{a}}\right), 131.8\left(\mathrm{~d},{ }^{2} \mathrm{~J}_{\mathrm{CP}}=10.2 \mathrm{~Hz}, 2 \mathrm{C}, o_{-}\right.$ $\mathrm{Ph}), 131.1\left(\mathrm{~d},{ }^{4} J_{\mathrm{CP}}=2.9 \mathrm{~Hz}, 2 \mathrm{C}, p-\mathrm{Ph}\right), 130.4\left(\mathrm{~d},{ }^{4} J_{\mathrm{CP}}=10.9 \mathrm{~Hz}, 1 \mathrm{C}, \mathrm{C}_{10}\right), 129.4$ $\left(d,{ }^{4} J_{C P}=10.8 \mathrm{~Hz}, 2 \mathrm{C}, C_{4,5}\right), 129.2\left(\mathrm{~d},{ }^{3} J_{\mathrm{CP}}=12.4 \mathrm{~Hz}, 4 \mathrm{C}, m-\mathrm{Ph}\right), 126.1$ (s, 2C, $\left.C_{2,7}\right), 125.7\left(s, 2 C, C_{1,8}\right), 125.5\left(s, 2 C, C_{3,6}\right), 124.0\left(d,{ }^{1} J_{C P}=90.9 \mathrm{~Hz}, 1 \mathrm{C}, C_{9}\right)$, $23.0\left(\mathrm{~s}, 1 \mathrm{C}, \mathrm{CH}_{2}\right), 16.2$ (s, $\left.1 \mathrm{C}, \mathrm{CH}_{3}\right)$.

\section{${ }^{31} \mathrm{P}\left\{{ }^{1} \mathrm{H}\right\}-N M R$}

$($ THF-d8 $121 \mathrm{MHz}) \quad \delta[\mathrm{ppm}]=31.69$.

\section{ESI-MS}

$\mathrm{m} / \mathrm{z}(\%)$ :

$423.2(100)[\mathrm{M}+\mathrm{H}]^{+}, 445.1(29)[\mathrm{M}+\mathrm{Na}]^{+}$. 


\section{HR-MS (EI+)}

$\mathrm{m} / \mathrm{z}$ (calculated): $\quad 423.1321(423.1331)$.

\section{Elemental analysis}

in \% (calculated): $\quad$ C: 79.24 (79.59), H: 5.45 (5.49), S: 8.00 (7.59).

\subsubsection{Synthesis of [9-(S)PPh$\left.-10-\mathrm{SiMe}_{3}-\left(\mathrm{C}_{14} \mathrm{H}_{8}\right)\right](16)$}

9-Bromo 10-trimethylsilylanthracene $\left(0.50 \mathrm{~g}, 1.5 \mathrm{mmol}, 1.0\right.$ eq.) was dissolved in $\mathrm{Et}_{2} \mathrm{O}(15 \mathrm{~mL})$ and cooled to $-78^{\circ} \mathrm{C}$. $n$ BuLi in hexane $(4.1 \mathrm{M}, 0.39 \mathrm{~mL}, 1.6 \mathrm{mmol}, 1.05 \mathrm{eq})$ was added dropwise over $10 \mathrm{~min}$ and the mixture stirred until it turned red (10 min). Chlorodiphenylphosphine (0.35 g, $0.29 \mathrm{~mL}, 1.6 \mathrm{mmol}, 1.05$ eq.) was added dropwise. The solution turned yellow again and was warmed to ambient temperature overnight, while a yellow solid precipitated. The solid was filtered off, dissolved in DCM $(10 \mathrm{~mL})$ and filtered again. The solvent was removed under reduced pressure and [9-PPh$\left.-\mathrm{SiMe}_{3}-\left(\mathrm{C}_{14} \mathrm{H}_{8}\right)\right]$ was obtained as a yellow powder and used without further purification. For oxidation the yellow solid was dissolved in Toluene $(10 \mathrm{~mL})$ and Sulfur $\mathrm{S}_{8}\left(0.05 \mathrm{~g}, 1.5 \mathrm{mmol}, 1.0 \mathrm{eq}\right.$.) was added. The mixture was heated to $80{ }^{\circ} \mathrm{C}$ for $6 \mathrm{~h}$. After cooling to ambient temperature, the mixture was filtered and the solvent removed under reduced pressure. The target compound could be obtained by recrystallization from DCM.

Yield:

Chemical formula:

Molecular weight:

$466.65 \mathrm{~g} / \mathrm{mol}$

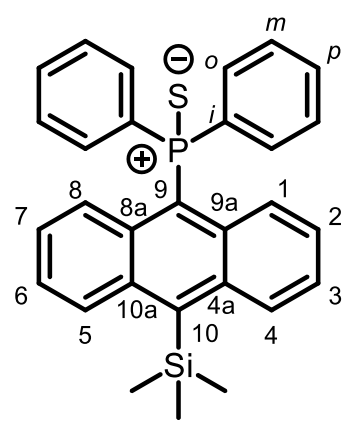

\section{${ }^{1} \mathrm{H}-\mathrm{NMR}$}

$\left(\mathrm{CDCl}_{3}, 400 \mathrm{MHz}\right)$

$\delta[\mathrm{ppm}]=8.34\left(\mathrm{~d},{ }^{3} J_{\mathrm{HH}}=8.9 \mathrm{~Hz}, 2 \mathrm{H}, \mathrm{H}_{4,5}\right), 8.11\left(\mathrm{~d},{ }^{3} \mathrm{~J}_{\mathrm{HH}}=8.9 \mathrm{~Hz}, 2 \mathrm{H}, \mathrm{H}_{1,8}\right)$, $7.76(\mathrm{~m}, 4 \mathrm{H}, o-\mathrm{Ph}), 7.36-7.26\left(\mathrm{~m}, 8 \mathrm{H}, \mathrm{H}_{3,6}, m-\mathrm{Ph}, p-\mathrm{Ph}\right), 7.03-6.99(\mathrm{~m}$, $\left.2 \mathrm{H}, \mathrm{H}_{2,7}\right), 0.74\left(\mathrm{~s}, 9 \mathrm{H}, \mathrm{CH}_{3}\right)$.

\section{${ }^{13} \mathrm{C}\left\{{ }^{1} \mathrm{H}\right\}-\mathrm{NMR}$}

$\left(\mathrm{CDCL}_{3}, 100 \mathrm{MHz}\right)$

$\delta[\mathrm{ppm}]=145.3\left(\mathrm{~d},{ }^{4} J_{\mathrm{CP}}=3.8 \mathrm{~Hz}, 1 \mathrm{C}, \mathrm{C}_{10}\right), 137.2\left(\mathrm{~d},{ }^{1} J_{\mathrm{CP}}=82.8 \mathrm{~Hz}, 2 \mathrm{C}, i-\mathrm{Ph}\right)$, $137.0\left(d,{ }^{2} J_{C P}=10.5 \mathrm{~Hz}, 2 \mathrm{C}, \mathrm{C}_{8 \mathrm{a}}, 9 \mathrm{a}\right), 132.3\left(\mathrm{~d},{ }^{3} \mathrm{JPP}_{\mathrm{CP}}=7.0 \mathrm{~Hz}, 2 \mathrm{C}, \mathrm{C}_{4 \mathrm{a}}, 10 \mathrm{a}\right), 131.0$ $\left(\mathrm{d},{ }^{2} J_{\mathrm{CP}}=10.4 \mathrm{~Hz}, 4 \mathrm{C}, o-\mathrm{Ph}\right.$ ), $130.6\left(\mathrm{~d},{ }^{4} J_{\mathrm{CP}}=3.0 \mathrm{~Hz}, 2 \mathrm{C}, p-\mathrm{Ph}\right), 129.0$ (s, 2C, 
$\left.\mathrm{C}_{4,5}\right), 128.6\left(\mathrm{~d},{ }^{3} \mathrm{~J}_{\mathrm{CP}}=12.5 \mathrm{~Hz}, 4 \mathrm{C}, m-\mathrm{Ph}\right), 128.0\left(\mathrm{~d},{ }^{3} \mathrm{~J}_{\mathrm{CP}}=10.9 \mathrm{~Hz}, 2 \mathrm{C}, \mathrm{C}_{1,8}\right)$, $124.9\left(\mathrm{~s}, 2 \mathrm{C}, \mathrm{C}_{2,7}\right), 124.9\left(\mathrm{~d},{ }^{1} \mathrm{~J}_{\mathrm{CP}}=88.5 \mathrm{~Hz}, \mathrm{C}_{9}\right), 124.3\left(\mathrm{~s}, 2 \mathrm{C}, \mathrm{C}_{3,6}\right), 4.3(\mathrm{~s}, 3 \mathrm{C}$, $\left.\mathrm{CH}_{3}\right)$.

\section{${ }^{31} \mathrm{P}\left\{{ }^{1} \mathrm{H}\right\}-\mathrm{NMR}$}

$\left(\mathrm{CDCl}_{3}, 121 \mathrm{MHz}\right) \quad \delta[\mathrm{ppm}]=34.7$.

\section{EI-MS}

$\mathrm{m} / \mathrm{z}(\%):$

$\left.466.1(100)[\mathrm{M}]^{+}, 357.1(30)\left[\mathrm{M}-(\mathrm{S}) \mathrm{C}_{6} \mathrm{H}_{5}\right)\right]^{+}, 266.1(43)\left[\mathrm{C}_{14} \mathrm{H}_{10} \mathrm{PSiMe}_{2}\right]^{+}$.

\section{HR-MS (EI $\left.{ }^{+}\right)$}

$\mathrm{m} / \mathrm{z}$ (calculated): $\quad 465.1269$ (465.1262).

\section{Elemental analysis}

in \% (calculated): $\quad$ C: 72.57 (74.64), H: 5.75 (5.83), S: 6.76 (6.87).

\subsubsection{Synthesis of [9-(S)PPh $\left.-10-P h-\left(\mathrm{C}_{14} \mathrm{H}_{8}\right)\right](17)$}

9-(diphenylphosphanyl)-10-phenylanthracene $(0.25 \mathrm{~g}, 0.57 \mathrm{mmol}, 1.0 \mathrm{eq})$ and $\mathrm{S}_{8}(0.035 \mathrm{~g}, 0.81 \mathrm{mmol}, 1.4$ eq) were dissolved in Toluene $(10 \mathrm{~mL})$ and heated to $80^{\circ} \mathrm{C}$ for $4 \mathrm{~h}$. After cooling to ambient temperature, the mixture was filtrated, and the solvent was removed under reduced pressure. The yellow powder was recrystallized from toluene affording the target compound.

Yield:

$0.21 \mathrm{~g}(0.45 \mathrm{mmol}, 79 \%)$

Chemical formula: $\quad \mathrm{C}_{32} \mathrm{H}_{23} \mathrm{PS}$

Molecular weight: $\quad 470.57 \mathrm{~g} / \mathrm{mol}$

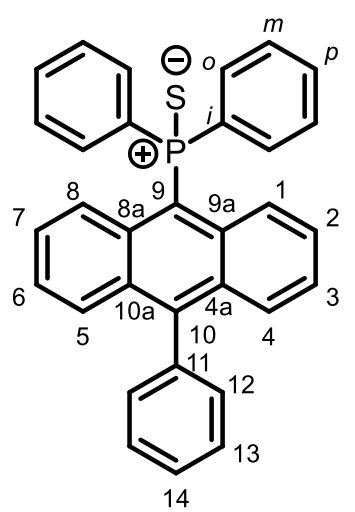

\section{${ }^{1}$ H-NMR}

$\left(\mathrm{CDCl}_{3}, 300 \mathrm{MHz}\right) \quad \delta[\mathrm{ppm}]=8.12-8.09\left(\mathrm{~m}, 2 \mathrm{H}, \mathrm{H}_{1,8}\right), 7.89-7.81(\mathrm{~m}, 4 \mathrm{H}, \mathrm{o}-\mathrm{Ph}), 7.67-7.57$ $\left(\mathrm{m}, 5 \mathrm{H}, \mathrm{H}_{4,5,12,14,16}\right), 7.47-7.44\left(\mathrm{~m}, 2 \mathrm{H}, \mathrm{H}_{13,15}\right), 7.37-7.28(\mathrm{~m}, 6 \mathrm{H}, p-\mathrm{Ph}$, $m-\mathrm{Ph}), 7.23-7.18\left(m, 2 \mathrm{H}, \mathrm{H}_{3,6}\right), 7.06-7.01\left(\mathrm{~m}, 2 \mathrm{H}, \mathrm{H}_{2,7}\right)$.

\section{${ }^{13} \mathrm{C}\left\{{ }^{1} \mathrm{H}\right\}-\mathrm{NMR}$}


$\left(\mathrm{CDCl}_{3}, 75 \mathrm{MHz}\right)$

$\delta[\mathrm{ppm}]=143.9\left(\mathrm{~s}, 1 \mathrm{C}, \mathrm{C}_{10}\right), 143.8\left(\mathrm{~s}, 1 \mathrm{C}, \mathrm{C}_{11}\right), 138.3\left(\mathrm{~s}, 2 \mathrm{C}, \mathrm{C}_{8 \mathrm{a}, 9 \mathrm{a}}\right), 137.3(\mathrm{~d}$, $\left.{ }^{1} J_{\mathrm{CP}}=82.7 \mathrm{~Hz}, 2 \mathrm{C}, i-\mathrm{Ph}\right), 133.0\left(\mathrm{~d},{ }^{3} \mathrm{~J}_{\mathrm{CP}}=7.7 \mathrm{~Hz}, 2 \mathrm{C}, \mathrm{C}_{4 \mathrm{a}}, 10 \mathrm{a}\right), 130.9\left(\mathrm{~d},{ }^{2} \mathrm{~J}_{\mathrm{CP}}=\right.$ $10.4 \mathrm{~Hz}, 4 \mathrm{C}, o-\mathrm{Ph}$ ), 130.8 (s, 2C, C 13,15$), 130.6$ (d, ${ }^{4} \mathrm{~J}_{\mathrm{CP}}=3.1 \mathrm{~Hz}, 2 \mathrm{C}, p-\mathrm{Ph}$ ) $128.5\left(\mathrm{~d},{ }^{3} \mathrm{~J}_{\mathrm{CP}}=12.9 \mathrm{~Hz}, 4 \mathrm{C}, \mathrm{m}-\mathrm{Ph}\right), 128.0\left(\mathrm{~s}, 3 \mathrm{C}, \mathrm{C}_{12,14,16}\right), 127.8\left(\mathrm{~s}, 2 \mathrm{C}, \mathrm{C}_{4,5}\right)$, $127.5\left(d,{ }^{2} J_{C P}=10.6 \mathrm{~Hz}, 2 \mathrm{C}, \mathrm{C}_{1,8}\right), 125.3\left(\mathrm{~s}, 2 \mathrm{C}, \mathrm{C}_{2,7}\right), 124.9\left(\mathrm{~s}, 2 \mathrm{C}, \mathrm{C}_{3,6}\right), 122.6$ $\left(d,{ }^{1} J_{C P}=88.7 \mathrm{~Hz}, C_{9}\right)$.

\section{${ }^{31} \mathrm{P}\left\{{ }^{1} \mathrm{H}\right\}-\mathrm{NMR}$}

$\left(\mathrm{CDCl}_{3}, 121 \mathrm{MHz}\right) \quad \delta[\mathrm{ppm}]=34.55$

\section{EI-MS}

$\mathrm{m} / \mathrm{z}(\%):$

$470(100)[\mathrm{M}]^{+}, 361(50)[\mathrm{M}-\mathrm{PhS}]^{+}, 285$ (80) [M-SPh] ${ }^{+}, 252(85)\left[\mathrm{M}-\mathrm{PSPh}_{2}\right]^{+}$.

\section{HR-MS (EI+)}

$\mathrm{m} / \mathrm{z}$ (calculated): $\quad 470.1250(470.1258)$.

\section{Elemental analysis}

in \% (calculated): $\quad$ C: 80.57 (81.68), H: 4.90 (4.93), S: 6.80 (6.81).

\subsubsection{Synthesis of 1-Chloroanthracene}

1-Chloroanthraquinone $\left(7.0 \mathrm{~g}, 28.8 \mathrm{mmol}, 1.0\right.$ eq.) was suspended $\mathrm{NH}_{3}$-solution $(25 \%, 80 \mathrm{~mL})$ and water $(60 \mathrm{~mL})$. At $0{ }^{\circ} \mathrm{C} Z \mathrm{n}$-dust $(30.0 \mathrm{~g}, 460 \mathrm{mmol}, 16$ eq.) was added in portions and the yellow mixture stirred for $15 \mathrm{~min}$, before it was heated to $75^{\circ} \mathrm{C}$ for $4 \mathrm{~h}$. The red suspension was cooled to ambient temperature, the grey solid was filtered off and washed with $\mathrm{iPrOH}$. The filtrate was extracted with $\mathrm{DCM}$ and the grey solid was dissolved in DCM. Removal of the solvent of the combined organic phases gave an orange solid, which was subsequently dissolved in conc. $\mathrm{HCl}(20 \mathrm{~mL})$ and $\mathrm{iProH}(20 \mathrm{~mL})$. The mixture was heated to reflux and $\mathrm{iPrOH}$ was added until the solid was dissolved completely. The target compound crystallizes upon cooling to ambient temperature as a yellow solid and could be filtered off. The crude product contains small amount of anthracene as impurity. If required, the product could be further purified by recrystallization from $i \mathrm{PrOH}$.

Yield:

$4.5 \mathrm{~g}(21.2 \mathrm{mmol}, 73 \%)$

Chemical formula: $\quad \mathrm{C}_{14} \mathrm{H}_{9} \mathrm{Cl}$

Molecular weight: $\quad 212.67 \mathrm{~g} / \mathrm{mol}$

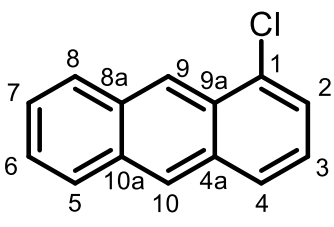




\section{${ }^{1} \mathrm{H}-\mathrm{NMR}$}

$\left(\mathrm{CDCl}_{3}, 400 \mathrm{MHz}\right) \quad \delta[\mathrm{ppm}]=8.86\left(\mathrm{~s}, 1 \mathrm{H}, \mathrm{H}_{9}\right), 8.45\left(\mathrm{~s}, 1 \mathrm{H}, \mathrm{H}_{10}\right), 8.11-7.93\left(\mathrm{~m}, 3 \mathrm{H}, \mathrm{H}_{4,5,8}\right), 7.59$ $-7.51\left(m, 3 H, H_{2,6,7}\right), 7.38-7.34\left(m, 1 H, H_{3}\right)$.

${ }^{13} \mathrm{C}\left\{{ }^{1} \mathrm{H}\right\}-N M R$

$\left(\mathrm{CDCl}_{3}, 100 \mathrm{MHz}\right) \quad \delta[\mathrm{ppm}]=132.3\left(\mathrm{~s}, 1 \mathrm{C}, \mathrm{C}_{10 \mathrm{a}}\right), 132.2\left(\mathrm{~s}, 1 \mathrm{C}, \mathrm{C}_{8 \mathrm{a}}\right), 132.0\left(\mathrm{~s}, 1 \mathrm{C}, \mathrm{C}_{9 \mathrm{a}}\right), 129.0(\mathrm{~s}$, 1C, $\mathrm{C}_{4 \mathrm{a}}$ ), 128.7 (s, 1C, $\left.\mathrm{C}_{4}\right), 128.2$ (s, 1C, $\left.\mathrm{C}_{1}\right), 127.9$ (s, 1C, $\left.\mathrm{C}_{5}\right), 127.6$ (s, 1C, $\left.\mathrm{C}_{8}\right), 126.9\left(\mathrm{~s}, 1 \mathrm{C}, \mathrm{C}_{10}\right), 126.2\left(\mathrm{~s}, 1 \mathrm{C}, \mathrm{C}_{7}\right), 126.0\left(\mathrm{~s}, 1 \mathrm{C}, \mathrm{C}_{6}\right), 125.3\left(\mathrm{~s}, 1 \mathrm{C}, \mathrm{C}_{2}\right)$, $124.8\left(s, 1 C, C_{3}\right), 123.6\left(s, 1 C, C_{9}\right)$.

\section{EI-MS}

$\mathrm{m} / \mathrm{z}(\%):$

$189.1(100)\left[\mathrm{C}_{15} \mathrm{H}_{9}\right]^{+}, 269.0(100)\left[\mathrm{M}-\mathrm{CH}_{3}\right]^{+}, 284.0(46)[\mathrm{M}]^{+}$.

HR-MS (EI+)

$\mathrm{m} / \mathrm{z}$ (calculated): $\quad 284.0199(284.0201)$.

\subsubsection{Synthesis of [1-PPh $\left.-\left(\mathrm{C}_{14} \mathrm{H}_{9}\right)\right](18)$}

1-Chloroanthracene $\left(0.48 \mathrm{~g}, 2.20 \mathrm{mmol}, 1.0\right.$ eq.) was suspended in THF $(10 \mathrm{~mL})$ and cooled to $0{ }^{\circ} \mathrm{C}$. $\mathrm{LiPPh}_{2}$ $(0.43 \mathrm{~g}, 2.20 \mathrm{mmol}, 1.0 \mathrm{eq}$.) in THF $(10 \mathrm{~mL})$ was added slowly over the period of $10 \mathrm{~min}$. After stirring at $0{ }^{\circ} \mathrm{C}$ for additional $30 \mathrm{~min}$ the mixture was allowed to warm to ambient temperature and stirred for another $3 \mathrm{~h}$. Afterwards the mixture was heated to reflux for $3 \mathrm{~h}$. Cooling to ambient temperature, filtration and subsequent removal of the solvent under reduced pressure yielded the product as an orange solid. Crystallization from DCM gave suitable crystals for X-Ray structure determination.

Yield:

$0.61 \mathrm{~g}(1.67 \mathrm{mmol}, 76 \%)$.

Chemical formula: $\quad \mathrm{C}_{26} \mathrm{H}_{19} \mathrm{P}$

Molecular weight: $\quad 362.41 \mathrm{~g} / \mathrm{mol}$

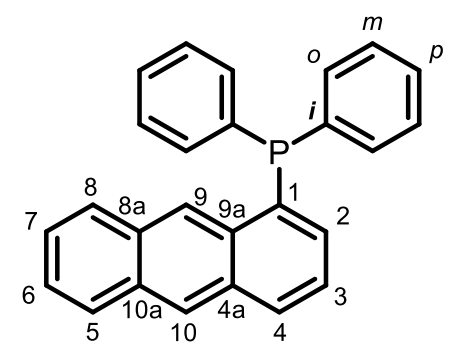

\section{${ }^{1} \mathrm{H}-\mathrm{NMR}$}

$\left(\mathrm{CDCl}_{3}, 300 \mathrm{MHz}\right) \quad \delta[\mathrm{ppm}]=9.00\left(\mathrm{~d}, J=4.3 \mathrm{~Hz}, 1 \mathrm{H}, \mathrm{H}_{9}\right), 8.46\left(\mathrm{~s}, 1 \mathrm{H}, \mathrm{H}_{10}\right), 8.03-7.98(\mathrm{~m}, 2 \mathrm{H}$, p-Ph), $7.95-7.92\left(m, 1 \mathrm{H}, \mathrm{H}_{8}\right), 7.49-7.42\left(\mathrm{~m}, 2 \mathrm{H}, \mathrm{H}_{3,6}\right), 7.40-7.32(\mathrm{~m}$, $\left.11 \mathrm{H}, o-\mathrm{Ph}, \mathrm{m}-\mathrm{Ph}, \mathrm{H}_{4,5,7}\right), 7.03-6,99\left(\mathrm{~m}, 1 \mathrm{H}, \mathrm{H}_{2}\right)$. 


\section{${ }^{13} \mathrm{C}\left\{{ }^{1} \mathrm{H}\right\}-\mathrm{NMR}$}

$\left(\mathrm{CDCl}_{3}, 75 \mathrm{MHz}\right)$

$\delta[\mathrm{ppm}]=136.2\left(\mathrm{~d}, 2 \mathrm{C},{ }^{1} J(\mathrm{C}, \mathrm{P})=9.1 \mathrm{~Hz}\right.$, ipso-Ph, $134.7\left(\mathrm{~d},{ }^{1} J(\mathrm{C}, \mathrm{P})=14.0 \mathrm{~Hz}\right.$, $\left.C_{1}\right), 134.2\left(d,{ }^{2} J(C, P)=19.8 \mathrm{~Hz}, o-P h\right), 133.0\left(d,{ }^{2} J(C, P)=21.9 \mathrm{~Hz}, C_{9 a}\right), 132.1$ $\left(\mathrm{s}, \mathrm{C}_{2}\right), 131.6\left(\mathrm{~d},{ }^{3} \mathrm{~J}(\mathrm{C}, \mathrm{P})=5.7 \mathrm{~Hz}, \mathrm{C}_{4 \mathrm{a}}\right), 131.5\left(\mathrm{~s}, \mathrm{C}_{8 \mathrm{a}}\right), 129.8\left(\mathrm{~s}, \mathrm{C}_{10 \mathrm{a}}\right), 128.9$ (s, $p$-Ph), $128.7\left(s, C_{5}\right), 128.6\left(d,{ }^{3} J(C, P)=7.2 \mathrm{~Hz}, m-P h\right), 127.7\left(s, C_{8}\right), 127.0$ $\left(d,{ }^{4} J(C, P)=1.8 \mathrm{~Hz}, C_{10}\right), 125.6\left(s, C_{6}\right), 125.5\left(s, C_{3}\right) 125.2$ (s, $\left.C_{5}\right), 124.9$ (d, $\left.{ }^{3} J(C, P)=1.7 \mathrm{~Hz}, C_{9}\right), 124.8\left(s, C_{4}\right)$.

\section{${ }^{31} \mathrm{P}\left\{{ }^{1} \mathrm{H}\right\}-N M R$}

$\left(\mathrm{CDCl}_{3}, 121 \mathrm{MHz}\right) \quad \delta[\mathrm{ppm}]=-14.1$

\section{EI-MS}

$\mathrm{m} / \mathrm{z}(\%):$

$362.1(100)[\mathrm{M}]^{+}, 283.1(50)[\mathrm{M}-(\mathrm{Ph}+2 \mathrm{H})]^{+}, 253.1(50)[\mathrm{M}-(\mathrm{PPh}+2 \mathrm{H})]^{+}$.

\section{HR-MS (EI+)}

$\mathrm{m} / \mathrm{z}$ (calculated): $\quad 362.1218(362.1224)$.

\section{Elemental analysis}

in \% (calculated): $\quad$ C: $85.42(86.17), 5.26(5.28)$.

\subsubsection{Synthesis of [1-(O)PPh $\left.-\left(\mathrm{C}_{14} \mathrm{H}_{9}\right)\right](19)$}

[1- $\left.\mathrm{PPh}_{2}-\left(\mathrm{C}_{14} \mathrm{H}_{9}\right)\right](18)(0.72 \mathrm{~g}, 2 \mathrm{mmol}, 1.0$ eq.) and hydrogen peroxide - urea (0.19 g, $2.0 \mathrm{mmol}, 1.0$ eq.) were dissolved in DCM $(10 \mathrm{~mL})$ and stirred for $16 \mathrm{~h} . \mathrm{H}_{2} \mathrm{O}(20 \mathrm{~mL})$ was added and the mixture extracted with DCM $(3 \times 20 \mathrm{~mL})$. The organic phase was extracted with DCM $(3 \times 20 \mathrm{~mL})$ and dried over $\mathrm{MgSO}_{4}$. After filtration the solvent was removed under reduced pressure and the target compound obtained as a yellow powder, which could be crystallized from toluene.

Yield: $0.66 \mathrm{~g}(1.74 \mathrm{mmol}, 87 \%)$.

Chemical formula: $\quad \mathrm{C}_{26} \mathrm{H}_{19} \mathrm{PO}$

Molecular weight: $\quad 378.41 \mathrm{~g} / \mathrm{mol}$

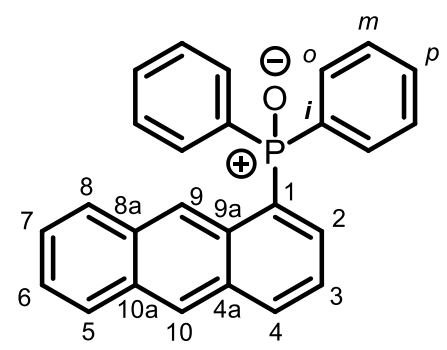

\section{${ }^{1} \mathrm{H}-\mathrm{NMR}$}

$\left(\mathrm{CDCl}_{3}, 300 \mathrm{MHz}\right)$

$\delta[p p m]=9.20\left(s, 1 H, H_{9}\right), 8.47\left(s, 1 H, H_{10}\right), 8.19-8.15\left(m, 1 \mathrm{H}, \mathrm{H}_{4}\right), 7.98-$ $7.95\left(m, 1 \mathrm{H}, \mathrm{H}_{5}\right), 7.91-7.88\left(\mathrm{~m}, 1 \mathrm{H}, \mathrm{H}_{8}\right), 7.78-7.70(\mathrm{~m}, 4 \mathrm{H}, \mathrm{o}-\mathrm{Ph}), 7.58-$ 
$7.51(\mathrm{~m}, 2 \mathrm{H}, p-\mathrm{Ph}), 7.49-7.41\left(\mathrm{~m}, 6 \mathrm{H}, m-\mathrm{Ph}, \mathrm{H}_{6,7}\right), 7.39-7.34\left(\mathrm{~m}, 1 \mathrm{H}, \mathrm{H}_{2}\right)$, $7.32-7.28\left(\mathrm{~m}, 1 \mathrm{H}, \mathrm{H}_{3}\right)$.

${ }^{13} \mathrm{C}\left\{{ }^{1} \mathrm{H}\right\}-\mathrm{NMR}$

$\left(\mathrm{CDCl}_{3}, 75 \mathrm{MHz}\right)$

$\delta[\mathrm{ppm}]=134.4\left(\mathrm{~d},{ }^{3} \mathrm{~J}(\mathrm{C}, \mathrm{P})=11.4 \mathrm{~Hz}, \mathrm{C}_{3}\right), 133.8\left(\mathrm{~d},{ }^{4} \mathrm{~J}(\mathrm{C}, \mathrm{P})=3.0 \mathrm{~Hz}, \mathrm{C}_{4}\right)$, $132.8\left(\mathrm{~d},{ }^{1} J(\mathrm{C}, \mathrm{P})=107.0 \mathrm{~Hz}, \mathrm{C}_{1}\right) .132 .1\left(\mathrm{~d},{ }^{2} J(\mathrm{C}, \mathrm{P})=9.9 \mathrm{~Hz}, \mathrm{o}-\mathrm{Ph}\right), 131.9$ (d, $\left.{ }^{4} J(C, P)=2.7 \mathrm{~Hz}, p-P h\right), 131.8\left(d,{ }^{3} J(C, P)=8.6 \mathrm{~Hz}, C_{4 a}\right), 131.5\left(s, C_{8 a}\right), 130.7$ $\left(d,{ }^{2} J(C, P)=8.1 \mathrm{~Hz}, C_{9 a}\right), 130.6\left(s, C_{10 a}\right), 129.1\left(s, C_{8}\right), 128.6\left(d,{ }^{3} J(C, P)=\right.$ $12.2 \mathrm{~Hz}, m-\mathrm{Ph}), 128.4$ (d, $\left.{ }^{1}(\mathrm{C}, \mathrm{P})=111.6 \mathrm{~Hz}, i-\mathrm{Ph}\right), 127.6$ (s, $\left.\mathrm{C}_{5}\right), 127.4$ (s, $\left.C_{10}\right), 127.1\left(d,{ }^{3} J(C, P)=5.5 \mathrm{~Hz}, C_{9}\right), 126.0\left(s, 2 C, C_{6,7}\right), 123.3\left(d,{ }^{2} J(C, P)=\right.$ $14.8 \mathrm{~Hz}, \mathrm{C}_{2}$ ).

\section{${ }^{31} \mathrm{P}\left\{{ }^{1} \mathrm{H}\right\}-\mathrm{NMR}$}

$\left(\mathrm{CDCl}_{3}, 121 \mathrm{MHz}\right) \quad \delta[\mathrm{ppm}]=32.44$.

\section{EI-MS}

$\mathrm{m} / \mathrm{z}(\%)$ :

$394.1(100)[\mathrm{M}]^{+}, 285.1(50)[\mathrm{M}-(\mathrm{S}) \mathrm{Ph}]^{+}, 185.1(15)\left[\mathrm{PPh}_{2}\right]^{+}$.

\section{HR-MS (EI $\left.{ }^{+}\right)$}

m/z (calculated): $\quad 394.0944$ (394.0945).

\section{Elemental analysis}

in \% (calculated): $\quad$ C: 82.24 (82.53), 5.11 (5.06).

\subsubsection{Synthesis of [1-(S)PPh $\left.-\left(\mathrm{C}_{14} \mathrm{H}_{9}\right)\right](20)$}

[1-PPh $2-\left(\mathrm{C}_{14} \mathrm{H}_{9}\right)$ ] (18) $(0.68 \mathrm{mmol}, 1.9 \mathrm{mmol}, 1.0 \mathrm{eq})$ and $\mathrm{S}_{8}(0.06 \mathrm{mg}, 0.24 \mathrm{mmol}, 1.0 \mathrm{eq})$ were dissolved in toluene $(15 \mathrm{~mL})$. The mixture was stirred and heated up to $80^{\circ} \mathrm{C}$ for $6 \mathrm{~h}$. After cooling to ambient temperature, a pale-yellow precipitate formed, which was filtered and dried under reduced pressure. Removing of the solvent of the filtrate afforded further product. Crystallization from toluene afforded the target compound in high purity.

Yield:

$0.57 \mathrm{~g}$ (1.4 mmol, $76 \%)$.

Chemical formula: $\quad \mathrm{C}_{26} \mathrm{H}_{19} \mathrm{PS}$

Molecular weight: $\quad 394.47 \mathrm{~g} / \mathrm{mol}$

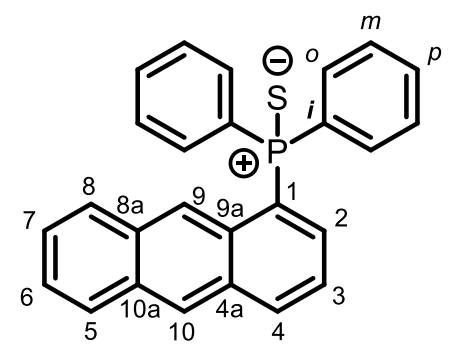




\section{${ }^{1} \mathrm{H}-\mathrm{NMR}$}

$\left(\mathrm{CDCl}_{3}, 300 \mathrm{MHz}\right)$

$\delta[p p m]=9.11\left(\mathrm{~s}, 1 \mathrm{H}, \mathrm{H}_{9}\right), 8.47\left(\mathrm{~s}, 1 \mathrm{H}, \mathrm{H}_{10}\right), 8.16-8.12\left(\mathrm{~m}, 1 \mathrm{H}, \mathrm{H}_{4}\right), 7.98$ $-7.94\left(m, 1 \mathrm{H}, \mathrm{H}_{5}\right), 7.92-7.94(\mathrm{~m}, 4 \mathrm{H}, \mathrm{o}-\mathrm{Ph}), 7.80-7.76\left(\mathrm{~m}, 1 \mathrm{H}, \mathrm{H}_{8}\right), 7.57$ - $7.37\left(m, 8 \mathrm{H}, p-\mathrm{Ph}, m-\mathrm{Ph}, \mathrm{H}_{6,7}\right), 7.35-7.30\left(\mathrm{~m}, 1 \mathrm{H}, \mathrm{H}_{2}\right), 7.28-7.18(\mathrm{~m}$, $\left.1 \mathrm{H}, \mathrm{H}_{3}\right)$.

\section{${ }^{13} \mathrm{C}\left\{{ }^{1} \mathrm{H}\right\}-N M R$}

$\left(\mathrm{CDCl}_{3}, 75 \mathrm{MHz}\right)$

$\delta[\mathrm{ppm}]=133.7\left(\mathrm{~d},{ }^{3} J(\mathrm{C}, \mathrm{P})=12.5 \mathrm{~Hz}, \mathrm{C}_{3}\right), 133.7\left(\mathrm{~s}, \mathrm{C}_{4}\right), 132.5\left(\mathrm{~d},{ }^{2} J(\mathrm{C}, \mathrm{P})=\right.$ $10.7 \mathrm{~Hz}, 0-\mathrm{Ph}), 132.5\left(\mathrm{~d},{ }^{1} J(\mathrm{C}, \mathrm{P})=84.9 \mathrm{~Hz}\right.$, ipso-Ph), 132.1 (d, ${ }^{3}(\mathrm{C}, \mathrm{P})=$ $\left.8.6 \mathrm{~Hz}, \mathrm{C}_{4 \mathrm{a}}\right), 131.6\left(\mathrm{~d},{ }^{4} \mathrm{~J}(\mathrm{C}, \mathrm{P})=3.2 \mathrm{~Hz}, p-\mathrm{Ph}\right), 131.6\left(\mathrm{~s}, \mathrm{C}_{8 \mathrm{a}}\right), 131,5\left(\mathrm{~s}, \mathrm{C}_{10 \mathrm{a}}\right)$, $129.7\left(d,{ }^{1} J(C, P)=84.6 \mathrm{~Hz}, C_{1}\right), 129.2\left(d,{ }^{2} J(C, P)=9.07 \mathrm{~Hz}, C_{9 a}\right), 129.0\left(s, C_{8}\right)$, $128.7\left(d, 3 J(C, P)=12.6 \mathrm{~Hz}, m-P h, 127.8\right.$ (d, J(C,P) = 7.1 Hz, $\left.C_{9}\right), 127.6$ (d, $\left.{ }^{4} J(C, P)=1.3 \mathrm{~Hz}, C_{10}\right), 127.6\left(s, C_{5}\right), 126.2\left(s, C_{7}\right), 125.9\left(s, C_{6}\right), 123.5$ (d, $\left.{ }^{2} J(C, P)=14.7 \mathrm{~Hz}, C_{2}\right)$.

\section{${ }^{31} \mathrm{P}\left\{{ }^{1} \mathrm{H}\right\}-\mathrm{NMR}$}

$\left(\mathrm{CDCl}_{3}, 121 \mathrm{MHz}\right) \quad \delta[\mathrm{ppm}]=42.1$.

\section{EI-MS}

$\mathrm{m} / \mathrm{z}(\%):$

$394.1(100)[\mathrm{M}]^{+}, 285.1(50)[\mathrm{M}-(\mathrm{S}) \mathrm{Ph}]^{+}, 185.1(15)\left[\mathrm{PPh}_{2}\right]^{+}$.

\section{HR-MS (EI+)}

m/z (calculated): $\quad 394.0944$ (394.0945).

\section{Elemental analysis}

in \% (calculated): $\quad$ C: 79.83 (79.17), H: 4.73 (4.86), S: 8.56 (8.13).

\subsubsection{Synthesis of 2-Bromoanthraquinone}

$\mathrm{CuBr}_{2}\left(6.1 \mathrm{~g}, 27.3 \mathrm{mmol}, 1.2\right.$ eq.) and $t \mathrm{BuNO}_{2}(3.5 \mathrm{~g}, 4.0 \mathrm{~mL}, 33.6 \mathrm{mmol}, 1.5$ eq.) were dispersed in $\mathrm{MeCN}$ and heated to $65^{\circ} \mathrm{C}$. 2-Aminoanthraquinone $(5.0 \mathrm{~g}, 22.5 \mathrm{mmol}, 1.0$ eq.) was added in portions and the mixture stirred for $16 \mathrm{~h}$ at $65{ }^{\circ} \mathrm{C}$. After to cooling to ambient temperature the mixture was poured into aqueous $\mathrm{HCl}(6 \mathrm{M}, 250 \mathrm{~mL})$. The precipitate was collected by suction filtration and washed with $\mathrm{EtOH}$ and $\mathrm{H}_{2} \mathrm{O}$. The solid was dissolved in DCM/Hexane $(1: 1,500 \mathrm{~mL})$ and washed with $\mathrm{H}_{2} \mathrm{O}(3 \times 250 \mathrm{~mL})$. The organic phase was dried over $\mathrm{MgSO}_{4}$, filtrated and the solvent removed under reduced pressure. The product was obtained as a brown powder and used without further purification. 
Yield:

$2.29 \mathrm{~g}(8.0 \mathrm{mmol}, 36 \%)$

Chemical formula: $\quad \mathrm{C}_{14} \mathrm{H}_{7} \mathrm{BrO}_{2}$

Molecular weight: $\quad 287.11 \mathrm{~g} / \mathrm{mol}$

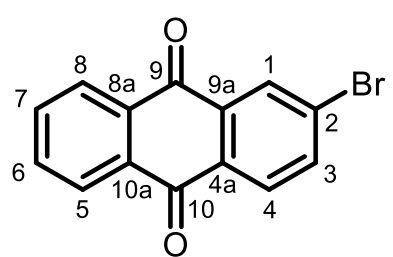

${ }^{1} \mathrm{H}-\mathrm{NMR}$

$\left(\mathrm{CDCl}_{3}, 300 \mathrm{MHz}\right)$

$\delta[p p m]=8.43\left(\mathrm{~d}, \mathrm{~J}_{\mathrm{HH}}=2.0 \mathrm{~Hz}, 1 \mathrm{H}, \mathrm{H}_{1}\right), 8.32-8.28\left(\mathrm{~m}, 2 \mathrm{H}, \mathrm{H}_{5,8}\right), 8.17(\mathrm{~d}$, $\left.\mathrm{J}_{\mathrm{HH}}=8.3 \mathrm{~Hz}, 1 \mathrm{H}, \mathrm{H}_{4}\right), 7.92\left(\mathrm{dd}, \mathrm{J}_{\mathrm{HH}}=8.3,2.0 \mathrm{~Hz}, 1 \mathrm{H}, \mathrm{H}_{3}\right), 7.83-7.80(\mathrm{~m}, 2 \mathrm{H}$, $\left.\mathrm{H}_{6,7}\right)$.

\section{${ }^{13} \mathrm{C}\left\{{ }^{1} \mathrm{H}\right\}-N M R$}

$\left(\mathrm{CDCl}_{3}, 75 \mathrm{MHz}\right)$

$\delta[\mathrm{ppm}]=182.3(\mathrm{~s}, 1 \mathrm{C}), 182.0(\mathrm{~s}, 1 \mathrm{C}), 137.2\left(\mathrm{~s}, 1 \mathrm{C}, \mathrm{C}_{3}\right), 134.5\left(\mathrm{~s}, 1 \mathrm{C}, \mathrm{C}_{9 \mathrm{a}}\right)$, 134.4 / 134.2 (s, 2C, $\left.C_{6,7}\right), 133.3$ (s, 1C, $C_{8 a}$ ), 133.1 (s, 1C, $\left.C_{10 a}\right), 132.0$ (s, 1C, C $\mathrm{C}_{4 \mathrm{a}} 130.2$ (s, 1C, $\left.\mathrm{C}_{1}\right), 129.7$ (), 129.0 (s, 1C, $\mathrm{C}_{4}$ ), 127.4 / 127.3 (s, 2C, $\mathrm{C}_{5,8}$ ),

\section{EI-MS}

$\mathrm{m} / \mathrm{z}(\%): \quad 287.9(100)[\mathrm{M}]^{+}$.

\section{HR-MS (EI+)}

$\mathrm{m} / \mathrm{z}$ (calculated): $\quad 285.9619$ (285.9629).

\subsubsection{Synthesis of 2-Bromoanthracene}

2-Bromoanthraquinone (1.9 g, $6.9 \mathrm{mmol}, 1.0$ eq.), $\mathrm{NaBH}_{4}(1.5 \mathrm{~g}, 41.4 \mathrm{mmol}, 6.0$ eq.) were dissolved in $i \operatorname{PrOH}(150 \mathrm{~mL})$ at ambient temperature and stirred for $16 \mathrm{~h}$. The suspension was poured onto ice-water $(100 \mathrm{~mL})$ and filtrated. The solid was dissolved in conc. $\mathrm{HCl}(25 \mathrm{~mL})$ and heated to $75 \mathrm{C}$ for $5 \mathrm{~h}$. After cooling to ambient temperature, the mixture was filtered and a brown solid was obtained, which was dissolved in iPrOH together with $\mathrm{NaBH}_{4}(1.5 \mathrm{~g}, 41.4 \mathrm{mmol}, 6.0 \mathrm{eq})$. After refluxing for $16 \mathrm{~h}$, conc $\mathrm{HCl}$ was added carefully at room temperature until gas evolution was completed. The mixture was stirred for $3 \mathrm{~h}$ at $75 \mathrm{C}$. The solid was filtered off washed with $\mathrm{H}_{2} \mathrm{O}$ and dried under reduced pressure. The product was obtained as a slight brown powder.

Yield:

$0.43 \mathrm{~g}(6.9 \mathrm{mmol}, 24 \%)$

Chemical formula: $\quad \mathrm{C}_{14} \mathrm{H}_{9} \mathrm{Br}$

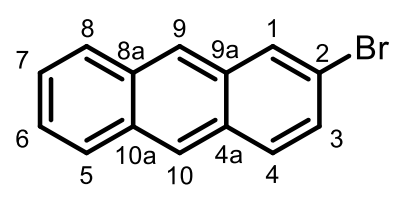

Molecular weight: $\quad 257.13 \mathrm{~g} / \mathrm{mol}$ 


\section{${ }^{1} \mathrm{H}-\mathrm{NMR}$}

$\left(\mathrm{CDCl}_{3}, 400 \mathrm{MHz}\right) \quad \delta[\mathrm{ppm}]=8.39\left(\mathrm{~s}, 1 \mathrm{H}, \mathrm{H}_{9}\right), 8.32\left(\mathrm{~s}, 1 \mathrm{H}, \mathrm{H}_{10}\right), 8.17\left(\mathrm{~s}, 1 \mathrm{H}, \mathrm{H}_{1}\right), 8.01-7.98(\mathrm{~m}$, $\left.2 \mathrm{H}, \mathrm{H}_{5,8}\right), 7.87\left(\mathrm{~d},{ }^{3} \mathrm{~J}_{\mathrm{HH}}=9.0 \mathrm{~Hz}, 1 \mathrm{H}, \mathrm{H}_{4}\right), 7.52-7.48\left(\mathrm{~m}, 3 \mathrm{H}, \mathrm{H}_{3,6,7}\right)$.

${ }^{13} \mathrm{C}\left\{{ }^{1} \mathrm{H}\right\}-\mathrm{NMR}$

$\left(\mathrm{CDCl}_{3}, 100 \mathrm{MHz}\right) \quad \delta[\mathrm{ppm}]=132.4\left(\mathrm{~s}, 1 \mathrm{C}, \mathrm{C}_{\mathrm{q}}\right), 132.3\left(\mathrm{~s}, 1 \mathrm{C}, \mathrm{C}_{\mathrm{q}}\right), 131.9\left(\mathrm{~s}, 1 \mathrm{C}, \mathrm{C}_{\mathrm{q}}\right), 130.1(\mathrm{~s}, 1 \mathrm{C}$, $\mathrm{C}_{4}$ ), 130.0 (s, 1C, C 1 ), 129.9 (s, 1C, Cq), 129.0 (s, 1C, C 3 ), 128.4 (s, 1C, C5), $128.3\left(\mathrm{~s}, 1 \mathrm{C}, \mathrm{C}_{8}\right), 126.7$ (s, 1C, $\left.\mathrm{C}_{9}\right), 126.2$ (s, 1C, $\left.\mathrm{C}_{7}\right), 125.9$ (s, 1C, $\left.\mathrm{C}_{6}\right), 125.5$ $\left(\mathrm{s}, 1 \mathrm{C}, \mathrm{C}_{10}\right), 120.0\left(\mathrm{~s}, 1 \mathrm{C}, \mathrm{C}_{2}\right)$.

\section{EI-MS}

$\mathrm{m} / \mathrm{z}(\%): \quad 258.0(85)[\mathrm{M}]^{+}, 177.1(100)[\mathrm{M}-\mathrm{Br}]^{+}$

HR-MS (EI+)

$\mathrm{m} / \mathrm{z}$ (calculated): $\quad 255.9885$ (255.9888).

\subsubsection{Synthesis of [2-PPh $\left.-\left(\mathrm{C}_{14} \mathrm{H}_{9}\right)\right](21)$}

2-Bromoanthracene $\left(0.40 \mathrm{~g}, 1.6 \mathrm{mmol}, 1.0\right.$ eq.) was suspended in $\mathrm{Et}_{2} \mathrm{O}(15 \mathrm{~mL})$ and cooled to $0{ }^{\circ} \mathrm{C}$. ${ }^{n}$ Butyllithium in hexane $(2.2 \mathrm{M}, 0.11 \mathrm{~g}, 1.7 \mathrm{mmol}, 1.1 \mathrm{eq}$.) was added slowly and the mixture stirred for $10 \mathrm{~min}$ and then allowed to reach ambient temperature. After the solid was completely dissolved the mixture was cooled to $0{ }^{\circ} \mathrm{C}$ again and Chlorodiphenylphosphine $(0.38 \mathrm{~g}, 0.31 \mathrm{~mL}, 2.4 \mathrm{mmol}, 1.1 \mathrm{eq}$.) was added dropwise. The solution turned red and a precipitate formed immediately. The mixture was warmed to ambient temperature and stirred for another $2 \mathrm{~h}$ and the precipitate was filtered off. The precipitate was dissolved in DCM $(10 \mathrm{~mL})$ and filtered again for removal of $\mathrm{LiCl}$. Removal of the solvent under reduced pressure gave the desired product as an orange powder.

Yield:

$0.17 \mathrm{~g}$ (0.47 mmol, $44 \%)$.

Chemical formula: $\quad \mathrm{C}_{26} \mathrm{H}_{19} \mathrm{P}$

Molecular weight: $\quad 362.41 \mathrm{~g} / \mathrm{mol}$

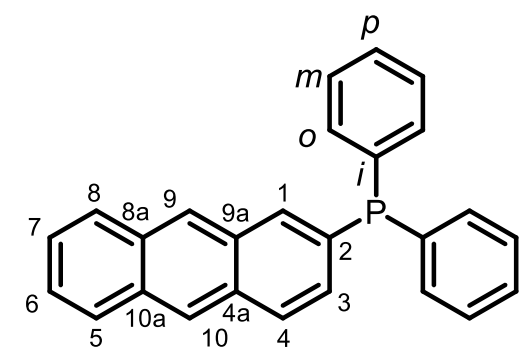

${ }^{1} \mathrm{H}-\mathrm{NMR}$

$\left(\mathrm{CDCl}_{3}, 300 \mathrm{MHz}\right) \quad \delta[\mathrm{ppm}]=8.39\left(\mathrm{~s}, 1 \mathrm{H}, \mathrm{H}_{9}\right), 8.32\left(\mathrm{~s}, 1 \mathrm{H}, \mathrm{H}_{10}\right), 8.01-7.92\left(\mathrm{~m}, 4 \mathrm{H}, \mathrm{H}_{1,3}, p-\mathrm{Ph}\right)$, $7.48-7.33\left(\mathrm{~m}, 13 \mathrm{H}, \mathrm{H}_{4-8}, m-\mathrm{Ph}, \mathrm{o}-\mathrm{Ph}\right)$. 


\section{${ }^{13} \mathrm{C}\left\{{ }^{1} \mathrm{H}\right\}-\mathrm{NMR}$}

$\left(\mathrm{CDCl}_{3}, 75 \mathrm{MHz}\right) \quad \delta[\mathrm{ppm}]=136.7\left(\mathrm{~d},{ }^{1} \mathrm{~J}_{\mathrm{CP}}=11.5 \mathrm{~Hz}, 2 \mathrm{C}, i-\mathrm{Ph}\right), 134.6\left(\mathrm{~d},{ }^{2} J_{\mathrm{CP}}=19.4 \mathrm{~Hz}, \mathrm{C}_{3}\right), 133.9$ $\left(d,{ }^{2} J_{\mathrm{CP}}=19.4 \mathrm{~Hz}, 4 \mathrm{C}, o-\mathrm{Ph}\right), 132.5\left(\mathrm{~d},{ }^{1} J_{\mathrm{CP}}=10.7 \mathrm{~Hz}, \mathrm{C}_{2}\right), 132.1\left(\mathrm{~d},{ }^{2} J_{\mathrm{CP}}=\right.$ $20.0 \mathrm{~Hz}, \mathrm{C}_{1}$ ), 131.6 (s, $\left.\mathrm{C}_{\mathrm{q}, \mathrm{anth}}\right), 131.5$ (s, $\left.\mathrm{C}_{\mathrm{q}, \text { anth }}\right), 129.2$ (s, $\left.\mathrm{C}_{q, \text { anth }}\right), 128.8$ (d, ${ }^{3} \mathrm{~J}_{\mathrm{CP}}$ $=7.3 \mathrm{~Hz}, 4 \mathrm{C}, m-\mathrm{Ph}$ ), $128.4(\mathrm{~s}, 2 \mathrm{C}, p-\mathrm{Ph}), 128.3\left(\mathrm{~s}, 4 \mathrm{C}, \mathrm{C}_{5-8}\right), 128.2$ (d, ${ }^{3} \mathrm{CP}_{\mathrm{CP}}=$ $\left.6.8 \mathrm{~Hz}, \mathrm{C}_{9 \mathrm{a}}\right), 126.7\left(\mathrm{~s}, \mathrm{C}_{10}\right), 126.2\left(\mathrm{~s}, \mathrm{C}_{9}\right), 125.7\left(\mathrm{~d},{ }^{3} \mathrm{~J}_{\mathrm{CP}}=10.5 \mathrm{~Hz}, \mathrm{C}_{4}\right)$.

${ }^{31} \mathrm{P}\left\{{ }^{1} \mathrm{H}\right\}-\mathrm{NMR}$

$\left(\mathrm{CDCl}_{3}, 121 \mathrm{MHz}\right) \quad \delta[\mathrm{ppm}]=-4.37$.

\section{EI-MS}

$m / z(\%):$

$362.1(100)[\mathrm{M}]^{+}, 283.1(37)[\mathrm{M}-(\mathrm{Ph}+2 \mathrm{H})]^{+}, 254.1(81)\left[\mathrm{M}-\left(\mathrm{Ph}+\mathrm{C}_{2} \mathrm{H}_{7}\right)\right]$.

HR-MS (EI+)

$\mathrm{m} / \mathrm{z}$ (calculated): $\quad 362.1214$ (362.1224).

Elemental analysis

in \% (calculated): $\quad$ C: 84.85 (86.17), H: 5.39 (5.28).

\subsubsection{Synthesis of [2-(S)PPh2- $\left.\left(\mathrm{C}_{14} \mathrm{H}_{9}\right)\right](22)$}

[2- $\left.\mathrm{PPh}_{2}-\left(\mathrm{C}_{14} \mathrm{H}_{9}\right)\right]$ (21) $\left(0.20 \mathrm{~g}, 0.55 \mathrm{~mol} 1.0\right.$ eq.) and $\mathrm{S}_{8}(0.026 \mathrm{~g}, 0.83 \mathrm{mmol}, 1.5$ eq. $)$ were dissolved in toluene $(10 \mathrm{~mL})$ and heated to $80^{\circ} \mathrm{C}$ over the period of $6 \mathrm{~h}$. Afterwards the mixture was filtered and the precipitate was recrystallized from toluene yielding the target compound as pale yellow crystals.

Yield:

$0.14 \mathrm{~g}(0.36 \mathrm{mmol}, 65 \%)$.

Chemical formula: $\quad \mathrm{C}_{26} \mathrm{H}_{19} \mathrm{PS}$

Molecular weight: $\quad 394.47 \mathrm{~g} / \mathrm{mol}$

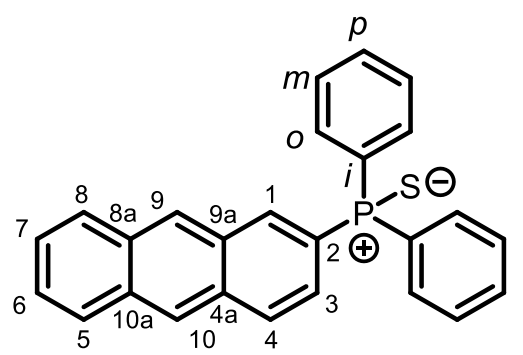

\section{${ }^{1} \mathrm{H}-\mathrm{NMR}$}

$\left(\mathrm{CDCl}_{3}, 300 \mathrm{MHz}\right) \quad \delta[\mathrm{ppm}] 8.45\left(\mathrm{~s}, 2 \mathrm{H}, \mathrm{H}_{9,10}\right), 8.41-8.35\left(\mathrm{~m}, 1 \mathrm{H}, \mathrm{H}_{1}\right), 8.06-7.98\left(\mathrm{~m}, 3 \mathrm{H}, \mathrm{H}_{3,5,8}\right)$ $7.85-7.78(\mathrm{~m}, 4 \mathrm{H}, o-\mathrm{Ph}), 7.71-7.64\left(\mathrm{~m}, 1 \mathrm{H}, \mathrm{H}_{4}\right), 7.58-7.44(\mathrm{~m}, 8 \mathrm{H}, m-\mathrm{Ph}$, $p$-Ph, $\left.\mathrm{H}_{6,7}\right)$.

\section{${ }^{13} \mathrm{C}\left\{{ }^{1} \mathrm{H}\right\}-\mathrm{NMR}$}


$\left(\mathrm{CDCl}_{3}, 75 \mathrm{MHz}\right)$

$\delta[p p m]=135.2\left(\mathrm{~d},{ }^{2} J(\mathrm{C}, \mathrm{P})=10.4 \mathrm{~Hz}, 1 \mathrm{C}, \mathrm{C}_{1}\right), 133.2\left({ }^{1} J(\mathrm{C}, \mathrm{P})=17.0 \mathrm{~Hz}, 1 \mathrm{C}, \mathrm{C}_{2}\right)$, $132.4\left(\mathrm{~d},{ }^{2} J(C, P)=10.7 \mathrm{~Hz}, 4 \mathrm{C}, 0-P h\right), 132.2\left(\mathrm{~s}, 1 \mathrm{C}, \mathrm{C}_{8 \mathrm{a}}\right), 131.7\left(\mathrm{~d},{ }^{3} J(C, P)=\right.$ $2.3 \mathrm{~Hz}, 1 \mathrm{C}, \mathrm{C}_{9 \mathrm{a}}$ ), 131.6 (d, $\left.{ }^{4} \mathrm{~J}(\mathrm{C}, \mathrm{P})=2.0 \mathrm{~Hz}, 2 \mathrm{C}, \mathrm{p}-\mathrm{Ph}\right) 130.2\left(\mathrm{~s}, 1 \mathrm{C}, \mathrm{C}_{4 \mathrm{a}}\right), 130.0$ $\left(d,{ }^{1} J(C, P)=11.4 \mathrm{~Hz}, 2 \mathrm{C}\right.$, ipso-Ph), $128.8\left(\mathrm{~d},{ }^{2} J(C, P)=19.8 \mathrm{~Hz}, 1 \mathrm{C}, \mathrm{C}_{3}\right), 128.0$ (s, 1C, $\left.C_{10 a}\right), 128.6\left(d,{ }^{3} J(C, P)=12.6 \mathrm{~Hz}, 4 C, m-P h\right), 128.4\left(s, 2 C, C_{5,8}\right), 128.2$ $\left(d,{ }^{4} J(C, P)=7.3 \mathrm{~Hz}, 1 \mathrm{C}, C_{9}\right), 126.3\left(\mathrm{~s}, 1 \mathrm{C}, \mathrm{C}_{10}\right), 126.3$ (s, 2C, $\left.\mathrm{C}_{6,7}\right), 125.6$ (d, $\left.{ }^{3} J(C, P)=11.4 \mathrm{~Hz}, 1 \mathrm{C}, \mathrm{C}_{4}\right)$.

${ }^{31} \mathrm{P}\left\{{ }^{1} \mathrm{H}\right\}-N M R$

$\left(\mathrm{CDCl}_{3}, 121 \mathrm{MHz}\right) \quad \delta[\mathrm{ppm}]=43.6$.

ESI-MS

$\mathrm{m} / \mathrm{z}(\%): \quad 395.1(100)[\mathrm{M}+\mathrm{H}]^{+}, 417.1(65)[\mathrm{M}+\mathrm{Na}]^{+}$.

HR-MS (EI+)

$\mathrm{m} / \mathrm{z}$ (calculated): $\quad 395.1014$ (395.1018).

Elemental analysis

in \% (calculated): $\quad$ C: 78.96 (79.17), H: 4.71 (4.86), S: 8.36 (8.13).

\subsubsection{Synthesis of 1,4-Dibromoanthraquinone}

1,4-Diaminoanthraquinone ( $2.80 \mathrm{~g}, 12.0 \mathrm{mmol}, 1.0$ eq.), Copper(II)bromide (6.80 g, $30.5 \mathrm{mmol}, 2.5$ eq.) and tert-Butylnitrite ( $3.70 \mathrm{~g}, 4.3 \mathrm{~mL}, 36 \mathrm{mmol}, 3.0$ eq.) were suspended in $\mathrm{MeCN}(200 \mathrm{~mL}$ ) and heated to $75^{\circ} \mathrm{C}$ over the period of $16 \mathrm{~h}$. After cooling to ambient temperature, the mixture was filtered and poured into a $\mathrm{HCl}$-solution $\left(6 \mathrm{M}, 200 \mathrm{~mL}\right.$ ). The formed precipitate was filtered and washed with deionized $\mathrm{H}_{2} \mathrm{O}$ several times. Drying under reduced pressure yielded a yellow-brown powder.

Yield:

Chemical formula: $\quad \mathrm{C}_{14} \mathrm{H}_{6} \mathrm{Br}_{2} \mathrm{O}_{2}$

Molecular weight: $\quad 366.01 \mathrm{~g} / \mathrm{mol}$

${ }^{1} \mathrm{H}-\mathrm{NMR}$

$\left(\mathrm{CDCl}_{3}, 300 \mathrm{MHz}\right)$

$\delta[p p m]=8.22-819\left(\mathrm{~m}, 2 \mathrm{H}, \mathrm{H}_{5,8}\right), 7.81\left(\mathrm{~s}, 2 \mathrm{H}, \mathrm{H}_{2,3}\right), 7.80-7.78(\mathrm{~m}, 2 \mathrm{H}$, $\left.H_{6,7}\right)$.

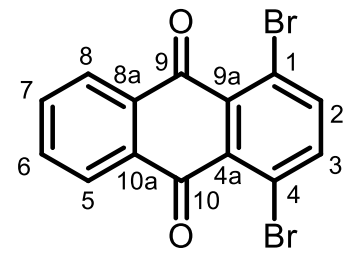

${ }^{13} \mathrm{C}\left\{{ }^{1} \mathrm{H}\right\}-\mathrm{NMR}$ 
$\left(\mathrm{CDCl}_{3}, 75 \mathrm{MHz}\right)$

$\delta[\mathrm{ppm}]=181.6(\mathrm{~s}, 2 \mathrm{C}, \mathrm{CO}), 140.63\left(\mathrm{~s}, 2 \mathrm{C}, \mathrm{C}_{2,3}\right), 134.2\left(2 \mathrm{C}, \mathrm{C}_{6,7}\right), 133.6$

$\left(C_{8 a}, 9 a\right), 133.5\left(C_{4 a, 10 a}\right), 126.9\left(s, 2 C, C_{6,7}\right), 122.06\left(s, 2 C, C_{1,4}\right)$.

\section{EI-MS}

$\mathrm{m} / \mathrm{z}(\%):$

$365.9(100)[\mathrm{M}]^{+}, 337.9(35)[\mathrm{M}-(\mathrm{CO})]^{+}, 309.9(33)[\mathrm{M}-(2 \mathrm{CO})]^{+}$.

HR-MS (EI+)

$\mathrm{m} / \mathrm{z}$ (calculated): $\quad 363.8745$ (363.8734).

\subsubsection{Synthesis of 1,4-Dibromoanthracene}

Method A: 1,4-Dibromoanthraquinone ( $1.10 \mathrm{~g}, 3.0 \mathrm{mmol}, 1.0$ eq.) and $\mathrm{NaBH}_{4}(0.45 \mathrm{~g}, 12.0 \mathrm{mmol}, 4.0$ eq.) were suspended in 2-Propanol $(10 \mathrm{~mL})$ and stirred for $16 \mathrm{~h}$. The mixture was added to ice water $(100 \mathrm{~mL})$ and filtered afterwards. The residue was dissolved in $\mathrm{HCl}(2 \mathrm{M}, 40 \mathrm{~mL})$ and stirred at $75{ }^{\circ} \mathrm{C}$ for $5 \mathrm{~h}$. After cooling to ambient temperature, the mixture was filtered again. To the brown solid $\mathrm{NaBH}_{4}(0.68 \mathrm{~g}$, $18 \mathrm{mmol}, 6$ eq.) and ${ }^{2} \mathrm{PrOH}(20 \mathrm{~mL})$ was added and the mixture was refluxed for $16 \mathrm{~h}$. After cooling to ambient temperature again $\mathrm{HCl}(2 \mathrm{M})$ was carefully added until gas-evolving ended. The mixture was then warmed to $75{ }^{\circ} \mathrm{C}$ for $3 \mathrm{~h}$. The precipitate was filtered and washed with $\mathrm{H}_{2} \mathrm{O}(2 \times 20 \mathrm{~mL})$ and dried under reduced pressure. Column chromatography using hexane/DCM (4:1) as eluent yielded a yellow powder.

Method B: 1,4-Dibromoanthraquinone $(4.0 \mathrm{~g}, 10.6 \mathrm{mmol}, 1.0 \mathrm{eq})$ was suspended in $\mathrm{iPrOH}(200 \mathrm{~mL})$ and cooled to $0{ }^{\circ} \mathrm{C} . \mathrm{NaBH}_{4}(1.15 \mathrm{~g}, 30 \mathrm{mmol}, 2.5 \mathrm{eq}$.) was added in portions and the red solution was stirred for $1.5 \mathrm{~h}$ at $0{ }^{\circ} \mathrm{C}$ and for $3 \mathrm{~h}$ at ambient temperature. The mixture was poured onto $\mathrm{H}_{2} \mathrm{O}(250 \mathrm{~mL})$ and stirred for further 30 min until the solution turned yellow. The grey solid was filtered off and washed with $\mathrm{H}_{2} \mathrm{O}$ and dried under reduced pressure. Afterwards the solid was suspended in $\mathrm{AcOH}(150 \mathrm{~mL})$ and $\mathrm{SnCl}_{2}(7.5 \mathrm{~g}$, $40 \mathrm{mmol}, 4$ eq.) was added. The reaction was heated to reflux for $2 \mathrm{~h}$ and $\mathrm{AcOH}$ was added until the solid was dissolved completely. Cooling to ambient temperature afforded the desired product as green-yellow needles, which were filtered off and washed with water and dried under reduced pressure.

Yield:

Method A: $0.43 \mathrm{~g} \mathrm{(1.3} \mathrm{mmol,} \mathrm{43 \% ),}$

Method B: $2.3 \mathrm{~g}(6.9 \mathrm{mmol}, 65 \%)$.

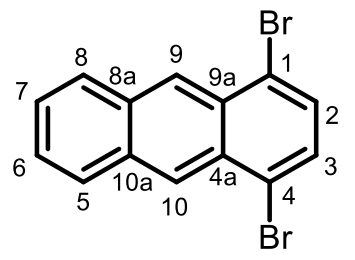

Chemical formula: $\quad \mathrm{C}_{14} \mathrm{H}_{8} \mathrm{Br}_{2}$

Molecular weight: $\quad 336.03 \mathrm{~g} / \mathrm{mol}$

${ }^{1} \mathrm{H}-\mathrm{NMR}$

$\left(\mathrm{CDCl}_{3}, 300 \mathrm{MHz}\right) \quad \delta[\mathrm{ppm}]=8.83\left(\mathrm{~s}, 2 \mathrm{H}, \mathrm{H}_{9,10}\right), 8.13-8.09\left(\mathrm{~m}, 2 \mathrm{H}, \mathrm{H}_{5,8}\right), 7.62\left(\mathrm{~s}, 2 \mathrm{H}, \mathrm{H}_{2,3}\right)$, $7.60-7.56\left(\mathrm{~m}, 2 \mathrm{H}, \mathrm{H}_{6,7}\right)$. 


\section{${ }^{13} \mathrm{C}\left\{{ }^{1} \mathrm{H}\right\}-\mathrm{NMR}$}
$\left(\mathrm{CDCl}_{3}, 75 \mathrm{MHz}\right)$
$\delta[p p m]=132.6\left(\mathrm{~s}, 2 \mathrm{C}, \mathrm{C}_{9 \mathrm{a}, 8 \mathrm{a}}\right), 130.4\left(\mathrm{~s}, 2 \mathrm{C}, \mathrm{C}_{4 \mathrm{a}, 10 \mathrm{a}}\right), 129.0\left(\mathrm{~s}, 2 \mathrm{C}, \mathrm{C}_{2,3}\right), 128.3$
$\left(s, 2 C, C_{5,8}\right), 127.3\left(s, 2 C, C_{9,19}\right), 126.9\left(s, 2 C, C_{6,7}\right), 122.7\left(s, 2 C, C_{1,4}\right)$.

\section{EI-MS}

m/z (\%): $\left.\quad 335.9(100)[\mathrm{M}]^{+}, 257.0(13)[\mathrm{M}-\mathrm{Br}]^{+}, 176.1(66)\left[\mathrm{M}-\mathrm{Br}_{2}\right)\right]^{+}$.

\section{HR-MS (EI+)}

$\mathrm{m} / \mathrm{z}$ (calculated): $\quad 333.8993(333.8993)$.

\subsubsection{Synthesis of [1-(S)PPh2-4-Et- $\left.\left(\mathrm{C}_{14} \mathrm{H}_{8}\right)\right](24)$}

1,4-Dibromoanthracene (1.016 g, $3.0 \mathrm{mmol}, 1.0$ eq.) was dissolved in THF (15 mL) and cooled to $-78^{\circ} \mathrm{C}$. ${ }^{n}$ Butyllithium in hexane $(2.5 \mathrm{M}, 1.3 \mathrm{~mL}, 3.2 \mathrm{mmol}, 1.05$ eq.) was added dropwise over the course of $10 \mathrm{~min}$ while the mixture turned red. After stirring for $20 \mathrm{~min}$ at $-78^{\circ} \mathrm{C} \mathrm{Etl}(0.70 \mathrm{~g}, 0.36 \mathrm{~mL}, 4.5 \mathrm{mmol}, 1.5 \mathrm{eq}$.) was added and the mixture was allowed to reach ambient temperature overnight. Afterwards the mixture was quenched with $\mathrm{H}_{2} \mathrm{O}$ and extracted with DCM $(2 \times 15 \mathrm{~mL})$. The combined organic phases were dried over $\mathrm{MgSO}_{4}$ and filtered. Removal of the solvent under reduced pressure yields 1-Bromo-4-ethylanthracene as a yellow-brown oil $(0.828 \mathrm{~g}, 2.9 \mathrm{mmol}, 97 \%)$, which was used without further purification.

1-Bromo-4-ethylanthracene was dissolved in THF (15 mL) and cooled to $-78{ }^{\circ} \mathrm{C}$. ${ }^{n}$ Butyllithium in hexane (2.5 M, $1.22 \mathrm{~mL}, 3.05 \mathrm{mmol}, 1.05$ eq.) was added and the mixture stirred for $20 \mathrm{~min}$ at $-78{ }^{\circ} \mathrm{C}$. Afterwards Chlorodiphenylphosphine $(0.702 \mathrm{~g}, 0.57 \mathrm{~mL}, 3.2 \mathrm{mmol}, 1.1 \mathrm{eq}$.) was added dropwise and the mixture stirred for $16 \mathrm{~h}$ and warmed slowly to ambient temperature. The solvent was removed under reduced pressure and the residue dissolved in Toluene $(10 \mathrm{~mL})$ and filtered. To the filtrate $\mathrm{S}_{8}(0.103 \mathrm{~g}, 3.2 \mathrm{mmol}$, 1.1 eq.) was added and the mixture was heated to $80^{\circ} \mathrm{C}$ for $6 \mathrm{~h}$. After cooling to ambient temperature, the solution was concentrated, and the target compound was precipitated through addition of Pentane as a yellow solid. Purification was achieved by crystallization from Toluene.

Yield:

Chemical formula: $\quad \mathrm{C}_{28} \mathrm{H}_{23} \mathrm{PS}$

Molecular weight: $\quad 422.53 \mathrm{~g} / \mathrm{mol}$

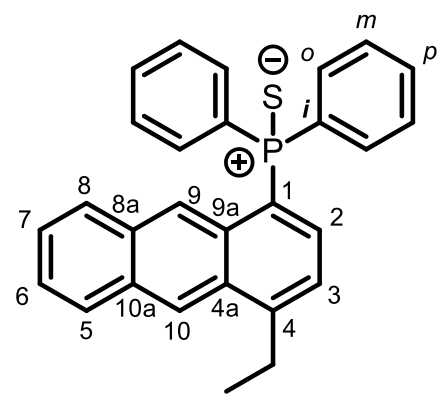

\section{${ }^{1} \mathrm{H}-\mathrm{NMR}$}


$\left(\mathrm{CDCl}_{3}, 300 \mathrm{MHz}\right)$

$\delta[p p m]=9.15\left(\mathrm{~s}, 1 \mathrm{H}, \mathrm{H}_{9}\right), 8.67\left(\mathrm{~s}, 1 \mathrm{H}, \mathrm{H}_{10}\right), 8.01\left(\mathrm{~d},{ }^{3} \mathrm{JHH}_{\mathrm{HH}}=8.4 \mathrm{~Hz}, 1 \mathrm{H}, \mathrm{H}_{5}\right)$, $7.93-7.83(\mathrm{~m}, 4 \mathrm{H}, \mathrm{o}-\mathrm{Ph}), 7.79\left(\mathrm{~d},{ }^{3} \mathrm{~J}_{\mathrm{HH}}=8.4,1 \mathrm{H}, \mathrm{H}_{8}\right), 7.55-7.42(\mathrm{~m}, 8 \mathrm{H}$, $\left.m-\mathrm{Ph}, p-\mathrm{Ph}, \mathrm{H}_{6,7}\right), 7.24-7.11\left(\mathrm{~m}, 2 \mathrm{H}, \mathrm{H}_{2,3}\right), 3.27\left(\mathrm{q},{ }^{3} \mathrm{~J}_{\mathrm{HH}}=7.5 \mathrm{~Hz}, 2 \mathrm{H}, \mathrm{CH}_{2}\right)$, $1.46\left(\mathrm{t}, 3^{3} \mathrm{HH}=7.5 \mathrm{~Hz}, 3 \mathrm{H}, \mathrm{CH}_{3}\right)$.

${ }^{13} \mathrm{C}\left\{{ }^{1} \mathrm{H}\right\}-N M R$

$\left(\mathrm{CDCl}_{3}, 100 \mathrm{MHz}\right)$

$\delta[\mathrm{ppm}]=146.1\left(\mathrm{~d}, J_{\mathrm{CP}}=3.2 \mathrm{~Hz}, 1 \mathrm{C}, \mathrm{C}_{4}\right), 134.1\left(\mathrm{~d},{ }^{2} J_{\mathrm{CP}}=10.3 \mathrm{~Hz}, 1 \mathrm{C}, \mathrm{C}_{2}\right)$, $132.8\left(\mathrm{~d},{ }^{1} J_{\mathrm{CP}}=85.1 \mathrm{~Hz}, 2 \mathrm{C}, i-\mathrm{Ph}\right), 132.7\left(\mathrm{~d},{ }^{2} J_{\mathrm{CP}}=10.7 \mathrm{~Hz}, 4 \mathrm{C}, o-\mathrm{Ph}\right), 132.3$ $\left(\mathrm{s}, 1 \mathrm{C}, \mathrm{C}_{8 \mathrm{~A}}\right), 131.5\left(\mathrm{~d},{ }^{4} \mathrm{~J}_{\mathrm{CP}}=3.0 \mathrm{~Hz}, p-\mathrm{Ph}\right), 131.1\left(\mathrm{~d},{ }^{2} J_{\mathrm{CP}}=20.4 \mathrm{~Hz}, \mathrm{C}_{9 \mathrm{a}}\right), 129.6$ $\left(d,{ }^{3} \mathrm{CP}_{2}=9.3 \mathrm{~Hz}, \mathrm{C}_{4 \mathrm{a}}\right.$ ), 129.0 (s, 1C, C $), 128.8$ (s, 1C, C $\left.10 \mathrm{a}\right), 128.6$ (d, $\left.{ }^{3} J_{\mathrm{CP}}=12.4 \mathrm{~Hz}, \mathrm{~m}-\mathrm{Ph}\right), 128.4\left(\mathrm{~d},{ }^{3} \mathrm{~J}_{\mathrm{CP}}=7.2 \mathrm{~Hz}, 1 \mathrm{C}, \mathrm{C}_{9}\right), 127.9\left(\mathrm{~s}, 1 \mathrm{C}, \mathrm{C}_{5}\right), 127.0$ $\left(d,{ }^{1} J_{C P}=86.6 \mathrm{~Hz}, 1 \mathrm{C}, \mathrm{C}_{1}\right), 126.1\left(\mathrm{~s}, \mathrm{C}_{6}\right), 125.9\left(\mathrm{~s}, \mathrm{C}_{7}\right), 123.5\left(\mathrm{~s}, 1 \mathrm{C}, \mathrm{C}_{10}\right), 122.3$ $\left(\mathrm{d},{ }^{3} \mathrm{~J}_{\mathrm{CP}}=14.7 \mathrm{~Hz}, \mathrm{C}_{3}\right), 26.3\left(\mathrm{~s}, 1 \mathrm{C}, \mathrm{CH}_{2}\right), 14.4\left(\mathrm{~s}, 1 \mathrm{C}, \mathrm{CH}_{3}\right)$.

\section{${ }^{31} \mathrm{P}\left\{{ }^{1} \mathrm{H}\right\}-\mathrm{NMR}$}

$\left(\mathrm{CDCl}_{3}, 121 \mathrm{MHz}\right) \quad \delta[\mathrm{ppm}]=42.3$.

\section{EI-MS}

$m / z(\%):$

$422.1250(63)[\mathrm{M}]^{+} ; 390.1525(100)[\mathrm{M}-\mathrm{S}]^{+}$.

\section{HR-MS (EI+)}

$\mathrm{m} / \mathrm{z}$ (calculated): $\quad 422.1250(422.1253)$.

\section{Elemental analysis}

in \% (calculated): $\quad$ C: 79.52 (79.59), H: 5.61 (5.49), S: 7.47 (7.59).

\subsubsection{Synthesis of $\left[1,4-\left(\mathrm{PPh}_{2}\right)_{2}-\left(\mathrm{C}_{14} \mathrm{H}_{8}\right)\right](25)$}

1,4-Dibromoanhtracene $(0.34 \mathrm{~g}, 1.0 \mathrm{mmol}, 1.0 \mathrm{eq})$ was dissolved in THF $(25 \mathrm{~mL})$ and cooled to $-78^{\circ} \mathrm{C}$. $n$ Butyllithium in hexane $(2.2 \mathrm{M}, 0.15 \mathrm{~g}, 2.2 \mathrm{mmol}, 2.2 \mathrm{eq})$ was added dropwise over $10 \mathrm{~min}$. The mixture was stirred for further $10 \mathrm{~min}$ until the solid was dissolved completely. Chlorodiphenylphosphine $(0.48 \mathrm{~g}$, $2.2 \mathrm{mmol}, 2.2$ eq.) was added slowly and the solution was allowed to warm to ambient temperature and stirred for $16 \mathrm{~h}$. Volatiles were removed under reduced pressure and the remaining solid was dissolved in DCM (15 mL) and filtrated. Removing the solvent of the filtrate yielded a brown-red solid, which could be crystallized from DCM.

Yield:

$0.37 \mathrm{~g}(0.67 \mathrm{mmol}, 67 \%)$ 
Chemical formula: $\quad \mathrm{C}_{38} \mathrm{H}_{28} \mathrm{P}_{2}$

Molecular weight: $\quad 546.59 \mathrm{~g} / \mathrm{mol}$

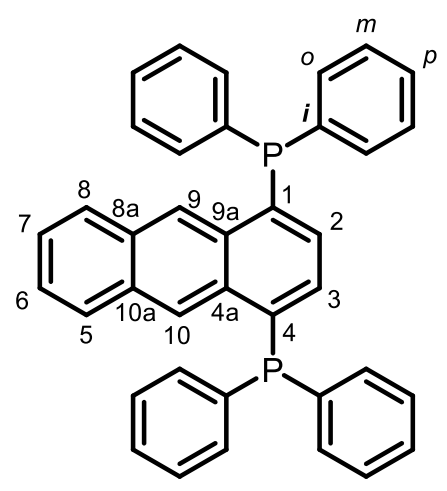

\section{${ }^{1} \mathrm{H}-\mathrm{NMR}$}

$\left(\mathrm{CDCl}_{3}, 300 \mathrm{MHz}\right)$

$\delta[p p m]=9.01\left(\mathrm{~d}, J_{\mathrm{HP}}=4.1 \mathrm{~Hz}, 2 \mathrm{H}, \mathrm{H}_{9,10}\right), 7.89\left(\mathrm{dd}, J_{\mathrm{HH}}=6.5,3.3 \mathrm{~Hz}, 2 \mathrm{H}\right.$, $\left.\mathrm{H}_{5,8}\right), 7.90-7.30\left(\mathrm{~m}, 22 \mathrm{H}, P h-\mathrm{H}, \mathrm{H}_{6,7}\right), 6.83\left(\mathrm{dd}, \mathrm{J}_{\mathrm{HP}}=3.4,2.3 \mathrm{~Hz}, 2 \mathrm{H}, \mathrm{H}_{2,3}\right)$.

${ }^{13} \mathrm{C}\left\{{ }^{1} \mathrm{H}\right\}-\mathrm{NMR}$

$\left(\mathrm{CDCl}_{3}, 75 \mathrm{MHz}\right)$

$\delta[p p m]=136.6\left(\mathrm{~d},{ }^{1} J_{\mathrm{CP}}=14.1 \mathrm{~Hz}, 2 \mathrm{C}, \mathrm{C}_{1,4}\right), 136.1\left(\mathrm{~d},{ }^{1} J_{\mathrm{CP}}=8.9 \mathrm{~Hz}, 4 \mathrm{C}, i-\mathrm{Ph}\right)$, $134.5\left(d,{ }^{2} J_{C P}=20.0 \mathrm{~Hz}, 8 \mathrm{C}, o-P h\right), 133.1\left(d, J_{C P}=3.6 \mathrm{~Hz}, 2 \mathrm{C}, \mathrm{C}_{8 \mathrm{a}}, \mathrm{C}_{10 \mathrm{a}}\right), 132.9$ $\left(d, J_{C P}=3.8 \mathrm{~Hz}, 2 \mathrm{C}, \mathrm{C}_{4 a}, \mathrm{C}_{9 \mathrm{a}}\right), 131.5\left(\mathrm{~d}, \mathrm{~J}_{\mathrm{CP}}=8.1 \mathrm{~Hz}, 2 \mathrm{C}, \mathrm{C}_{2,3}\right), 129.1$ (s, 4C, $p$ $\mathrm{Ph}), 128.8\left(\mathrm{~d},{ }^{3} \mathrm{~J}_{\mathrm{CP}}=7.3 \mathrm{~Hz}, 8 \mathrm{C}, m-\mathrm{Ph}\right), 128.5\left(\mathrm{~s}, 2 \mathrm{C}, \mathrm{C}_{5,8}\right) 125.9\left(\mathrm{~s}, 2 \mathrm{C}, \mathrm{C}_{6,7}\right)$, $125.8\left(\mathrm{~d}, \mathrm{~J}_{\mathrm{CP}}=25.6 \mathrm{~Hz}, 2 \mathrm{C}, \mathrm{C}_{9,10}\right)$.

${ }^{31} \mathrm{P}\left\{{ }^{1} \mathrm{H}\right\}-\mathrm{NMR}$

$\left(\mathrm{CDCl}_{3}, 121 \mathrm{MHz}\right) \quad \delta[\mathrm{ppm}]=-13.3$.

ESI-MS

$\mathrm{m} / \mathrm{z}(\%): \quad 547.2(100)[\mathrm{M}+\mathrm{H}]^{+}$.

HR-MS (ESI $\left.{ }^{+}\right)$

$\mathrm{m} / \mathrm{z}$ (calculated): $\quad 547.1737(547.1739)$.

Elemental analysis

in \% (calculated): $\quad$ C: $82.67(83.50), \mathrm{H}: 5.18(5.16)$.

\subsubsection{Synthesis of 1,5-Dichloroanthracene}

1,5-Dichloroanthraquinone $(5.0 \mathrm{~g}, 18.0 \mathrm{mmol}, 1.0 \mathrm{eq})$ was suspended in aq. $\mathrm{NH}_{3}$-solution $(25 \%, 60 \mathrm{~mL})$ and $\mathrm{H}_{2} \mathrm{O}(45 \mathrm{~mL})$ and cooled to $0{ }^{\circ} \mathrm{C}$. Zinc-dust $(25 \mathrm{~g}, 380 \mathrm{mmol}, 21 \mathrm{eq}$.) was added carefully in portions. The yellow mixture turned red and was allowed to reach ambient temperature and afterwards heated to $75{ }^{\circ} \mathrm{C}$ 
for $2 \mathrm{~h}$. At ambient temperature the mixture was filtered, and the grey solid washed with DCM. The filtrate was extracted with DCM $(2 \times 100 \mathrm{~mL})$ and the solvent of the combined organic phases were evaporated. Conc. $\mathrm{HCl}(30 \mathrm{~mL})$ was added to the remaining yellow solid and heated to reflux. $\mathrm{PrOH}$ was added in portions until the solid was dissolved completely. A yellow powder formed while cooling to ambient temperature, which was collected by filtration and washed with $\mathrm{H}_{2} \mathrm{O}$.

Yield:

Chemical formula:

Molecular weight:

${ }^{1} \mathrm{H}-\mathrm{NMR}$

$\left(\mathrm{CDCl}_{3}, 300 \mathrm{MHz}\right)$

${ }^{13} \mathrm{C}\left\{{ }^{1} \mathrm{H}\right\}-\mathrm{NMR}$

$\left(\mathrm{CDCl}_{3}, 75 \mathrm{MHz}\right)$
$2.30 \mathrm{~g}(9.5 \mathrm{mmol}, 50 \%)$.

$\mathrm{C}_{14} \mathrm{H}_{8} \mathrm{Cl}_{2}$

$247.12 \mathrm{~g} / \mathrm{mol}$

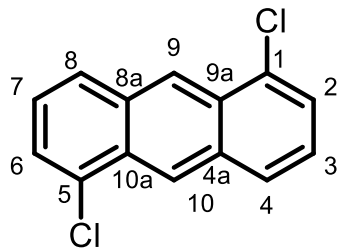

$\left.\delta[p p m]=8.84\left(\mathrm{~s}, 2 \mathrm{H}, \mathrm{H}_{9,10}\right), 8.00\left(\mathrm{~d},{ }^{3}\right)_{\mathrm{HH}}=8.5 \mathrm{~Hz}, 2 \mathrm{H}, \mathrm{H}_{4,8}\right), 7.61(\mathrm{~d}$, $\left.{ }^{3} J_{\mathrm{HH}}=7.2 \mathrm{~Hz}, 2 \mathrm{H}, \mathrm{H}_{2,6}\right), 7.41\left(\mathrm{dd},{ }^{3} J_{\mathrm{HH}}=8.5 \mathrm{~Hz}, 7.2 \mathrm{~Hz}, 2 \mathrm{H}, \mathrm{H}_{3,7}\right)$.

$\delta[p p m]=132.8\left(\mathrm{~s}, 2 \mathrm{C}, \mathrm{C}_{9 \mathrm{a}, 10 \mathrm{a}}\right), 131.8\left(\mathrm{~s}, \mathrm{C}_{4 \mathrm{a}, 8 \mathrm{a}}\right), 129.4\left(\mathrm{~s}, 2 \mathrm{C}, \mathrm{C}_{1,5}\right), 128.0$ (s, 2C, $\left.C_{4,8}\right), 126.1\left(\mathrm{~s}, 2 \mathrm{C}, \mathrm{C}_{2,6}\right), 125.5\left(\mathrm{~s}, 2 \mathrm{C}, \mathrm{C}_{3,7}\right), 124.3\left(\mathrm{~s}, 2 \mathrm{C}, \mathrm{C}_{9,10}\right)$.

\section{EI-MS}

$\mathrm{m} / \mathrm{z}(\%)$ :

$246.0(100)[\mathrm{M}]^{+}, 176.1(45)\left[\mathrm{M}-\mathrm{Cl}_{2}\right]^{+}$.

HR-MS (EI+)

$\mathrm{m} / \mathrm{z}$ (calculated): $\quad 246.0003(246.0003)$.

\subsubsection{Synthesis of $\left[1,5-\left(\mathrm{PPh}_{2}\right)_{2}-\left(\mathrm{C}_{14} \mathrm{H}_{8}\right)\right](26)$}

1,5-Dichloroanthracene $(0.25 \mathrm{~g}, 1.0 \mathrm{mmol}, 1.0 \mathrm{eq})$ was dissolved in THF (15 mL) and cooled to $0{ }^{\circ} \mathrm{C}$. $\mathrm{LiPPh}_{2}$ in THF (0.5 M, $4.2 \mathrm{~mL}, 2.1 \mathrm{mmol}, 2.1$ eq.) was added slowly. The mixture was allowed to warm to ambient temperature overnight, while a pale-yellow precipitate formed. To the mixture an EtOH/ $\mathrm{H}_{2} \mathrm{O}$-solution (5:1, $30 \mathrm{~mL}$ ) was added and stirred for further $15 \mathrm{~min}$ to complete precipitation. The solid was filtered off, washed with water and dried under reduced pressure. Crystallization from DCM yielded crystals suitable for structure determination.

Yield:

$0.33 \mathrm{~g}(0.6 \mathrm{mmol}, 60 \%)$.

Chemical formula: $\quad \mathrm{C}_{38} \mathrm{H}_{28} \mathrm{P}_{2}$ 
Molecular weight: $\quad 546.59 \mathrm{~g} / \mathrm{mol}$

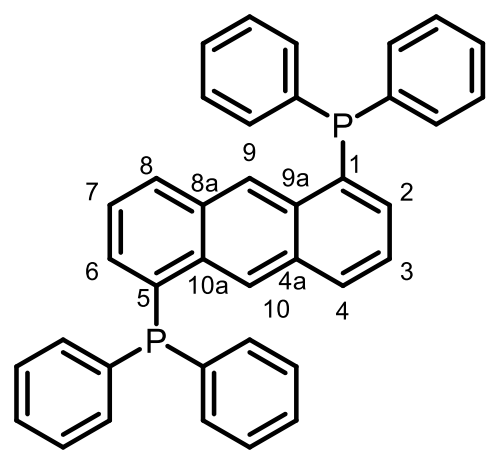

${ }^{1} \mathrm{H}-\mathrm{NMR}$

$\left(\mathrm{CDCl}_{3}, 400 \mathrm{MHz}\right) \quad \delta[\mathrm{ppm}]=9.0\left(\mathrm{~d},{ }^{4} \mathrm{~J}_{\mathrm{PH}}=4.0 \mathrm{~Hz}, 2 \mathrm{H}, \mathrm{H}_{9,10}\right), 7.92\left(\mathrm{~d},{ }^{3} \mathrm{~J}_{\mathrm{HH}}=8.5 \mathrm{~Hz}, 2 \mathrm{H}, \mathrm{H}_{4,8}\right), 7.37$

$-7.33(\mathrm{~m}, 20 \mathrm{H}, \mathrm{Ph}), 7.30-7.28\left(\mathrm{~m}, 2 \mathrm{H}, \mathrm{H}_{3,7}\right), 6.99\left(\mathrm{t}, \mathrm{J}_{\mathrm{HH}}=6.0 \mathrm{~Hz}, 2 \mathrm{H}, \mathrm{H}_{2,6}\right)$.

${ }^{13} \mathrm{C}\left\{{ }^{1} \mathrm{H}\right\}-\mathrm{NMR}$

$\left(\mathrm{CDCl}_{3}, 75 \mathrm{MHz}\right)$

$\delta[\mathrm{ppm}]=134.2\left(\mathrm{~d},{ }^{2} \mathrm{~J}_{\mathrm{CP}}=20.0 \mathrm{~Hz}, 8 \mathrm{C}, \mathrm{o}-\mathrm{Ph}\right), 132.4\left(\mathrm{~s}, 2 \mathrm{C}, \mathrm{C}_{2,6}\right), 130.3(\mathrm{~s}, 2 \mathrm{C}$, $\left.\mathrm{C}_{4,8}\right), 128.8\left(\mathrm{~d},{ }^{3} \mathrm{~J}_{\mathrm{CP}}=24.4 \mathrm{~Hz}, 8 \mathrm{C}, \mathrm{m}-\mathrm{Ph}\right), 128.6$ (s, 4C, $\left.p-\mathrm{Ph}\right), 125.8$ (d, $\left.{ }^{3} J_{C P}=26.1 \mathrm{~Hz}, 2 \mathrm{C}, \mathrm{C}_{9,10}\right), 125.1\left(\mathrm{~s}, 2 \mathrm{C}, \mathrm{C}_{3,7}\right)$.

${ }^{31} \mathrm{P}\left\{{ }^{1} \mathrm{H}\right\}-\mathrm{NMR}$

$\left(\mathrm{CDCl}_{3}, 121 \mathrm{MHz}\right) \quad \delta[\mathrm{ppm}]=-14.58$.

ESI-MS

$\mathrm{m} / \mathrm{z}(\%)$ :

$547.2(100)[\mathrm{M}+\mathrm{H}]^{+}$.

HR-MS (ESI $\left.{ }^{+}\right)$

$\mathrm{m} / \mathrm{z}$ (calculated): $\quad 547.1737$ (547.1739).

Elemental analysis

in \% (calculated): $\quad$ C: 81.52 (83.50), H: $5.01(5.16)$.

\subsubsection{Synthesis of 1,8-Dichloroanthracene}

1,8-Dichloroanthraquinone $\left(5.0 \mathrm{~g}, 18.0 \mathrm{mmol}, 1.0\right.$ eq.) was suspended in aqueous $\mathrm{NH}_{3}(25 \%, 60 \mathrm{~mL})$ and $\mathrm{H}_{2} \mathrm{O}(45 \mathrm{~mL})$ and cooled to $0{ }^{\circ} \mathrm{C}$. Zinc-dust ( $25 \mathrm{~g}, 380 \mathrm{mmol}, 21 \mathrm{eq}$.) was added in portions under stirring. The yellowish suspension turned red and was allowed to warm to ambient temperature and afterwards heated to $75^{\circ} \mathrm{C}$ for $3 \mathrm{~h}$. After cooling to ambient temperature, the mixture was filtrated and the solid was washed with DCM $(5 \times 20 \mathrm{~mL})$ and the filtrate extracted with DCM $(3 \times 30 \mathrm{~mL})$. Solvent of the combined organic phases were evaporated and conc. $\mathrm{HCl}(35 \%, 30 \mathrm{~mL})$ was added to the yellow powder. The mixture was heated to reflux and $i \mathrm{PrOH}$ was added until the solid was dissolved completely. While cooling to 
ambient temperature the product was obtained as yellow needles which were filtered off and dried under reduced pressure.

Yield:

Chemical formula:

Molecular weight:

${ }^{1} \mathrm{H}-\mathrm{NMR}$

$\left(\mathrm{CDCl}_{3}, 400 \mathrm{MHz}\right)$

${ }^{13} \mathrm{C}\left\{{ }^{1} \mathrm{H}\right\}-N M R$

$\left(\mathrm{CDCl}_{3}, 100 \mathrm{MHz}\right)$
$2.69 \mathrm{~g}$ (10.9 mmol, 61 \%).

$\mathrm{C}_{14} \mathrm{H}_{8} \mathrm{Cl}_{2}$

$247.12 \mathrm{~g} / \mathrm{mol}$

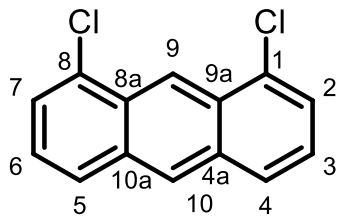

$\delta[p p m]=9.23\left(\mathrm{~s}, 1 \mathrm{H}, \mathrm{C}_{9}\right), 8.43\left(\mathrm{~s}, 1 \mathrm{H}, \mathrm{C}_{10}\right), 7.92\left(\mathrm{~d},{ }^{3} \mathrm{~J}_{\mathrm{HH}}=8.5 \mathrm{~Hz}, 2 \mathrm{H}, \mathrm{H}_{4,5}\right)$

$7.62\left(d,{ }^{3} \mathrm{~J}_{\mathrm{HH}}=7.1 \mathrm{~Hz}, 2 \mathrm{H}, \mathrm{H}_{2,7}\right), 7.40\left(\mathrm{~m}, 2 \mathrm{H}, \mathrm{H}_{3,6}\right)$.

$\delta[p p m]=132.7\left(\mathrm{~s}, 2 \mathrm{C}, \mathrm{C}_{4 \mathrm{a}, 10 \mathrm{a}}\right), 132.6\left(\mathrm{~s}, 2 \mathrm{C}, \mathrm{C}_{8 \mathrm{a}, 9 \mathrm{a}}\right), 129.6\left(\mathrm{~s}, 2 \mathrm{C}, \mathrm{C}_{1,8}\right), 127.7$

$\left(\mathrm{s}, 1 \mathrm{C}, \mathrm{C}_{10}\right), 127.4\left(\mathrm{~s}, 2 \mathrm{C}, \mathrm{C}_{4,5}\right), 126.1\left(\mathrm{~s}, 2 \mathrm{C}, \mathrm{C}_{2,7}\right), 125.8\left(\mathrm{~s}, 2 \mathrm{C}, \mathrm{C}_{3,6}\right), 121.1$ (s, 1C, $\left.C_{9}\right)$.

\section{EI-MS}

$\mathrm{m} / \mathrm{z}(\%):$

$246.0(100)[\mathrm{M}]^{+}, 176.1(40)\left[\mathrm{M}-\mathrm{Cl}_{2}\right]^{+}$.

\section{HR-MS (EI+)}

$\mathrm{m} / \mathrm{z}$ (calculated): $\quad 246.0011(246.0003)$.

\subsubsection{Synthesis of $\left[1,8-\left(\mathrm{PPh}_{2}\right)_{2}-\left(\mathrm{C}_{14} \mathrm{H}_{8}\right)\right](27)$}

1,8 Dichloroanthracene $\left(0.49 \mathrm{~g}, 2.0 \mathrm{mmol}, 1.0\right.$ eq.) was dissolved in THF (10 mL) and cooled to $0{ }^{\circ} \mathrm{C}$. A solution of $\mathrm{LiPPh}_{2}$ in THF $(0.5 \mathrm{M}, 8.4 \mathrm{~mL}, 4.2 \mathrm{mmol})$ was slowly added and the mixture was stirred at $0{ }^{\circ} \mathrm{C}$ for $30 \mathrm{~min}$. Afterwards the solution was allowed to reach ambient temperature and stirred for further $16 \mathrm{~h}$. The reaction was quenched with $\mathrm{H}_{2} \mathrm{O}(1 \mathrm{~mL})$ and $\mathrm{EtOH} / \mathrm{H}_{2} \mathrm{O}$-solution (5:1) was added until a yellow precipitate formed. The suspension was stirred for $10 \mathrm{~min}$, filtrated and the solid was washed with $\mathrm{EtOH} / \mathrm{H}_{2} \mathrm{O}$. Recrystallized from DCM afforded the target compound as a yellow crystalline solid.

Yield: $\quad 0.99 \mathrm{~g}(1.8 \mathrm{mmol}, 90 \%)$

Chemical formula: $\quad \mathrm{C}_{38} \mathrm{H}_{28} \mathrm{P}_{2}$ 
Molecular weight: $\quad 610.71 \mathrm{~g} / \mathrm{mol}$

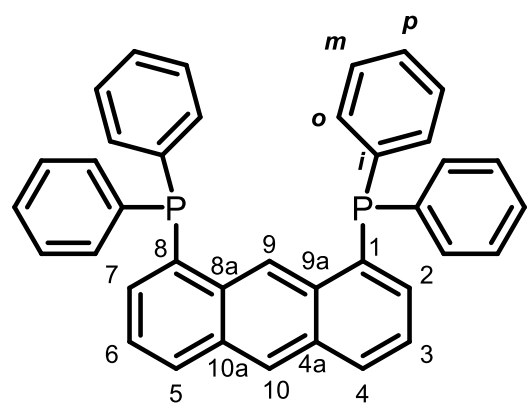

\section{${ }^{1} \mathrm{H}-\mathrm{NMR}$}

$\left(\mathrm{CDCl}_{3}, 300 \mathrm{MHz}\right)$

$\delta[\mathrm{ppm}]=9.82\left(\mathrm{t},{ }^{4} \mathrm{~J}_{\mathrm{HP}}=5.3 \mathrm{~Hz}, 1 \mathrm{H}, \mathrm{H}_{9}\right), 8.47\left(\mathrm{~s}, 1 \mathrm{H}, \mathrm{H}_{10}\right), 7.99\left(\mathrm{~d},{ }^{3} \int_{\mathrm{HH}}=8.5 \mathrm{~Hz}\right.$, $\left.2 \mathrm{H}, \mathrm{H}_{4,5}\right), 7.37-7.32\left(\mathrm{~m}, 2 \mathrm{H}, \mathrm{H}_{2,7}\right), 7.32-7.22(\mathrm{~m}, 20 \mathrm{H}, \mathrm{Ph}), 7.04\left(\mathrm{~m}, 2 \mathrm{H}, \mathrm{H}_{3,6}\right)$.

\section{${ }^{13} \mathrm{C}\left\{{ }^{1} \mathrm{H}\right\}-N M R$}

$\left(\mathrm{CDCl}_{3}, 75 \mathrm{MHz}\right)$

$\delta[\mathrm{ppm}]=136.5\left(\mathrm{~d},{ }^{1} \mathrm{~J}_{\mathrm{CP}}=10.3 \mathrm{~Hz}, 4 \mathrm{C}, i-\mathrm{Ph}\right), 136.3\left(\mathrm{~d},{ }^{1} J_{\mathrm{CP}}=15.2 \mathrm{~Hz}, 2 \mathrm{C}, \mathrm{C}_{1,8}\right)$ $134.3\left(\mathrm{~d}, \mathrm{~J}_{\mathrm{CP}}=20.0 \mathrm{~Hz}, o-\mathrm{Ph}\right), 133.3\left(\mathrm{~d},{ }^{2} \mathrm{~J}_{\mathrm{CP}}=22.6 \mathrm{~Hz}, 2 \mathrm{C}, \mathrm{C}_{8 \mathrm{a}, 9 \mathrm{a}}\right.$ ), $132.2(\mathrm{~s}, 2 \mathrm{C}$, $\left.\mathrm{C}_{3,6}\right), 131.7\left(\mathrm{~d},{ }^{3} \mathrm{JP}^{\prime}=-3.9 \mathrm{~Hz}, 2 \mathrm{C}, \mathrm{C}_{41,10 \mathrm{a}}\right), 129.4\left(\mathrm{~s}, 2 \mathrm{C}, \mathrm{C}_{4,5}\right), 128.8(\mathrm{~s}, 4 \mathrm{C}, \mathrm{p}-\mathrm{Ph})$, $128.5\left(\mathrm{~d},{ }^{3} \mathrm{~J}_{\mathrm{CP}}=6.9 \mathrm{~Hz}, 8 \mathrm{C}, \mathrm{m}-\mathrm{Ph}\right), 127.8\left(\mathrm{~s}, 1 \mathrm{C}, \mathrm{C}_{10}\right) .125 .5\left(\mathrm{~s}, 2 \mathrm{C}, \mathrm{C}_{2,7}\right) 124.2(\mathrm{t}$, $\left.{ }^{3} J_{C P}=27.8 \mathrm{~Hz}, 1 \mathrm{C}, \mathrm{C}_{9}\right)$.

\section{${ }^{31} \mathrm{P}\left\{{ }^{1} \mathrm{H}\right\}-\mathrm{NMR}$}

$\left(\mathrm{CDCl}_{3}, 121 \mathrm{MHz}\right) \quad \delta[\mathrm{ppm}]=-14.76$

\section{EI-MS}

$\mathrm{m} / \mathrm{z}(\%):$

$546.2(100)[\mathrm{M}]^{+}$.

\section{HR-MS (ESI $\left.{ }^{+}\right)$}

$\mathrm{m} / \mathrm{z}$ (calculated): $\quad 546.1667(547.1666)$.

\section{Elemental analysis}

in \% (calculated): $\quad$ C: 83.29 (83.50), H: 5.23 (5.15).

\subsubsection{Synthesis of 2,6-Dibromoanthraquinone}

2,6-Diaminoanthraquinone (5.0 g $20.7 \mathrm{mmol}, 1.0$ eq.) $\mathrm{CuBr}_{2}(12.4 \mathrm{~g}, 55.6 \mathrm{mmol}, 2.7$ eq.) and $t \mathrm{BuNO}_{2}(5.7 \mathrm{~g}, 6.5 \mathrm{~mL}, 55.6 \mathrm{mmol}, 2.7$ eq.) were dissolved in MeCN $(150 \mathrm{~mL})$. the mixture was heated to $65^{\circ} \mathrm{C}$ for $16 \mathrm{~h}$. After cooling to ambient temperature, the mixture was poured into $\mathrm{HCl}(6 \mathrm{M}, 400 \mathrm{~mL})$ and the precipitate was filtered off, washed with $\mathrm{H}_{2} \mathrm{O}$ and dried. The product was obtained as a greyyellow solid, which could be purified by crystallization from 1,4-Dioxane. 
Yield:

$6.3 \mathrm{~g}(17.2 \mathrm{mmol}, 83 \%)$.

Chemical formula: $\quad \mathrm{C}_{14} \mathrm{H}_{6} \mathrm{Br}_{2} \mathrm{O}_{2}$

Molecular weight: $\quad 366.0 \mathrm{~g} / \mathrm{mol}$

${ }^{1} \mathrm{H}-\mathrm{NMR}$

$\left(\mathrm{CDCl}_{3}, 400 \mathrm{MHz}\right)$

$\delta[p p m]=8.46\left(\mathrm{~d}, \mathrm{~J}_{\mathrm{HH}}=2.0 \mathrm{~Hz}, 2 \mathrm{H}, \mathrm{H}_{1,5}\right), 8.20\left(\mathrm{~d}, \mathrm{~J}_{\mathrm{HH}}=8.3 \mathrm{~Hz}, 2 \mathrm{H}, \mathrm{H}_{4,8}\right), 7.97$

$\left(\mathrm{dd}, \mathrm{J}_{\mathrm{HH}}=8.3,2.0 \mathrm{~Hz}, 2 \mathrm{H}, \mathrm{H}_{3,7}\right)$.

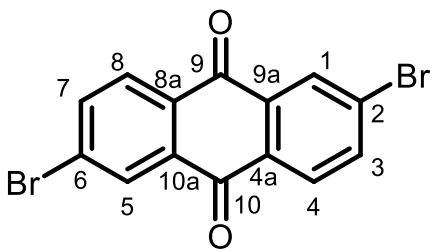

${ }^{13} \mathrm{C}\left\{{ }^{1} \mathrm{H}\right\}-\mathrm{NMR}$

$\left(\mathrm{CDCl}_{3}, 100 \mathrm{MHz}\right)$

$\delta[p p m]=181.5\left(\mathrm{~s}, 2 \mathrm{C}, \mathrm{C}_{9,10}\right), 137.6\left(\mathrm{~s}, 2 \mathrm{C}, \mathrm{C}_{3,7}\right), 134.6\left(\mathrm{~s}, 2 \mathrm{C}, \mathrm{C}_{9 \mathrm{a}, 10 \mathrm{a}}\right), 131.9$

$\left(\mathrm{s}, 2 \mathrm{C}, \mathrm{C}_{4 a, 8 \mathrm{a}}\right), 130.5\left(\mathrm{~s}, 2 \mathrm{C}, \mathrm{C}_{1,5}\right), 130.3\left(\mathrm{~s}, 2 \mathrm{C}, \mathrm{C}_{2,6}\right), 129.3\left(\mathrm{C}_{4,8}\right)$.

\section{EI-MS}

$\mathrm{m} / \mathrm{z}(\%):$

$365.9(100)[\mathrm{M}]^{+}, 150.0(70)$.

\section{HR-MS (ESI $\left.{ }^{+}\right)$}

m/z (calculated): $\quad 363.8730(363.8735)$.

\subsubsection{Synthesis of 2,6-Dibromoanthracene}

2,6-Dibromoanthraquinone ( $3.5 \mathrm{~g}, 9.6 \mathrm{mmol}, 1.0$ eq.) was suspended in $\mathrm{AcOH}(100 \mathrm{~mL})$. $\mathrm{HI}(57 \%, 15.0 \mathrm{~mL})$ and $\mathrm{H}_{3} \mathrm{PO}_{2}(50 \%, 8 \mathrm{~mL})$ were added under stirring. The mixture was heated to reflux for $16 \mathrm{~h}$ and a fluffy precipitate formed. The mixture was cooled to ambient temperature and ice-water was added ( $150 \mathrm{~mL}$ ). The yellow solid was filtered off and washed with $\mathrm{H}_{2} \mathrm{O}$ and dired under reduced pressure to give 2,6dibromo-9,10-dihydroanthracene. The solid (0.82 g, $2.4 \mathrm{mmol}$ ) was dissolved in 1,4-dioxane (50 mL). oChloranile $(2.4 \mathrm{~g}, 9.6 \mathrm{mmol})$ was added and the mixture heated to $80^{\circ} \mathrm{C}$ for $16 \mathrm{~h}$. After cooling to ambient temperature, the formed solid was filtered off and washed with $\mathrm{H}_{2} \mathrm{O}$. Recrystallization from toluene afforded 2,6-dibromoanthracene.

Yield:

$1.1 \mathrm{~g},(3.3 \mathrm{mmol}, 34 \%)$

Chemical formula: $\quad \mathrm{C}_{14} \mathrm{H}_{8} \mathrm{Br}_{2}$

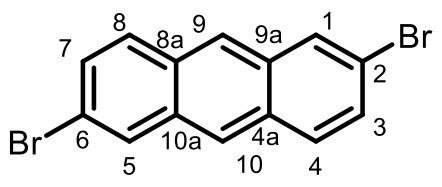

Molecular weight: $\quad 336.03$

${ }^{1} \mathrm{H}-\mathrm{NMR}$ 
$\left(\mathrm{CDCl}_{3}, 400 \mathrm{MHz}\right)$

$\delta[\mathrm{ppm}]=8.30\left(\mathrm{~s}, 2 \mathrm{H}, \mathrm{H}_{9.10}\right), 8.16\left(\mathrm{~d}, J_{\mathrm{HH}}=2.0 \mathrm{~Hz}, 2 \mathrm{H}, \mathrm{H}_{1,5}\right), 7.87(\mathrm{~d}$, $\left.J_{H H}=9.0 \mathrm{~Hz}, 2 \mathrm{H}, \mathrm{H}_{4,8}\right), 7.53\left(\mathrm{dd}, J_{\mathrm{HH}}=9.0,2.0 \mathrm{~Hz}, 2 \mathrm{H}, \mathrm{H}_{3,7}\right)$.

${ }^{13} \mathrm{C}\left\{{ }^{1} \mathrm{H}\right\}-\mathrm{NMR}$

due to poor solubility of the compound, no reliable ${ }^{13} \mathrm{C}$-data could be obtained.

\section{EI-MS}

$\mathrm{m} / \mathrm{z}(\%): \quad 335.8(100)[\mathrm{M}]^{+}, 176.0(80)\left[\mathrm{M}-\mathrm{Br}_{2}\right]^{+}$.

\section{HR-MS (EI+)}

$\mathrm{m} / \mathrm{z}$ (calculated): $\quad 333.8992(333.8993)$.

\subsubsection{Synthesis of $\left[2,6-\left(\mathrm{PPh}_{2}\right)_{2}-\left(\mathrm{C}_{14} \mathrm{H}_{8}\right)\right](28)$}

2,6-Dibromoanthracene $(0.34 \mathrm{~g}, 1.0 \mathrm{mmol}, 1.0 \mathrm{eq})$ was dissolved in $\mathrm{Et}_{2} \mathrm{O}(30 \mathrm{~mL})$ and cooled to $-20^{\circ} \mathrm{C}$. nBuLi in hexane $(2.2 \mathrm{M}, 0.15 \mathrm{~g}, 2.2 \mathrm{mmol}, 2.2 \mathrm{eq})$ was added over the course of $10 \mathrm{~min}$, while the color of the suspension changed from orange over green to light yellow. The mixture was stirred for $10 \mathrm{~min}$ at $20^{\circ} \mathrm{C}$ and for another $10 \mathrm{~min}$ at ambient temperature. After cooling again to $-20^{\circ} \mathrm{C}$ Chlorodiphenylphosphine $(0.48 \mathrm{~g}, 0.39 \mathrm{~mL}, 2.2 \mathrm{mmol}, 2.2$ eq.) was added and the mixture allowed to warm to ambient temperature over night. The yellow precipitate was filtered off and washed with $\mathrm{Et}_{2} \mathrm{O}$. Crystallization through diffusion of $n$-hexane into a nenzene solution afforded yellow crystals.

Yield:

$0.12 \mathrm{~g}(0.22 \mathrm{mmol}, 22 \%)$.

Chemical formula: $\quad \mathrm{C}_{38} \mathrm{H}_{28} \mathrm{P}_{2}$

Molecular weight: $546.59 \mathrm{~g} / \mathrm{mol}$

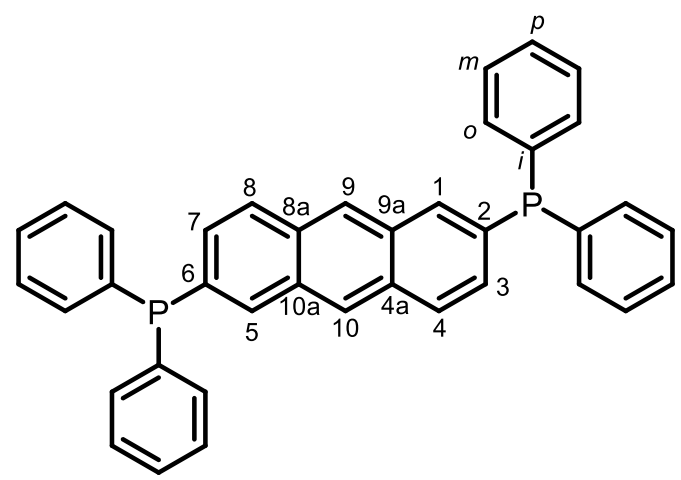

\section{${ }^{1} \mathrm{H}-\mathrm{NMR}$}

$\left(\mathrm{CDCl}_{3}, 300 \mathrm{MHz}\right) \quad \delta[\mathrm{ppm}]=8.27\left(\mathrm{~s}, 2 \mathrm{H}, \mathrm{H}_{9,10}\right), 7.92-7.88\left(\mathrm{~m}, 4 \mathrm{H}, \mathrm{H}_{1,4,5,8}\right), 7.43-7.34(\mathrm{~m}, 22 \mathrm{H}$, $\left.\mathrm{H}_{3,7}, \mathrm{H}_{\mathrm{Ph}}\right)$.

\section{${ }^{13} \mathrm{C}\left\{{ }^{1} \mathrm{H}\right\}-\mathrm{NMR}$}

$\left(\mathrm{CDCl}_{3}, 100 \mathrm{MHz}\right) \quad \delta[\mathrm{ppm}]=136.9\left(\mathrm{~d},{ }^{1} J_{\mathrm{CP}}=10.8 \mathrm{~Hz}, 2 \mathrm{C}, i-\mathrm{Ph}\right), 135.0\left(\mathrm{~d},{ }^{1} J_{\mathrm{CP}}=11.6 \mathrm{~Hz}, 2 \mathrm{C}, \mathrm{C}_{2,6}\right)$, $134.5\left(\mathrm{~d},{ }^{2} J_{\mathrm{CP}}=21.7 \mathrm{~Hz}, 2 \mathrm{C}, \mathrm{C}_{1,8}\right), 134.1\left(\mathrm{~d},{ }^{2} \mathrm{~J}_{\mathrm{CP}}=19.5 \mathrm{~Hz}, 8 \mathrm{C}, 0-\mathrm{Ph}\right), 132.0$ (d, $\left.{ }^{3} J_{C P}=7.9 \mathrm{~Hz}, 2 \mathrm{C}, \mathrm{C}_{9 a}, 10 \mathrm{a}\right), 131.7\left(\mathrm{~s}, 2 \mathrm{C}, \mathrm{C}_{8 \mathrm{a}, 9 \mathrm{a}}\right), 129.3\left(\mathrm{~d},{ }^{2} \mathrm{~J}_{\mathrm{CP}}=18.1 \mathrm{~Hz}, 2 \mathrm{C}, \mathrm{C}_{3,7}\right)$, 
129.1 (s, 4C, $p-\mathrm{Ph}$ ), 128.8 (d, $\left.{ }^{3} \mathrm{CP}_{\mathrm{CP}}=7.0 \mathrm{~Hz}, 8 \mathrm{C}, m-\mathrm{Ph}\right), 128.3$ (d, ${ }^{3} \mathrm{~J}_{\mathrm{CP}}=7.0 \mathrm{~Hz}$, $\left.2 \mathrm{C}, \mathrm{C}_{4,8}\right), 126.6\left(\mathrm{~s}, 2 \mathrm{C}, \mathrm{C}_{9,10}\right)$.

\section{${ }^{31} \mathrm{P}\left\{{ }^{1} \mathrm{H}\right\}-\mathrm{NMR}$}

$\left(\mathrm{CDCl}_{3}, 121 \mathrm{MHz}\right) \quad \delta[\mathrm{ppm}]=-4.37$.

\section{EI-MS}

$\mathrm{m} / \mathrm{z}(\%): \quad 546.2(100)[\mathrm{M}]^{+}, 176.1(45)\left[\mathrm{M}-\left(\mathrm{PPh}_{2}\right)_{2}\right]^{+}$.

\section{HR-MS (ESI $\left.{ }^{+}\right)$}

$\mathrm{m} / \mathrm{z}$ (calculated): $\quad 547.1747$ (547.1739).

\section{Elemental analysis}

in \% (calculated): $\quad$ C: 80.05 (83.50), H: 5.05 (5.15).

\subsubsection{Synthesis of $\left[1,4-\left((\mathrm{S}) \mathrm{PPh}_{2}\right)_{2}-\left(\mathrm{C}_{14} \mathrm{H}_{8}\right)\right](30)$}

[1,4- $\left.\left(\mathrm{PPh}_{2}\right)_{2}-\left(\mathrm{C}_{14} \mathrm{H}_{8}\right)\right](0.37 \mathrm{~g}, 0.70 \mathrm{mmol}, 1.0 \mathrm{eq})$ and $\mathrm{S}_{8}(0.09 \mathrm{~g}, 2.7 \mathrm{mmol}, 4.0$ eq. $)$ were dissolved in toluene $(10 \mathrm{~mL})$ and heated to $80^{\circ} \mathrm{C}$ for $4 \mathrm{~h}$. After cooling to ambient temperature, the mixture was filtrated. Removing the solvent of the filtrate afforded the target compound. It could be recrystallized from from EtOAc or Toluene to give yellow crystals.

Yield:

Chemical formula: $\quad \mathrm{C}_{38} \mathrm{H}_{28} \mathrm{P}_{2} \mathrm{~S}_{2}$

Molecular weight: $\quad 610.71 \mathrm{~g} / \mathrm{mol}$

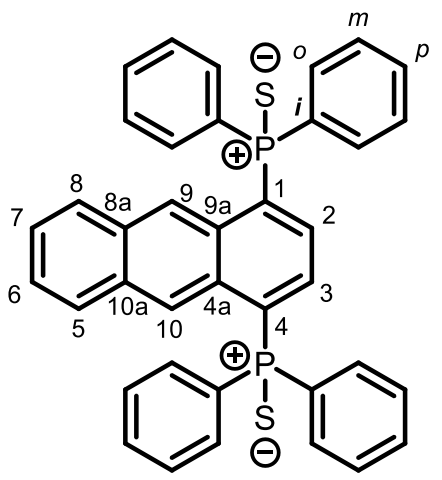

\section{${ }^{1} \mathrm{H}-\mathrm{NMR}$}

$\left(\mathrm{CDCl}_{3}, 300 \mathrm{MHz}\right)$

$\delta[\mathrm{ppm}]=9.22\left(\mathrm{~s}, 2 \mathrm{H}, \mathrm{H}_{9,10}\right), 7.89-7.82(\mathrm{~m}, 8 \mathrm{H}, o-\mathrm{Ph}), 7.70\left(\mathrm{dd}, J_{\mathrm{HH}}=\right.$ $\left.14.5 \mathrm{~Hz}, 4.5 \mathrm{~Hz}, 2 \mathrm{H}, \mathrm{H}_{5,8}\right), 7.55-7.43(\mathrm{~m}, 12 \mathrm{H}, m-\mathrm{Ph}, p-\mathrm{Ph}), 7.37$ (dd, $J_{\mathrm{HH}}=$ $\left.14.5 \mathrm{~Hz}, 4.5 \mathrm{~Hz}, 2 \mathrm{H}, \mathrm{H}_{6,7}\right), 7.00$ (dd, $\mathrm{J}_{\mathrm{PH}}=14.5 \mathrm{~Hz}, 5.4 \mathrm{~Hz}, 2 \mathrm{H}, \mathrm{H}_{2,3}$ ).

${ }^{13} \mathrm{C}\left\{{ }^{1} \mathrm{H}\right\}-\mathrm{NMR}$

$\left(\mathrm{CDCl}_{3}, 75 \mathrm{MHz}\right) \quad \delta[\mathrm{ppm}]=134.8\left(\mathrm{~d},{ }^{1} \mathrm{~J}_{\mathrm{CP}}=77.9 \mathrm{~Hz}, 132.5\left(\mathrm{~d},{ }^{2} \mathrm{~J}_{\mathrm{CP}}=11.3 \mathrm{~Hz}, 8 \mathrm{C}, \mathrm{o}-\mathrm{Ph}\right), 132.4\right.$ $\left(d,{ }^{2} J_{C P}=11.3 \mathrm{~Hz}, C_{4 a}, 9 a\right), 131.9(s, 4 C, p-P h), 131.2\left(d,{ }^{4} J_{C P}=4.2 \mathrm{~Hz}, 2 C\right.$, 
$\left.\mathrm{C}_{8 \mathrm{a}, 10 \mathrm{a}}\right), 130.9\left(\mathrm{~d},{ }^{2} \mathrm{~J}_{\mathrm{CP}}=12.5 \mathrm{~Hz}, 2 \mathrm{C}, \mathrm{C}_{2,3}\right), 128.9\left(\mathrm{~d},{ }^{3} \mathrm{JPP}_{\mathrm{CP}}=15.4 \mathrm{~Hz}, 2 \mathrm{C}, \mathrm{C}_{9,10}\right)$ $128.8\left(\mathrm{~d},{ }^{3} \mathrm{JPP}_{\mathrm{CP}}=13.4 \mathrm{~Hz}, 8 \mathrm{C}, m-\mathrm{Ph}\right), 128.4\left(\mathrm{~s}, 2 \mathrm{C}, \mathrm{C}_{8,4}\right), 126.7\left(\mathrm{~s}, 2 \mathrm{C}, \mathrm{C}_{6,7}\right)$.

${ }^{31} \mathrm{P}\left\{{ }^{1} \mathrm{H}\right\}-N M R$

$\left(\mathrm{CDCl}_{3}, 121 \mathrm{MHz}\right) \quad \delta[\mathrm{ppm}]=42.47$.

EI-MS

$\mathrm{m} / \mathrm{z}(\%):$

$611.1(100)[\mathrm{M}+\mathrm{H}]^{+}, 633.1(60)[\mathrm{M}+\mathrm{Na}]^{+}$.

HR-MS (EI+)

$\mathrm{m} / \mathrm{z}$ (calculated): $\quad 611.1167(611.1180)$.

Elemental analysis

in \% (calculated): $\quad$ C: 77.05 (76.90), $\mathrm{H}: 5.41$ (5.16), S: 8.38 (9.12); calcd. for $\mathrm{C}_{38} \mathrm{H}_{28} \mathrm{P}_{2} \mathrm{~S}_{2} \times \mathrm{C}_{7} \mathrm{H}_{8}$. 


\section{Crystallographic Section}

\subsection{Crystal Selection}

Crystals of the presented compounds were selected from the mother liquor and transferred into perfluorated oil on a microscope slide. With the polarizer microscope suitable crystals for X-ray diffraction analysis were evaluated and separated. The chosen crystals were mounted to the tip of a MiTeGen ${ }^{\odot}$ MicroMount, applied to the goniometer head of the diffractometer and shock cooled to $100 \mathrm{~K}$ in a flow of nitrogen in the crystal cooling device. ${ }^{[231]}$

\subsection{Data collection and processing}

Data collection was performed on BRUKER Smart APEX diffractometers equipped with either an Incoatec Mo-I $\mu$ S Microsource $(\lambda=0.71073 \AA)$, a BRUKER Mo-TXS rotating anode $(\lambda=0.71073 \AA)$ or an Incoatec AgI $\mu$ S Microsource $(\lambda=0.56086 \AA) .{ }^{[232]}$ Cooling of the crystals during the measurement was done with Oxford Crysosystems crystal cooling device. Apex II CCD detectors and either Incoatec Quazar or Helios mirror optics mounted on a D8-goniometer, were used for data collection.

Diffractometers were controlled by the APEX2 program package. ${ }^{[233]}$ Crystal quality and cell parameters were determined by a matrix-scan before the measurement. Verification of known polymorphs and cocrystals were also performed via matrix-scans of several crystals from the sample. Data collection was performed in the $\omega$-scan mode with $0.5^{\circ}$ steps.

The collected data were integrated with $S A I N T^{[234]}$ and as semi-empirical absorption correction was applied with SADABS. ${ }^{[235]}$ A $3 \lambda$ correction was applied if necessary. ${ }^{[236]}$ Data statistics and preliminary space group determination and file setup for structure-solution was done with XPREP. ${ }^{[237]}$

\subsection{Structure solution and refinement}

The obtained structure was solved by direct methods using SHELXT within the SHELX software package. ${ }^{[238]}$ The C-bonded hydrogen atoms were refined isotropically on calculated positions. Refinement results were evaluated by comparison of calculated and observed structure factors, with commonly used criteria $R 1$ and $w R 2$. The goodness of fit (GooF) shows the relation between deviation of calculated and observed structure factors. Structures with disordered moieties or solvent molecules were refined with distance restraints and restraints for the anisotropic displacement parameters. Disordered groups were modelled with DSR. ${ }^{[239]}$ Graphics were generated with the XP or Mercury ${ }^{[240]}$ program. The anisotropic displacement parameters were depicted at the $50 \%$ probability level if not otherwise noted. 


\subsection{Structures determined during this work}

\subsubsection{Crystal structure of $\left[9-\mathrm{PPh}_{2}-\left(\mathrm{C}_{14} \mathrm{H}_{9}\right)\right]$ (1)}
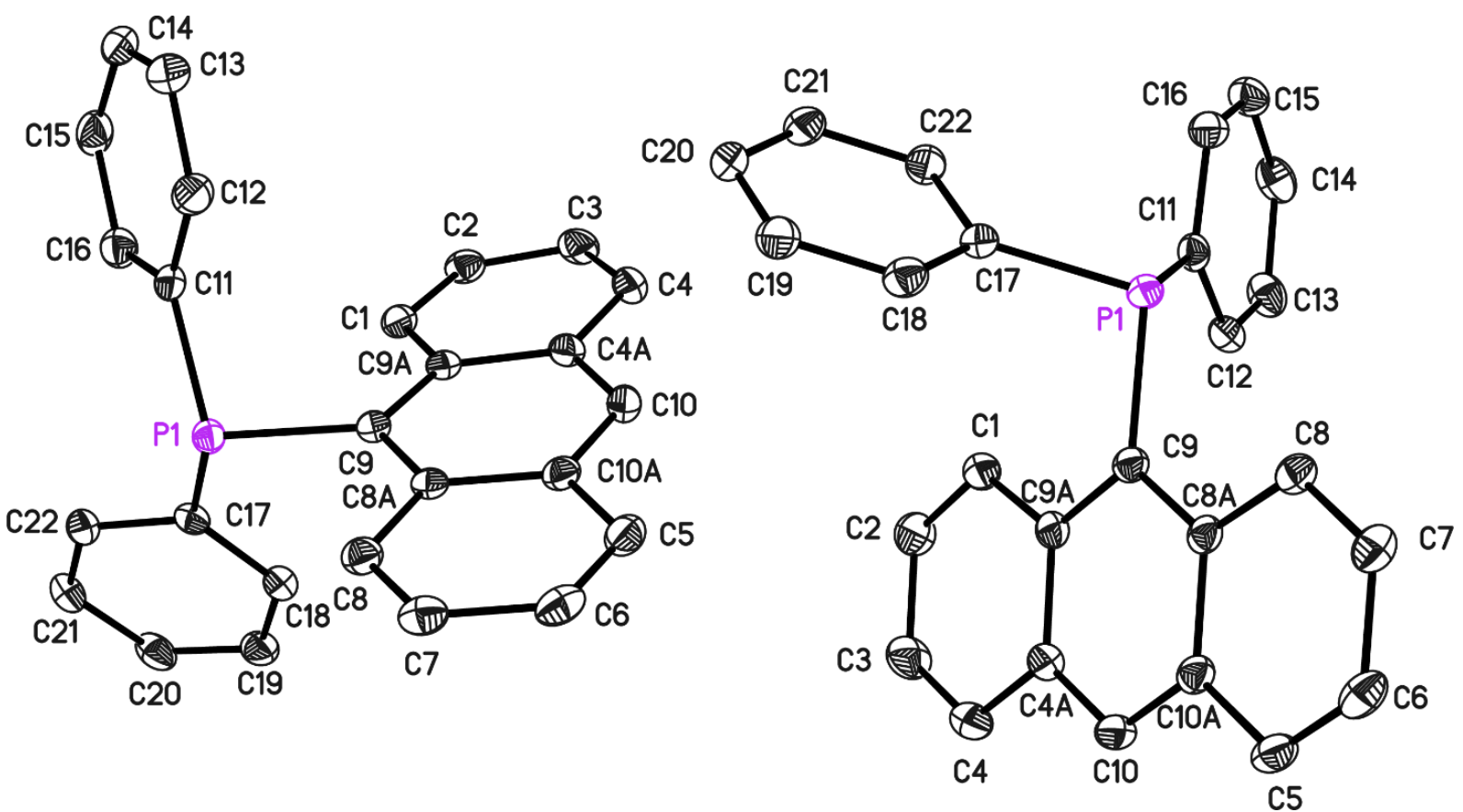

Figure 80. Asymmetric unit of 1 . The anisotropic displacement parameters are depicted at the $50 \%$ probability level. Hydrogen atoms are omitted for clarity.

Table 20. Crystallographic data of 1.

Structure Code
Empirical formula
Formula weight $[\mathrm{g} / \mathrm{mol}]$
Temperature $[\mathrm{K}]$
Wavelength $[\AA]$
Crystal system
space group
a $[\AA \AA$
$\mathrm{b}[\AA]$
$\mathrm{c}[\AA]$
$\alpha\left[^{\circ}\right]$
$\beta\left[^{\circ}\right]$
$\gamma\left[^{\circ}\right]$
Volume $\left[\AA^{3}\right]$

\begin{tabular}{|l} 
ts 135 \\
$\mathrm{C}_{26} \mathrm{H}_{19} \mathrm{P}$ \\
362.38 \\
$100(2)$ \\
0.71073 \\
triclinic \\
$P \overline{\mathbf{1}}$ \\
$10.826(2)$ \\
$12.274(2)$ \\
$14.531(3)$ \\
$74.58(4)$ \\
$88.28(4)$ \\
$89.36(5)$ \\
$1860.5(7)$
\end{tabular}

$Z$
$\rho_{\text {calc }}$
$\mu\left[\mathrm{mm}^{-1}\right]$
$\mathrm{F}(000)$
$\theta$ range $\left[^{\circ}\right]$
Reflections collected
Independent reflections
$\mathrm{R}($ int)
Max. / min. transmission
Restraints / parameter
GooF
R1 / wR2 (I > 2 $\sigma(I))$
R1 / wR2 (all data)
max. diff peak / hole [e $\left.\AA^{-3}\right]$

\begin{tabular}{|l}
4 \\
1.294 \\
0.155 \\
760 \\
1.454 to 26.440 \\
83493 \\
7675 \\
0.0464 \\
$0.7454 / 0.6943$ \\
$0 / 487$ \\
1.032 \\
$0.0357 / 0.0839$ \\
$0.0422 / 0.0877$ \\
$0.370 /-0.327$
\end{tabular}




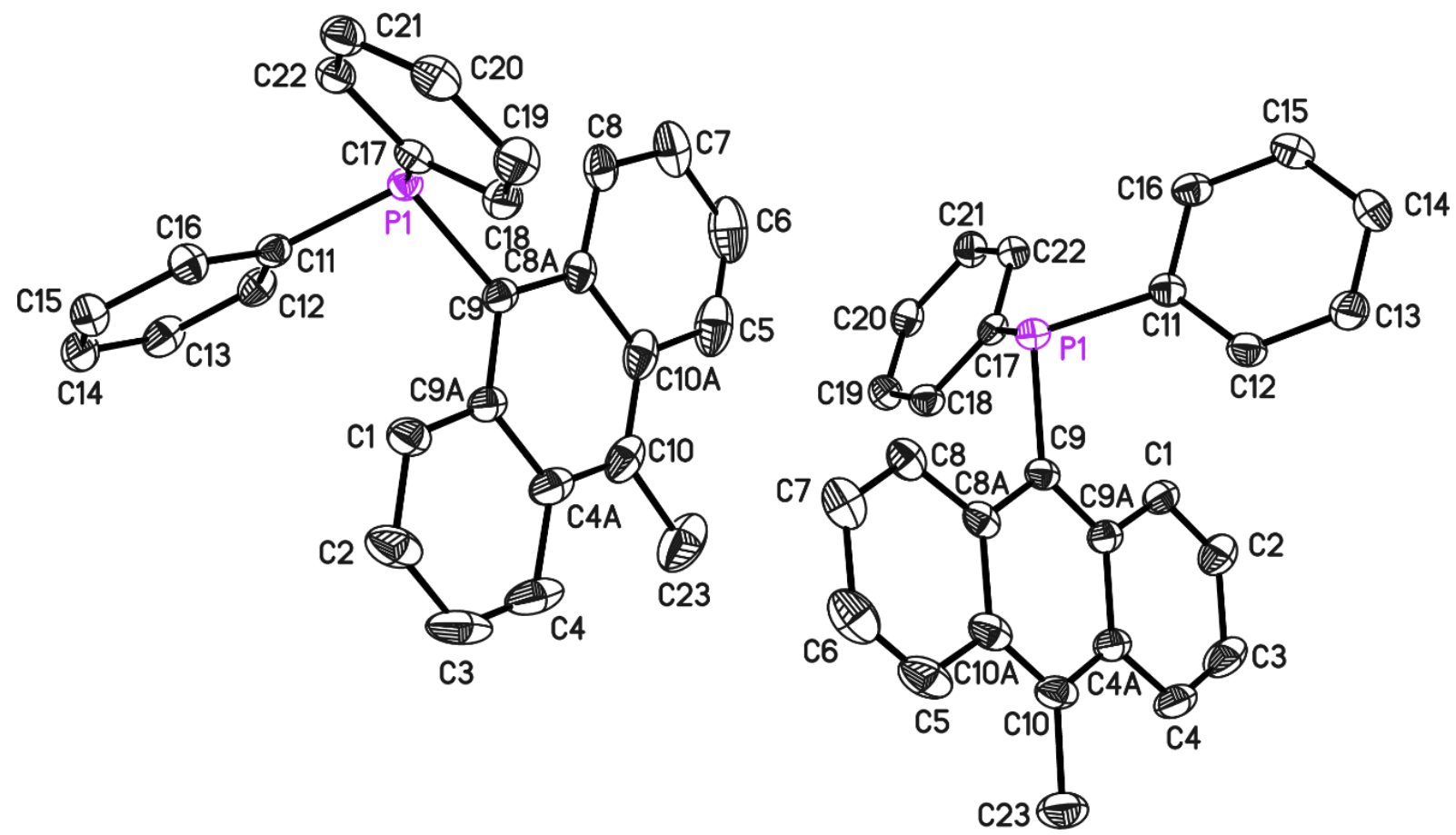

Figure 81. Asymmetric unit of 3. The anisotropic displacement parameters are depicted at the $50 \%$ probability level. Hydrogen atoms are omitted for clarity. Hydrogen atoms attached to each C23 are disordered over two positions. The occupancies of the main positions refined to $0.70(3)$ and $0.75(3)$ respectively.

Table 21. Crystallographic data of 3.

Structure Code
Empirical formula
Formula weight $[\mathrm{g} / \mathrm{mol}]$
Temperature $[\mathrm{K}]$
Wavelength $[\AA]$
Crystal system
space group
a $[\AA \AA]$
$\mathrm{b}[\AA]$
$\mathrm{c}[\AA]$
$\alpha\left[\left[^{\circ}\right]\right.$
$\beta\left[\left[^{\circ}\right]\right.$
$\gamma\left[{ }^{\circ}\right]$
Volume $\left[\AA^{3}\right]$

\begin{tabular}{|l} 
ts 158 \\
$\mathrm{C}_{27} \mathrm{H}_{21} \mathrm{P}$ \\
376.41 \\
$100(2)$ \\
0.71073 \\
triclinic \\
$P \overline{\mathbf{1}}$ \\
$10.899(2)$ \\
$12.476(2)$ \\
$14.596(3)$ \\
$93.83(2)$ \\
$97.89(3)$ \\
$90.27(2)$ \\
$1961.3(4)$
\end{tabular}

$Z$
$\rho_{\text {calc }}$
$\mu\left[\mathrm{mm}^{-1}\right]$
$\mathrm{F}(000)$
$\theta$ range $\left[^{\circ}\right]$
Reflections collected
Independent reflections
$\mathrm{R}($ int)
Max. / min. transmission
Restraints / parameter
GooF
R1 / wR2 (I > 2 $\sigma(I))$
R1 / wR2 (all data)
max. diff peak / hole [e $\left.\AA^{-3}\right]$

4
1.275
0.150
792
1.412 to 26.381
47776
8046
0.0347
0.7454 / 0.6942
$0 / 509$
1.008
$0.0353 / 0.0876$
$0.0431 / 0.0930$
$0.380 /-0.314$




\subsubsection{Crystal structure of [9-PPh $\left.-10-\mathrm{Et}-\left(\mathrm{C}_{14} \mathrm{H}_{8}\right)\right](4)$}

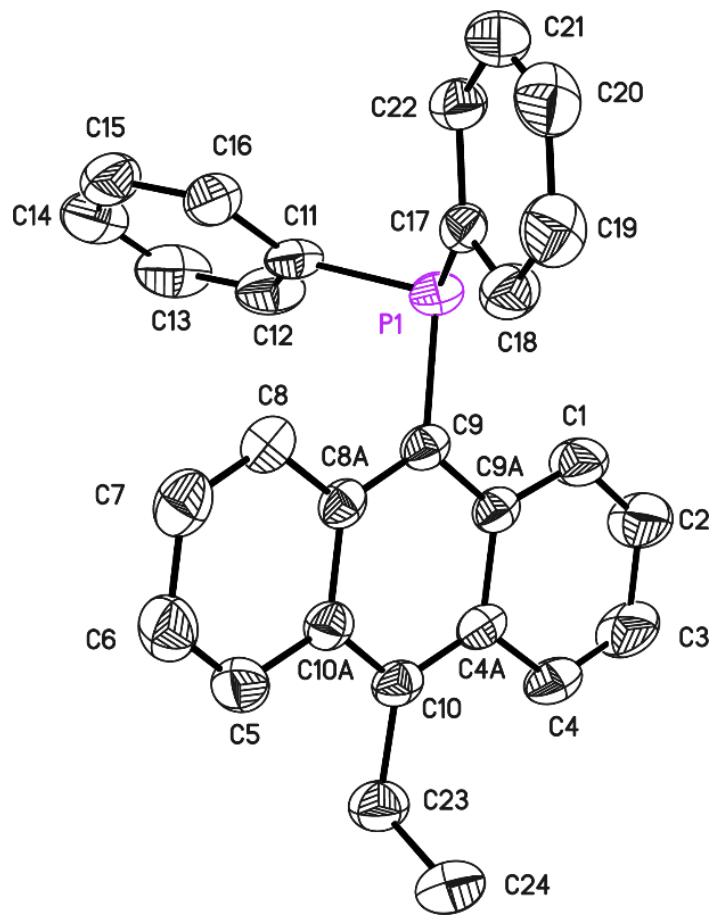

Figure 82. Asymmetric unit of 4. The anisotropic displacement parameters are depicted at the $50 \%$ probability level. Hydrogen atoms are omitted for clarity.

Table 22. Crystallographic data of 4.

\begin{tabular}{|c|c|c|c|}
\hline Structure Code & ts129_dcm & Z & 2 \\
\hline Empirical formula & $\mathrm{C}_{28} \mathrm{H}_{23} \mathrm{P}$ & $\rho_{\text {calc }}$ & 1.234 \\
\hline Formula weight $[\mathrm{g} / \mathrm{mol}]$ & 390.43 & $\mu\left[\mathrm{mm}^{-1}\right]$ & 0.082 \\
\hline Temperature $[\mathrm{K}]$ & $100(2)$ & $F(000)$ & 412 \\
\hline Wavelength $[\AA]$ & 0.56086 & $\theta$ range $\left[{ }^{\circ}\right]$ & 1.263 to 20.596 \\
\hline Crystal system & Triclinic & Reflections collected & 79436 \\
\hline space group & & Independent reflections & 4339 \\
\hline a $[\AA ̊]$ & $9.009(2)$ & $\mathrm{R}$ (int) & 0.1773 \\
\hline $\mathrm{b}[\AA ̊]$ & $9.649(2)$ & Max. / min. transmission & $0.7445 / 0.7252$ \\
\hline$c[\AA]$ & $13.132(3)$ & Restraints / parameter & $0 / 263$ \\
\hline$\alpha\left[^{\circ}\right]$ & $94.89(2)$ & GooF & 1.078 \\
\hline$\beta\left[^{\circ}\right]$ & $101.22(2)$ & $\mathrm{R} 1 / w \mathrm{R} 2(\mathrm{I}>2 \sigma(\mathrm{I}))$ & $0.0474 / 0.1381$ \\
\hline$\gamma\left[^{\circ}\right]$ & $108.23(3)$ & $\mathrm{R} 1 /$ wR2 (all data) & $0.0577 / 0.1455$ \\
\hline Volume $\left[\AA^{3}\right]$ & $1050.4(3)$ & max. diff peak / hole $\left[e \AA^{-3}\right]$ & $0.284 /-0.355$ \\
\hline
\end{tabular}




\subsubsection{Crystal structure of [9-PPh2-10-Ph- $\left.\left(\mathrm{C}_{14} \mathrm{H}_{8}\right)\right](6)$}

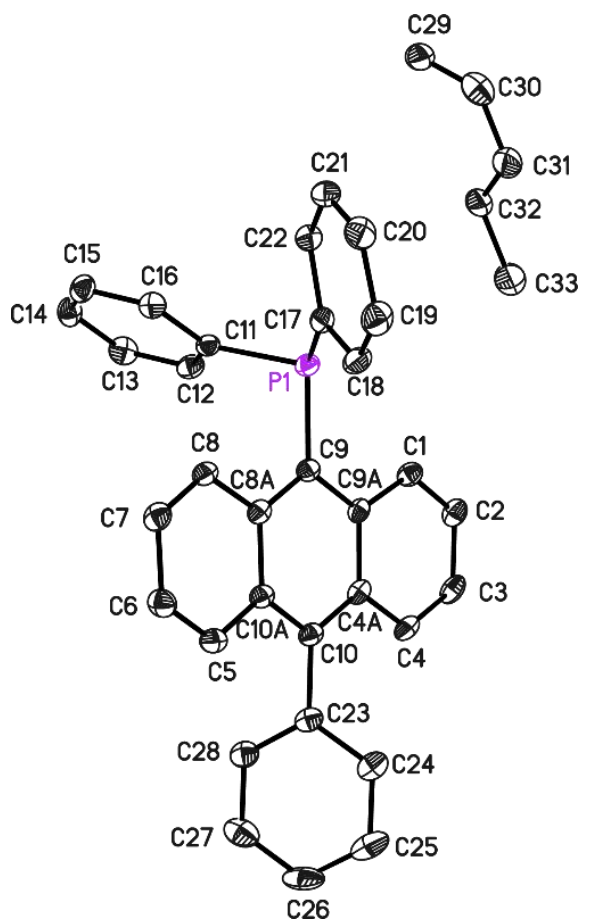

Figure 83. Asymmetric unit of 6 . The anisotropic displacement parameters are depicted at the $50 \%$ probability level. Hydrogen atoms are omitted for clarity. The toluene molecule is situated on a two-fold axis leading to disorder of the methyl hydrogen atoms.

Table 23. Crystallographic data of 6 .

Structure Code
Empirical formula
Formula weight $[\mathrm{g} / \mathrm{mol}]$
Temperature $[\mathrm{K}]$
Wavelength $[\AA \AA$
Crystal system
space group
a $[\AA \AA]$
$\mathrm{b}[\AA]$
$\mathrm{c}[\AA]$
$\alpha\left[\left[^{\circ}\right]\right.$
$\beta\left[^{\circ}\right]$
$\gamma\left[{ }^{\circ}\right]$
Volume $\left[\AA^{3}\right]$

\begin{tabular}{|l} 
ts 199 \\
$\mathrm{C}_{71} \mathrm{H}_{54} \mathrm{P}_{2}$ \\
969.08 \\
$100(2)$ \\
0.71073 \\
monoclinic \\
$C 2 / \mathrm{c}$ \\
$15.942(2)$ \\
$11.129(2)$ \\
$29.140(4)$ \\
90 \\
$102.79(3)$ \\
90 \\
$5041.7(14)$
\end{tabular}

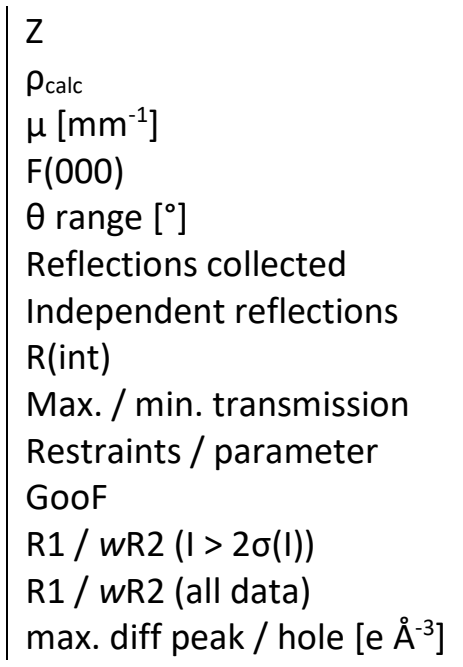

4

1.277

0.133

2040

1.433 to 26.438

25605

5177

0.0653

0.7454 / 0.6671

$0 / 332$

1.059

0.0467 / 0.1133

$0.0660 / 0.1239$

0.513 / -0.383 


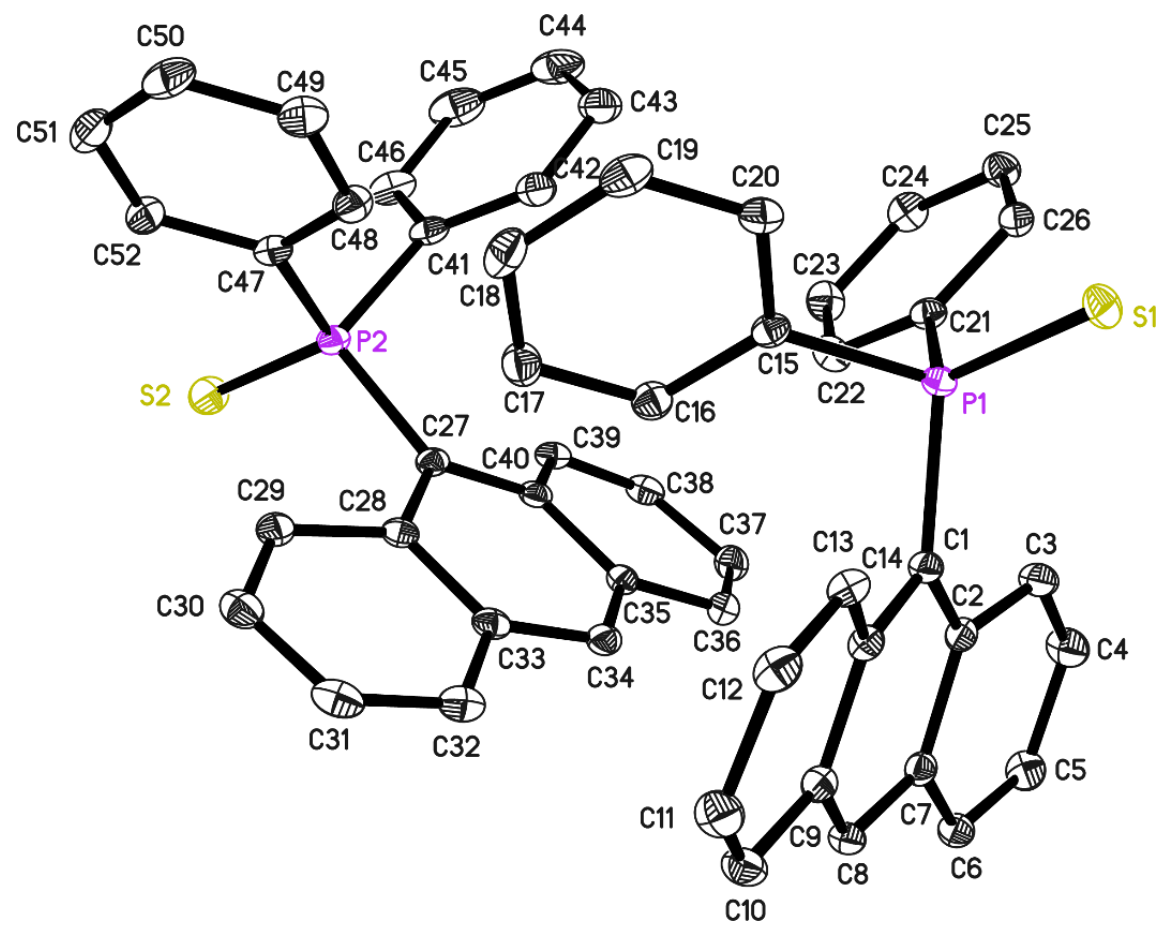

Figure 84. Asymmetric unit of 8. The anisotropic displacement parameters are depicted at the $50 \%$ probability level. Hydrogen atoms are omitted for clarity.

Table 24. Crystallographic data of 8.

\begin{tabular}{l|l|l|l} 
Structure Code & ts 106 & $\mathrm{Z}$ & 4 \\
Empirical formula & $\mathrm{C}_{26} \mathrm{H}_{19} \mathrm{PS}$ & $\rho_{\text {calc }}$ & 1.323 \\
Formula weight $[\mathrm{g} / \mathrm{mol}]$ & 394.44 & $\mu\left[\mathrm{mm}^{-1}\right]$ & 0.253 \\
Temperature $[\mathrm{K}]$ & $100(2) \mathrm{K}$ & $\mathrm{F}(000)$ & 824 \\
Wavelength $[\AA]$ & $0.71073 \AA$ & $\theta$ range $\left[^{\circ}\right]$ & 1.203 to 27.502 \\
Crystal system & Triclinic & Reflections collected & 52179 \\
space group & $P \overline{\mathbf{1}}$ & Independent reflections & 9063 \\
$\mathrm{a}[\AA \AA$ & $10.2318(7)$ & $\mathrm{R}($ int $)$ & 0.0277 \\
$\mathrm{~b}[\AA]$ & $12.3354(8)$ & Max. / min. transmission & $0.7456 / 0.7041$ \\
$\mathrm{c}[\AA]$ & $17.3784(11)$ & Restraints / parameter & $0 / 505$ \\
$\alpha\left[^{\circ}\right]$ & $101.6060(10)$ & GooF & 1.045 \\
$\beta\left[^{\circ}\right]$ & $91.2080(10)$ & R1 / wR2 $(\mathrm{I}>2 \sigma(\mathrm{I}))$ & $0.0306 / 0.0788$ \\
$\gamma\left[^{\circ}\right]$ & $112.0000(10)$ & R1 $/ w R 2($ all data) & $0.0353 / 0.0823$ \\
Volume $\left[\AA^{3}\right]$ & $1980.8(2)$ & max. diff peak / hole $\left[\mathrm{e} \AA^{-3}\right]$ & $0.410 /-0.353$
\end{tabular}




\subsubsection{Crystal structure of [9-BMes2- $\left.\left(\mathrm{C}_{14} \mathrm{H}_{9}\right)\right]$ (9)}

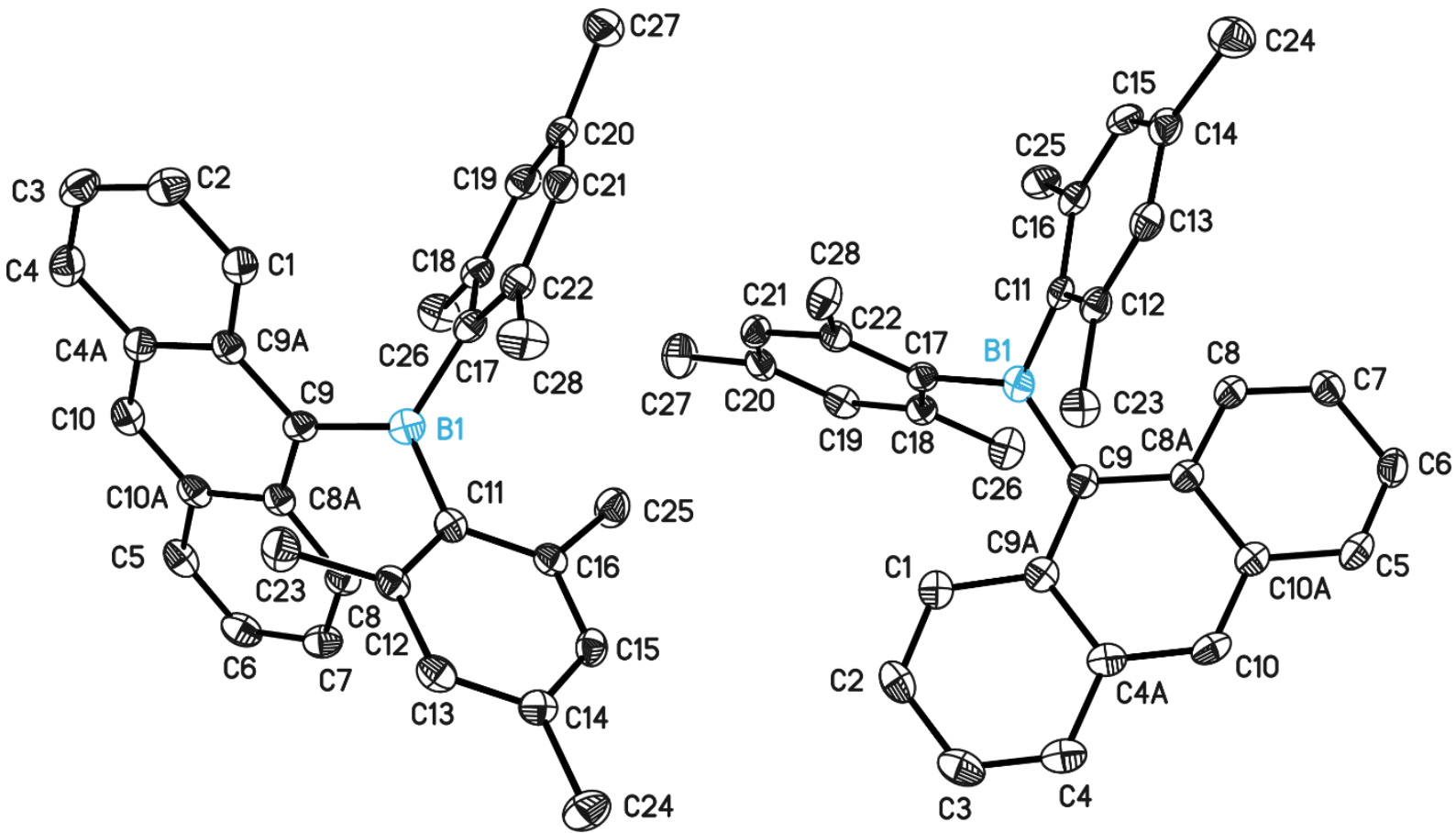

Figure 85. Asymmetric unit of 9. The anisotropic displacement parameters are depicted at the $50 \%$ probability level. Hydrogen atoms are omitted for clarity.

Table 25. Crystallographic data of $\mathbf{9 .}$

\begin{tabular}{|c|c|c|c|}
\hline Structure Code & ts345 & Z & 8 \\
\hline Empirical formula & $\mathrm{C}_{23} \mathrm{H}_{31} \mathrm{~B}$ & $\rho_{\text {calc }}$ & 1.185 \\
\hline Formula weight $[\mathrm{g} / \mathrm{mol}]$ & 426.38 & $\mu\left[\mathrm{mm}^{-1}\right]$ & 0.066 \\
\hline Temperature $[\mathrm{K}]$ & $100(2)$ & $F(000)$ & 1824 \\
\hline Wavelength $[\AA ̊]$ & 0.71073 & $\theta$ range $\left[{ }^{\circ}\right]$ & 1.874 to 25.404 \\
\hline Crystal system & monoclinic & Reflections collected & 89018 \\
\hline space group & $P 2_{1} / \mathrm{n}$ & Independent reflections & 8779 \\
\hline $\mathrm{a}[\AA]$ & $8.391(2)$ & $\mathrm{R}$ (int) & 0.1035 \\
\hline $\mathrm{b}[\AA]$ & $46.316(3)$ & Max. / min. transmission & 0.7452 / 0.6822 \\
\hline$c[\AA]$ & $12.317(2)$ & Restraints / parameter & 0 / 607 \\
\hline$\alpha\left[^{\circ}\right]$ & 90 & GooF & 1.027 \\
\hline$\beta\left[^{\circ}\right]$ & $92.85(2)$ & $\mathrm{R} 1 / w \mathrm{R} 2(\mathrm{I}>2 \sigma(\mathrm{I}))$ & $0.0541 / 0.1044$ \\
\hline$\gamma\left[{ }^{\circ}\right]$ & 90 & R1 / wR2 (all data) & 0.0989 / 0.1185 \\
\hline Volume $\left[\AA^{3}\right]$ & $4780.9(14)$ & max. diff peak / hole $\left[e \AA^{-3}\right]$ & $0.230 /-0.235$ \\
\hline
\end{tabular}




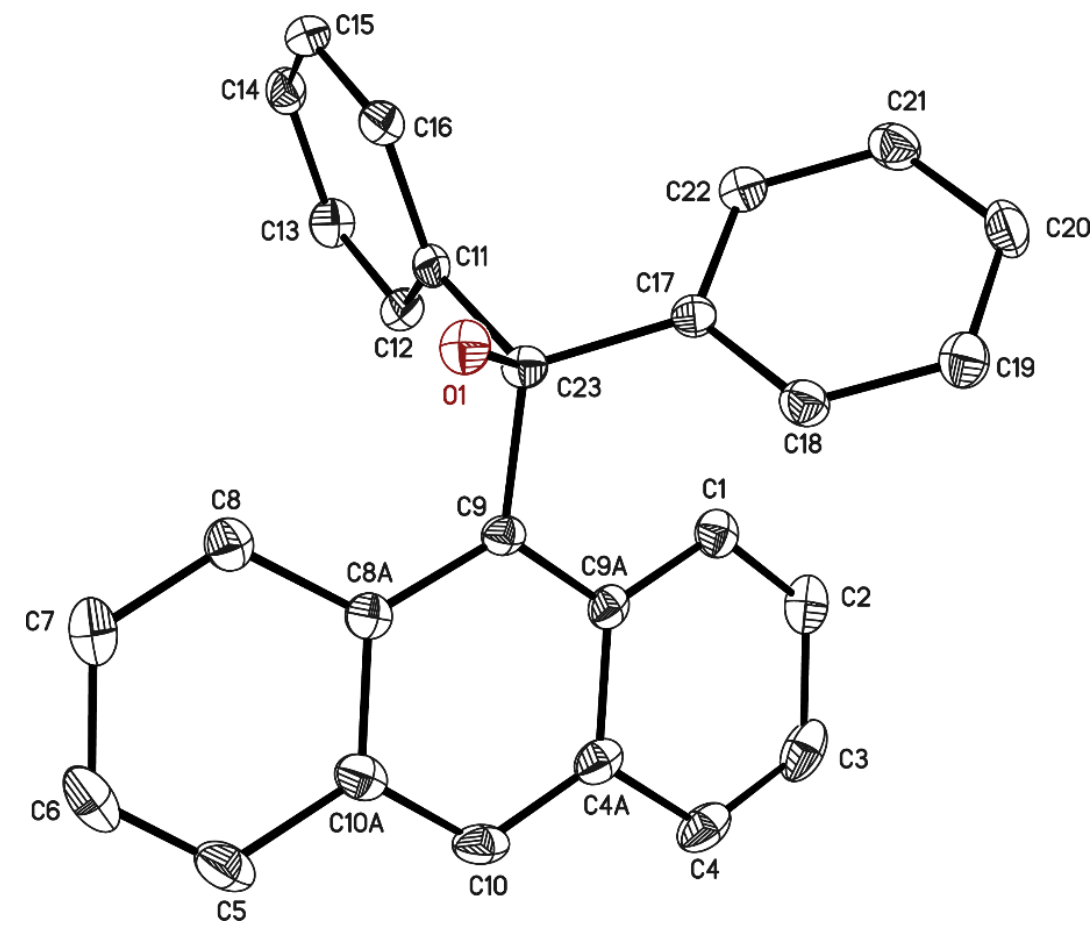

Figure 86. Asymmetric unit of 10 . The anisotropic displacement parameters are depicted at the $50 \%$ probability level. Hydrogen atoms are omitted for clarity.

Table 26. Crystallographic data of 10.

\begin{tabular}{|c|c|c|c|}
\hline Structure Code & ts354 & Z & 4 \\
\hline Empirical formula & $\mathrm{C}_{27} \mathrm{H}_{20} \mathrm{O}$ & $\rho_{\text {calc }}$ & 1.274 \\
\hline Formula weight $[\mathrm{g} / \mathrm{mol}]$ & 360.43 & $\mu\left[\mathrm{mm}^{-1}\right]$ & 0.076 \\
\hline Temperature $[\mathrm{K}]$ & $100(2)$ & $F(000)$ & 760 \\
\hline Wavelength $[\AA]$ & 0.71073 & $\theta$ range $\left[{ }^{\circ}\right]$ & 2.178 to 26.356 \\
\hline Crystal system & monoclinic & Reflections collected & 13852 \\
\hline space group & $P 2_{1} / \mathrm{n}$ & Independent reflections & 3837 \\
\hline a $[\AA ̊]$ & $10.585(3)$ & $\mathrm{R}$ (int) & 0.0364 \\
\hline $\mathrm{b}[\AA ̊]$ & $12.223(3)$ & Max. / min. transmission & 0.7454 / 0.6877 \\
\hline$c[\AA]$ & $14.695(4)$ & Restraints / parameter & $0 / 257$ \\
\hline$\alpha\left[^{\circ}\right]$ & 90 & GooF & 1.018 \\
\hline$\beta\left[^{\circ}\right]$ & $98.86(2)$ & $\mathrm{R} 1 / w \mathrm{R} 2(\mathrm{I}>2 \sigma(\mathrm{I}))$ & $0.0380 / 0.0868$ \\
\hline$\gamma\left[^{\circ}\right]$ & 90 & R1 / wR2 (all data) & $0.0497 / 0.0931$ \\
\hline Volume $\left[\AA^{3}\right]$ & $1878.6(9$ & max. diff peak / hole $\left[e \AA^{-3}\right]$ & $0.284 /-0.212$ \\
\hline
\end{tabular}




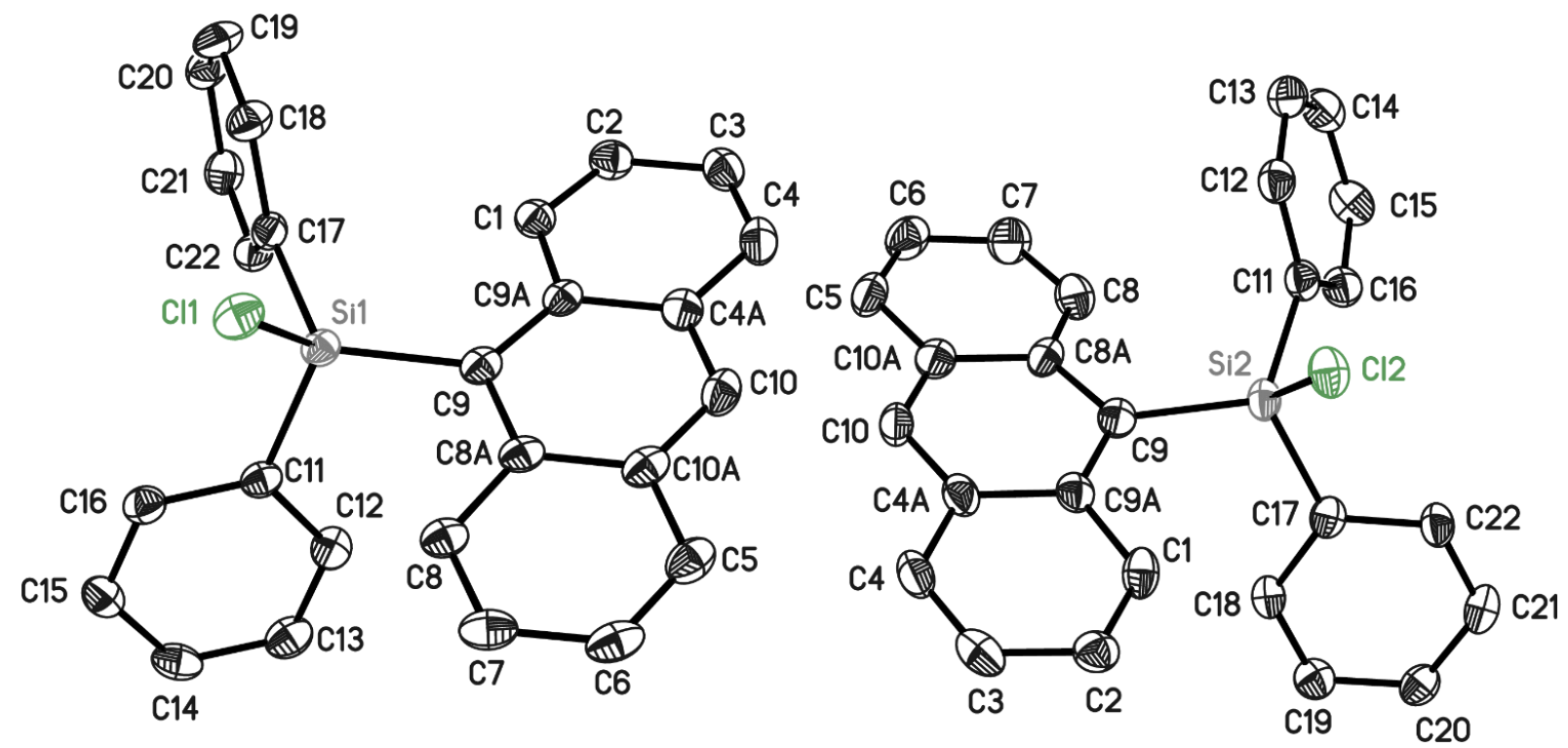

Figure 87. Asymmetric unit of 11 . The anisotropic displacement parameters are depicted at the $50 \%$ probability level. Hydrogen atoms are omitted for clarity.

Table 27. Crystallographic data of 11.

\begin{tabular}{|c|c|c|c|}
\hline Structure Code & ts 223 & Z & 4 \\
\hline Empirical formula & $\mathrm{C}_{26} \mathrm{H}_{19} \mathrm{ClSi}$ & $\rho_{\text {calc }}$ & 1.305 \\
\hline Formula weight $[\mathrm{g} / \mathrm{mol}]$ & 394.95 & $\mu\left[\mathrm{mm}^{-1}\right]$ & 0.258 \\
\hline Temperature [K] & $100(2)$ & $F(000)$ & 824 \\
\hline Wavelength $[\AA ̊ 丿]$ & 0.70173 & $\theta$ range $\left[^{\circ}\right]$ & 1.073 to 25.692 \\
\hline Crystal system & triclinic & Reflections collected & 51549 \\
\hline space group & $P \overline{1}$ & Independent reflections & 7611 \\
\hline $\mathrm{a}[\AA]$ & $9.858(2)$ & $R$ (int) & 0.0690 \\
\hline $\mathrm{b}[\AA]$ & $10.877(2)$ & Max. / min. transmission & $0.7453 / 0.6918$ \\
\hline$c[\AA ̊]$ & $19.668(4)$ & Restraints / parameter & $0 / 505$ \\
\hline$\alpha\left[^{\circ}\right]$ & $74.89(2)$ & GooF & 1.041 \\
\hline$\beta\left[^{\circ}\right]$ & $89.05(3)$ & $\mathrm{R} 1 / w \mathrm{R} 2(\mathrm{I}>2 \sigma(\mathrm{I}))$ & 0.0446 / 0.1017 \\
\hline$\gamma\left[^{\circ}\right]$ & $81.00(2)$ & $\mathrm{R} 1 / w \mathrm{R} 2$ (all data) & $0.0682 / 0.1126$ \\
\hline Volume $\left[\AA^{3}\right]$ & $2010.3(7)$ & max. diff peak / hole $\left[e \AA^{-3}\right]$ & $0.913 /-0.431$ \\
\hline
\end{tabular}




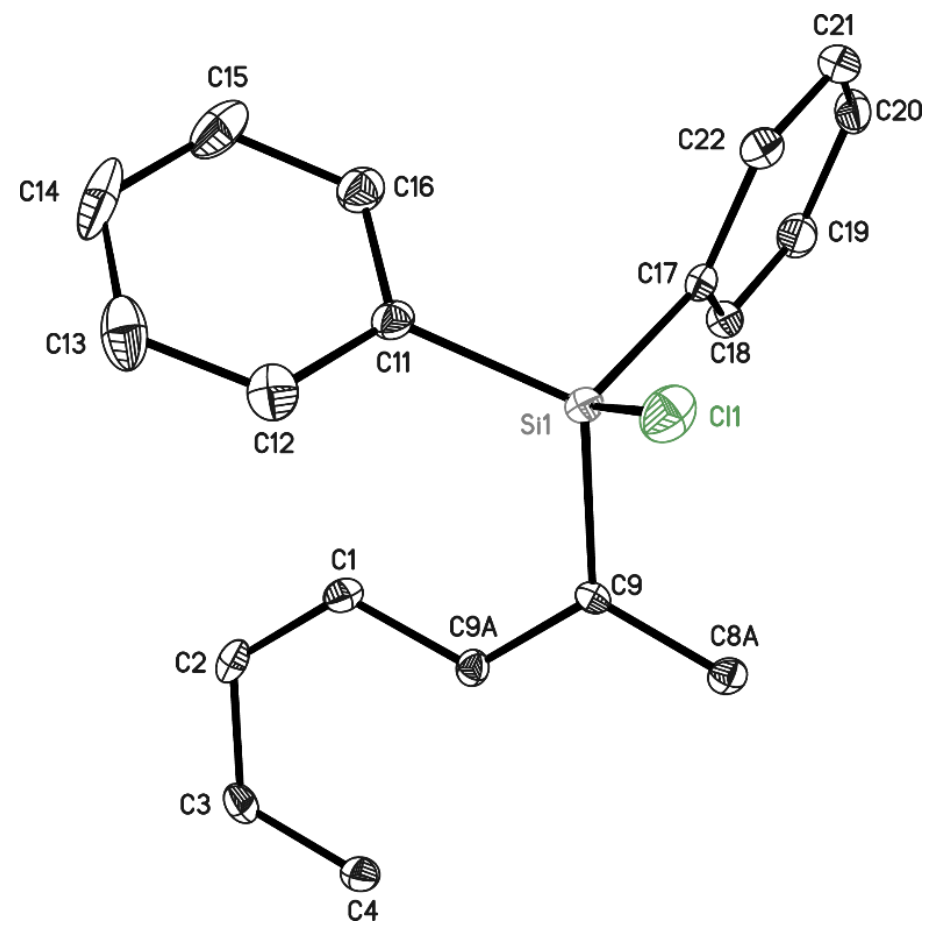

Figure 88. Asymmetric unit of 12. The anisotropic displacement parameters are depicted at the 50\% probability level. Hydrogen atoms are omitted for clarity.

Table 28. Crystallographic data of 12.

\begin{tabular}{|c|c|c|c|}
\hline Structure Code & ts230_2 & Z & 2 \\
\hline Empirical formula & $\mathrm{C}_{38} \mathrm{H}_{28} \mathrm{Cl}_{2} \mathrm{Si}_{2}$ & $\rho_{\text {calc }}$ & 1.357 \\
\hline Formula weight $[\mathrm{g} / \mathrm{mol}]$ & 611.68 & $\mu\left[\mathrm{mm}^{-1}\right]$ & 0.173 \\
\hline Temperature $[\mathrm{K}]$ & $100(2)$ & $F(000)$ & 636 \\
\hline Wavelength $[\AA ̊]$ & 0.56086 & $\theta$ range $\left[{ }^{\circ}\right]$ & 1.829 to 20.116 \\
\hline Crystal system & monoclinic & Reflections collected & 13646 \\
\hline space group & $P 2_{1} / \mathrm{c}$ & Independent reflections & 2889 \\
\hline $\mathrm{a}[\AA ̊]$ & $6.839(2)$ & $\mathrm{R}$ (int) & 0.0613 \\
\hline $\mathrm{b}[\AA]$ & $12.457(2)$ & Max. / min. transmission & 0.7444 / 0.7039 \\
\hline$c[\AA]$ & $17.834(3)$ & Restraints / parameter & $0 / 190$ \\
\hline$\alpha\left[^{\circ}\right]$ & 90 & GooF & 1.027 \\
\hline$\beta\left[^{\circ}\right]$ & $99.8(2)$ & $\mathrm{R} 1 / w \mathrm{R} 2(\mathrm{I}>2 \sigma(\mathrm{I}))$ & $0.0412 / 0.0896$ \\
\hline$\gamma\left[^{\circ}\right]$ & 90 & $\mathrm{R} 1 /$ wR2 (all data) & $0.0624 / 0.0980$ \\
\hline Volume $\left[\AA^{3}\right]$ & $1496.9(6)$ & max. diff peak / hole $\left[e \AA^{-3}\right]$ & $0.333 /-0.291$ \\
\hline
\end{tabular}




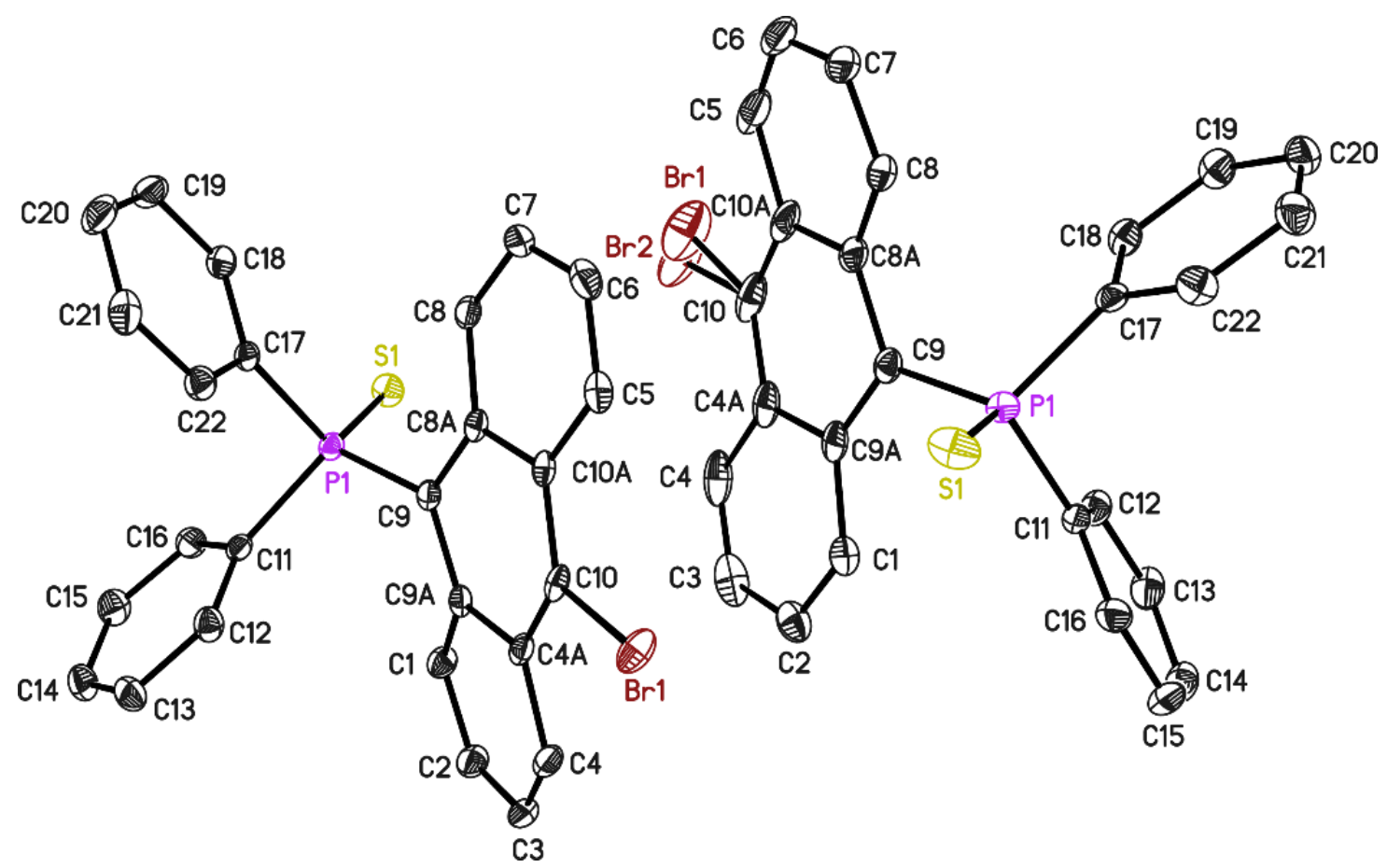

Figure 89. Asymmetric unit of 13 . The anisotropic displacement parameters are depicted at the $50 \%$ probability level. Hydrogen atoms are omitted for clarity. One $\mathrm{Br}$ atom is disordered over a second position.

Table 29. Crystallographic data of 13.

\begin{tabular}{l|l|l|l} 
Structure Code & ts034 & $\mathrm{Z}$ & 8 \\
Empirical formula & $\mathrm{C}_{26} \mathrm{H}_{18} \mathrm{BrPS}$ & $\rho_{\text {calc }}$ & 1.511 \\
Formula weight $[\mathrm{g} / \mathrm{mol}]$ & 473.34 & $\mu\left[\mathrm{mm}^{-1}\right]$ & 2.164 \\
Temperature $[\mathrm{K}]$ & $100(2)$ & $\mathrm{F}(000)$ & 1920 \\
Wavelength $[\AA]$ & 0.17073 & $\theta$ range $\left[^{\circ}\right]$ & 1.661 to 27.768 \\
Crystal system & Monoclinic & Reflections collected & 108626 \\
space group & $\mathrm{P} 2{ }_{1} / \mathrm{n}$ & Independent reflections & 9784 \\
a $[\AA \AA$ & $17.226(2)$ & R(int) & 0.0857 \\
$\mathrm{~b}[\AA]$ & $13.937(2)$ & Max. $/$ min. transmission & $0.7456 / 0.6606$ \\
$\mathrm{c}[\AA]$ & $17.329(3)$ & Restraints / parameter & $880 / 533$ \\
$\alpha\left[^{\circ}\right]$ & 90 & GooF & 1.0021 \\
$\beta\left[^{\circ}\right]$ & $90.42(2)$ & R1 $/$ wR2 $(\mathrm{I}>2 \sigma(\mathrm{I}))$ & $0.0348 / 0.0701$ \\
$\gamma\left[^{\circ}\right]$ & 90 & R1 $/$ wR2 (all data) & $0.0560 / 0.0770$ \\
Volume $\left[\AA^{\circ}\right]$ & $4160.2(11)$ & max. diff peak / hole $\left[\mathrm{e} \AA^{-3}\right]$ & $0.426 /-0.481$
\end{tabular}




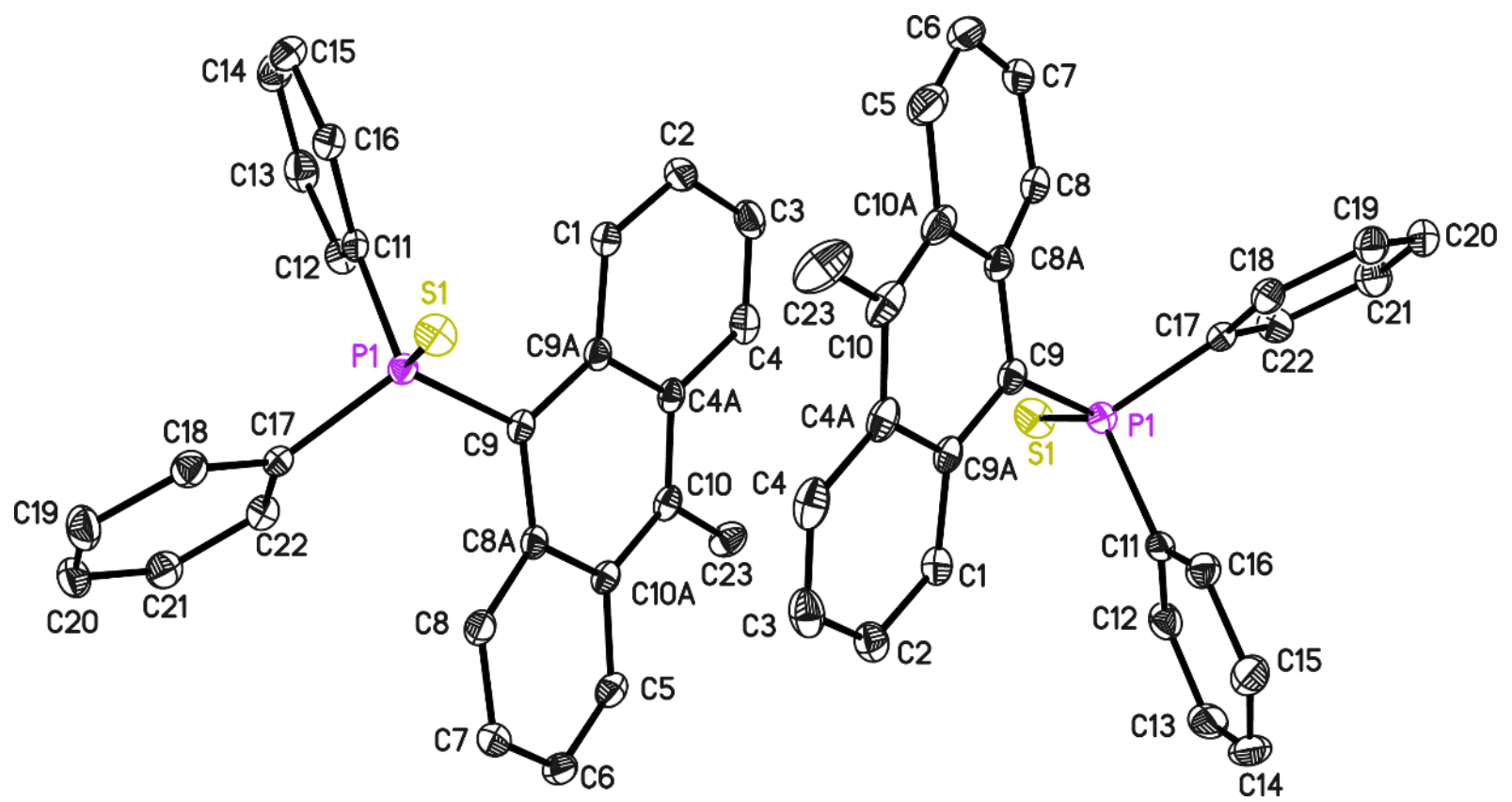

Figure 90. Asymmetric unit of 14. The anisotropic displacement parameters are depicted at the 50\% probability level. Hydrogen atoms are omitted for clarity. Hydrogen atoms attached to one $\mathrm{C} 23$ are disordered over two positions. Occupancy of the major part refines to $0.76(2)$.

Table 30. Crystallographic data of 14.

Structure Code
Empirical formula
Formula weight $[\mathrm{g} / \mathrm{mol}]$
Temperature $[\mathrm{K}]$
Wavelength $[\AA]$
Crystal system
space group
a $[\AA \AA$
$\mathrm{b}[\AA]$
$\mathrm{c}[\AA]$
$\alpha\left[^{\circ}\right]$
$\beta\left[^{\circ}\right]$
$\gamma\left[^{\circ}\right]$
Volume $\left[\AA^{3}\right]$

tsO66
$\mathrm{C}_{27} \mathrm{H}_{21} \mathrm{PS}$
408.47
$100(2) \mathrm{K}$
$0.71073 \AA$
Monoclinic
$\mathrm{P} 21 / \mathrm{n}$
$17.1280(10))$
$13.7960(10$
$17.412(2)$
90
$90.070(10$
90
$4114.4(6)$

\begin{tabular}{|l}
$Z$ \\
$\rho_{\text {calc }}$ \\
$\mu\left[\mathrm{mm}^{-1}\right]$ \\
$\mathrm{F}(000)$ \\
$\theta$ range $\left[^{\circ}\right]$ \\
Reflections collected \\
Independent reflections \\
R(int) \\
Max. / min. transmission \\
Restraints / parameter \\
GooF \\
R1 / wR2 (I > 2 $\sigma(I))$ \\
R1 / wR2 (all data) \\
max. diff peak / hole $\left[e \AA^{-3}\right]$
\end{tabular}

8

1.319

0.246

1712

1.667 to 26.399

168507

8439

0.0479

0.7454 / 0.6960

$0 / 527$

1.025

$0.0308 / 0.0771$

$0.0373 / 0.0812$

$0.348 /-0.304$ 


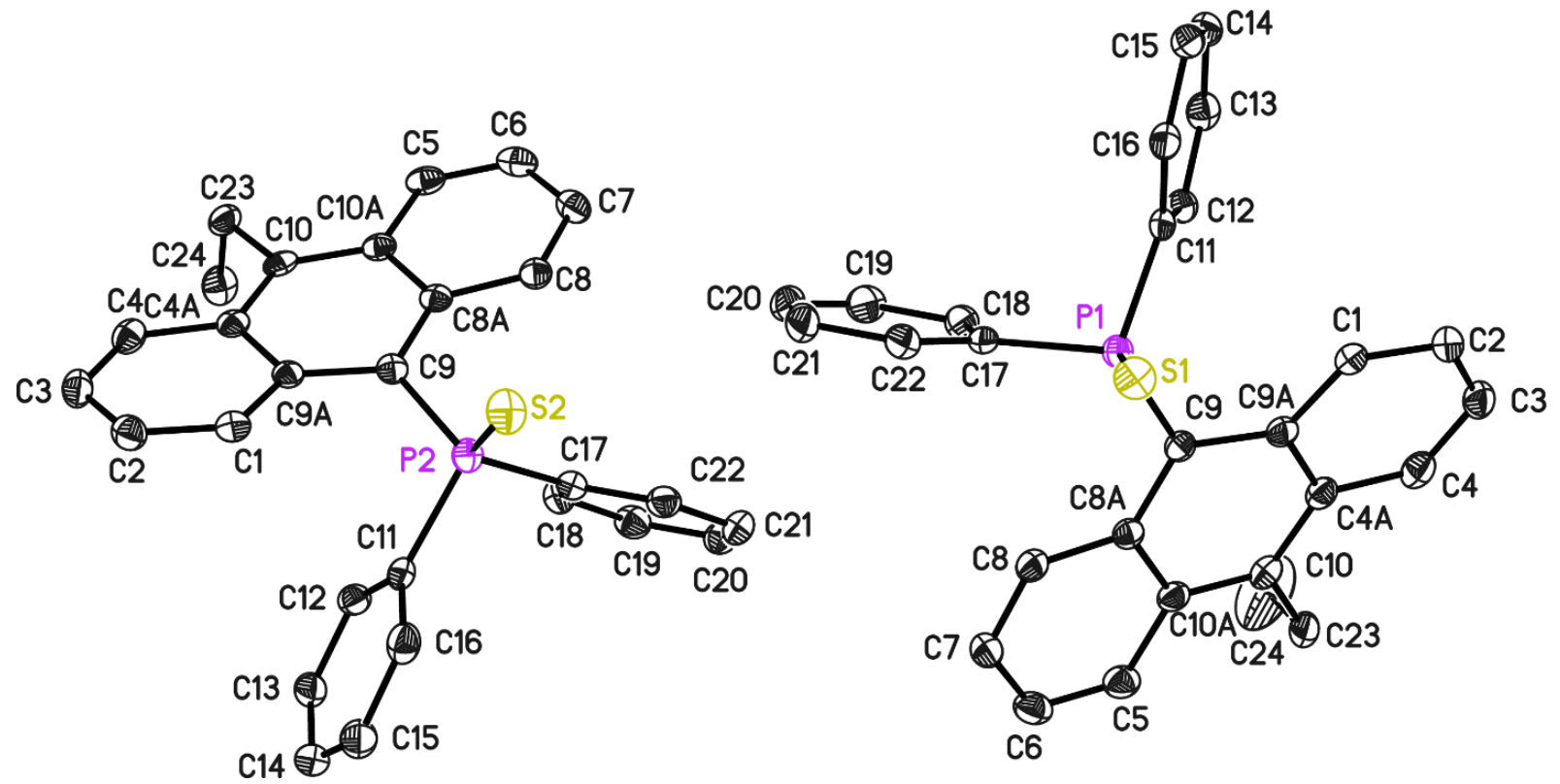

Figure 91. Asymmetric unit of 15 . The anisotropic displacement parameters are depicted at the $50 \%$ probability level. Hydrogen atoms are omitted for clarity.

Table 31. Crystallographic data of 15.

\begin{tabular}{|c|c|c|c|}
\hline Structure Code & ts_etanps_ee & Z & 2 \\
\hline Empirical formula & $\mathrm{C}_{28} \mathrm{H}_{23} \mathrm{PS}$ & $\rho_{\text {calc }}$ & 1.290 \\
\hline Formula weight $[\mathrm{g} / \mathrm{mol}]$ & 422.53 & $\mu\left[\mathrm{mm}^{-1}\right]$ & 0.235 \\
\hline Temperature $[\mathrm{K}]$ & $100(2) \mathrm{K}$ & $F(000)$ & 888 \\
\hline Wavelength $[\AA ̊]$ & $0.71073 \AA$ & $\theta$ range $\left[{ }^{\circ}\right]$ & 1.180 to 26.429 \\
\hline Crystal system & Triclinic & Reflections collected & 48428 \\
\hline space group & $\mathrm{P} \overline{\mathbf{1}}$ & Independent reflections & 8859 \\
\hline $\mathrm{a}[\AA \AA]$ & $9.791(2)$ & $\mathrm{R}$ (int) & 0.0741 \\
\hline$b[\AA]$ & $13.338(2)$ & Max. / min. transmission & $0.7454 / 0.7046$ \\
\hline$c[\AA]$ & $17.308(3)$ & Restraints / parameter & $0 / 543$ \\
\hline$\alpha\left[^{\circ}\right]$ & $85.72(2)$ & GooF & 0.949 \\
\hline$\beta\left[^{\circ}\right]$ & $87.71(3)$ & $\mathrm{R} 1 / w \mathrm{R} 2(\mathrm{I}>2 \sigma(\mathrm{I}))$ & $0.0377 / 0.0851$ \\
\hline$\gamma\left[^{\circ}\right]$ & $74.84(2)$ & $\mathrm{R} 1 / w \mathrm{R} 2$ (all data) & $0.0630 / 0.0923$ \\
\hline Volume $\left[\AA^{3}\right]$ & $2175-1(7)$ & max. diff peak / hole $\left[\mathrm{e} \AA^{-3}\right]$ & $0.555 /-0.291$ \\
\hline
\end{tabular}




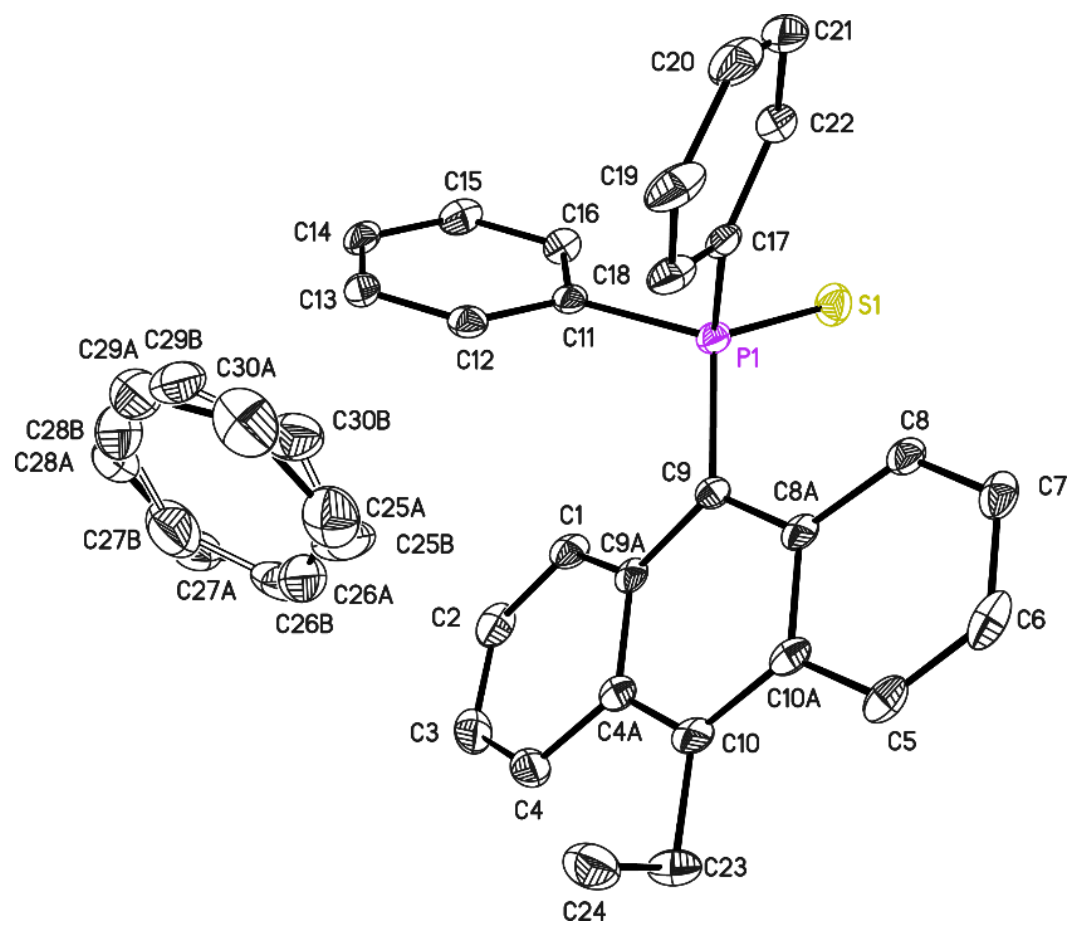

Figure 92. Asymmetric unit of 15a. The anisotropic displacement parameters are depicted at the $50 \%$ probability level. Hydrogen atoms are omitted for clarity. The benzene molecule is disordered over two positions. It was refined with distance restraints and restraints for the anisotropic displacement parameters. The occupancy of the major component refined to $0.61(2)$.

Table 32. Crystallographic data of 15a.

\begin{tabular}{|c|c|c|c|}
\hline Structure Code & ts182_c6h6 & Z & 2 \\
\hline Empirical formula & $\mathrm{C}_{34} \mathrm{H}_{29} \mathrm{PS}$ & $\rho_{\text {calc }}$ & 1.272 \\
\hline Formula weight $[\mathrm{g} / \mathrm{mol}]$ & 500.60 & $\mu\left[\mathrm{mm}^{-1}\right]$ & 0.207 \\
\hline Temperature $[\mathrm{K}]$ & $100(2)$ & $F(000)$ & 528 \\
\hline Wavelength $[\AA ̊ 丿]$ & 0.71073 & $\theta$ range $\left[{ }^{\circ}\right]$ & 1.367 to 26.375 \\
\hline Crystal system & triclinic & Reflections collected & 31351 \\
\hline space group & $\mathrm{P} \overline{\mathbf{1}}$ & Independent reflections & 5354 \\
\hline$a[\AA ̊]$ & $9.218(2)$ & $\mathrm{R}$ (int) & 0.1604 \\
\hline $\mathrm{b}[\AA ̊]$ & $10.152(2)$ & Max. / min. transmission & 0.7454 / 0.6763 \\
\hline$c[\AA]$ & $15.728(3)$ & Restraints / parameter & 390 / 381 \\
\hline$\alpha\left[^{\circ}\right]$ & $72.15(2)$ & GooF & 0.971 \\
\hline$\beta\left[^{\circ}\right]$ & $78.83(3)$ & $\mathrm{R} 1 / w \mathrm{R} 2(\mathrm{I}>2 \sigma(\mathrm{I}))$ & 0.0412 / 0.0964 \\
\hline$\gamma\left[^{\circ}\right]$ & $70.10(2)$ & $\mathrm{R} 1 /$ wR2 (all data) & $0.0646 / 0.1040$ \\
\hline Volume $\left[\AA^{3}\right]$ & $1307.3(5)$ & max. diff peak / hole $\left[e \AA^{-3}\right.$ ] & $0.502 /-0.333$ \\
\hline
\end{tabular}




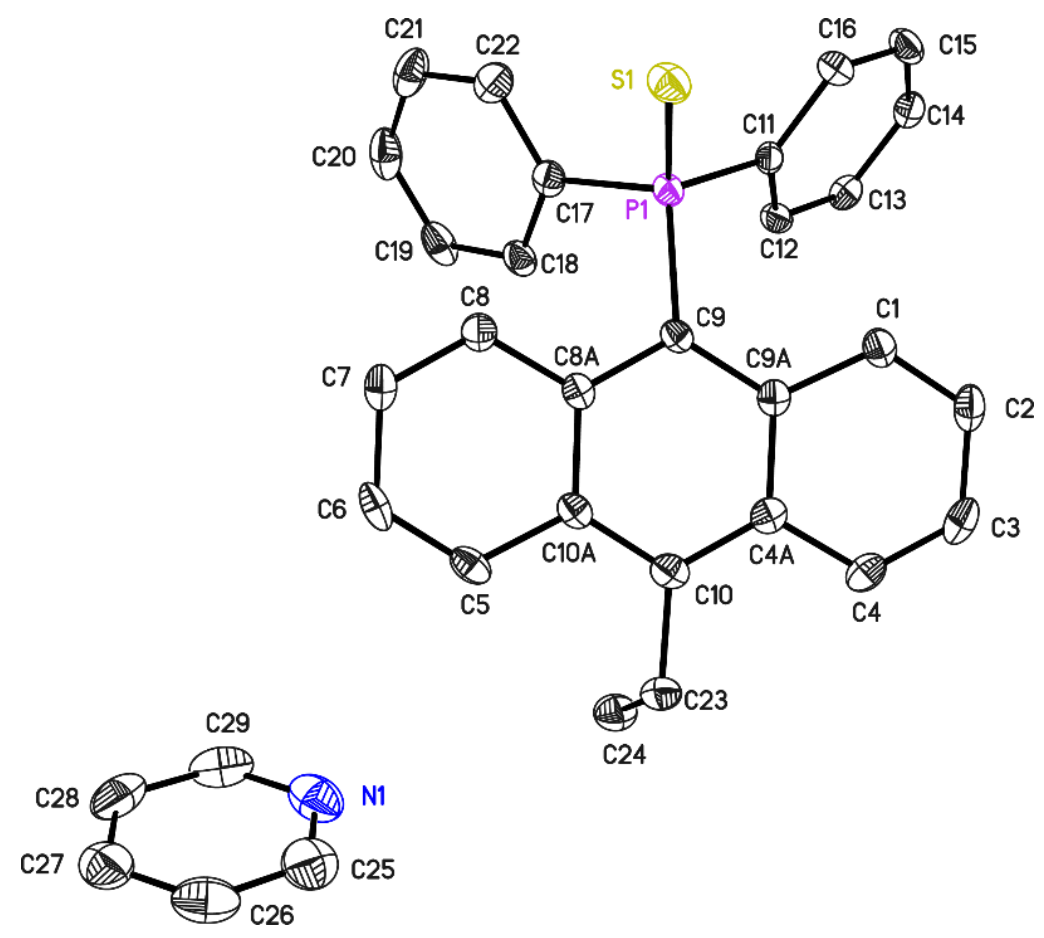

Figure 93. Asymmetric unit of $\mathbf{1 5 b}$. The anisotropic displacement parameters are depicted at the $50 \%$ probability level. Hydrogen atoms are omitted for clarity.

Table 33. Crystallographic data of 15b.

\begin{tabular}{l|l|l|l} 
Structure Code & ts182_c5h5n & $\mathrm{Z}$ & 2 \\
Empirical formula & $\mathrm{C}_{33} \mathrm{H}_{28} \mathrm{NPS}$ & $\rho_{\text {calc }}$ & 1.294 \\
Formula weight $[\mathrm{g} / \mathrm{mol}]$ & 501.59 & $\mu\left[\mathrm{mm}^{-1}\right]$ & 0.211 \\
Temperature $[\mathrm{K}]$ & $100(2)$ & $\mathrm{F}(000)$ & 528 \\
Wavelength $[\AA]$ & 0.71073 & $\theta$ range $\left[^{\circ}\right]$ & 1.368 to 25.714 \\
Crystal system & triclinic & Reflections collected & 30155 \\
space group & $\mathrm{P} \overline{1}$ & Independent reflections & 4920 \\
$\mathrm{a}[\AA \AA$ & $\mathrm{R}($ int) & 0.0568 \\
$\mathrm{~b}[\AA]$ & $9.160(4)$ & Max. $/$ min. transmission & $0.7453 / 0.6915$ \\
$\mathrm{c}[\AA]$ & $10.087(4)$ & Restraints / parameter & $0 / 326$ \\
$\alpha\left[^{\circ}\right]$ & $15.699(7)$ & GooF & 1.036 \\
$\beta\left[^{\circ}\right]$ & $72.31(2)$ & $\mathrm{R} 1 / w \mathrm{R} 2(\mathrm{I}>2 \sigma(\mathrm{I}))$ & $0.0397 / 0.0881$ \\
$\gamma\left[^{\circ}\right]$ & $78.83(3)$ & $\mathrm{R} 1 / w \mathrm{wR} 2(\mathrm{all}$ data) & $0.0578 / 0.0966$ \\
Volume $\left[\AA^{3}\right]$ & $69.42(2)$ & max. diff peak / hole $\left[\mathrm{e} \AA^{-3}\right]$ & $0.741 /-0.385$
\end{tabular}




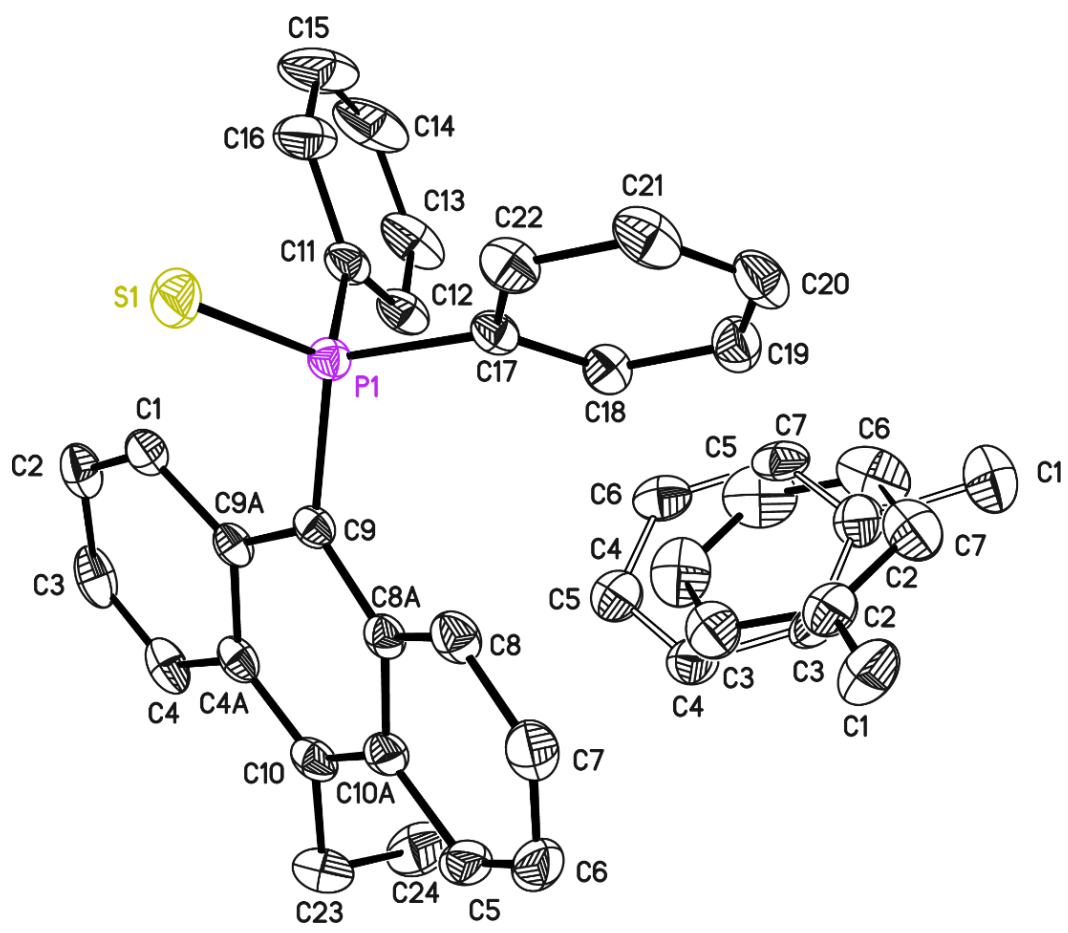

Figure 94. Asymmetric unit of 15c. The anisotropic displacement parameters are depicted at the 50\% probability level. Hydrogen atoms are omitted for clarity. The toluene is disordered over two positions. It was refined with distance restraints and restraints for the anisotropic displacement parameters. The occupancy of the major component refined to $0.662(4)$.

Table 34. Crystallographic data of $\mathbf{1 5 c}$.

\begin{tabular}{|c|c|c|c|}
\hline Structure Code & $\mathrm{ts} 041$ & Z & 2 \\
\hline Empirical formula & $\mathrm{C}_{35} \mathrm{H}_{31} \mathrm{PS}$ & $\rho_{\text {calc }}$ & 1.267 \\
\hline Formula weight $[\mathrm{g} / \mathrm{mol}]$ & 514.63 & $\mu\left[\mathrm{mm}^{-1}\right]$ & 0.202 \\
\hline Temperature [K] & $100(2)$ & $F(000)$ & 544 \\
\hline Wavelength $[\AA]$ & 0.71073 & $\theta$ range $\left[{ }^{\circ}\right]$ & 2.209 to 26.372 \\
\hline Crystal system & Triclinic & Reflections collected & 26376 \\
\hline space group & $P \overline{1}$ & Independent reflections & 5505 \\
\hline a $[\AA ̊]$ & $9.411(2)$ & $\mathrm{R}$ (int) & 0.0187 \\
\hline $\mathrm{b}[\AA ̊]$ & $10.333(2)$ & Max. / min. transmission & $0.7454 / 0.7170$ \\
\hline c $[\AA]$ & $15.806(3)$ & Restraints / parameter & 450 / 401 \\
\hline$\alpha\left[^{\circ}\right]$ & $71.39(2)$ & GooF & 1.034 \\
\hline$\beta\left[^{\circ}\right]$ & $88.10(3)$ & $\mathrm{R} 1 / w \mathrm{R} 2(\mathrm{I}>2 \sigma(\mathrm{I}))$ & $0.0343 / 0.0867$ \\
\hline$\gamma\left[{ }^{\circ}\right]$ & $68.50(2)$ & R1 / wR2 (all data) & $0.0373 / 0.0889$ \\
\hline Volume $\left[\AA^{3}\right]$ & $1349.2(5)$ & max. diff peak / hole $\left[\right.$ e $\left.\AA^{-3}\right]$ & $0.412 /-0.494$ \\
\hline
\end{tabular}




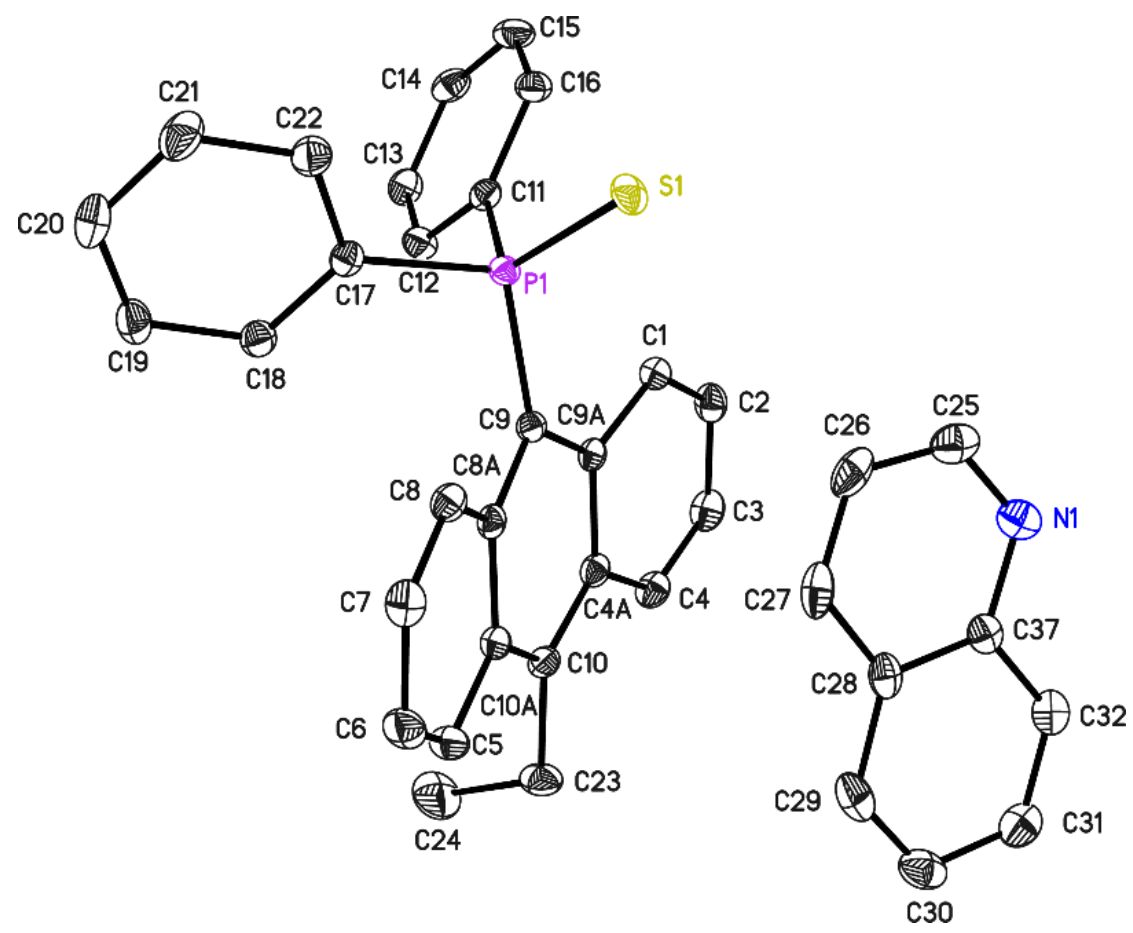

Figure 95. Asymmetric unit of $\mathbf{1 5 d}$. The anisotropic displacement parameters are depicted at the $50 \%$ probability level. Hydrogen atoms are omitted for clarity.

Table 35. Crystallographic data of 15d.

Structure Code

Empirical formula

Formula weight $[\mathrm{g} / \mathrm{mol}]$

Temperature [K]

Wavelength $[\AA ̊]$

Crystal system

space group

a $[\AA]$

b $[\AA]$

c $[\AA ̊]$

$\alpha\left[^{\circ}\right]$

$\beta\left[^{\circ}\right]$

$\gamma\left[{ }^{\circ}\right]$

Volume $\left[\AA^{3}\right]$

\begin{tabular}{|l} 
ts182_c9h7n \\
$\mathrm{C}_{37} \mathrm{H}_{30} \mathrm{NPS}$ \\
551.65 \\
$100(2)$ \\
0.71073 \\
monoclinic \\
$\mathrm{P} 2_{1} / \mathrm{c}$ \\
$10.068(2)$ \\
$12.168(2)$ \\
$23.120(3)$ \\
90 \\
$93.35(2)$ \\
90 \\
$2827.5(8)$
\end{tabular}

4

1.296

0.199

1160

1.765 to 26.405

66973

5801

0.0460

0.7454 / 0.6740

$0 / 362$

1.023

$0.0295 / 0.0762$

$0.0323 / 0.0785$

0.347 / -0.337 


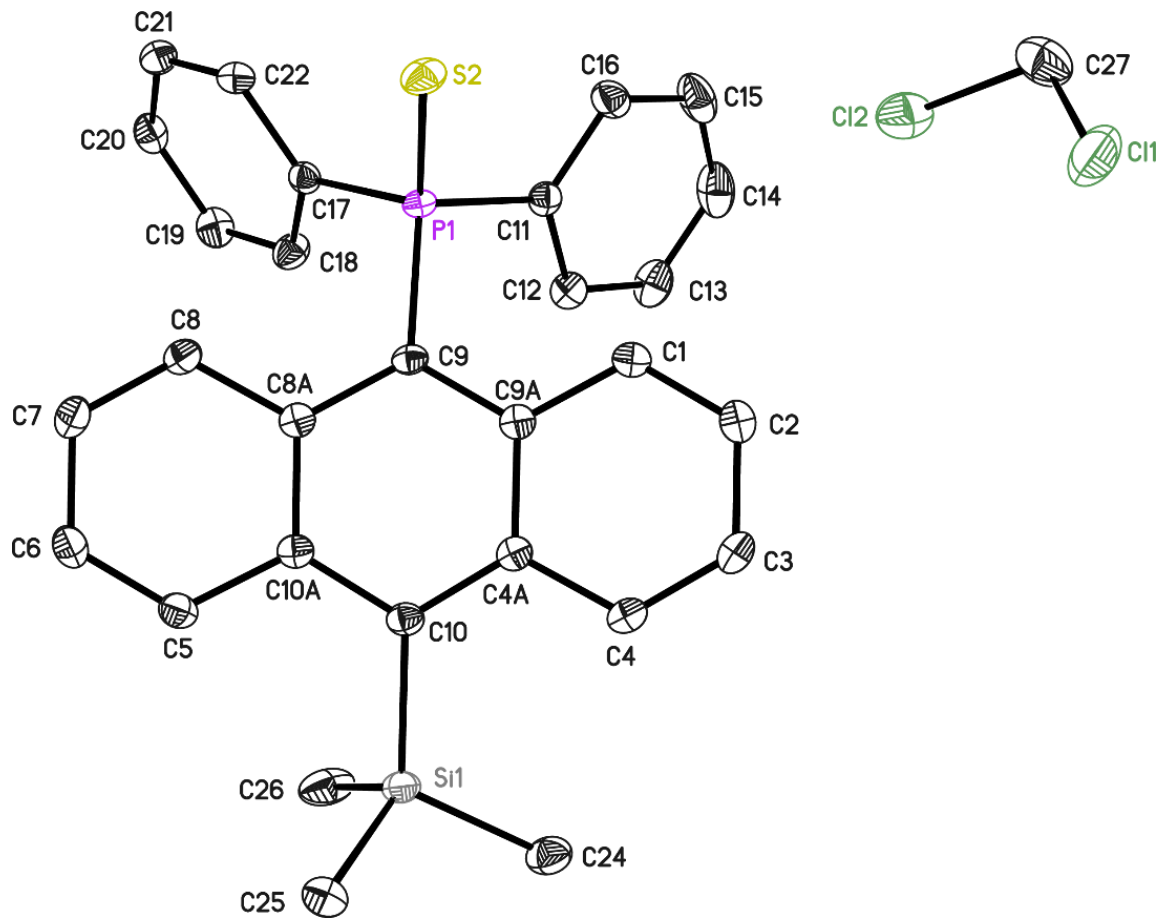

Figure 96. Asymmetric unit of 16 . The anisotropic displacement parameters are depicted at the $50 \%$ probability level. Hydrogen atoms are omitted for clarity.

Table 36. Crystallographic data of 16.

\begin{tabular}{|c|c|c|c|}
\hline Structure Code & ts113_1 & Z & 2 \\
\hline Empirical formula & $\mathrm{C}_{30} \mathrm{H}_{29} \overline{\mathrm{Cl}}_{2} \mathrm{PSSi}$ & $\rho_{\text {calc }}$ & 1.340 \\
\hline Formula weight $[\mathrm{g} / \mathrm{mol}]$ & 551.55 .53 & $\mu\left[\mathrm{mm}^{-1}\right]$ & 0.435 \\
\hline Temperature $[\mathrm{K}]$ & $100(2) \mathrm{K}$ & $F(000)$ & 576 \\
\hline Wavelength $[\AA]$ & $0.71073 \AA$ & $\theta$ range $\left[{ }^{\circ}\right]$ & 1.767 to 26.381 \\
\hline Crystal system & Triclinic & Reflections collected & 28603 \\
\hline space group & $\mathrm{P} \overline{\mathbf{1}}$ & Independent reflections & 5590 \\
\hline $\mathrm{a}[\AA \AA]$ & $10.067(2)$ & $\mathrm{R}$ (int) & 0.0211 \\
\hline $\mathrm{b}[\AA]$ & $11.783(2)$ & Max. / min. transmission & 0.7454 / 0.7068 \\
\hline c $[\AA]$ & $13.301(3)$ & Restraints / parameter & $0 / 319$ \\
\hline$\alpha\left[^{\circ}\right]$ & $111.73(2)$ & GooF & 1.039 \\
\hline$\beta\left[^{\circ}\right]$ & $108.95(3)$ & $\mathrm{R} 1 / w \mathrm{R} 2(\mathrm{I}>2 \sigma(\mathrm{I}))$ & $0.0323 / 0.0828$ \\
\hline$\gamma\left[^{\circ}\right]$ & $91.52(29$ & R1 / wR2 (all data) & $0.0360 / 0.0852$ \\
\hline Volume $\left[\AA^{3}\right]$ & $1366.7(6)$ & max. diff peak / hole $\left[e \AA^{-3}\right]$ & $0.784 /-0.369$ \\
\hline
\end{tabular}






Figure 97. Asymmetric unit of 17 . The anisotropic displacement parameters are depicted at the $50 \%$ probability level. Hydrogen atoms are omitted for clarity.

Table 37. Crystallographic data of 17.

\begin{tabular}{l|l|l|l} 
Structure Code & ts187_anisol & $\mathrm{Z}$ & 4 \\
Empirical formula & $\mathrm{C}_{32} \mathrm{H}_{23} \mathrm{PS}$ & $\rho_{\text {calc }}$ & 1.291 \\
Formula weight $[\mathrm{g} / \mathrm{mol}]$ & 470.53 & $\mu\left[\mathrm{mm}^{-1}\right]$ & 0.219 \\
Temperature $[\mathrm{K}]$ & $100(2) \mathrm{K}$ & $\mathrm{F}(000)$ & 984 \\
Wavelength $[\AA]$ & $0.71073 \AA$ & $\theta$ range $\left[^{\circ}\right]$ & 1.562 to 26.394 \\
Crystal system & Orthorhombic & Reflections collected & 33453 \\
space group & $\mathrm{P} 212121$ & Independent reflections & 4985 \\
a $[\AA \AA]$ & $6.746(2)$ & $\mathrm{R}($ int $)$ & 0.0645 \\
$\mathrm{~b}[\AA]$ & $16.049(2)$ & Max. / min. transmission & $0.7454 / 0.6168$ \\
$\mathrm{c}[\AA]$ & $22.369(3)$ & Restraints / parameter & $0 / 308$ \\
$\alpha\left[^{\circ}\right]$ & 90 & GooF & 1.051 \\
$\beta\left[^{\circ}\right]$ & 90 & $\mathrm{R} 1 / w R 2(\mathrm{I}>2 \sigma(\mathrm{I}))$ & $0.0400 / 0.0970$ \\
$\gamma\left[^{\circ}\right]$ & 90 & $\mathrm{R} 1 / w R 2($ all data) & $0.0500 / 0.1031$ \\
Volume $\left[\AA^{3}\right]$ & $2421.8(8)$ & max. diff peak / hole $\left[\mathrm{e} \AA^{-3}\right]$ & $0.458 /-0.299$
\end{tabular}




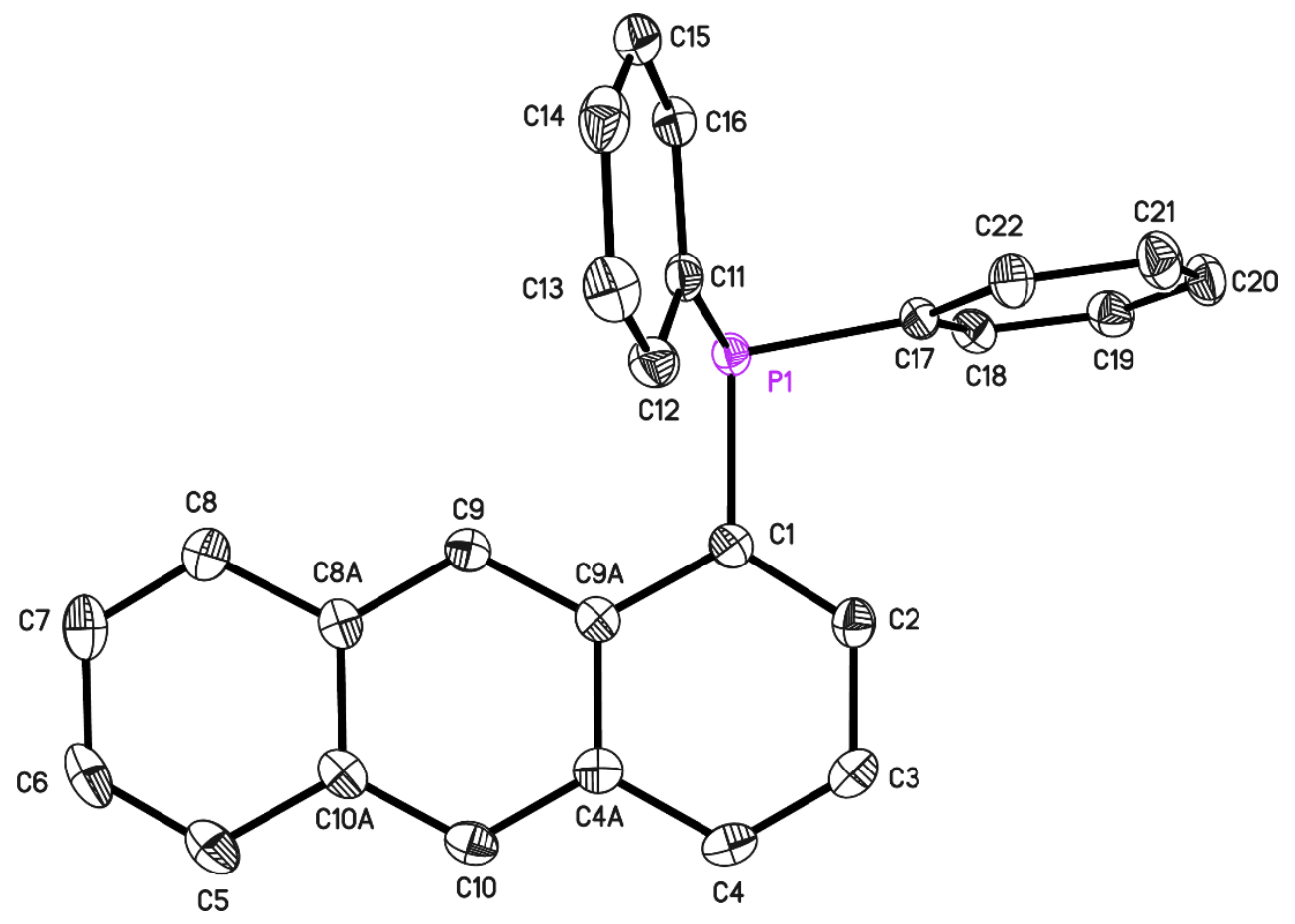

Figure 98. Asymmetric unit of 18. The anisotropic displacement parameters are depicted at the 50\% probability level. Hydrogen atoms are omitted for clarity.

Table 38. Crystallographic data of 18.

\begin{tabular}{|c|c|c|c|}
\hline Structure Code & ts181_dcm & Z & 2 \\
\hline Empirical formula & $\mathrm{C}_{26} \mathrm{H}_{19} \mathrm{P}$ & $\rho_{\text {calc }}$ & 1.276 \\
\hline Formula weight $[\mathrm{g} / \mathrm{mol}]$ & 362.38 & $\mu\left[\mathrm{mm}^{-1}\right]$ & 0.153 \\
\hline Temperature $[\mathrm{K}]$ & $100(2)$ & $F(000)$ & 380 \\
\hline Wavelength $[\AA]$ & 0.71073 & $\theta$ range $\left[{ }^{\circ}\right]$ & 1.937 to 26.473 \\
\hline Crystal system & triclinic & Reflections collected & 22799 \\
\hline space group & $P \overline{\mathbf{1}}$ & Independent reflections & 3894 \\
\hline a $[\AA]$ & $9.492(2)$ & $\mathrm{R}$ (int) & 0.0284 \\
\hline $\mathrm{b}[\AA]$ & $10.216(2)$ & Max. / min. transmission & $0.7454 / 0.6565$ \\
\hline$c[\AA]$ & $10.582(2)$ & Restraints / parameter & $0 / 244$ \\
\hline$\alpha\left[^{\circ}\right]$ & $86.87(3)$ & GooF & 1.055 \\
\hline$\beta\left[^{\circ}\right]$ & $83.46(2)$ & $\mathrm{R} 1 / w \mathrm{R} 2(\mathrm{I}>2 \sigma(\mathrm{I}))$ & $0.0335 / 0.0867$ \\
\hline$\gamma\left[^{\circ}\right]$ & $67.74(2)$ & $\mathrm{R} 1 /$ wR2 (all data) & $0.0387 / 0.0906$ \\
\hline Volume $\left[\AA^{3}\right]$ & $943.4(3)$ & max. diff peak / hole $\left[\mathrm{e} \AA^{-3}\right]$ & $0.467 /-0.259$ \\
\hline
\end{tabular}




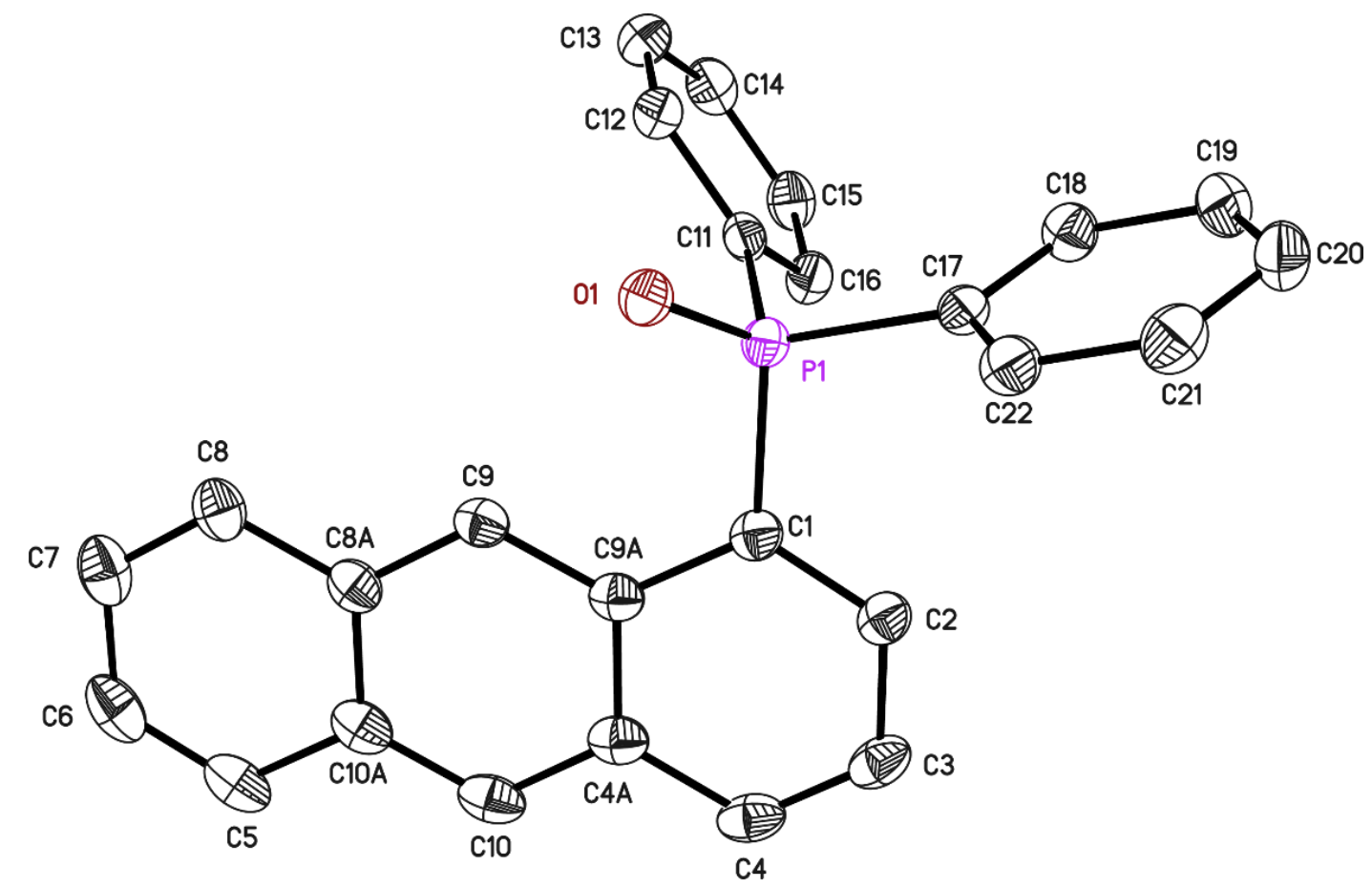

Figure 99. Asymmetric unit of 19. The anisotropic displacement parameters are depicted at the $50 \%$ probability level. Hydrogen atoms are omitted for clarity.

Table 39. Crystallographic data of 19.

Structure Code
Empirical formula
Formula weight $[\mathrm{g} / \mathrm{mol}]$
Temperature $[\mathrm{K}]$
Wavelength $[\AA \AA$
Crystal system
space group
a $[\AA \AA]$
$\mathrm{b}[\AA]$
$\mathrm{c}[\AA]$
$\alpha\left[\left[^{\circ}\right]\right.$
$\beta\left[^{\circ}\right]$
$\gamma\left[{ }^{\circ}\right]$
Volume $\left[\AA^{3}\right]$

\begin{tabular}{|l} 
ts225 \\
$\mathrm{C}_{26} \mathrm{H}_{19} \mathrm{PO}$ \\
378.38 \\
$100(2)$ \\
0.71073 \\
Triclinic \\
$P \overline{\mathbf{1}}$ \\
$8.596(2)$ \\
$10.929(2)$ \\
$11.081(3)$ \\
$96.87(3)$ \\
$91.41(3)$ \\
$110.50(4)$ \\
$962.6(5)$
\end{tabular}

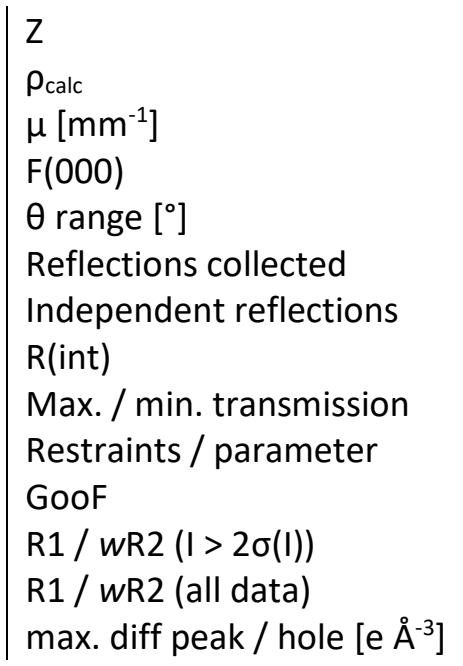

2

1.305

0.157

396

1.856 to 26.434

22061

3955

0.0431

0.7454 / 0.6972

$0 / 253$

1.035

0.0385 / 0.0912

$0.0541 / 0.1003$

$0.350 /-0.374$ 


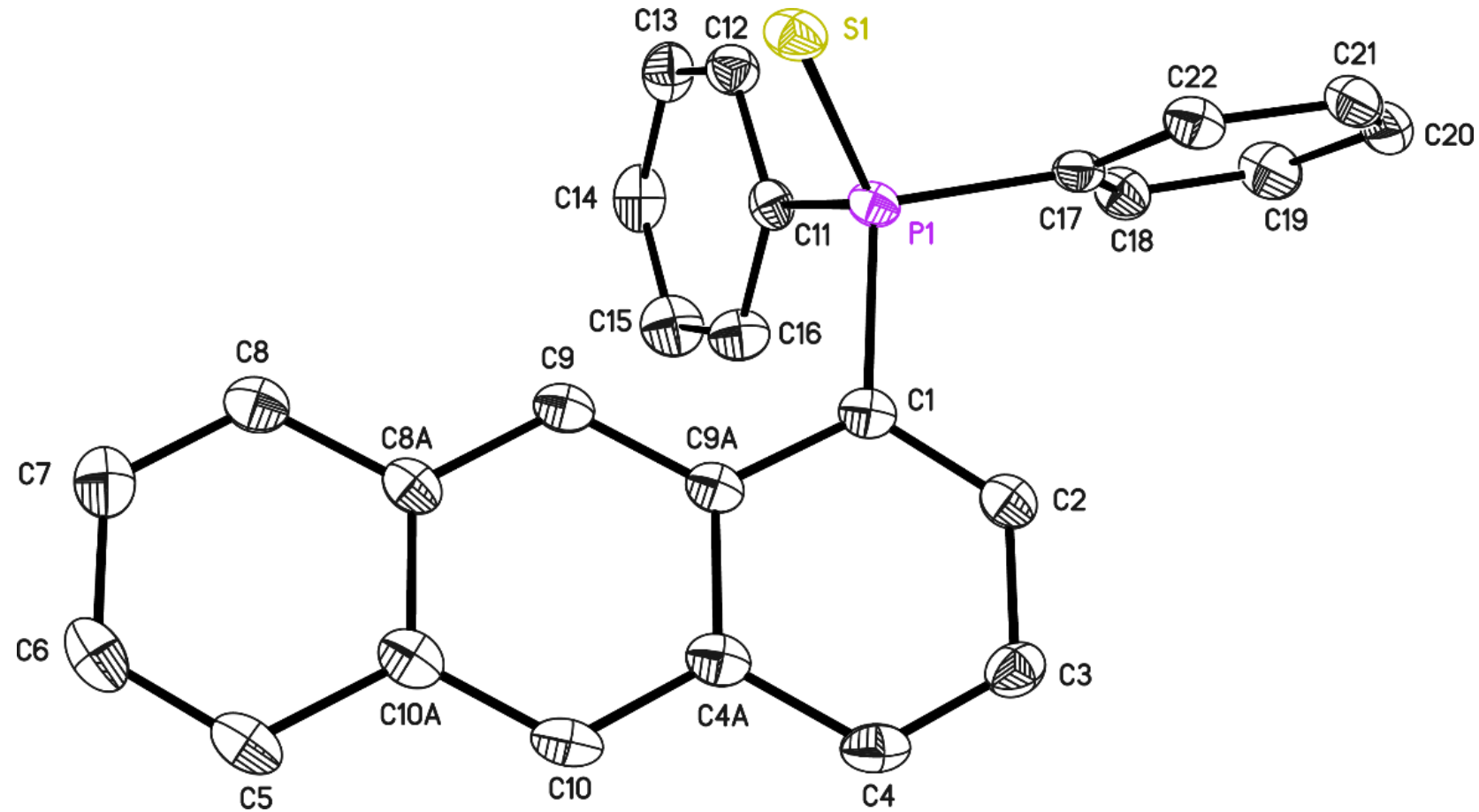

Figure 100. Asymmetric unit of $\alpha-20$. The anisotropic displacement parameters are depicted at the $50 \%$ probability level. Hydrogen atoms are omitted for clarity.

Table 40. Crystallographic data of $\alpha-20$.

\begin{tabular}{|c|c|c|c|}
\hline Structure Code & ts232_toluene & Z & 2 \\
\hline Empirical formula & $\mathrm{C}_{26} \mathrm{H}_{19} \mathrm{PS}$ & $\rho_{\text {calc }}$ & 1.334 \\
\hline Formula weight $[\mathrm{g} / \mathrm{mol}]$ & 394.44 & $\mu\left[\mathrm{mm}^{-1}\right]$ & 0.138 \\
\hline Temperature $[\mathrm{K}]$ & $100(2)$ & $F(000)$ & 412 \\
\hline Wavelength $[\AA]$ & 0.56086 & $\theta$ range $\left[{ }^{\circ}\right]$ & 1.734 to 20.557 \\
\hline Crystal system & Triclinic & Reflections collected & 40821 \\
\hline space group & $P \overline{\mathbf{1}}$ & Independent reflections & 4051 \\
\hline a $[\AA]$ & $9.948(2)$ & $\mathrm{R}$ (int) & 0.0550 \\
\hline $\mathrm{b}[\AA]$ & $10.252(2)$ & Max. / min. transmission & $0.7445 / 0.7163$ \\
\hline$c[\AA]$ & $10.815(3)$ & Restraints / parameter & $0 / 253$ \\
\hline$\alpha\left[^{\circ}\right]$ & $83.72(4)$ & GooF & 1.054 \\
\hline$\beta\left[^{\circ}\right]$ & $80.19(3)$ & $\mathrm{R} 1 / w \mathrm{R} 2(\mathrm{I}>2 \sigma(\mathrm{I}))$ & $0.0363 / 0.0906$ \\
\hline$\gamma\left[^{\circ}\right]$ & $64.76(3)$ & $\mathrm{R} 1 /$ wR2 (all data) & $0.0500 / 0.0980$ \\
\hline Volume $\left[\AA^{3}\right]$ & $982.3(5)$ & max. diff peak / hole $\left[\mathrm{e} \AA^{-3}\right]$ & $0.314 /-0.362$ \\
\hline
\end{tabular}




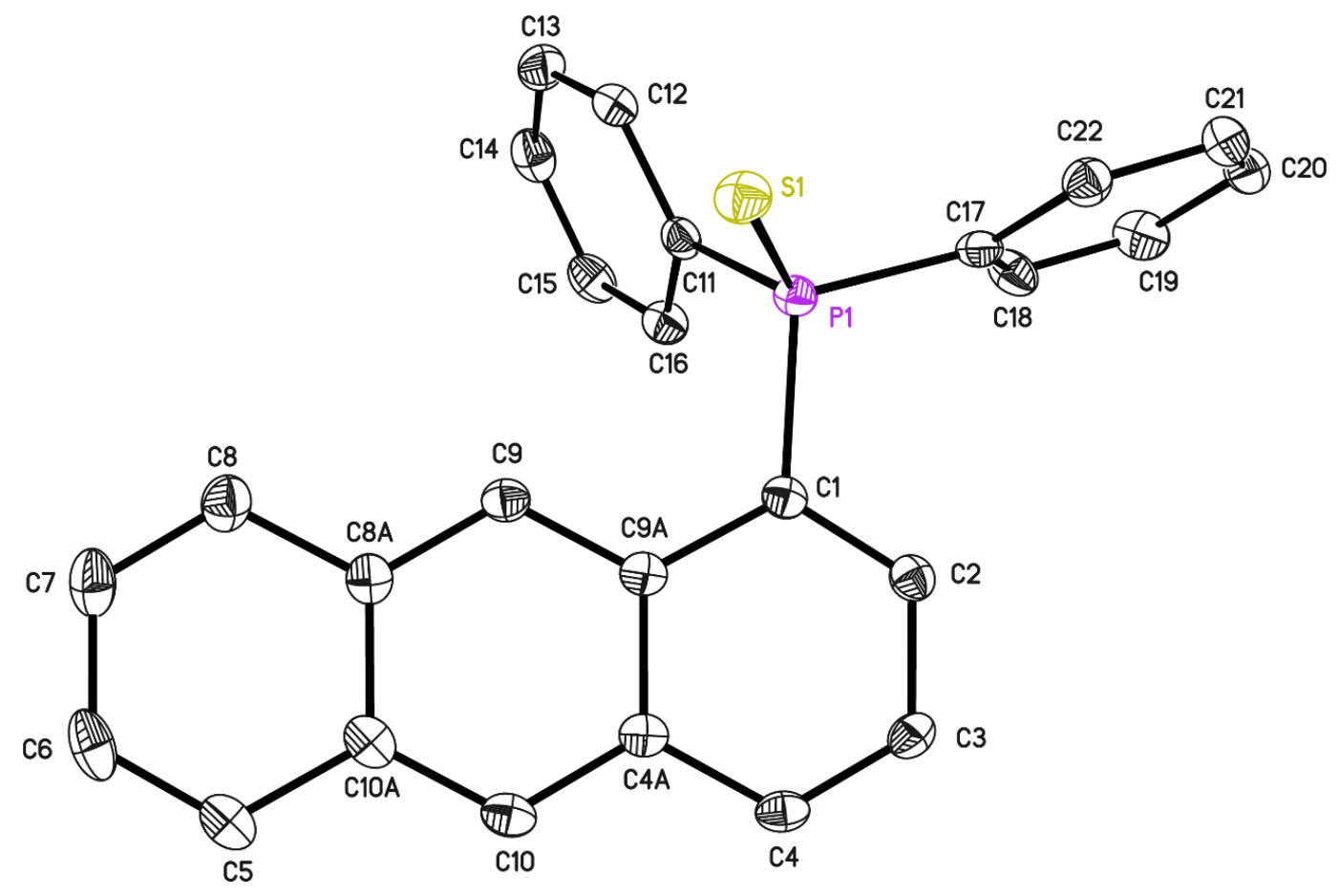

Figure 101. Asymmetric unit of Asymmetric unit of $\beta-20$. The anisotropic displacement parameters are depicted at the $50 \%$ probability level. Hydrogen atoms are omitted for clarity.

Table 41. Crystallographic data of $\beta-20$.

Structure Code

Empirical formula

Formula weight $[\mathrm{g} / \mathrm{mol}]$

Temperature [K]

Wavelength $[\AA ̊]$

Crystal system

space group

a $[\AA]$

$\mathrm{b}[\AA \AA]$

c $[\AA]$

$\alpha\left[^{\circ}\right]$

$\beta\left[^{\circ}\right]$

$\gamma\left[^{\circ}\right]$

Volume $\left[\AA^{3}\right]$

\begin{tabular}{|l} 
ts181_toluene \\
$\mathrm{C}_{26} \mathrm{H}_{19} \mathrm{PS}$ \\
394.44 \\
$100(2)$ \\
0.71073 \\
Monoclinic \\
$\mathrm{P} 2_{1} / \mathrm{c}$ \\
$13.967(2)$ \\
$8.892(2)$ \\
$17.201(3)$ \\
90 \\
$110.46(4)$ \\
90 \\
$2001.5(8)$
\end{tabular}

4

1.309

0.250

824

1.556 to 26.440

47173

4113

0.0776

0.7454 / 0.7083

$0 / 253$

1.032

0.0337 / 0.0809

$0.0475 / 0.0860$

0.349 / - 0.317 


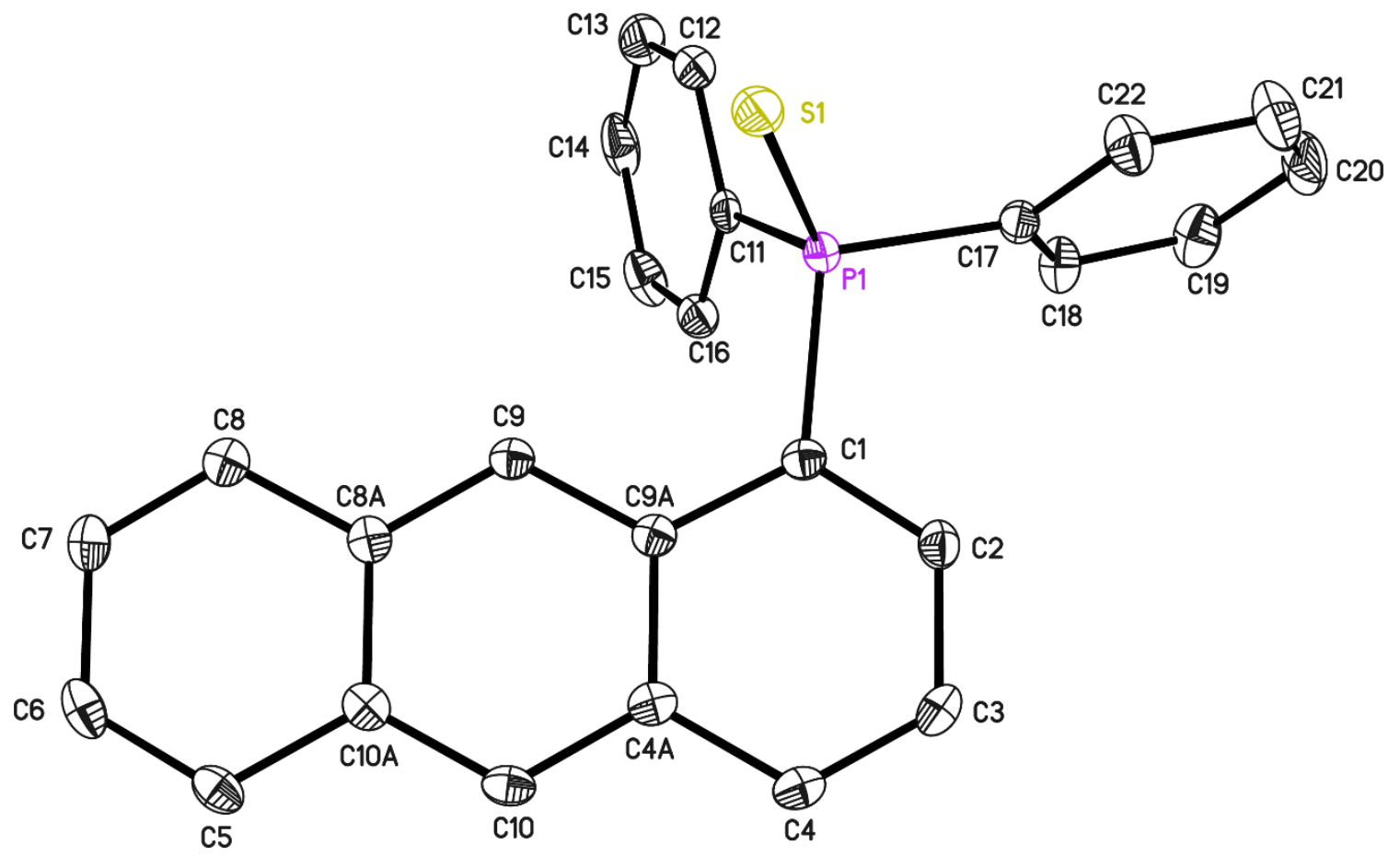

Figure 102. Asymmetric unit of Asymmetric unit of $y$-20. The anisotropic displacement parameters are depicted at the $50 \%$ probability level. Hydrogen atoms are omitted for clarity.

Table 42. Crystallographic data of $\gamma-\mathbf{2 0}$.

\begin{tabular}{l|l|l|l} 
Structure Code & ts232_dmf & $\mathrm{Z}$ & 4 \\
Empirical formula & $\mathrm{C}_{26} \mathrm{H}_{19} \mathrm{PS}$ & $\rho_{\text {calc }}$ & 1.333 \\
Formula weight $[\mathrm{g} / \mathrm{mol}]$ & 394.44 & $\mu\left[\mathrm{mm}^{-1}\right]$ & 0.138 \\
Temperature $[\mathrm{K}]$ & $100(2)$ & $\mathrm{F}(000)$ & 824 \\
Wavelength $[\AA]$ & 0.56086 & $\theta$ range $\left[^{\circ}\right]$ & 1.891 to 20.537 \\
Crystal system & Monoclinic & Reflections collected & 51656 \\
space group & $\mathrm{P} 2{ }_{1} / \mathrm{c}$ & Independent reflections & 4022 \\
$\mathrm{a}[\AA \AA$ & $17.662(3)$ & $\mathrm{R}(\mathrm{int})$ & 0.0525 \\
$\mathrm{~b}[\AA]$ & $7.158(2)$ & Max. $/$ min. transmission & $0.7445 / 0.7265$ \\
$\mathrm{c}[\AA]$ & $17.051(2)$ & Restraints $/$ parameter & $0 / 253$ \\
$\alpha\left[^{\circ}\right]$ & 90 & GooF & 1.074 \\
$\beta\left[^{\circ}\right]$ & $114.250(2)$ & $\mathrm{R} 1 / w R 2(\mathrm{I}>2 \sigma(\mathrm{I}))$ & $0.0318 / 0.0793$ \\
$\gamma\left[^{\circ}\right]$ & 90 & $\mathrm{R} 1 / w R 2($ all data) & $0.0410 / 0.0847$ \\
Volume $\left[\AA^{3}\right]$ & $1965.5(7)$ & max. diff peak / hole $\left[\mathrm{e} \AA^{-3}\right]$ & $0.337 /-0.283$
\end{tabular}




\subsubsection{Crystal structure of $\mathrm{C}_{6} \mathrm{H}_{6} @\left[1-(\mathrm{S}) \mathrm{PPh}_{2}-\left(\mathrm{C}_{14} \mathrm{H}_{9}\right)\right](20 \mathrm{a})$}

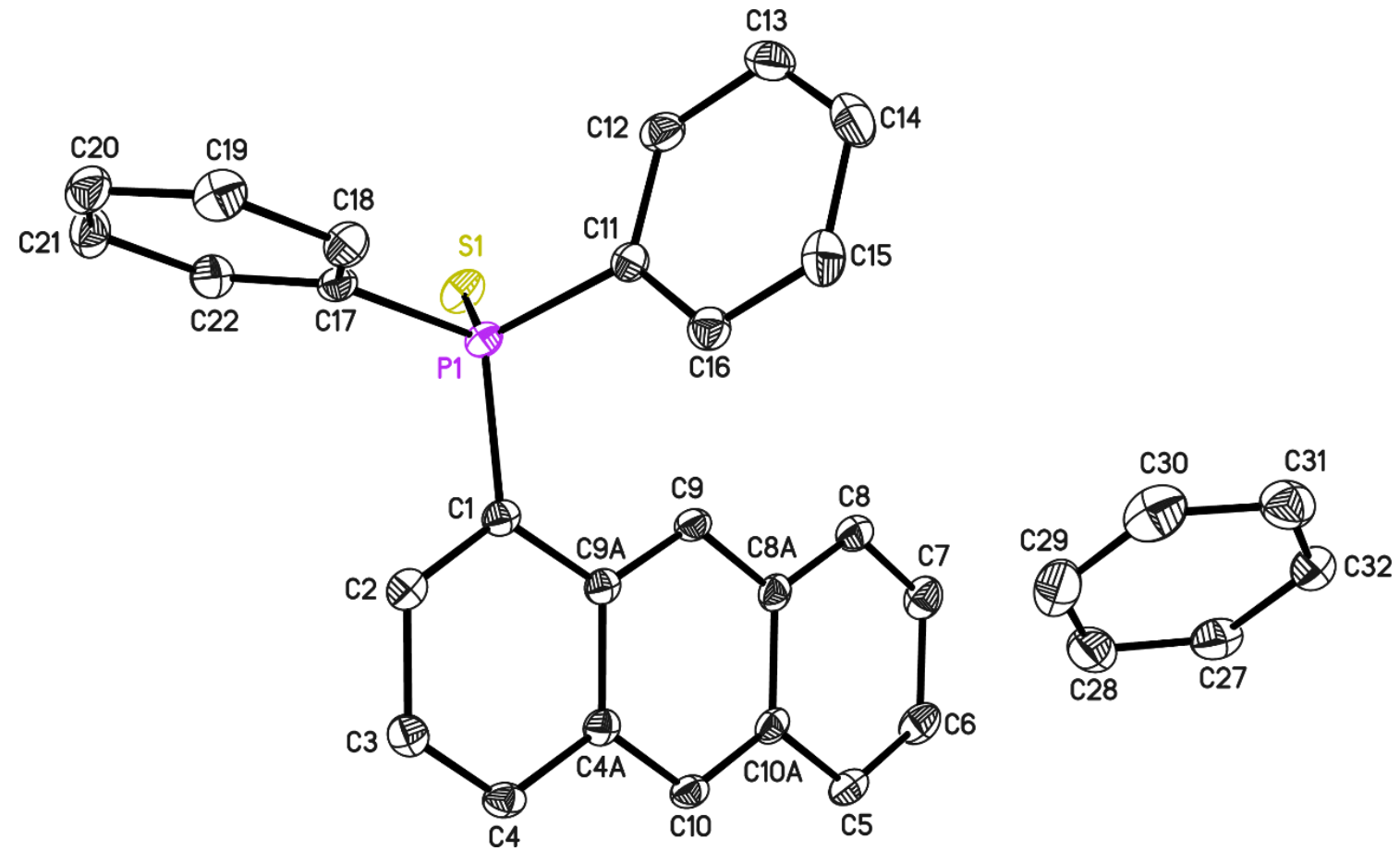

Figure 103. Asymmetric unit of 20a. The anisotropic displacement parameters are depicted at the 50\% probability level. Hydrogen atoms are omitted for clarity.

Table 43. Crystallographic data of 20a.

\begin{tabular}{|c|c|c|c|}
\hline Structure Code & ts232_c6h6 & Z & 2 \\
\hline Empirical formula & $\mathrm{C}_{32} \mathrm{H}_{25} \mathrm{PS}$ & $\rho_{\text {calc }}$ & 1.291 \\
\hline Formula weight $[\mathrm{g} / \mathrm{mol}]$ & 472.55 & $\mu\left[\mathrm{mm}^{-1}\right]$ & 0.120 \\
\hline Temperature [K] & $100(2)$ & $F(000)$ & 496 \\
\hline Wavelength $[\AA ̊]$ & 0.56086 & $\theta$ range $\left[{ }^{\circ}\right]$ & 1.763 to 20.594 \\
\hline Crystal system & Triclinic & Reflections collected & 101888 \\
\hline space group & $P \overline{1}$ & Independent reflections & 4993 \\
\hline $\mathrm{a}[\AA]$ & $9.448(2)$ & $\mathrm{R}$ (int) & 0.0638 \\
\hline $\mathrm{b}[\AA]$ & $10.737(2)$ & Max. / min. transmission & $0.7445 / 0.7190$ \\
\hline$c[\AA]$ & $12.653(3)$ & Restraints / parameter & $0 / 307$ \\
\hline$\alpha\left[^{\circ}\right]$ & $78.97(2)$ & GooF & 1.036 \\
\hline$\beta\left[^{\circ}\right]$ & $86.69(3)$ & $\mathrm{R} 1 / w \mathrm{R} 2(\mathrm{I}>2 \sigma(\mathrm{I}))$ & $0.0324 / 0.0770$ \\
\hline$\gamma\left[^{\circ}\right]$ & $74.95(2)$ & $\mathrm{R} 1 / w \mathrm{R} 2$ (all data) & $0.0423 / 0.0819$ \\
\hline Volume $\left[\AA^{3}\right]$ & $1215.3(5)$ & max. diff peak / hole $\left[e \AA^{-3}\right]$ & 0.327 / - 0.302 \\
\hline
\end{tabular}




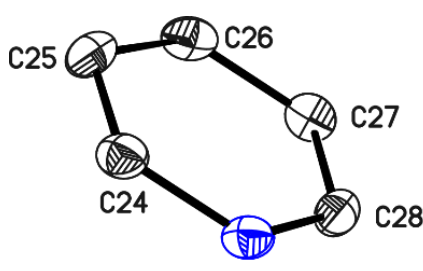

N1
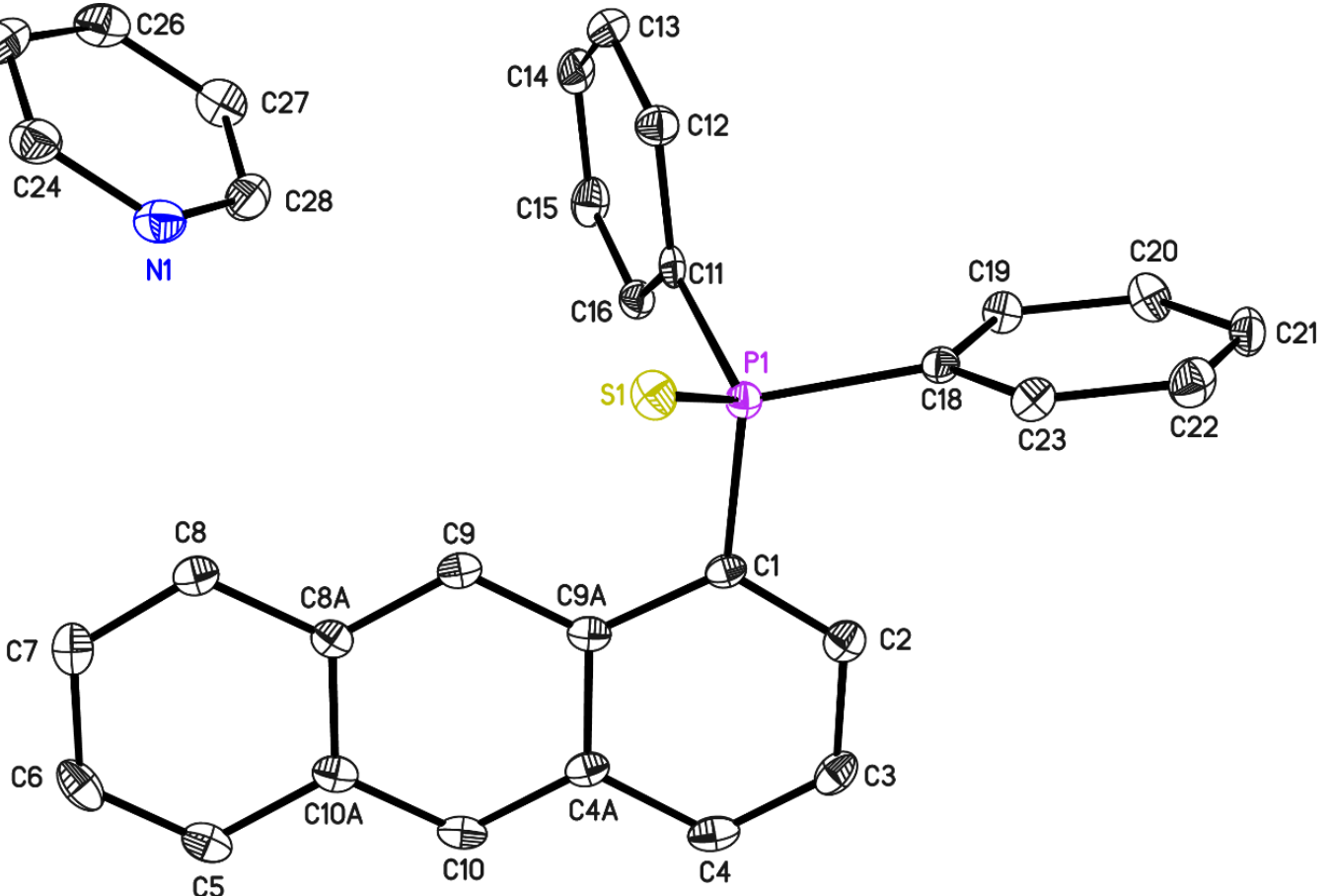

Figure 104. Asymmetric unit of 20b. The anisotropic displacement parameters are depicted at the 50\% probability level. Hydrogen atoms are omitted for clarity.

Table 44. Crystallographic data of $20 \mathrm{~b}$.

\begin{tabular}{|c|c|c|c|}
\hline Structure Code & ts232_c6h5n & Z & 2 \\
\hline Empirical formula & $\mathrm{C}_{31} \mathrm{H}_{24} \bar{N} \mathrm{NS}$ & $\rho_{\text {calc }}$ & 1.335 \\
\hline Formula weight $[\mathrm{g} / \mathrm{mol}]$ & 473.54 & $\mu\left[\mathrm{mm}^{-1}\right]$ & 0.124 \\
\hline Temperature $[\mathrm{K}]$ & $100(2)$ & $F(000)$ & 496 \\
\hline Wavelength $[\AA ̊]$ & 0.56086 & $\theta$ range $\left[{ }^{\circ}\right]$ & 1.730 to 20.599 \\
\hline Crystal system & Triclinic & Reflections collected & 34641 \\
\hline space group & & Independent reflections & 4883 \\
\hline $\mathrm{a}[\AA]$ & $9.230(2)$ & $\mathrm{R}$ (int) & 0.1359 \\
\hline $\mathrm{b}[\AA]$ & $9.706(2)$ & Max. / min. transmission & $0.7445 / 0.7065$ \\
\hline$c[\AA ̊]$ & $13.905(3)$ & Restraints / parameter & $0 / 308$ \\
\hline$\alpha\left[^{\circ}\right]$ & $78.17(2)$ & GooF & 0.913 \\
\hline$\beta\left[^{\circ}\right]$ & $81.21(3)$ & $\mathrm{R} 1 / w \mathrm{R} 2(\mathrm{I}>2 \sigma(\mathrm{I}))$ & $0.0442 / 0.0909$ \\
\hline$\gamma\left[^{\circ}\right]$ & $76.45(2)$ & $\mathrm{R} 1 /$ wR2 (all data) & $0.0718 / 0.0983$ \\
\hline Volume $\left[\AA^{3}\right]$ & $1178.1(5)$ & max. diff peak / hole $\left[e \AA^{-3}\right]$ & $0.353 /-0.433$ \\
\hline
\end{tabular}




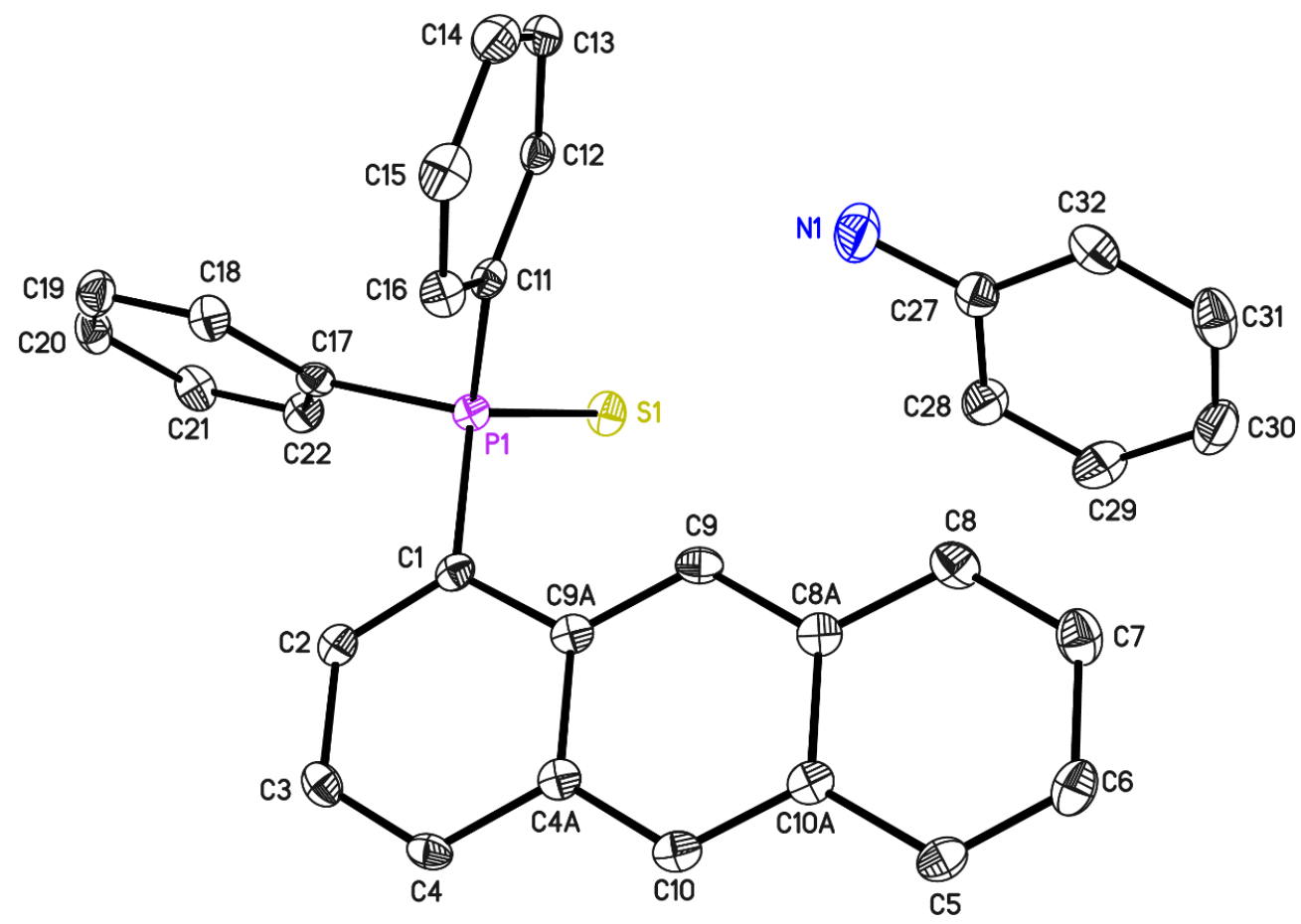

Figure 105. Asymmetric unit of 20c. The anisotropic displacement parameters are depicted at the $50 \%$ probability level. Hydrogen atoms are omitted for clarity.

Table 45. Crystallographic data of 20c.

Structure Code

Empirical formula

Formula weight $[\mathrm{g} / \mathrm{mol}]$

Temperature [K]

Wavelength $[\AA ̊]$

Crystal system

space group

a $[\AA ̊]$

b $[\AA]$

c $[\AA]$

$\alpha\left[^{\circ}\right]$

$\beta\left[^{\circ}\right]$

$\gamma\left[^{\circ}\right]$

Volume $\left[\AA^{3}\right]$

\begin{tabular}{|l} 
ts232_aniline \\
$\mathrm{C}_{32} \mathrm{H}_{26} \mathrm{NPS}$ \\
487.57 \\
$100(2)$ \\
0.56086 \\
Triclinic \\
$P \overline{1}$ \\
$9.397(2)$ \\
$10.926(2)$ \\
$12.969(3)$ \\
$79.18(2)$ \\
$88.58(3)$ \\
$74.97(2)$ \\
$1236.2(5)$
\end{tabular}

2

1.310

0.120

512

1.771 to 20.301

31092

4918

0.0766

0.7445 / 0.6941

$1 / 325$

1.017

$0.0421 / 0.0859$

$0.0673 / 0.0964$

0.308 / - 0.364 

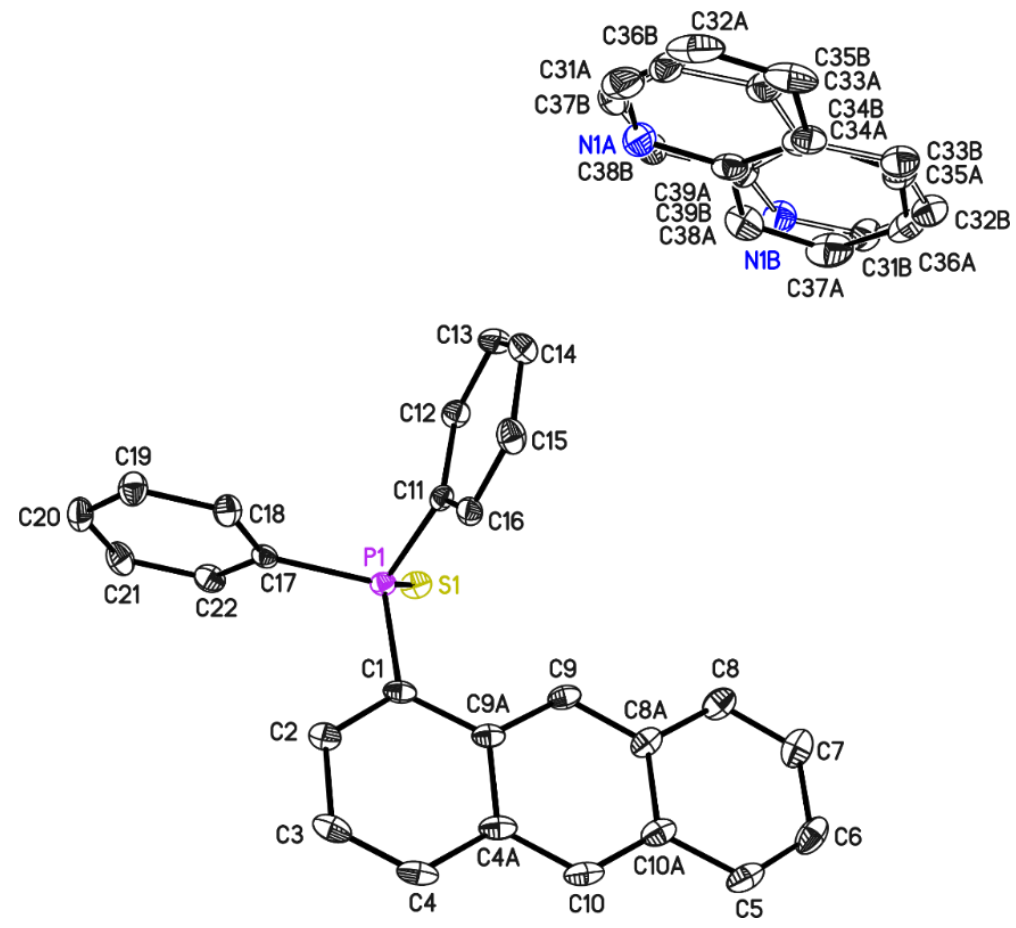

Figure 106. Asymmetric unit of 20d. The anisotropic displacement parameters are depicted at the $50 \%$ probability level. Hydrogen atoms are omitted for clarity. The quinoline molecule is disordered over two positions. It was refined with distance restraints and restraints for the anisotropic displacement parameters. The occupancy of the major component refined to $0.792(7)$.

Table 46. Crystallographic data of $20 \mathrm{~d}$.

\begin{tabular}{l|l|l|l} 
Structure Code & ts181_quinoline & $\mathrm{Z}$ & 2 \\
Empirical formula & $\mathrm{C}_{35} \mathrm{H}_{26} \mathrm{NPS}$ & $\rho_{\text {calc }}$ & 1.327 \\
Formula weight $[\mathrm{g} / \mathrm{mol}]$ & 523.60 & $\mu\left[\mathrm{mm}^{-1}\right]$ & 0.116 \\
Temperature $[\mathrm{K}]$ & $100(2)$ & $\mathrm{F}(000)$ & 548 \\
Wavelength $[\AA]$ & 0.56086 & $\theta$ range $\left[^{\circ}\right]$ & 1.622 to 19.782 \\
Crystal system & Triclinic & Reflections collected & 33656 \\
space group & $P \overline{1}$ & Independent reflections & 4830 \\
$\mathrm{a}[\AA \AA$ & $\mathrm{A}]$ & $\mathrm{R}($ int) & 0.0669 \\
$\mathrm{~b}[\AA]$ & $10.544(2)$ & Max. $/$ min. transmission & $0.7444 / 0.7077$ \\
$\mathrm{c}[\AA]$ & $11.403(2)$ & Restraints $/$ parameter & $531 / 434$ \\
$\alpha\left[^{\circ}\right]$ & $12.930(3)$ & GooF & 1.021 \\
$\beta\left[^{\circ}\right]$ & $102.89(2)$ & $\mathrm{R} 1 / w R 2(\mathrm{I}>2 \sigma(\mathrm{I}))$ & $0.0384 / 0.0821$ \\
$\gamma\left[^{\circ}\right]$ & $103.98(2)$ & $\mathrm{R} 1 / w R 2($ all data) & $0.0620 / 0.0910$ \\
Volume $\left[\AA^{3}\right]$ & $112.01(3)$ & max. diff peak / hole $\left[\mathrm{e} \AA^{-3}\right]$ & $0.286 /-0.342$
\end{tabular}




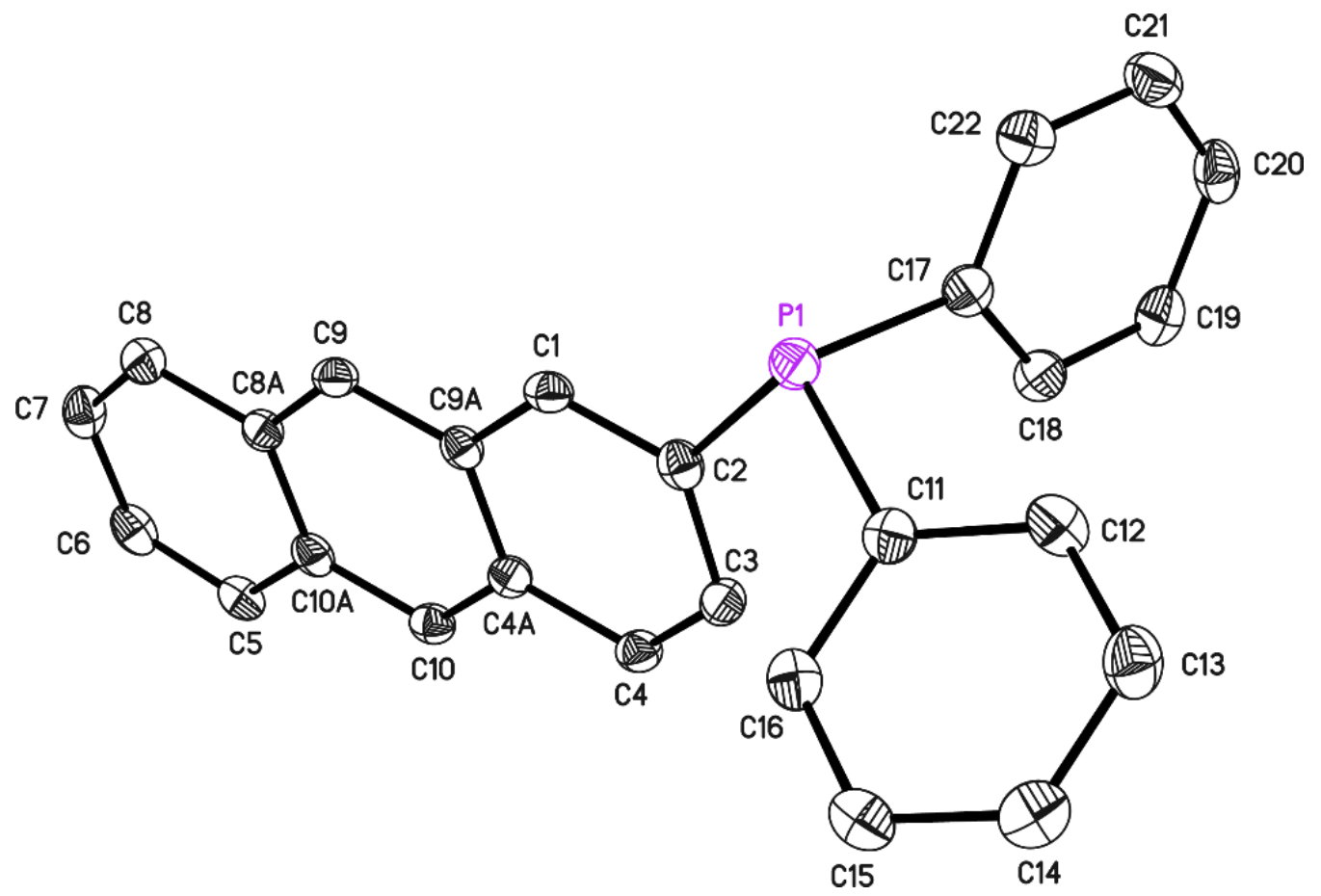

Figure 107. Asymmetric unit of 21. The anisotropic displacement parameters are depicted at the $50 \%$ probability level. Hydrogen atoms are omitted for clarity.

Table 47. Crystallographic data of 21.

\begin{tabular}{|c|c|c|c|}
\hline Structure Code & ts303 & Z & 8 \\
\hline Empirical formula & $\mathrm{C}_{26} \mathrm{H}_{19} \mathrm{P}$ & $\rho_{\text {calc }}$ & 1.307 \\
\hline Formula weight $[\mathrm{g} / \mathrm{mol}]$ & 362.38 & $\mu\left[\mathrm{mm}^{-1}\right]$ & 0.157 \\
\hline Temperature [K] & $100(2)$ & $F(000)$ & 1520 \\
\hline Wavelength $[\AA]$ & 0.71073 & $\theta$ range $\left[{ }^{\circ}\right]$ & 2.142 to 25.729 \\
\hline Crystal system & Monoclinic & Reflections collected & 38072 \\
\hline space group & $\mathrm{C} 2 / \mathrm{c}$ & Independent reflections & 3522 \\
\hline $\mathrm{a}[\AA]$ & $24.510(4)$ & $\mathrm{R}$ (int) & 0.1119 \\
\hline $\mathrm{b}[\AA]$ & $6.044(2)$ & Max. / min. transmission & $0.7453 / 0.6845$ \\
\hline$c[\AA]$ & $25.237(4)$ & Restraints / parameter & $0 / 244$ \\
\hline$\alpha\left[^{\circ}\right]$ & 90 & GooF & 1.020 \\
\hline$\beta\left[^{\circ}\right]$ & $99.76(2)$ & $\mathrm{R} 1 / w \mathrm{R} 2(\mathrm{I}>2 \sigma(\mathrm{I}))$ & $0.0446 / 0.0866$ \\
\hline$\gamma\left[^{\circ}\right]$ & 90 & $\mathrm{R} 1 / w \mathrm{R} 2$ (all data) & $0.0824 / 0.1015$ \\
\hline Volume $\left[\AA^{3}\right]$ & $3684.5(15)$ & max. diff peak / hole $\left[e \AA^{-3}\right]$ & $0.322 /-0342$ \\
\hline
\end{tabular}




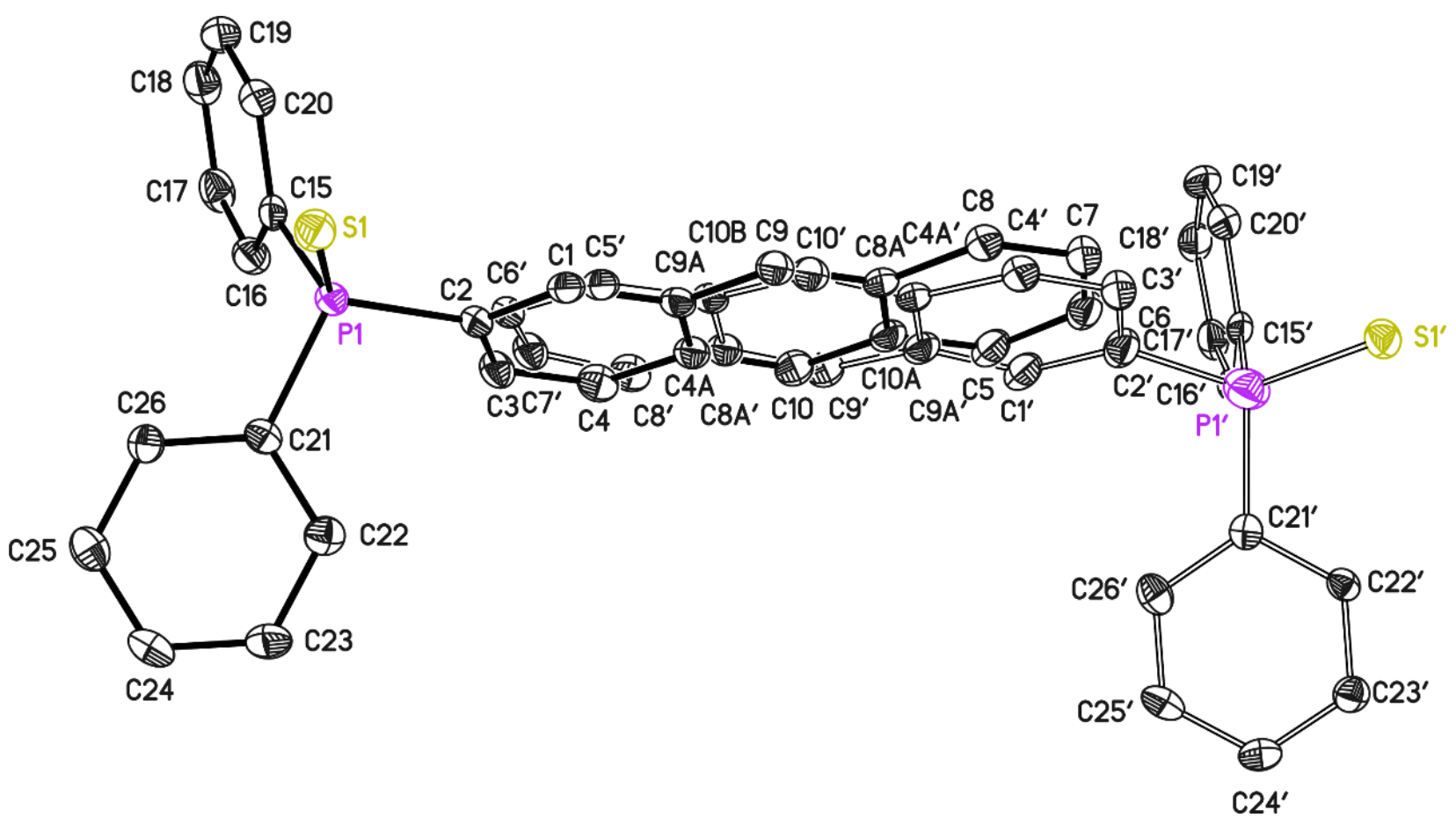

Figure 108. Asymmetric unit of 22. The anisotropic displacement parameters are depicted at the $50 \%$ probability level. Hydrogen atoms are omitted for clarity. The whole molecule is disordered over two positions, whereas the substituents adopts a different orientation by rotation of around $180^{\circ}$. The occupancy of the minor component refines to $0.0652(15)$.

Table 48. Crystallographic data of 22.

\begin{tabular}{|c|c|c|c|}
\hline Structure Code & ts175 & Z & 4 \\
\hline Empirical formula & $\mathrm{C}_{26} \mathrm{H}_{19} \mathrm{PS}$ & $\rho_{\text {calc }}$ & 1.342 \\
\hline Formula weight $[\mathrm{g} / \mathrm{mol}]$ & 394.44 & $\mu\left[\mathrm{mm}^{-1}\right]$ & 0.257 \\
\hline Temperature $[\mathrm{K}]$ & $100(2)$ & $F(000)$ & 824 \\
\hline Wavelength $[\AA ̊]$ & 0.71073 & $\theta$ range $\left[{ }^{\circ}\right]$ & 2.057 to 26.743 \\
\hline Crystal system & Monoclinic & Reflections collected & 40972 \\
\hline space group & $\mathrm{P} 2{ }_{1} / \mathrm{n}$ & Independent reflections & 4151 \\
\hline $\mathrm{a}[\AA]$ & $9.649(2)$ & $\mathrm{R}$ (int) & 0.0884 \\
\hline $\mathrm{b}[\AA ̊]$ & $16.139(3)$ & Max. / min. transmission & 0.7454 / 0.7056 \\
\hline$c[\AA]$ & $13.295(3)$ & Restraints / parameter & $695 / 344$ \\
\hline$\alpha\left[^{\circ}\right]$ & 90 & GooF & 1.047 \\
\hline$\beta\left[^{\circ}\right]$ & $109.43(2)$ & $\mathrm{R} 1 / w \mathrm{R} 2(\mathrm{I}>2 \sigma(\mathrm{I}))$ & $0.0426 / 0.0975$ \\
\hline$\gamma\left[^{\circ}\right]$ & 90 & R1 / wR2 (all data) & 0.0559 / 0.1017 \\
\hline Volume $\left[\AA^{3}\right]$ & $1952.5(7)$ & max. diff peak / hole $\left[\right.$ e $\AA^{-3}$ ] & $0.336 /-0.370$ \\
\hline
\end{tabular}




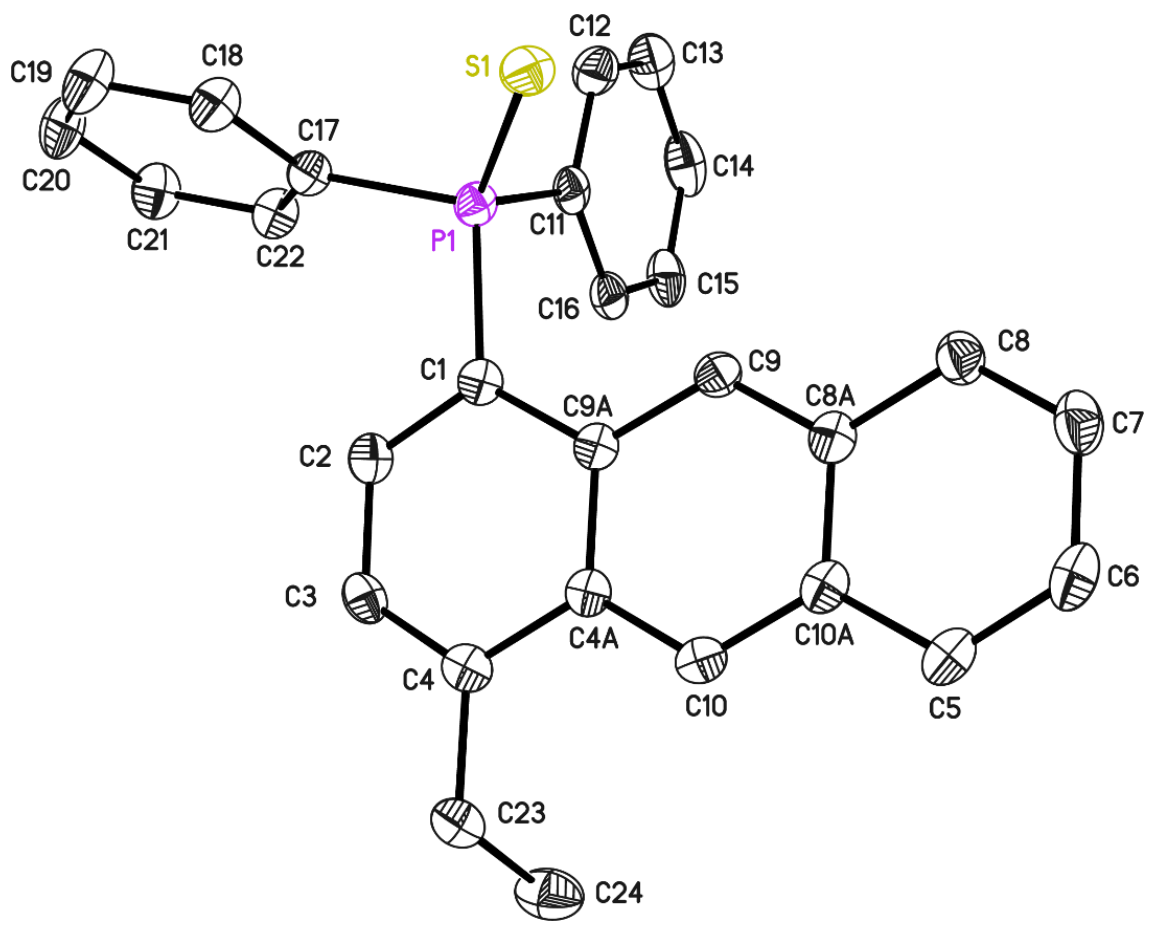

Figure 109. Asymmetric unit of 24. The anisotropic displacement parameters are depicted at the $50 \%$ probability level. Hydrogen atoms are omitted for clarity.

Table 49. Crystallographic data of 24.

\begin{tabular}{l|l|l|l} 
Structure Code & ts361_tol & $\mathrm{Z}$ & 4 \\
Empirical formula & $\mathrm{C}_{28} \mathrm{H}_{23} \mathrm{PS}$ & $\rho_{\text {calc }}$ & 1.298 \\
Formula weight $[\mathrm{g} / \mathrm{mol}]$ & 422.49 & $\mu\left[\mathrm{mm}^{-1}\right]$ & 0.237 \\
Temperature $[\mathrm{K}]$ & $100(2)$ & $\mathrm{F}(000)$ & 888 \\
Wavelength $[\AA]$ & 0.71073 & $\theta$ range $\left[^{\circ}\right]$ & 2.217 to 26.658 \\
Crystal system & monoclinic & Reflections collected & 45044 \\
space group & $P 2_{1} / \mathrm{n}$ & Independent reflections & 4420 \\
$\mathrm{a}[\AA]$ & $11.7111(2)$ & $\mathrm{R}($ int $)$ & 0.0749 \\
$\mathrm{~b}[\AA]$ & $10.962(2)$ & Max. $/$ min. transmission & $0.7454 / 0.6785$ \\
$\mathrm{c}[\AA]$ & $17.881(3)$ & Restraints $/$ parameter & $0 / 272$ \\
$\alpha\left[^{\circ}\right]$ & 90 & GooF & 1.013 \\
$\beta\left[^{\circ}\right]$ & $109.63(2)$ & $\mathrm{R} 1 / w R 2(\mathrm{I}>2 \sigma(\mathrm{I}))$ & $0.0415 / 0.0913$ \\
$\gamma\left[^{\circ}\right]$ & 90 & $\mathrm{R} 1 / w R 2($ all data) & $0.0714 / 0.1034$ \\
Volume $\left[\AA^{3}\right]$ & $2162.1(7)$ & max. diff peak / hole $\left[\mathrm{e} \AA^{-3}\right]$ & $0.443 /-0.297$
\end{tabular}




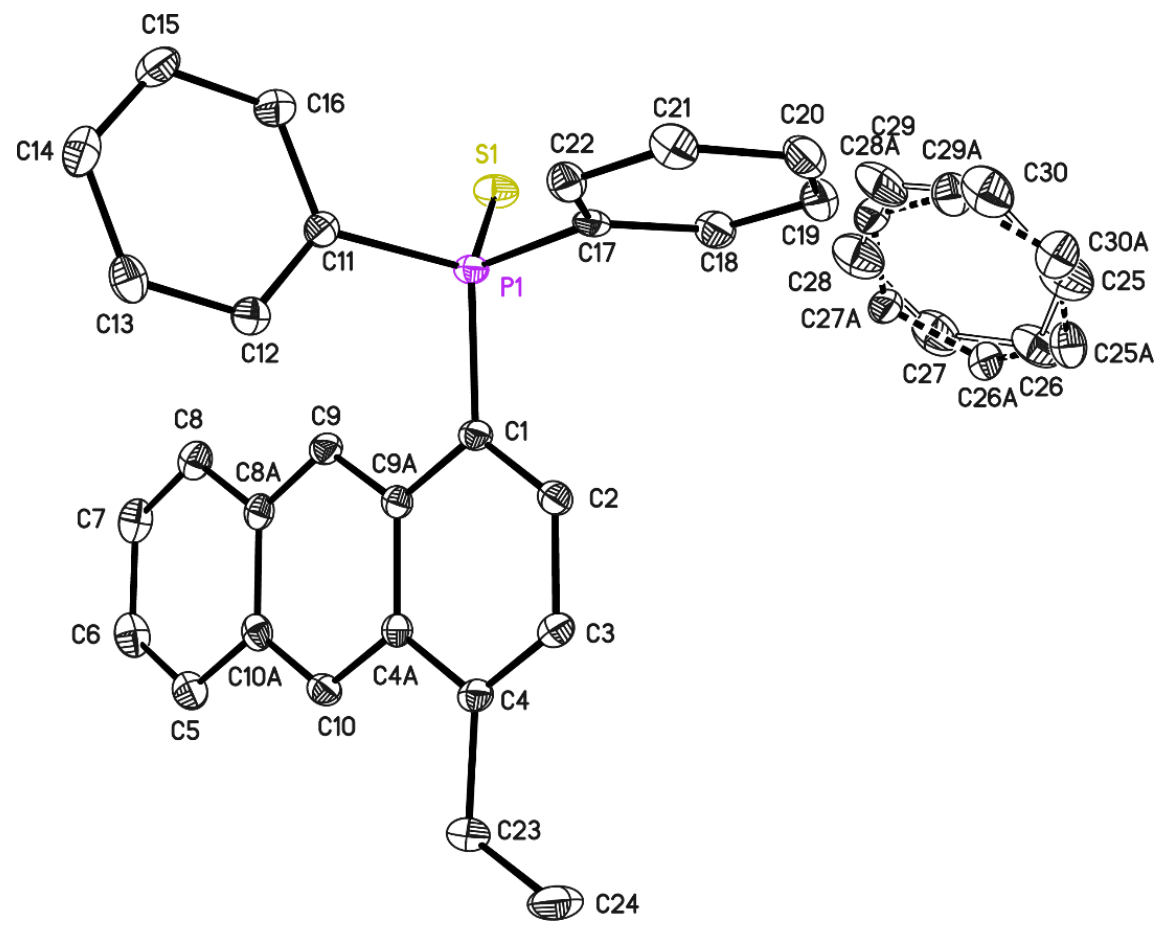

Figure 110. Asymmetric unit of 24a. The anisotropic displacement parameters are depicted at the $50 \%$ probability level. Hydrogen atoms are omitted for clarity. The benzene molecule is disordered over four positions two each related by an inversion center. It was refined with distance restraints and restraints for the anisotropic displacement parameters. The occupancy of the major component refined to $0.368(11)$.

Table 50. Crystallographic data of $\mathbf{2 4 a}$.

\begin{tabular}{|c|c|c|c|}
\hline Structure Code & ts360_c6h62 & Z & 8 \\
\hline Empirical formula & $\mathrm{C}_{28} \mathrm{H}_{23} \mathrm{PS} \cdot 0.5 \mathrm{C}_{6} \mathrm{H}_{6}$ & $\rho_{\text {calc }}$ & 1.278 \\
\hline Formula weight $[\mathrm{g} / \mathrm{mol}]$ & 461.55 & $\mu\left[\mathrm{mm}^{-1}\right]$ & 0.219 \\
\hline Temperature $[\mathrm{K}]$ & $100(2)$ & $F(000)$ & 1944 \\
\hline Wavelength $[\AA]$ & 0.71073 & $\theta$ range $\left[{ }^{\circ}\right]$ & 1.946 to 26.393 \\
\hline Crystal system & monoclinic & Reflections collected & 65041 \\
\hline space group & $C 2 / c$ & Independent reflections & 4925 \\
\hline $\mathrm{a}[\AA \AA]$ & $16.448(2)$ & $\mathrm{R}$ (int) & 0.0534 \\
\hline $\mathrm{b}[\AA]$ & $14.176(2)$ & Max. / min. transmission & 0.7454 / 0.7147 \\
\hline$c[\AA]$ & $21.798(3)$ & Restraints / parameter & $390 / 381$ \\
\hline$\alpha\left[^{\circ}\right]$ & 90 & GooF & 1.042 \\
\hline$\beta\left[^{\circ}\right]$ & $109.73(2)$ & $\mathrm{R} 1 / w \mathrm{R} 2(\mathrm{I}>2 \sigma(\mathrm{I}))$ & $0.0316 / 0.0766$ \\
\hline$\gamma\left[^{\circ}\right]$ & 90 & $\mathrm{R} 1 / w \mathrm{R} 2$ (all data) & $0.0393 / 0.0813$ \\
\hline Volume $\left[\AA^{3}\right]$ & $4795.8(12)$ & max. diff peak / hole $\left[e \AA^{-3}\right]$ & $0.343 /-0.307$ \\
\hline
\end{tabular}




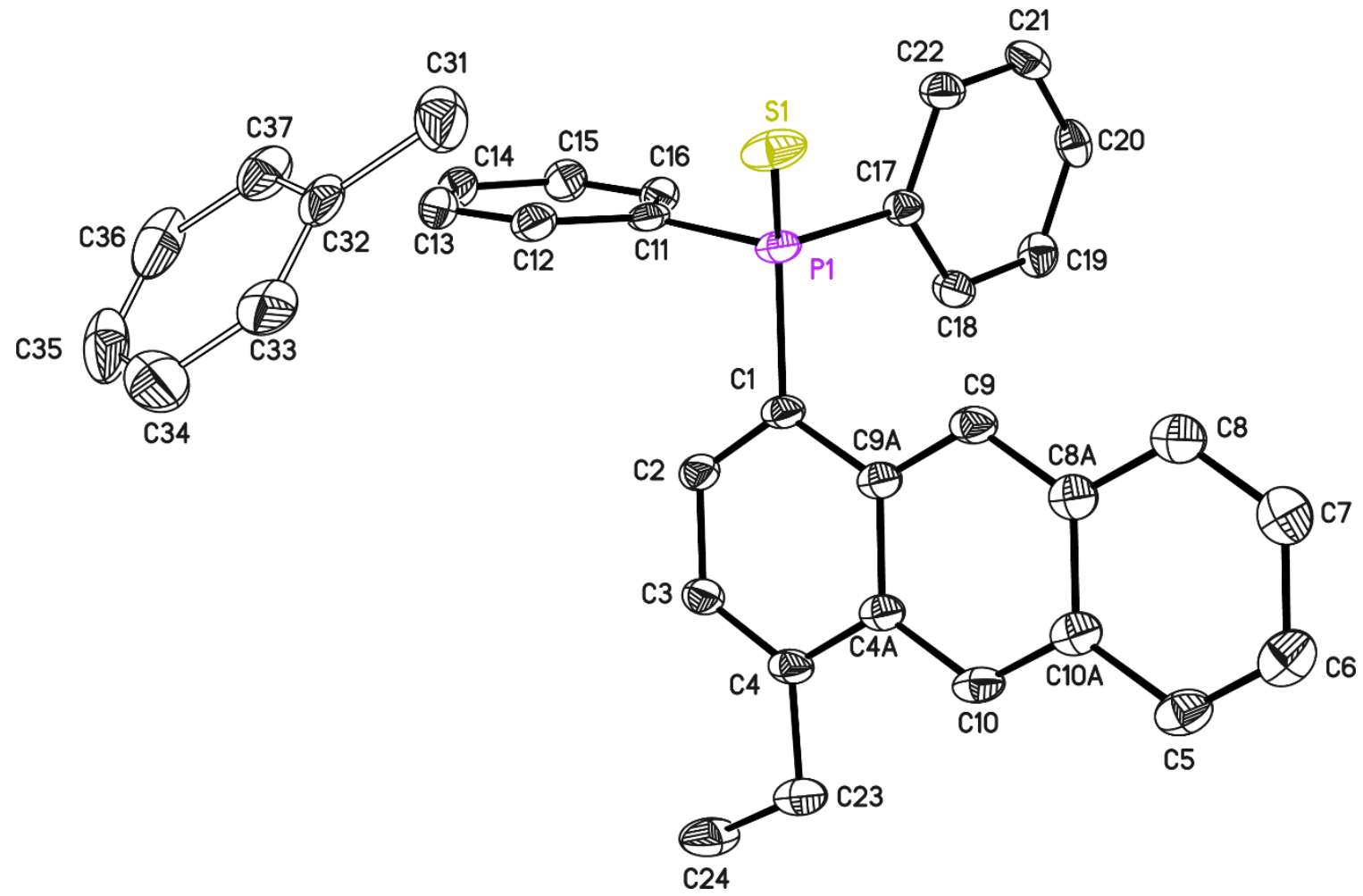

Figure 111. Asymmetric unit of 24b. The anisotropic displacement parameters are depicted at the $50 \%$ probability level. Hydrogen atoms are omitted for clarity. The toluene molecule is disordered over an inversion center. It was refined with distance restraints and restraints for the anisotropic displacement parameters.

Table 51. Crystallographic data of 24b.

\begin{tabular}{l|l|l|l} 
Structure Code & ts360_toluol & $\mathrm{Z}$ & 8 \\
Empirical formula & $\mathrm{C}_{28} \mathrm{H}_{23} \mathrm{PS} \cdot 0.5 \mathrm{C}_{7} \mathrm{H}_{8}$ & $\rho_{\text {calc }}$ & 1.284 \\
Formula weight $[\mathrm{g} / \mathrm{mol}]$ & 468.56 & $\mu\left[\mathrm{mm}^{-1}\right]$ & 0.218 \\
Temperature $[\mathrm{K}]$ & $100(2)$ & $\mathrm{F}(000)$ & 1976 \\
Wavelength $[\AA]$ & 0.71073 & $\theta$ range $\left[^{\circ}\right]$ & 1.962 to 26.445 \\
Crystal system & monoclinic & Reflections collected & 64607 \\
space group & $C 2 / \mathrm{c}$ & Independent reflections & 4996 \\
$\mathrm{a}[\AA \AA]$ & $16.506(2)$ & $\mathrm{R}($ int) & 0.0509 \\
$\mathrm{~b}[\AA]$ & $14.293(2)$ & Max. $/$ min. transmission & $0.7454 / 0.7165$ \\
$\mathrm{c}[\AA]$ & $21.854(3)$ & Restraints / parameter & $97 / 336$ \\
$\alpha\left[^{\circ}\right]$ & 90 & GooF & 1.030 \\
$\beta\left[^{\circ}\right]$ & $109.93(2)$ & R1 $/ w R 2(\mathrm{I}>2 \sigma(\mathrm{I}))$ & $0.0328 / 0.0794$ \\
$\gamma\left[^{\circ}\right]$ & 90 & R1 $/ w R 2($ all data) & $0.0450 / 0.0864$ \\
Volume $\left[\AA^{3}\right]$ & $4847.0(13)$ & max. diff peak / hole $\left[\mathrm{e} \AA^{-3}\right]$ & $0.295 /-0.310$
\end{tabular}




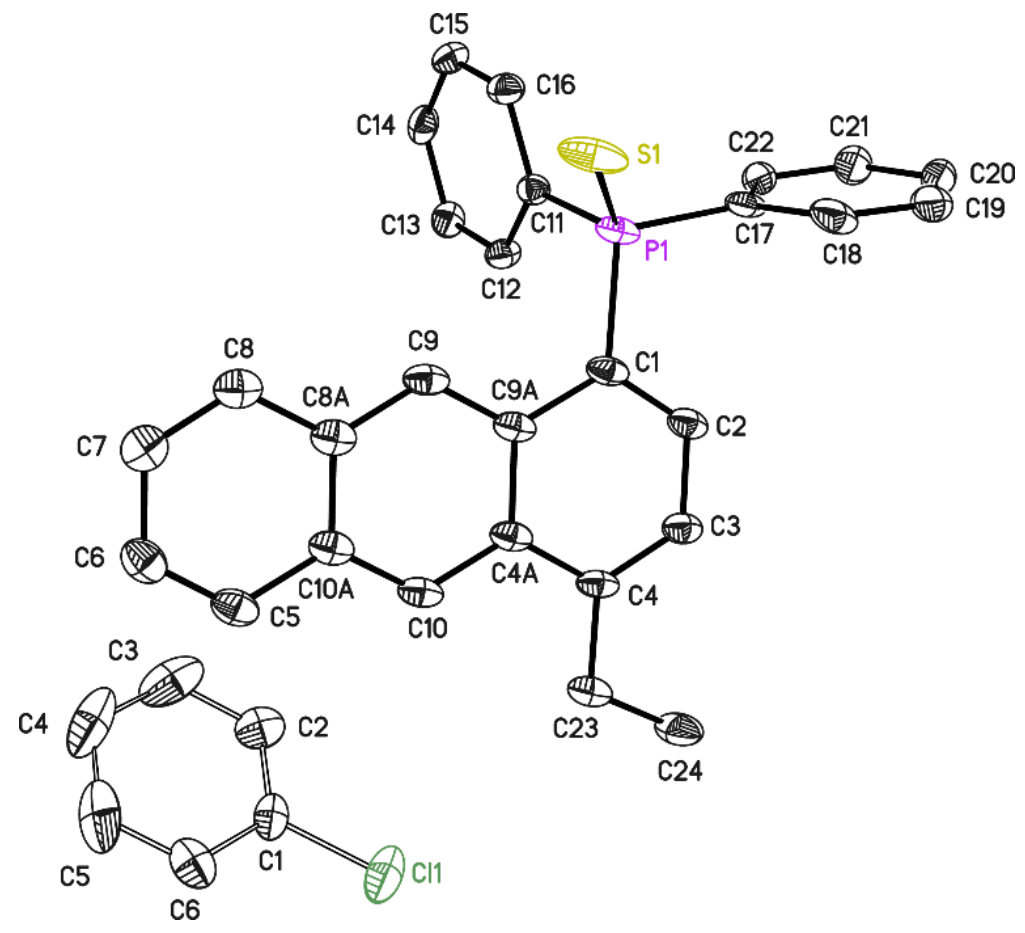

Figure 112. Asymmetric unit of 24c. The anisotropic displacement parameters are depicted at the $50 \%$ probability level. Hydrogen atoms are omitted for clarity. The chlorobenzene molecule is disordered over an inversion center. It was refined with distance restraints and restraints for the anisotropic displacement parameters.

Table 52. Crystallographic data of 24c.

\begin{tabular}{l|l} 
Structure Code & ts $361 \_c 6 h 5 c l$ \\
Empirical formula & $\mathrm{C}_{28} \mathrm{H}_{23} \mathrm{PS} \cdot 0.5 \mathrm{C}_{6} \mathrm{H}_{5} \mathrm{Cl}$ \\
Formula weight $[\mathrm{g} / \mathrm{mol}]$ & 957.53 \\
Temperature $[\mathrm{K}]$ & $100(2)$ \\
Wavelength $[\AA]$ & 0.71073 \\
Crystal system & monoclinic \\
space group & $\mathrm{C} / \mathrm{c}$ \\
a $[\AA]$ & $16.557(2)$ \\
$\mathrm{b}[\AA]$ & $14.273(2)$ \\
$\mathrm{c}[\AA]$ & $21.811(3)$ \\
$\alpha\left[^{\circ}\right]$ & 90 \\
$\beta\left[^{\circ}\right]$ & $109.69(2)$ \\
$\gamma\left[^{\circ}\right]$ & 90 \\
Volume $\left[\AA^{3}\right]$ & $4853.0(13)$
\end{tabular}

\begin{tabular}{|l|l}
$Z$ & 4 \\
$\rho_{\text {calc }}$ & 1.311 \\
$\left.\mu \mathrm{mm}^{-1}\right]$ & 0.273 \\
$\mathrm{~F}(000)$ & 2008 \\
$\theta$ range $\left[^{\circ}\right]$ & 1.935 to 26.454 \\
Reflections collected & 42666 \\
Independent reflections & 4997 \\
R(int) & 0.0439 \\
Max. / min. transmission & $0.7454 / 0.7167$ \\
Restraints / parameter & $119 / 335$ \\
GooF & 1.021 \\
R1 / wR2 (I > 2 $\sigma(I))$ & 0.0372 / 0.0888 \\
R1 / wR2 (all data) & $0.0510 / 0.0966$ \\
max. diff peak / hole [e $\left.\AA^{-3}\right]$ & $0.475 /-0.436$
\end{tabular}



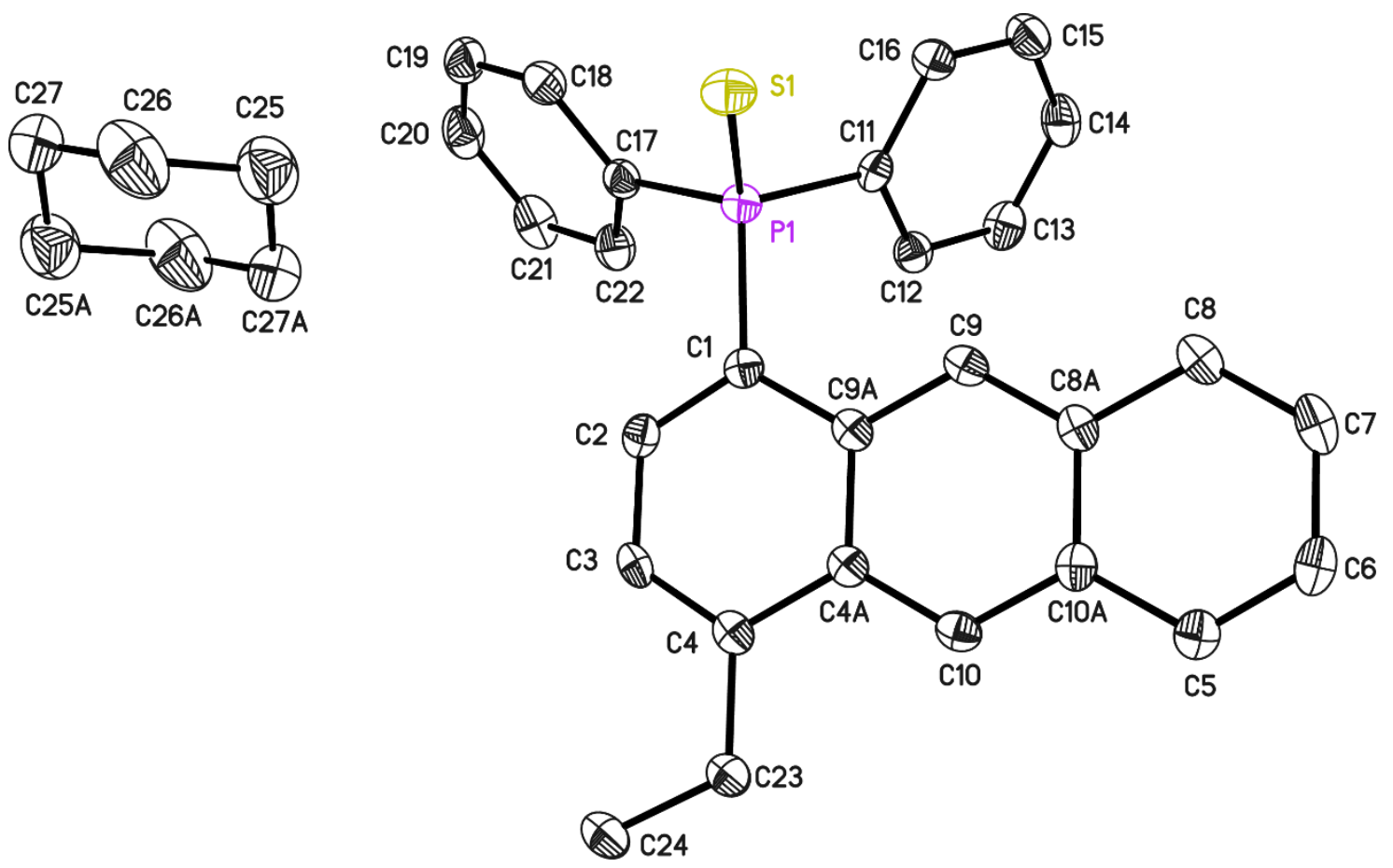

Figure 113. Asymmetric unit of $\mathbf{2 4 d}$. The anisotropic displacement parameters are depicted at the $50 \%$ probability level. Hydrogen atoms are omitted for clarity. The second half of the cyclohexane molecule is generated by an inversion center.

Table 53. Crystallographic data of $\mathbf{2 4 d .}$

Structure Code
Empirical formula
Formula weight $[\mathrm{g} / \mathrm{mol}]$
Temperature $[\mathrm{K}]$
Wavelength $[\AA \AA$
Crystal system
space group
a $[\AA]$
$\mathrm{b}[\AA \AA]$
$\mathrm{c}[\AA]$
$\alpha\left[^{\circ}\right]$
$\beta\left[^{\circ}\right]$
$\gamma\left[^{\circ}\right]$
Volume $\left[\AA^{3}\right]$

\begin{tabular}{|l} 
ts361_c6h12 \\
$\mathrm{C}_{28} \mathrm{H}_{23} \mathrm{PS} \cdot 0.5 \mathrm{C}_{6} \mathrm{H}_{12}$ \\
464.57 \\
$100(2)$ \\
0.71073 \\
triclinic \\
$P \overline{1}$ \\
$7.338(3)$ \\
$10.836(4)$ \\
$15.917(6)$ \\
$76.05(2)$ \\
$84.53(2)$ \\
$88.18(3)$ \\
$1222.7(8)$
\end{tabular}

\begin{tabular}{|l|l}
$Z$ & 2 \\
$\rho_{\text {calc }}$ & 1.262 \\
$\mu\left[\mathrm{mm}^{-1}\right]$ & 0.215 \\
$\mathrm{~F}(000)$ & 492 \\
$\theta$ range $\left[^{\circ}\right]$ & 1.937 to 25.641 \\
Reflections collected & 26054 \\
Independent reflections & 4548 \\
$\mathrm{R}($ int) & 0.0787 \\
Max. / min. transmission & 0.7452 / 0.6835 \\
Restraints / parameter & $0 / 299$ \\
GooF & 1.015 \\
R1 / wR2 (I > 2 $\sigma(I))$ & $0.0443 / 0.0945$ \\
R1 / wR2 (all data) & $0.0817 / 0.1099$ \\
max. diff peak / hole [e $\left.\AA^{-3}\right]$ & $0.326 /-0.276$
\end{tabular}




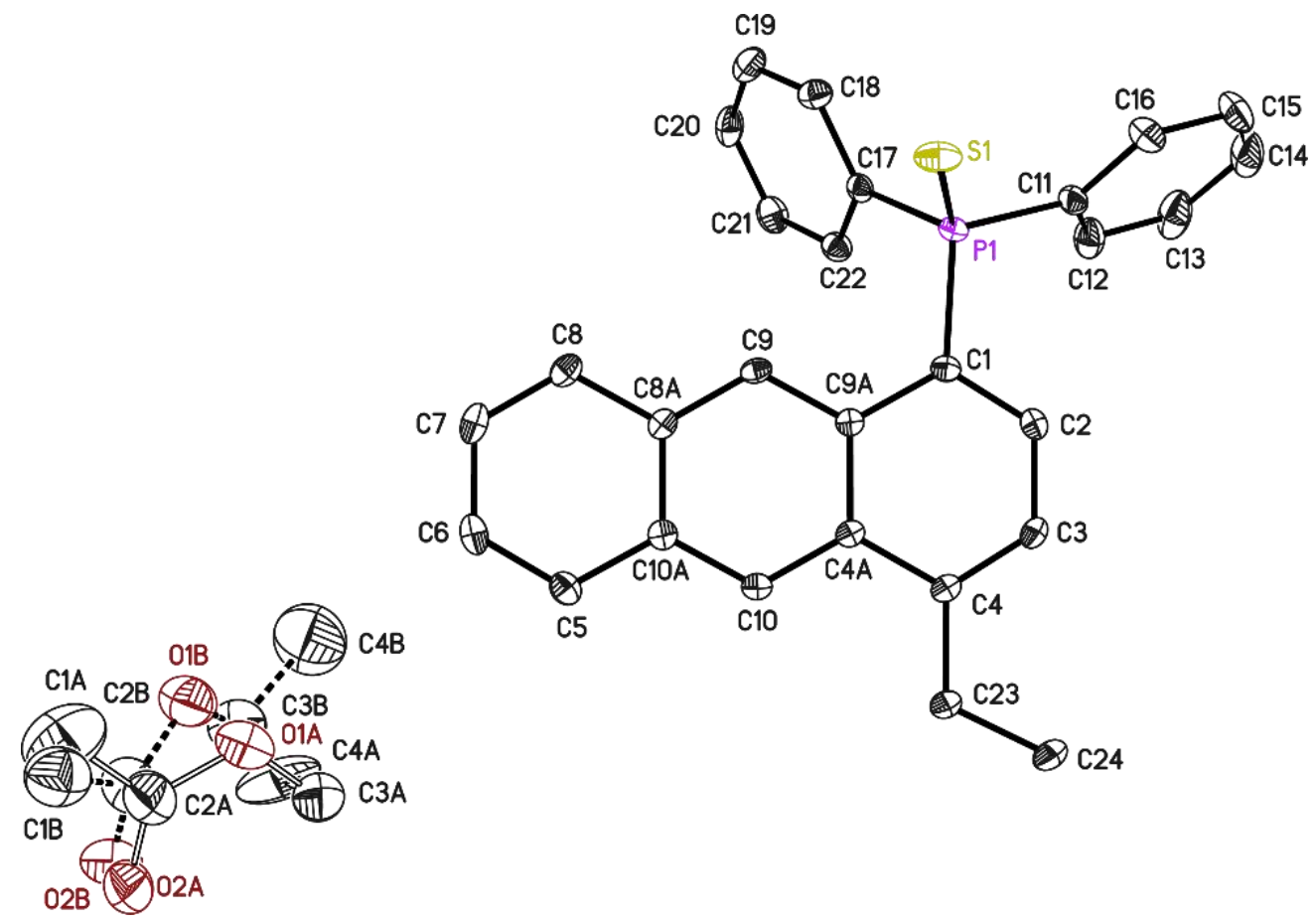

Figure 114. Asymmetric unit of 24e. The anisotropic displacement parameters are depicted at the $50 \%$ probability level. Hydrogen atoms are omitted for clarity. The ethyl acetate molecule is disordered over four positions two each related by an inversion center. It was refined with distance restraints and restraints for the anisotropic displacement parameters. The occupancy of the major component refined to $0.430(3)$.

Table 54. Crystallographic data of $24 e$.

\begin{tabular}{|c|c|c|c|}
\hline Structure Code & ts360_etoac & Z & 2 \\
\hline Empirical formula & $\mathrm{C}_{28} \mathrm{H}_{23} \mathrm{PS} \cdot 0.5 \mathrm{C}_{4} \mathrm{H}_{8} \mathrm{O}_{2}$ & $\rho_{\text {calc }}$ & 1.274 \\
\hline Formula weight $[\mathrm{g} / \mathrm{mol}]$ & 466.54 & $\mu\left[\mathrm{mm}^{-1}\right]$ & 0.220 \\
\hline Temperature $[\mathrm{K}]$ & $100(2)$ & $F(000)$ & 492 \\
\hline Wavelength $[\AA ̊]$ & 0.71073 & $\theta$ range $\left[{ }^{\circ}\right]$ & 1.940 to 26.418 \\
\hline Crystal system & triclinic & Reflections collected & 26439 \\
\hline space group & $P \overline{1}$ & Independent reflections & 4992 \\
\hline $\mathrm{a}[\AA \AA]$ & $7.371(2)$ & $\mathrm{R}$ (int) & 0.0257 \\
\hline $\mathrm{b}[\AA]$ & $10.765(2)$ & Max. / min. transmission & 0.7454 / 07139 \\
\hline$c[\AA]$ & $15.783(3)$ & Restraints / parameter & $208 / 382$ \\
\hline$\alpha\left[^{\circ}\right]$ & $77.27(2)$ & GooF & 1.074 \\
\hline$\beta\left[^{\circ}\right]$ & $84.98(2)$ & $\mathrm{R} 1 / w \mathrm{R} 2(\mathrm{I}>2 \sigma(\mathrm{I}))$ & $0.0316 / 0.0833$ \\
\hline$\gamma\left[{ }^{\circ}\right]$ & $87.70(3)$ & R1 / wR2 (all data) & $0.0339 / 0.0853$ \\
\hline Volume $\left[\AA^{3}\right]$ & $1216.6(5)$ & max. diff peak / hole $\left[\mathrm{e} \AA^{-3}\right.$ ] & $0.360 /-0.298$ \\
\hline
\end{tabular}




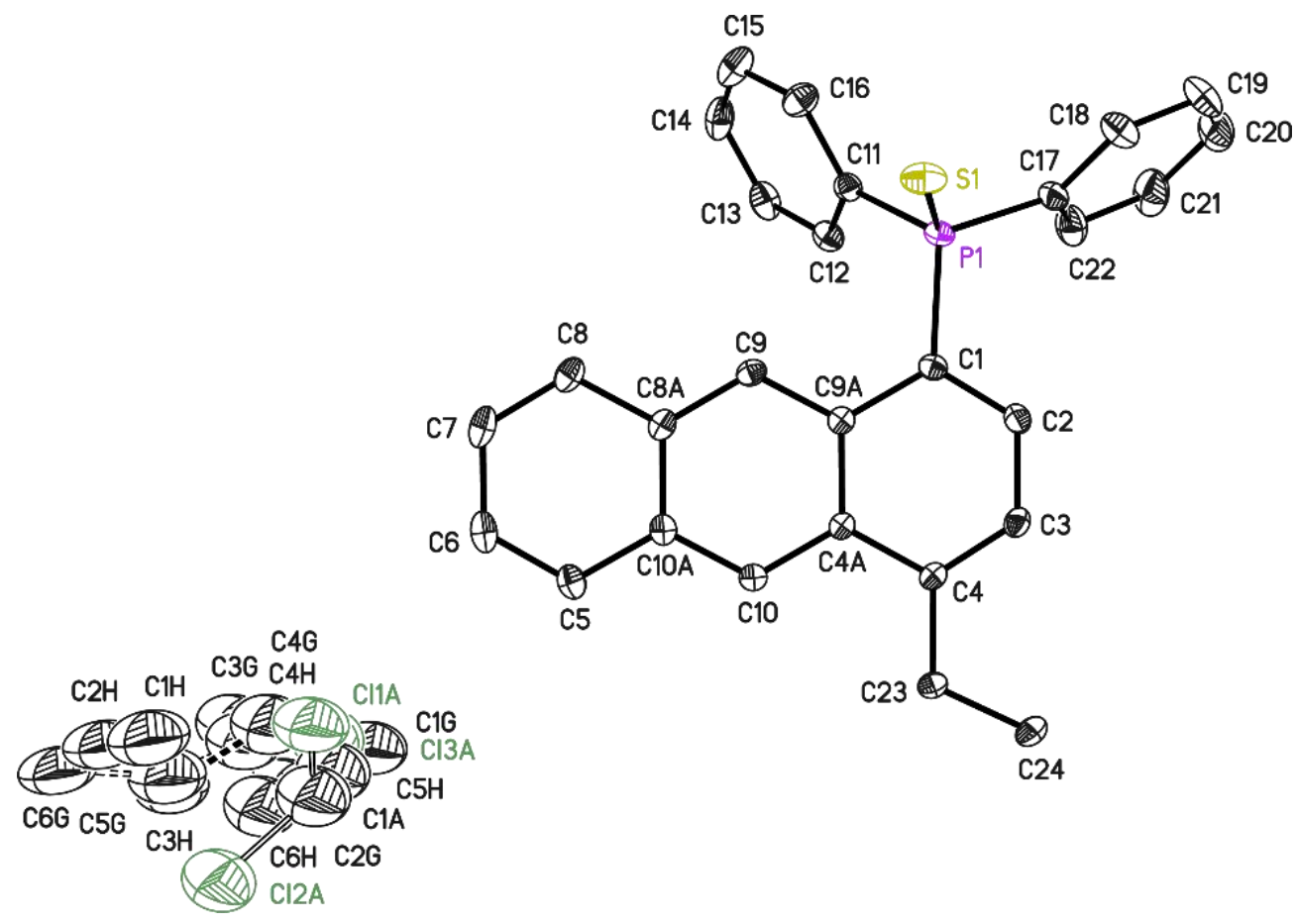

Figure 115. Asymmetric unit of $\mathbf{2 4 f}$. The anisotropic displacement parameters are depicted at the $50 \%$ probability level. Hydrogen atoms are omitted for clarity. The residual density in the solvent region about an inversion center was refined with two positions of hexane with refined occupancies of $0.208(3)$ and $0.158(2)$ and one position of chloroform with refined occupancy of $0.1346(17)$. They were refined with distance restraints and restraints for the anisotropic displacement parameters.

Table 55. Crystallographic data of $24 f$.

\begin{tabular}{|c|c|c|c|}
\hline Structure Code & ts361_chcl3 & Z & 2 \\
\hline Empirical formula & $\begin{array}{l}\mathrm{C}_{28} \mathrm{H}_{23} \mathrm{PS} \cdot 0.37 \\
\mathrm{C}_{6} \mathrm{H}_{14} \cdot 0.13 \mathrm{CHCl}_{3}\end{array}$ & $\rho_{\text {calc }}$ & 1.267 \\
\hline Formula weight $[\mathrm{g} / \mathrm{mol}]$ & 469.42 & $\mu\left[\mathrm{mm}^{-1}\right]$ & 0.257 \\
\hline Temperature [K] & $100(2)$ & $F(000)$ & 495 \\
\hline Wavelength $[\AA ̊ \Omega]$ & 0.71073 & $\theta$ range $\left[{ }^{\circ}\right]$ & 1.935 to 28.424 \\
\hline Crystal system & triclinic & Reflections collected & 29281 \\
\hline space group & $P \overline{1}$ & Independent reflections & 6160 \\
\hline $\mathrm{a}[\AA]$ & $7.363(2)$ & $\mathrm{R}$ (int) & 0.0277 \\
\hline $\mathrm{b}[\AA]$ & $10.855(2)$ & Max. / min. transmission & $0.7457 / 0.7130$ \\
\hline$c[\AA ̊]$ & $15.939(3)$ & Restraints / parameter & $401 / 420$ \\
\hline$\alpha\left[^{\circ}\right]$ & $75.87(2)$ & GooF & 1.035 \\
\hline$\beta\left[^{\circ}\right]$ & $84.83(3)$ & $\mathrm{R} 1 / w \mathrm{R} 2(\mathrm{I}>2 \sigma(\mathrm{I}))$ & $0.0391 / 0.1007$ \\
\hline$\gamma\left[^{\circ}\right]$ & $87.88(2)$ & $\mathrm{R} 1 / w \mathrm{R} 2$ (all data) & $0.0432 / 0.1046$ \\
\hline Volume $\left[\AA^{3}\right]$ & $1230.2(5)$ & max. diff peak / hole $\left[e \AA^{-3}\right]$ & $0.545 /-0.518$ \\
\hline
\end{tabular}




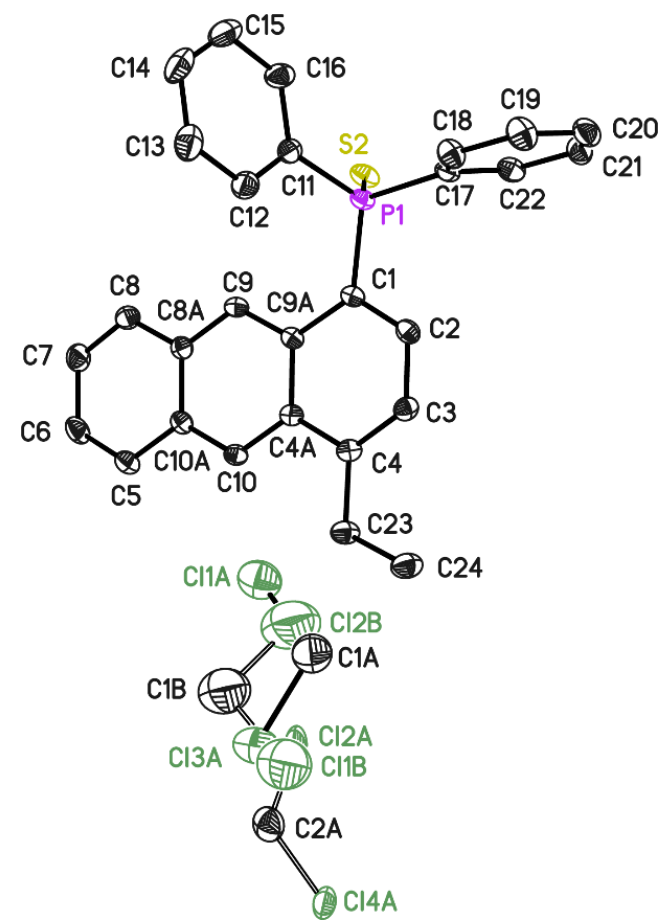

Figure 116. Asymmetric unit of $\mathbf{2 4 g}$. The anisotropic displacement parameters are depicted at the $50 \%$ probability level. Hydrogen atoms are omitted for clarity. One DCM molecule is disordered over two positions. It was refined with distance restraints and restraints for the anisotropic displacement parameters. The occupancy of the major component refined to $0.909(2)$.

Table 56. Crystallographic data of $\mathbf{2 4 g}$.

\begin{tabular}{l|l} 
Structure Code & ts $361 \_d c m$ \\
Empirical formula & $\mathrm{C}_{28} \mathrm{H}_{23} \mathrm{PS} \cdot 1.4 \mathrm{C}_{2} \mathrm{H}_{2} \mathrm{Cl}{ }_{2}$ \\
Formula weight $[\mathrm{g} / \mathrm{mol}]$ & 541.39 \\
Temperature $[\mathrm{K}]$ & $100(2)$ \\
Wavelength $[\AA]$ & 0.71073 \\
Crystal system & monoclinic \\
space group & $P 21 / \mathrm{n}$ \\
a $[\AA \AA$ & $11.559(2)$ \\
$\mathrm{b}[\AA]$ & $16.633(3)$ \\
$\mathrm{c}[\AA]$ & $13.886(2)$ \\
$\alpha\left[^{\circ}\right]$ & 90 \\
$\beta\left[^{\circ}\right]$ & $100.74(2)$ \\
$\gamma\left[^{\circ}\right]$ & 90 \\
Volume $\left[\AA^{3}\right]$ & $2623.0(8)$
\end{tabular}

$Z$
$\rho_{\text {calc }}$
$\mu\left[\mathrm{mm}^{-1}\right]$
$\mathrm{F}(000)$
$\theta$ range $\left[^{\circ}\right]$
Reflections collected
Independent reflections
$\mathrm{R}($ int $)$
Max. / min. transmission
Restraints / parameter
GooF
R1 / wR2 (I > 2 $\sigma(I))$
R1 / wR2 (all data)
max. diff peak / hole $\left[e \AA^{-3}\right]$

4

1.371

0.487

1123

1.931 to 26.539

53374

5452

0.0327

0.7454 / 0.7111

$111 / 354$

1.035

0.0395 / 0.1061

$0.0470 / 0.1117$

$0.657 /-0.622$ 


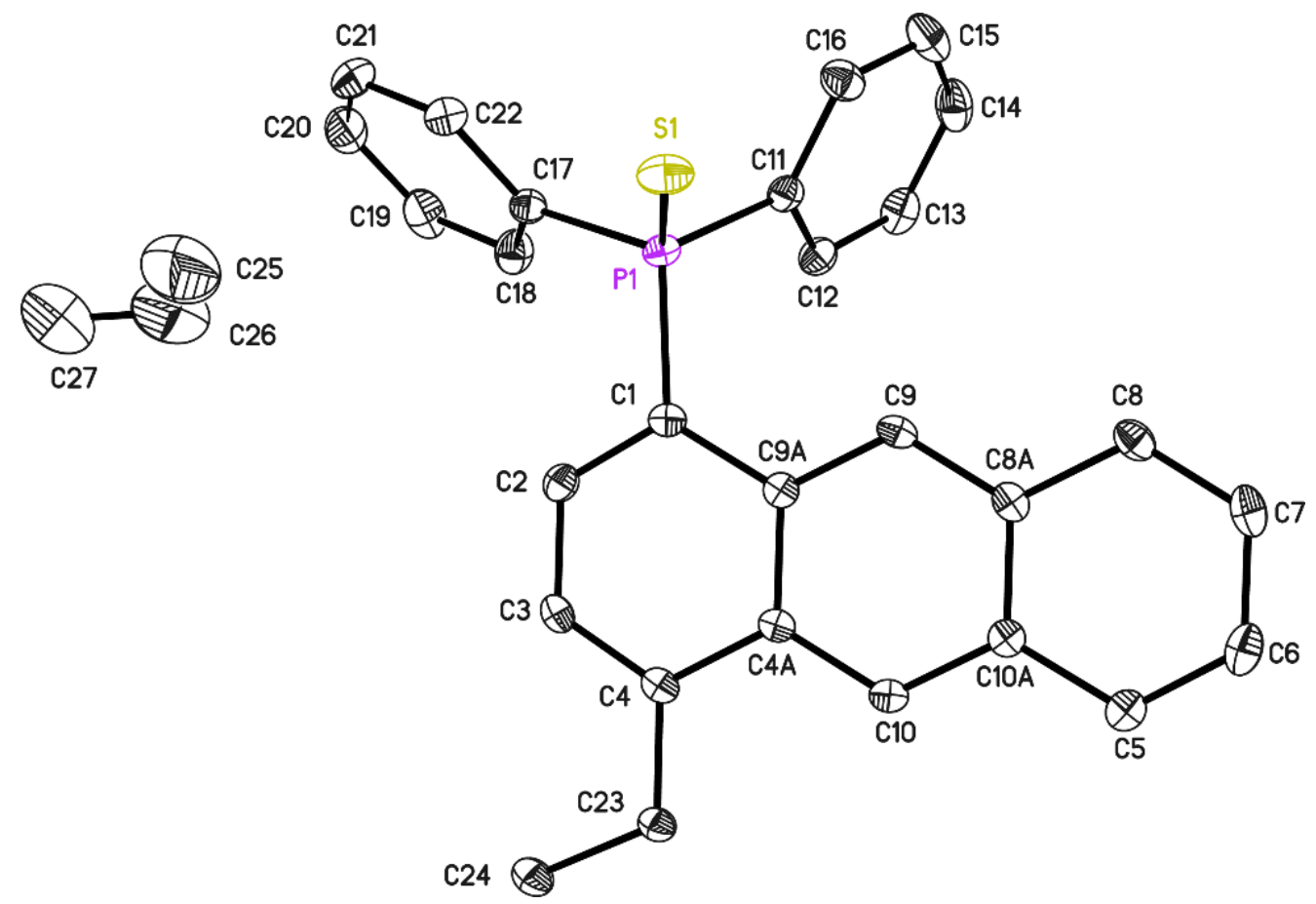

Figure 117. Asymmetric unit of $\mathbf{2 4 h}$. The anisotropic displacement parameters are depicted at the $50 \%$ probability level. Hydrogen atoms are omitted for clarity. The second half of the benzene molecule is generated by an inversion center.

Table 57. Crystallographic data of $24 \mathrm{~h}$.

Structure Code
Empirical formula
Formula weight $[\mathrm{g} / \mathrm{mol}]$
Temperature $[\mathrm{K}]$
Wavelength $[\AA \AA$
Crystal system
space group
a $[\AA]$
$\mathrm{b}[\AA \AA]$
$\mathrm{c}[\AA \AA]$
$\alpha\left[^{\circ}\right]$
$\beta\left[^{\circ}\right]$
$\gamma\left[^{\circ}\right]$
Volume $\left[\AA^{3}\right]$

\begin{tabular}{|l} 
ts361_c6h12 \\
$\mathrm{C}_{28} \mathrm{H}_{23} \mathrm{PS} \cdot 0.5 \mathrm{C}_{6} \mathrm{H}_{6}$ \\
461.55 \\
$100(2)$ \\
0.71073 \\
triclinic \\
$P \overline{1}$ \\
$7.326(2)$ \\
$10.848(2)$ \\
$15.858(3)$ \\
$76.03(2)$ \\
$85.08(2)$ \\
$88.53(3)$ \\
$1218.5(5)$
\end{tabular}

\begin{tabular}{|l|l}
$Z$ & 2 \\
$\rho_{\text {calc }}$ & 1.258 \\
$\mu\left[\mathrm{mm}^{-1}\right]$ & 0.216 \\
$\mathrm{~F}(000)$ & 486 \\
$\theta$ range $\left[^{\circ}\right]$ & 1.328 to 26.387 \\
Reflections collected & 29447 \\
Independent reflections & 4988 \\
$\mathrm{R}($ int) & 0.0458 \\
Max. / min. transmission & $0.7454 / 0.7124$ \\
Restraints / parameter & $0 / 299$ \\
GooF & 1.034 \\
R1 / wR2 (I > 2 $\sigma(I))$ & $0.0385 / 0.0926$ \\
R1 / wR2 (all data) & $0.0508 / 0.0999$ \\
max. diff peak / hole $\left[e \AA^{-3}\right]$ & $0.488 /-0.320$
\end{tabular}




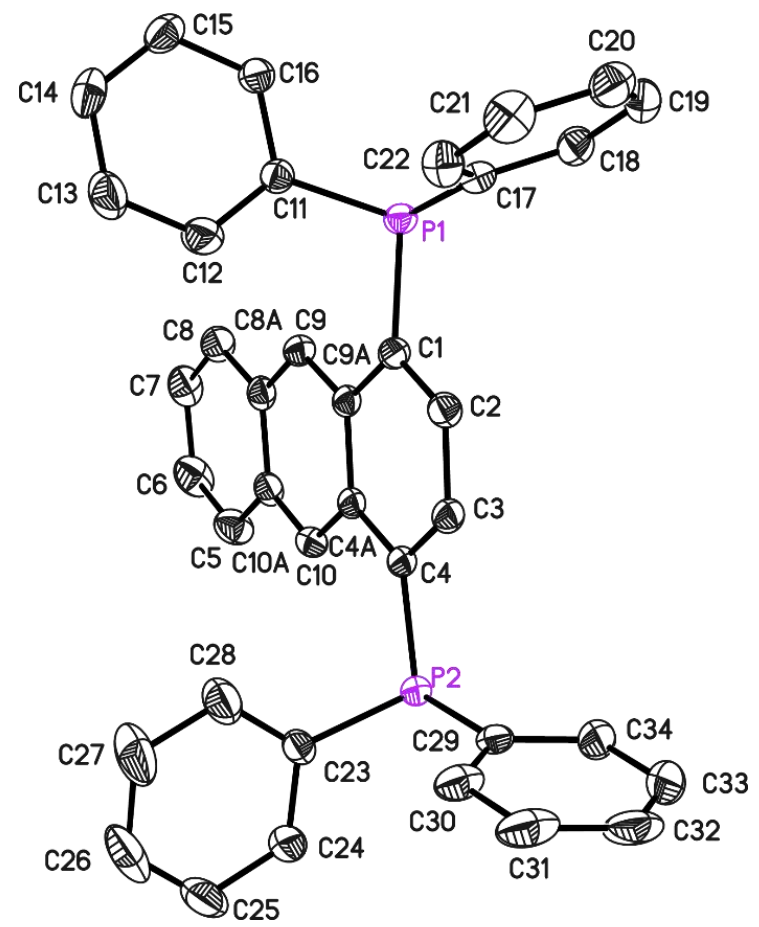

Figure 118. Asymmetric unit 25. The anisotropic displacement parameters are depicted at the $50 \%$ probability level. Hydrogen atoms are omitted for clarity.

Table 58. Crystallographic data of 25.

\begin{tabular}{|c|c|c|c|}
\hline Structure Code & ts370_tol & Z & 2 \\
\hline Empirical formula & $\mathrm{C}_{38} \mathrm{H}_{28} \mathrm{P}_{2}$ & $\rho_{\text {calc }}$ & 1.273 \\
\hline Formula weight $[\mathrm{g} / \mathrm{mol}]$ & 546.54 & $\mu\left[\mathrm{mm}^{-1}\right]$ & 0.179 \\
\hline Temperature [K] & $100(2)$ & $F(000)$ & 572 \\
\hline Wavelength $[\AA ̊]$ & 0.71073 & $\theta$ range $\left[{ }^{\circ}\right]$ & 1.311 to 26.550 \\
\hline Crystal system & triclinic & Reflections collected & 38593 \\
\hline space group & & Independent reflections & 5849 \\
\hline $\mathrm{a}[\AA ̊ \Omega]$ & $9.436(2)$ & $\mathrm{R}$ (int) & 0.0257 \\
\hline $\mathrm{b}[\AA ̊]$ & $10.125(2)$ & Max. / min. transmission & 0.7454 / 0.7195 \\
\hline$c[\AA]$ & $15.531(3)$ & Restraints / parameter & $0 / 362$ \\
\hline$\alpha\left[^{\circ}\right]$ & $89.89(3)$ & GooF & 1.046 \\
\hline$\beta\left[^{\circ}\right]$ & $88.80(2)$ & $\mathrm{R} 1 / w \mathrm{R} 2(\mathrm{I}>2 \sigma(\mathrm{I}))$ & $0.0374 / 0.0928$ \\
\hline$\gamma\left[^{\circ}\right]$ & $73.90(2)$ & R1 / wR2 (all data) & $0.0424 / 0.0979$ \\
\hline Volume $\left[\AA^{3}\right]$ & $1425.3(5)$ & max. diff peak / hole $\left[\mathrm{e} \AA^{-3}\right]$ & $0.400 /-0.295$ \\
\hline
\end{tabular}




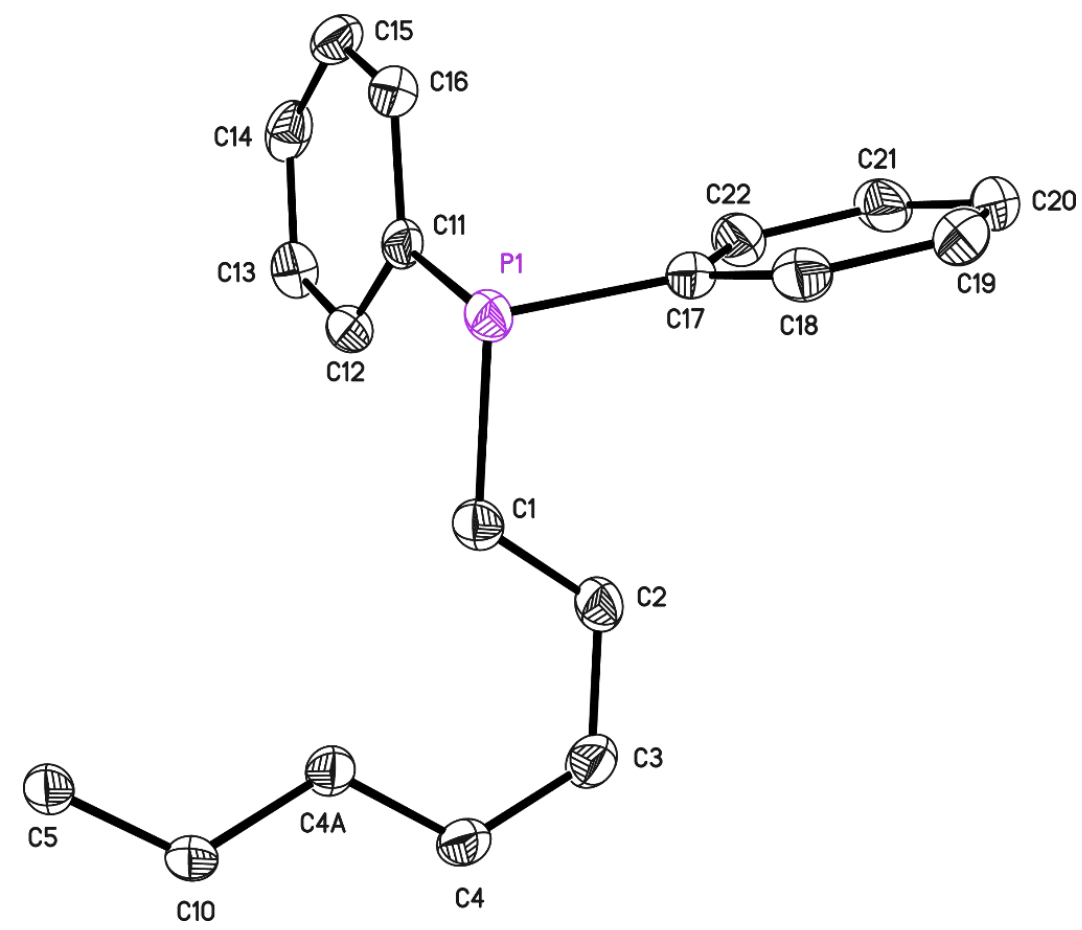

Figure 119. Asymmetric unit of 26. The anisotropic displacement parameters are depicted at the $50 \%$ probability level. Hydrogen atoms are omitted for clarity. A small amount of residual electron density can be found at the P1 atom, which could be refined as oxygen with an occupancy of around $5 \%$ indicating a slight oxidation of the phosphorous during the crystallization process.

Table 59. Crystallographic data of 26.

\begin{tabular}{l|l} 
Structure Code & ts 241 \\
Empirical formula & $\mathrm{C}_{38} \mathrm{H}_{28} \mathrm{P}_{2}$ \\
Formula weight $[\mathrm{g} / \mathrm{mol}]$ & 546.54 \\
Temperature $[\mathrm{K}]$ & $100(2)$ \\
Wavelength $[\AA]$ & 0.71073 \\
Crystal system & triclinic \\
space group & $P \overline{1}$ \\
a $[\AA \AA]$ & $9.073(2)$ \\
$\mathrm{b}[\AA]]$ & $9.121(2)$ \\
$\mathrm{c}[\AA]$ & $9.679(3)$ \\
$\alpha\left[^{\circ}\right]$ & $74.99(3)$ \\
$\beta\left[^{\circ}\right]$ & $72.53(2)$ \\
$\gamma\left[{ }^{\circ}\right]$ & $73.90(2)$ \\
Volume $\left[\AA^{3}\right]$ & $720.4(3)$
\end{tabular}

\begin{tabular}{|c|c|}
\hline Z & 1 \\
\hline$\rho_{\text {calc }}$ & 1.260 \\
\hline$\mu\left[\mathrm{mm}^{-1}\right]$ & 0.177 \\
\hline$F(000)$ & 286 \\
\hline$\theta$ range $\left[{ }^{\circ}\right]$ & 2.248 to 26.356 \\
\hline Reflections collected & 14580 \\
\hline Independent reflections & 2940 \\
\hline $\mathrm{R}$ (int) & 0.0393 \\
\hline Max. / min. transmission & 0.7454 / 0.6845 \\
\hline Restraints / parameter & $0 / 181$ \\
\hline GooF & 1.056 \\
\hline $\mathrm{R} 1 / w \mathrm{R} 2(\mathrm{I}>2 \sigma(\mathrm{I}))$ & $0.0433 / 0.1073$ \\
\hline R1 / wR2 (all data) & $0.0575 / 0.1164$ \\
\hline max. diff peak / hole $\left[\mathrm{e} \AA^{-3}\right]$ & $0.830 /-0.250$ \\
\hline
\end{tabular}




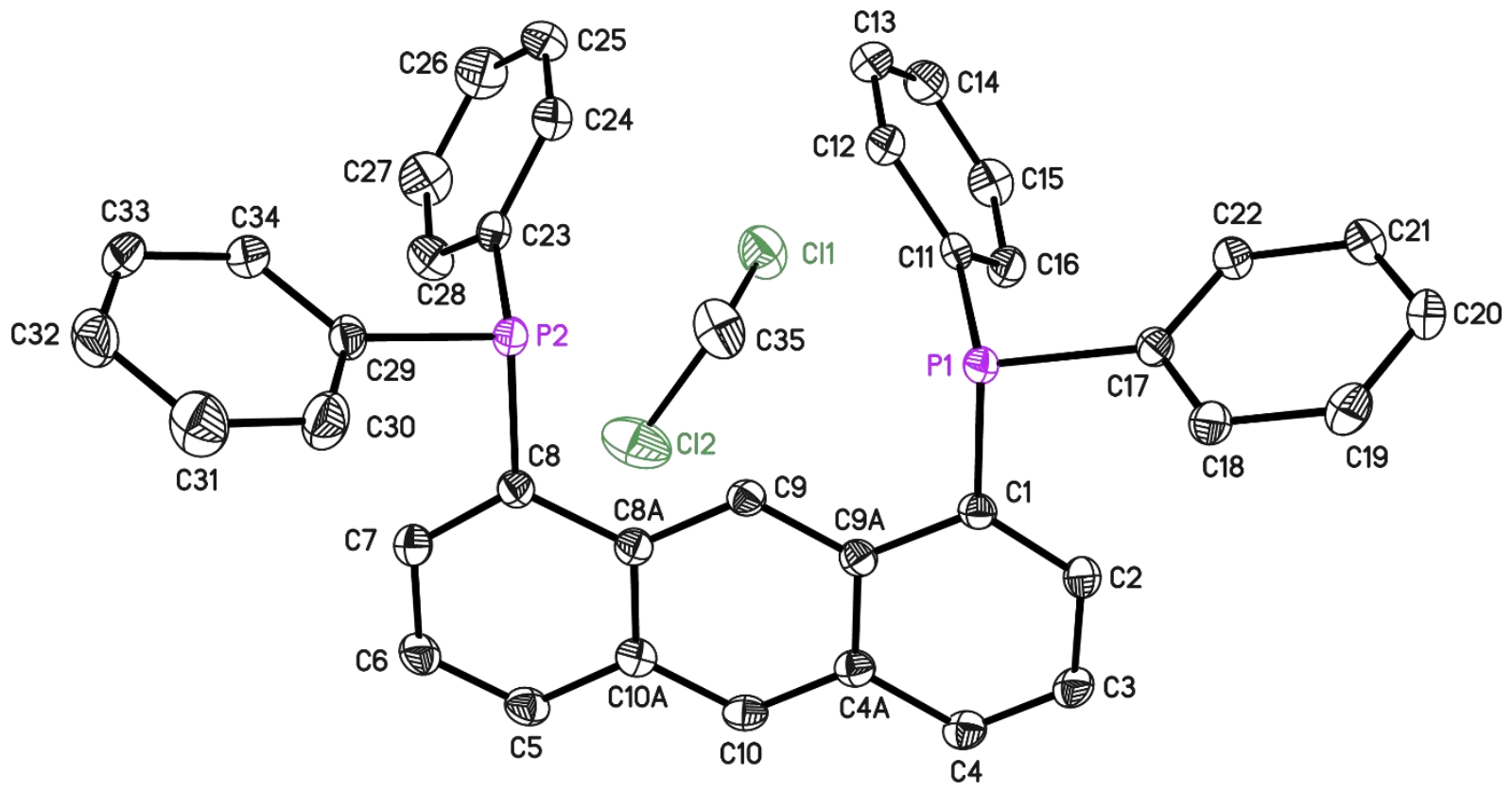

Figure 120. Asymmetric unit of 27. The anisotropic displacement parameters are depicted at the 50\% probability level. Hydrogen atoms are omitted for clarity.

Table 60. Crystallographic data of 27.

\begin{tabular}{|c|c|c|c|}
\hline Structure Code & ts218 & Z & 4 \\
\hline Empirical formula & $\mathrm{C}_{38} \mathrm{H}_{28} \mathrm{P}_{2} \mathrm{CH}_{2} \mathrm{Cl}_{2}$ & $\rho_{\text {calc }}$ & 1.333 \\
\hline Formula weight $[\mathrm{g} / \mathrm{mol}]$ & 546.54 & $\mu\left[\mathrm{mm}^{-1}\right]$ & 0.336 \\
\hline Temperature $[\mathrm{K}]$ & $100(2)$ & $F(000)$ & 1312 \\
\hline Wavelength $[\AA ̊]$ & 0.71073 & $\theta$ range $\left[{ }^{\circ}\right]$ & 1.492 to 26.387 \\
\hline Crystal system & Monoclinic & Reflections collected & 74811 \\
\hline space group & $P 21 / \mathrm{n}$ & Independent reflections & 6444 \\
\hline $\mathrm{a}[\AA ̊]$ & $8.298(2)$ & $\mathrm{R}$ (int) & 0.0649 \\
\hline $\mathrm{b}[\AA]$ & $21.359(3)$ & Max. / min. transmission & $0.7454 / 0.6662$ \\
\hline$c[\AA]$ & $17.839(2)$ & Restraints / parameter & $0 / 390$ \\
\hline$\alpha\left[^{\circ}\right]$ & 90 & GooF & 1.070 \\
\hline$\beta\left[^{\circ}\right]$ & $95.67(2)$ & $\mathrm{R} 1 / w \mathrm{R} 2(\mathrm{I}>2 \sigma(\mathrm{I}))$ & $0.0460 / 0.1221$ \\
\hline$\gamma\left[^{\circ}\right]$ & 90 & $\mathrm{R} 1 /$ wR2 (all data) & $0.0562 / 0.1304$ \\
\hline Volume $\left[\AA^{3}\right]$ & $3146.2(6)$ & max. diff peak / hole $\left[e \AA^{-3}\right]$ & 0.909 / -0.489 \\
\hline
\end{tabular}




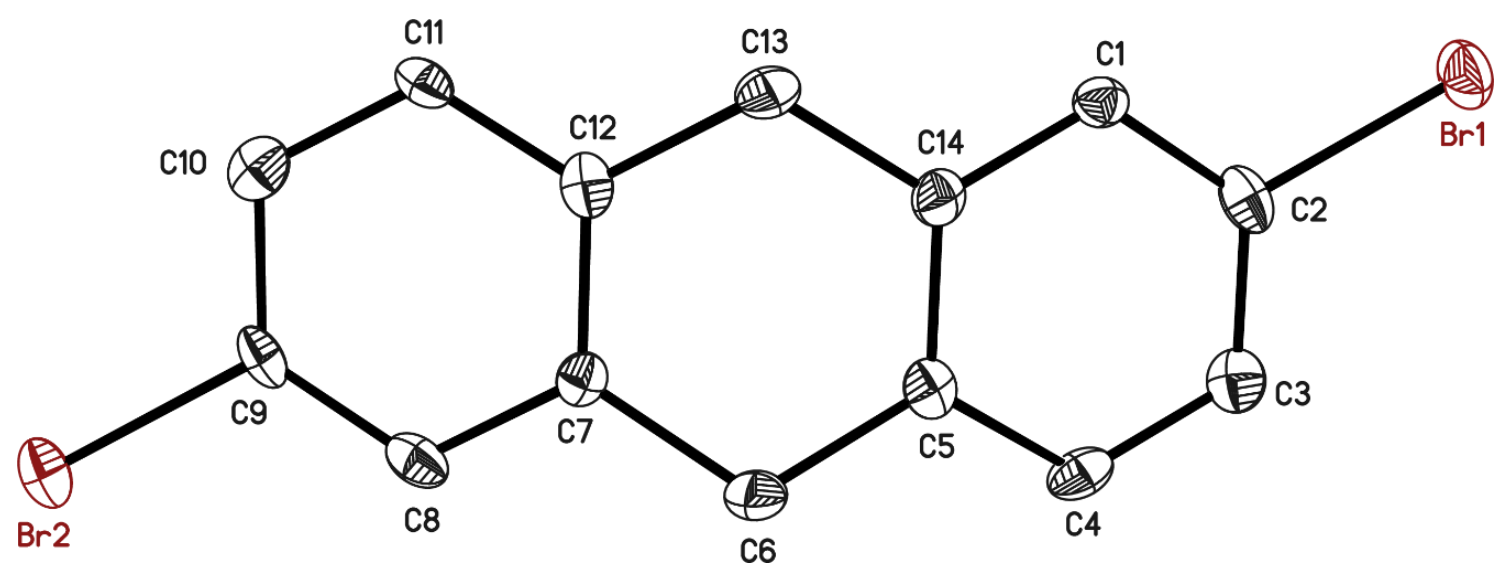

Figure 121. Asymmetric unit of 2,6-dibromo-9,10-dihydroanthracene. The anisotropic displacement parameters are depicted at the $50 \%$ probability level. Hydrogen atoms are omitted for clarity.

Table 61. Crystallographic data of 2,6-dibromo-9,10-dihydroanthracene.

Structure Code
Empirical formula
Formula weight $[\mathrm{g} / \mathrm{mol}]$
Temperature $[\mathrm{K}]$
Wavelength $[\AA \AA$
Crystal system
space group
a $[\AA \AA$
$\mathrm{b}[\AA]$
$\mathrm{c}[\AA]$
$\alpha\left[^{\circ}\right]$
$\beta\left[^{\circ}\right]$
$\gamma\left[^{\circ}\right]$
Volume $\left[\AA^{3}\right]$

\begin{tabular}{|l} 
ts 294 \\
$\mathrm{C}_{14} \mathrm{H}_{10} \mathrm{Br}_{2}$ \\
338.04 \\
$100(2)$ \\
0.56086 \\
Orthorhombic \\
Pna $2_{1}$ \\
$10.379(2)$ \\
$14.203(3)$ \\
$7.972(2)$ \\
90 \\
90 \\
90 \\
$1175.2(4)$
\end{tabular}

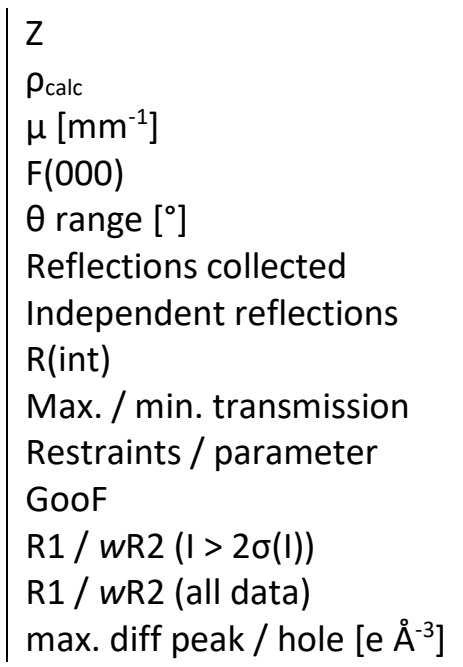

4
1.911
3.680
656
1.918 to 20.542
11597
2394
0.04841
$0.7445 / 0.6532$
$1 / 145$
1.002
$0.0261 / 0.0425$
$0.0319 / 0.0438$
$0.352 /-0.298$




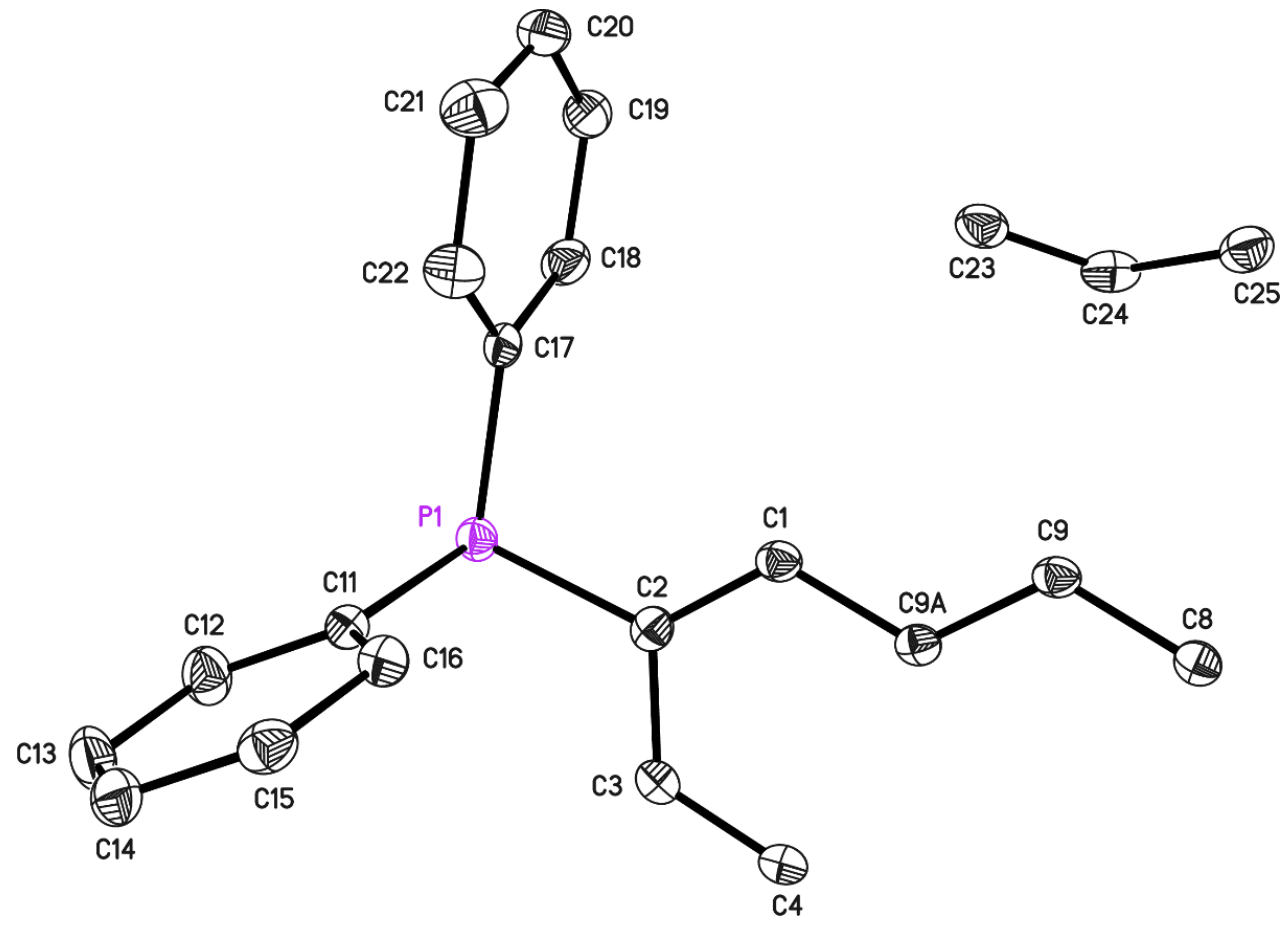

Figure 122. Asymmetric unit of 28. The anisotropic displacement parameters are depicted at the $50 \%$ probability level. Hydrogen atoms are omitted for clarity. The second half of the benzene molecule is generated by an inversion center.

Table 62. Crystallographic data of 28.

\begin{tabular}{l|l|l|l} 
Structure Code & $\mathrm{ts} 331$ & $\mathrm{Z}$ & 1 \\
Empirical formula & $\mathrm{C}_{38} \mathrm{H}_{28} \mathrm{P}_{2} \cdot 0.5 \mathrm{C}_{6} \mathrm{H}_{6}$ & $\rho_{\text {calc }}$ & 1.253 \\
Formula weight $[\mathrm{g} / \mathrm{mol}]$ & 624.65 & $\mu\left[\mathrm{mm}^{-1}\right]$ & 0.163 \\
Temperature $[\mathrm{K}]$ & $100(2)$ & $\mathrm{F}(000)$ & 328 \\
Wavelength $[\AA]$ & 0.71073 & $\theta$ range $\left[^{\circ}\right]$ & 2.235 to 26.379 \\
Crystal system & Triclinic & Reflections collected & 16776 \\
space group & $P \overline{1}$ & Independent reflections & 3362 \\
$\mathrm{a}[\AA \AA$ & $\mathrm{A}]$ & $\mathrm{R}(\mathrm{int})$ & 0.0366 \\
$\mathrm{~b}[\AA]$ & $9.528(2)$ & Max. $/$ min. transmission & $0.7454 / 0.7010$ \\
$\mathrm{c}[\AA]$ & $10.219(2)$ & Restraints $/$ parameter & $0 / 208$ \\
$\alpha\left[^{\circ}\right]$ & $10.479(3)$ & GooF & 1.047 \\
$\beta\left[^{\circ}\right]$ & $61.15(2)$ & $\mathrm{R} 1 / w R 2(\mathrm{I}>2 \sigma(\mathrm{I}))$ & $0.0366 / 0.0881$ \\
$\gamma\left[^{\circ}\right]$ & $85.70(3)$ & $\mathrm{R} 1 / w R 2($ all data) & $0.0433 / 0.0921$ \\
Volume $\left[\AA^{3}\right]$ & $68.90(2)$ & max. diff peak / hole $\left[\mathrm{e} \AA^{-3}\right]$ & $0.424 /-0.333$
\end{tabular}




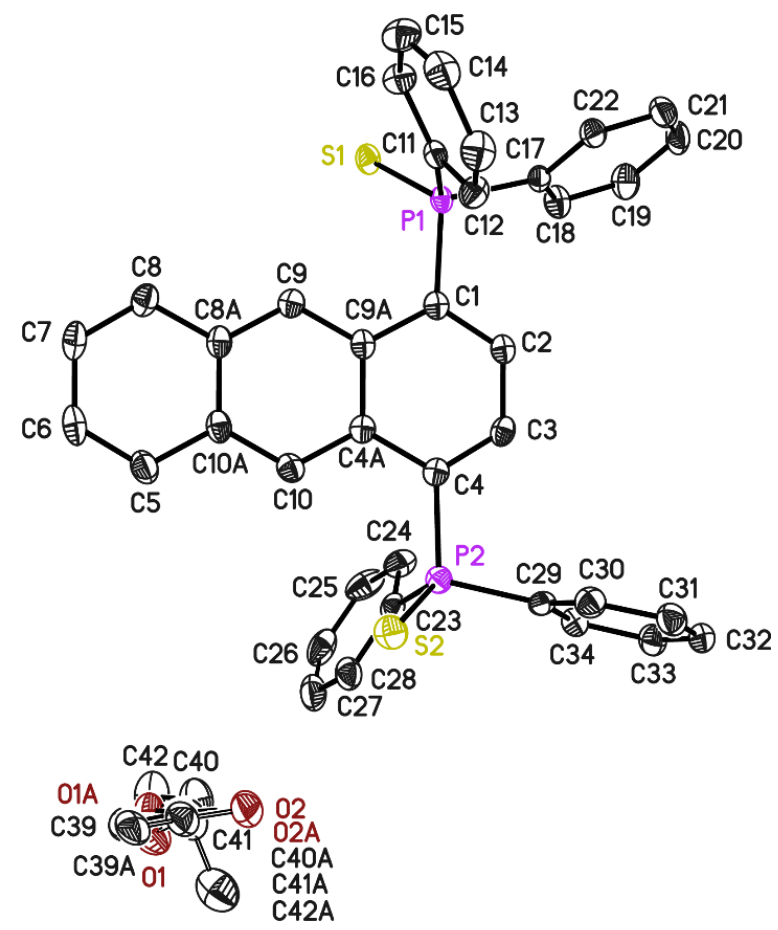

Figure 123. Asymmetric unit of 30a. The anisotropic displacement parameters are depicted at the $50 \%$ probability level. Hydrogen atoms are omitted for clarity. The ethyl acetate molecule is disordered over two positions. It was refined with distance restraints and restraints for the anisotropic displacement parameters.

Table 63. Crystallographic data of 30a.

\begin{tabular}{l|l|l|l} 
Structure Code & ts274_etoac & $\mathrm{Z}$ & 4 \\
Empirical formula & $\mathrm{C}_{42} \mathrm{H}_{36} \mathrm{O}_{2} \mathrm{P}_{2} \mathrm{~S}_{2}$ & $\rho_{\text {calc }}$ & 1.267 \\
Formula weight $[\mathrm{g} / \mathrm{mol}]$ & 698.77 & $\mu\left[\mathrm{mm}^{-1}\right]$ & 0.268 \\
Temperature $[\mathrm{K}]$ & $100(2)$ & $\mathrm{F}(000)$ & 1464 \\
Wavelength $[\AA]$ & 0.71073 & $\theta$ range $\left[^{\circ}\right]$ & 1.312 to 25.351 \\
Crystal system & monoclinic & Reflections collected & 77634 \\
space group & $P 2_{1} / \mathrm{c}$ & Independent reflections & 6702 \\
a $[\AA]$ & $15.884(2)$ & $\mathrm{R}($ int) & 0.1226 \\
$\mathrm{~b}[\AA]$ & $14.366(2)$ & Max. / min. transmission & $0.7454 / 0.7125$ \\
$\mathrm{c}[\AA]$ & $16.433(3)$ & Restraints / parameter & $226 / 490$ \\
$\alpha\left[^{\circ}\right]$ & 90 & GooF & 1.035 \\
$\beta\left[^{\circ}\right]$ & $102.27(2)$ & R1 $/ w R 2(\mathrm{I}>2 \sigma(\mathrm{I}))$ & $0.0417 / 0.1078$ \\
$\gamma\left[^{\circ}\right]$ & 90 & R1 / wR2 (all data) & $0.0607 / 0.1155$ \\
Volume $\left[\AA^{3}\right]$ & $3664.2(10)$ & max. diff peak / hole $\left[\mathrm{e} \AA^{-3}\right]$ & $0.604 /-0.282$
\end{tabular}




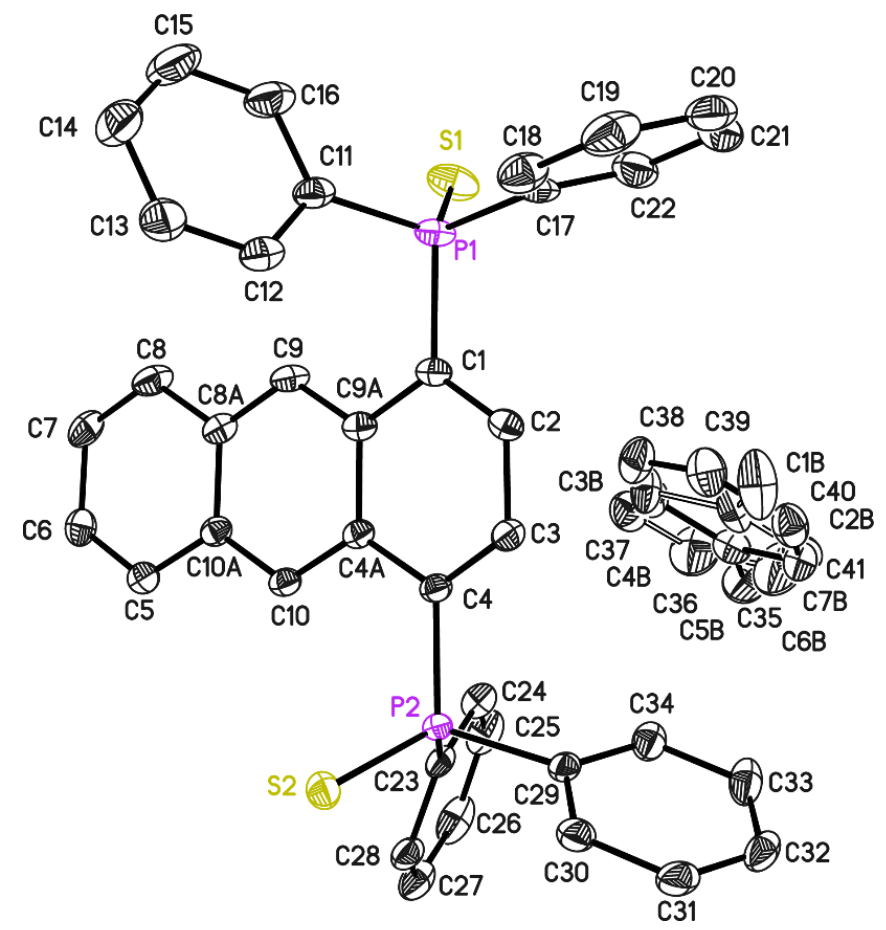

Figure 124. Asymmetric unit of 30b. The anisotropic displacement parameters are depicted at the 50\% probability level. Hydrogen atoms are omitted for clarity. The ethyl acetate molecule is disordered over two positions. It was refined with distance restraints and restraints for the anisotropic displacement parameters.

Table 64. Crystallographic data of 30b.

\begin{tabular}{l|l|l|l} 
Structure Code & ts274_tol & $\mathrm{Z}$ & 4 \\
Empirical formula & $\mathrm{C}_{45} \mathrm{H}_{36} \mathrm{P}_{2} \mathrm{~S}_{2}$ & $\rho_{\text {calc }}$ & 1.280 \\
Formula weight $[\mathrm{g} / \mathrm{mol}]$ & 702.80 & $\mu\left[\mathrm{mm}^{-1}\right]$ & 0.266 \\
Temperature $[\mathrm{K}]$ & $100(2)$ & $\mathrm{F}(000)$ & 1472 \\
Wavelength $[\AA]$ & 0.71073 & $\theta$ range $\left[^{\circ}\right]$ & 1.660 to 26.397 \\
Crystal system & monoclinic & Reflections collected & 86128 \\
space group & $P 2{ }_{1} / \mathrm{c}$ & Independent reflections & 7473 \\
$\mathrm{a}[\AA]$ & $12.475(2)$ & $\mathrm{R}(\mathrm{int})$ & 0.0399 \\
$\mathrm{~b}[\AA]$ & $16.696(3)$ & Max. $/$ min. transmission & $0.7454 / 0.7125$ \\
$\mathrm{c}[\AA]$ & $17.807(3)$ & Restraints $/$ parameter & $459 / 508$ \\
$\alpha\left[^{\circ}\right]$ & 90 & GooF & 1.046 \\
$\beta\left[^{\circ}\right]$ & $100.49(2)$ & $\mathrm{R} 1 / w R 2(\mathrm{I}>2 \sigma(\mathrm{I}))$ & $0.0328 / 0.0827$ \\
$\gamma\left[^{\circ}\right]$ & 90 & $\mathrm{R} 1 / w R 2($ all data) & $0.0393 / 0.0874$ \\
Volume $\left[\AA^{3}\right]$ & $3646.9(11)$ & max. diff peak / hole $\left[\mathrm{e} \AA^{-3}\right]$ & $0.419 /-0.401$
\end{tabular}




\section{Literature}

[1] J. R. Lakowicz, Principles of fluorescence spectroscopy, Springer, New York, 2006.

[2] E. M. Goldys (Ed.) Fluorescence applications in biotechnology and life sciences, Wiley-Blackwell, Hoboken, NJ, 2009.

[3] B. Pedras (Ed.) Fluorescence in Industry, Springer Nature, Switzerland, 2019.

[4] R. Y. Tsien, Annu. Rev. Biochem. 1998, 67, 509.

[5] O. Shimomura, Angew. Chem. 2009, 48, 5590.

[6] a) B. O. Leung, K. C. Chou, Appl. Spectrosc. 2011, 65, 967; b) N. Ji, Nat. Methods 2017, 14, 374; c) J. W. Lichtman, J.-A. Conchello, Nat. Methods 2005, 2, 910.

[7] a) S. W. Hell, J. Wichmann, Opt. Lett. 1994, 19, 780; b) T. A. Klar, S. W. Hell, Opt. Lett. 1999, $24,954$.

[8] Eric Betzig, George H. Patterson, Rachid Sougrat, O. Wolf Lindwasser, Scott Olenych, Juan S. Bonifacino, Michael W. Davidson, Jennifer Lippincott-Schwartz, Harald F. Hess, Science 2006, 313, 1642.

[9] a) E. Betzig, Rev. Mod. Phys. 2015, 87, 1153; b) S. W. Hell, Rev. Mod. Phys. 2015, 87, 1169; c) W. E. Moerner, Rev. Mod. Phys. 2015, 87, 1183.

[10] a) A. Kitai, Luminescent materials and applications, John Wiley, Chichester, England, Hoboken, NJ, 2008; b) M. Y. Wong, E. Zysman-Colman, Adv. Mater. 2017, 29.

[11] a) S.-J. Zou, Y. Shen, F.-M. Xie, J.-D. Chen, Y.-Q. Li, J.-X. Tang, Mater. Chem. Front. 2020, 4, 788; b) H. Sasabe, J. Kido, Chem. Mater. 2011, 23, 621.

[12] A. Moliton, J.-M. Nunzi, Polym. Int. 2006, 55, 583.

[13] S. Allard, M. Forster, B. Souharce, H. Thiem, U. Scherf, Angew. Chem. 2008, 47, 4070.

[14] a) J. Gierschner, S. Varghese, S. Y. Park, Adv. Opt. Mater. 2016, 4, 348; b) S. Chénais, S. Forget, Polym. Int. 2012, 61, 390; c) G. Kranzelbinder, G. Leising, Nature 2000, 63, 729; d) A. J. C. Kuehne, M. C. Gather, Chem. Rev. 2016, 116, 12823.

[15] a) Y. Liu, K.-R. Wang, D.-S. Guo, B.-P. Jiang, Adv. Funct. Mater. 2009, 19, 2230; b) M. Kaur, M. J. Cho, D. H. Choi, Dyes Pigment. 2016, 125, 1.

[16] A. Bergh, G. Craford, A. Duggal, R. Haitz, Phys. Today 2001, 54, 42.

[17] D. Chitnis, N. Thejo kalyani, H. C. Swart, S. J. Dhoble, Renew. Sustain. Energy Rev. 2016, 64, 727.

[18] S. P. Anthony, ChemPlusChem 2012, 77, 518.

[19] N. Thejo kalyani, S. J. Dhoble, Renew. Sustain. Energy Rev. 2012, 16, 2696.

[20] J.-H. Lee, C.-H. Chen, P.-H. Lee, H.-Y. Lin, M. Leung, T.-L. Chiu, C.-F. Lin, J. Mater. Chem. C 2019, 7, 5874.

[21] Z. Chen, C.-L. Ho, L. Wang, W.-Y. Wong, Adv. Mater. 2020, 32, 1903269.

[22] N. J. Turro, V. Ramamurthy, J. C. Scaiano, Modern Molecular Photochemistry of Organic Molecules, University Science Books, Sausalito, 2010.

[23] M. Klessinger, J. Michl, Excited states and photochemistry of organic molecules, VCH, New York, 1995. 
[24] S. R. Forrest, M. A. Baldo, D. F. O'Brien, Y. You, A. Shoustikov, S. Sibley, M. E. Thompson, Nature 1998, 395, 151.

[25] J.-H. Jou, S. Kumar, A. Agrawal, T.-H. Li, S. Sahoo, J. Mater. Chem. C 2015, 3, 2974.

[26] H. Yersin (Ed.) Highly efficient OLEDs. Materials based on thermally activated delayed fluorescence, Wiley-VCH, Weinheim, Germany, 2019.

[27] M. A. Baldo, S. Lamansky, P. E. Burrows, M. E. Thompson, S. R. Forrest, Appl. Phys. Lett. 1999, 75, 4.

[28] J. B. Birks, Photophysics of aromatic molecules, Wiley-Interscience, London, 1970.

[29] L. F. Gildea, J. Williams in Woodhead Publishing Series in Electronic and Optical Materials, Vol. 36 (Ed.: A. Buckley), Woodhead Publ, Oxford, 2013, pp. 77-113.

[30] a) R. J. Holmes, S. R. Forrest, Y.-J. Tung, R. C. Kwong, J. J. Brown, S. Garon, M. E. Thompson, Appl. Phys. Lett. 2003, 82, 2422; b) K. Udagawa, H. Sasabe, C. Cai, J. Kido, Advanced Materials 2014, 26, 5062; c) G. Li, T. Fleetham, E. Turner, X.-C. Hang, J. Li, Adv. Opt. Mater. 2015, 3, 390.

[31] Z. Yang, Z. Mao, Z. Xie, Y. Zhang, S. Liu, J. Zhao, J. Xu, Z. Chi, M. P. Aldred, Chem. Soc. Rev. 2017, 46, 915.

[32] Y. Im, M. Kim, Y. J. Cho, J.-A. Seo, K. S. Yook, J. Y. Lee, Chem. Mater. 2017, 29, 1946.

[33] R. Czerwieniec, M. J. Leitl, H. H. Homeier, H. Yersin, Coord. Chem. Rev. 2016, 325, 2.

[34] H. Uoyama, K. Goushi, K. Shizu, H. Nomura, C. Adachi, Nature 2012, 492, 234.

[35] M. Y. Wong, E. Zysman-Colman, Adv. Mater. 2017, 29, 1605444.

[36] S. Varghese, S. Das, J. Phys. Chem. Lett. 2011, 2, 863.

[37] N. S. S. Kumar, S. Varghese, C. H. Suresh, N. P. Rath, S. Das, J. Phys. Chem. C 2009, 113, 11927.

[38] H. Langhals, T. Potrawa, H. Nöth, G. Linti, Angew. Chem. 1989, 28, 478.

[39] K. Shirai, M. Matsuoka, K. Fukunishi, Dyes Pigm. 1999, 42, 95.

[40] Q. Li, Z. Li, Acc. Chem. Res. 2020, 53, 962.

[41] C. Kaufmann, D. Bialas, M. Stolte, F. Würthner, J. Am. Chem. Soc. 2018, 140, 9986.

[42] F. Würthner, Angew. Chem. 2020, 59, 14192.

[43] G. C. Schmidt, Ann. Phys. Chem. 1896, 294, 103.

[44] G. Oster, Y. Nishijima, J. Am. Chem. Soc. 1956, 78, 1581.

[45] D. A. Shultz, M. A. Fox, J. Am. Chem. Soc. 1989, 111, 6311.

[46] a) H.-J. Egelhaaf, M. Brun, S. Reich, D. Oelkrug, J. Mol. Struc. THEOCHEM 1992, 267, 297; b) D.

Oelkrug, A. Tompert, H.-J. Egelhaaf, M. Hanack, E. Steinhuber, M. Hohloch, H. Meier, U. Stalmach, Synth. Met. 1996, 83, 231; c) D. Oelkrug, A. Tompert, J. Gierschner, H.-J. Egelhaaf, M. Hanack, M. Hohloch, E. Steinhuber, J. Phys. Chem. B 1998, 102, 1902.

[47] J. Luo, Z. Xie, J. W. Y. Lam, L. Cheng, H. Chen, C. Qiu, H. S. Kwok, X. Zhan, Y. Liu, D. Zhu et al., Chem. Commun. 2001, 0, 1740.

[48] H. Tong, Y. Hong, Y. Dong, M. Häussler, J. W. Y. Lam, Z. Li, Z. Guo, Z. Guo, B. Z. Tang, Chem. Commun. 2006, 3705.

[49] X. Cai, B. Liu, Angew. Chem. 2020, 59, 9868. 
[50] a) J. Huang, Y. Jiang, J. Yang, R. Tang, N. Xie, Q. Li, H. S. Kwok, B. Z. Tang, Z. Li, J. Mater. Chem. C 2014, 2, 2028; b) J. Huang, R. Tang, T. Zhang, Q. Li, G. Yu, S. Xie, Y. Liu, S. Ye, J. Qin, Z. Li, Chem. Eur. J. 2014, 20, 5317.

[51] J. Chen, C. C. W. Law, J. W. Y. Lam, Y. Dong, S. M. F. Lo, I. D. Williams, D. Zhu, B. Z. Tang, Chem. Mater. 2003, 15, 1535.

[52] Y. Ren, J. W. Y. Lam, Y. Dong, B. Z. Tang, K. S. Wong, J. Phys. Chem. B 2005, 109, 1135.

[53] Y. Hong, J. W. Y. Lam, B. Z. Tang, Chem. Commun. 2009, 4332.

[54] N. L. C. Leung, N. Xie, W. Yuan, Y. Liu, Q. Wu, Q. Peng, Q. Miao, J. W. Y. Lam, B. Z. Tang, Chem. Eur. J. 2014, 20, 15349.

[55] J. Mei, N. L. C. Leung, R. T. K. Kwok, J. W. Y. Lam, B. Z. Tang, Chem. Rev. 2015, 115, 11718.

[56] J. Mei, Y. Hong, J. W. Y. Lam, A. Qin, Y. Tang, B. Z. Tang, Adv. Mater. 2014, 26, 5429.

[57] Y. Hong, J. W. Y. Lam, B. Z. Tang, Chem. Soc. Rev. 2011, 40, 5361.

[58] E. E. Jelley, Nature 1936, 138, 1009.

[59] E. E. Jelley, Nature 1937, 139, 631.

[60] G. Scheibe, L. Kandler, H. Ecker, Naturwissenschaften 1937, 25, 75.

[61] G. Scheibe, Angew. Chem. 1937, 50, 212.

[62] F. Würthner, T. E. Kaiser, C. R. Saha-Möller, Angew. Chem. Int. Ed. 2011, 50, 3376.

[63] J. Gierschner, L. Lüer, B. Milián-Medina, D. Oelkrug, H.-J. Egelhaaf, J. Phys. Chem. Lett. 2013, 4, 2686.

[64] M. Kasha, H. R. Rawls, M. Ashraf El-Bayoumi, Pure Appl. Chem. 1965, 11, 371.

[65] T. Förster, K. Kasper, Z. Phys. Chem. 1954, 1, 275.

[66] J. B. Birks, L. G. Christophorou, Nature 1962, 194, 442.

[67] J. B. Birks, Rep. Prog. Phys. 1975, 38, 903.

[68] Y. Shen, Z. Zhang, H. Liu, Y. Yan, S. Zhang, B. Yang, Y. Ma, J. Phys. Chem. C 2019, 123, 13047.

[69] a) C. Wang, D. Hashizume, M. Nakano, T. Ogaki, H. Takenaka, K. Kawabata, K. Takimiya, Chem. Sci. 2020, 107, 926; b) J.-M. Lehn, Supramolecular chemistry. Concepts and perspectives, VCH, Weinheim, New York, 1995.

[70] a) S. Grimme, Angew. Chem. Int. Ed. 2008, 47, 3430; b) E. C. Lee, D. Kim, P. Jurecka, P. Tarakeshwar, P. Hobza, K. S. Kim, J. Phys. Chem. A 2007, 111, 3446; c) M. L. Waters, Curr. Opin. Chem. Biol. 2002, 6, 736; d) K. Carter-Fenk, J. M. Herbert, Phys. Chem. Chem. Phys. 2020, 22, 24870.

[71] C. R. Martinez, B. L. Iverson, Chem. Sci. 2012, 3, 2191.

[72] C. A. Hunter, J. K. M. Sanders, J. Am. Chem. Soc. 1990, 112, 5525.

[73] M. O. Sinnokrot, C. D. Sherrill, J. Phys. Chem. A 2006, 110, 10656.

[74] a) B. W. Gung, J. C. Amicangelo, J. Org. Chem. 2006, 71, 9261; b) W. Wang, Y. Zhang, Y.-B. Wang, Int. J. Quantum Chem. 2017, 117, e25345.

[75] F. Cozzi, M. Cinquini, R. Annuziata, J. S. Siegel, J. Am. Chem. Soc. 1993, 115, 5330.

[76] E. A. Meyer, R. K. Castellano, F. Diederich, Angew. Chem. Int. Ed. 2003, 42, 1210.

[77] S. E. Wheeler, K. N. Houk, J. Am. Chem. Soc. 2008, 130, 10854.

[78] S. Grimme, Angew. Chem., Int. Ed. 2008, 47, 3430. 
[79] a) S. K. Burley, G. A. Petsko, Science 1985, 229, 23; b) P. Mignon, S. Loverix, J. Steyaert, P. Geerlings, Nucleic Acids Res. 2005, 33, 1779.

[80] J. W. G. Bloom, S. E. Wheeler, Angew. Chem., Int. Ed. 2011, 50, 7847.

[81] M. Nishio, Y. Umezawa, J. Fantini, M. S. Weiss, P. Chakrabarti, Phys. Chem. Chem. Phys. 2014, 16, 12648.

[82] M. Nishio, Phys. Chem. Chem. Phys. 2011, 13, 13873.

[83] S. Tsuzuki, K. Honda, T. Uchimaru, M. Mikami, K. Tanabe, J. Am. Chem. Soc. 2000, 122, 3746.

[84] a) D. A. Dougherty, Acc. Chem. Res. 2013, 46, 885; b) J. C. Ma, D. A. Dougherty, Chem. Rev. 1997, 97, 1303; c) A. Frontera, P. Gamez, M. Mascal, T. J. Mooibroek, J. Reedijk, Angew. Chem. Int. Ed. 2011, 50, 9564; d) B. L. Schottel, H. T. Chifotides, K. R. Dunbar, Chem. Soc. Rev. 2008, 37, 68.

[85] C. Wang, H. Dong, L. Jiang, W. Hu, Chem. Soc. Rev. 2018, 47, 422.

[86] a) T. Kumari, S. M. Lee, S.-H. Kang, S. Chen, C. Yang, Energy Environ. Sci. 2017, 10, 258; b) L. Wang, S. Guo, K. Zhou, W. Ma, Sustain. Energy Fuels. 2020, 4, 4934.

[87] T. Chen, M. Li, J. Liu, Cryst. Growth. Des. 2018, 18, 2765.

[88] J. E. Anthony, Chem. Rev. 2006, 106, 5028.

[89] a) P. Majumdar, F. Tharammal, J. Gierschner, S. Varghese, ChemPhysChem 2020, 21, 616; b) S.

Varughese, J. Mater. Chem. C 2014, 2, 3499.

[90] A. R. Horrocks, F. Wilkinson, Proc. R. Soc. Lond. A 1968, 306, 257.

[91] Lord Rayleigh, F. R. S, Proc. R. Soc. Lond. A 1927, 116, 702.

[92] J. J. Hopfield, Phys. Rev. 1930, 35, 1133.

[93] M. H. R. Hutchinson, Appl. Phys. 1980, 21, 95.

[94] B. Stevens, E. Hutton, Nature 1960, 186, 1045.

[95] T. Förster, Angew. Chem. Int. Ed. 1969, 8, 333.

[96] J. B. Birks, M. D. Lumb, I. H. Munro, B. H. Flowers, Proc. R. Soc. Lond. A 1964, 280, 289.

[97] C. A. Parker, C. G. Hatchard, Proc. R. Soc. Lond. A 1962, 269, 574.

[98] G. F. Moore, Nature 1966, 212, 1452.

[99] R. L. Barnes, J. B. Birks, Proc. R. Soc. Lond. A 1966, 291, 570.

[100] J. Ferguson, A.-H. Mau, Mol. Phys. 1974, 27, 377.

[101] a) T. M. Cardozo, A. P. Galliez, I. Borges, F. Plasser, A. J. A. Aquino, M. Barbatti, H. Lischka, Phys. Chem. Chem. Phys. 2019, 21, 13916; b) N. O. Dubinets, A. A. Safonov, A. A. Bagaturyants, J. Phs. Chem. A 2016, 120, 2779.

[102] E. S. S. Iyer, A. Sadybekov, O. Lioubashevski, A. I. Krylov, S. Ruhman, J. Phys. Chem. A 2017, 121, 1962.

[103] K. Diri, A. I. Krylov, J. Phys. Chem. A 2012, 116, 653.

[104] a) S. Shirai, S. Iwata, T. Tani, S. Inagaki, J. Phys. Chem. A 2011, 115, 7687; b) M. Kołaski, C. R.

Arunkumar, K. S. Kim, J. Chem. Theory. Comput. 2012, 9, 847.

[105] T. Hirata, H. Ikeda, H. Saigusa, J. Phys. Chem. A 1999, 103, 1014.

[106] J. B. Birks, A. A. Kazzaz, Proc. R. Soc. Lond. A 1968, 304, 291.

[107] B. Stevens, Spectrochim. Acta 1962, 18, 439. 
[108] P. F. Jones, M. Nicol, J. Chem. Phys. 1965, 43, 3759.

[109] J. B. Birks, A. A. Kazzaz, T. A. King, Proc. R. Soc. Lond. A 1966, 291, 556.

[110] D. Basting, G. Marowsky, Excimer Laser Technology, Springer-Verlag Berlin Heidelberg, Berlin, Heidelberg, 2005.

[111] a) A. Reynolds, J. E. Moore, S. A. Naroo, C. B. T. Moore, S. Shah, Clin. Experiment. Ophthalmol. 2010, 38, 168; b) M. Rothschild, J. Vac. Sci. Technol. B 1988, 6, 1.

[112] a) S. Nishizawa, Y. Kato, N. Teramae, J. Am. Chem. Soc. 1999, 121, 9463; b) F. D. Lewis, Y. Zhang, R. L. Letsinger, J. Am. Chem. Soc. 1997, 119, 5451; c) P. L. Paris, J. M. Langenhan, E. T. Kool, Nucleic Acids Res. 1998, 26, 3789.

[113] S. Chen, L. Wang, N. E. Fahmi, S. J. Benkovic, S. M. Hecht, J. Am. Chem. Soc. 2012, 134, 18883.

[114] a) C. J. Yang, S. Jockusch, M. Vicens, N. J. Turro, W. Tan, P. Natl. Acad. Sci. USA 2005, 102, 17278;

b) P. Conlon, C. J. Yang, Y. Wu, Y. Chen, K. Martinez, Y. Kim, N. Stevens, A. A. Marti, S. Jockusch, N. J. Turro et al., J. Am. Chem. Soc. 2008, 130, 336; c) S. Karuppannan, J.-C. Chambron, Chem. Asian J. 2011, 6, 964.

[115] J. Vollbrecht, New J. Chem. 2018, 42, 11249.

[116] C. Wei, M. Gao, F. Hu, J. Yao, Y. S. Zhao, Adv. Opt. Mater. 2016, 4, 1009.

[117] P. E. Hartnett, A. Timalsina, H. S. S. R. Matte, N. Zhou, X. Guo, W. Zhao, A. Facchetti, R. P. H.

Chang, M. C. Hersam, M. R. Wasielewski et al., J. Am. Chem. Soc. 2014, 136, 16345.

[118] E. A. Margulies, L. E. Shoer, S. W. Eaton, M. R. Wasielewski, Phys. Chem. Chem. Phys. 2014, 16, 23735.

[119] K. T. Kamtekar, A. P. Monkman, M. R. Bryce, Adv. Mater. 2010, 22, 572.

[120] M. Mazzeo, V. Vitale, F. Della Sala, M. Anni, G. Barbarella, L. Favaretto, G. Sotgiu, R. Cingolani, G. Gigli, Adv. Mater. 2005, 17, 34.

[121] S. Tao, Y. Zhou, C.-S. Lee, S.-T. Lee, D. Huang, X. Zhang, J. Mater. Chem. 2008, 18, 3981.

[122] Y.-H. Chen, K.-C. Tang, Y.-T. Chen, J.-Y. Shen, Y.-S. Wu, S.-H. Liu, C.-S. Lee, C.-H. Chen, T.-Y. Lai, S.H. Tung et al., Chem. Sci. 2016, 7, 3556.

[123] Y. Zhang, H. Ma, S. Wang, Z. Li, K. Ye, J. Zhang, Y. Liu, Q. Peng, Y. Wang, J. Phys. Chem. C 2016, 120, 19759.

[124] Y. Zhang, Y. Miao, X. Song, Y. Gao, Z. Zhang, K. Ye, Y. Wang, J. Phys. Chem. Lett. 2017, 8, 4808.

[125] T. Fleetham, L. Huang, J. Li, Adv. Funct. Mater. 2014, 24, 6066.

[126] S. Kumar, Y. Hisamatsu, Y. Tamaki, O. Ishitani, S. Aoki, Inorg. Chem. 2016, 55, 3829.

[127] L. Murphy, P. Brulatti, V. Fattori, M. Cocchi, J. A. G. Williams, Chem. Commun. 2012, 48, 5817.

[128] M. Bachmann, D. Suter, O. Blacque, K. Venkatesan, Inorg. Chem. 2016, 55, 4733.

[129] H. Y. Zhang, Z. L. Zhang, K. Q. Ye, J. Y. Zhang, Y. Wang, Adv. Mater. 2006, 18, 2369.

[130] B. Dong, M. Wang, C. Xu, Q. Feng, Y. Wang, Cryst. Growth Des. 2012, 12, 5986.

[131] S. Hisamatsu, H. Masu, M. Takahashi, K. Kishikawa, S. Kohmoto, Cryst. Growth Des. 2015, 15, 2291.

[132] Y. Shen, H. Liu, J. Cao, S. Zhang, W. Li, B. Yang, Phys. Chem. Chem. Phys. 2019, 21, 14511. 
[133] H. Liu, D. Cong, B. Li, L. Ye, Y. Ge, X. Tang, Y. Shen, Y. Wen, J. Wang, C. Zhou et al., Cryst. Growth Des. 2017, 17, 2945.

[134] Y. Shen, H. Liu, S. Zhang, Y. Gao, B. Li, Y. Yan, Y. Hu, L. Zhao, B. Yang, J. Mater. Chem. C 2017, 5, 10061.

[135] H. Liu, L. Yao, B. Li, X. Chen, Y. Gao, S. Zhang, W. Li, P. Lu, B. Yang, Y. Ma, Chem. Commun. 2016, $52,7356$.

[136] A. Garci, Y. Beldjoudi, M. S. Kodaimati, J. E. Hornick, M. T. Nguyen, M. M. Cetin, C. L. Stern, I. Roy, E. A. Weiss, J. F. Stoddart, J. Am. Chem. Soc. 2020, 142, 7956.

[137] A. Das, A. Danao, S. Banerjee, A. M. Raj, G. Sharma, R. Prabhakar, V. Srinivasan, V. Ramamurthy, P. Sen, J. Am. Chem. Soc. 2021, 143, 2025.

[138] Y. Ge, Y. Wen, H. Liu, T. Lu, Y. Yu, X. Zhang, B. Li, S.-T. Zhang, W. Li, B. Yang, J. Mater. Chem. C 2020, 8, 11830.

[139] a) H.-T. Feng, J. W. Lam, B. Z. Tang, Coord. Chem. Rev. 2020, 406, 213142; b) J. Li, J. Wang, H. Li, N. Song, D. Wang, B. Z. Tang, Chem. Soc. Rev. 2020, 49, 1144; c) B. Z. Tang, Z. Zhao, H. Zhang, J. W. Y. Lam, Angew. Chem. Int. Ed. 2020, 59, 9888; d) R. Hu, N. L. C. Leung, B. Z. Tang, Chem. Soc. Rev. 2014, 43, 4494.

[140] J. B. Briks, Photophysics of Aromatic Molecules, Wiley - Interscience, London, New York, Sydney, Toronto, 1970.

[141] D. D. La, S. V. Bhosale, L. A. Jones, S. V. Bhosale, ACS Appl. Mater. Interfaces 2018, 10, 12189.

[142] Z. Zhao, B. He, B. Z. Tang, Chem. Sci. 2015, 6, 5347.

[143] a) Z. Li, Y. Dong, B. Mi, Y. Tang, M. Häussler, H. Tong, Y. Dong, J. W. Y. Lam, Y. Ren, H. H. Y. Sung et al., J. Phys. Chem. B 2005, 109, 10061; b) K. Nikitin, H. Müller-Bunz, Y. Ortin, J. Muldoon, M. J.

McGlinchey, Org. Lett. 2011, 13, 256.

[144] Q. Li, L. Blancafort, Chem. Commun. 2013, 49, 5966.

[145] X.-L. Peng, S. Ruiz-Barragan, Z.-S. Li, Q.-S. Li, L. Blancafort, J. Mater. Chem. C 2016, 4, 2802.

[146] R. Crespo-Otero, Q. Li, L. Blancafort, Chem. Asian J. 2019, 14, 700.

[147] Y. Cai, L. Du, K. Samedov, X. Gu, F. Qi, H. H. Y. Sung, B. O. Patrick, Z. Yan, X. Jiang, H. Zhang et al., Chem. Sci. 2018, 9, 4662.

[148] S. Suzuki, S. Sasaki, A. S. Sairi, R. Iwai, B. Z. Tang, G. Konishi, Angew. Chem. Int. Ed. 2020.

[149] S. Sasaki, K. Igawa, G. Konishi, J. Mater. Chem. C 2015, 3, 5940.

[150] S. Sasaki, S. Suzuki, W. M. C. Sameera, K. Igawa, K. Morokuma, G. Konishi, J. Am. Chem. Soc. 2016, 138, 8194.

[151] Z. Fei, N. Kocher, C. J. Mohrschladt, H. Ihmels, D. Stalke, Angew. Chem. Int. Ed. 2003, 42, 783.

[152] D. Stern, PhD Thesis, Georg-August-Universität, Göttingen, 2009.

[153] N. Finkelmeier, PhD Thesis, Georg-August-Universität, Göttingen, 2013.

[154] G. Schwab, Diploma Thesis, Julius-Maximilians-Universität, Würzburg, 2004.

[155] G. Schwab, PhD Thesis, Georg-August-Universität, Göttingen, 2008.

[156] L. Krause, PhD Thesis, Georg-August-Universität, Göttingen, 2017.

[157] D. Stern, Diploma Thesis, Georg-August-Universität, Göttingen, 2006. 
[158] a) Y. Chen, D.-G. Chen, Y.-A. Chen, C.-H. Wu, K.-H. Chang, F.-Y. Meng, M.-C. Chen, J.-A. Lin, C.-Y. Huang, J. Su et al., Chem. Eur. J. 2019; b) Y. Tu, J. Liu, H. Zhang, Q. Peng, J. W. Y. Lam, B. Z. Tang, Angew. Chem. Int. Ed. 2019, 131, 15053.

[159] J. Tang, M. A. Robichaux, K.-L. Wu, J. Pei, N. T. Nguyen, Y. Zhou, T. G. Wensel, H. Xiao, J. Am. Chem. Soc. 2019, 141, 14699.

[160] T. Sanji, A. Fuchigami, M. Tanaka, Organometallics 2018, 37, 350.

[161] G. Schwab, D. Stern, D. Leusser, D. Stalke, Z. Naturforsch. B 2007, 62, 711.

[162] a) J. Shi, C. W. Tang, Appl. Phys. Lett. 2002, 80, 3201; b) M. Chen, L. Yan, Y. Zhao, I. Murtaza, H. Meng, W. Huang, J. Mater. Chem. C 2018, 13, 945.

[163] O. L. Wright, L. E. Mura, J. Chem. Educ. 1966, 43, 150.

[164] a) P. B. D. de La Mare, Electrophilic additions to unsaturated systems, Elsevier Scientific Pub. Co, Amsterdam, New York, 1982; b) J. Kong, B. Galabov, G. Koleva, J.-J. Zou, H. F. Schaefer, P. v. R.

Schleyer, Angew. Chem. Int. Ed. 2011, 123, 6941.

[165] P. B. D. De La Mare, J. H. Ridd, Aromatic Substitution: Nitration and Halogenation, Butterworths Scientific Publications, London, 1959.

[166] K. S. Jang, H. Y. Shin, D. Y. Chi, Tetrahedron 2008, 64, 5666.

[167] R. H. Mitchell, Y. Chen, J. Zhang, Organic Preparations and Procedures International 1997, 29, 715.

[168] H. O. House, N. I. Ghali, J. L. Haack, D. VanDerveer, J. Org. Chem. 1980, 45, 1807.

[169] R. Ghorbani-Vaghei, H. Jalili, Synthesis 2005, 1099.

[170] a) C. Li, T. Chen, B. Li, G. Xiao, W. Tang, Angew. Chem. Int. Ed. 2015, 54, 3792; b) Siegel, Tobe, Shinkai (Eds.) Category 6, Compounds with All-Carbon Functions, Georg Thieme Verlag, Stuttgart, 2009.

[171] M. Hesse, H. Meier, B. Zeeh, Spektroskopische Methoden in der organischen Chemie, Thieme, Stuttgart, New York, 2005.

[172] L. D. Quin, G. S. Quin, A guide to organophosphorus chemistry, Wiley, New York, 2000.

[173] S. Sasaki, K. Hattori, K. Igawa, G. Konishi, J. Phys. Chem. A 2015, 119, 4898.

[174] K. Akasaka, T. Suzuki, H. Ohrui, H. Meguro, Anal. Lett. 1987, 20, 731.

[175] M. Onoda, S. Uchiyama, A. Endo, H. Tokuyama, T. Santa, K. Imai, Org. Lett. 2003, 5, 1459.

[176] B. Valeur, Coord. Chem. Rev. 2000, 205, 3.

[177] A. P. de Silva, H. Q. N. Gunaratne, T. Gunnlaugsson, A. J. M. Huxley, C. P. McCoy, J. T. Rademacher, T. E. Rice, Chem. Rev. 1997, 97, 1515.

[178] a) R. Lin, J. H. K. Yip, K. Zhang, L. L. Koh, K.-Y. Wong, K. P. Ho, J. Am. Chem. Soc. 2004, 126, 15852; b) F. Yang, P. E. Fanwick, C. P. Kubiak, Inorg. Chem. 2002, 41, 4805; c) R. Lin, J. H. K. Yip, Inorg. Chem. 2006, 45, 4423; d) J. Hu, R. Lin, J. H. K. Yip, K.-Y. Wong, D.-L. Ma, J. J. Vittal, Organometallics 2007, $26,6533$.

[179] Z. Jiang, M. Xu, F. Li, Y. Yu, J. Am. Chem. Soc. 2013, 135, 16446.

[180] R. Tao, J. Zhao, F. Zhong, C. Zhang, W. Yang, K. Xu, Chem. Commun. 2015, 51, 12403.

[181] T. A. Albright, W. J. Freeman, E. E. Schweizer, J. Org. Chem. 1975, 40, 3437. 
[182] R. A. Dhokale, S. B. Mhaske, Org. Lett. 2013, 15, 2218.

[183] a) G. Baccolini, C. Boga, M. Mazzacurati, J. Org. Chem. 2005, 70, 4774; b) M. Hayashi, T. Matsuura, I. Tanaka, H. Ohta, Y. Watanabe, Org. Lett. 2013, 15, 628.

[184] A. T. Breshears, A. C. Behrle, C. L. Barnes, C. H. Laber, G. A. Baker, J. R. Walensky, Polyhedron 2015, 100, 333.

[185] J. F. Blount, P. Finocchiaro, D. Gust, K. Mislow, J. Am. Chem. Soc. 1973, 95, 7019.

[186] C. Förster, W. Seichter, A. Schwarzer, E. Weber, Supramol. Chem. 2010, 22, 571.

[187] T. Nishiuchi, R. Ito, A. Takada, Y. Yasuda, T. Nagata, E. Stratmann, T. Kubo, Chemistry - An Asian Journal 2019, 14, 1830.

[188] K. Katagiri, Y. Yamamoto, Y. Takahata, R. Kishibe, N. Fujimoto, Tetrahedron Lett 2019, 60, 2026.

[189] T. Schillmöller, P. N. Ruth, R. Herbst-Irmer, Dietmar Stalke, Chem. Eur. J. 2020, 26, 17390.

[190] a) J. M. Rowe, J. M. Hay, W. A. Maza, R. C. Chapleski, E. Soderstrom, D. Troya, A. J. Morris, Journal of Photochemistry and Photobiology A: Chemistry 2017, 337, 207; b) S. Abou-Hatab, V. A. Spata, S. Matsika, J. Phys. Chem. A 2017, 121, 1213; c) Y. Dawei, Z. Xiaojuan, W. Zhiming, Y. Bing, M. Yuguang, P. Yuyu, RSC Adv. 2018, 8, 27979.

[191] R. Teerasarunyanon, L. C. Wilkins, G. Park, F. P. Gabbaï, Dalton Trans. 2019, 48, 14777.

[192] A. M. Brouwer, Pure Appl. Chem. 2011, 83.

[193] A. Ito, K. Kawanishi, E. Sakuda, N. Kitamura, Chem. Eur. J. 2014, 20, 3940.

[194] a) B. W. Rawe, C. P. Chun, D. P. Gates, Chem. Sci. 2014, 5, 4928; b) B. W. Rawe, M. R. Scott, C. M. Brown, H. K. MacKenzie, D. P. Gates, Macromolecules 2017, 50, 8916.

[195] A. P. de Silva, J. Phys. Chem. Lett. 2011, 2, 2865.

[196] a) J. M. Goldberg, S. Batjargal, B. S. Chen, E. J. Petersson, J. Am. Chem. Soc. 2013, 135, 18651; b)

J. M. Goldberg, L. C. Speight, M. W. Fegley, E. J. Petersson, J. Am. Chem. Soc. 2012, 134, 6088; c) J.

M. Goldberg, S. Batjargal, E. J. Petersson, J. Am. Chem. Soc. 2010, 132, 14718.

[197] S. Kyushin, M. Ikarugi, M. Goto, H. Hiratsuka, H. Matsumoto, Organometallics 1996, 15, 1067.

[198] T. Karatsu, R. Hazuku, M. Asuke, A. Nishigaki, S. Yagai, Y. Suzuri, H. Kita, A. Kitamura, Org.

Electron. 2007, 8, 357.

[199] R. Haruki, Y. Sasaki, K. Masutani, N. Yanai, N. Kimizuka, Chem. Commun. 2020.

[200] M. Bukala, personal communication.

[201] T. Noda, H. Ogawa, Y. Shirota, Adv. Mater. 1999, 11, 283.

[202] a) T. Noda, Y. Shirota, J. Am. Chem. Soc. 1998, 120, 9714; b) G. Turkoglu, M. E. Cinar, T. Ozturk, Molecules 2017, 22.

[203] a) Y. Shirota, M. Kinoshita, T. Noda, K. Okumoto, T. Ohara, J. Am. Chem. Soc. 2000, 122, 11021; b) Z. M. Hudson, S. Wang, Acc. Chem. Res. 2009, 42, 1584.

[204] a) T.-T. Bui, F. Goubard, M. Ibrahim-Ouali, D. Gigmes, F. Dumur, Beilstein J. Org. Chem. 2018, 14, 282; b) T.-T. Bui, F. Goubard, M. Ibrahim-Ouali, D. Gigmes, F. Dumur, Appl. Sci. 2018, 8, 494.

[205] Y. S. Han, S. Jeong, S. C. Ryu, E. J. Park, E. J. Lyu, G. Kwak, L. S. Park, Mol. Cryst. Liq. Cryst. 2009, $513,163$. 
[206] M. Munakata, L. P. Wu, T. Kuroda-Sowa, M. Maekawa, Y. Suenaga, T. Ohta, H. Konaka, Inorg. Chem. 2003, 42, 2553.

[207] a) A. K. Narsaria, J. Poater, C. Fonseca Guerra, A. W. Ehlers, T. A. Hamlin, K. Lammertsma, F. M. Bickelhaupt, Chem. Eur. J. 2019, 26, 2080; b) R. Kimura, H. Kuramochi, P. Liu, T. Yamakado, A. Osuka, T. Tahara, S. Saito, Angew. Chem. Int. Ed. 2020, 59; c) A. Srivastava, R. Kumar, H. Aggarwal, Chem. Eur. J. 2020, 21, 10653.

[208] M. N. Akhtar, J. G. Hamilton, D. R. Boyd, A. Braunstein, H. E. Seifried, D. M. Jerina, J. Chem. Soc., Perkin Trans. 1 1979, 1442.

[209] J. Netka, S. L. Crump, B. Rickborn, J. Org. Chem. 1986, 51, 1189.

[210] N. P. Tsvetkov, E. Gonzalez-Rodriguez, A. Hughes, G. Dos Passos Gomes, F. D. White, F. Kuriakose, I. V. Alabugin, Angew. Chem. Int. Ed. 2018, 57, 3651.

[211] S. Bringmann, S. Ahmed, R. Hartmann, J. Mattay, Synthesis 2011, 2011, 2291.

[212] a) D. Heijnen, J.-B. Gualtierotti, V. Hornillos, B. L. Feringa, Chem. Eur. J. 2016, 22, 3991; b) P. Y. Yeung, C. M. So, C. P. Lau, F. Y. Kwong, Org. Lett. 2011, 13, 648.

[213] H. Zorn, H. Schindlbauer, H. Hagen, Monatsh. Chem. 1964, 95, 422.

[214] M. W. Haenel, S. Oevers, J. Bruckmann, J. Kuhnigk, C. Krüger, Synlett 1998, 1998, 301.

[215] M. W. Haenel, D. Jakubik, C. Krüger, P. Betz, Chem. Ber. 1991, 124, 333.

[216] K. Ito, T. Suzuki, Y. Sakamoto, D. Kubota, Y. Inoue, F. Sato, S. Tokito, Angew. Chem. Int. Ed. 2003, 42, 1159.

[217] A. J. Cruz-Cabeza, S. M. Reutzel-Edens, J. Bernstein, Chem. Soc. Rev. 2015, 44, 8619.

[218] S. P. Thomas, P. R. Spackman, D. Jayatilaka, M. A. Spackman, J. Chem. Theory Comput. 2018, 14, 1614.

[219] S. P. Thomas, M. A. Spackman, Aust. J. Chem. 2018, 71, 279.

[220] a) W. Ostwald, Z. Phys. Chem. 1897, 22U; b) R. A. van Santen, J. Phys. Chem. 1984, 88, 5768.

[221] Y. Gao, H. Liu, S. Zhang, Q. Gu, Y. Shen, Y. Ge, B. Yang, Phys. Chem. Chem. Phys. 2018, 20, 12129.

[222] a) E. Li, K. Jie, M. Liu, X. Sheng, W. Zhu, F. Huang, Chem. Soc. Rev. 2020, 49, 1517; b) H. Liu, Y.

Shen, Y. Yan, C. Zhou, S. Zhang, B. Li, L. Ye, B. Yang, Adv. Funct. Mater. 2019, 29, 1901895.

[223] A. M. Christianson, F. P. Gabbaï, Inorg. Chem. 2016, 55, 5828.

[224] N. Agarwal, M. Patil, M. Patil, RSC Adv. 2015, 5, 98447.

[225] N. Soh, O. Sakawaki, K. Makihara, Y. Odo, T. Fukaminato, T. Kawai, M. Irie, T. Imato, Bioorg. Med. Chem. 2005, 13, 1131.

[226] a) T. Hofbeck, U. Monkowius, H. Yersin, J. Am. Chem. Soc. 2015, 137, 399; b) C. L. Linfoot, M. J. Leitl, P. Richardson, A. F. Rausch, O. Chepelin, F. J. White, H. Yersin, N. Robertson, Inorg. Chem. 2014, 53, 10854.

[227] N. I. Georgiev, V. V. Bakov, V. B. Bojinov, ChemistrySelect 2019, 4, 4163.

[228] Y. Harabuchi, T. Taketsugu, S. Maeda, Phys Chem Chem Phys 2015, 17, 22561.

[229] G. R. Fulmer, A. J. M. Miller, N. H. Sherden, H. E. Gottlieb, A. Nudelman, B. M. Stoltz, J. E. Bercaw, K. I. Goldberg, Organometallics 2010, 29, 2176. 
[230] a) G. Bodenhausen, D. J. Ruben, Chem. Phys. Lett. 1980, 69, 185; b) A. Bax, M. F. Summers, J. Am. Chem. Soc. 1986, 108, 2093.

[231] T. Kottke, R. J. Lagow, D. Stalke, J. Appl. Cryst. 1996, 29, 465.

[232] T. Schulz, K. Meindl, D. Leusser, D. Stern, J. Graf, C. Michaelsen, M. Ruf, G. M. Sheldrick, D.

Stalke, J. Appl. Cryst. 2009, 42, 885.

[233] APEX2 v2.2012.2-0. APEX2, WI, USA, Madison, 2012.

[234] Bruker AXS Inc., SAINT V8.30C, Madison, USA, 2013.

[235] L. Krause, R. Herbst-Irmer, G. M. Sheldrick, D. Stalke, J. Appl. Cryst. 2015, 48, 3.

[236] L. Krause, R. Herbst-Irmer, D. Stalke, J. Appl. Cryst. 2015, 48, 1907.

[237] G. M. Sheldrick, XPREP in SHELXTL 2014/2, Göttingen, 2014.

[238] G. M. Sheldrick, Acta Crystallogr. 2015, A71, 3.

[239] a) D. Kratzert, I. Krossing, J. Appl. Cryst. 2018, 51, 928; b) D. Kratzert, J. J. Holstein, I. Krossing, J. Appl. Cryst. 2015, 48, 933.

[240] CCDC, Mercury CSD, Cambridge (UK), 2016. 


\section{Acknowledgements}

Zuerst möchte ich mich bei Prof. Dr. Dietmar Stalke für die interessante und herausfordernde Themenstellung, für die gewährten Freiheiten beim Anfertigen dieser Arbeit und für die Unterstützung jeglicher Art während der gesamten Zeit in der Arbeitsgruppe bedanken.

Weiterhin bedanke ich mich bei Prof. Dr. Sven Schneider für die Übernahme des Korreferats und den wissenschaftlichen Austausch im Rahmen der Fortschrittsberichte.

Bei den weiteren Mitgliedern der Prüfungskommission, Prof. Dr. Manuel Alcarazo, Prof. Dr. Konrad Koszinowski, Prof. Dr. Ricardo Mata und Jun-Prof. Dr. Anna Krawczuk möchte ich mich ebenfalls bedanken.

Zudem möchte ich mich bei allen Mitarbeitern in den Werkstätten sowie den Hausmeistern für die Unterstützung bei verschiedenen technischen Problemen und Wünschen und die schnelle und unkomplizierte Umsetzung bedanken.

Heike danke ich für die Zusammenarbeit bei organisatorischen Dingen jeglicher Art und deine Hilfe bei unterschiedlichsten Fragen und Problemen. Bei Martin, Tobias und Laura bedanke ich mich ebenfalls für die Hilfe bei der schnellen Beschaffung von Chemikalien und Gerätschaften und sonstigen Fragestellungen.

Außerdem danke ich Regine für die Unterstützung beim Lösen und Verfeinern von Kristallstrukturen sowie den vielfältigen Tipps und Ratschlägen.

Allen aktuellen und ehemaligen Mitgliedern des Arbeitskreises danke ich für die Hilfe und Diskussionen bei Problemen jeglicher Art und die gemeinsamen Aktivitäten außerhalb des Forschungsalltags (Dartung!). Besonders bedanken möchte ich mich bei Johannes, Alex, Anne, Helena, Nico, Christina, Daniel, Peter, Niklas, Xiaobai, Annika, Tobias und den Christians.

Meinen Freunden in Göttingen danke ich für die schöne, gemeinsame Zeit hier. Besonders der ein oder andere Abend beim Ernest, das jährliche Fußballturnier (Fehling 1!), die Dartungen, der Besuch von einigen Wohnheimsfeiern und alle anderen außeruniversitären Aktivitäten werden immer in guter Erinnerung bleiben.

Natürlich danke ich auch meinen Freunden in Lohne. Auch wenn die Treffen nicht mehr so regelmäßig sind, freue ich mich auf jeden Besuch in der Heimat!

Und natürlich möchte ich mich auch bei Dir, Hanna, von ganzem Herzen bedanken. Danke, dass du mich immer unterstützt und immer für mich da bist!

Bei meiner Familie bedanke ich mich für die großartige Unterstützung in jeglicher Hinsicht von Anfang bis Ende. Danke, dass ihr immer für mich da seid. 
